This item was submitted to Loughborough's Research Repository by the author.

Items in Figshare are protected by copyright, with all rights reserved, unless otherwise indicated.

\title{
An alternative approach to aeroservoelastic design and clearance
}

PLEASE CITE THE PUBLISHED VERSION

PUBLISHER

(C) R. Taylor 1995

LICENCE

CC BY-NC-ND 4.0

REPOSITORY RECORD

Taylor, Richard. 2013. "An Alternative Approach to Aeroservoelastic Design and Clearance". figshare. https://hdl.handle.net/2134/13600. 


\section{Loughborough University}

This item was submitted to Loughborough University as a PhD thesis by the author and is made available in the Institutional Repository

(https://dspace.lboro.ac.uk/) under the following Creative Commons Licence conditions.

\section{cc) creative}

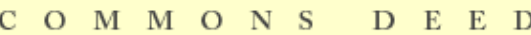

Attribution-NonCommercial-NoDerivs 2.5

You are free:

- to copy, distribute, display, and perform the work

Under the following conditions:

Attribution. You must attribute the work in the manner specified by the author or licensor.

Noncommercial. You may not use this work for commercial purposes.

No Derivative Works. You may not alter, transform, or build upon this work.

- For any reuse or distribution, you must make clear to others the license terms of this work.

- Any of these conditions can be waived if you get permission from the copyright holder.

Your fair use and other rights are in no way affected by the above.

This is a human-readable summary of the Leqal Code (the full license).

Disclaimer 민

For the full text of this licence, please go to: http://creativecommons.org/licenses/by-nc-nd/2.5/ 


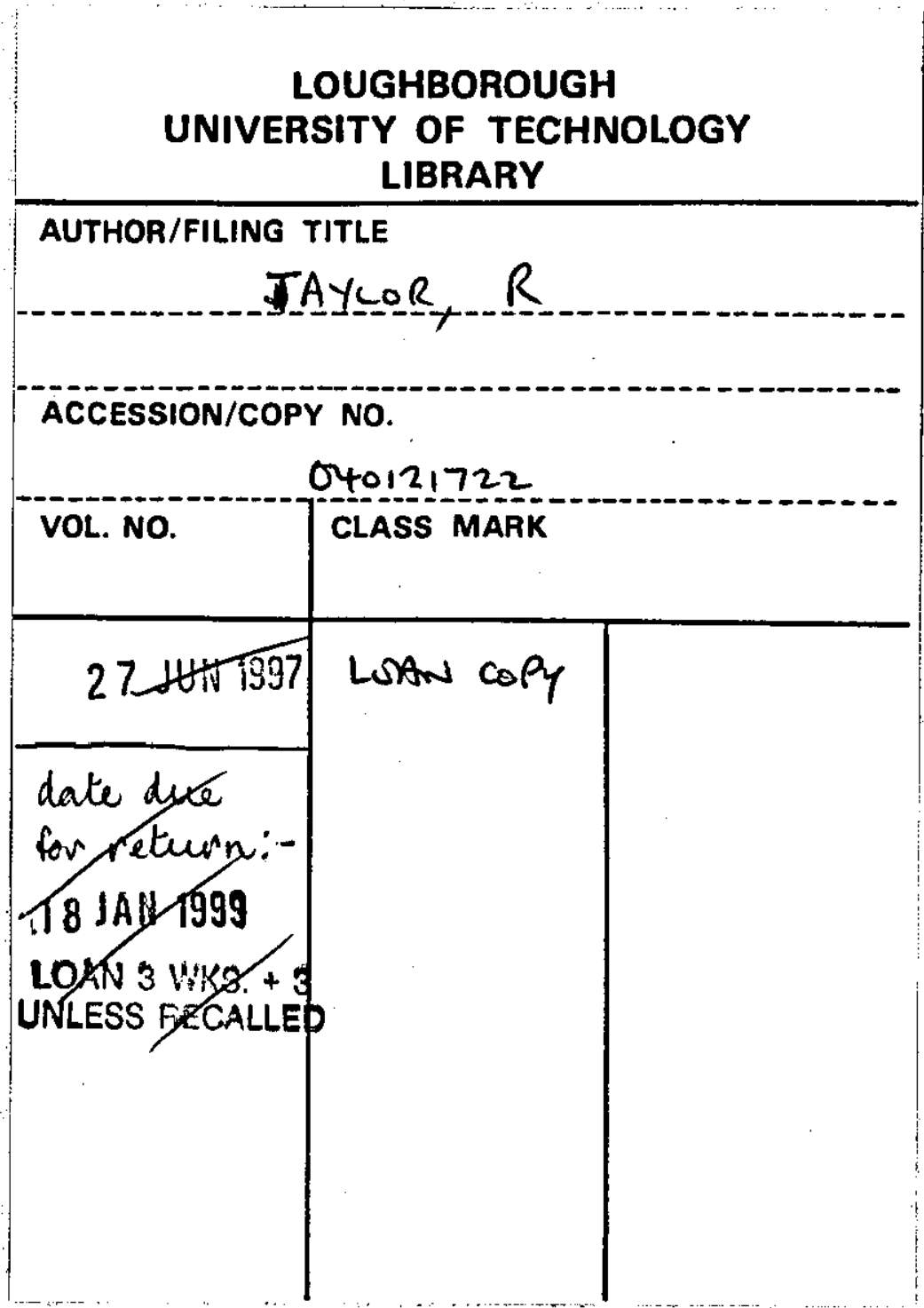




\title{
AN ALTERNATIVE APPROACH TO AEROSERVOELASTIC DESIGN AND CLEARANCE
}

\author{
by \\ Richard Taylor
}

A Doctoral Thesis

Submitted in partial fulfilment of the requirements for the award of Doctor of Philosophy of the Loughborough Univertsity of Technology

September 1995

(c) by R. Taylor 1995 


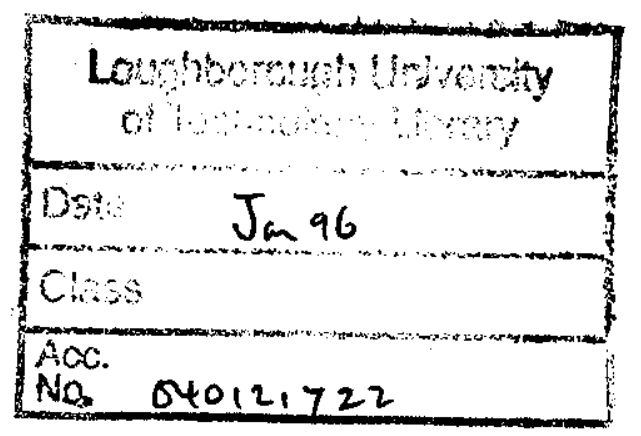

$q 3086232$ 


\section{Abstract}

The interaction between an aircraft's structural dynamics, unsteady aerodynamics and flight control system is known as aeroservoelasticity. The problem can occur because the control system sensors are of sufficient bandwidth to sense the structural vibrations as well as the rigid-body motion of the aircraft. This sensed structural vibration can result in further excitation of the structure through both aerodynamic and inertial excitation, leading to a potential closed-loop instability. At present, such an unstable interaction is prevented by the inclusion of notch filters within the feedback path which have a detrimental effect on the aircraft's rigid-body performance.

The current clearance procedure is restricted by a poor understanding of the array of complex issues involved. The aim of the project was to develop a clearer understanding of the interactions between system components leading to a reduction in the clearance requirements.

Work has concentrated on the effects of system nonlinearities and on the digital nature of modern control systems. A major source of nonlinearities within the control system are the servo-hydraulic actuators. Through detailed actuator modelling confirmed by rig testing of actual hardware, these nonlinearities are analysed and a method for predicting the response of the actuators in the presence of two input signals proposed. As a result, it is demonstrated that an unstable structural oscillation would cause a limit-cycle oscillation as opposed to an unbounded response. Through nonlinear system theory the criteria for the existence of such limit-cycles are obtained, enabling them to be predicted and therefore prevented.

Consideration of the true nonlinear nature of the aeroservoelastic system has enabled an alternative design and clearance procedure to be proposed which reduces the attenuation requirements of the structural-mode filters whilst ensuring satisfactory aircraft performance even in the presence of modelling errors. This design procedure is demonstrated on both a model of the aircraft system and a simple test system enabling verification of the nonlinear analysis and comparison between the current and proposed alternative procedures. As a result, it is demonstrated that consideration of the true nonlinear nature of the aeroservoelastic interaction has the potential for allowing a significant reduction in structural filter attenuation requirements. Consequently, a reduction in the phase lag due to the filters is possible resulting in an improvement in closed-loop system performance whilst ensuring the safe operation of the aircraft. 


\section{Acknowledgements}

This research was completed in the Department of Aeronautical and Automotive Engineering and Transport Studies, Loughborough University of Technology and was funded by British Aerospace Defence Ltd. (Military Aircraft) as part of its Centres of Excellence programme.

The author would like to thank his supervisor for the project, Mr. R. W. Pratt, with whom it has been a pleasure to work. His advice, encouragement and enthusiasm in all aspects of the project has been beyond value. In addition, the author would also like to thank his industrial supervisor, Mr. B. D. Caldwell, for his tireless efforts at British Aerospace on behalf of this work and for his advice throughout the project.

Within the Department of Aeronautical and Automotive Engineering itself, the author would like to thank all of the academic, technical, secretarial and research staff and students for their help and advice. In particular, Mr. P. Karia, for his help and support during the many hours of experimental testing, and Mr. N. Randall, Mr. K. Coulthard, Mr. T. Newbold and Mr. A. Morby for their skill and experience in the development of the test rig.

As well as all the members of the Flight Control System Design Group at British Aerospace, the author would also like to thank Mr. J. Steed of Dowty Boulton Paul Ltd. for his help and advice in the design and commissioning of the test rig.

Finally, the author would like to thank his family and friends for their support and encouragement, in particular Mum, Dad and Samantha for their confidence, love and understanding. 


\section{Contents}

\section{Chapter 1}

\section{Chapter 2}

Chapter 3

\section{Introduction 1}

Aeroelasticity 2

Aeroservoelasticity 3

Effect of actuator dynamics 5

Digital effects 6

Project aims 6

\section{Current Design Assumptions 9}

Introduction 10

Description of current solution method 10

Current design assumptions 12

Introduction 12

Flight control system 12

Sensors 12

Actuation 13

Structural modelling 13

Unsteady Aerodynamic modelling 14

Rigid-body dynamics 15

Summary of design assumptions 15

Conclusions 15

\section{Modelling of the Flexible Aircraft 17}

Introduction 18

Structural dynamics 18

Introduction 18

Derivation of equations of motion for a simple undamped system 19

Derivation of equations of motion for idealised aircraft structure 23

Selection of a suitable generalised coordinate system 27

Inclusion of structural damping into the equations of motion 30

Aerodynamic effects 31

Introduction 31

Incorporation of aerodynamic effects into the equations of motion 32

Incremental aerodynamic forces on a vibrating structure 34

Rigid-body dynamics 37

Introduction 37

Derivation of rigid-body equations of motion 38

Control surface dynamics 39

Introduction. 39

Derivation of equations of motion for control surfaces 40

Assembling the flexible aircraft model 42

Introduction 42

Assembly of the full inertia matrix 43

Assembly of the full structural stiffness matrix 44

Conversion to a generalised coordinate system 45

Assembly of the Aerodynamic stiffness matrix 47

Assembly of the Aerodynamic damping matrix 48

Full flexible aircraft model 49 
Reduction of the flexible aircraft model 50 Introduction 50 Selection of suitable normal modes 50

Conversion of the flexible aircraft model into state-space form 51 Introduction 51

Conversion of the flexible aircraft equations into state-space form 51

Verification of the flexible aircraft model 56

Conclusions 56

\section{Chapter 4}

\section{Chapter 5}

\section{Chapter 6}

\section{Investigation of Current Design Method 57}

Introduction 58

Creation of a full system model 58

Introduction 58

Creation of the system model 58

Demonstration of aeroservoelastic effects 63

Prevention of the aeroservoelastic interaction using currently applied methods and assumptions 64

Introduction 64

Description of the current solution procedure 65

Production of the structural filter attenuation requirements 65.

Design of the structural-mode filters 70

Conclusions 77

\section{Digital Sampling Effects and Sensor Modelling 78}

Introduction 79

Analysis of the sampling process 79

Introduction 79

Mathematical analysis of the sampling process 80

Sampling of a sinusoidal input signal 83

Aliasing 87

Effect of the zero-order-hold 87

Introduction 87

Transfer function of the zero-order-hold 88

Sensor modelling 92

Introduction 92

Modelling of the digital aircraft motion sensor unit 92

Application of digital effects to a typical system 95

Introduction 95

Application of digital effects to an aeroservoelastic system 96

Conclusions 100

Inclusion of Digital Effects and Sensor Dynamics $\underline{101}$

Introduction 102

Application of digital effects to an aeroservoelastic system 102

Introduction 102

Design of the digital fight control system 103

Inclusion of zero-order-hold attenuation 104

Effect of aliasing 106

Production of structural-mode filter attenuation requirements 108

Inclusion of sensor dynamics 111 
Application of structural-mode filters 112

Effect of zero-order-hold and sensor phase lag 113

Conclusions 114

Evaluation of alternative design assumptions 114

Introduction 114

Aliasing effects on structural-mode attenuation requirements 114

Consideration of Phase effects 118

Effect of consideration of signal path phase on structural-mode attenuation requirements 120

Conclusions 123

\section{Chapter 7}

\section{Effect of Structural Feedback Signals on Actuator Performance 125}

Introduction 126

Actuation system modelling 126

Introduction 126

Basic actuation system components 127

Main-valve actuation 128

Main-valve dynamics 129

Main-Ram Dynamics 131

Actuator Control System 131

Model Simplification 131

Comparison of actuation system model with experimental results 135

Introduction 135

Description of experimental rig 135

Comparison of test results with predicted frequency response 136

Effect of structural-mode feedback signals on actuator performance 138

Introduction 138

Linearity boundary for non-linear actuation system model 138

Effect of structural-mode signals on low-frequency actuator performance 141

Effect of structural feedback signals on software rate limiting 145

Dual input response of software rate limiter in isolation 148

Effect of main-valve port profile on actuator performance in the presence of structural feedback signals 149

Conclusion 153

Prediction of actuator performance changes in the presence of structural feedback signals 153

Introduction 153

Prediction of actuator performance changes in the presence of structural feedback signals 154

Conclusion 158

Comparison of actuator model with experimental results in the presence of structural feedback signals 159

Introduction 159

Actuator performance changes in the presence of a $50 \mathrm{~Hz}$ structural feedback signal 159

Actuator performance changes in the presence of a $7 \mathrm{~Hz}$ structural feedback signal 163

Conclusions 164

Effect Of subharmonic generation on aircraft response 164

Introduction 164

Use of the dual input describing function to predict subharmonic generation 164

Effect of subharmonic components on aircraft response 167 
Effect of higher frequency structural-modes on subharmonic generation 168

Subharmonic generation in a digital system 169

Conclusion 169

Performance boundary for nonlinear actuation system 170

Introduction 170

Actuator performance boundary for analogue system 170

Actuator performance boundary for a digital system 172

Conclusion 175

Conclusions 175

\section{Chapter 8}

\section{Limit-cycle Prediction and Specification of} Alternative Clearance Requirements 177

Introduction 178

Description of limit-cycle prediction technique 178

Introduction 178

Limit-cycle criteria and prediction 179

Derivation of describing function for rate limit function 179

Prediction of limit-cycles in an example system 183

Limit-cycle prediction in an aircraft system 184

Conclusions 187

Prediction of limit-cycles in the presence of phase uncertainty 187

Introduction 187

Limit-cycle prediction in the presence of phase uncertainty 188

Application of the prediction of limit-cycle amplitude to an aircraft

system 189

Conclusions 191

Prevention of limit-cycles 191

Introduction 191

Criteria for the prevention of limit-cycles 192

Specification of an alternative clearance procedure 194

Introduction 194

Specification of an alternative clearance procedure 194

Demonstration of alternative clearance procedure on analogue aircraft system 197

Introduction 197

Design of structural-mode filters 199

Limit-cycle prediction in the presence of system modelling errors 202

Estimation of the effect of a limit cycling condition on rigid aircraft stability 205

Use of time domain system simulations in the assessment of the effect of limit cycling oscillations 208

Experimental verification of the effects of the limit-cycles on actuator performance 216

Conclusions 221

Effect of digital representation 222

Introduction 222

Limit-cycle prediction in digital system 222

Demonstration of alternative clearance procedure on digital aircraft system 223

Introduction 223

Design of structural-mode filters 223

Limit-cycle prediction for digital system in the presence of modelling errors 225

Assessment of the effect of limit-cycle oscillations within the digital system 227 


\section{Chapter 9}

\section{Experimental Evaluation of Alternative Clearance}

\section{Requirements 237}

Introduction 238

Description of the test rig 238

Derivation of a system model 239

Introduction 239

Analytical derivation of a system model 240

Comparison of simulated open-loop frequency response with test results 241

Limit-cycle prediction for the test system 242

Introduction 242

Limit-cycle prediction for test system 243

Design of structural-mode filters 247

Introduction 247

Design of structural-mode filters 247

Implementation of structural-mode filtering 248

Effect of structural-mode filters and limit-cycles on rigid-body performance 250

Conclusions 252

Prediction of limit-cycles in the presence of modelling error 253 Introduction 253

Prediction of limit-cycles in the presence of modelling error 253

Effect of limit-cycle on system performance in the presence of modelling errors 255

Conclusions 257

Inclusion of digital effects 258

Conclusions 258

\section{Chapter 10}

Appendix A

\section{Conclusions 260}

Aeroservoelasticity 261

Discussion of the current design process 261

Effect of digital nature of control system on the current design procedure 263

Actuator nonlinearities 264

Discussion of the alternative design procedure 266

Recommendations 268

Future Work 269

\section{References 272}

\section{Structure of the Flexible Aircraft model 278}

Introduction 279

Structure of the Flexible Aircraft Model 279

Structure of the actuation system model 281 
Chapter 1

\section{Introduction}




\subsection{Aeroelasticity}

For many years aeroelasticity has been a vital part of the aircraft design procedure. The interaction between the structural dynamics and aerodynamics of an aircraft in flight has been the subject of a great deal of research. As the drive to produce lighter and stronger aircraft structures results in a more flexible structure, the problems of aeroelasticity become more apparent. In addition, the expansion of the flight envelope into the hypersonic speed ranges increases the likelihood of such interactions taking place. In order to face such challenges the aerospace community has developed many complex analytic tools for the prediction of the aeroelastic phenomena, and the prevention of its unwanted effects.

Aeroelasticity manifests itself in various forms, such as static divergence and more commonly flutter. Static divergence of an aircraft lifting or control surface is rarely encountered, but has been known to occur ${ }^{1}$. It is only recently that advances in materials and structural design has made the use of a forward swept wing, as in the X29 aircraft, a viable proposition. Without these advanced materials and structural design methods, a forward swept wing of conventional construction would be liable to a static aeroelastic divergence ${ }^{1}$.

As the name suggests, flutter is a structural oscillatory response brought about by an interaction between the unsteady aerodynamics of an aircraft, and its structural dynamics. Where flutter occurs, the energy needed to sustain the structural vibration is provided by the unsteady aerodynamic effects. It is the duty of the aerodynamic and structural engineer to ensure that an unstable response is prevented. If an unstable flutter response of the aircraft structure is not predicted and prevented, the resulting oscillation can have dramatic and destructive consequences ${ }^{1}$.

A more localised form of the aeroelastic phenomena exists in the form of panel flutter. This form of flutter is limited to the outer panels of the aircraft structure, and is a phenomena under particular scrutiny at present due to its importance in the field of supersonic and hypersonic vehicles. This is due to the fact that the flutter speed of such panels is beyond the range of normal aircraft operation, but falls within the flight envelope of such supersonic and hypersonic vehicles. Several techniques of avoiding the flutter of panels is being investigated, one of which is the inclusion of piezoelectric actuators within the panel surface resulting in so called smart structures ${ }^{2}$. Such actuators can be combined with a suitable control system in order to prevent the unwanted flutter condition arising within the flight envelope. 


\subsection{Aeroservoelasticity}

With the increasing use of high-authority, high-gain active control systems to augment the stability of the rigid aircraft, interactions between an aircraft's structural dynamics, unsteady aerodynamics and flight control systems have emerged as a further design consideration ${ }^{3}$. Such interactions have become known as aeroservoelastic $^{4-6}$ interactions. As with flutter, the energy required to sustain the resulting structural oscillation comes in part from the unsteady aerodynamic effects. In this case, energy is also provided from the flight control system by means of the control surface actuation systems.

The three elements of the aeroservoelastic problem can be represented diagrammatically by Figure $1.1^{5}$. Here the interactions between the three elements of the problem, namely the structural dynamics, aerodynamics and flight control system dynamics, are represented. The left arm of the diagram represents the interaction between the aerodynamics and structural dynamics of the aircraft, namely aeroelasticity, whereas the right arm represents the interaction between the structural dynamics and flight control system of the aircraft, namely servoelasticity. The lower arm represents the interaction between the aerodynamics and the flight control system of the aircraft, here given the name aeroservodynamics. Finally, the centre of the diagram represents the interaction between all three elements; the aeroservoelastic interaction.

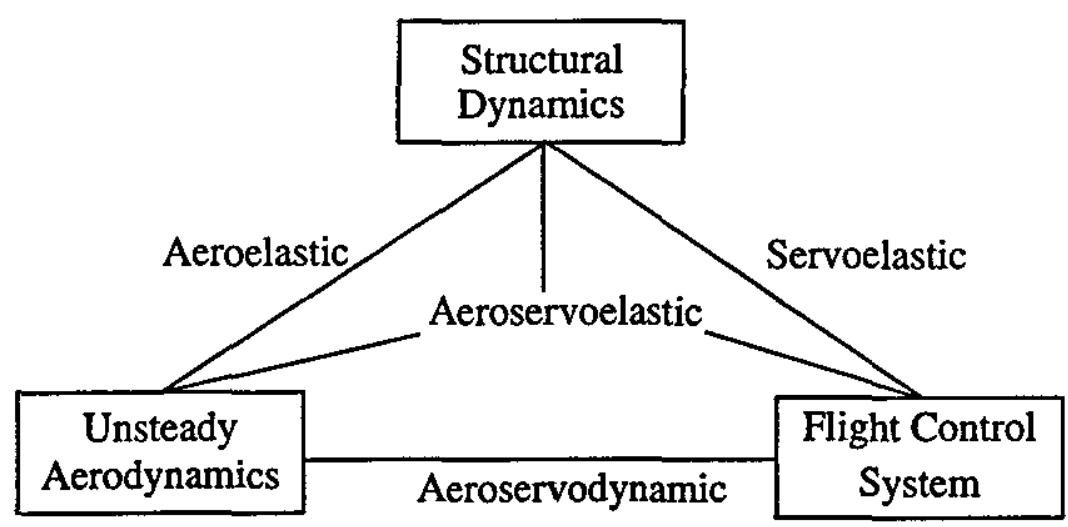

Figure 1.1 - Interaction triangle 5

It is possible to consider the three outer arms of Figure 1.1 as specific cases of the central aeroservoelastic interaction. The servoelastic interaction for example can be considered as the zero speed aeroservoelastic case with the energy required to sustain the oscillation being provided entirely by the flight control system. The aeroservodynamic interaction can be considered as the rigid-body aeroservoelastic interaction, which represents classical, rigid-body flight control design considerations.

Historically, there have been many occurrences of aeroservoelastic interactions. 
Reference 5 describes many such examples, from that involving a B-36 aircraft in 1948 to interactions encountered on the YF-17 aircraft in the early 1970's. The YF-17 for example suffered from both aeroservodynamic and servoelastic interactions as well as an aeroservoelastic interaction ${ }^{7}$. In addition, more recent examples of interactions have also been reported on the YF-16 ${ }^{8}, \mathrm{~B}-2^{9}$ and F-16 aircraft ${ }^{10}$.

The mechanism of an aeroservoelastic interaction can be represented by the block diagram of Figure $1.2^{11-12}$. Here, an initial energy input to the aircraft structural dynamics would result in the vibrational modes of the aircraft structure being excited. Such an initial energy input to the system could result from a sudden gust load changing the aerodynamics of the system, or an inertial load as a result of stores release or control surface motion. Once an oscillatory response of the structure has been initiated, its amplification or attenuation by the aircraft system as a whole depends on the dynamics of the aerodynamics and the control system. Considering the aerodynamics, a vibrational structural motion will set up an unsteady wake resulting in oscillatory aerodynamic loads over the entire aircraft structure. If these unsteady aerodynamic loads result in an amplification of the initial structural response, the classic flutter condition has been achieved. As a result, the structural vibration will grow in amplitude, with the aerodynamic loading effectively resulting in a closed-loop system with the obvious potential for instability.

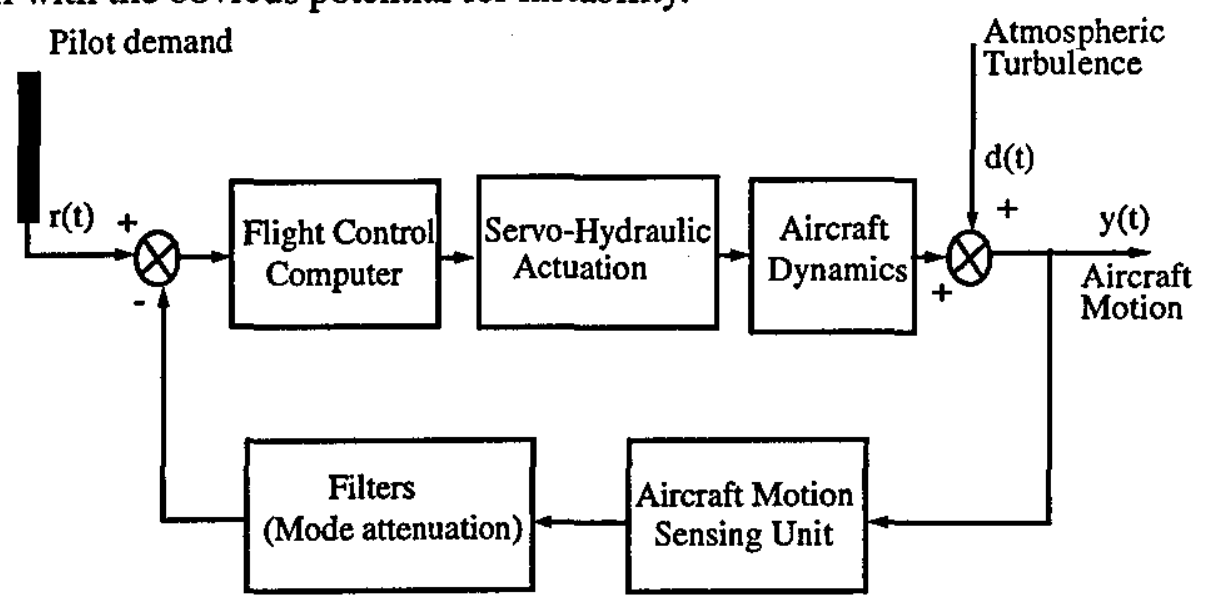

Figure 1.2 - Aircraft system components for aeroservoelastic interactions

Considering the effect of the initial structural response on the flight control system, the structural response will be sensed by the aircraft motion sensor mounted to the aircraft structure itself. The output from such a sensor, which would now contain the vibrational response of the structure as well as the rigid-body motion of the aircraft, is fed to the flight control computers for analysis as in Figure 1.2. Provided that the frequency of the structural vibration is within the bandwidth of the flight control system, the control surfaces will be moved by the actuators in an attempt to control not only the rigid-body motion as demanded by the pilot, but also the sensed structural vibrations. If the motion of the control surfaces, in an attempt to control 
these structural vibrations, results in the vibrations being amplified then an unstable closed loop system has resulted. Such further excitation of the structure due to control surface motion is possible because the centre of gravity of the surface is generally offset from the hinge line. As a result, motion of the control surface by the actuation system results in inertial excitation of the attachment structure. In addition, motion of the control surface will effect the aerodynamic loading of the remainder of the structure, which could in turn lead to further structural excitation.

Although the example described here highlights the negative consequences of interactions between the system elements, in many cases, such interactions have been applied to give beneficial results. One example of this is in the development of gust load alleviation and ride control systems ${ }^{13}$. These systems use a closed loop controller and suitable control surfaces to reduce the loads within the structure by altering the aerodynamic loading of the structure itself. This has the effect of reducing the structural excitation due to aerodynamic loading and/or increasing the structural damping. It is interesting to note that the development of such systems encountered some undesirable coupling of the flight control system, aerodynamics and structural dynamics ${ }^{13}$. Such interactions were prevented by including notch filters in the feedback path between sensors and flight control system.

An additional application where the interaction between the three system components can be used to advantage is in the field of panel flutter as has already been described. In such smart structures, the control system and actuators can be used to push the flutter speed beyond the flight envelope of the vehicle.

\subsection{Effect of actuator dynamics}

The inclusion of the actuators as a separate block within Figure 1.2 is intended to emphasise the importance of this element on the aeroservoelasticity problem. The frequency response of the actuators is obviously of great importance, both for their ability to attenuate the high-frequency structural vibration signals and their role in maintaining satisfactory rigid-body control. It is important to realise however that hydraulic actuators are highly nonlinear systems involving, for example, valve travel limits and nonlinear fluid flow. One result of this is that high-frequency structural feedback signals could lead to valve saturation, seriously diminishing the performance of the actuation system to low-frequency control system demands. This could in turn lead to an inability of the control system to maintain satisfactory control of the rigidbody aircraft. In addition, saturation of valve travel limits can cause a limit cycling condition to arise. An example of such an effect was seen where an Atlas rocket suffered an interaction between its control system and structural modes ${ }^{1}$. This was as a result of the effect of a single structural mode on the control actuator's servo-valve, 
resulting in limit cycling of the servo system at the frequency of the structural mode. As a result, further excitation of the structural vibration occurred leading to catastrophic failure of the vehicle structure.

\subsection{Digital effects}

The increasing use of fully digital flight control systems has introduced a further element to the aeroservoelastic problem. Before the introduction of digital flight control systems, only those structural vibration modes within the bandwidth of the flight control system could result in significant control surface motion leading to further possible structural excitation. The effect of the sampling process within the digital fight control system is to aliase the high-frequency structural modes down to within the bandwidth of the flight control system ${ }^{14}$. Although there will be a certain amount of attenuation of these high-frequency aliases by the sample-and-hold process, such aliasing can cause an aeroservoelastic interaction to take place. Such an interaction occurred on the X-29 aircraft ${ }^{15}$. The need to consider the digital nature of the control system as part of the aeroservoelastic analysis is therefore evident.

\subsection{Project aims}

It is the aim of this research to develop a greater understanding of the component parts of the aeroservoelastic problem introduced here. Once this has been achieved, the way in which these components interact can be analysed. As a result it will be possible to improve the current design methods which reflect the present uncertainties in the interaction process. Such an improvement in design methods or relaxation of the clearance requirements could result in both significant cost savings during the design process and an improvement in aircraft performance.

Ideally, the results of this work should be in a generic form, and not limited in scope to an application to a particular aircraft system. In order to achieve such an understanding of the problem in general terms however, it is essential that a typical system be examined in detail before generalizations can be made. As a result, the following work is based on a generic combat aircraft system. The aircraft itself is typical of a modern combat aircraft, being of canard-delta configuration, and with unstable longitudinal rigid-body dynamics. In order to simplify significantly the analysis, only the longitudinal motion is considered. The study examines the taileron actuation system of the Jaguar Fly-By-Wire (FBW) aircraft. This actuator is representative of current combat aircraft actuation systems, having a high bandwidth, and being digitally controlled. In addition, such an actuator was available for experimental verification of the results. 
The experimental rig used for the verification of the modelling results consists of a Jaguar FBW taileron actuator driving a load made up of a pivoted mass-spring system as shown in Figure 1.3. The LVDT shown in Figure 1.3 was set so as to measure the deflection of the spring. This signal was then combined with the actuator ram deflection within the rig controller before feedback to the actuator input signal. Although the rig itself is simple in comparison with an entire aircraft system, it exhibits many characteristics of the full system. The nonlinear actuator is driving a load which exhibits a structural mode. This structural mode is sensed by the control system and fed back to the actuator input as in the aircraft.

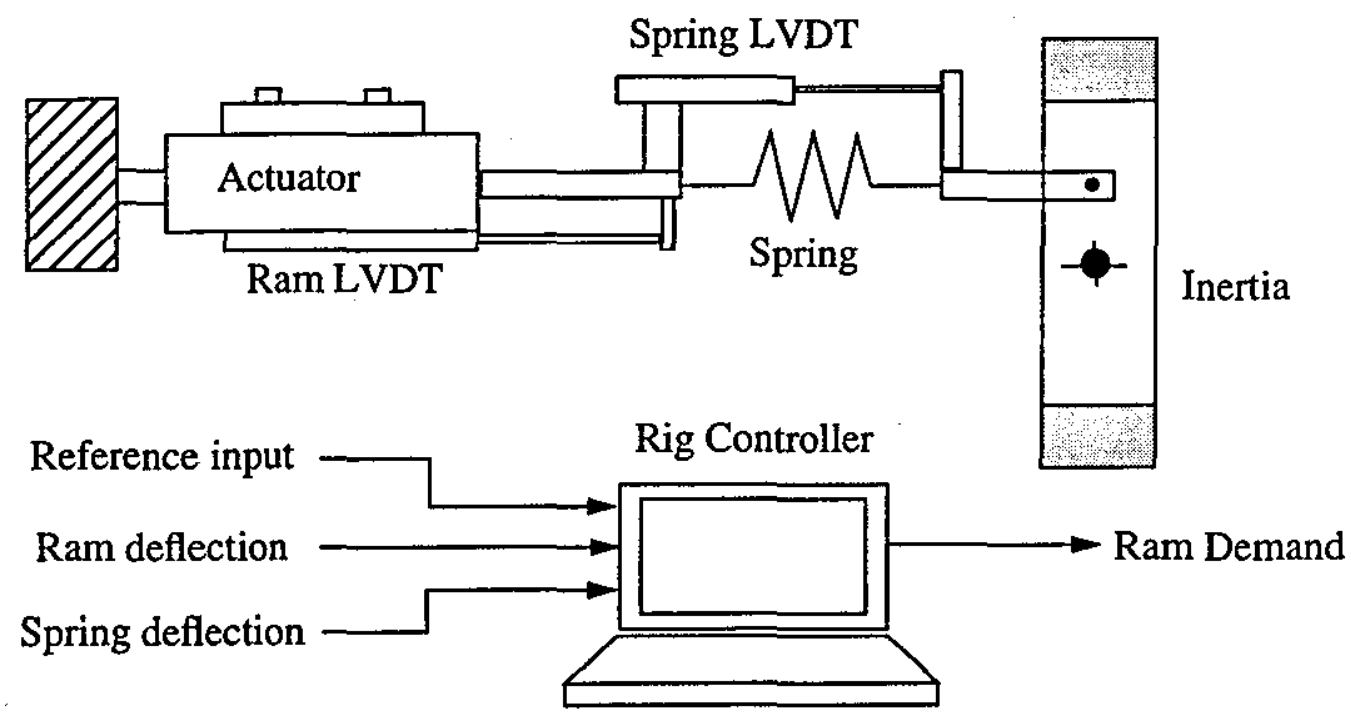

Figure 1.3- Schematic of test rig

In the following chapters, the results of the research are examined. In Chapter 2 , discussion is made of the current method of solution of the aeroservoelasticity problem, with particular emphasis being made on the current design assumptions employed by British Aerospace Defence Ltd. (Military Aircraft).

In Chapter 3, a linear flexible aircraft model is developed which will include the elements of structural dynamics, unsteady aerodynamics, rigid-body motion and control surface dynamics. This model will be in a form suitable for incorporation into the MATLAB/SIMULINK analysis package which will be the main analysis tool used throughout this work.

Chapter 4 contains a description of the current aeroservoelasticity solution techniques using the model developed in the Chapter 3 as an example. A full system model is developed assuming the flight control system to be analogue, and the actuation systems to be adequately represented by low-order linear transfer functions.

In Chapter 5, discussion is made of the digital nature of modern control systems and its effect on the aeroservoelasticity problem in general terms. The effect of sampling high-frequency signals in terms of aliasing and phase changes is investigated 
along with the effect of introducing the modelling of the sensor dynamics to the problem.

In Chapter 6, the results obtained in Chapter 5 are applied to the system model developed in Chapter 3 in order to evaluate the current design assumptions regarding the effect of the digital nature of the flight control system on the aeroservoelasticity problem.

Chapter 7 begins with a discussion of actuator modelling techniques leading to the production of a full nonlinear model of the actuation system. The performance of the actuation system in the presence of high-frequency noise signals is analysed with the results verified from experimental tests on the actuator itself.

In Chapter 8, the affect of the nonlinear nature of the actuation system on the aeroservoelastic problem is discussed, and an alternative method of specifying the clearance requirements proposed. The consequences of adopting such an alternative clearance requirement is demonstrated for the aircraft system model. In addition, the effect of the digital nature of the control system as discussed in Chapter 5 on the alternative clearance procedure is investigated, with the consequences demonstrated on the system model.

In Chapter 9 a simplified single-input, single-output model of a system containing just one structural mode is described. Such a simplified system is representative of the available hardware for experimental verification of the results. Results from the testing of the simplified system are presented which examine the current and proposed clearance requirements for both analogue and digital flight control systems following the discussion of Chapter 8 .

Finally, in Chapter 10 the results presented in the earlier chapters are discussed, with particular emphasis being given to the alternative clearance requirements proposed in Chapter 8. Possible areas of future work are also introduced. 
Chapter 2

\section{Current Design \\ Assumptions}




\subsection{Introduction}

Aeroservoelasticity has been introduced in Chapter 1 , with the mechanism behind the phenomena being discussed in some detail. The importance of the aeroservoelastic interaction on the design of the flight control system for modern aircraft has been highlighted ${ }^{11,12}$, without mention of a method of solution.

It is the aim of this chapter to describe the current method of solution of the aeroservoelasticity problem, paying particular attention to the assumptions that are applied to the design of this solution and the reasoning behind those assumptions. Although the method of solution is similar for many different aircraft programmes $^{7,8,15}$, the discussion made in the following chapter is based on those assumptions made by British Aerospace in its design procedures ${ }^{16,17}$.

A fuller description and example of the current method will be given in Chapter 4 .

\subsection{Description of current solution method}

The mechanism of an aeroservoelastic interaction has been discussed in Chapter 1, from where it can be seen that the problem can be considered as that of the propagation of structural signals around the closed-system loop. One method of solution is to prevent this propagation of the structural signals around the closed-loop by the use of suitable signal filtering, and this is the method used in most design cases. The actual design of these structural filters depends naturally on the system in question, and its susceptibility to aeroservoelastic interactions. This may be due to a particularly flexible structure, high-gain flight control system or more commonly, a combination of the two. The basic components of the aircraft system can be seen from Figure 1.2, with each component of the system having an effect on the aeroservoelastic problem. In addition to the components shown in the figure, as discussed in Chapter 1, the digital nature of modern flight control systems will also have an effect on the problem.

In order to design suitable structural-mode filtering, the designer must first take into account all components of the aircraft system, from where, using the applicable design assumptions and clearance requirements, it is possible to calculate the attenuation requirements of the structural-mode filters. Once the attenuation requirements of the filters have been obtained, it only remains to design the required filters to achieve those attenuation requirements. Unfortunately, there exist certain constraints which makes the design of these filters more than a simple case of introducing a low-pass filter into the feedback path in order to attenuate all of the structural modes. Clearly, the introduction of a low-pass filter would result in 
additional phase lag at the rigid-body frequencies. This additional phase lag could possibly result in the failure of the aircraft system to meet rigid-body stability margin clearance requirements ${ }^{16}$.

As will be shown in Chapter 4, there generally exist low-frequency structural modes (less than $10 \mathrm{~Hz}$ ), such that the use of a low-pass filter to attenuate such modes would have a serious effect on the aircraft's rigid-body response. An additional effect of the introduction of a phase lag into the system can be seen when the lag is considered as a pure time delay, which will have a detrimental effect on the aircraft handling qualities. The design of suitable structural-mode filters is therefore a case of balancing the requirements of both rigid-body and structural-mode stability. In addition, the minimum order of structural-mode filtering should be used in order to simplify their implementation and reduce the computational delay in the case of digitally implemented filters.

A further consideration in the design of the filters is that they must be robust to changes in the configuration of the aircraft. Differing fuel states and stores configurations can have a large effect on the structural modes, and such affects should be taken into consideration. Ideally, one set of structural-mode filters should be designed for all possible aircraft configurations. Switching between different sets of filters can have serious consequences in terms of robustness and implementation ${ }^{18}$.

One possibility in the design of the structural-mode filters is the use of optimization techniques to design the optimum structural-mode filters for a given set of criteria ${ }^{19-21}$, although work in this field is at a relatively early stage.

There exists no viable alternative method of solution at present to the aeroservoelastic problem despite work being carried out into more advanced methods of solution including digital filtering techniques and non-linear attenuators ${ }^{16}$.

Clearly, the level of understanding in the modelling of the aeroservoelastic system and its multivariable nature makes it an ideal candidate for the application of robust control techniques ${ }^{22-25}$. In order for this to be possible however, a greater level of understanding of the aeroservoelastic interaction needs to be obtained, which is one of the aims of this research.

In order to design a suitable set of structural-mode filters it is necessary to have a good understanding of the various elements of the aeroservoelastic problem, and a knowledge on the limits of this understanding. The following section describes the assumptions that must be made when calculating the structural-mode attenuation requirements, and the reasoning behind these assumptions. 


\subsection{Current design assumptions}

\subsubsection{Introduction}

The above section has briefly described the current method of solution of the aeroservoelasticity problem, and the problems that have to be faced when designing suitable structural-mode filters for clearance into flight.

The necessity to meet stringent clearance requirements for both the rigid-body and structural-mode stability, means that it is necessary to make certain assumptions in order to account for the current level of uncertainty in the modelling of the various aspects of the system. These assumptions are described in the following section, giving, where possible, the reasoning behind the assumptions.

From Figure 1.2 the elements of the aeroservoelastic problem can be identified and considered in turn, each element having an effect on the design assumptions that must be made.

\subsubsection{Flight control system}

Compared to the other elements of the aircraft system, the flight control system represents the one in which most confidence can be given. The effect of the flight control system on the propagation of the structural-mode signals is well understood. One problem however is that the nature of the digital flight control system can result in the aliasing of high-frequency signals. This introduces a further element to the aeroservoelastic problem. Theoretically, there exist an infinite number of aliased lobes, which are folded back onto the low-frequency range. As a result, it is necessary to make an assumption regarding the frequency range to be considered. Fortunately, the attenuation of other elements within the system, particularly the attenuation of the actuation system, results in the effect of the very high-frequency lobes as being negligible. The effect of the lower frequency alias lobes requires investigation however, and this will be discussed in Chapter 6 .

An additional problem introduced by the digital nature of the flight control system, is the generation of high-frequency signal components as a result of the sample-andhold process, as will be shown in Chapter 4. These high-frequency signal components introduced by the flight control computer to the actuator demand signal, for example, could have serious effect on the performance of the actuation system ${ }^{26}$. Such affects will be investigated further in Chapter 7 .

\subsubsection{Sensors}

As for the fiight control system, the sensor dynamics can also be modelled with confidence. Compared to the other elements within the system, the dynamics of the 
sensors are relatively simple. Once again however, the sensor systems in use on modern aircraft are usually digital in nature, introducing the problem of aliasing once again. In this case however, the sampling frequency of typical sensors are usually high enough for all aliasing effects to be discounted due to the attenuation of the very highfrequency signals by the actuation system.

\subsubsection{Actuation}

The actuation system represents one of the two elements of Figure 1.2 which introduce most uncertainty into the aeroservoelastic problem. As a highly non-linear system, the behaviour of the typical actuation system to signals consisting of multiple frequency inputs is difficult to predict. In addition, the saturation limits that exist within the actuation system itself can produce additional non-linear effects such as harmonic/subharmonic signal generation and performance limitations ${ }^{27-30}$.

The area of actuation system modelling is a large one in its own right. Existing work provides the use of comprehensive actuation system models for aeroservoelastic analysis ${ }^{31,32}$. Additionally, other work concerning the effect of the actuator modelling on aeroservoelastic analysis at high aircraft incidence has been completed ${ }^{33,34}$. For an aircraft at high incidence, the conventional aerodynamic control surfaces lose their effectiveness, particularly at flight conditions corresponding to low dynamic pressures $^{35}$. This can result in an increase in flight control system gain and in the saturation of the control surfaces with a consequent increase in the coupling between the structural dynamics and the actuation system. As a result, accurate actuation system modelling becomes paramount at such flight conditions ${ }^{33}$.

Clearly, the actuation system has a large effect on the aeroservoelastic process, and the use of adequate models is important ${ }^{36}$. The usual method in the design of the structural-mode filters is to use a low-order linear transfer function model for the actuator, matched to actual test data. This is the method that will be used in the analysis of Chapter 4.

The ability of the low-order model to represent accurately the response of the actuator is limited however, and as such, a certain amount of uncertainty in the validity of the system analysis is introduced. This uncertainty is allowed for in the conservatism of current clearance requirements for the structural modes. In later chapters, the effect of introducing nonlinear actuation system models to the analysis will be investigated.

\subsubsection{Structural modelling}

The modelling of the aircraft structure is part of the requirements for the modelling of the aircraft component block in Figure 1.2, the other components being the 
unsteady aerodynamics and rigid-body dynamics.

Considering the uncertainties involved with the modelling of the aircraft structure, it is clear that it would be almost impossible to model accurately every element of the aircraft structure. As a result, any structural model will be an approximation of the true aircraft structure, the structure being idealised using finite element packages such as NASTRAN into a form suitable for derivation of its equations of motion ${ }^{37,38}$.

In addition to this approximation, there exists a further major problem introduced by the modelling of the structural dynamics. For the aircraft structure under consideration, a generic agile combat aircraft with multiple inputs and multiple outputs, the frequency response of the system to inputs on several control surfaces at once is not simple to find. In fact, the robustness of the phase response of the system represents a very difficult problem, especially at varying flight conditions and aircraft configurations $^{16}$.

The structural modelling may well be validated using appropriate ground tests on the actual aircraft, but as will be shown later, for certain structural modes, the in-flight situation represents the worst case in terms of modal stability. Since in-flight structural mode testing has only recently become possible ${ }^{26,39,40}$, reliance is still placed on the mathematical modelling of unsteady aerodynamics. As a result, the phase response of the system is generally neglected, the various gain responses for the signal paths representing each input to output being added algebraically to result in a response envelope for the worst case.

This assumption of an in-phase response will also have repercussions in terms of the digital system, where it will be necessary to assume in-phase addition of all frequency aliases as they are folded down onto the low-frequency ranges.

\subsubsection{Unsteady Aerodynamic modelling}

The modelling of the unsteady aerodynamic characteristics of an aircraft at a particular flight condition is a large topic in its own right, and it is not intended to go into much detail here. As will be discussed later, in order to linearise the equations describing the unsteady aerodynamics, it is necessary to make certain assumptions regarding the aircraft incidence and nature of the structural oscillation. Work has been completed into applying a more accurate representation of the unsteady aerodynamics whilst minimising the size of the model, but such results will not be applied in this case $^{38,41,42}$. In addition, some work has been completed regarding the aeroservoelastic characteristics of aircraft at high incidence ${ }^{33,34}$, and into the use of non-linear unsteady aerodynamics within aeroservoelasticity ${ }^{43}$. In order to simplify the analysis however, these methods will not be applied here.

Clearly, as with the structural modelling above, there exists a certain amount of 
uncertainty in the modelling of the unsteady aerodynamics. Recent advances in the use of in-flight structural-mode testing methods ${ }^{26}$ have enabled to some extent the modelling of the unsteady aerodynamic effects to be validated for the low-frequency structural modes. These low-frequency modes are of particular importance to the design of the structural-mode filters. Any validation of the phase response at these frequencies can be used to reduce the attenuation of the structural-mode filters. Such a method of ensuring the stability of these low-frequency structural modes as a result of modelling confidence at these frequencies is under investigation. This method is known as phase stabilisation of the structural modes ${ }^{44-46}$.

In the analysis carried out in Chapter 4 , it will be assumed that the same assumptions regarding modelling reliability will be used as are used in the design of the structural-mode filters at present.

\subsubsection{Rigid-body dynamics}

There is generally a large amount of confidence in the modelling of the rigid-body dynamics of the aircraft due in part to the availability of wind tunnel and flight test data. As a result, there is no need to make any assumptions about the modelling of this element of the problem.

\subsubsection{Summary of design assumptions}

In summary, the design assumptions that will be applied in the analysis of Chapter 4 will match those that are currently applied in the production of the structural-mode attenuation requirements by British Aerospace. All signal paths will be assumed to act in-phase such that the phase response of the system will not be used as a measure of stability. In order to take account of the variation in the flight control system, structural dynamics and unsteady aerodynamics with different flight conditions and aircraft configuration, the design will be based on the worst-case. This worst-case is produced from a consideration of all possible combinations of flight condition, fuel state and stores layout.

In addition, a clearance requirement of a maximum open-loop structural-mode gain of $-9 \mathrm{~dB}$ for the worst case will be used to take into consideration the uncertainty of the modelling techniques and as a suitable safety margin for clearance into flight. This level of $-9 \mathrm{~dB}$ compares with that specified by the MILSPEC military standard ${ }^{47}$ which specifies a $-8 \mathrm{~dB}$ maximum level and 60 degrees phase margin.

\subsection{Conclusions}

The current method of solution of the aeroservoelastic problem has been described. It has been shown that this method takes into account the present level of 
uncertainty in the process of an aeroservoelastic interaction. In addition, the current design process allows for errors in the modelling of the system by ensuring a $-9 \mathrm{~dB}$ maximum open-loop gain at structural frequencies. 
Chapter 3

Modelling of the Flexible Aircraft 


\subsection{Introduction}

It has been stated in Chapter 1 that there are three main elements to the aeroservoelastic problem, namely those of aircraft dynamics, actuator dynamics and flight control system dynamics ${ }^{48-50}$. Clearly, in order to obtain an understanding of the structural coupling problem, it is necessary to first obtain an understanding of these three elements. This chapter is dedicated towards this aim.

It is intended to develop a single model in the following chapter which will represent the aircraft dynamics in the analysis of the later chapters. Such a model of the aircraft dynamics must incorporate the elements of structural dynamics and unsteady aerodynamics, along with the more usual elements of rigid-body aircraft dynamics and control surface dynamics. A representation of the required elements is shown in Figure 3.1.

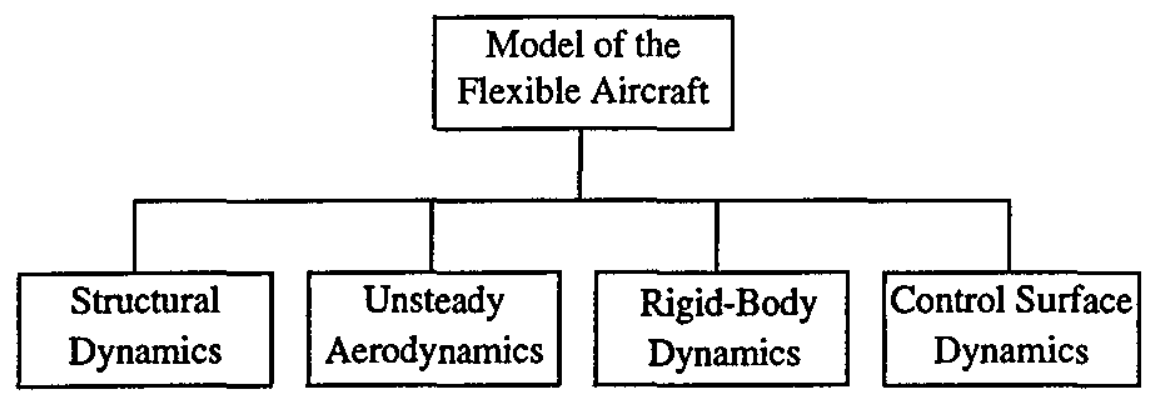

Figure 3.1 - Elements of a flexible aircraft model

The process described in this chapter will result in a model of the flexible aircraft describing all of the above components in a form suitable for analysis in MATLAB/ SIMULINK.

Throughout the following analysis, it is assumed that all motions involve only a small perturbation about a nominal steady state condition, and that all forces generated as a result of these motions are linear functions of these small displacements.

As has been discussed in Chapter 2, the consequences of such assumptions are under investigation, particularly for aircraft at high incidence and for nonlinear aerodynamic effects. Such topics are large in their own right however, and in order to simplify the following analysis, these considerations will be neglected.

\subsection{Structural dynamics}

\subsubsection{Introduction}

The following section describes the theoretical background to the modelling of the structural dynamics of the aircraft as highlighted in Figure 3.2. 
In order to simplify the following analysis significantly, the structure of the aircraft is idealised by breaking it down into many discrete masses. Each mass represents a local inertia of the aircraft structure which is interconnected by massless springs and dampers. Such a representation of the aircraft structure is shown in Figure 3.3. The structural dynamics are thus defined by the motion of these masses in a superposition of the separate vibrational modes of the complete structure ${ }^{51}$.

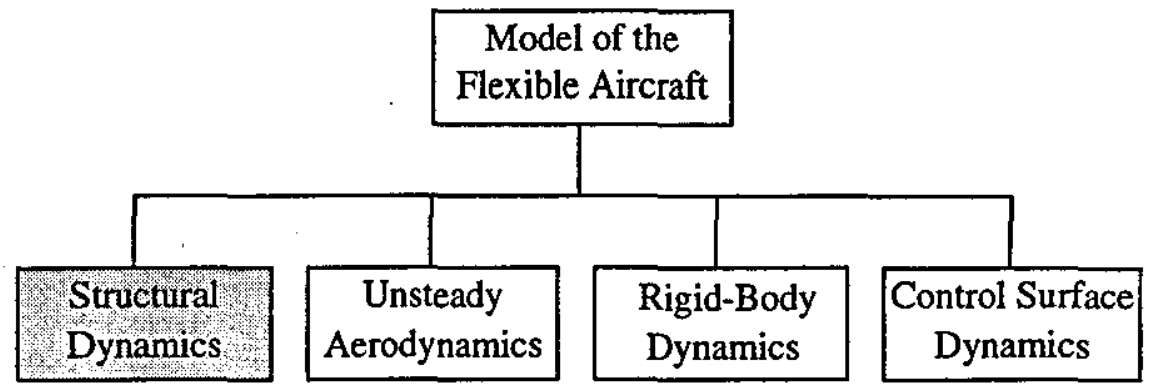

Figure 3.2 - Elements of a flexible aircraft model: structural dynamics

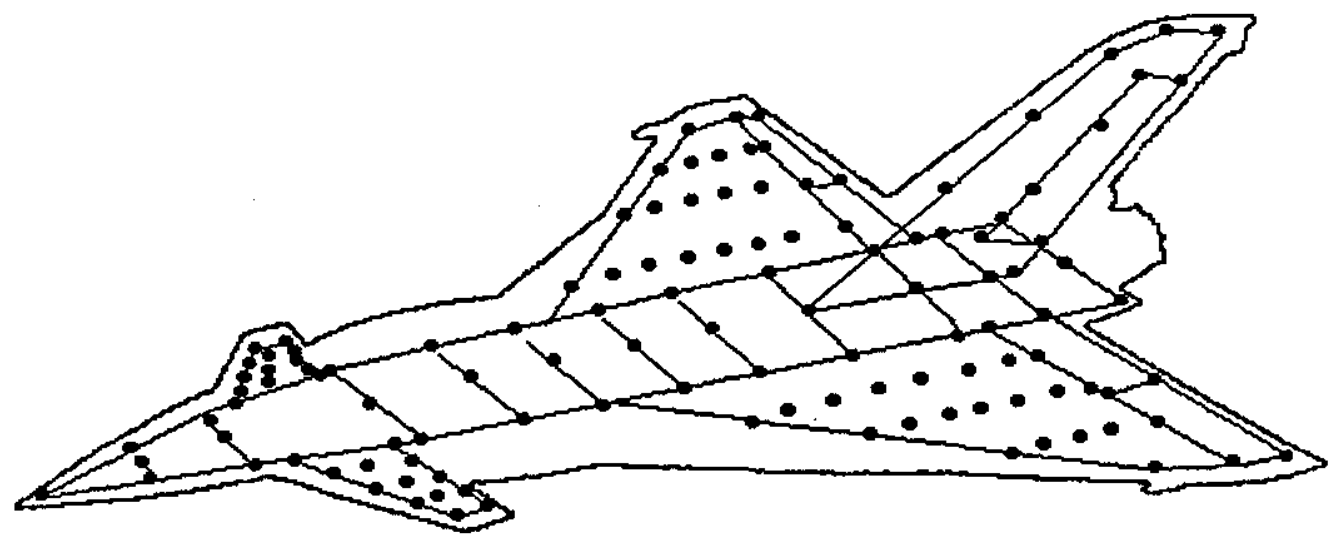

Figure 3.3 - Schematic representation of the discrete mass structural idealization

\subsubsection{Derivation of equations of motion for a simple undamped system}

In order for the equations of motion of these masses to be derived, it is necessary to first define a suitable coordinate system.

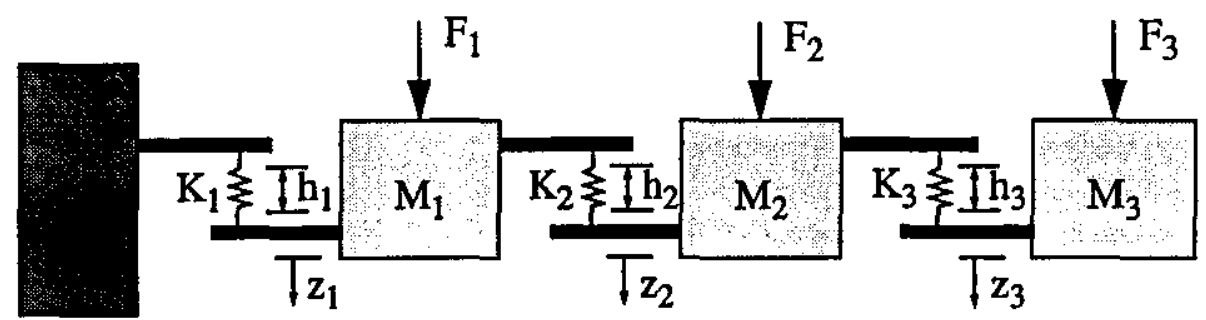

Figure 3.4 - Simple three-degree of freedom mass-spring system

In any dynamic system such as that of the aircraft structure, there are many suitable coordinate systems that could be used. For example, consider the simple 3- 
degree-of-freedom mass-spring system as shown in Figure 3.4. This system could be considered as the mass distribution along a wing for example. In order to obtain the equations of motion of this system, consider the case where the mass displacements are of particular interest. This leads to the following equations of motion taking unit displacements of the masses as the basic modes

$$
\left[\begin{array}{ccc}
\mathrm{M}_{1} & 0 & 0 \\
0 & \mathrm{M}_{2} & 0 \\
0 & 0 & \mathrm{M}_{3}
\end{array}\right] \times\left[\begin{array}{c}
\ddot{z}_{1} \\
\ddot{z}_{2} \\
\ddot{z}_{3}
\end{array}\right]+\left[\begin{array}{ccc}
\mathrm{K}_{1}+\mathrm{K}_{2} & -\mathrm{K}_{2} & 0 \\
-\mathrm{K}_{2} & \mathrm{~K}_{2}+\mathrm{K}_{3}-\mathrm{K}_{3} \\
0 & -\mathrm{K}_{3} & \mathrm{~K}_{3}
\end{array}\right] \times\left[\begin{array}{l}
\mathrm{z}_{1} \\
\mathrm{z}_{2} \\
\mathrm{z}_{3}
\end{array}\right]=\left[\begin{array}{l}
\mathrm{F}_{1} \\
\mathrm{~F}_{2} \\
\mathrm{~F}_{3}
\end{array}\right]
$$

which could be rewritten as

$$
\mathbf{M}_{\mathrm{m}} \ddot{\mathbf{q}}_{\mathrm{m}}+\mathbf{K}_{\mathrm{m}} \mathbf{q}_{\mathrm{m}}=\mathbf{F}
$$

where

$$
\mathbf{q}_{\mathbf{m}}=\left[\begin{array}{l}
z_{1} \\
z_{2} \\
z_{3}
\end{array}\right]
$$

Such a representation allows the effect of the mass values on the dynamics of the system to be easily evaluated, the inertia matrix in this case being a diagonal matrix of the masses. The stiffness matrix on the other hand contains terms that are not direct representations of the spring stiffnesses, and it would not be as simple to examine the effect of changing the spring stiffnesses on the dynamics of the system in this case.

If the equations of motion of the system are now obtained taking the spring extensions as the basic states, the result is

$$
\left[\begin{array}{ccc}
M_{1}+M_{2}+M_{3} & M_{2}+M_{3} & M_{3} \\
M_{2}+M_{3} & M_{2}+M_{3} & M_{3} \\
M_{3} & M_{3} & M_{3}
\end{array}\right] \times\left[\begin{array}{c}
\ddot{h_{1}} \\
\ddot{h_{2}} \\
\ddot{h_{3}}
\end{array}\right]+\left[\begin{array}{ccc}
K_{1} & 0 & 0 \\
0 & K_{2} & 0 \\
0 & 0 & K_{3}
\end{array}\right] \times\left[\begin{array}{l}
h_{1} \\
h_{2} \\
h_{3}
\end{array}\right]=\left[\begin{array}{lll}
1 & 1 & 1 \\
0 & 1 & 1 \\
0 & 0 & 1
\end{array}\right] \times\left[\begin{array}{c}
F_{1} \\
F_{2} \\
F_{3}
\end{array}\right]
$$

which could be rewritten as

$$
M_{k} \ddot{q}_{k}+K_{k} q_{k}=Z_{m k} F
$$

where

$$
\mathbf{q}_{\mathbf{k}}=\left[\begin{array}{l}
\mathrm{h}_{1} \\
\mathrm{~h}_{2} \\
\mathrm{~h}_{3}
\end{array}\right]
$$

In the representation of equation (3.4), the elements of the stiffness matrix are now identical to the spring stiffnesses, whilst the elements of the inertia matrix bear only an 
obscure relationship to the true mass values. In addition, the forcing function on the right hand side of equation (3.4) has become more complex than that of the original equations of motion shown in equation (3.1).

Therefore, equations (3.1) to (3.6) show that the form of the equations and their consequent ease of interpretation is governed by the choice of states, $\mathbf{q}$.

Clearly, the conversion between equations (3.1) and (3.4) can be achieved by the transformation matrix, $\mathbf{Z}_{\mathrm{mk}}$ where

$$
\mathbf{q}_{m}=\left[\begin{array}{lll}
1 & 0 & 0 \\
1 & 1 & 0 \\
1 & 1 & 1
\end{array}\right] \times \mathbf{q}_{k}=\mathbf{Z}_{m k}^{\mathbf{r}} \mathbf{q}_{k}
$$

This results in

$$
\mathbf{Z}_{m k}^{T} M_{m} \ddot{q}_{m}+\mathbf{Z}_{m k}^{T} K_{m} \mathbf{q}_{m}=\mathbf{Z}_{m k}^{T} \mathbf{F}
$$

and using equation (3.7), this becomes

$$
Z_{m k}^{T} M_{m} Z_{m k} \ddot{q}_{k}+Z_{m k}^{T} K_{m} Z_{m k} q_{k}=Z_{m k}^{T} F
$$

Since

$$
M_{k}=Z_{m k}^{T} M_{m} Z_{m k} \quad \text { and } \quad K_{k}=Z_{m k}^{T} K_{m} Z_{m k}
$$

then equation (3.4) is the result.

The two representations of the system are identical, as would be expected as they describe the same physical system. The transformation matrix $\mathbf{Z}_{\mathrm{mk}}$ is the link between the two representations. In performing the transformation however, it is important that all of the degrees of freedom are maintained.

The importance of this demonstration, is that it is possible to chose any suitable states for the derivation of the equations of motion, and that any further representations can be obtained by using a suitable transformation matrix such as $\mathbf{Z}_{\mathrm{mk}}$ in this case. 


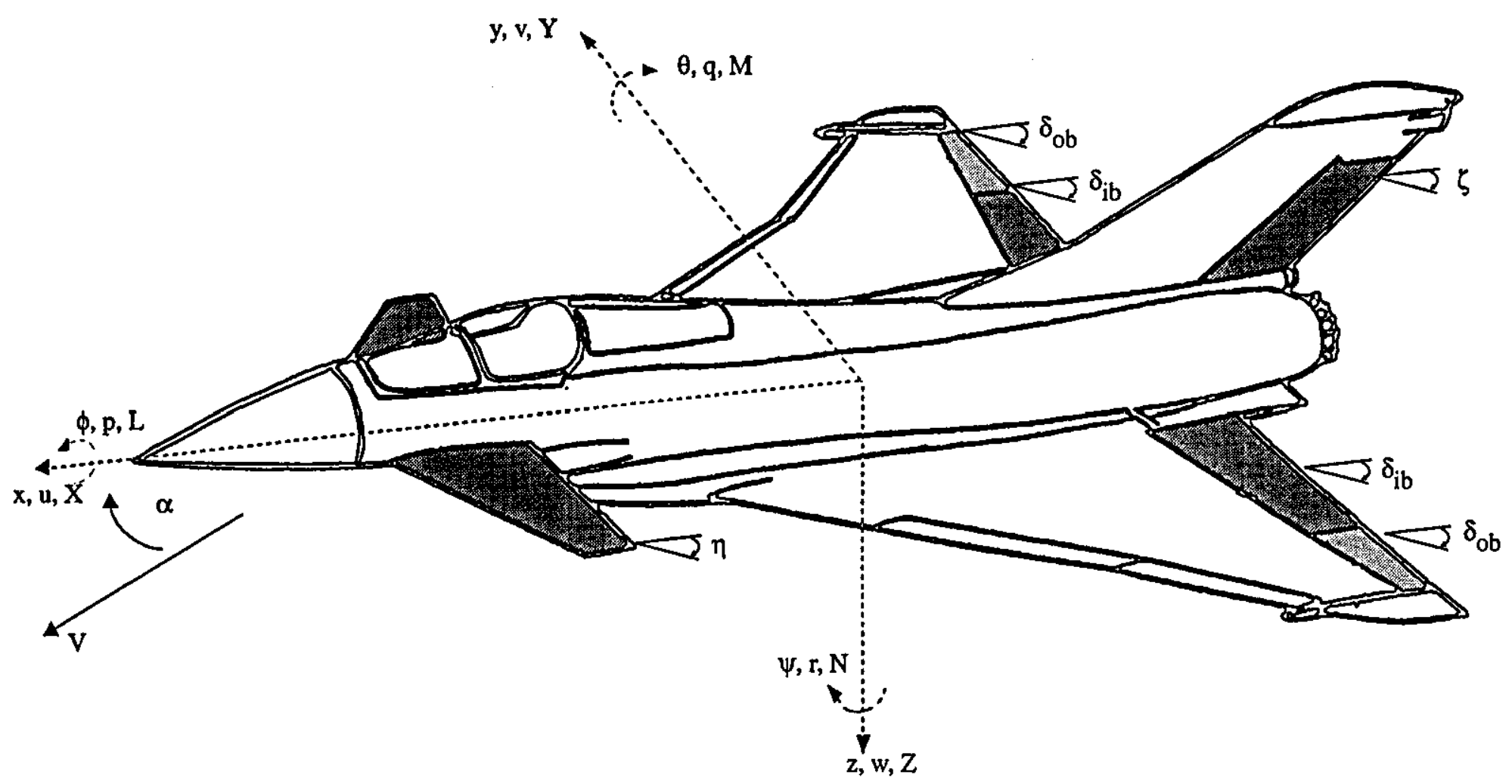

Figure 3.5- Coordinate system for generic canard delta aircraft 


\subsubsection{Derivation of equations of motion for idealised aircraft structure}

Considering now the idealisation of the aircraft structure as shown in Figure 3.3, each mass has 6-degrees-of-freedom, namely translation in the three axes, and rotation about the three axes, such that a displacement vector, $d$, can be defined as

$$
\mathbf{d}=\left[\begin{array}{l}
\mathrm{x} \\
\mathrm{y} \\
\mathrm{z} \\
\phi \\
\theta \\
\psi
\end{array}\right]
$$

with the terms as defined in Figure 3.5. To simplify the following derivation, the displacements of the masses will be limited to just 2-degrees-of-freedom, namely translation along the $z$-axis, $z$, and rotation about the $y$-axis, $\theta$. Such a simplification is consistent with the decision to consider the longitudinal motion of the aircraft only. This is because the longitudinal motion is not significantly coupled to the lateral motion for small perturbations, and the short period motion of the rigid-body aircraft is dominated by the $\mathrm{z}$ and $\theta$ terms. This reduces the displacement vector to

$$
\mathbf{d}=\left[\begin{array}{l}
\mathrm{z} \\
\theta
\end{array}\right]
$$

It is now possible to express this displacement vector in terms of a transformation vector and a set of arbitrary states or generalised coordinates. The choice of these generalised coordinates and subsequent transformation matrix is not relevant at present, but from the earlier considerations, it is clear that any suitable coordinate system can be used. A transformation to any other coordinate system is possible, whilst maintaining the validity of the representation. The mass displacements can therefore be defined as

$$
\mathbf{d}=\left[\begin{array}{l}
\mathbf{z} \\
\theta
\end{array}\right]=\left[\begin{array}{c}
\mathbf{S} \\
\partial \mathrm{S} \\
\partial \mathrm{x}
\end{array}\right] \times \mathbf{q}=\mathbf{Z q}
$$

where at any point, the rotation about the $y$-axis, $\theta$, can be equated to the derivative of the $\mathrm{z}$-axis displacement with respect to the $\mathrm{X}$-axis. The transformation matrices, $S$ and $\mathbf{Z}$ will be defined according to the choice of the coordinate system. The choice of the coordinate system can be arbitrary as has been shown, but there will be significant advantages to be gained if a coordinate system is devised which simplifies the problem. Such a coordinate system is based on the normal modes of vibration of the structure. Development of equations of motion for these normal modes results in both the inertia and stiffness matrices being diagonal. The development of this coordinate 
system will be discussed later in the chapter.

The equations of motion for the aircraft structure can now be derived, using an application of Lagrange's equation. Lagrange's equation is an energy based approach to system dynamics and is based on a limited degree-of-freedom application of Hamilton's principle. It is not intended to include the proof of Lagrange's equation here, as this is covered in many standard texts ${ }^{51}$.

From Reference 51, Lagrange's equation can be defined as

$$
\frac{d}{d t}\left(\frac{\partial \mathbf{T}}{\partial \dot{\mathbf{q}}}\right)-\frac{\partial \mathbf{T}}{\partial \mathbf{q}}+\frac{\partial \mathbf{U}}{\partial \mathbf{q}}=\mathbf{Q}
$$

where,

$\mathbf{T}$, is the system kinetic energy associated with the system inertia

$\mathbf{U}$ is the potential energy of the conservative forces, namely the elasticity of the system in this case,

$\mathbf{Q}$ are the generalised forces applied to the system excluding those included in $\mathbf{U}$, $\mathbf{q}$ are a set of generalised coordinates as described above.

In this case, it is possible to expand the term representing the generalised forces $\mathbf{Q}$ to give

$$
\frac{d}{d t}\left(\frac{\partial \mathbf{T}}{\partial \dot{\mathbf{q}}}\right)-\frac{\partial \mathbf{T}}{\partial \mathbf{q}}+\frac{\partial \mathbf{U}}{\partial \mathbf{q}}=\mathbf{Q}=\frac{\delta \mathbf{W}}{\delta \mathbf{q}}=\mathbf{L} \frac{\partial \mathbf{z}}{\partial \mathbf{q}}+\mathbf{M} \frac{\partial \theta}{\partial \mathbf{q}}
$$

where,

$\delta \mathrm{W}$ is the work done by the generalised forces in the virtual displacements $\delta q$,

$\mathbf{L}, \mathbf{M}$ are the generalised forces and moments contributing to $\mathbf{Q}$.

Considering the kinetic energy, $\mathbf{T}$, in equation (3.15), for the idealised structure it is possible to write

$$
\mathbf{T}=\frac{1}{2}\left(\sum_{1}^{p} m_{i} \dot{z}_{i}^{2}+\sum_{1}^{p} I_{\theta_{i}} \dot{\theta}_{i}^{2}\right)
$$

where,

$\mathrm{m}_{\mathrm{i}}$ is an individual mass value in the idealisation,

$\mathrm{I}_{0 \mathrm{i}}$ is an individual moment of inertia about the $y$-axis of the idealised $\mathrm{i}^{\text {th }}$ mass

$\mathrm{p}$ is the number of discrete masses in the idealisation

The total kinetic energy of the system is therefore equated to the sum of the individual kinetic energies of the discrete masses in the idealisation.

Equation (3.16) can be written in matrix form as 


$$
2 \mathbf{T}=\left[\begin{array}{ll}
\dot{z} & \dot{\theta}
\end{array}\right]\left[\begin{array}{ll}
\mathbf{m} & 0 \\
0 & \mathbf{I}
\end{array}\right]\left[\begin{array}{l}
\dot{z} \\
\dot{\theta}
\end{array}\right]=\dot{d}^{\mathbf{T}} \mathbf{M} \dot{d}
$$

Applying a suitable transformation matrix $\mathbf{Z}$, equation (3.17) can be expressed in terms of the generalised coordinate system, q. Substituting equation (3.13) in (3.17) gives

$$
\mathbf{T}=\frac{1}{2}\left(\dot{\mathbf{q}}^{\mathbf{T}} \mathbf{Z}^{\mathbf{T}} \mathbf{M Z} \mathbf{q}\right)
$$

where,

$$
\mathbf{d}=\mathbf{Z q}
$$

This gives an expression for the kinetic energy of the system in terms of the generalised coordinate system. In order to obtain the equations of motion of the idealised aircraft structure, equation (3.18) can be applied to the kinetic energy terms in Lagrange's equation (3.15). From equation (3.18),

$$
\begin{gathered}
\frac{\partial \mathbf{T}}{\partial \dot{\mathbf{q}}}=\mathbf{Z}^{\mathbf{T}} \mathbf{M Z \dot { q }} \\
\frac{\partial \mathbf{T}}{\partial \mathbf{q}}=\mathbf{0}
\end{gathered}
$$

which results in the kinetic terms of Lagrange's equation (3.15) becoming

$$
\frac{d}{d t}\left(\frac{\partial \mathbf{T}}{\partial \dot{\mathbf{q}}}\right)-\frac{\partial \mathbf{T}}{\partial \mathbf{q}}=\mathbf{Z}^{\mathbf{T}} \mathbf{M Z} \ddot{\mathbf{q}}=\mathbf{A} \ddot{\mathbf{q}}
$$

where $\mathbf{A}$ can be considered as a generalised inertia matrix for the structure in terms of the generalised coordinate system $\mathbf{q}$. $\mathbf{A}$ is generated from the system inertia matrix $\mathbf{M}$ using the coordinate transformation matrix, $\mathbf{Z}$, according to equation (3.21).

Consider now the term for potential energy in Lagrange's equation (3.15). The potential energy stored in the system due to the elasticity of the structure, can be expressed in terms of the displacements of the discrete masses as follows

$$
\mathbf{U}=\frac{1}{2}\left(\sum_{1}^{\mathrm{p}} \mathrm{k}_{\mathrm{i}_{\mathrm{zz}}} \mathbf{z}_{\mathrm{i}}^{2}+\sum_{1}^{\mathrm{p}} \mathrm{k}_{\mathrm{i}_{\mathrm{x}}} \theta_{\mathrm{i}}^{2}+\sum_{1}^{\mathrm{p}} \mathrm{k}_{\mathrm{i}_{\mathrm{ze}}} \mathbf{z}_{\mathrm{i}}^{2}+\sum_{\mathrm{i}}^{\mathrm{p}} \mathrm{k}_{\mathrm{i}_{\theta \theta}} \theta_{\mathrm{i}}^{2}\right)
$$

where, $k_{i}$ is an individual stiffness for the $i^{\text {th }}$ discrete mass incorporated in the structural idealisation. The four stiffness values for each mass represent the stiffness in each degree of freedom. Since the system has 2-degrees-of-freedom there will be four stiffness values to take into consideration. These stiffnesses are known as influence coefficients and can be considered as the loads and moments induced at the discrete masses by unit displacements at each other mass location in turn whilst keeping all 
other masses fixed.

Expressing equation (3.23) in matrix algebra form gives

$$
2 U=\left[\begin{array}{ll}
z & \theta
\end{array}\right]\left[\begin{array}{ll}
k_{z z} & k_{z \theta} \\
k_{\theta z} & k_{\theta \theta}
\end{array}\right]\left[\begin{array}{l}
z \\
\theta
\end{array}\right]=d^{T} K d
$$

and converting this expression for the potential energy of the system into the generalised coordinate system using equation (3.13) gives

$$
\mathbf{U}=\frac{1}{2}\left(\mathbf{q}^{\mathbf{T}} \mathbf{Z}^{\mathbf{T}} \mathbf{K Z \mathbf { Z }}\right)
$$

Applying this result in terms of the generalised coordinates to the potential energy term in Lagrange's equation (3.15) results in

$$
\frac{\partial \mathbf{U}}{\partial \mathbf{q}}=\mathbf{Z}^{\mathbf{T}} \mathbf{K Z q}=\mathbf{E q}
$$

where $\mathbf{E}$ can be considered as a generalised stiffness matrix for the structure in terms of the generalised coordinate system $\mathbf{q}$. $\mathbf{E}$ is generated from the system stiffness matrix $\mathbf{K}$ using the coordinate transformation matrix, $\mathrm{Z}$, according to equation (3.25).

By substituting equations (3.22) and (3.26) into Lagrange's equation (3.15), it is possible to construct the equations of motion of the system in the generalised coordinate system chosen. The resulting equation is

$$
\mathbf{A} \ddot{\mathbf{q}}+\mathbf{E q}=\mathbf{Q}
$$

Now, from Lagrange's equation as expressed in (3.15), the generalised force vector $\mathbf{Q}$ can be expressed as

$$
\mathbf{Q}=\frac{\delta \mathbf{W}}{\delta \mathbf{q}}=\mathbf{L} \frac{\partial \mathbf{z}}{\partial \mathbf{q}}+\mathbf{M} \frac{\partial \theta}{\partial \mathbf{q}}
$$

and from equation (3.13)

$$
\mathbf{d}=\left[\begin{array}{l}
\mathrm{z} \\
\theta
\end{array}\right]=\left[\begin{array}{c}
\mathbf{s} \\
\partial \mathrm{S} \\
\partial \mathrm{x}
\end{array}\right] \times \mathbf{q}=\mathbf{Z q}
$$

so that,

$$
\frac{\partial \mathbf{z}}{\partial \mathbf{q}}=\mathbf{S}
$$

and,

$$
\frac{\partial \theta}{\partial q}=\frac{\partial S}{\partial x}
$$


The generalised force vector $\mathbf{Q}$ can be defined therefore as

$$
Q=\left[S \frac{\partial S}{\partial x}\right]\left[\begin{array}{l}
\mathbf{L} \\
\mathbf{M}
\end{array}\right]=\mathbf{Z}^{\mathbf{T}} \mathbf{F}
$$

The generalised equation of motion for the undamped aircraft structure can therefore be defined as

$$
\mathbf{A q}+\mathbf{E q}=\mathbf{Q}
$$

where the generalised inertia, stiffness and forcing matrices are defined in equations (3.22), (3.26) and (3.32) respectively.

Compare the above equations for the aircraft with those for the simple example system, (3.2) and (3.5), and in particular with the equations describing the transformation between the two chosen derivations of the equations of motion for the simple system, equations (3.9) and (3.10). It can be seen that the equations are very similar in form, as would be expected, since they all describe similar undamped systems. The important point to note is that equation (3.33) is very general in nature, the generalised coordinate system and transformation matrix being undefined as yet. Even though this is the case, from the earlier example it is clear that whatever coordinate system is chosen, this equation will produce a valid set of equations of motion.

\subsubsection{Selection of a suitable generalised coordinate system}

The above derivation leads to the selection of a suitable coordinate system in which to express the equations of motion. In most vibration studies, the system used relies on the normal modes of the undamped structure. Use of normal modes to represent the dynamics of the structure reduces the complexity of the representation. The true vibrational modes are then a superposition of these normal modes. The normal modes of a structure can be found as follows.

Consider a system described by equations of motion similar to those of equation (3.33), but with no forcing function, $\mathbf{F}$. This results in equations of motion in the matrix form of

$$
\mathbf{M d}+\mathbf{K d}=\mathbf{0}
$$

Re-arranging the above equation gives -

$$
\mathbf{d}=-\mathbf{M}^{-1} \mathbf{K d}
$$

If the eigenvalue decomposition of $\mathbf{M}^{-1} \mathbf{K}$ is produced such that

$$
\mathbf{M}^{-1} \mathbf{K}=\mathbf{V} \Lambda \mathbf{V}^{-1}
$$


where,

$\mathbf{V}$ is the matrix of eigenvectors of $\mathbf{M}^{-1} \mathbf{K}$,

$\Lambda$ is the diagonal matrix of the eigenvalues of $\mathbf{M}^{-1} \mathbf{K}$.

Re-arranging equation (3.36) gives

$$
\mathbf{K}=\mathbf{M V} \Lambda \mathbf{V}^{-1}
$$

hence,

$$
\mathbf{K V}=\mathbf{M V \Lambda}
$$

and,

$$
\mathrm{KV} \Lambda^{-1}=\mathbf{M V}
$$

giving finally,

$$
\mathrm{V}^{\mathrm{T}} \mathrm{KV} \Lambda^{-1}=\mathbf{V}^{\mathrm{T}} \mathrm{MV}
$$

It is a property of the eigensolution that $\mathbf{V}^{\mathrm{T}} \mathbf{K V}$ is a diagonal matrix, that is to say that the eigensolution diagonalises the problem. The same applies to $\mathbf{V}^{\mathrm{T}} \mathbf{M V}$. By renormalising the matrices in this way the problem is greatly simplified. The various vibrational modes are decoupled to produce the diagonal matrix description. It is the eigenvectors contained in the $\mathbf{V}$ matrix which are known as the normal modes of the system, and from the above analysis, the original vibrational modes of the system are simply obtained from these normal modes by superposition.

Suppose that this method of diagonalisation is used on the aircraft equations of motion developed earlier. The equations of motion for the undamped aircraft used a set of generalised coordinates $\mathbf{q}$, and a transformation matrix $\mathbf{Z}$. Suppose that the eigenvectors of $\mathbf{M}^{-1} \mathbf{K}$ for the aircraft structure were used to generate $\mathbf{Z}$ and $\mathbf{q}$, such that from equation (3.36),

$$
\mathbf{M}^{-1} \mathbf{K}=\mathbf{Z} \Lambda \mathbf{Z}^{-1}
$$

and,

$$
\mathbf{d}=\left[\begin{array}{l}
\mathbf{z} \\
\theta
\end{array}\right]=\mathbf{Z q}
$$

as defined in equation (3.13). This would result in the generalised equations of motion for the aircraft structure as given in equation (3.33) being diagonal in nature, whilst still maintaining their validity from the earlier reasoning.

Considering the physical significance of this development, the diagonalisation of the equations of motion has resulted in the equations being formulated in terms of the 
normal modes of the system, with these modes being used to transform from the generalised coordinate system to the true displacements. In this case, the eigenvectors of $\mathbf{M}^{-1} \mathbf{K}$ represent the modeshapes of these normal modes, for unit amplitude. The resulting generalised coordinates represent the actual amplitudes of these normal modes, and it is for these amplitudes that the generalised equations of motion as given in equation (3.33) may be solved. Once a solution for these normal mode amplitudes has been obtained, the actual displacements for the discrete masses can be calculated from an application of equation (3.42), the final true displacements being a superposition of the separate normal modes.

Returning to the example of the three connected masses as shown in Figure 3.4, suppose that the inertia and stiffness matrices were given by -

$$
\begin{gathered}
\mathbf{M}=\left[\begin{array}{lll}
1 & 0 & 0 \\
0 & 2 & 0 \\
0 & 0 & 3
\end{array}\right] \\
\mathbf{K}=\left[\begin{array}{ccc}
300 & -200 & 0 \\
-200 & 500 & -300 \\
0 & -300 & 300
\end{array}\right]
\end{gathered}
$$

which is given from equation (3.1) with $M_{1}=1 \mathrm{Kg}, M_{2}=2 \mathrm{Kg}, M_{3}=3 \mathrm{~kg}$,

$$
\mathrm{K}_{1}=100 \mathrm{~N} / \mathrm{m}, \mathrm{K}_{2}=200 \mathrm{~N} / \mathrm{m}, \mathrm{K}_{3}=300 \mathrm{~N} / \mathrm{m}
$$

and the displacement vector being

$$
\mathbf{d}=\left[\begin{array}{l}
z_{1} \\
z_{2} \\
z_{3}
\end{array}\right]
$$

The eigensolution of these inertia and stiffness matrices is given by

$$
\begin{gathered}
\mathbf{V}=\left[\begin{array}{ccc}
-0.8105 & 0.4176 & -0.8165 \\
0.5617 & 0.6026 & -0.4082 \\
-0.1659 & 0.6801 & 0.4082
\end{array}\right] \\
\Lambda=\left[\begin{array}{ccc}
438.6 & 0 & 0 \\
0 & 11.4 & 0 \\
0 & 0 & 200.0
\end{array}\right]
\end{gathered}
$$

which results in the equations of motion in generalised coordinates becoming,

$$
\mathbf{A} \ddot{\mathbf{q}}+\mathbf{E q}=\mathbf{Q}
$$

where, from equations (3.22) and (3.26) 


$$
\begin{gathered}
\mathbf{A}=\mathbf{V}^{\mathrm{T}} \mathbf{M V}=\left[\begin{array}{ccc}
1.3705 & 0 & 0 \\
0 & 2.2882 & 0 \\
0 & 0 & 1.5
\end{array}\right] \\
\mathbf{E}=\mathbf{V}^{\mathrm{T}} \mathbf{K V}=\left[\begin{array}{ccc}
601 & 0 & 0 \\
0 & 26 & 0 \\
0 & 0 & 300
\end{array}\right]
\end{gathered}
$$

and,

$$
\mathbf{d}=\mathbf{V q}
$$

Thus by using the eigensolution of the inertia and stiffness matrices, the equations of motion have been diagonalised.

\subsubsection{Inclusion of structural damping into the equations of motion}

In reality, the structure of the aircraft will have some inherent damping, and it is necessary to include this into the equations of motion for the structure. Unfortunately, the type of damping present in this type of structure is extremely difficult to describe analytically, and it is usual to assume a certain level of damping for the structure as a whole. This value of damping may be incorporated into equation (3.33) by considering the form of this equation. The equation is a second order matrix differential equation, and as a result, the frequency of the various unforced modes can be easily calculated. Assuming that the equations have been derived using the above method, resulting in a set of diagonalised equations of motion, there is no need to calculate a further eigensolution. The normal mode frequencies can simply be calculated from the generalised inertia and stiffness matrices $\mathbf{A}$ and $\mathbf{E}$. It can be seen from equation (3.40) that,

$$
\mathbf{V}^{\mathrm{T}} \mathrm{KV} \Lambda^{-1}=\mathbf{V}^{\mathrm{T}} \mathbf{M V}
$$

or alternatively,

$$
\mathbf{E} \Lambda^{-1}=\mathbf{A}
$$

which, since $\mathbf{E}, \mathbf{A}$ and $\Lambda$ are all diagonal matrices, equation (3.53) can be expressed in terms of the elements, such that

$$
e_{1 i} \lambda^{-1}{ }_{11}=a_{11}
$$

Thus, each modal frequency can be derived from,

$$
\omega_{i}=\sqrt{\frac{e_{11}}{a_{i 1}}}
$$

Assuming viscous damping, it is possible to calculate the terms in a damping 
matrix, $\mathbf{D}$, for the aircraft structure assuming a value of the damping ratio, $\zeta$, so that,

$$
\mathrm{d}_{\mathrm{ij}}=2 \zeta \omega_{\mathrm{i}}
$$

where the modal frequency can be calculated from equation (3.55). The amount of structural damping usually assumed for the aircraft structural modes is $1 \%$ such that $\zeta=0.01$.

Equation (3.33) can therefore be expanded to include viscous damping of the structural modes, so that the equations of motion in terms of the generalised coordinates becomes,

$$
\mathbf{A} \ddot{\mathbf{q}}+\mathbf{D} \dot{q}+\mathbf{E q}=\mathbf{Q}
$$

with the generalised damping matrix, $\mathbf{D}$, being generated from equations (3.55) and (3.56). The existence of off-diagonal damping terms in the generalised stiffness matrix is ignored.

Returning to the example of Figure 3.4, assuming a value of $\zeta=0.01$, applying equations (3.55) and (3.56) results in,

$$
\omega=\left[\begin{array}{ccc}
20.9428 & 0 & 0 \\
0 & 3.3764 & 0 \\
0 & 0 & 14.1421
\end{array}\right] \quad \frac{\text { Rads }}{\text { Sec }}
$$

and hence,

$$
\mathrm{D}=\left[\begin{array}{ccc}
0.2094 & 0 & 0 \\
0 & 0.0338 & 0 \\
0 & 0 & 0.1414
\end{array}\right]
$$

\subsection{Aerodynamic effects}

\subsubsection{Introduction}

In the above section, the theoretical basis for the derivation of the equations of motion of the aircraft structure has been given, assuming that the structure is unloaded. In reality, there will be significant loads on the structure due to aerodynamics effects, gust loading and control surface deflection. It is intended to introduce the theoretical background to the first of these loading effects in the following section. The analysis presented in the following section will therefore form the second section of the flexible aircraft model as highlighted in Figure 3.6.

The analytical derivation of aerodynamic loads is a very large subject in itself $^{52}$, the derivation of aerodynamic loads on an oscillating structure being just a small specialisation of a much larger topic. One of the main problems facing the use of 
aerodynamic loads in this way is that the loads vary with aircraft flight condition and structural oscillation frequency. In the case of aeroelastic studies, there are many ways in which the variation of aerodynamic characteristics are accounted for, the usual method being the use of a non-dimensional frequency parameter or reduced frequency to match the aerodynamic derivatives to a particular range of oscillation frequencies ${ }^{53}$.

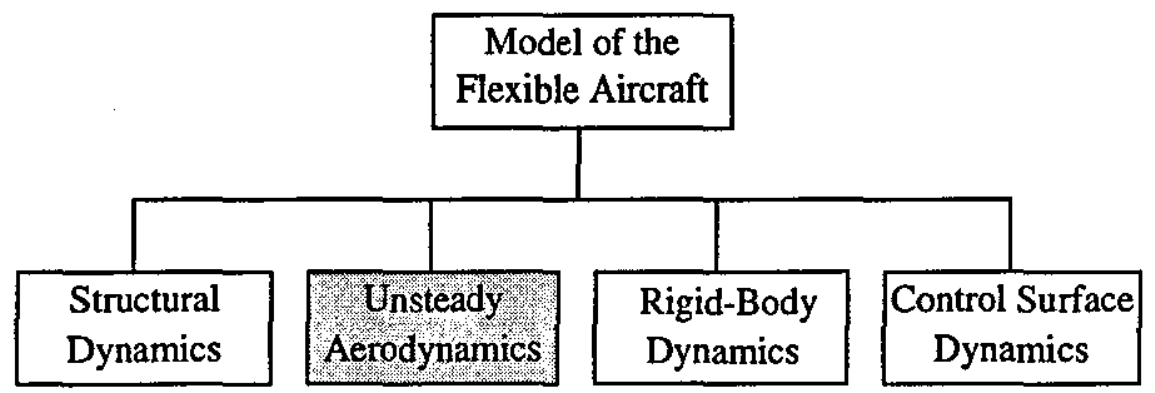

Figure 3.6 - Elements of a flexible aircraft model: unsteady aerodynamics

In more complete aeroelastic studies, the variation of these aerodynamic derivatives as a result of varying flight condition and oscillatory frequency is modelled by the use of Padé and least-squares approximations, the aerodynamic derivatives becoming functions of the frequency parameter and Mach number ${ }^{54}$. The resulting aeroelastic equation for the structure can then be solved as the equivalent airspeed varies for assumed values of Mach number and frequency parameter. This results in the prediction of an airspeed at which the damping of particular modes of the structure is zero, indicating a flutter condition.

In the following analysis, and subsequent use of the aerodynamic loads in the flexible aircraft model, the aerodynamic loads have been formulated for a single flight condition and frequency parameter. As a result, the aerodynamic model will only be strictly valid for oscillations corresponding to the value of frequency parameter, but this approximation is considered adequate for these purposes.

\subsubsection{Incorporation of aerodynamic effects into the equations of motion}

The equations of motion generated so far were given in equation (3.57), which states

$$
\mathbf{A} \ddot{\mathbf{q}}+\mathbf{D q}+\mathbf{E q}=\mathbf{Q}
$$

The loading of the structure other than the conservative forces previously included in the derivation for the potential energy, (3.23), is incorporated into the generalised force vector, $\mathbf{Q}$. This force vector can be considered to be made up of two main components as shown in Figure 3.7. The generalised force vector can be expressed as

$$
\mathbf{Q}=\mathbf{Q}_{\mathbf{A}}+\mathbf{Q}_{\mathbf{0}}
$$


where,

$\mathbf{Q}_{\mathrm{A}}$ is the vector of Aerodynamic forces, and

$\mathbf{Q}_{\mathrm{O}}$ is the vector of External forces, such as forces due to landing, taxiing, stores release etc.

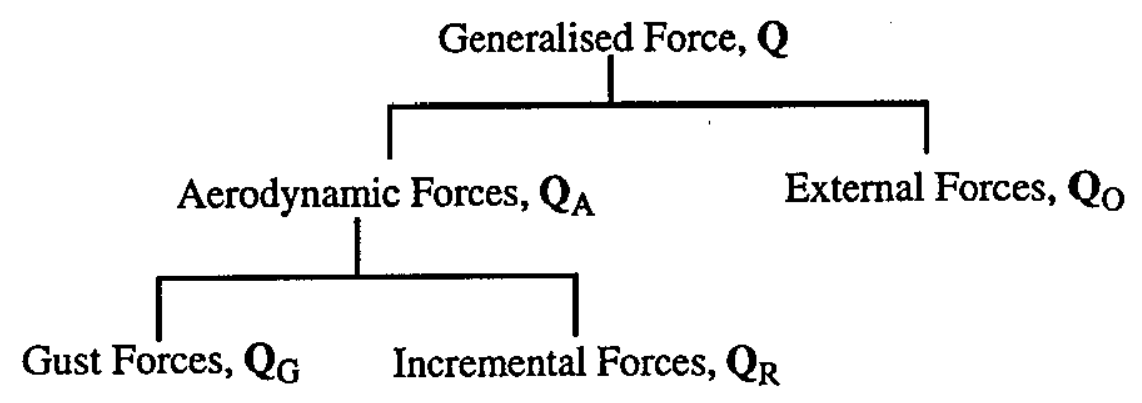

Figure 3.7 - Elements of the generalized loading vector

Assuming that $\mathbf{Q}_{0}$ is zero, then $\mathbf{Q}$ can be considered to consist of purely aerodynamic forces.

It is possible to split the aerodynamic force vector into two further components so that

$$
\mathbf{Q}_{\mathrm{A}}=\mathbf{Q}_{\mathrm{G}}+\mathbf{Q}_{\mathbf{R}}
$$

where,

$Q_{G}$ is the vector of gust forces on the structure, and

$\mathbf{Q}_{\mathrm{R}}$ is the vector of incremental aerodynamic forces on the structure due to the deflection of the structure itself.

Considering the case where the gust loads on the aircraft structure can be considered to be zero, then the generalised force vector $\mathbf{Q}$ can be considered to be made up of entirely incremental aerodynamic forces, so that equation (3.60) can be rewritten as

$$
A \ddot{q}+\mathbf{D} \dot{q}+\mathbf{E q}=\mathbf{Q}_{R}
$$

From equation (3.32), the generalised force vector can be expressed as a transformed version of the true load vector. In this case, the transformation vector, $\mathbf{Z}$, is the matrix of the eigenvectors of the true mass and stiffness matrices as derived earlier. This indicates that in order to generate the generalised force vector under the assumptions concerning the nature of the aerodynamic loads, it is only necessary to obtain the incremental forces and moments on the idealised structure due to the deflection of the structure itself. 


\subsubsection{Incremental aerodynamic forces on a vibrating structure}

The aerodynamic forces which act on the aircraft structure as a result of its response, $\mathbf{Q}_{\mathbf{R}}$,can be split into two distinct types, namely, aerodynamic stiffness and aerodynamic damping ${ }^{55}$. Aerodynamic stiffnesses are proportional to the structural displacements whereas aerodynamic damping forces are proportional to the normal structural velocity.

In order to obtain a suitable expression for these aerodynamic stiffness and damping forces, it is necessary to make some assumptions. Firstly, it is assumed that the aircraft structure is vibrating with simple harmonic motion, so that at any point, the displacement, $\mathrm{z}$, can be expressed as a function of time, where

$$
z=z_{0} e^{i \omega t}
$$

and hence, the velocity of the point can be expressed as

$$
\dot{z}=i \omega z_{0} e^{i \omega t}=i \omega z
$$

and similarly

$$
\begin{gathered}
\theta=\theta_{0} \mathrm{e}^{\mathrm{j} \omega t} \\
\dot{\theta}=\mathrm{i} \omega \theta_{0} \mathrm{e}^{\mathrm{i} \omega t}=\mathrm{i} \omega \theta
\end{gathered}
$$

Strictly, making this assumption means that the following derivation is only valid at the flutter speed, where the structural oscillations are undamped, and hence the motion can be considered as simple harmonic.

The second assumption, is that the aircraft structural vibration has existed for a time sufficient for an oscillatory downwash to be established. This would lead to the existence of constant aerodynamic derivatives provided that the forces are linear in nature. The assumption of the constant nature of the aerodynamic derivatives greatly simplifies the problem.

Since the structure is assumed to be vibrating with simple harmonic motion, the forces acting on the structure will not be the same as in the steady state. The forces vary as a function of the oscillatory frequency, $\omega$. As mentioned earlier, this dependence on the oscillatory frequency is encompassed within a parameter, $v$, the frequency parameter, which is a non-dimensional quantity defined as

$$
v=\frac{\omega l_{T}}{V_{T}}
$$

where,

$\mathbb{I}_{T}$ is the aircraft reference length in metres, and 
$\mathrm{V}_{\mathrm{T}}$ is the true airspeed of the aircraft in $\mathrm{m} / \mathrm{s}$

From Reference 55, the lift force and moment on an oscillating structure can be defined as

$$
\begin{gathered}
\mathrm{L}=\rho \mathrm{V}^{2} \mathrm{~S}\left(\left(\mathrm{ivl_{z }}+\mathrm{l}_{\mathrm{z}}\right) \frac{\mathrm{z}}{\mathrm{l}_{\mathrm{T}}}+\left(\mathrm{ivl_{ \theta }}+\mathrm{l}_{\theta}\right) \theta\right) \\
\mathrm{M}=\rho \mathrm{V}^{2} 1_{\mathrm{T}} \mathrm{S}\left(\left(\mathrm{ivm_{z }}+\mathrm{m}_{\mathrm{z}}\right) \frac{\mathrm{z}}{1_{\mathrm{T}}}+\left(\mathrm{ivm_{ \theta }}+\mathrm{m}_{\theta}\right) \theta\right)
\end{gathered}
$$

where,

$\rho$ is the airstream density $\left(\mathrm{kg} / \mathrm{m}^{3}\right)$,

$\mathrm{V}$ is the airspeed $(\mathrm{m} / \mathrm{s})$,

$\mathrm{l}_{\mathrm{T}}$ is the reference length $(\mathrm{m})$,

$\mathrm{S}$ is the surface area $\left(\mathrm{m}^{2}\right)$,

$\mathrm{z}, \theta$ are the displacements ( $\mathrm{m}$ and radians),

$l_{2}, l_{z}, l_{\theta}, l_{\theta}$ are lift force derivatives due to the vertical velocity, vertical displacement, rotational velocity and rotational displacement respectively,

$\mathrm{m}_{\mathrm{z}}, \mathrm{m}_{\mathrm{z}}, \mathrm{m}_{\theta}, \mathrm{m}_{\theta}$ are moment derivatives due to the vertical velocity, vertical displacement, rotational velocity and rotational displacement respectively

The imaginary terms in the above equations describe the phase relationships between the forces dependent on displacement and those dependent on velocity.

It should be noted that, in reality, there will also be contributions to the lift force and moment as a result of the accelerations in both the $\mathrm{z}$ and $\theta$ directions, which can be considered as aerodynamic inertia terms in the above equations. These aerodynamic inertias represent small positive additions to the structural inertias ${ }^{55}$, but they will be assumed to be negligible.

From Reference 55, these equations have been derived for a single aerofoil section oscillating in the airstream with oscillatory frequency $\omega$. Extending equations (3.69) and (3.70) for the idealised model of the aircraft structure, and assuming that the forces at any of the discrete points are functions of the displacements and velocities at all other points, the above two equations can be rewritten as

$$
\begin{aligned}
& \mathbf{L}=\sum_{k=1}^{p} \sigma V_{T}^{2} S_{k}\left(\sum_{n=1}^{p}\left(i v l_{t_{k s}}+l_{z_{k n}}\right) \frac{z_{n}}{l_{T_{2}}}+\left(i v l_{\theta_{k s}}+l_{\theta_{k e}}\right) \theta_{n}\right) \\
& \mathbf{M}=\sum_{k=1}^{p} \sigma V_{T}^{2} 1_{T_{k}} S_{k}\left(\sum_{n=1}^{p}\left(i v m_{t_{k=0}}+m_{z_{k=1}}\right) \frac{z_{n}}{1_{T_{k}}}+\left(i v m_{\theta_{k=0}}+m_{\theta_{k=1}}\right) \theta_{n}\right)
\end{aligned}
$$

where, 
$l_{k}$ is the reference length associated with discrete mass $k$,

$\sigma$ is the relative density,

$S_{k}$ is the reference surface area used in the normalisation of the derivatives for discrete mass $\mathrm{k}$

$\mathrm{p}$ is the number of discrete masses in the representation as earlier,

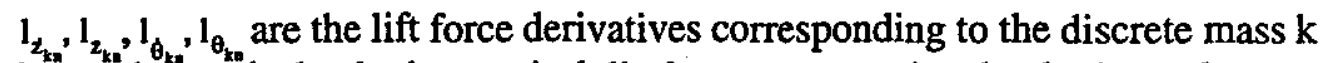
due to the vertical velocity, vertical displacement, rotational velocity and rotational displacement at discrete mass $\mathrm{n}$,

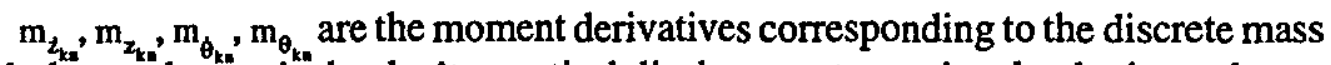
$\mathrm{k}$ due to the vertical velocity, vertical displacement, rotational velocity and rotational displacement at discrete mass $\mathbf{n}$

The derivatives have been normalised originally by $\sigma \mathrm{V}_{\mathrm{T}}{ }^{2}$ which is the convention used by British Aerospace.

Substituting in equations (3.69) and (3.70) for the frequency parameter $v$ as given in equation (3.68) and expanding gives,

$$
\begin{aligned}
& \mathbf{L}=\sum_{k=1}^{p} \sigma V_{T}^{2} S_{k}\left(\sum_{n=1}^{p}\left(i \frac{\omega l_{T_{k}}}{V_{T}} l_{t_{k n}}+l_{z_{k n}}\right) \frac{z_{n}}{1_{T_{k}}}+\left(i \frac{\omega l_{T_{k}}}{V_{T}} l_{\theta_{k n}}+l_{\theta_{k n}}\right) \theta_{n}\right) \\
& \mathbf{M}=\sum_{k=1}^{p} \sigma V_{T}^{2} 1_{T_{k}} S_{k}\left(\sum_{n=1}^{p}\left(i \frac{\omega l_{T_{k}}}{V_{T}} m_{t_{k 0}}+m_{z_{k n}}\right) \frac{z_{n}}{I_{T_{k}}}+\left(i \frac{\omega l_{T_{k}}}{V_{T}} m_{\theta_{k s}}+m_{\theta_{k n}}\right) \theta_{n}\right)
\end{aligned}
$$

Cancelling terms and substituting for $i \omega z$ and $i \omega \theta$ according to equations (3.65) and (3.64) respectively gives,

$$
\begin{aligned}
& \mathbf{L}=\sum_{k=1}^{p} \sigma V_{T} S_{k}\left(\sum_{n=1}^{p} 1_{t_{k n}} \dot{n}_{n}+V_{T} 1_{z_{k n}} \frac{z_{n}}{T_{k}}+l_{\theta_{k a}} \dot{\theta}_{n}+V_{T} 1_{\theta_{k a}} \theta_{n}\right) \\
& \mathbf{M}=\sum_{k=1}^{p} \sigma V_{T} 1_{T_{k}} S_{k}\left(\sum_{n=1}^{p} m_{k_{k n}} \dot{z}_{n}+V_{T} m_{z_{k a}} z_{n}+1_{T_{k}} m_{\theta_{k n}} \dot{\theta}_{n}+V_{T} m_{\theta_{k n}} \theta_{n}\right)
\end{aligned}
$$

Equations (3.75) and (3.75) can be rewritten and combined into a single matrix equation, such that,

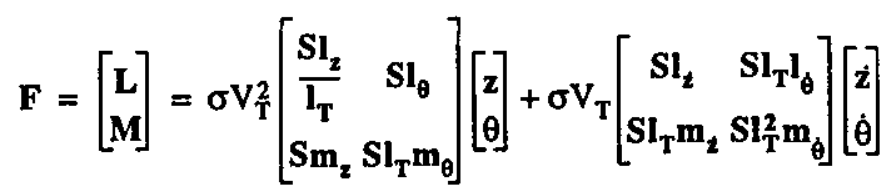

The values of the derivatives can be generated from various aerodynamic theories along with wind tunnel testing of suitable models ${ }^{56}$. Derivatives are generally obtained for displacements at points dictated by the aerodynamic theory, and these points will not in general coincide with the discrete mass points of the structural representation. The displacements used in the derivation of the derivatives however, 
are simply extrapolated from those at the discrete mass location, with the extrapolations being incorporated within the derivative values used in the equation (3.76).

Substituting for the displacement and velocity matrices from equation (3.13), and using equation (3.32), the generalised incremental force matrix due to the response of the structure can be derived as

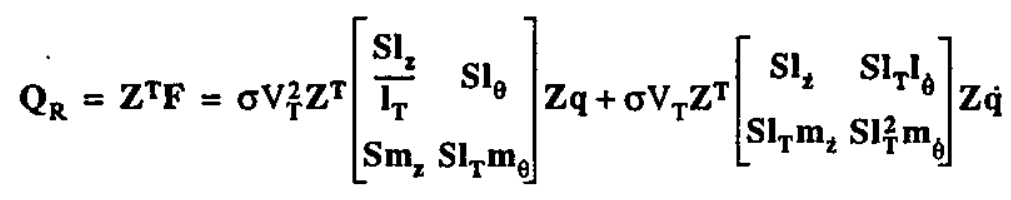

which can be simplified as,

$$
Q_{R}=\sigma V_{T} B \dot{q}+\sigma V_{T}^{2} \mathbf{C q}
$$

where,

$B$ is defined as the generalised aerodynamic damping matrix, and

$\mathbf{C}$ is defined as the generalised aerodynamic stiffness matrix

Substituting for $\mathbf{Q}_{\mathrm{R}}$ from equation (3.79) in equation (3.60) and taking the sign change within the $\mathbf{B}$ and $\mathbf{C}$ matrices gives the generalised equations of motion for the aircraft structure under incremental aerodynamic loading of frequency $\omega$,

$$
\mathbf{A} \ddot{\mathbf{q}}+\left(\mathbf{D}+\sigma \mathrm{V}_{\mathrm{T}} \mathbf{B}\right) \dot{\mathbf{q}}+\left(\mathbf{E}+\sigma \mathrm{V}_{\mathrm{T}}^{2} \mathbf{C}\right) \mathbf{q}=0
$$

As it stands, the above result represents the classic form of the aeroelastic equation. If further flight conditions were considered, with the variations in the aerodynamic derivatives being expressed as a function of frequency parameter and airspeed, it would be possible to calculate the speed at which certain structural modes would become unstable. These speeds would be the flutter speeds for the aircraft.

In this case however, the interactions between the structural dynamics, aerodynamics and flight control system are of interest. It is necessary therefore to include further elements to the classic aeroelasticity problem.

\subsection{Rigid-body dynamics}

\subsubsection{Introduction}

The equations of motion for the aircraft derived so far describe the dynamics of the aircraft structure with incremental loading as a result of the structural response. In order to obtain a full model of the aircraft, it is necessary to include in the equations suitable expressions to describe the rigid-body motion of the aircraft itself, with 
loading from suitable control surfaces.

The following section is dedicated to establishing the rigid-body equations of motion for the aircraft, and to incorporate these equations into the existing representation. As a result, the third element of the flexible aircraft model will be derived as shown in Figure 3.8.

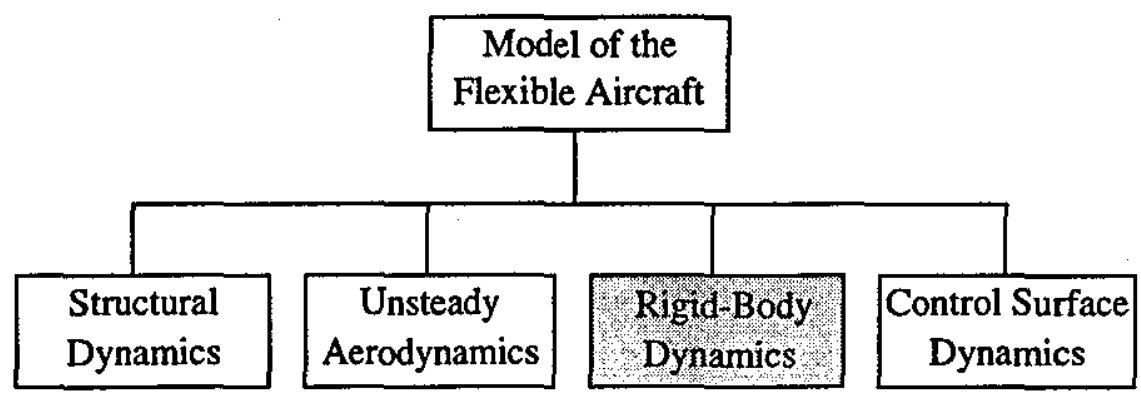

Figure 3.8 - Elements of a flexible aircraft model: rigid-body dynamics

\subsubsection{Derivation of rigid-body equations of motion}

The derivation of equations of motion for the rigid-body motion of an aircraft is an extremely well documented process, and it is not intended to give a full description of the process here. There are many suitable texts containing such a description, and the interested reader should consult one of these for a further description ${ }^{57}$.

For the configuration of the aircraft under consideration, the longitudinal equations of motion for straight and level flight can be written as ${ }^{57}$

$$
\begin{gathered}
\mathrm{m}_{\mathrm{T}} \dot{\mathrm{u}}-\mathrm{X}_{\mathrm{u}} \mathrm{u}-\mathrm{X}_{\dot{w}} \dot{\mathrm{w}}-\mathrm{X}_{\mathrm{w}} \mathrm{w}-\mathrm{X}_{\mathrm{q}} \mathrm{q}=\mathrm{X}_{\delta_{\mathrm{ib}}} \delta_{\mathrm{ib}}+\mathrm{X}_{\delta_{\mathrm{ob}}} \delta_{\mathrm{ob}}+\mathrm{X}_{\eta} \eta \\
\mathrm{m}_{\mathrm{T}} \dot{\mathrm{w}}-\mathrm{Z}_{\mathrm{u}} \mathrm{u}-\mathrm{Z}_{\mathrm{w}} \dot{\mathrm{w}}-\mathrm{Z}_{\mathrm{w}} \mathrm{w}-\mathrm{Z}_{\mathrm{q}} \mathrm{q}-\mathrm{m}_{\mathrm{T}} \mathrm{V}_{\mathrm{T}} \mathrm{q}=\mathrm{Z}_{\delta_{\mathrm{bb}}} \delta_{\mathrm{ib}}+\mathrm{Z}_{\delta_{\mathrm{ob}}} \delta_{\mathrm{ob}}+\mathrm{Z}_{\eta} \eta \\
\mathrm{I}_{\mathrm{y}_{\mathrm{T}}} \dot{\mathrm{q}}-\mathrm{M}_{\mathrm{u}} \mathrm{u}-\mathrm{M}_{\mathrm{w}} \dot{\mathrm{w}}-\mathrm{M}_{\mathrm{w}} \mathrm{w}-\mathrm{M}_{\mathrm{q}} \mathrm{q}=\mathrm{M}_{\delta_{\mathrm{ib}}} \delta_{\mathrm{ib}}+\mathrm{M}_{\delta_{\mathrm{ob}}} \delta_{o b}+\mathrm{M}_{\eta} \eta
\end{gathered}
$$

where,

$\mathrm{m}_{\mathrm{T}}$ is the total aircraft mass $(\mathrm{kg})$

$\mathrm{u}, \mathrm{w}, \mathrm{q}$ are the incremental translational and rotational velocities according to the axis system $(\mathrm{m} / \mathrm{s})$

$\delta_{i b}, \delta_{o b}, \eta$ are the inboard flap, outboard flap and foreplane surface deflections (Rads)

$\mathrm{I}_{\mathrm{yT}}$ is the aircraft second moment of inertia about the $y$-axis $\left(\mathrm{kgm}^{2}\right)$

$\mathrm{X}_{\mathrm{u}}, \mathrm{X}_{\mathrm{w}}, \mathrm{X}_{\mathrm{w}}, \mathrm{X}_{\mathrm{q}}$ are the $\mathrm{X}$-axis force derivatives due to incremental changes in the respective velocities and accelerations

$Z_{v}, Z_{w}, Z_{w}, Z_{q}$ are the $\mathbf{Z}$-axis force derivatives due to incremental changes in the respective velocities and accelerations

$\mathrm{M}_{\mathrm{u}}, \mathrm{M}_{w}, \mathrm{M}_{\mathrm{w}}, \mathrm{M}_{q}$ are the moment derivatives about the $\mathrm{y}$-axis due to incremental changes in the respective velocities and accelerations 
$\mathrm{X}_{\delta_{\mathrm{ib}}}, \mathrm{X}_{\delta_{\mathrm{o}}}, \mathrm{X}_{\eta}$ are the $\mathrm{x}$-axis force derivatives due to displacement of the inboard flap, outboard flap and foreplane surfaces

$Z_{\delta_{10}}, Z_{\delta_{0 b}}, Z_{\eta}$ are the $z$-axis force derivatives due to displacement of the inboard flap, outboard flap and foreplane surfaces

$\mathrm{M}_{\delta_{10}}, \mathbf{M}_{\delta_{\mathrm{ab}}}, \mathbf{M}_{\eta}$ are the moment derivatives about the $\mathrm{y}$-axis due to displacement of the inboard flap, outboard flap and foreplane surfaces

The above three equations thus represent the equations of motion for the canarddelta configuration shown in Figure 3.5. In order to simplify the analysis, and as the longitudinal motion of the aircraft is dominated by the short period rigid-body mode, the equation of motion along the $\mathrm{x}$-axis can be discarded. Eliminating equation (3.81) and terms in $u$ in equations (3.82) and (3.83), and reposing these equations in terms of the chosen states, $z$ and $\theta$, gives

$$
\begin{gathered}
\mathrm{m}_{\mathrm{T}} \ddot{z}-Z_{z} \ddot{z}-Z_{z} \dot{z}-Z_{\dot{\theta}} \dot{\theta}-m_{T} V_{T} \dot{\theta}=Z_{\delta_{b b}} \delta_{i b}+Z_{\delta_{o b}} \delta_{o b}+Z_{\eta} \eta \\
I_{y_{T}} \ddot{\theta}-M_{z} \ddot{z}-M_{z} \dot{z}-M_{\theta} \dot{\theta}=M_{\delta_{i b}} \delta_{i b}+M_{\delta_{o b}} \delta_{o b}+M_{\eta} \eta
\end{gathered}
$$

Writing equations (3.83) and (3.85) in matrix form results in

$$
\left[\begin{array}{cc}
\mathrm{m}_{\mathrm{T}}-\mathrm{Z}_{z} & 0 \\
-\mathrm{M}_{z} & \mathrm{I}_{\mathrm{y}_{\tau}}
\end{array}\right]\left[\begin{array}{l}
\ddot{z} \\
\ddot{\theta}
\end{array}\right]+\left[\begin{array}{cc}
-\mathrm{Z}_{\dot{z}}-\mathrm{Z}_{\dot{\theta}}-\mathrm{m}_{\mathrm{T}} \mathrm{V}_{\mathrm{T}} \\
-\mathrm{M}_{z} & -\mathrm{M}_{\dot{\theta}}
\end{array}\right]\left[\begin{array}{l}
\dot{z} \\
\dot{\theta}
\end{array}\right]=\left[\begin{array}{ccc}
\mathrm{z}_{\delta_{\mathrm{b}}} & \mathrm{z}_{\delta_{\mathrm{ob}}} & \mathrm{Z}_{\eta} \\
\mathrm{M}_{\delta_{\mathrm{ib}}} & \mathrm{M}_{\delta_{\mathrm{ob}}} & \mathrm{M}_{\eta}
\end{array}\right]\left[\begin{array}{c}
\delta_{\mathrm{ib}} \\
\delta_{\mathrm{ob}} \\
\eta
\end{array}\right]
$$

which can be written as

$$
M_{R} \dot{d}_{R}+D_{R} d_{R}=A_{S_{R}} d_{c}
$$

where the matrices are defined as in equation (3.86).

Equation (3.87) thus represents the equations of motion for the rigid-body aircraft in response to inputs in control surface deflection. To improve the model for use in the future work, it is necessary to add further equations of motion to describe the dynamics of the control surfaces themselves.

\subsection{Control surface dynamics}

\subsubsection{Introduction.}

It was stated in the above section that it is desirable to derive the equations describing the dynamics of the three control surfaces themselves. As for the aircraft structure in general, these control surfaces have inertia, stiffness and damping terms associated with them. In addition, they are undergoing forced motion initiated by hydraulic actuators against opposing aerodynamic forces. It is important therefore that equations describing their motion and effect on the other elements within the flexible 
aircraft model are derived. It will then be possible to incorporate these equations along with those for the rigid-body, equation (3.87), into the generalised equations describing the structural dynamics and structurally induced aerodynamic effects, equation (3.80). The following section forms the final part of the flexible aircraft model as shown in Figure 3.9 and will describe the derivation of these equations of motion in a form suitable for inclusion into the full flexible aircraft model.

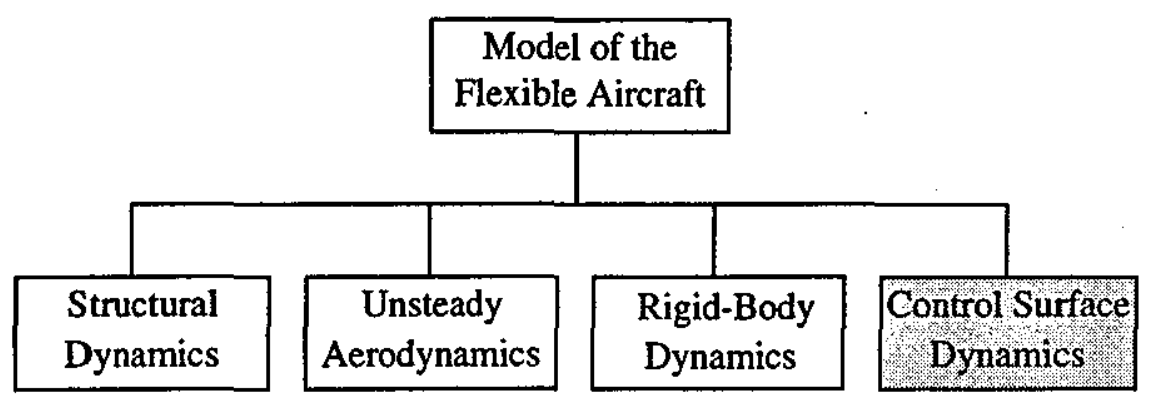

Figure 3.9 - Elements of a flexible aircraft model: control surface dynamics

\subsubsection{Derivation of equations of motion for control surfaces}

Considering an arbitrary control surface, the loading of the control surface can be split into two distinct components. The hydraulic actuation of the control surface is applying a force to the control surface which is opposed by the aerodynamic loading of the surface. Considering the action of the actuator, and assuming that the control surface moves as a rigid-body in rotation only, the equation of motion of the control surface can be derived as

$$
\mathrm{I} \ddot{\delta}+\mathrm{d} \dot{\delta}+\mathrm{k} \delta=\mathrm{k} \delta_{\mathrm{dem}}+\mathrm{d} \dot{\delta}_{\mathrm{dem}}
$$

where,

I is the rotational inertia of the control surface

$\mathrm{d}$ is the viscous damping associated with rotation of the control surface

$\mathrm{k}$ is the stiffness associated with rotation of the control surface

$\delta$ is the actual deflection of the control surface

$\delta_{\mathrm{dem}}$ is the demanded deflection of the control surface as dictated by the motion of the ram of the hydraulic actuator

Considering the aerodynamic effects of the motion of the control surface, there will be additional terms involved in the incremental loading of the aircraft structure, equations (3.69) and (3.70), due to the motion of the control surface. There will also be loading of the control surface itself as a result of both the structural response and of the control surface motion.

Considering the additional terms involved in the loading of the aircraft structure as 
a result of the control surface motion, suitable equations can be derived as ${ }^{55}$

$$
\begin{gathered}
\mathrm{L}=\rho \mathrm{V}^{2} \mathrm{~S}\left(\mathrm{ivl_{ \delta }}+\mathrm{l}_{\delta}\right) \delta \\
\mathrm{M}=\rho \mathrm{V}^{2} \mathrm{l}_{\mathrm{T}} \mathrm{S}\left(\mathrm{ivm}_{\delta}+\mathrm{m}_{\delta}\right) \delta
\end{gathered}
$$

where,

$\rho$ is the airstream density $\left(\mathrm{kg} / \mathrm{m}^{3}\right)$,

$V$ is the airspeed $(\mathrm{m} / \mathrm{s})$

$v$ is the frequency parameter

$\mathrm{l}_{\mathrm{T}}$ is the reference length $(\mathrm{m})$,

$S$ is the surface area $\left(\mathrm{m}^{2}\right)$,

$\delta$ are the displacements of the control surface (rads),

$l_{\delta}, l_{\delta}$ are lift force derivatives due to the rotational velocity and rotational displacement of the control surface

$\mathrm{m}_{\delta}, \mathrm{m}_{\delta}$ are moment derivatives due to the rotational velocity and rotational displacement of the control surface

which can be rearranged as in section 3.3.3 above resulting in a matrix equation for the additional loading in the form of

$$
\left[\begin{array}{l}
\mathrm{L} \\
\mathrm{M}
\end{array}\right]=\sigma \mathrm{V}_{\mathrm{T}}\left[\begin{array}{c}
\mathrm{Sl}_{\mathrm{T}} \mathrm{l}_{\delta} \\
\mathrm{Sl}_{\mathrm{T}} \mathrm{m}_{\delta}
\end{array}\right] \delta+\sigma \mathrm{V}_{\mathrm{T}}^{2}\left[\begin{array}{c}
\mathrm{Sl}_{\delta} \\
\mathrm{Sl}_{\mathrm{T}} \mathrm{m}_{\delta}
\end{array}\right] \delta
$$

where the derivatives have been normalised by the true airspeed, $V_{T}$, and the relative density, $\sigma$.

Equation (3.91) can be seen to be of a form similar to that for the existing incremental aerodynamic loading due to structural response as in equation (3.77). In effect, when these additional forces are added to this equation, the control surface can be considered as being just an additional structural element in the original derivation. It has been assumed here, that the motion of this arbitrary control surface can also be assumed to be simple harmonic in nature.

Considering the loading of the control surface itself due its motion and the response of the aircraft structure, the hinge moment equation for the control surface could be written as ${ }^{55}$

$$
H=\rho V^{2} I_{T} S\left(\left(i v h_{t}+h_{Z}\right) \frac{z}{l_{T}}+\left(i v h_{\theta}+h_{\theta}\right) \theta+\left(i v h_{\delta}+h_{\delta}\right) \delta\right)
$$

where,

$h_{i}, h_{2}, h_{\theta}, h_{\theta}$ are the hinge moment derivatives due to the vertical velocity, vertical displacement, rotational velocity and rotational displacement of the struc- 
ture respectively

$h_{\delta}, h_{\delta}$ are the hinge moment derivatives due to the control surface velocity and displacement

Rewriting this equation in a form suitable for describing the discrete nature of the aircraft structure, and performing the substitutions as in section 3.3.3 above, the matrix equation describing the aerodynamic loading of the arbitrary control surface can be written as

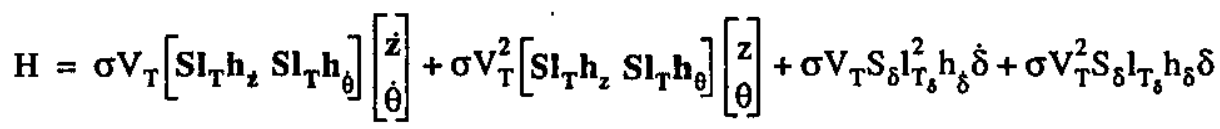

where,

$S_{\delta}$ is the reference area used for the normalisation of the derivatives for the arbitrary control surface, and

$I_{T_{8}}$ is the reference length of the arbitrary control surface.

Equation (3.93) thus represents the hinge moment produced on an arbitrary control surface, due to the response of the aircraft structure, and also due to the motion of the control surface itself.

It is possible then to combine equation (3.88) with equation (3.93) to produce the equation of motion for an arbitrary control surface,

$$
\begin{gathered}
I \ddot{\delta}+d \delta+k \delta-\sigma V_{T}\left[S_{T} h_{z} S l_{T} h_{\dot{\theta}}\right]\left[\begin{array}{l}
\ddot{z} \\
\dot{\theta}
\end{array}\right]-\sigma V_{T}^{2}\left[S l_{T} h_{z} S l_{T} h_{\theta}\right]\left[\begin{array}{l}
z \\
\theta
\end{array}\right] \\
-\sigma V_{T} S_{\delta} l_{T_{\delta}}^{2} h_{\delta} \delta-\sigma V_{T}^{2} S_{\delta} l_{T_{\delta}} h_{\delta} \delta=k \delta_{d e m}
\end{gathered}
$$

It has been assumed that the loading of the control surface due to the demanded rate, $\dot{\delta}_{\text {dem }}$ is negligible when compared with that due to the demanded displacement, $\delta_{\text {dem }}$.

It can be seen that the form of dynamics of the arbitrary control surface above match those for the structural dynamics derived earlier, in that there are terms of structural damping and stiffness, and also terms of aerodynamic damping and stiffness

\subsection{Assembling the flexible aircraft model}

\subsubsection{Introduction}

In order to obtain a useful flexible aircraft model, the equations of motion derived so far all need to be combined into a single set of equations. These equations will thus describe the response of the aircraft in terms of its rigid-body motion, structural response and control surface response to an input in the form of an actuator demanded 
control surface angle.

In the following section, the equations which have been derived will be assembled to give the full model for the flexible aircraft.

\subsubsection{Assembly of the full inertia matrix}

The inertia matrix for the system can be built up from the separate inertia matrices of the aircraft structure, control surfaces and rigid-body motion respectively. From equation (3.17), the inertia matrix for the aircraft structure can be assembled from suitable finite element modelling on packages such as NASTRAN. This will produce a discretised inertia matrix for the aircraft structure, $\mathbf{M}_{\mathbf{s}}$ in the form shown in equation (3.17),

$$
M_{S}=\left[\begin{array}{cc}
m_{S} & 0 \\
0 & I_{S}
\end{array}\right]
$$

The mass matrix, $\mathbf{m}_{\mathbf{s}}$, and inertia matrix, $\mathbf{I}_{\mathbf{s}}$, correspond to a displacement matrix for the structure of the form used in section 3.2.3. In fact, this inertia matrix is not used in this form, but is rearranged to correspond to a structural displacement vector, $d_{S}$ of the form

$$
d_{s}=\left[\begin{array}{c}
z_{1} \\
\theta_{1} \\
z_{2} \\
\theta_{2} \\
\cdots \\
\cdots \\
z_{p} \\
\theta_{p}
\end{array}\right]
$$

The inertia matrix for the control surfaces can be derived from the equation for the arbitrary control surface equations of motion, equation (3.94), so that

$$
\mathbf{M}_{\mathbf{C}}=\left[\begin{array}{ccc}
\mathrm{I}_{\delta_{1 b}} & 0 & 0 \\
0 & \mathrm{I}_{\delta_{\mathrm{ob}}} & 0 \\
0 & 0 & \mathrm{I}_{\eta}
\end{array}\right]
$$

where the inertias correspond to the second moment of inertia of the three control surfaces about their hinge lines.

The final component of the inertia matrix for the whole aircraft consists of the inertias corresponding to the rigid-body motion of the aircraft. This can be derived from equation (3.87) with the rigid aircraft inertia matrix, $\mathbf{M}_{R}$, being as defined.

To obtain the full inertia matrix for the aircraft, it is only a matter of assembling 
the above three inertia matrices. This results in the following inertia matrix and true displacement vector for the aircraft model

$$
\begin{gathered}
M=\left[\begin{array}{ccc}
M_{S} & 0 & 0 \\
0 & M_{C} & 0 \\
0 & 0 & M_{R}
\end{array}\right] \\
d_{t}=\left[\begin{array}{c}
z_{1} \\
\theta_{1} \\
z_{2} \\
\cdots \\
\cdots \\
z_{p} \\
\theta_{p} \\
\delta_{i b} \\
\delta_{o b} \\
\eta \\
z_{R} \\
\theta_{R}
\end{array}\right]
\end{gathered}
$$

where,

$z_{1}, \theta_{1}, z_{2}, \theta_{2}, \ldots . z_{p}, \theta_{p}$ are the displacements of the $p$ masses making up the discrete aircraft structure ( $\mathrm{m}$ and radians),

$\delta_{\mathrm{ib}}, \delta_{\mathrm{ob}}, \eta$ are the inboard flap, outboard flap and foreplane surface deflections (radians), and

$\mathrm{z}_{\mathrm{R}}, \theta_{\mathrm{R}}$ are the rigid-body displacement ( $\mathrm{m}$ and radians)

\subsubsection{Assembly of the full structural stiffness matrix}

Using a similar approach to that used for the inertia matrix above, it is possible to generate the full structural stiffness matrix for the aircraft model. Once again the stiffness matrix for the aircraft structure is obtained from a NASTRAN model of the discretised aircraft structure, and is arranged to match the new displacement vector, $\mathbf{d}_{\mathrm{s}}$, as shown in equation (3.96) above. This results in a structural stiffness matrix of the form

$$
\mathbf{K}_{\mathbf{s}}=\left[\begin{array}{cccccc}
\mathbf{k}_{z_{1} z_{1}} & \mathbf{k}_{z_{1} \theta_{1}} & \ldots & \ldots & k_{z_{1} z_{p}} & k_{z_{1} \theta_{p}} \\
\cdots & \cdots & \cdots & \cdots & \cdots & \cdots \\
\cdots & \cdots & \cdots & \cdots & \cdots & \cdots \\
\cdots & \cdots & \cdots & \cdots & \cdots & \cdots \\
\cdots & \cdots & \cdots & \cdots & \cdots & \cdots \\
k_{\theta_{p} z_{1}} & k_{\theta_{p} \theta_{1}} & \cdots & \cdots & k_{\theta_{p} z_{p}} & k_{\theta_{p} \theta_{d}}
\end{array}\right]
$$

This may be combined with the control surface stiffness matrix generated from the 
equations of motion for the arbitrary control surface,(3.94), where

$$
\mathbf{K}_{\mathbf{C}}=\left[\begin{array}{ccc}
\mathrm{k}_{\delta_{\mathrm{ib}}} & 0 & 0 \\
0 & \mathrm{k}_{\delta_{\mathrm{ob}}} & 0 \\
0 & 0 & \mathrm{k}_{\eta}
\end{array}\right]
$$

As there is no body stiffness terms in the equations of motion for the rigid-body aircraft, the aircraft stiffness matrix can be assembled from equations (3.100) and (3.101) such that

$$
K=\left[\begin{array}{ccc}
K_{s} & 0 & 0 \\
0 . & \mathbf{K}_{C} & 0 \\
0 & 0 & 0
\end{array}\right]
$$

\subsubsection{Conversion to a generalised coordinate system}

The conversion from the true displacement coordinate system to that of a generalised coordinate system has been covered in some detail in section 3.2.4. It has been stated that the usual practice is to convert to a set of coordinates which describe the uncoupled or normal modes of the dynamic system, which has the effect of diagonalising the resultant generalised inertia and stiffness matrices, reducing the complexity of the problem significantly. In this case, it is desirable to maintain the true displacements as modes for the control surface dynamics and rigid-body motion. This maintains a simple relationship between the inertia and stiffness matrix elements and the physical problem for the control surface modes and rigid-body motion.

It is now possible to generalise the inertia and stiffness matrices according to section 3.2.4. In order to maintain the coordinate system for the control surface modes and rigid-body dynamics however, the transformation matrix, $\mathbf{Z}$, must be constructed as follows.

In order to diagonalise the structural response terms, the eigensolution of the inertia and stiffness matrices for the structural dynamics is found so that

$$
\mathbf{M}_{\mathrm{S}}^{-1} \mathbf{K}_{\mathrm{S}}=\mathbf{Z}_{\mathrm{S}} \Lambda_{\mathrm{S}} \mathbf{Z}_{\mathrm{S}}^{-1}
$$

Thus in order to diagonalise the structural response, whilst maintaining the coordinate system for the control surface response and rigid-body dynamics, the transformation matrix $\mathbf{Z}$ could be defined as

$$
Z=\left[\begin{array}{ccc}
Z_{s} & 0 & 0 \\
0 & I & 0 \\
0 & 0 & I
\end{array}\right]
$$

This transformation matrix does not take into account the interaction between any 
rigid-body motion and the structural motion however.

In order to generate the correct transformation matrix, $\mathbf{Z}$, it is also necessary to consider the effect of the addition of the rigid aircraft motion to the structural response. The original equations for the structural response were derived using a set of displacements of the discrete masses, d, relative to an axis fixed on the aircraft centre of gravity. When the rigid-body motion is added to the system, it is important to add the effects of this motion on the motion of the structure itself, effectively transforming the displacements to a fixed inertial axis. In other words, for any particular mass location, the true displacement of the mass is a superposition of its displacements due to the structural response and its displacements due the rigid-body motion. This effect can be described for an arbitrary mass location, assuming small displacements, by equations of the form

$$
\begin{gathered}
z_{t}=z+z_{R}+x \theta \\
\theta_{t}=\theta+\theta_{R}
\end{gathered}
$$

where,

$z_{t}$ is the total displacement of the discrete mass point along the $z$-axis (m), $\mathrm{z}$ is the displacement of the discrete mass point along the $\mathrm{z}$-axis due to the structural response $(\mathrm{m})$

$\mathrm{z}_{\mathrm{R}}$ is the displacement of the centre of gravity of the aircraft due to the rigid-body dynamics $(\mathrm{m})$

$\mathrm{x}$ is the distance along the $\mathrm{x}$-axis from the centre of gravity of the aircraft to the discrete mass point (m)

$\theta_{t}$ is the total rotation about the $y$-axis of the discrete mass point (rads),

$\theta$ is the rotation about the $y$-axis of the discrete mass point due to the structural response (rads), and,

$\theta_{R}$ is the rotation of the rigid aircraft about its centre of gravity due to the rigidbody motion (rads).

Rewriting equations (3.105) and (3.106) in matrix form for the complete aircraft gives 


$$
\mathbf{d}_{t}=\left[\begin{array}{c}
z_{1} \\
\theta_{1} \\
z_{2} \\
\cdots \\
\cdots \\
z_{p} \\
\theta_{p} \\
\delta_{i b} \\
\delta_{o b} \\
\eta \\
z_{R} \\
\theta_{R}
\end{array}\right]=d+\left[\begin{array}{cc}
1 & x_{1} \\
0 & 1 \\
1 & x_{2} \\
0 & 1 \\
\cdots & \cdots \\
1 & x_{p} \\
0 & 1 \\
0 & 0 \\
0 & 0 \\
0 & 0 \\
0 & 0 \\
0 & 0
\end{array}\right]\left[\begin{array}{c}
z_{R} \\
\theta_{R}
\end{array}\right]=d+\Delta\left[\begin{array}{c}
z_{R} \\
\theta_{R}
\end{array}\right]
$$

Therefore, these effects can be incorporated into the transformation matrix, such that equation (3.104) should read

$$
Z=\left[\begin{array}{ccc}
\mathbf{Z}_{S} & 0 & \Delta \\
0 & \mathbf{I} & 0 \\
0 & 0 & \mathbf{I}
\end{array}\right]
$$

Therefore, the transformation matrix above can be used to generate a suitable generalised coordinate system. This system diagonalises the structural dynamics, producing expressions for the decoupled or normal modes of the structure, whilst maintaining the original coordinate systems for the control surface dynamics and rigid-body dynamics.

Thus the generalised inertia and stiffness matrices for the full aircraft model can be obtained using equations (3.22) and (3.26). Taking the full inertia and stiffness matrices from equations (3.98) and (3.102), and using the transformation matrix as given in equation (3.108) results in

$$
\begin{aligned}
& \mathbf{A}=\mathbf{Z}^{\mathbf{T}} \mathbf{M Z} \\
& \mathbf{E}=\mathbf{Z}^{\mathbf{T}} \mathbf{K Z}
\end{aligned}
$$

which are similar to those shown in equation (3.108), but include terms for the control surface dynamics and rigid-body motion.

\subsubsection{Assembly of the Aerodynamic stiffness matrix}

It has been shown in sections 3.3.3,3.4 and 3.5 that the aerodynamic forces on the aircraft can be split into two distinct components - those proportional to velocity, and those proportional to displacement. The forces proportional to displacement can be considered as aerodynamic stiffness terms, with contributions from the structural response, control surface dynamics and rigid-body motion. 
Consider the forces which are proportional to displacement. The displacements of the structure, control surfaces and rigid-body motion all produce force increments which will be due to the changes in the aerodynamic loading across the aircraft. The results obtained for these forces in equations such as (3.77), (3.86) and (3.93) show that a change in any one displacement will have an effect on the aerodynamic loading at all other points. This may be expressed as a full aerodynamic stiffness matrix for the aircraft, which contains submatrices describing the aerodynamic stiffnesses for the structural response, control surface dynamics and rigid-body motion. In addition, matrices describing the cross-coupling of these stiffness terms between (say) the structural response and the control surface dynamics will also be included. The aerodynamic forces on the aircraft as a result of the displacement of the aircraft structure, control surfaces and rigid-body motion could thus be combined in a single equation such that

$$
\mathbf{F}_{\mathrm{A}_{s}}=\sigma \mathrm{V}_{\mathrm{T}}^{2} \mathbf{K}_{\mathrm{A}} \mathbf{d}_{\mathrm{t}}
$$

where the derivatives contained within the aerodynamic stiffness matrix are obtained from analysis and wind tunnel testing as mentioned earlier.

From equation (3.32), these forces can be transformed into the generalised coordinate system such that

$$
\mathbf{Q}_{\mathbf{A}_{\mathbf{s}}}=\mathbf{Z}^{\mathbf{T}} \mathbf{F}_{\mathbf{A}_{\mathbf{s}}}
$$

such that

$$
\mathbf{Q}_{A_{\mathbf{s}}}=\sigma \mathbf{V}_{\mathrm{T}}^{2} \mathbf{Z}^{\mathbf{T}} \mathbf{K}_{\mathrm{A}} \mathbf{Z q}
$$

or

$$
\mathbf{Q}_{\mathbf{A}_{s}}=\sigma \mathrm{V}_{\mathrm{T}}^{2} \mathbf{C q}
$$

where, $\mathbf{C}$ is the generalised aerodynamic stiffness matrix, as shown in equation (3.80), but which now includes terms for the control surface dynamics and rigid-body motion.

\subsubsection{Assembly of the Aerodynamic damping matrix}

The assembly of the aerodynamic damping matrix follows exactly the same reasoning of section 3.6.5, where the terms obtained for aerodynamic forces which are proportional to velocity from equations (3.77), (3.86) and (3.93) can be combined and extended to include further cross-coupling effects in a single equation of the form

$$
\mathbf{F}_{\mathbf{A}_{\mathbf{D}}}=\sigma \mathrm{V}_{\mathrm{T}} \mathbf{D}_{\mathbf{A}} \mathbf{d}_{\mathbf{t}}
$$


where the derivatives contained within the aerodynamic stiffness matrix have again been obtained from analysis and wind tunnel testing.

Converting to the generalised coordinate system gives,

$$
\mathbf{Q}_{\mathbf{A}_{\mathrm{D}}}=\sigma \mathrm{V}_{\mathrm{T}} \mathbf{Z}^{\mathrm{T}} \mathbf{D}_{\mathbf{A}} \mathbf{Z q}
$$

so that the generalised aerodynamic damping matrix can be defined for the full aircraft model as

$$
\mathbf{B}=\mathbf{Z}^{\mathbf{T}} \mathbf{D}_{\mathbf{A}} \mathbf{Z}
$$

\subsubsection{Full flexible aircraft model}

It is now possible to assemble the full equations of motion for the flexible aircraft in the chosen generalised coordinate system, by applying the full model inertia, stiffness, damping, aerodynamic damping and aerodynamic stiffness matrices as derived in section 3.6 to the equation for the structural dynamics alone as derived in equation (3.80). So that,

$$
\mathbf{A} \ddot{q}+\left(\mathbf{D}+\sigma \mathrm{V}_{\mathbf{T}} \mathbf{B}\right) \dot{q}+\left(\mathbf{E}+\sigma \mathrm{V}_{\mathrm{T}}^{2} \mathbf{C}\right) \mathbf{q}=\mathbf{0}
$$

This represents the equations of motion for the aircraft structure, control surface dynamics, and rigid-body motion at a particular flight condition and oscillatory aerodynamic state used in the derivation of the aerodynamic terms.

In order for the equation to be of real use, the forcing function must also be included on the right hand side of the above equation. This forcing function describes the input to the system, which for this formulation is expressed in terms of the desired control surface angles as dictated by the actuator ram positions. From the equation for the arbitrary control surface dynamics, equation (3.94), the forcing function to the whole system can be seen to be

$$
\text { Input Force to System }=k_{\delta_{i b}} \delta_{i b_{\text {dem }}}+k_{\delta_{o b}} \delta_{o b_{d e x}}+k_{\eta} \eta_{\text {dem }}
$$

which could be incorporated into equation (3.118) as the forcing function so that the final equation for the flexible aircraft model is

$$
\mathbf{A} \ddot{q}+\left(\mathbf{D}+\sigma \mathrm{V}_{\mathbf{T}} \mathbf{B}\right) \dot{q}+\left(\mathbf{E}+\sigma \mathrm{V}_{\mathrm{T}}^{2} \mathbf{C}\right) \mathbf{q}=\mathbf{F}\left[\begin{array}{l}
\delta_{i b_{\text {dem }}} \\
\delta_{o b_{\text {dem }}} \\
\eta_{\text {dem }}
\end{array}\right]
$$

which can be written as

$$
A \ddot{q}+\left(\mathbf{D}+\sigma V_{T} \mathbf{B}\right) \dot{q}+\left(\mathbf{E}+\sigma V_{T}^{2} \mathbf{C}\right) \mathbf{q}=\mathbf{F u}
$$


where,

$\mathbf{u}$ is as defined in equation (3.118), and

$\mathbf{F}$ is defined by

$$
\mathbf{F}=\left[\begin{array}{ccc}
0 & 0 & 0 \\
0 & 0 & 0 \\
\cdots & \cdots & \cdots \\
\cdots & \cdots & \cdots \\
0 & 0 & 0 \\
\mathrm{k}_{\delta_{i b}} & 0 & 0 \\
0 & \mathrm{k}_{\delta_{o b}} & 0 \\
0 & 0 & \mathrm{k}_{\eta} \\
0 & 0 & 0 \\
0 & 0 & 0
\end{array}\right]
$$

Strictly speaking, the forcing function should be generalised to conform with the generalised nature of the right hand side of the equations of motion. Since the control surface deflections have been maintained within the generalised representation however, the forcing function is already in a form suitable for inclusion. If for example, the forcing function matrix, $\mathbf{F}$, was generalised using the transformation $\mathbf{Z}$, the forcing function matrix would be unchanged.

\subsection{Reduction of the flexible aircraft model}

\section{$\underline{3.7 .1 \text { Introduction }}$}

Equation (3.118) above represents the equations of motion for the aircraft in the form that will be used in subsequent work. One of the problems that remains however is that the equation in its full form is very large, with several hundred discrete masses being used to idealise the structure. For 200 discrete mass points for example, the resulting inertia, stiffness and damping matrices in equation (3.118) would each be of the order of 405 by 405 for an aircraft with three control surfaces. Clearly, it is desirable to reduce the order of the model to reduce the computational burden whilst maintaining a realistic representation.

\subsubsection{Selection of suitable normal modes}

In the above example, with an aircraft idealised by 200 discrete mass points, the eigensolution of the structural inertia and stiffness matrices would result in the identification of 400 normal modes of the system. The eigenvectors describe the normalised displacements at each of the mass points for each of the 400 modes, thus resulting in a full transformation matrix of order 405 by 405 .

Many of the normal modes identified by the eigensolution would be at frequencies 
much higher than of interest in the aeroservoelastic problem however, and it is possible to delete the eigenvectors corresponding to these modes from the transformation matrix $\mathbf{Z}$. This would reduce the order of the transformation matrix to the order of 405 by 60 , where (say) 55 normal modes of the structure have been retained along with the representations of the control surface modes and rigid aircraft dynamics. Thus, using the reduced transformation matrix in this form, the generalised inertia, stiffness and damping matrices (both dynamic and aerodynamic) would be reduced to the order of 60 by 60 whilst maintaining the accuracy of the model within the bandwidth of interest.

The ability to delete normal modes in this way shows one of the great advantages of expressing the equations of motion in such a generalised form. By generalising the equations in this way, it is possible to decouple the modes, so that upon deletion of one of the modes, the remaining modes are unaffected.

In the reduced form, the equations of motion may be solved for the 55 normal modes selected. The displacements at the original 200 discrete mass points due to the 55 normal modes, control surface dynamics and rigid-body motion, are then resolvable using the reduced $\mathbf{Z}$ matrix.

\subsection{Conversion of the flexible aircraft model into state-space form}

\subsubsection{Introduction}

The matrices for the flexible aircraft model were provided initially by British Aerospace in a form suitable for inclusion within equation (3.118). In order to incorporate the reduced order flexible aircraft model into a full system model in the MATLAB/SIMULINK environment, it is necessary to convert equation (3.118) into a state-space form. It is also necessary to convert the outputs of the flexible aircraft model into meaningful flight control system variables as measured by a sensor rigidly mounted on the aircraft at a particular point.

\subsubsection{Conversion of the flexible aircraft equations into state-space form}

Pre-multiplying equation (3.118) by $\mathbf{A}^{-1}$ and rearranging gives,

$$
\ddot{q}=-A^{-1}\left(D+\sigma V_{T} B\right) \dot{q}-A^{-1}\left(E+\sigma V_{T}^{2} C\right) q+A^{-1} F u
$$

Letting,

$$
\begin{aligned}
& x_{1}=\dot{q} \\
& x_{2}=q
\end{aligned}
$$


then equation (3.123) can be written as two first order equations such that,

$$
\begin{gathered}
\dot{x}_{1}=-A^{-1}\left(D+\sigma V_{T} B\right) x_{1}-A^{-1}\left(E+\sigma V_{T}^{2} C\right) x_{2}+A^{-1} F u \\
\dot{x}_{2}=x_{1}
\end{gathered}
$$

Which can be written in matrix form as,

$$
\left[\begin{array}{l}
\dot{x}_{1} \\
\dot{x}_{2}
\end{array}\right]=\left[\begin{array}{c}
-A^{-1}\left(\mathbf{D}+\sigma V_{T} \mathbf{B}\right)-A^{-1}\left(\mathbf{E}+\sigma V_{T}^{2} \mathbf{C}\right) \\
\mathbf{I}
\end{array}\right]\left[\begin{array}{l}
\mathbf{x}_{1} \\
\mathbf{x}_{2}
\end{array}\right]+\left[\begin{array}{c}
\mathbf{A}^{-1} \mathbf{F} \\
0
\end{array}\right] \mathbf{u}
$$

which is partially in the standard state-space form suitable for implementation into a MATLAB model. In order to complete the representation, it is necessary to derive the c and $\mathbf{d}$ matrices such that the standard state-space representation

$$
\begin{aligned}
& \dot{x}=a x+b u \\
& y=c x+d u
\end{aligned}
$$

is satisfied. Now, in order to transform from the generalised coordinates, $\mathbf{q}$, to the actual displacements of the aircraft discrete mass points, the transformation matrix, $\mathbf{Z}$ can be used as in equation (3.42), so that the displacement of the aircraft structure at any one of the mass points can be deduced from $\mathbf{q}$. In reality, the matrices for the flexible aircraft model were provided by British Aerospace along with a reduced order transformation matrix. This transformation matrix enabled the displacements of twenty points along the aircraft fuselage to be calculated from the generalised coordinate vector $\mathbf{q}$. The locations of these twenty points along the fuselage are as shown in Figure 3.10, such that

$$
\mathbf{d}_{F}=\left[\begin{array}{c}
z_{1} \\
\theta_{1} \\
z_{1} \\
\theta_{2} \\
\cdots \\
\cdots \\
z_{20} \\
\theta_{20}
\end{array}\right]=Z^{\prime} \mathbf{q}
$$

where $\mathbf{Z}$ is the reduced transformation matrix as supplied by British Aerospace. 


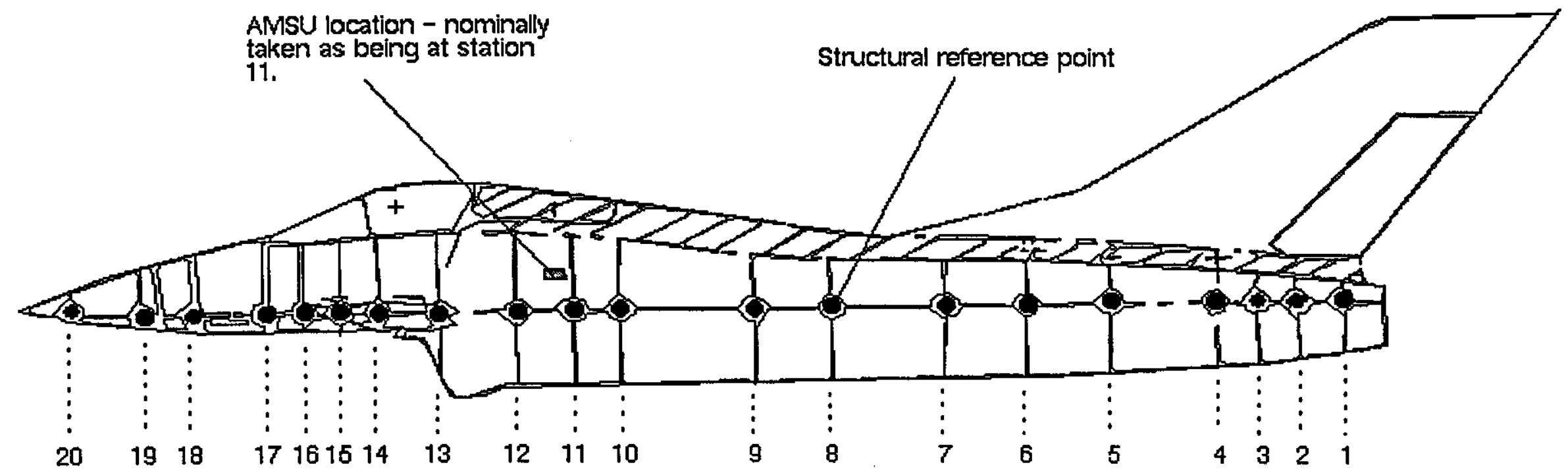

Figure 3.10 - Fuselage centre-line structural node locations

\begin{tabular}{|c|c|c|c|c|c|c|c|c|c|c|c|c|c|c|c|c|c|c|c|c|}
\hline $\begin{array}{c}\text { Fuselage centre-line struc- } \\
\text { tural node }\end{array}$ & 20 & 19 & 18 & 17 & 16 & 15 & 14 & 13 & 12 & 11 & 10 & 9 & 8 & 7 & 6 & 5 & 4 & 3 & 2 & 1 \\
\hline $\begin{array}{c}\text { Distance from structural } \\
\text { reference point (m) }\end{array}$ & 8.509 & 7.582 & 6.980 & 6.210 & 5.812 & 5.388 & 4.955 & 4.233 & 3.388 & 2.810 & 2.340 & 0.870 & 0.0 & -1.08 & -1.99 & -3.01 & -4.00 & -4.49 & -4.94 & -5.39 \\
\hline $\begin{array}{c}\text { Z matrix rows correspond- } \\
\text { ing to z, } \theta \text { deflections }\end{array}$ & 39,40 & 37,38 & 35,36 & 33,34 & 31,32 & 29,30 & 27,28 & 25,26 & 23,24 & 21,22 & 19,20 & 17,18 & 15,16 & 13,14 & 11,12 & 9,10 & 7,8 & 5,6 & 3,4 & 1,2 \\
\hline
\end{tabular}


Although the flexible aircraft model was supplied by British Aerospace initially, it was found that some work was needed to make the model more representative of the real aircraft. This modification of the flexible aircraft model will be discussed later in the chapter.

Considering the flight control system feedback variables which are the required output variables from the state-space flexible aircraft model, the equations are given by

$$
\begin{gathered}
\mathfrak{q}=\left[\theta_{\text {sensor }}\right] \mathbf{q} \\
\alpha=\left[\theta_{\text {sensor }}\right] q+\frac{\left[z_{\text {sensor }}\right] \dot{q}}{\mathrm{~V}_{\mathrm{T}}} \\
\mathrm{n}_{\mathrm{Z}}=\left[\mathrm{z}_{\text {sensor }}\right] \ddot{\mathbf{q}}
\end{gathered}
$$

where, the aircraft motion variables measured by the sensor are

$q$, the aircraft pitch rate,

$\alpha$, the aircraft incidence,

$\mathrm{n}_{\mathrm{z}}$, the normal acceleration,

$\left[\mathrm{z}_{\text {sensor }}\right],\left[\theta_{\text {sensor }}\right]$ correspond to the respective rows of the $\mathbf{Z}$ ' transformation matrix at the defined sensor location.

Considering the first desired output of pitch rate, and a sensor location at fuselage structural node, p, on the aircraft centre-line according to Figure 3.10, then from equation (3.132),

$$
\mathbf{q}=\mathbf{S}_{\mathbf{\theta}} \mathbf{Z}^{\prime} \mathbf{q}
$$

where,

$S_{\theta}$ is a 40 by 1 vector such that $S_{\theta}(2 p)=1$ and all other elements are zero.

In order to convert this expression to a form suitable for inclusion into the statespace description it necessary to create a new transformation vector, $\mathbf{Z}_{\mathrm{q}}$, where

$$
z_{q}=\left[z^{\prime} 0\right]
$$

such that,

$$
\mathbf{Z}_{\mathbf{q}} \mathbf{x}=\mathbf{Z}^{\prime} \dot{\mathbf{q}}
$$

so that the output equation for the pitch rate as sensed by a sensor at location $\mathrm{p}$ is given by

$$
q=S_{\theta} Z_{\mathbf{q}} \mathbf{X}
$$


Using a similar method to that above, it is possible to derive expressions for the incidence and normal acceleration outputs such that,

$$
\alpha=\left(\mathbf{S}_{\theta} \mathbf{Z}_{\mathbf{q}}+\frac{\mathbf{S}_{\mathbf{z}} \mathbf{Z}_{\mathbf{q}}}{\mathrm{V}_{\mathrm{T}}}\right) \mathbf{x}
$$

where,

$S_{z}$ is a 40 by 1 vector such that $S_{z}(2 p-1)=1$ and all other elements are zero, and $z_{q}$ is given by

$$
\mathbf{Z}_{\mathbf{q}}=\left[\begin{array}{ll}
0 & Z^{\prime}
\end{array}\right]
$$

such that,

$$
\mathbf{Z}_{\mathbf{q}} \mathbf{x}=\mathbf{Z}^{\prime} \dot{\mathbf{q}}
$$

Considering the output of normal acceleration, given from equation (3.134), and noting that from equation (3.128)

$$
\ddot{q}=\left[-A^{-1}\left(D+\sigma V_{T} B\right)-A^{-1}\left(E+\sigma V_{T}^{2} C\right)\right] x+\left[A^{-1} F\right] u
$$

the normal acceleration measured by a sensor mounted to the structure at point $p$ is given by

$$
\mathrm{n}_{\mathrm{Z}}=\mathrm{S}_{\mathbf{Z}} \mathbf{Z}^{\prime}\left[-\mathbf{A}^{-1}\left(\mathbf{D}+\sigma \mathrm{V}_{\mathrm{T}} \mathbf{B}\right)-\mathbf{A}^{-1}\left(\mathbf{E}+\sigma \mathrm{V}_{\mathrm{T}}^{2} \mathbf{C}\right)\right] \mathbf{x}+\mathbf{S}_{\mathbf{Z}} \mathbf{Z}^{\prime}\left[\mathbf{A}^{-1} \mathbf{F}\right] \mathbf{u}
$$

It is now possible to assemble the full state-space equations for the flexible aircraft model giving the desired outputs from the model of sensed pitch rates, incidence and normal acceleration. From equations (3.128),(3.138),(3.139) and (3.143) the statespace model becomes

$$
\begin{aligned}
& \dot{x}=\left[\begin{array}{cc}
-A^{-1}\left(D+\sigma V_{T} B\right) & -A^{-1}\left(E+\sigma V_{T}^{2} C\right) \\
I & 0
\end{array}\right] x+\left[\begin{array}{c}
A^{-1} F \\
0
\end{array}\right] u
\end{aligned}
$$

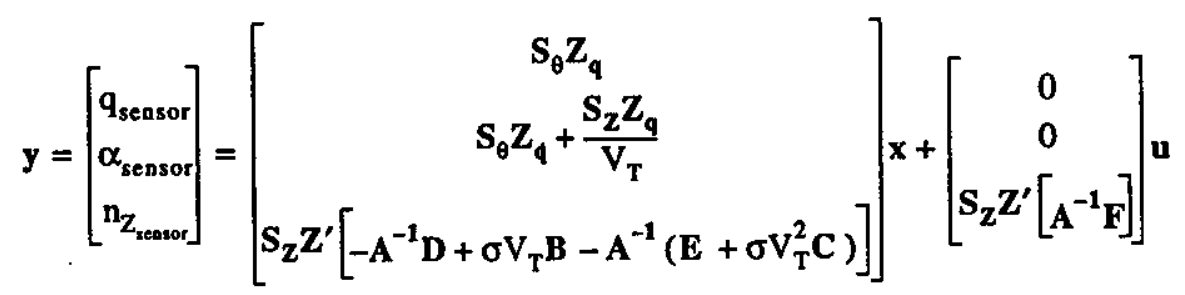




\subsection{Verification of the flexible aircraft model}

As has been discussed earlier, the flexible aircraft model was initially provided by British Aerospace in a form suitable for inclusion within the flutter equation, (3.118). In addition, the model reduction had already been performed leading to a system containing 55 structural modes along with the 3 control surface and 2 rigid-body modes. In order to verify that the model had been converted correctly to a state-space environment, results from the state-space model were compared with results from analysis performed at British Aerospace. These comparisons examined both time and frequency responses of the system. It was found that the state-space realisation matched exactly that of the original model as expected.

As a further confirmation of the validity of the flexible aircraft model however, comparisons were made with tests results from a representative aircraft. Although these comparisons cannot be reproduced here, it was felt that the flexible aircraft model was not sufficiently representative of the real aircraft for this research. As a result, it was necessary to modify the state-space model, as part of this research, in order to achieve a more representative response. These changes involved the inclusion of 2 additional high-frequency structural modes along with the modification of the remainder of the available model. In particular, the excitation of individual modes by the separate control surfaces was revised. Once these modifications were completed, it was felt that the flexible aircraft model was sufficiently representative of a real aircraft.

\subsection{Conclusions}

In this chapter, all the main elements have been drawn together to generate a model of the flexible aircraft. Furthermore, this model has been reduced to a tractable size whilst still giving an accurate representation in the bandwidth of interest. Finally, the model has been converted to a state-space form for analysis within the MATLAB/ SIMULINK environment. 
Chapter 4

\section{Investigation of Current Design Method}




\subsection{Introduction}

The earlier chapters have introduced the problem of aeroservoelasticity in general terms; discussing current design methods and assumptions. The development of a model of the flexible aircraft makes it possible to investigate these current methods further, in an attempt to identify areas where the current design methods may be improved.

The following chapter makes use of the earlier flexible aircraft model to demonstrate the problem of aeroservoelasticity when combined with suitable models of the other system components. Initially, these models of the flight control system and actuation will be simple linear models as is assumed in the current design methodologies. In later chapters, these assumptions will be analysed in more detail, with comprehensive models being applied for both the actuation and digital effects.

\subsection{Creation of a full system model}

\subsubsection{Introduction}

In order to demonstrate the current design solution to the problem of aeroservoelasticity, it is necessary to develop a model of the full aircraft system. This model will incorporate the existing model of the flexible aircraft, with representations of the flight control system and actuation. Such a full system model will be developed in the following section.

\subsubsection{Creation of the system model}

The main elements of a typical aircraft system are as shown in Figure 4.1. The model of the flexible aircraft has been developed in Chapter 3, which leaves only the flight control system and actuator models to be added. The representation of the flight control system and actuation system used in the following example will be simple linear models. Investigation of the consequences of the true nature of the actuators and flight control system will be made in later chapters.

Considering first the actuation system, in order to enable later comparisons to be made, the baseline actuator model will be a linear representation of the Jaguar FBW taileron actuator. This model is based on a linearisation of actual test results ${ }^{58}$. The model is

$$
\begin{aligned}
& \frac{\text { ram extension }(s)}{\text { ram demand }(s)}=\frac{(1+3.3 e-4 s)(1+3.9 e-3 s)\left(1+3.46 e-4 s+1.19 e-5 s^{2}\right)}{(1+3.31 e-4 s)(1+1.76 e-3 s)(1+3.79 e-3 s)(1+3.62 e-2 s)} \\
& \quad \times \frac{1}{\left(1+3.07 e-3 s+1.05 e-5 s^{2}\right)\left(1+5.47 e-3 s+2.54 e-5 s^{2}\right)}
\end{aligned}
$$


which represents the transfer function between demanded ram position and actual ram position.

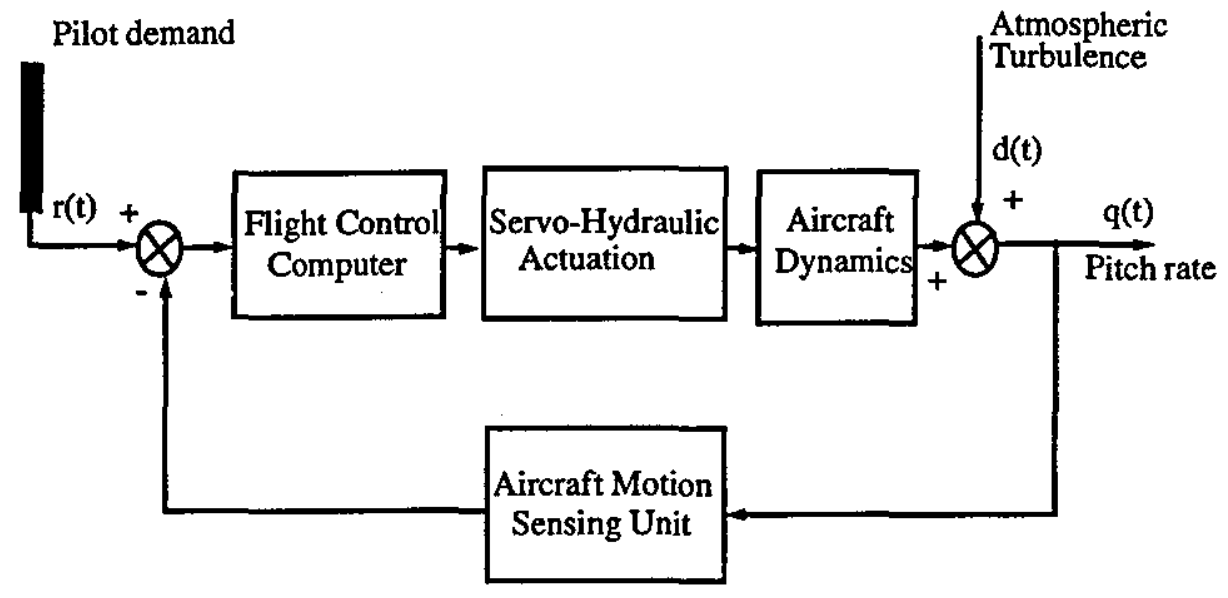

Figure 4.1 - Basic aircraft model block diagram

Since the demanded control surface position is linearly related to the ram position, the output from the actuator model can be used to drive the flexible aircraft model developed in Chapter 3. The relationship between the flight control surface demands and actuator ram position is shown in Figure 4.2.

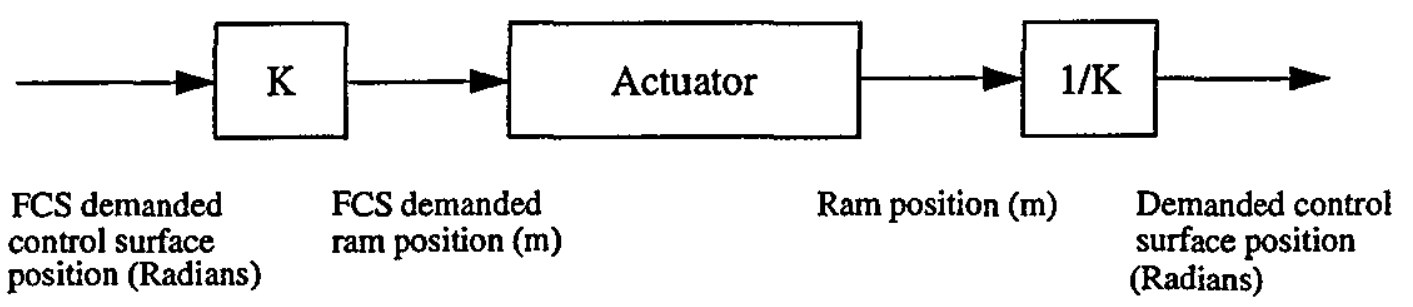

\section{Figure 4.2 - Relationship between FCS demands and actuator ram position}

For the purpose of an initial modelling exercise, the structural modes of the aircraft model will be neglected, leaving only the rigid-body dynamics and control surface dynamics within the model.

The output of the aircraft model created in Chapter 3 has been derived as being pitch rate, normal acceleration and incidence. In this case, only pitch rate will be used as a feedback variable to the flight control laws. The sensor in the actual aircraft is required to measure this rigid-body pitch rate for feedback to the flight control computer, and in the baseline model, the sensor dynamics will be neglected.

This leaves only the flight control laws for definition for the baseline model to be complete. It will be assumed that the three actuation systems required for control of the generic canard-delta aircraft under consideration are identical. The definition of the flight control system is obtained by completing a "classical" controller design on the baseline model, designing the controller to meet a specification set down by 
British Aerospace.

The basic rigid-body aircraft model as defined in Chapter 3, using aerodynamic derivative data from British Aerospace, describes a longitudinally unstable aircraft. Combined with the model for the actuation system, and assuming a fully analogue control system, the resulting model shows clear instability as shown in Figure 4.3. The response to an input of the form 1-Cos(t) with amplitude of 1 degree on the outboard flaperon actuator is clearly unsatisfactory with the pitch rate building up to almost 7000 degrees per second within the first four seconds.
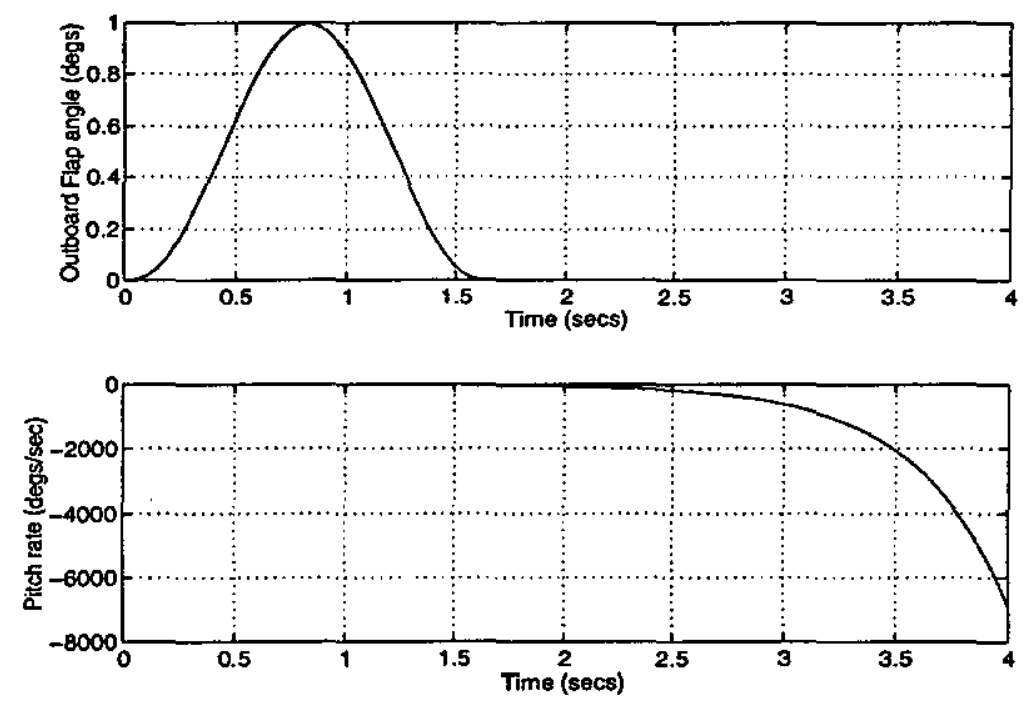

Figure 4.3 - Actuation and flexible aircraft model uncontrolled step response (M= 0.8, $36000 \mathrm{ft}$.)

A suitable control system is therefore required both to stabilise the aircraft, and meet the performance requirements for the system. The design of such a control system has been completed using "classical" design methods. It is not intended to reiterate the methods used here, "classical" control theory being covered adequately in many suitable texts ${ }^{56,57}$.

In this case, the resulting controller, as shown in Figure 4.4, is of the proportional plus integral type, with phase advance filtering in the feedback path. The controller meets the required specifications as shown in the Nichols plot of the open-loop system included as Figure 4.5. The required stability-margins are defined by the boundary as shown. This boundary is the current stability-margin boundary as required for clearance of an aircraft to flight, and must not be crossed by the frequency response of the open-loop system. From the Figure, the performance of the control system appears to meets the required specification, the frequency response of the system remaining safely outside of clearance boundary.

It is now possible to define the baseline aircraft system model that will be used for the following investigation of the aeroservoelastic process. The basic model will use the simplified models of aircraft structure and actuation systems as described above, 
and incorporate the controller as specified in Figure 4.4. It should be noted that a negative gain in the foreplane control path is simply due to the sign conventions adopted by British Aerospace in the development of the flexible aircraft model. An overview of the full system model is included in Figure 4.6.

The time response for the closed-loop system to a representative pilot input demand of form 1-Cos $(t)$ is shown in Figure 4.7. It can clearly be seen that the aircraft has been stabilised, with the response being satisfactory.

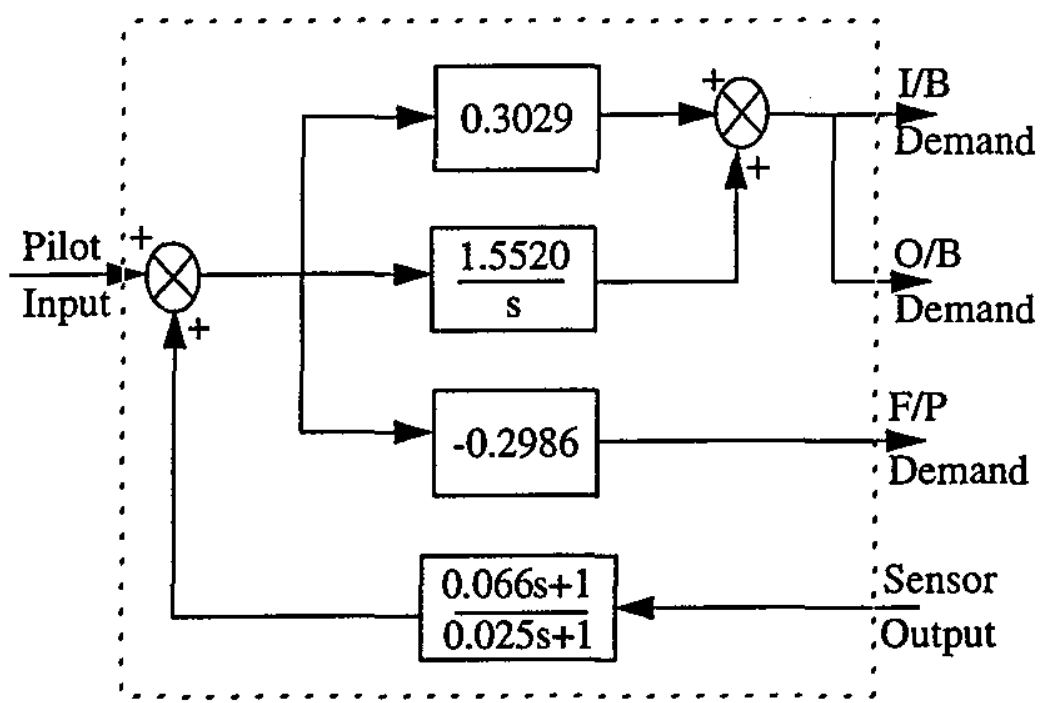

Figure 4.4 - Flight control laws for baseline model

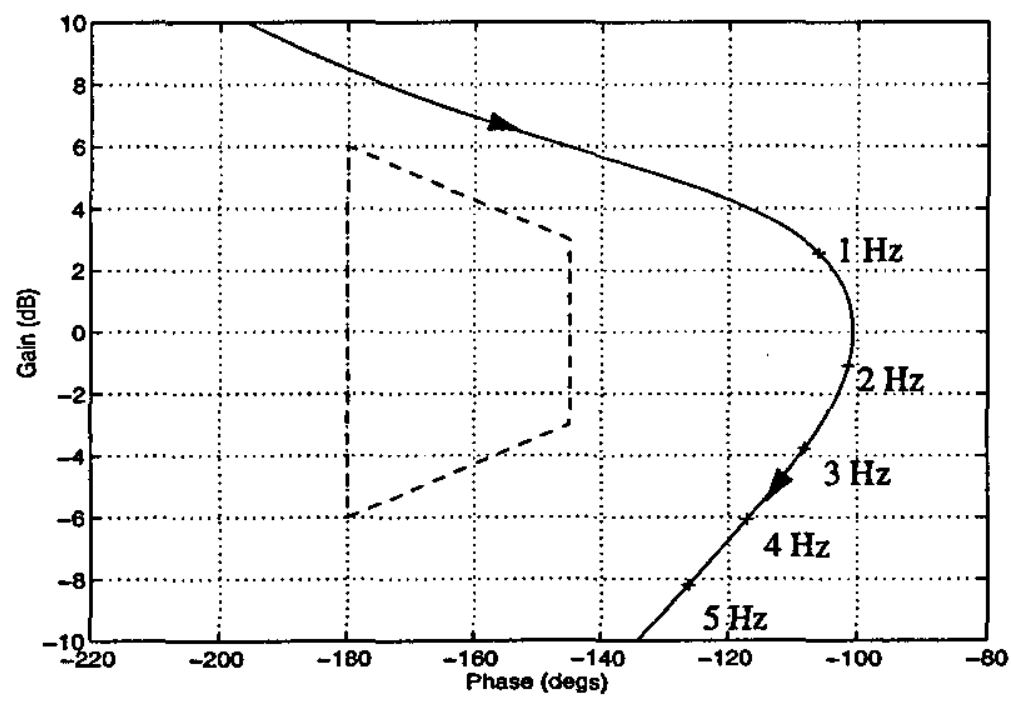

Figure 4.5 - Open-loop Nichols plot showing error signal stability-margins of complete baseline aircraft system $(M=0.8,36000 \mathrm{ft}$.) 


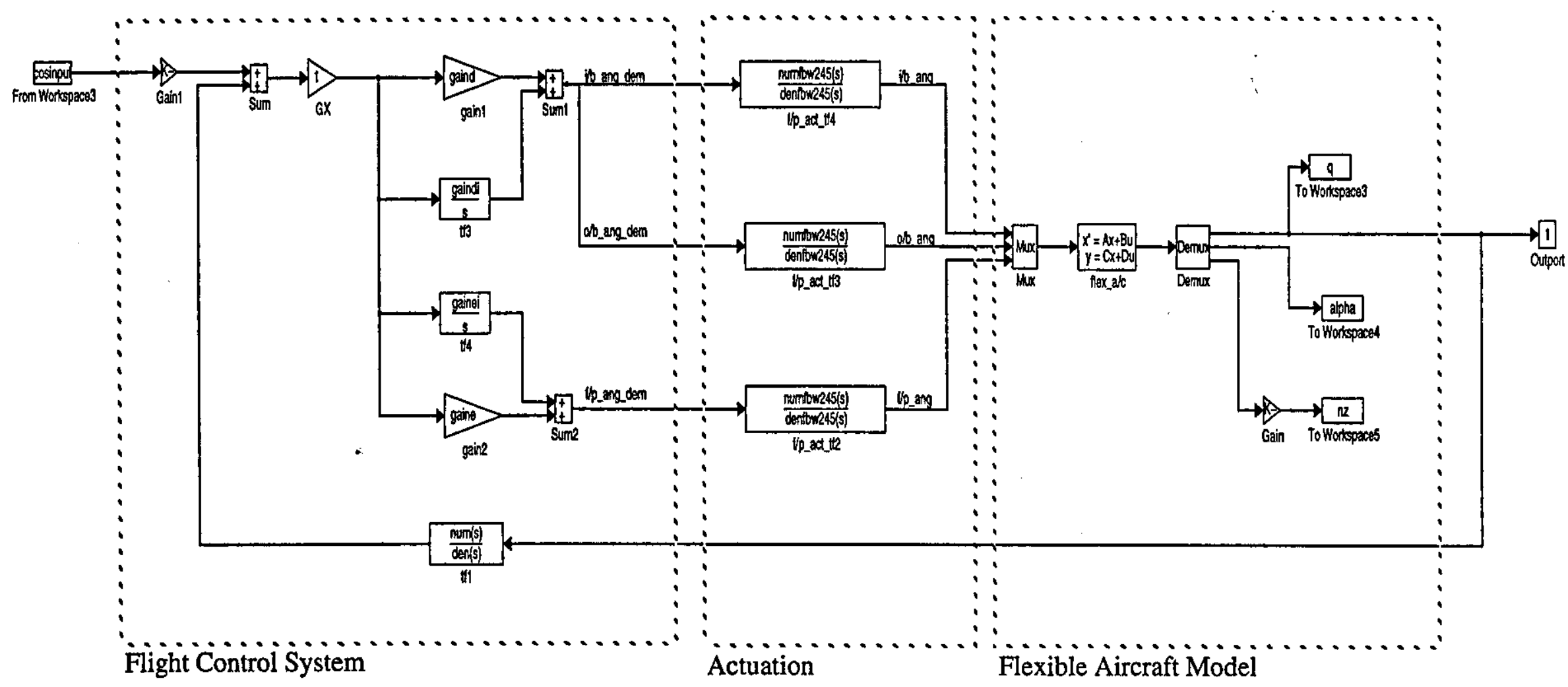

Figure 4.6 - System block diagram 

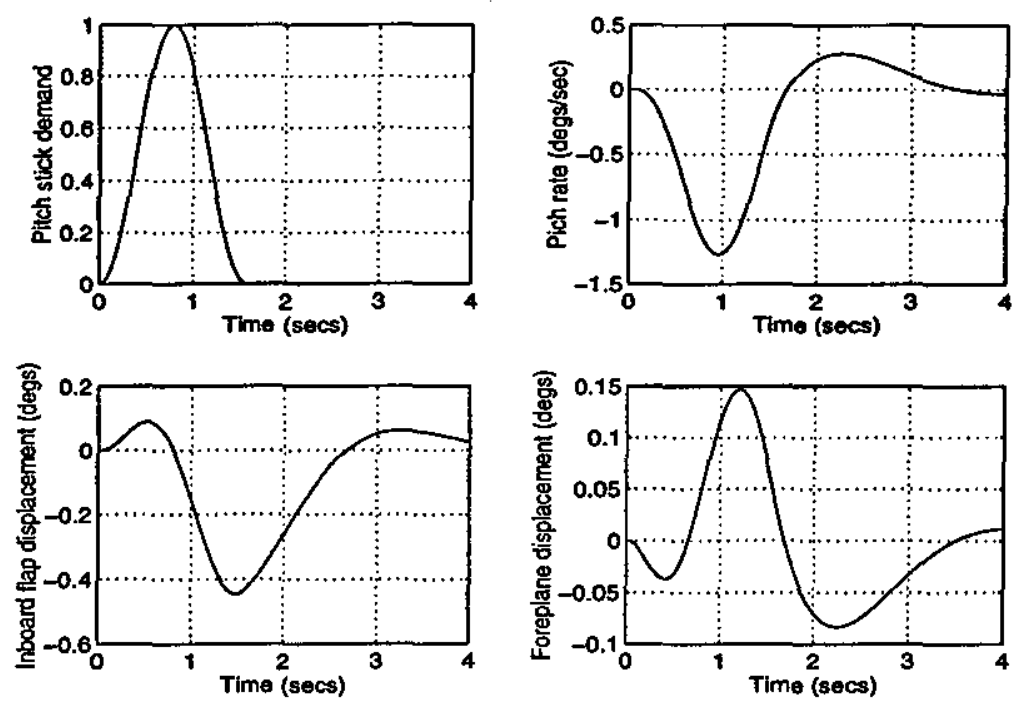

Figure 4.7 - Time response for baseline model $(M=0.8,36000 \mathrm{ft}$.

\subsubsection{Demonstration of aeroservoelastic effects}

The baseline model as described above includes only a representation of the rigidbody dynamics of the aircraft, and the control surface dynamics themselves. In order to demonstrate the effect of the inclusion of the flexible body dynamics on the stability of the aircraft, it is necessary to rework the aircraft model of the baseline system to include some of the vibrational modes of the aircraft structure. Due to the normalisation of the aircraft model discussed in Chapter 3, this is simply a matter of including in the state-space model of the aircraft those partitions corresponding to the desired normal modes of the structure.

Incorporating the seven lowest frequency normal modes of the structure into the aircraft model, it is possible to produce a time response for the same input and flight conditions as were used in Figure 4.7. Comparing this time response as shown in Figure 4.8 with that for the rigid aircraft system shows clearly the effect of the structural modes on the response of the aircraft. It should be noted that in the case of Figure 4.8, the time base has been shortened considerably due to the presence of the structural instability. The response in pitch rate in Figure 4.8 shows that the result of the high-frequency structural mode being sensed by the flight control system is a clear system instability at the frequency of the structural mode.

Examining the time responses for control surface deflection in Figure 4.8, shows that the high-frequency pitch rate component is being mirrored in the control surface defiection responses as expected. This is due to the flight control system responding to the high-frequency pitch rate component, resulting in the unstable response.

The open- loop Nichols plot for this system, given in Figure 4.9, shows that the system frequency response now crosses the stability-margin clearance boundary at a 
frequency of approximately $14 \mathrm{~Hz}$, indicating an unsatisfactory response. The introduction of just a few structural modes has therefore resulted in a unstable system response.
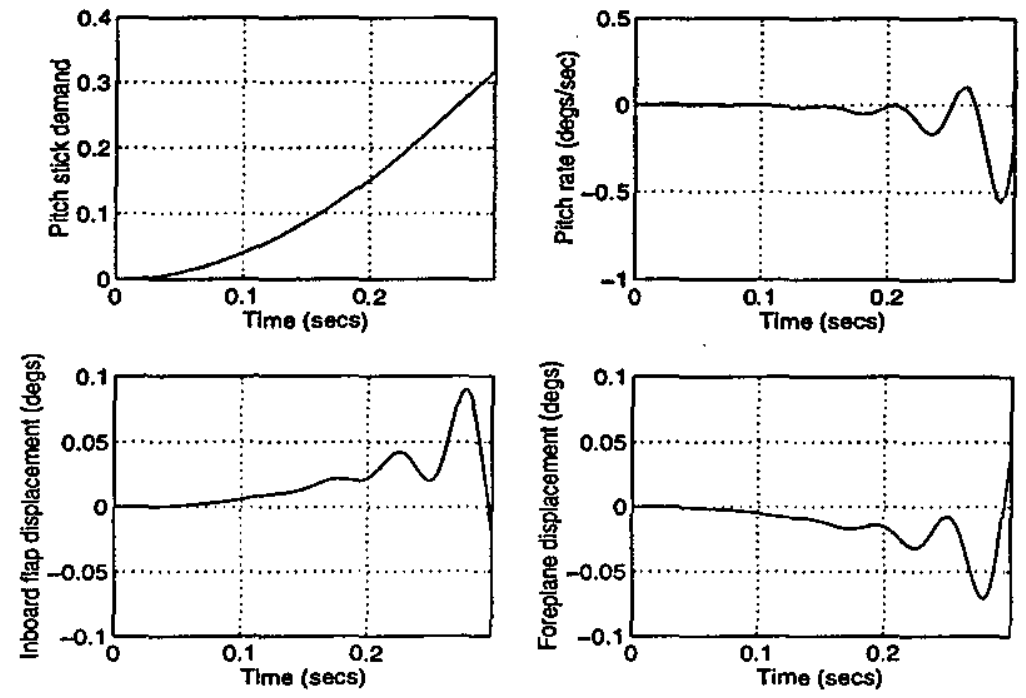

Figure 4.8 - Time response for baseline model incorporating the first seven structural modes $(\mathrm{M}=0.8,36000 \mathrm{ft}$.)

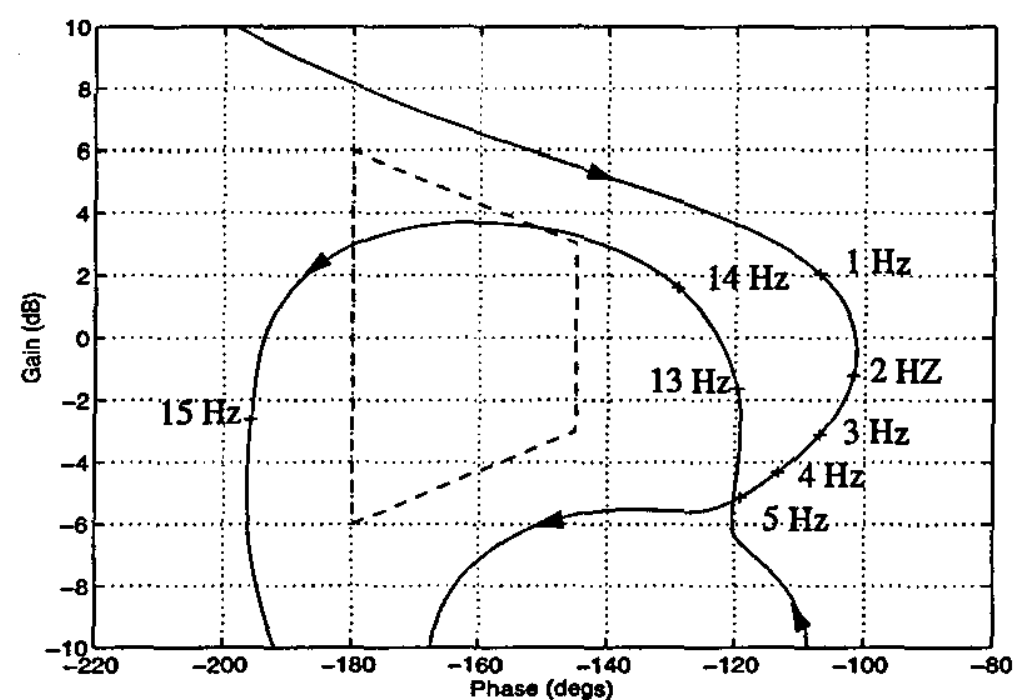

Figure 4.9- Open-loop Nichols plot showing error signal stability-margins for aircraft system incorporating 7 structural modes $(M=0.8,36000 \mathrm{ft}$.)

\subsection{Prevention of the aeroservoelastic interaction using currently applied methods and assumptions}

\subsubsection{Introduction}

The previous section has demonstrated the nature of the aeroservoelastic problem as applied to a basic system model. It remains to demonstrate the current method of the prevention of such an interaction as used by British Aerospace. The design assumptions as applied to the aeroservoelastic problem have been discussed in Chapter 2. These assumptions will now be employed in the prevention of the 
aeroservoelastic problem for the model developed in the proceeding section. Initially, the current procedure will be demonstrated using the baseline system model as developed earlier, but with the flexible aircraft model containing all 57 of the available structural modes.

\subsubsection{Description of the current solution procedure}

The current solution to the aeroservoelastic problem employed by British Aerospace and the majority of other aircraft manufacturers, makes use of feedback filtering to attenuate the feedback of the sensed structural modes to the flight control computers. The design procedure for these filters as used by British Aerospace involves the determination of the required level of attenuation. This is completed according to the current assumptions and clearance requirements, taking into account the digital effects of the sensor unit and flight control computers. The design is then verified from a comprehensive series of ground tests.

A fuller description of the design procedure as employed by British Aerospace is given in Reference 17. The design procedure will now be applied to the system model developed in the previous section.

\subsubsection{Production of the structural filter attenuation requirements}

The first step in the design procedure for the structural filter is to produce the attenuation requirements, taking into account the current assumptions and clearance requirements. In the case of a real aircraft design, the production of this envelope would rely initially on a structural model similar to that developed earlier. In addition, a model of the unsteady aerodynamic effects is required as discussed in Chapter 3. As an aircraft project develops however, the structural model can be replaced by actual ground test data. The production of the attenuation requirements would then rely only on the modelling of the aerodynamic effects, which can be improved with flight testing when available.

In this case, where ground test data of the actual aircraft is not available, the production of the attenuation requirements will rely on the structural model, which is considered to be sufficiently representative for this investigation.

The following calculation of the structural filter attenuation requirements will be made assuming the system to be fully analogue, using the baseline system model as developed earlier. This model will incorporate the full 57 structural modes available. The two fight conditions available for analysis are the ground static condition, and an in flight condition of Mach 0.8 at 36000 feet altitude. The ground static condition effectively represents the servoelastic effect as discussed in Chapter 1 . The in-flight condition is available for demonstration and analysis of the aerodynamic affect. 
Using the system model in SIMULINK form as shown in Figure 4.6, for the ground static condition, it is possible to produce the open-loop frequency responses for the system for each of the three inputs to the control surface actuators. This results in the gain responses as shown in Figure 4.10, Figure 4.11 and Figure 4.12.

Only the gain responses have been included initially as it is assumed that accurate phase information is unavailable for the system. This is the current assumption used in the structural-filter design procedure employed by British Aerospace. The reasoning behind this assumption has been discussed in Chapter 2. As the phase response of the system is assumed to be unreliable, any frequency for which the gain is greater than 0 $\mathrm{db}$ represents a possible instability.

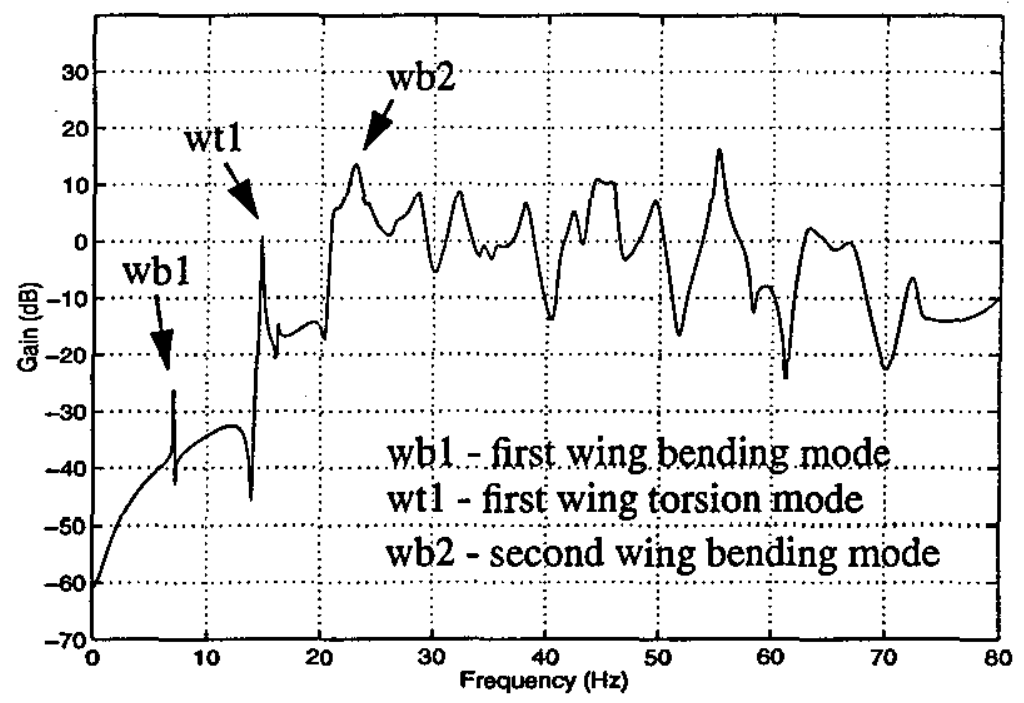

Figure 4.10 - Open-loop frequency response for input to the inboard flaperon actuator for the aircraft system model including the full-order flexible aircraft model $(\mathrm{M}=\mathbf{0}, \mathbf{0} \mathrm{ft}$.)

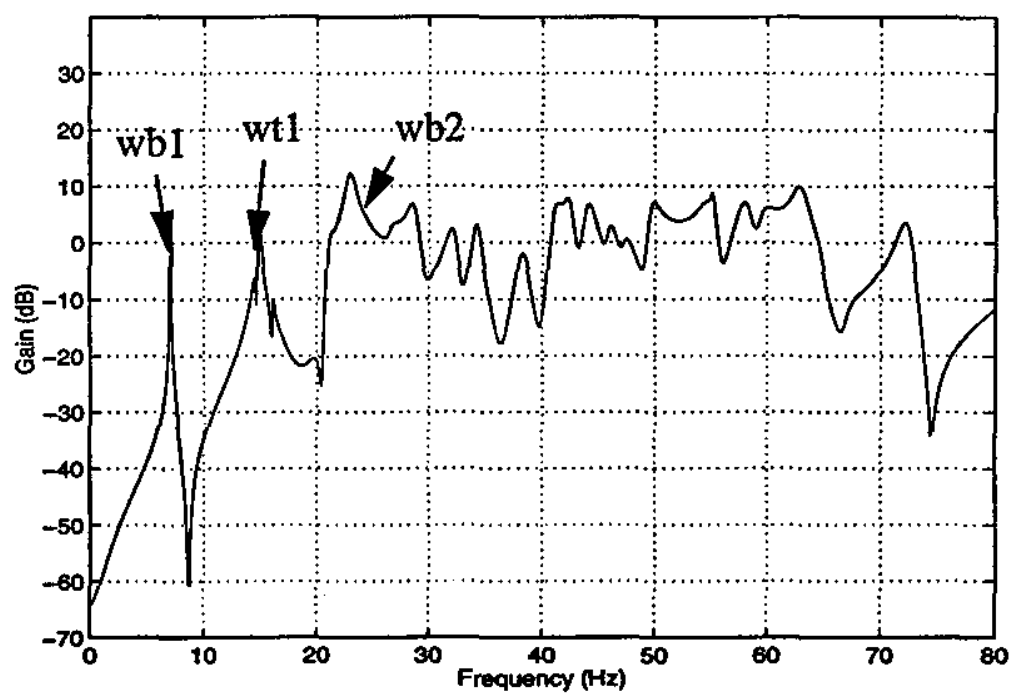

Figure 4.11 - Open-loop frequency response for input to the outboard flaperon actuator for the aircraft system model including the full-order flexible aircraft model $(M=0,0$ ft.)

The relative contribution of the individual control surfaces to a particular modal 
response can be deduced from the frequency responses. For example, the mode at approximately $7 \mathrm{~Hz}$ is excited almost exclusively by the outboard flaperons. Examination of the modeshape reveals that it represents the first wing bending mode, and as such, represents a mode that should be excited in the main by the outboard flaperons, since these control surfaces have a greater moment arm. It is clear that in this case at least, the relative contributions of the three control surfaces to the excitation of the mode corresponds well with the real situation.

Some of the other structural modes have been indicated on the responses of Figure 4.10, Figure 4.11 and Figure 4.12, and as with the consideration of the first wing bending mode, the contributions of the each control surface input to a particular modal response is evident.

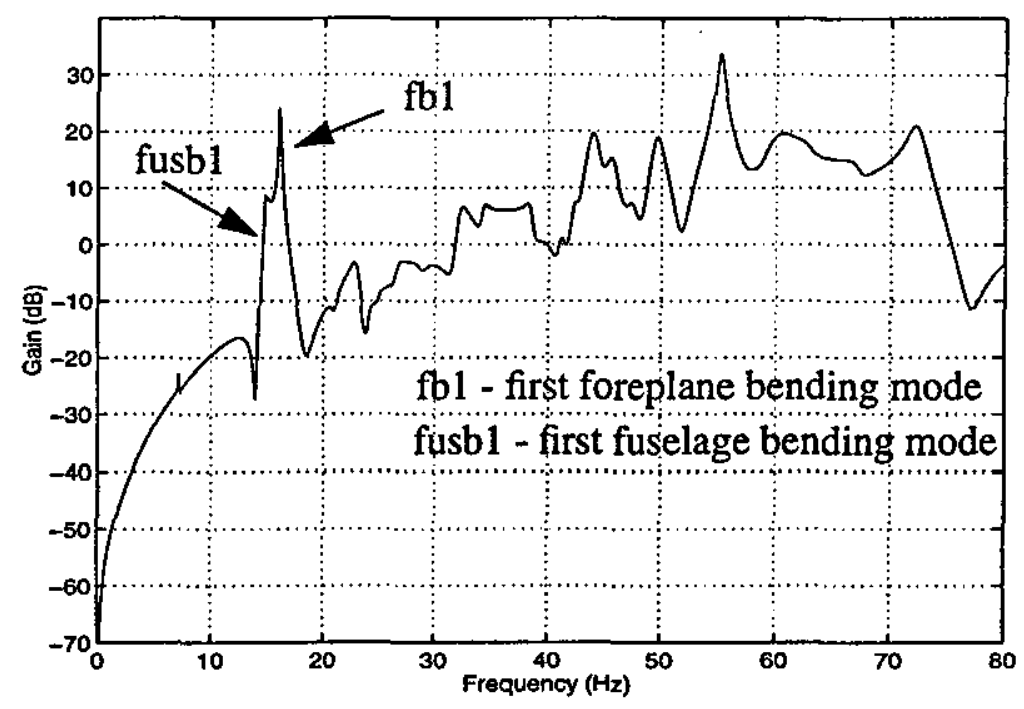

\section{Figure 4.12 - Open-loop frequency response for input to the foreplane actuator for the aircraft system model including the full-order flexible aircraft model $(\mathrm{M}=\mathbf{0}, \mathbf{0} \mathrm{ft}$.)}

The above responses thus relate to the servoelastic interaction between the flight control, actuation systems, and the aircraft structure. The energy required to excite the modal responses is provided entirely by the flight control system as there are no aerodynamic effects.

In order to specify the structural filter attenuation requirements for the full system, it is necessary to take into account the effect of the aerodynamics on the structural response for the aircraft in flight. In Chapter 3, it has been shown that there exists aerodynamic stiffness and aerodynamic damping terms in the equations of motion of the aircraft as a result of the unsteady aerodynamics of the vibrating structure. The addition of the aerodynamic terms will therefore involve a change to the response of the aircraft structure, with some structural modes being attenuated by the inclusion of the aerodynamic effects, whilst other modes may be amplified.

It is necessary, therefore, to reproduce the open-loop responses for the aircraft 
system for each flight condition available. The maximum envelope of these responses for each mode being taken as the worst case that could exist. In an actual aircraft design, this use of multiple flight conditions to obtain the maximum envelope of the response is combined with analysis involving changes to the configuration of the aircraft due to differing stores arrangements and fuel states. Each configuration will have an effect on the structural dynamics of the system. For example, a store with a high mass situated at the wing tip, will have a large effect on the wing bending mode. Eventually, the maximum envelope can be obtained, which represents the maximum gain of each structural mode for all flight conditions and aircraft configurations. In this case, information is only available for one flight condition apart from the ground static condition.

Producing the open-loop frequency responses for the system at a flight condition of Mach 0.8 with an altitude of 36000 feet for inputs to the three actuator results in the maximum envelope corresponding to each actuator input as shown in Figure 4.13, Figure 4.14 and Figure 4.15. The addition of the aerodynamic effects can be seen to result in increased responses for several modes. This is as a result of the differing contributions of the inertial and aerodynamic forces on the aircraft structure.

The maximum response envelope for each control surface actuator input has therefore been obtained, the ground static case being used as the baseline response for inter-modal frequencies. Only the response at modal frequencies are compared to obtain the maximum response envelope.

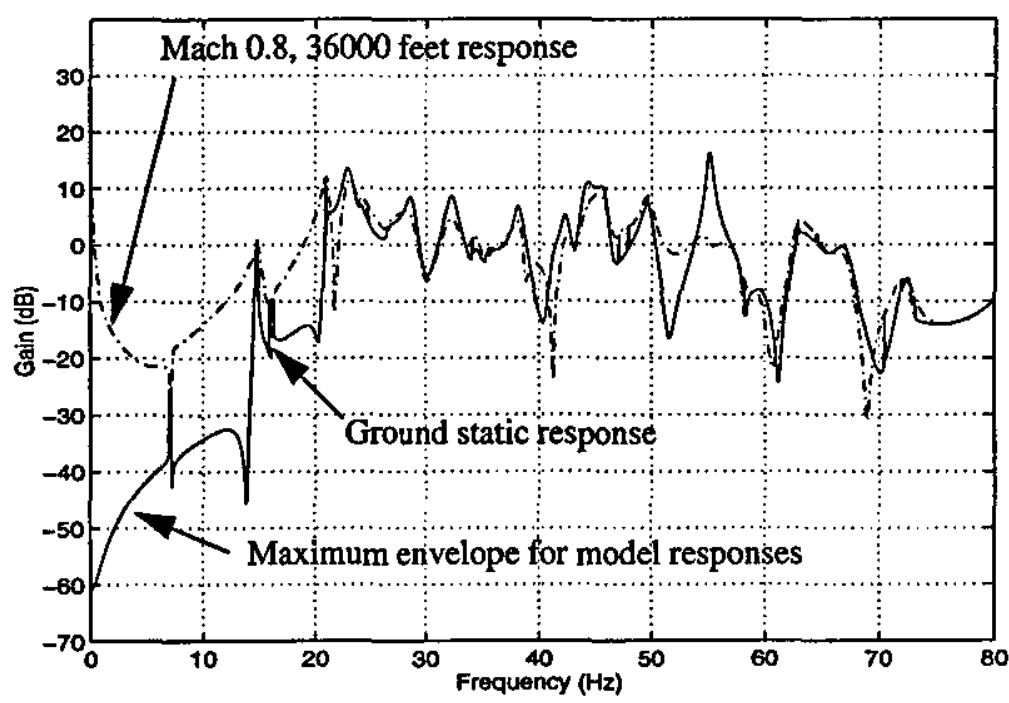

\section{Figure 4.13 - Open-loop frequency response maximum envelope for inboard flaperon actuator input}

The envelopes thus represent the maximum response of the system to an input on each of the control surface actuators. The problem now is that, in a real flight case, the control system will inevitably be using all three control surfaces. As a result, the aircraft structure will be responding to the excitation of all surfaces together. In order 
to obtain the attenuation requirements for the structural filters, it is necessary to combine the above maximum envelopes, to obtain a maximum envelope for the system as a whole.

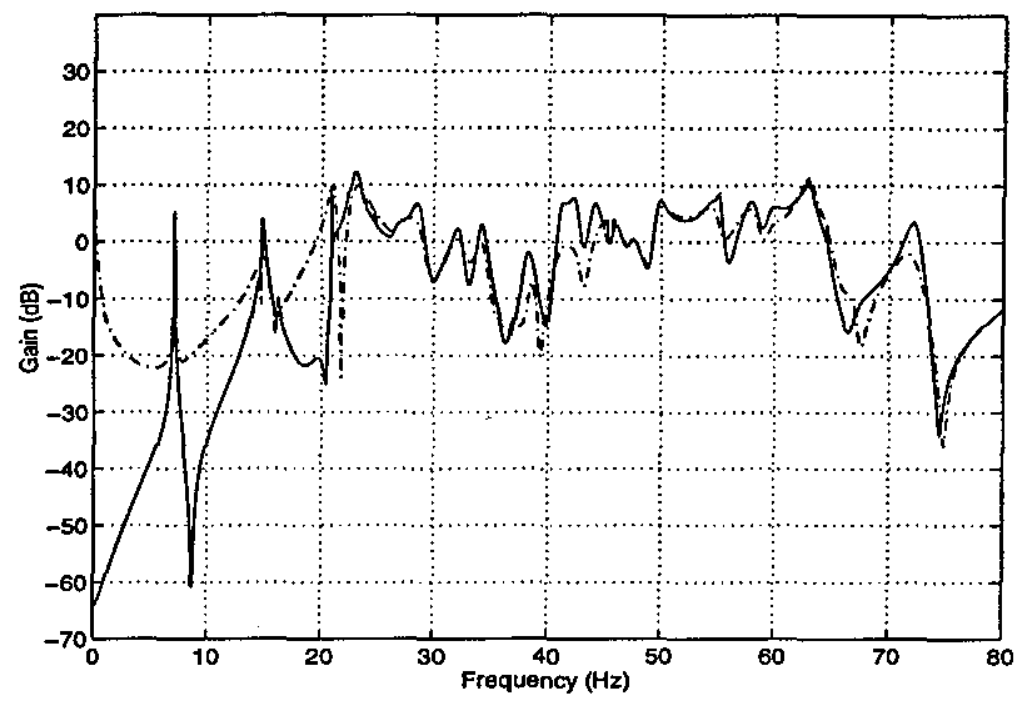

Figure 4.14 - Open-loop frequency response maximum envelope for outboard flaperon actuator input

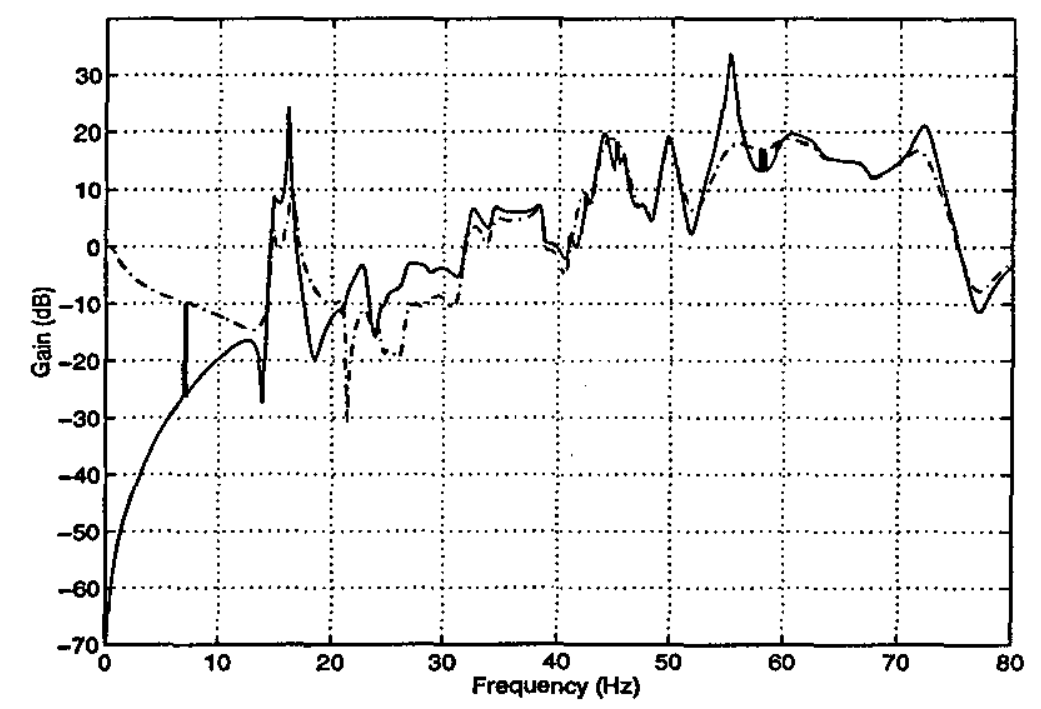

Figure 4.15 - Open-loop frequency response maximum envelope for foreplane actuator input

In reality, the structural responses to the three inputs will have phase differences associated with the response, so that a particular modal response to, say, the foreplane input might cancel with the same modal response to the inboard flaperon input. Unfortunately, under the assumptions about the reliability of phase modelling which are applicable at present, such cancelling cannot be assumed when producing the attenuation requirements for the structural-mode filters. In addition, the current clearance requirements ${ }^{47}$ specify that all possible combinations of phase between the three signal paths would have to be taken into consideration. Naturally, this would involve a significant amount of design work. As a result, it is necessary to add algebraically the maximum gain envelopes of Figure 4.13, Figure 4.14 and Figure 
4.15 in order to obtain the maximum envelope for the system as a whole. This effectively assumes the worst case, where all modal responses to the three actuator inputs act exactly in-phase in producing the structural response. Performing this algebraic addition of the individual control surface path envelopes, results in the maximum system gain envelope as shown in Figure 4.16. This envelope thus represents the maximum modal response of the aircraft for all flight conditions, assuming in-phase addition of the signal paths corresponding to the three actuator inputs.

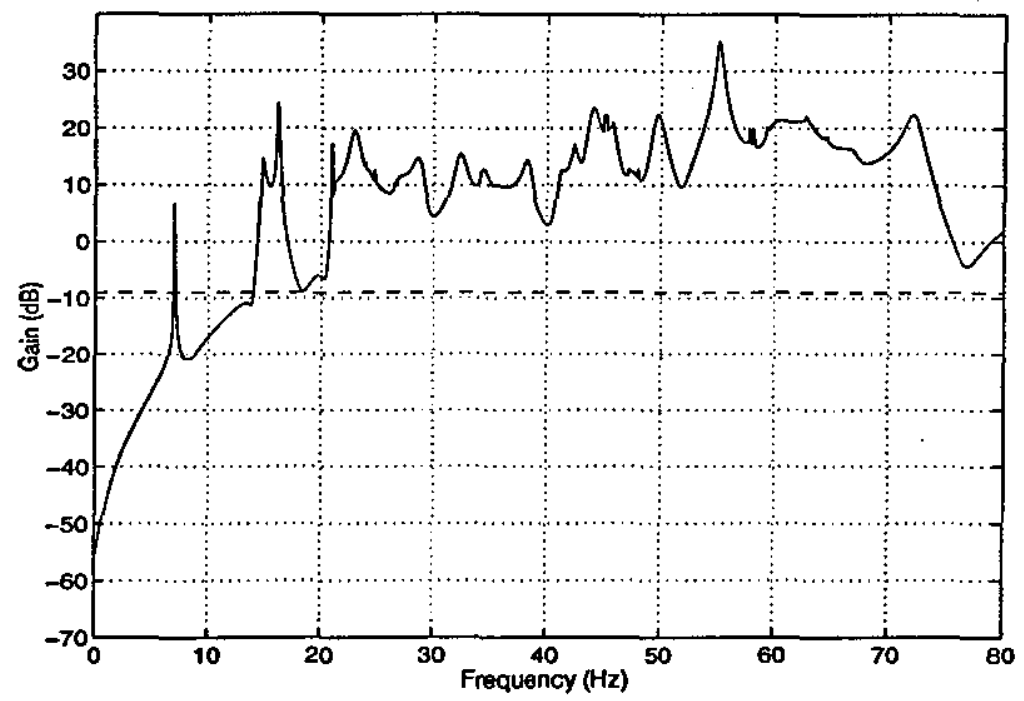

\section{Figure 4.16 - Maximum open-loop modal response envelope for full flexible aircraft system model.}

The final step in defining the attenuation requirements for the structural filters is to specify the maximum allowable open-loop gain for the structural modes.

Current clearance requirements as specified by British Aerospace demand an open-loop structural mode gain of no greater then $-9 \mathrm{~dB}$. Theoretically of course, this level would be set at $0 \mathrm{~dB}$, only structural modes with a gain of greater than $0 \mathrm{db}$ being amplified around the loop. Due to safety margin requirements, and uncertainty in the accuracy of structural and aerodynamic modelling however, the present requirement of $-9 \mathrm{~dB}$ must be met. This maximum gain boundary is indicated in Figure 4.16, and it can be clearly seen that many of the structural modes exceed this boundary.

\subsubsection{Design of the structural-mode filters}

In the above section, the attenuation requirements of the structural-mode filters have been developed following the current design procedure used by British Aerospace. In order to satisfy these requirements it is necessary to design structuralmode filters to be placed within the control system, such that the structural modes are attenuated where required around the system loop.

The design of these filters is restricted however by several factors. One of these is 
the limitations on the rigid aircraft stability-margin boundary. From Figure 4.5, which represents the open-loop Nichols plot for the rigid-body aircraft combined with the flight control system and actuator models, the frequency response of the basic aircraft is close to the stability-margin clearance boundary. Excessive phase lags introduced by the filters could result in the response of Figure 4.8 being shift too far to the left, resulting in a violation of the clearance boundary by the frequency response. It is clear that there is a limit on the effect of the structural-mode filters on the rigid-body frequency response of the aircraft. As a result, the design of the structural filters has to be a balance between achieving the structural-mode attenuation requirements and satisfying the clearance requirements for the rigid-body aircraft. In addition to these limitations, the structural filters must be of minimum order, and be implementable in a real system. The balance of all these elements must be made in the design of the structural-mode filters.

The limitations on the implementation of the filter result from practical experience gained in the implementation of flight control systems ${ }^{18}$. The limitations in the case of second-order filters of the form

$$
\frac{1+a s+b s^{2}}{1+c s+d s^{2}}
$$

are that the damping of the numerator must be greater than approximately 0.007 , and that the difference between the $s^{2}$ terms in the numerator and denominator must be greater than $2 \%$. These limitations are based on experience of filter implementation gained by British Aerospace. Combining these limitations and requirements, it is possible to design suitable structural-mode filters.

From Figure 4.16, the attenuation requirements for the structural-mode filters can be considered as having two main elements. Firstly, the attenuation required for the three main modes below $20 \mathrm{~Hz}$, and secondly, the attenuation of the remaining modes above $20 \mathrm{~Hz}$. An advantage of this approach is that the break frequency of the lowpass filter is maximised. This has the effect of minimising the phase lag introduced by such a low-pass filter, which tends to make up the majority of lag introduced by the structural-mode filters as a whole at low-frequencies. This results in the need to design four structural-mode filters; three band stop filters to attenuate particular lowfrequency modes, and a low-pass filter to attenuate the remaining structural-modes.

First, consider first the design of the bandstop filters. The initial step is to identify the frequencies that need to be attenuated, and then to decide on the level of attenuation required.

For the full-order system model, the frequencies at which the bandstop filters need to be centred can be read from Figure 4.16. A more accurate specification can be 
obtained from an eigensolution of the flexible aircraft model. In this case, the centre frequencies of the three bandstop filters are;

$44.9 \mathrm{rads} / \mathrm{sec}=7.15 \mathrm{~Hz}$ (First wing bending mode)

$101.2 \mathrm{rads} / \mathrm{sec}=16.11 \mathrm{~Hz}$ (Foreplane bending mode)

$92.9 \mathrm{rads} / \mathrm{sec}=14.79 \mathrm{~Hz}$ (Fuselage bending mode)

Considering the first these three filters, it is possible to design a suitable filter using the MATLAB signal processing toolbox. The filter is based on a Butterworth analogue low-pass filter prototype converted to a second-order bandstop filter of bandwidth $7 \mathrm{rads} / \mathrm{sec}$ using the appropriate toolbox command ${ }^{59}$. The bandwidth of the filter is chosen so as to control the attenuation of the filter. After suitable corrections to meet the implementation considerations given earlier, the resulting filter can be represented by the transfer function

$$
G_{s f l}(s)=\frac{s^{2}+0.65 s+2018}{s^{2}+7 s+1976}
$$

which corresponds to a frequency response as shown in Figure 4.17.

From Figure 4.17, the attenuation of the filter to a signal of frequency $7.1 \mathrm{~Hz}$ is evident. The resultant penalty in terms of additional phase lag at rigid-body frequencies is clearly visible in the phase response of the filter. One point to note is that in reality, with the differing aircraft configurations and fuel states being taken into account, it may be necessary to produce a filter with a wider notch than that shown in Figure 4.17. This is due to the frequency of a particular structural mode changing slightly for different fuel states and configurations.

The same approach can be applied to the design of the remaining two bandstop filters. A centre frequency of $101.2 \mathrm{rads} / \mathrm{sec}$ and bandwidth of $20 \mathrm{rads} / \mathrm{sec}$ for the second filter and a centre frequency of $92.9 \mathrm{rads} / \mathrm{sec}$ and bandwidth of $10 \mathrm{rads} / \mathrm{sec}$ for the final bandstop filter satisfies the requirements. The transfer function of the second and third bandstop filters are given by

$$
\begin{aligned}
& G_{s f 2}(s)=\frac{s^{2}+1.42 s+10240}{s^{2}+20 s+10450} \\
& G_{s f 3}(s)=\frac{s^{2}+1.31 s+8630}{s^{2}+10 s+8810}
\end{aligned}
$$

Combining the magnitude frequency responses of the three bandstop filters defined in equations (4.3),(4.4) and (4.5) with the attenuation requirements of the aircraft system as defined in Figure 4.16, results in Figure 4.18. This represents the attenuation requirements of the remaining low-pass filter assuming that the three bandstop filters have been applied to all signal paths. 

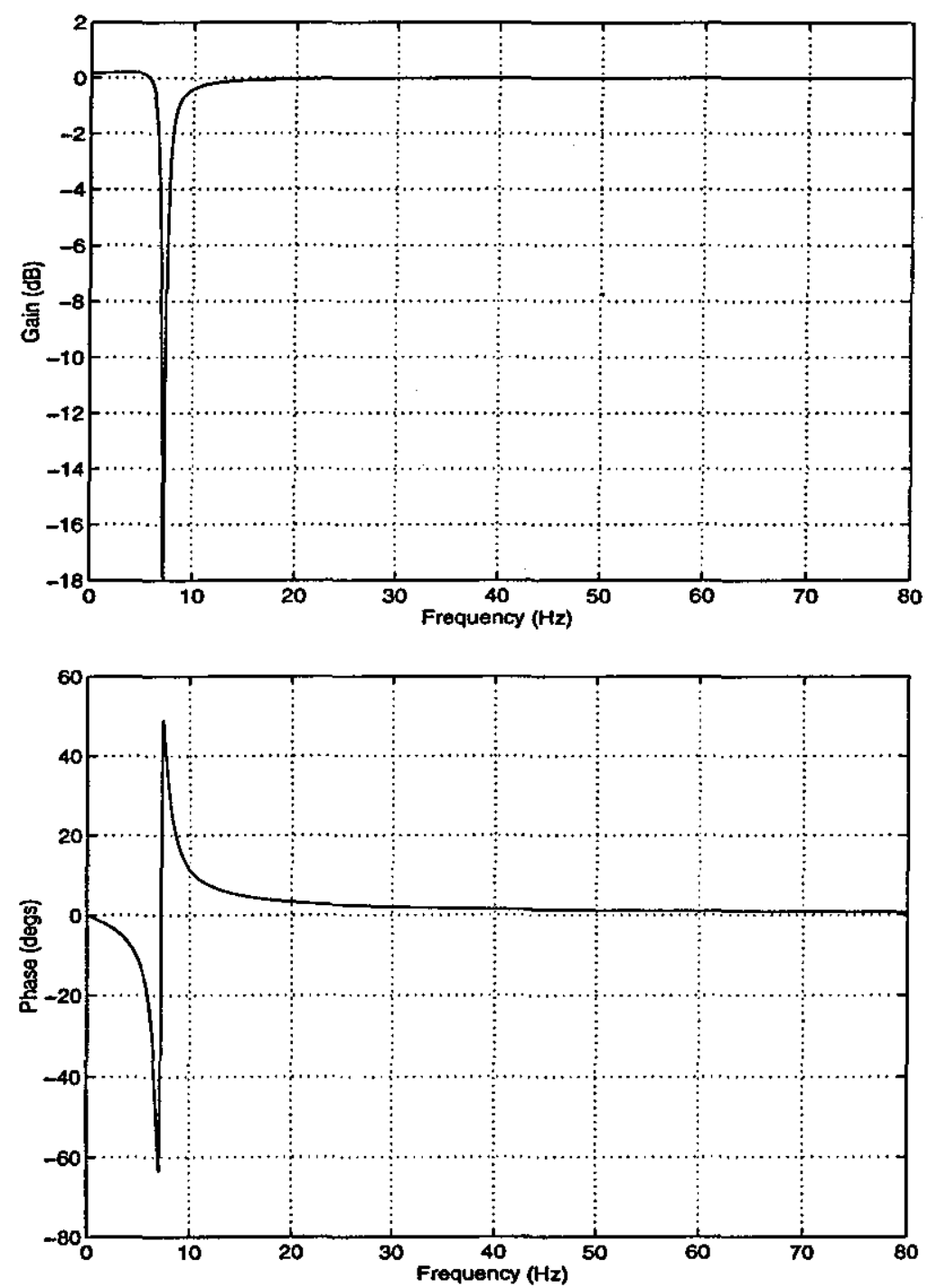

Figure 4.17 - Frequency response of first analogue bandstop filter

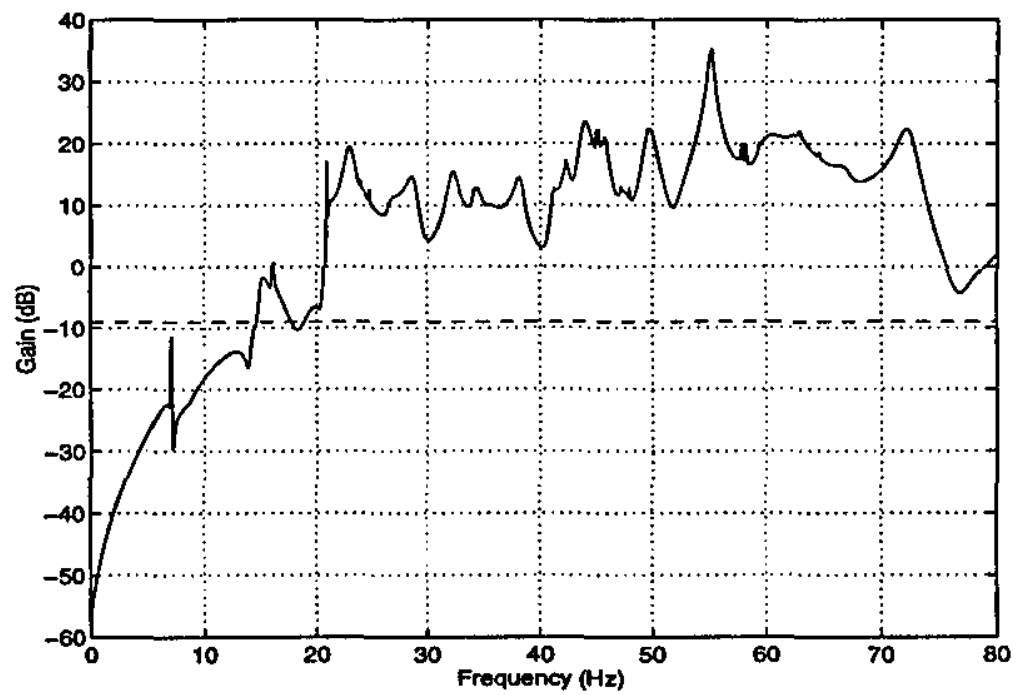

Figure 4.18 - Attenuation requirements for low-pass filter after application of bandstop filters to the original attenuation requirements 
Comparing Figure 4.16 with Figure 4.18 reveals the effect of the three bandstop filters on the maximum envelope response of the system. The three main modes below $20 \mathrm{~Hz}$ have been attenuated significantly. The inability of the second two bandstop filters to attenuate the two modes at 17.79 and $16.10 \mathrm{~Hz}$ to below the required level is intentional. The low-pass filter will provide additional attenuation of these modes as a result of its attenuation of the modes at a frequency of approximately $20 \mathrm{~Hz}$. This effect will be visible in later results.

Considering the design of the low-pass filter, it is possible to design a suitable filter using the MATLAB signal processing toolbox, basing the design on a fourth order Chebyshev type II analogue low-pass prototype ${ }^{59}$. This prototype allows specification of the amount of attenuation in the stop band. In this case, after several iterations, the break frequency was chosen as $122 \mathrm{rads} / \mathrm{sec}(19.42 \mathrm{~Hz})$, with a stop band attenuation of $26.5 \mathrm{~dB}$, resulting in a fourth order filter which satisfied all requirements. The need to interate the design of the filter to obtain the best specification in terms of phase lag and attenuation highlights the need for some form of optimization of the filter design process.

In order to allow the implementation of such a filter, it was factorised into two second-order filters. Each of these second-order components was corrected to meet the limitations set out earlier. The resultant filter which meets all requirements can be described by the transfer function

$$
\mathrm{G}_{\mathrm{sf4}}(\mathrm{s})=\frac{0.046\left(\mathrm{~s}^{2}+4.47 \mathrm{~s}+101630\right)\left(\mathrm{s}^{2}+1.85 \mathrm{~s}+17437\right)}{\left(\mathrm{s}^{2}+186 \mathrm{~s}+11363\right)\left(\mathrm{s}^{2}+49.9 \mathrm{~s}+7380\right)}
$$

The frequency response of the above filter is as shown in Figure 4.19. Comparison of the two frequency responses shows that the low-pass filter introduces a much greater phase lag than the band-stop filters. Compare for example, a phase lag of 25.3 degrees at $3 \mathrm{~Hz}$ from the low-pass filter with a total of 7.3 degrees at the same frequency from the three band-stop filters. Clearly, there is a major advantage to be gained by maximising the break frequency of the low-pass filter, since this component makes the largest contribution to the phase lag created by the structural-mode filters.

Implementing all of the structural-mode filters results in the maximum open-loop magnitude response for the system as shown in Figure 4.20. From the figure, it can be seen that the required structural-mode attenuation has been achieved.

Assuming that the filters will act on all signal paths, it is possible to produce the time response of the full flexible aircraft system to a pitch stick demand signal similar to that used in Figure 4.8, the results being as shown in Figure 4.21. Clearly, the response is now satisfactory. 

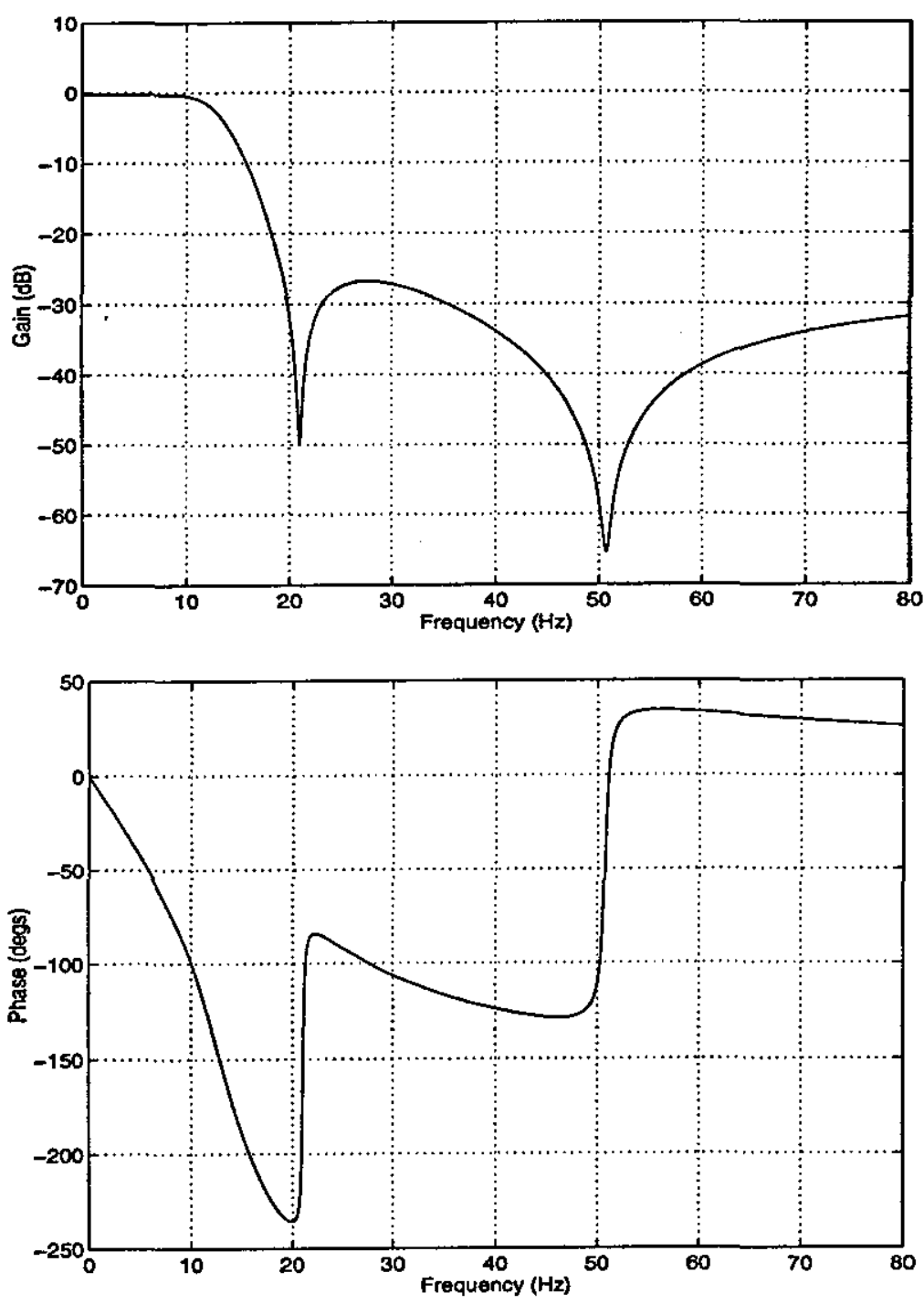

Figure 4.19 - Frequency response for fourth order low-pass filter

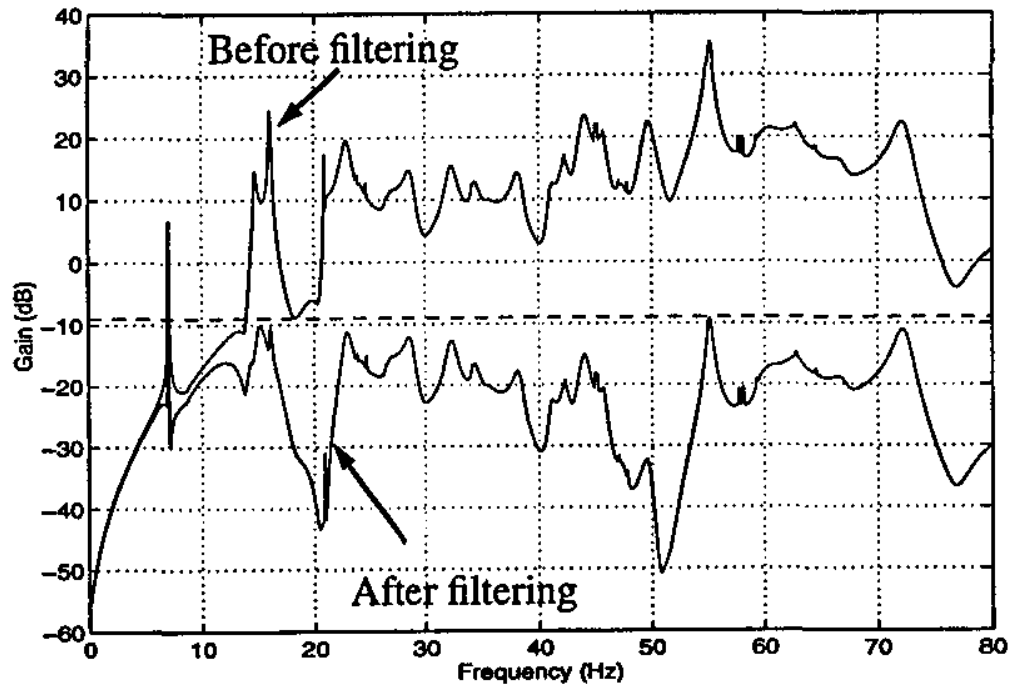

Figure 4.20 - Maximum open-loop response for full-order aircraft system incorporating the structural-mode filters 

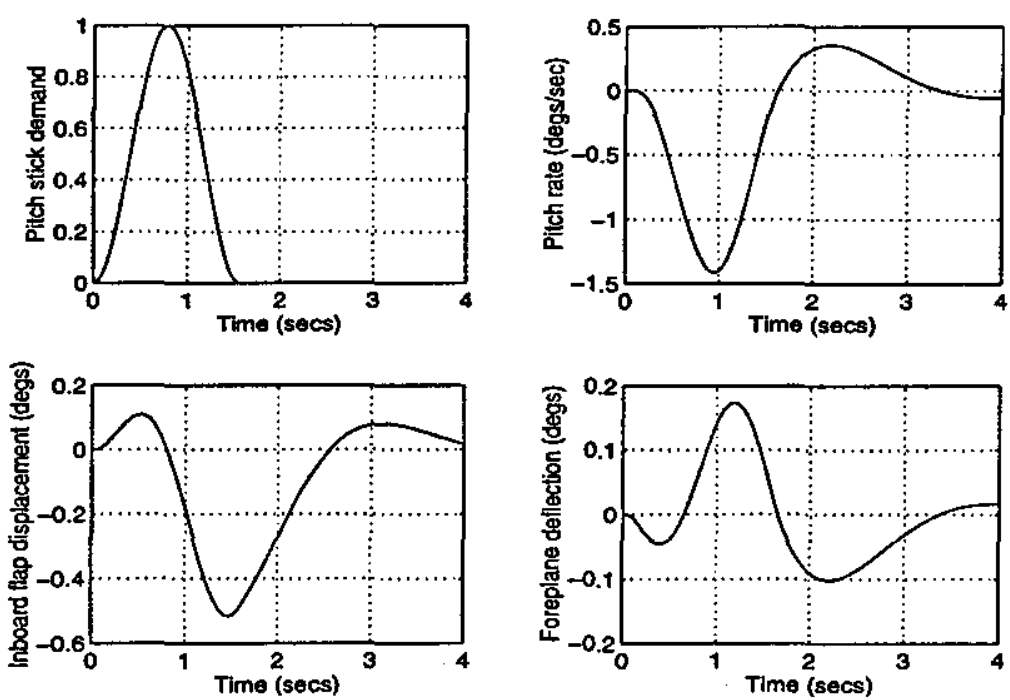

Figure 4.21 - Time response for full-order flexible aircraft system with structural-mode filters $(\mathrm{M}=0.8,36000 \mathrm{ft}$.)

Examining the effects of the structural-mode filters on the frequency response of the aircraft at rigid-body frequencies, the open-loop Nichols plot for the system incorporating the structural-mode filters is as shown in Figure 4.22. It can be seen that although the structural-mode filters have added significant phase lag to the system, the aircraft system still satisfies the clearance boundary requirements. The results as shown above were calculated for the case where all structural filters are in the feedback path, such that they act on all three signal paths. It would be possible however to take the contribution of each signal path toward a particular mode into consideration when designing the filters, such that the filter for a particular mode is only placed in the relevant path. This would reduce the phase lag introduced into the system as a whole. It can be seen however that all control surfaces play a small part in the excitation of each mode, so care would have to be taken when designing structural-mode filters for particular signal paths. Such an approach would also increase the complexity of the flight control system. 


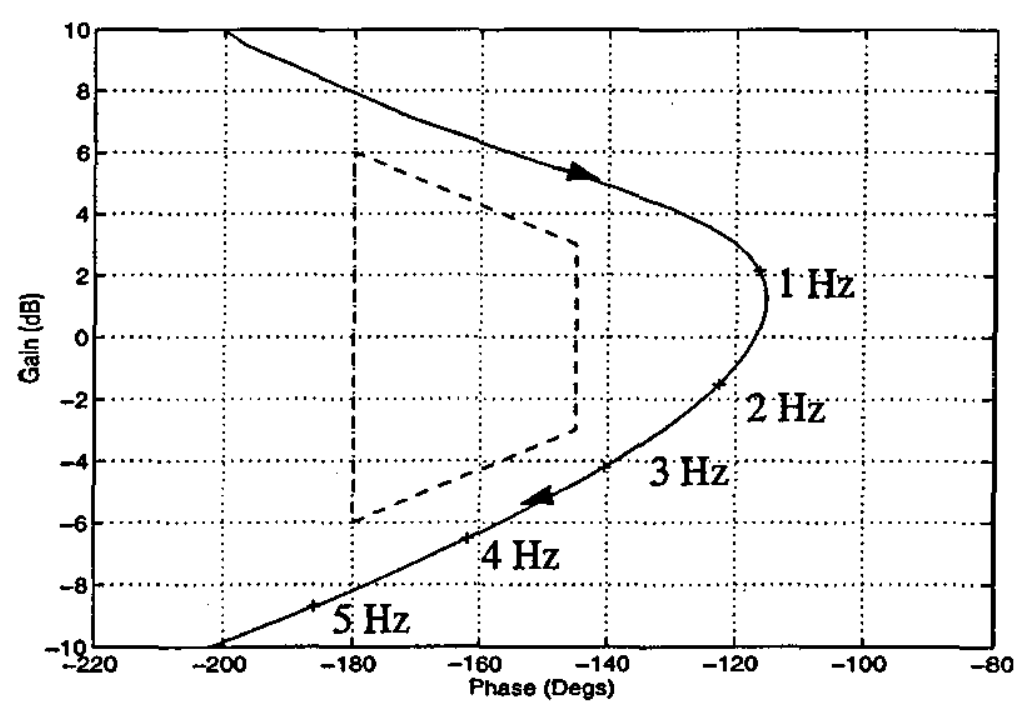

Figure 4.22 - Open-loop Nichols plot showing error signal stability-margins for aircraft system at rigid aircraft frequencies incorporating structural-mode filters $(\mathrm{M}=0.8,36000 \mathrm{ft}$. $)$

\subsection{Conclusions}

The problem of aeroservoelasticity has been demonstrated and the current method of solution examined. It is clear that the design of suitable structural-mode filters is a complex process as a result of the many conflicting requirements. Although the example system used here is relatively simple in that only two flight conditions are examined, it demonstrates the level of conservatism in the current design process.

In the following chapters, some of the assumptions applied in the filter design procedure will be examined. In addition, the assumptions regarding the linearity of the system will be discussed. 


\section{Chapter 5}

\section{Digital Sampling Effects and Sensor Modelling}




\subsection{Introduction}

In recent years, the flight control systems of aircraft have made an increasing use of digital computers for the processing of the flight control algorithms. This use of digital systems introduces a new element to the aeroservoelasticity problem. In some cases an aeroservoelastic interaction has occurred in the digital system when the analogue equivalent would show no such interaction ${ }^{15}$. The problem occurs because the flexible aircraft structure may contain structural-modes whose frequency is above the Nyquist frequency, resulting in aliasing of the response to within the bandwidth of the flight control system ${ }^{14}$.

This chapter develops the background to digital effects in the context of the aeroservoelastic problem, and uses this material to achieve a greater understanding of the overall interaction. In particular, consideration will be given to a situation whereby the system plant contains modes above the Nyquist frequency as may be the case in an aeroservoelastic system.

In order to assess the impact of the digital nature of the control system on the aeroservoelastic problem, it is necessary to consider the three main elements as shown in Figure 5.1.

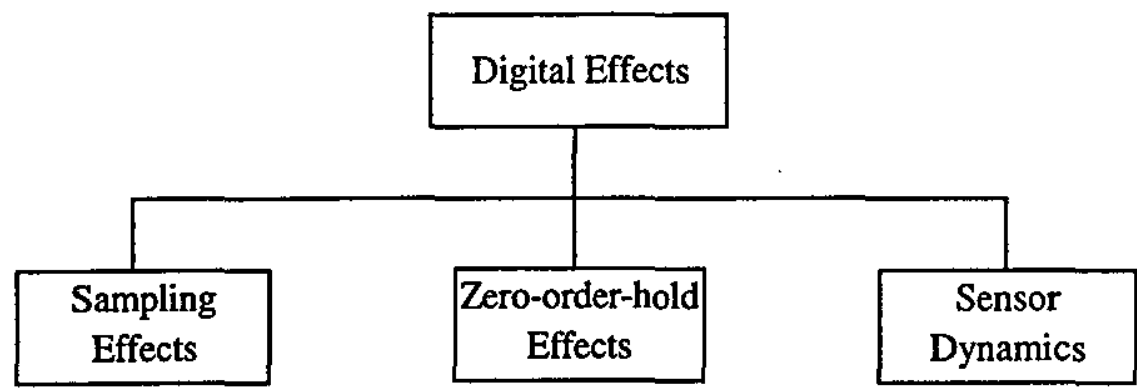

Figure 5.1 - Elements of the digital flight control system

\subsection{Analysis of the sampling process}

\section{$\underline{5.2 .1 \text { Introduction }}$}

The use of digital computers in modern aircraft to process the flight control algorithms introduces an additional factor into the aeroservoelastic problem. In order to understand the effect of the digital nature of the system in general, it is necessary to first obtain an insight into the sampling process itself.

The following section will examine the sampling process as highlighted in Figure 5.2, whilst paying particular attention to its effect on the aeroservoelastic interaction. 


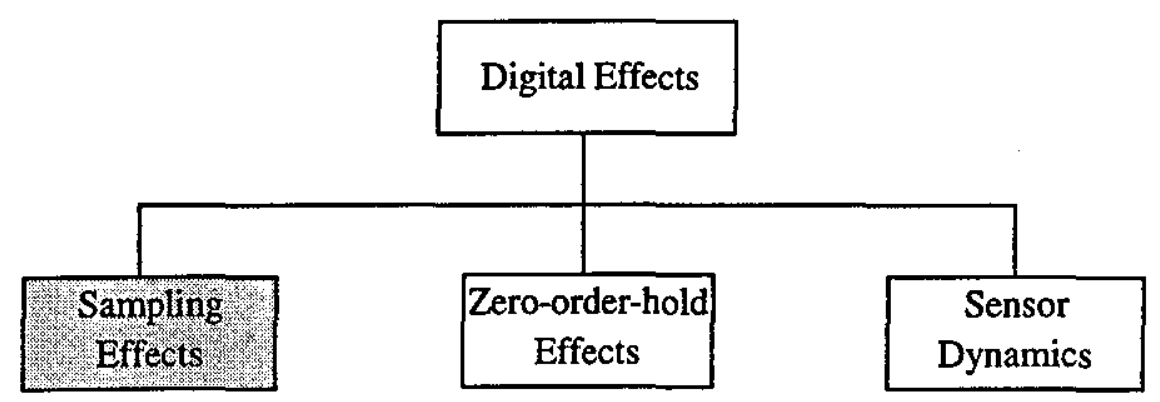

Figure 5.2 - Elements of the digital flight control system: sampling effects

\subsubsection{Mathematical analysis of the sampling process}

The mathematical theory of signal sampling has been discussed in many texts ${ }^{60-}$ ${ }^{62}$, but it is worth giving an overview of the analysis in a form more dedicated to the problem of aeroservoelasticity.

The sampling of a continuous signal is often ideally represented by the amplitude modulation of a unit impulse train ${ }^{60}$. The unit impulse train is defined as a train of unit area impulses at the sampling frequency, $\mathrm{T}$, as shown in Figure 5.3.

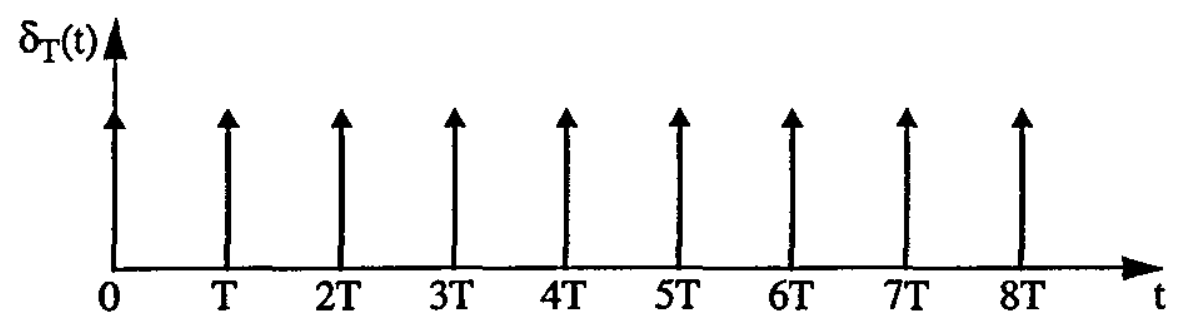

Figure 5.3 - Unit impulse train.

As the unit impulse train is a periodic function of frequency $2 \pi / \mathrm{T}$, it is possible to express the unit impulse train as a Fourier series, such that

$$
\delta_{T}(t)=\sum_{k=-\infty}^{\infty} \delta(t-k T)=\sum_{n=-\infty}^{\infty} C_{n} e^{j\left(\frac{2 \pi n}{T}\right) t}
$$

where the Fourier coefficients are given by,

$$
C_{n}=\frac{1}{T} \int_{-\frac{T}{2}}^{\frac{T}{2}} \delta_{T}(t) e^{-j n\left(\frac{2 \pi t}{T}\right)} d t
$$

so that in this case, 


$$
C_{n}=\frac{1}{T} \int_{-\frac{T}{2}}^{\frac{T}{2}} \sum_{k=-\infty}^{\infty} \delta(t-k T) e^{-j n\left(\frac{2 \pi t}{T}\right)} d t
$$

Since the only part of the impulse train that exists within the integral range is the unit impulse at $t=0$, the equation (5.3) reduces to

$$
C_{n}=\frac{1}{T} \int_{-\frac{T}{2}}^{\frac{T}{2}} \delta(t) e^{-j n\left(\frac{2 \pi t}{T}\right)} d t
$$

In order to evaluate this integral, it is necessary to make use of a property of the unit impulse known as the sifting property ${ }^{60}$. This can be expressed as

$$
\int_{-\infty}^{\infty} f(t) \delta(t-T)=f(T)
$$

Applying this result to equation (5.4) gives

$$
\int_{-\frac{T}{2}}^{\frac{T}{2}} \delta(t) e^{-j n\left(\frac{2 \pi t}{T}\right)} d t=\left.e^{-j n\left(\frac{2 \pi t}{T}\right)}\right|_{t=0}=1
$$

It is possible therefore to express the unit impulse train as

$$
\delta_{T}(t)=\frac{1}{T} \sum_{n=-\infty}^{\infty} e^{j\left(\frac{2 \pi n}{T}\right) t}
$$

Consideration of the above result reveals that the impulse train can be considered as an infinite sum of harmonically related cosine waves. This is the case since the impulse train is an even function which results in the sine terms in the expansion of the exponential term in equation (5.7) cancelling out.

If thought is given to a sum of harmonically related cosine waves, then it becomes clear that the waves will reinforce at $t=0, T, 2 T, \ldots, n T$, whilst cancelling out at all other time points, with the result that an impulse train will be produced if sufficient harmonics are added to the primary component. Now that an expression has been derived for the unit impulse train, it becomes possible to obtain expressions to describe a sampled signal.

As mentioned above, the sampling process can be considered to be the amplitude modulation of an unit impulse train by a continuous waveform. Therefore, the impulse train can be considered to be a carrier signal which is modulated by the continuous 
signal.

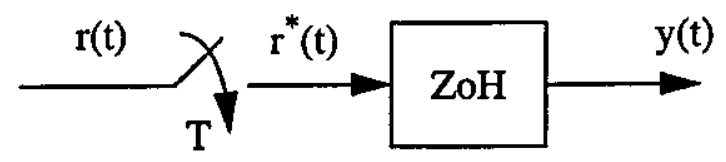

Figure 5.4- Block diagram of basic sampler and zero-order-hold

For a digital system as shown in Figure 5.4, the sampled signal can be expressed as

$$
r^{*}(t)=\frac{1}{T} \sum_{n=-\infty}^{\infty} r(t) e^{j\left(\frac{2 \pi n}{T}\right) t}
$$

Consider the Fourier transform of the sampled signal, which can be defined as

$$
R^{*}(j \omega)=\int_{-\infty}^{\infty} r^{*}(t) e^{-j \omega t} d t
$$

Substituting equation (5.8) into the above equation results in

$$
R^{*}(j \omega)=\frac{1}{T} \int_{-\infty}^{\infty} \sum_{=-\infty}^{\infty} r(t) e^{j\left(\frac{2 \pi n}{T}\right) t} e^{-j \omega t} d t
$$

Rearranging the order of the integration and summation, results in

$$
R^{*}(j \omega)=\frac{1}{T} \sum_{n=-\infty}^{\infty} \int_{-\infty}^{\infty} r(t) e^{j\left(\frac{2 \pi n}{T}\right) t} e^{-j \omega t} d t
$$

Denoting the Fourier transform of the continuous signal as

$$
\mathcal{F}_{\{r(t)\}}=R(j \omega)=\int_{-\infty}^{\infty} r(t) e^{-j \omega t} d t
$$

and noting that according to the frequency shift theorem

$$
\mathcal{F}_{\left\{e^{\mathrm{at}} r(\mathrm{t})\right\}}=\mathrm{R}(\mathrm{j} \omega-\mathrm{a})
$$

then the spectrum of the sampled signal can be defined as

$$
R^{*}(j \omega)=\frac{1}{T} \sum_{n=-\infty}^{\infty} R\left(j \omega-j n \omega_{s}\right)
$$

where $\omega_{\mathrm{s}}$ is the sampling frequency.

It can be seen from equation (5.14) that in terms of a given continuous signal 
spectra, $R(j \omega)$, the sampling process results in a spectra with an infinite number of side lobes. Each lobe is an attenuated version of the continuous signal spectrum centred on integer values of the sampling frequency.

The resulting process can be represented diagrammatically as shown in Figure 5.5.
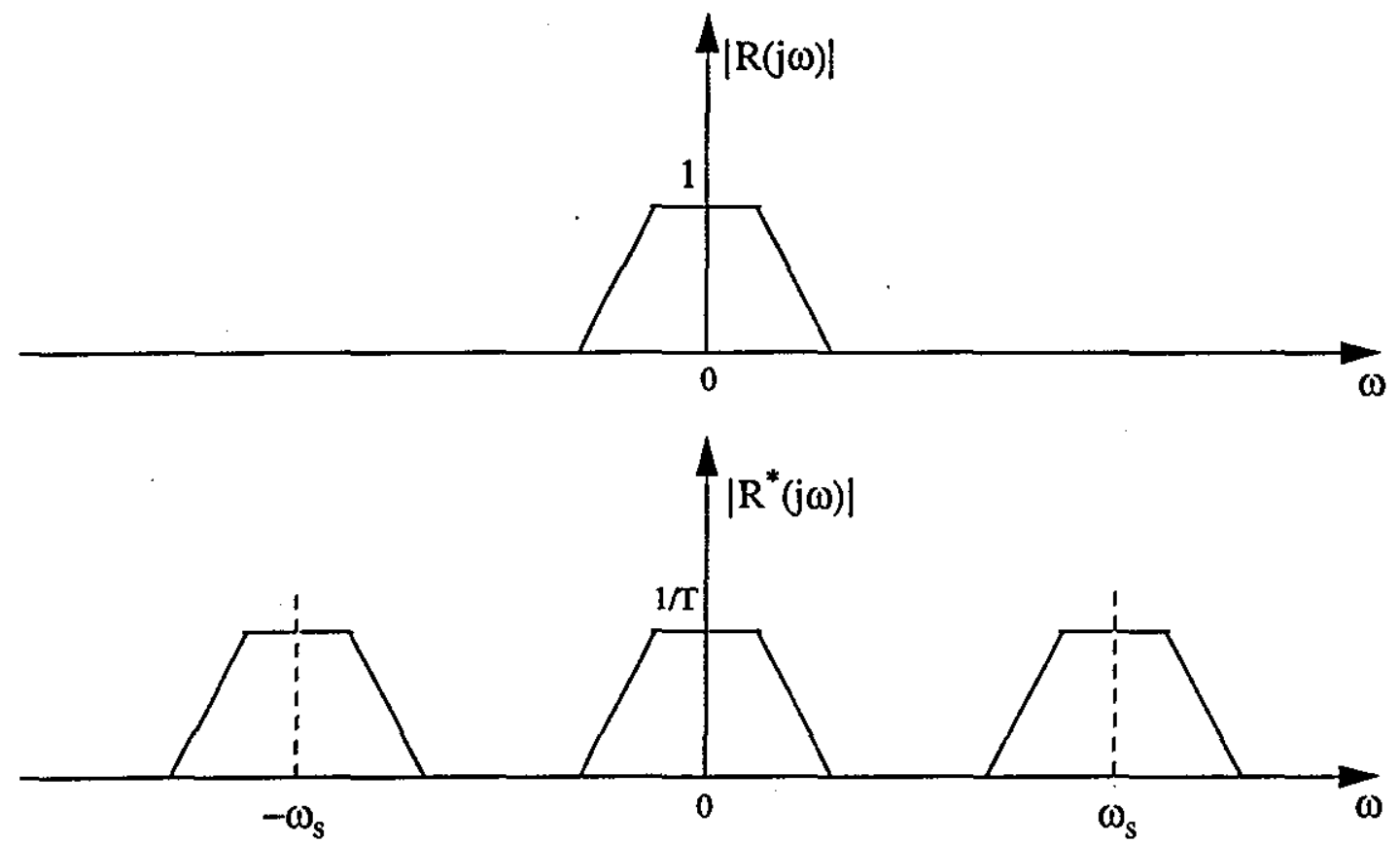

Figure 5.5 - Frequency spectra of arbitrary continuous and sampled signals

For a given spectra of a continuous signal, it is possible to derive the spectra of the sampled signal. Although the above result has been derived assuming perfect sampling which implies the width of the unit impulses is infinitely small, a similar procedure can be followed for a unit impulse train with finite pulse widths ${ }^{60}$.

The importance of this derivation is that it demonstrates the production of an infinite number of high-frequency signals by the sampling process. In an aeroservoelastic system for example, the sampled nature of the control system will result in high-frequency signals at the actuator input. This fact will be demonstrated later in the chapter.

\subsubsection{Sampling of a sinusoidal input signal}

So far, the theory has been derived in a general sense, with no specific form of input signal being considered. However, in the field of control system design the input signal considered is often a sinusoid. To apply the above theory to the design of control systems, it would be useful to obtain the frequency spectra of an arbitrary sinusoidal input signal.

The basis for the following derivation can be found in reference 62 , which calculates the frequency spectra for an arbitrary sinusoidal input signal of the form $r(t)=A \operatorname{Cos}\left(\omega_{0}+\phi\right)$. The derivation will be included here for completeness. 
Expressing the input signal as a sum of exponential terms gives

$$
I(t)=\frac{A}{2}\left(e^{j \omega_{0} t+j \phi}+e^{-j \omega_{0} t-j \phi}\right)
$$

Applying the derivation of the Fourier transform gives

$$
\mathcal{F}\{r(t)\}=R(j \omega)=\frac{A}{2} \int_{-\infty}^{\infty} e^{-j \omega t}\left(e^{j \omega_{0} t+j \phi}+e^{-j \omega_{0} t-j \phi}\right) d t
$$

which can be rewritten as

$$
R(j \omega)=\frac{A e^{j \phi}}{2} \int_{-\infty}^{\infty} e^{j t\left(\omega_{0}-\omega\right)} d t+\frac{A e^{-j \phi}}{2} \int_{-\infty}^{\infty} e^{j t\left(-\omega_{0}-\omega\right)} d t
$$

Consider now the sifting property of the impulse function as described earlier, which can be represented by

$$
\int_{-\infty}^{\infty} \delta(t) e^{-j \omega t} d t=1
$$

The inverse Fourier transform for an arbitrary signal $f(t)$ can be defined as

$$
f(t)=\frac{1}{2 \pi} \int_{-\infty}^{\infty} F(j \omega) e^{j \omega t} d \omega
$$

which, if $f(t)=\delta(t)$ and $F(j \omega)=1$, enables the unit impulse function to be expressed as

$$
\delta(t)=\frac{1}{2 \pi} \int_{-\infty}^{\infty} e^{j \omega t} d \omega
$$

so that by applying a change of variable and rearranging, an impulse in the frequency domain can be expressed as

$$
2 \pi \delta(\omega)=\int_{-\infty}^{\infty} e^{j \omega t} d t
$$

Applying this result to the expression for the frequency spectra of the sinusoidal signal as shown in equation (5.17) results in

$$
R(j \omega)=\pi A\left(e^{j \phi} \delta\left(\omega_{0}-\omega\right)+e^{-j \phi} \delta\left(-\omega_{0}-\omega\right)\right)
$$

and since the impulse function is an even function, the spectra can be expressed as 


$$
R(j \omega)=\pi A\left(e^{j \phi} \delta\left(\omega-\omega_{0}\right)+e^{-j \phi} \delta\left(\omega+\omega_{0}\right)\right)
$$

which represents two impulses in the frequency domain at frequencies of $\omega_{0}$ and $-\omega_{0}$ of intensity $\pi \mathrm{A}$ and phase $\phi$ and $-\phi$ respectively, where the phase angle, $\phi$, is with respect to a cosine wave input signal.

Diagrammatically, the spectrum for a sinusoid can be represented as shown in Figure 5.6 below.

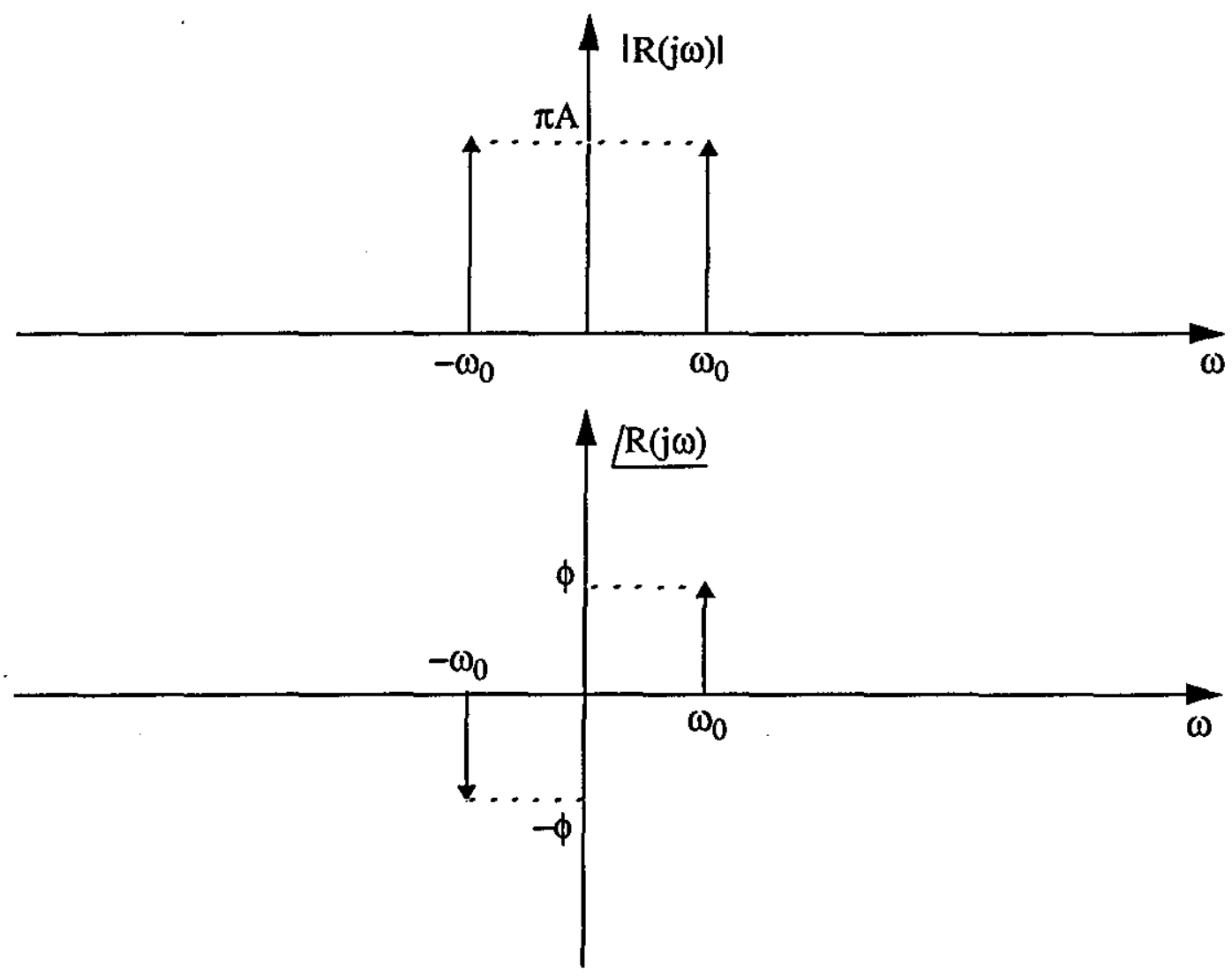

Figure 5.6 - Frequency spectrum for sinusoidal signal

The above spectrum therefore represents the frequency content of the continuous signal $r(t)$ in Figure 5.4. From equation (5.14), it is possible to construct the frequency spectra of the sampled version of this arbitrary sinusoidal input signal, which can be expressed mathematically as

$$
R^{*}(j \omega)=\frac{\pi A}{T} \sum_{n=-\infty}^{\infty} e^{j \phi} \delta\left(\omega-\left\{n \omega_{s}+\omega_{0}\right\}\right)+e^{-j \phi} \delta\left(\omega+\left\{n \omega_{g}+\omega_{0}\right\}\right)
$$

This can be represented diagrammatically as shown in Figure 5.7. From the diagram, it can be seen that the spectrum of the continuous signal has been repeated at intervals of the sampling frequency resulting in an infinite number of spectral lines.

Consider the spectral lines of the primary component in Figure 5.7. For this component, it is clear from the above derivation that the two spectral lines in the 
frequency domain represent a sinusoidal signal in the time domain of amplitude $A / T$ and phase $\phi$. The primary component of the sampled input signal can therefore be represented by

$$
r_{p}^{*}(t)=\frac{A}{T} \cos \left(\omega_{0} t+\phi\right)
$$
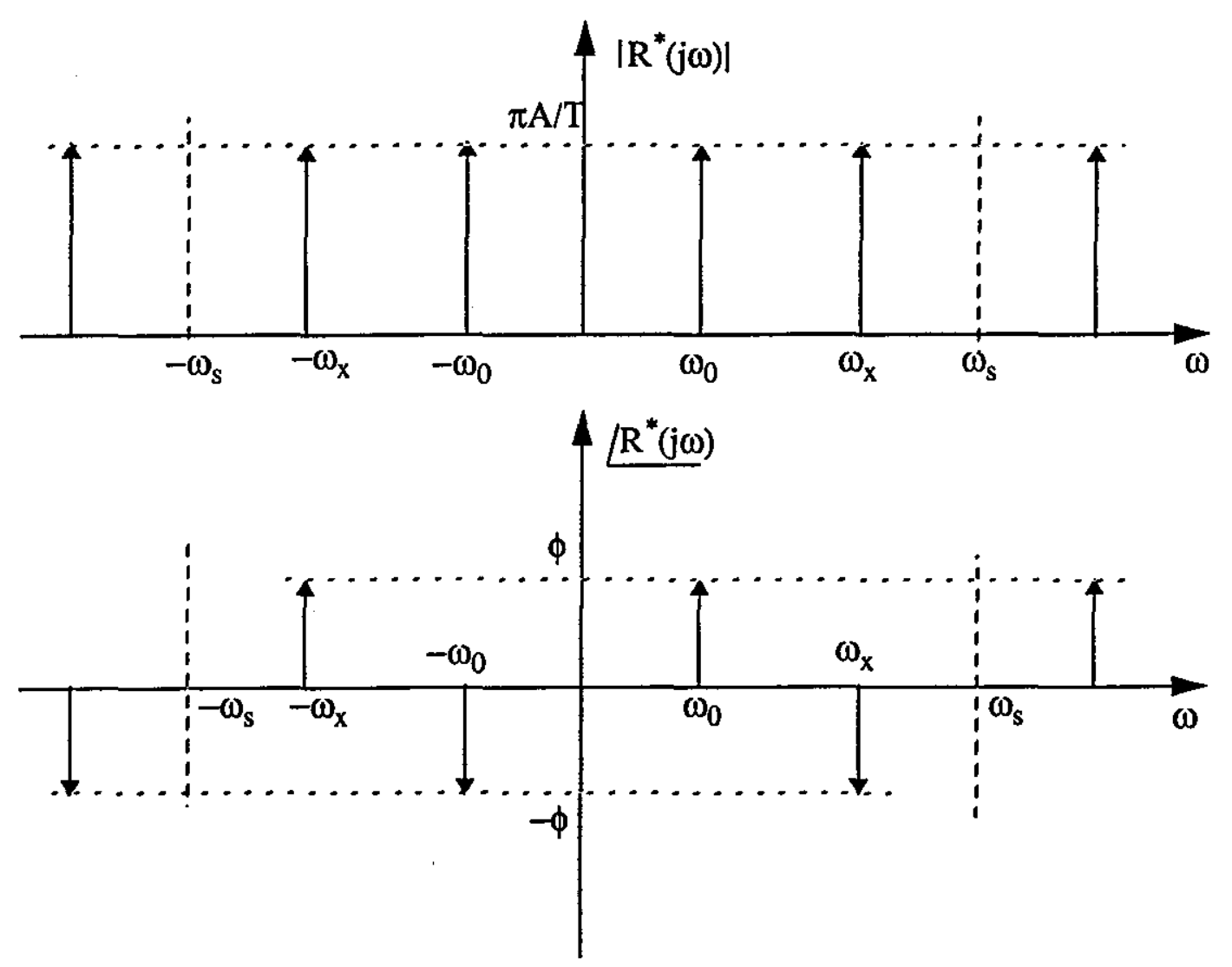

Figure 5.7 - Frequency spectrum for sampled arbitrary sinusoid

Consider the secondary component spectral lines at frequencies of $\omega_{x}$ and $-\omega_{x}$, where $\omega_{x}=\omega_{s}-\omega_{0}$ as shown in Figure 5.7. It can be seen from the earlier derivation that the spectrum of this secondary component signal will represent a sinusoidal signal of amplitude $A / T$, frequency $\omega_{x}$ and phase $-\phi$. This component signal can be represented by

$$
r_{1}^{*}(t)=\frac{A}{T} \cos \left(\left(\omega_{s}-\omega_{0}\right) t-\phi\right)
$$

Extending this method, it is possible to obtain an expression for the sampled signal, $\mathrm{r}^{*}(\mathrm{t})$ as shown in Figure 5.4, giving

$$
r^{*}(t)=\frac{A}{T}\left(\cos \left(\omega_{0} t+\phi\right)+\sum_{n=1}^{\infty} \cos \left(\left\{n \omega_{s}-\omega_{0}\right\} t-\phi\right)+\cos \left(\left\{n \omega_{s}+\omega_{0}\right\} t+\phi\right)\right)
$$

The sampled signal can be considered to be made up of an infinite number of harmonic components of identical amplitude and with phases alternating between $\phi$ 
and $-\phi$.

This derivation highlights the generation of high-frequency aliases of the primary input frequency by the sampling process. Clearly, these high-frequency aliases would have the potential for excitation of the aircraft structure if they are not sufficiently attenuated by the zero-order-hold and actuator dynamics.

\subsubsection{Aliasing}

Consider now the situation where the frequency of the continuous signal is greater than half the sampling frequency. Consequently, the secondary component spectral lines at a frequency of $\omega_{x}$ in Figure 5.7 would be at a frequency lower than the continuous signal. This is representative of the aliasing of a high-frequency signal by the sampler. In the case of an aeroservoelastic system, where a high-frequency structural-mode could be beyond the bandwidth of the control system, such aliasing could result in an unstable response as a result of the digital nature of the control system. Further consideration of the aliasing of high-frequency structural response will be given later in the chapter.

\subsection{Effect of the zero-order-hold}

\subsubsection{Introduction}

The previous section has developed in some detail the theory relating to the sampled nature of the control system. The most important points to note are that highfrequency component signals are introduced within the system, and that the aliasing of high-frequency response to within the bandwidth of the flight control system is possible.

As the interface between the sampled and continuous elements of an aeroservoelastic system, the zero-order-hold represents one of the three main components of the digital system as highlighted in Figure 5.8.

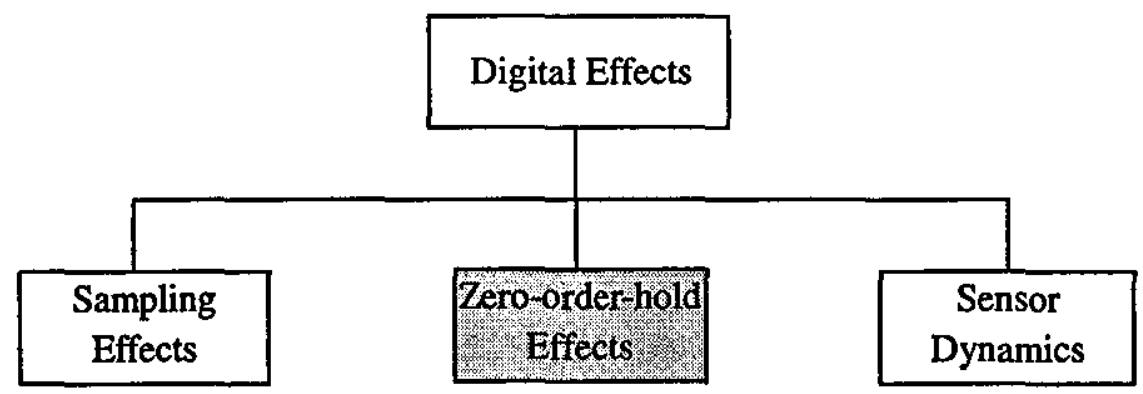

Figure 5.8 - Elements of the digital flight control system: zero-order-hold effects

The following section will analyse the effect of the zero-order-hold function on the components of the sampled signal, enabling the components of the output signal from 
the zero-order-hold to be predicted.

\subsubsection{Transfer function of the zero-order-hold}

The transfer function of the zero-order-hold block can be derived (reference 62) as

$$
G_{\mathrm{ZOH}}(j \omega)=\frac{1-e^{-j \omega T}}{j \omega}
$$

which can be expressed as

$$
G_{Z O H}(j \omega)=e^{-j \frac{\omega T}{2}}\left(\frac{e^{j \frac{\omega T}{2}}-e^{-j \frac{\omega T}{2}}}{2 j}\right) \frac{2 j}{j \omega}
$$

This can be rewritten as

$$
G_{\mathrm{ZOH}}(\mathrm{j} \omega)=\mathrm{Te} \mathrm{j}^{-\mathrm{j} \frac{\omega T}{2} \sin \frac{\omega T}{2}} \frac{\frac{\omega T}{2}}{2}
$$
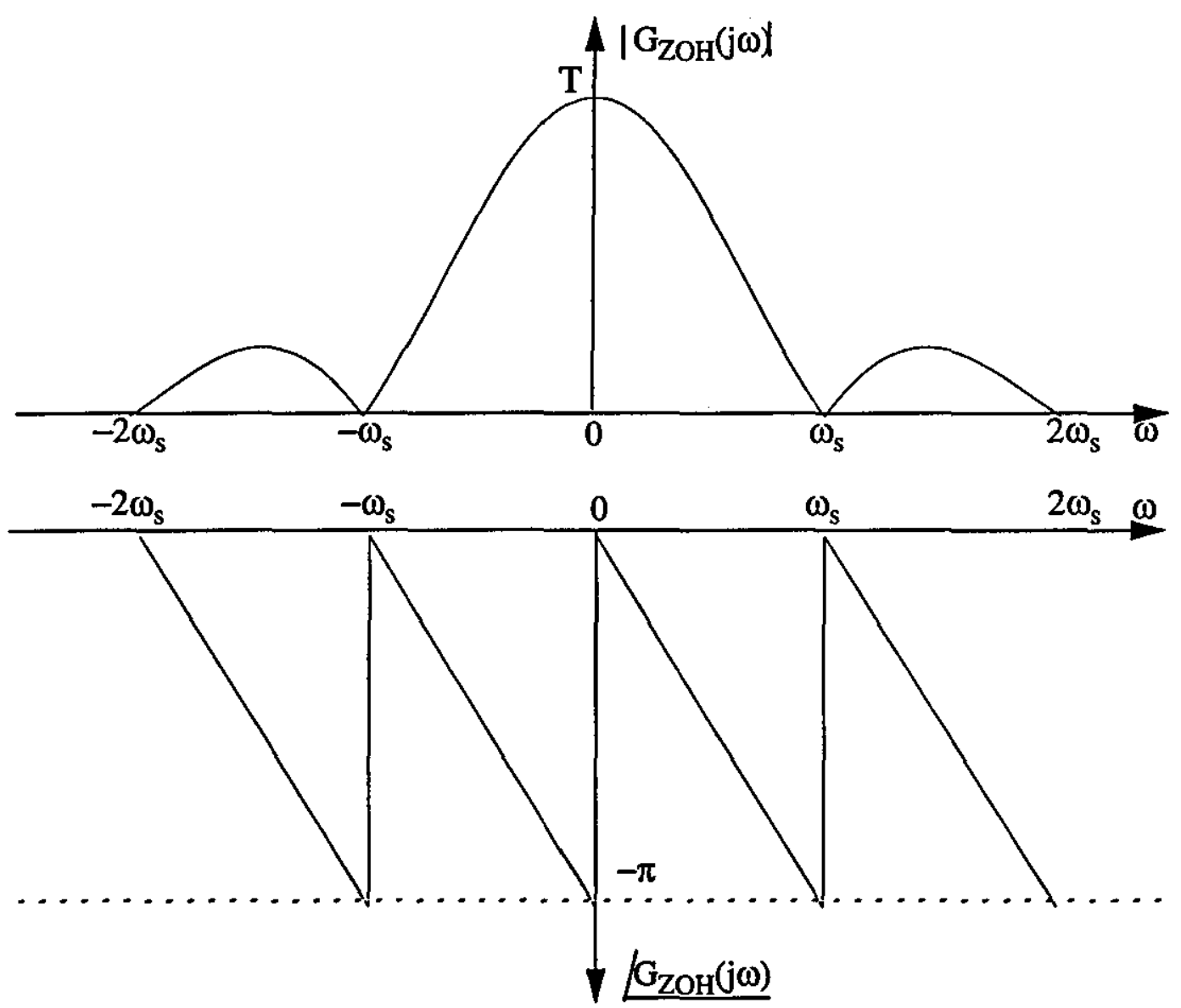

Figure 5.9 - Frequency response of Zero-order-hold function up to twice the sampling frequency

The frequency response of the zero-order-hold function can be represented on a 
Bode plot as shown in Figure 5.9. This frequency response may be applied to the component signals of the sampled sinusoidal input, resulting in the component signals of the zero-order-hold output signal.

It is possible therefore, to express the zero-order-hold output signal as a sum of weighted harmonic component signals as given by

$$
\begin{aligned}
y(t)=f\left(\omega_{0}\right) & \cos \left(\omega_{0} t+\phi-\frac{\omega_{0} T}{2}\right)+\sum_{n=1}^{\infty} f\left(n \omega_{s}-\omega_{0}\right) \cos \left(\left(n \omega_{s}-\omega_{0}\right) t-\phi-\pi+\frac{\omega_{0} T}{2}\right) \\
& +\sum_{n=1}^{\infty} f\left(n \omega_{s}+\omega_{0}\right) \cos \left(\left(n \omega_{s}+\omega_{0}\right) t+\phi-\frac{\omega_{0} T}{2}\right)
\end{aligned}
$$

where the amplitude of each component is given by the frequency dependent function

$$
f(\omega)=A\left|\frac{\sin \frac{\omega T}{2}}{\frac{\omega T}{2}}\right|
$$

A simple way to visualise the effect of the sampling and zero-order-hold on the sinusoidal input signal is to consider the input signal of form $r(t)=A \cos \left(\omega_{0} t+\phi\right)$ as being represented by an amplitude and phase diagrams as shown in Figure 5.10.

From the earlier theory, it is clear that the sampling of this arbitrary sinusoid will result in a signal whose components can be represented as shown in Figure 5.11, which is a diagrammatic representation of equation (5.27).

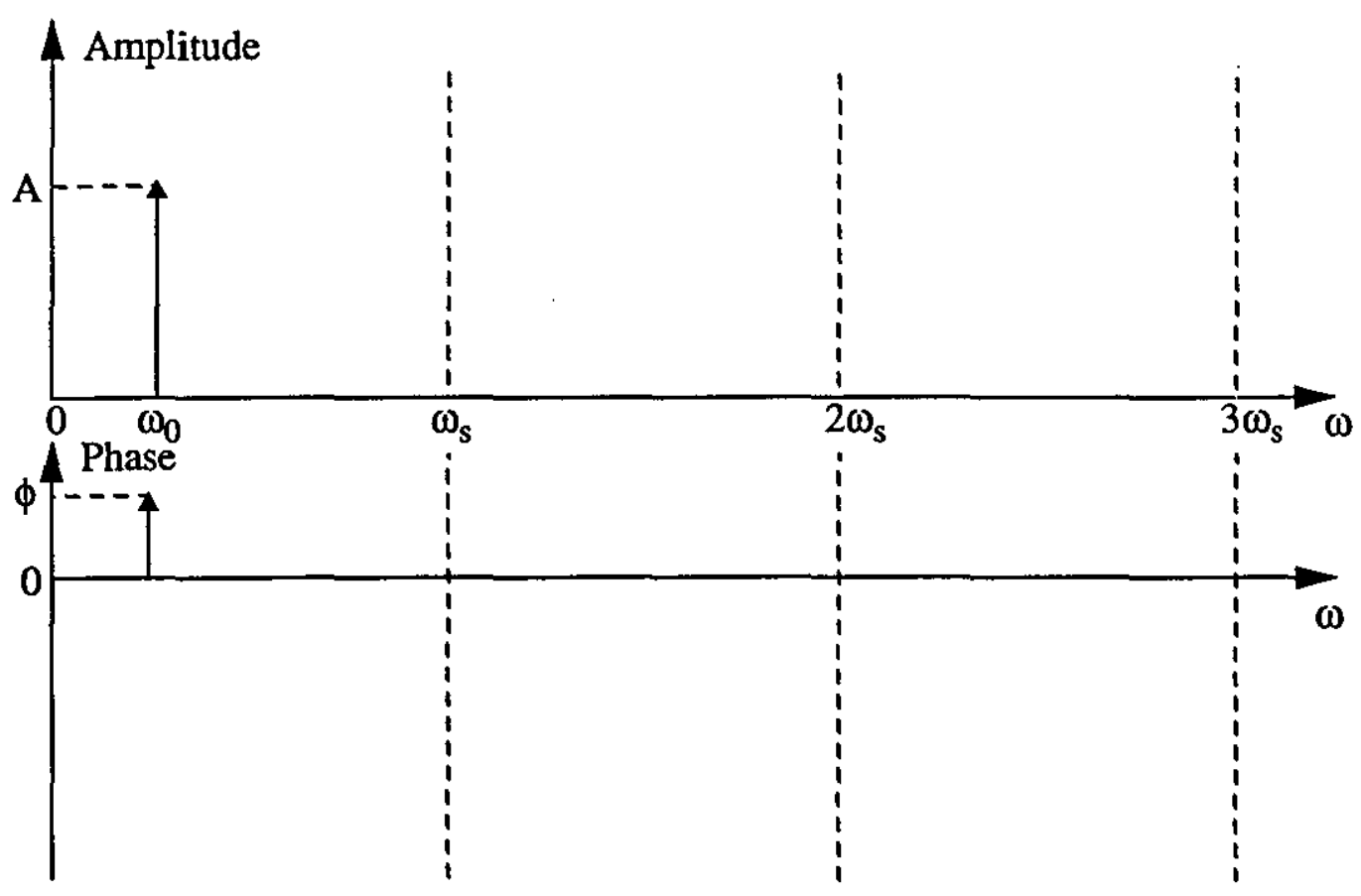

Figure 5.10- Representation of continuous time sinusoidal input signal 


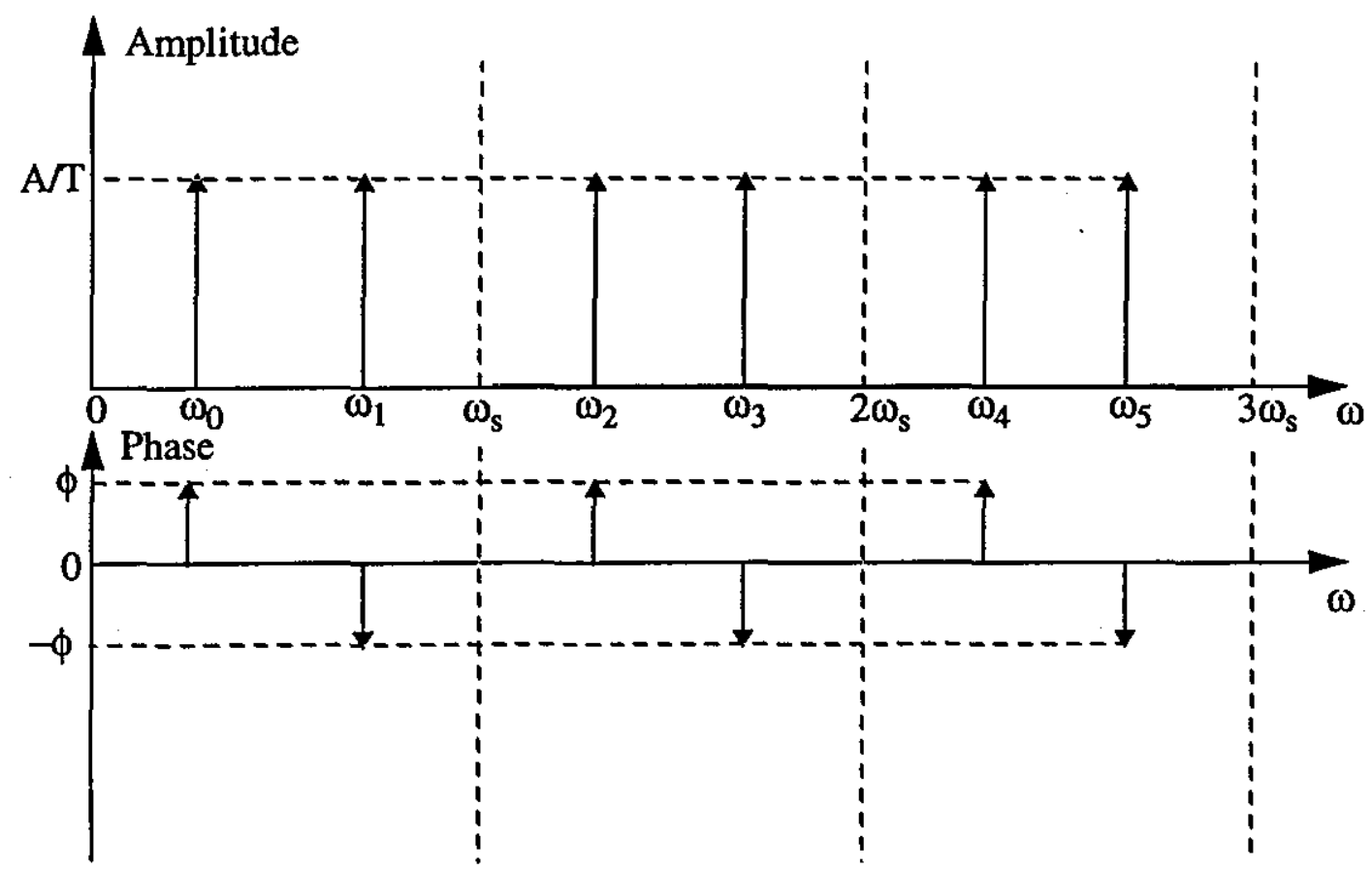

Figure 5.11 - Representation of sampled sinusoidal input signal

Applying the zero-order-hold response to each of these signal components results in the components of the zero-order-hold output, which can be represented as shown in Figure 5.12. It is possible, therefore, to determine the amplitudes and phases of the component signals that make up both the sampled input signal, and zero-order-hold output signal.

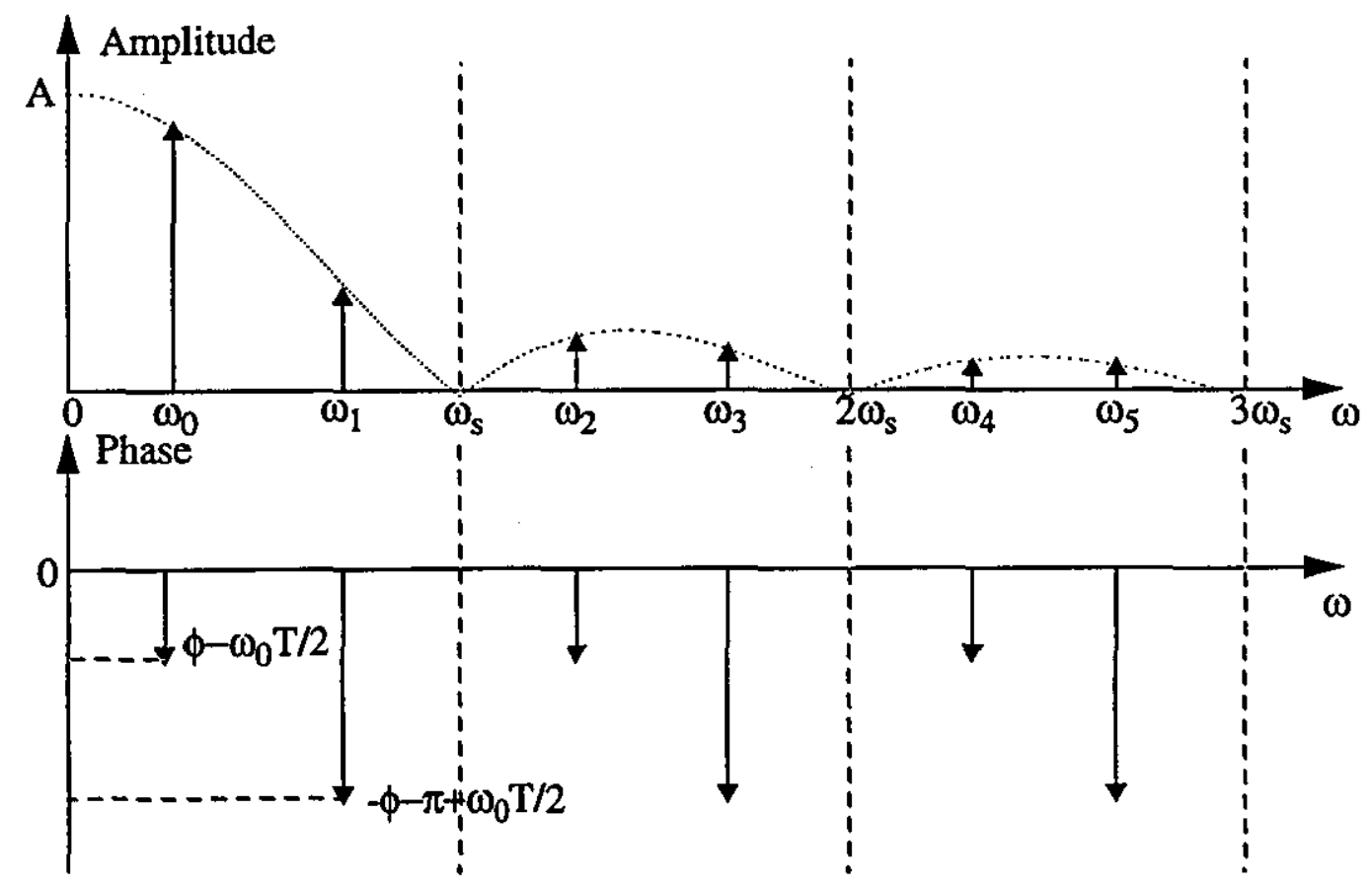

Figure 5.12- Representation of Zero-order-hold output signal

As an example, consider an arbitrary sampled sinusoid as shown in Figure 5.13 
being sampled with a sampling frequency of $10 \mathrm{~Hz}$. The true analogue version of this sampled signal, as would be created downstream of the zero-order-hold is as shown in Figure 5.14.

Using the earlier results of Fourier analysis as applied to sampled signals, it is possible to generate the components of the sampled signal, which can then be used to reconstruct the zero-order-hold output signal. There are an infinite number of these components, but it is possible to obtain a reasonably accurate reconstruction of the signal from relatively few components. Figure 5.14, shows a reconstruction of the zero-order-hold output signal using only five of the theoretical component signals. It can be seen that even with relatively few components, the reconstructed signal is a reasonable match to the actual signal.

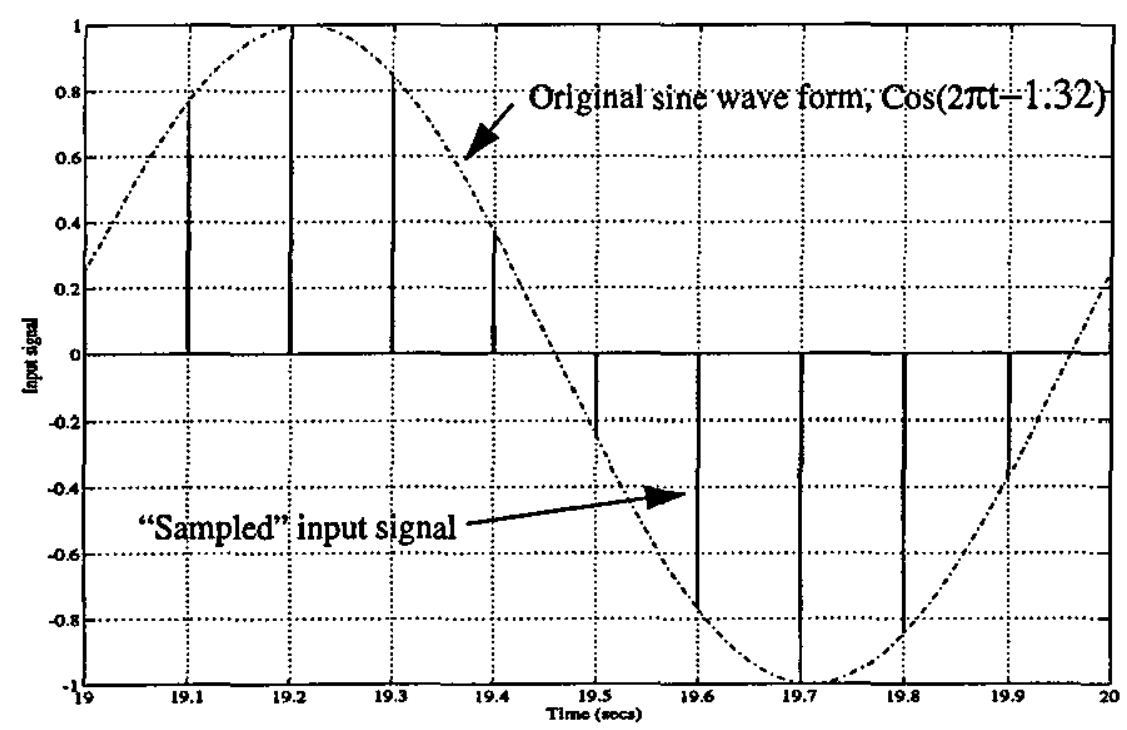

Figure 5.13 - Original and sampled version of arbitrary input signal

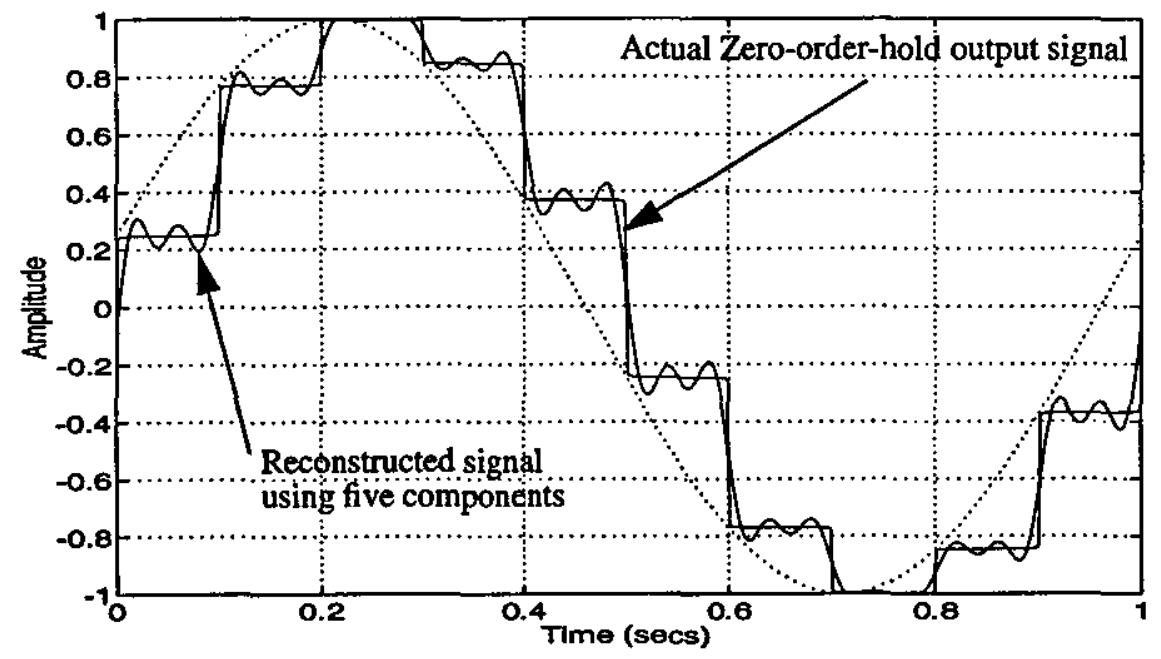

Figure 5.14 - Actual and reconstructed Zero-order-hold output signal

The importance of this derivation is that it demonstrates that the digital nature of 
the control system results in an infinite number of high-frequency components in the output signal of the zero-order-hold. Since it is this signal that drives the actuators, which in turn excite the aircraft structure, the potential for excitation of a structuralmode is clear. Importantly, however, it has been shown that the zero-order-hold provides a high-level of attenuation to these high-frequency signals, demonstrating that this attenuation should be taken into consideration within any analysis of a digital aeroservoelastic system.

\subsection{Sensor modelling}

\subsubsection{Introduction}

The role played by the sensor in the aeroservoelastic problem has already been discussed in some detail in Chapter 2, with the need for accurate modelling of the system being identified. In this section a suitable model of a typical sensor is developed, forming the third of the additional elements introduced by the digital nature of the control system as shown in Figure 5.15.

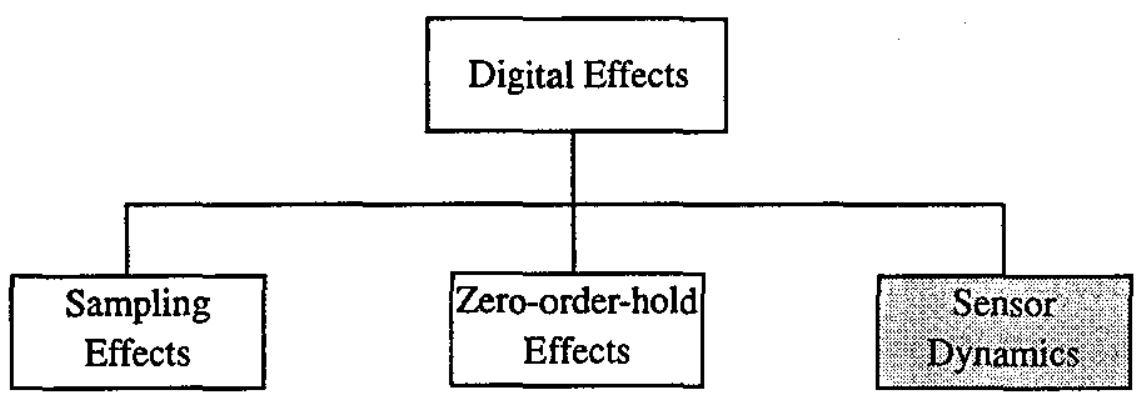

Figure 5.15 - Elements of the digital flight control system: sensor dynamics

In modern aircraft, the primary flight control sensors are generally rate gyros located in a single unit within the airframe of the aircraft, neglecting any multiplexing of the unit for reliability reasons. This unit, the Aircraft Motion Sensor Unit (AMSU) will generally consist of a set of laser ring gyros and the associated power and signal processing units. It is this unit which senses the structural response of the aircraft in addition to the rigid-body dynamics of the aircraft, passing the sensed rates to the flight control computers for processing of the flight control algorithms.

\subsubsection{Modelling of the digital aircraft motion sensor unit}

In this case, the aircraft motion sensor unit is a digital system as shown in Figure 5.16. The aircraft response is sensed by laser gyros, one rate gyro being aligned in each aircraft body axis. The output from the rate gyros is then sampled before input to the signal processing system. This system averages out the response of the rate gyros before passing the result to the flight control computer via a voting/monitoring system. 


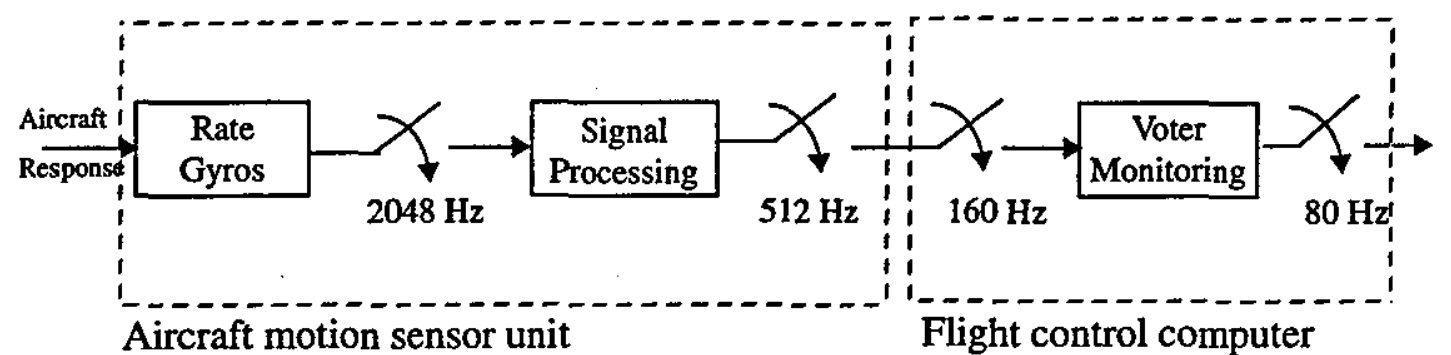

Figure 5.16 - Block diagram of the aircraft motion sensor unit

Neglecting the voting/monitoring process as having little if no effect on the system dynamics under normal operating conditions, and assuming that the rate gyros are capable of perfectly sensing the aircraft response, it is possible to obtain a model of the aircraft motion sensor unit by considering the signal processing unit alone.

A timing diagram representing the signal processing interface between the rate gyros and flight control computer is included as Figure $5.17^{63}$, where the computational time delay of the averaging process has been neglected.

From Figure 5.17, the signal processing within the aircraft motion sensor unit takes an average of 24 of the $2048 \mathrm{~Hz}$ gyro output samples. The result is then output at a frequency of $512 \mathrm{~Hz}$. The flight computer samples these AMSU output signals at a sampling rate of $160 \mathrm{~Hz}$ for the voting/monitoring system, the flight control algorithms being executed at a sampling rate of $80 \mathrm{~Hz}$.

The averaging process of the AMSU can be expressed as a $\mathrm{z}$-domain transfer function

$$
C(z)=\frac{\left(z^{-1}+z^{-2}+z^{-3}+z^{-4}+\ldots+z^{-23}+z^{-24}\right)}{24} R(z)
$$

which can be expressed as

$$
\frac{C}{R}(z)=\frac{\sum_{r=0}^{23} z^{r}}{24 z^{24}}
$$

Neglecting the sampling effects of the 2048 and $512 \mathrm{~Hz}$ sampling, it is possible to produce a frequency response for the AMSU as shown in Figure 5.18. This can be thought of as the frequency response for the AMSU from aircraft response to sensor output under the assumptions made at present. 

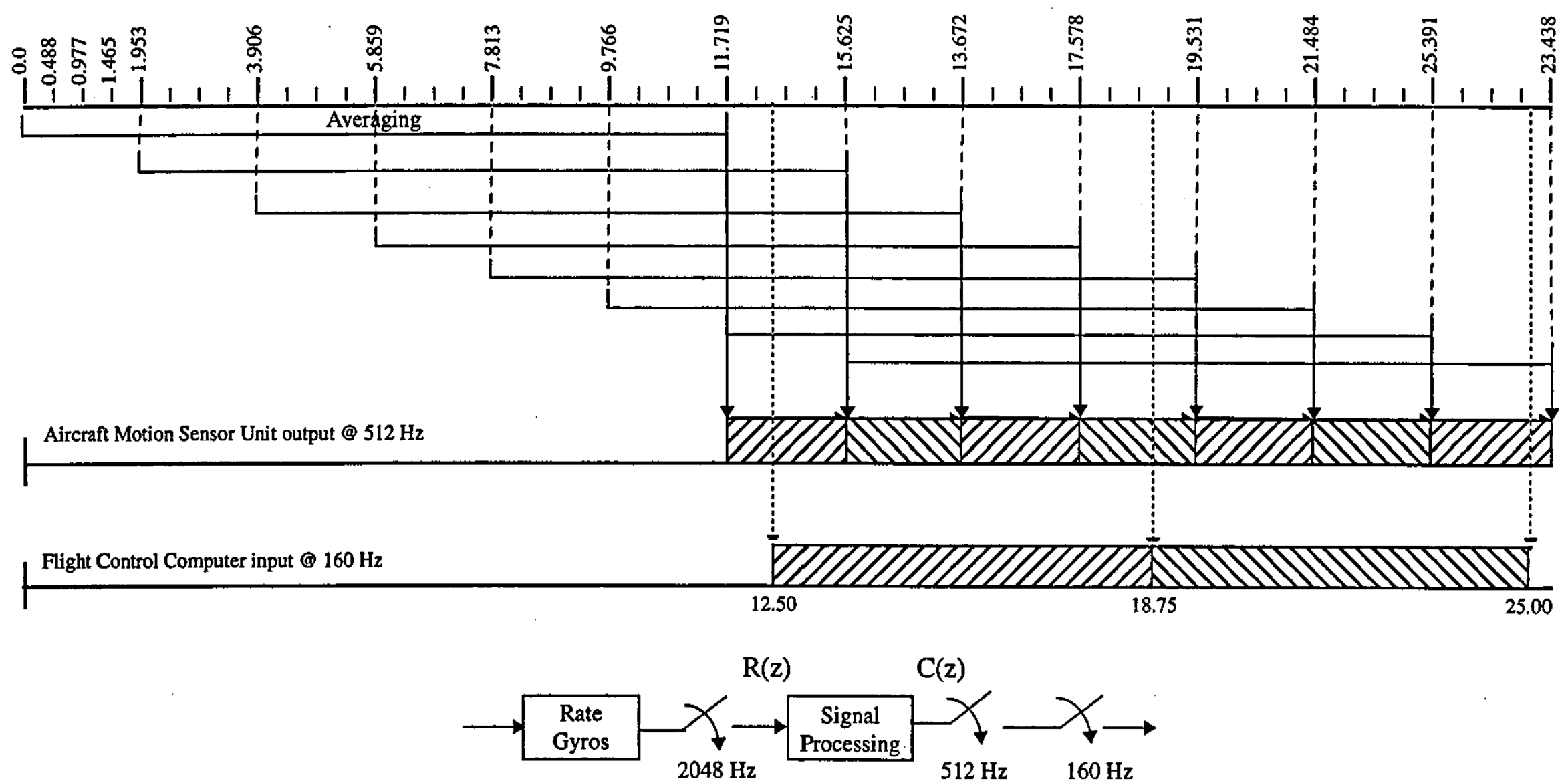

Times in msec

Figure 5.17 - Timing diagram for rate gyro/flight control computer signal processing interface 


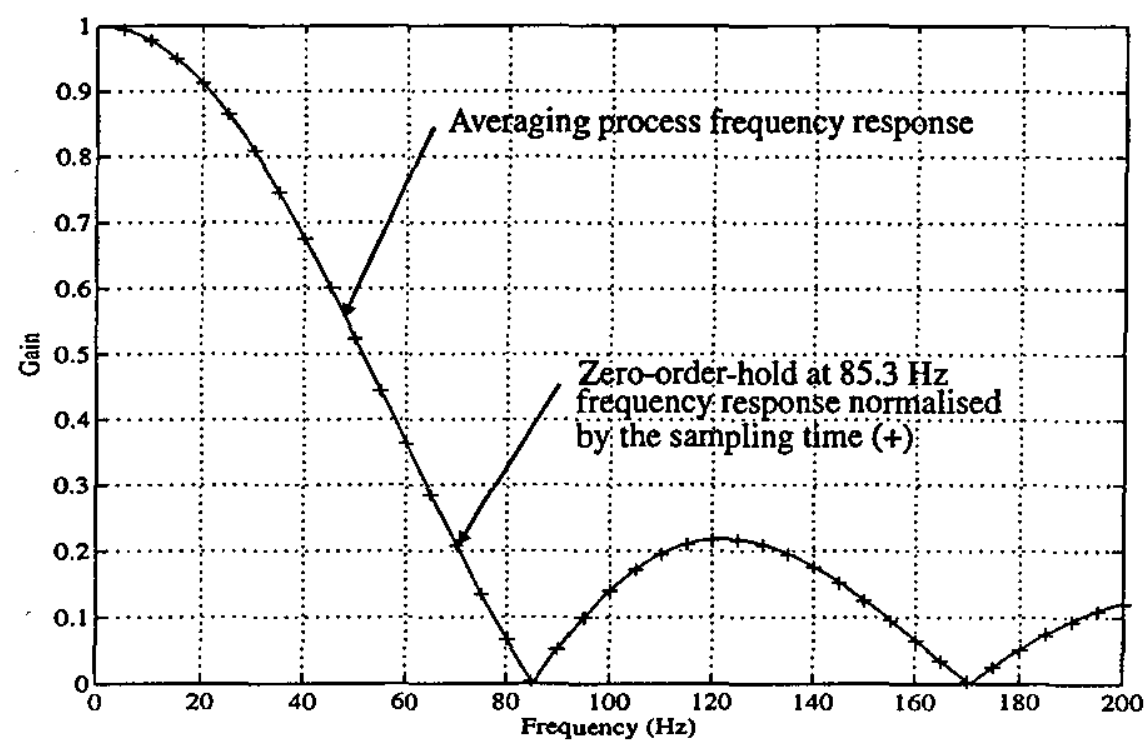

Figure 5.18 - Frequency response for Aircraft motion sensor unit

Comparing Figure 5.18 with the frequency response of the zero-order-hold as shown in Figure 5.9 demonstrates that the sensor sampling can be considered as a zero-order-hold process operating at a sampling frequency of approximately $85 \mathrm{~Hz}$. This can be seen from Figure 5.18, where the gain response for a $85.3 \mathrm{~Hz}$ zero-orderhold has been included.

It is clear that the under the assumption made above, the sensor dynamics can be represented by the averaging $z$-domain transfer function as given in equation (5.34), producing the gain response as shown in Figure 5.18. Naturally, there will be a computational time delay produced as a result of the digital averaging of the rate gyros output signals, but in addition to this delay, there exists a time dependent "asynchronous delay" as a result of the difference in sampling rates between the AMSU output and flight control computer input. For simplicity, these computational delays will be neglected in this case. It should be noted however that such delays will cause additional phase lags within the system and should be taken into account in the initial flight control system design.

\subsection{Application of digital effects to a typical system}

\subsubsection{Introduction}

The ability to calculate the magnitude and phase of the components of both the output of the sampler and the output of the zero-order-hold is very useful as a means of analysing the propagation of signals through a typical linear system under the control of a digital controller. In the case of a typical aeroservoelastic system, where the plant may show significant response at frequencies above the Nyquist frequency, these effects are of particular concern. 
The following section will demonstrate the application of the earlier results to a example typical of an aeroservoelastic system.

\subsubsection{Application of digital effects to an aeroservoelastic system}

Consider the block diagram for a typical aeroservoelastic system as shown in Figure 5.19.

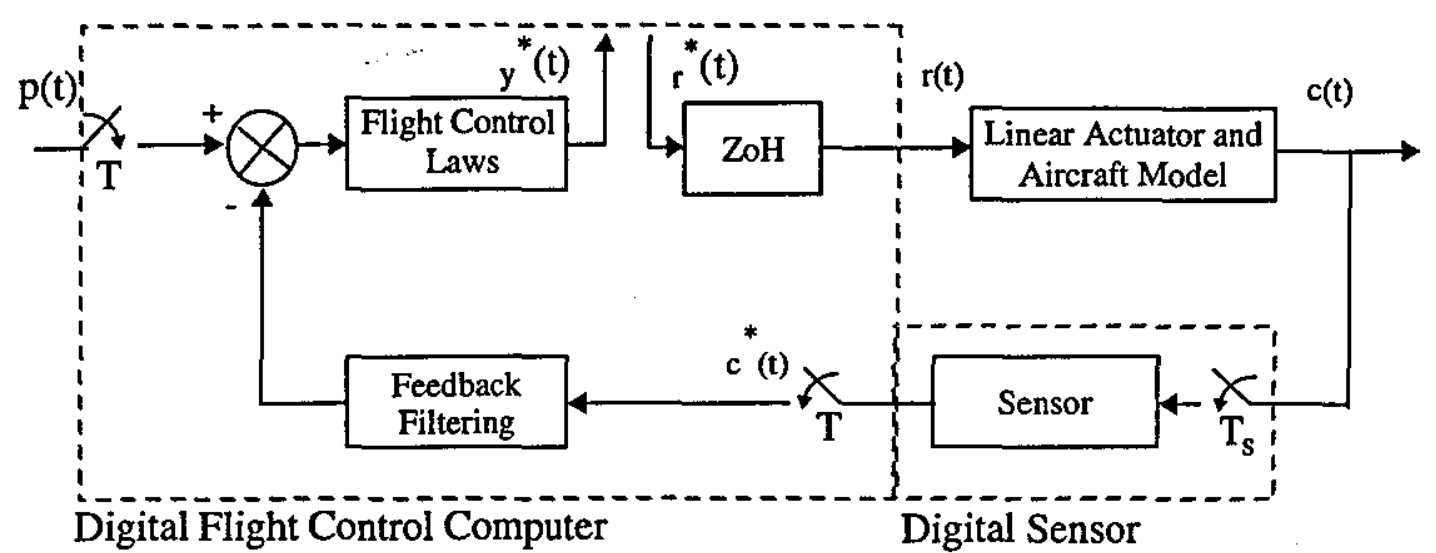

Figure 5.19 - Signal flow diagram through analogue linear system

Here, the input to the system, $\mathrm{r}^{*}(\mathrm{t})$, can be considered as being the output from a digital controller, prior to the zero-order-hold block.

Consider that the output from the zero-order-hold block, $r(t)$ is the input to a linear plant. Since the plant is linear it is possible to derive the amplitudes and phases of the components of the plant output signal, $c(t)$, from the components of the plant input signal, $r(t)$.

In most cases, the linear plant would be low-pass in nature, resulting in the attenuation of the high-frequency components. This is not the case in an aeroservoelastic system however, where there may be significant system response at high-frequencies. In particular, there may be structural-modes beyond the Nyquist frequency.

In the case of an aeroservoelastic system and in fact for all closed-loop digital systems, the output of the plant will be sampled for feedback to the digital controller as in Figure 5.19. Neglecting any sensor dynamics, and assuming that the sampling frequency remains the same as for the initial controller sampler, then it is possible to derive the component signals of $c^{*}(t)$ in Figure 5.19. Importantly, the sampling of the plant output signal will result in the aliasing of all of the component signals on to the frequency of the original continuous signal. To examine the effect of foldback more carefully, consider the frequency spectrum for a plant output signal as represented by Figure 5.20. Such an output signal spectra would be typical for the case of a primary 
input signal of frequency $\omega_{0}$. In addition, suppose that the plant itself possessed a structural-mode at the frequency $\omega_{1}$. This would result in a significant component in the output signal at that frequency.

The theory leading to equation (5.14) shows that after sampling, the spectrum of the sampled plant output signal can be derived from that of the continuous input signal by repeating the continuous spectrum at integer multiples of the sampling frequency, and attenuating the resulting spectrum by $1 / T$. This process has been demonstrated in Figure 5.5. If the continuous signal spectra contains elements at frequencies greater than half of the sampling frequency, then each of the lobes in the sampled version will interfere. In this case, where the spectrum of the continuous signal extends over an infinite bandwidth due to the initial sampling, the individual spectra centred on multiples of the sampling frequency will all interfere, and it is this interference that results in the aliasing of the response.

For this example, consider the spectrum as shown in Figure 5.20, but centred on the frequency $\omega_{\mathrm{s}}$. In this case, the spectral line at $-\omega_{1}$ in the original spectrum would fall on to that at $\omega_{0}$ on the spectrum centred at $\omega=0$. The same effect would apply for the spectrum centred at $-\omega_{s}$ where the spectral line at $\omega_{1}$ on the original spectrum would fall on to that at $-\omega_{0}$ on the spectrum centred at $\omega=0$. This would also be true for the spectral lines at $\omega_{0}$ and $-\omega_{0}$ being shifted to fall on those at $\omega_{1}$ and $-\omega_{1}$.
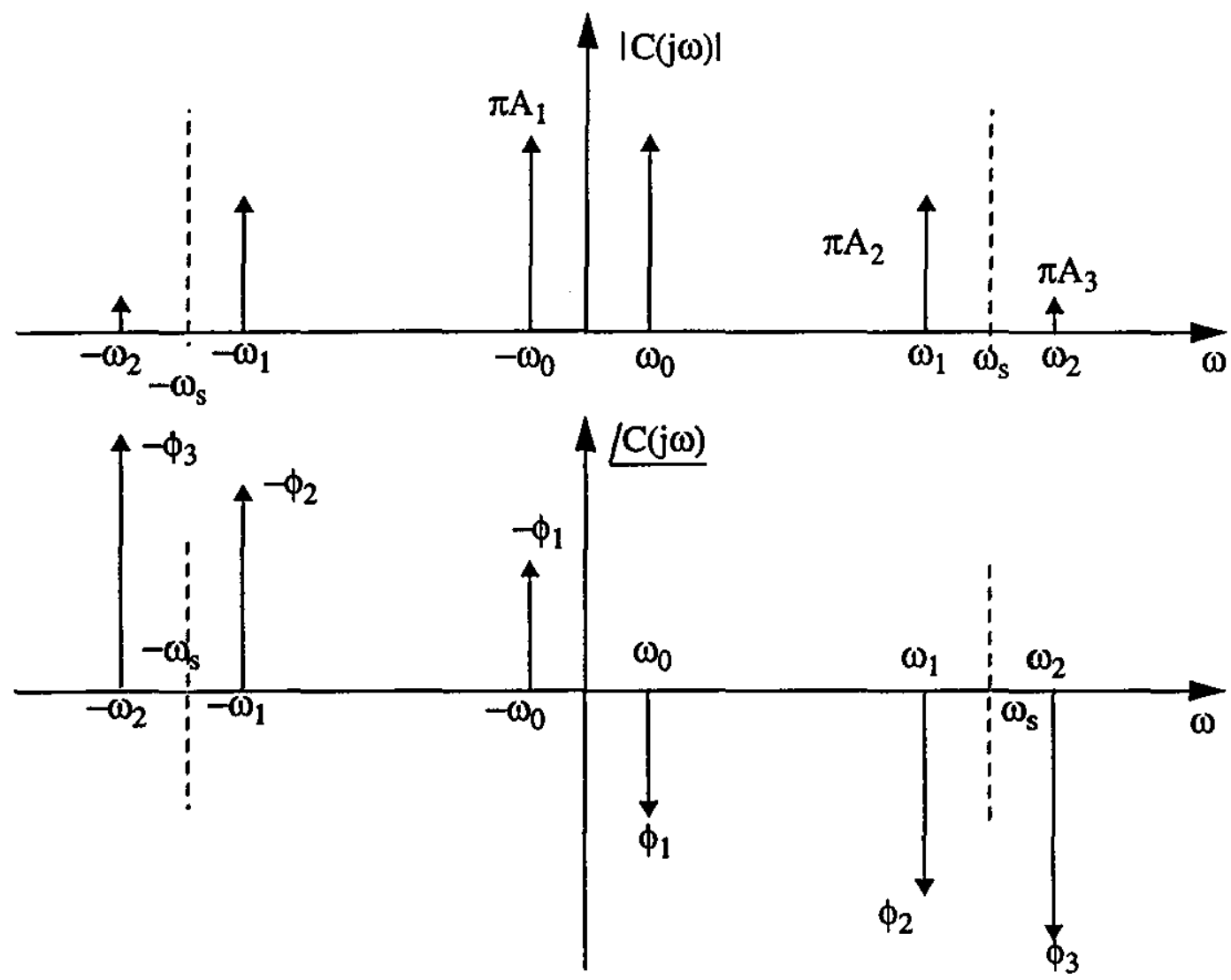
Figure 5.20- Frequency spectrum for example plant output signal

Repeating this consideration for an infinite number of secondary spectral lobes would thus result in the frequency spectrum of the sampled signal. The primary lobe (and hence all other secondary lobes) would be given by a combination of the component spectral lines shown above.

In this case, the spectral line located at a frequency of $\omega_{0}$ would be given by a phasor combination of those in the continuous spectrum at $\omega_{0},-\omega_{1}$ and $\omega_{2}$ with attenuation by the factor $1 / \mathrm{T}$. Thus the sampled signal spectrum would be as shown in Figure 5.21, where the component magnitude and phases are given by

$$
D e^{j \gamma}=A_{1} e^{j \phi 1}+A_{2} e^{-j \phi 2}+A_{3} e^{j \phi 3}
$$
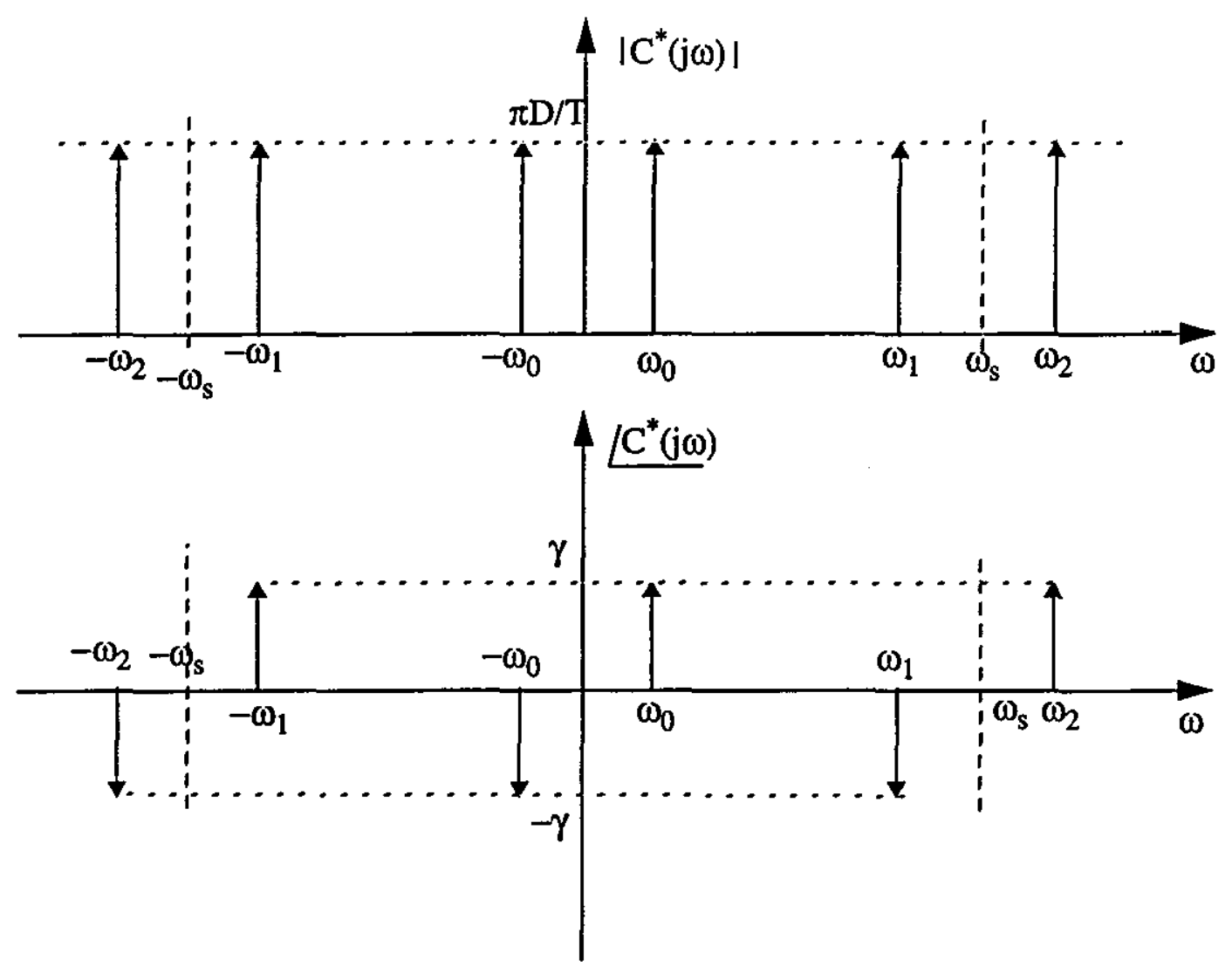

\section{Figure 5.21 - Sampled example plant output frequency spectra}

From classical stability considerations, any component of the input signal, $\mathbf{r}^{*}(t)$, that is amplified around the loop may result in an unstable oscillation at that frequency. If $r^{*}(t)$ was representative of an input signal at frequency $\omega_{0}$, then the zeroorder-hold output signal, $r(t)$ would contain components of frequency $\omega_{0}, \omega_{1}, \omega_{2}$ etc. Assuming that the actuator is operating within its linear region, then the earlier considerations apply, enabling the components of the plant output signal, $c(t)$ to be derived. If the plant has a structural-mode at one of the component frequencies then 
the plant output signal could contain a significant contribution from one of the highfrequency components.

After sampling of the plant output signal, all signals present at frequencies higher than the Nyquist frequency will be folded back onto the primary frequency range. In this case, since the plant is assumed linear, all of the frequencies present in the output signal will be aliases of the primary input signal frequency. Hence all components will fold back on to the primary signal frequency. It is possible therefore to obtain an openloop frequency response for the system taking into account the sample and hold effects.

From the above diagram, it is clear that the closed-loop stability of the system depends on the frequency response between signals $r^{*}(t)$ and $y^{*}(t)$. By examining the gain and phase changes between the primary components of these two signals, the open-loop frequency response can be deduced.

In terms of the aeroservoelastic system, it is possible to obtain the open-loop frequency response for the "analogue" system from a combination of the frequency responses of both the plant and the zero-order-hold function. The resulting "analogue" system frequency response can then be aliased on the low-frequency range to take account of the sampling effects, observing the fact that the components of frequency $n \omega_{s}-\omega_{0}$ should have their phase negated prior to combination with the primary component.

Clearly, it would be possible for a plant with resonant modes of frequencies above the Nyquist frequency to cause a system instability as a result of the digital implementation of the control system. It is necessary therefore to take all digital effects fully into account when assessing the stability of the system. Theoretically, this would entail accounting for all signal components in the input to the plant. As has been shown earlier, attenuation of signal components as a result of the zero-order-hold frequency response and, more significantly, of the plant itself, means that most highfrequency components can be neglected. This is obviously dependent on the frequency response of the plant being low-pass in nature which is the case for most engineering dynamic systems, but not necessarily the case in the aeroservoelastic system.

It is necessary therefore to assess the effect of the plant and the zero-order-hold on the higher frequency input signal components, it being possible to neglect those components which are attenuated to a suitable level. Such an investigation will be carried out in the following chapter.

Although the effect of the sensor dynamics has not been included in this example, it is clear from Figure 5.18 that the high level of attenuation provided at high 
frequencies will aid in reducing the effect of aliasing. It is important therefore that the dynamics of the sensor are included in the aeroservoelastic analysis.

In addition to the problem of high-frequency input components causing possible aeroservoelasticity instabilities, their presence can have a detrimental effect on the performance of the actuator. It will be demonstrated in Chapter 7 that the presence of a high-frequency input signal superimposed on a low-frequency demand signal can result in a reduction in the performance of the actuator. Also, high-frequency input components may cause undue wear within the actuator and undesired sub-harmonic generation. These effects will be discussed in Chapter 7.

\subsection{Conclusions}

The theoretical background to the propagation of sampled signals through a typical aeroservoelastic system has been derived. The possible excitation of a structural-mode as a result of the digital nature of the control system has been discussed In addition, the potential for instability as a result of the aliasing of highfrequency response has been demonstrated. The importance of the attenuation of the zero-order-hold and sensor dynamics has been highlighted, it being shown that both components should be included in any aeroservoelastic analysis.

In the following chapter, the theory derived here will be applied to the aircraft system described in Chapter 4 . The assumptions regarding the digital nature of the modern flight control system and their effect on the aeroservoelastic problem will then be examined. 
Chapter 6

\section{Inclusion of Digital Effects and Sensor Dynamics}




\subsection{Introduction}

In Chapter 4, the design of the structural-mode filters assuming analogue operation of the flight control computer was described. In addition, it was assumed that the effect of any sensor dynamics could be neglected. Considering the first assumption, it has been shown in Chapter 5 that the digital nature of the system can affect the aeroservoelastic problem. An example of an in-flight aeroservoelastic interaction as a result of the digital nature of the control system was discussed in Chapter 1 . This example occurred as a result of the aliasing of a high-frequency structural-mode onto the low-frequency rigid-body response. The combination of low-frequency response and aliased high-frequency mode resulting in the instability ${ }^{15}$. It is necessary therefore to take any digital processing of the control signals into account when designing the structural-mode attenuation.

The following chapter extends the analysis of Chapter 4 to include the digital nature of the control system and the sensor dynamics as described in Chapter 5. The current design assumptions regarding the effect of the digital nature of the control system are investigated. In addition, the effect of relaxing some of these design criteria on the attenuation requirements of the structural filters is investigated.

\subsection{Application of digital effects to an aeroservoelastic system}

\subsubsection{Introduction}

The previous chapter has highlighted the need to consider three main elements when examining the effect of the digital nature of the control system on the aeroservoelastic interaction. These three elements are as shown in Figure 6.1.

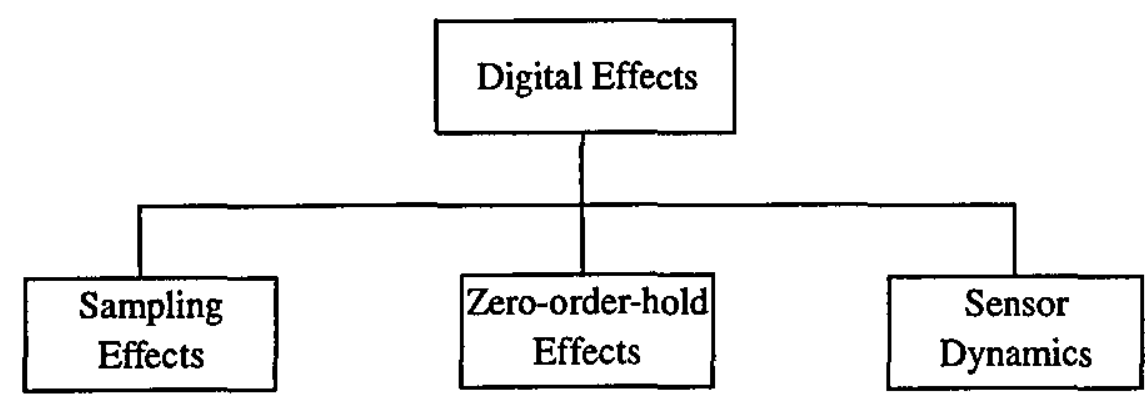

Figure 6.1 - Elements of the digital flight control system

The following section will consider each of these elements in turn, applying their effect to the aircraft model developed in Chapters 3 and 4. Before this can be achieved however, it is necessary to consider a digital implementation of the flight control system. 


\subsubsection{Design of the digital flight control system}

For the system under consideration, the flight control system for the rigid-body aircraft needs to be transformed into the z-domain for incorporation into a digital description of the aircraft system. This can be accomplished using the bilinear transformation ${ }^{60}$

$$
s=\frac{2}{T} \times \frac{z-1}{z+1}
$$

and performing pre-warping of the transfer functions to match digital frequency response to analogue frequency response. This process results in the digital fight control system for the rigid aircraft shown in Figure 6.2, with the system matching the time response characteristics for the analogue system. Conversion of the analogue flight control system into the digital from assumes that the digital implementation of the control system will cause no additional problems in terms of rigid-body control. It has been discussed in Chapter 5 that the zero-order-hold function and sensor dynamics can introduce phase lags into the system. In addition, the computational delays associated with a digital implementation of a control system can introduce further phase lags. In order for a simple comparison to be made between the requirements for the analogue and digital strategies, the flight control system will not be modified to take account of these effects.

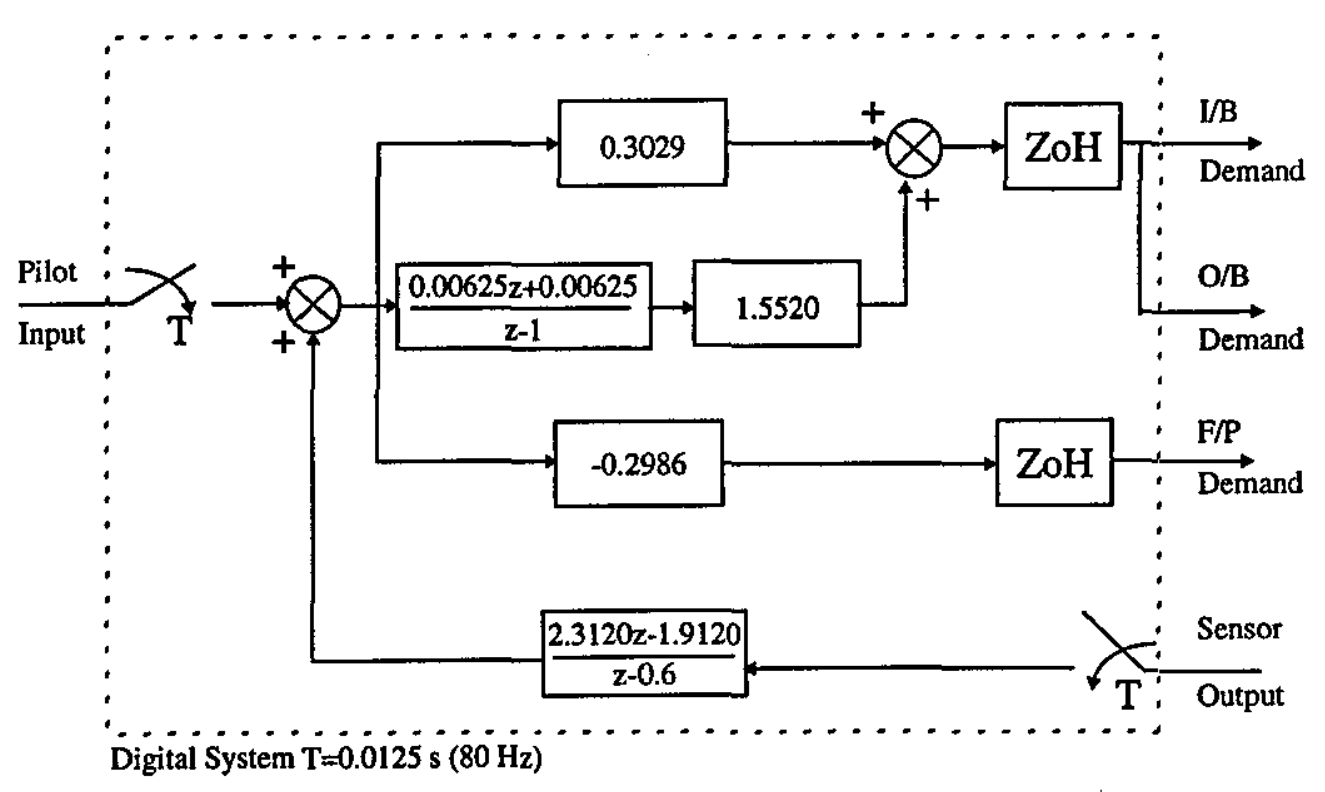

\section{Figure 6.2 - Rigid-body flight control laws for digital system}

In order to be able to design suitable structural-mode filters for the digital system, it is necessary to produce the maximum open-loop envelope as for the analogue system. In this case, the maximum open-loop envelope for the system is effected by the attenuation of the zero-order-hold process and sensor dynamics. In addition, the effect of the sampling in terms of the aliasing of the response must be taken into 
consideration as shown in Figure 6.1.

\subsubsection{Inclusion of zero-order-hold attenuation}

Consider the block diagram for the system as shown in Figure 6.3. The zero-orderholds act between the digital controller and the actuator. As a result, the highfrequency components of the actuator demand signals are attenuated., and it is this attenuation that must be taken into consideration when producing the open-loop frequency response of the system.

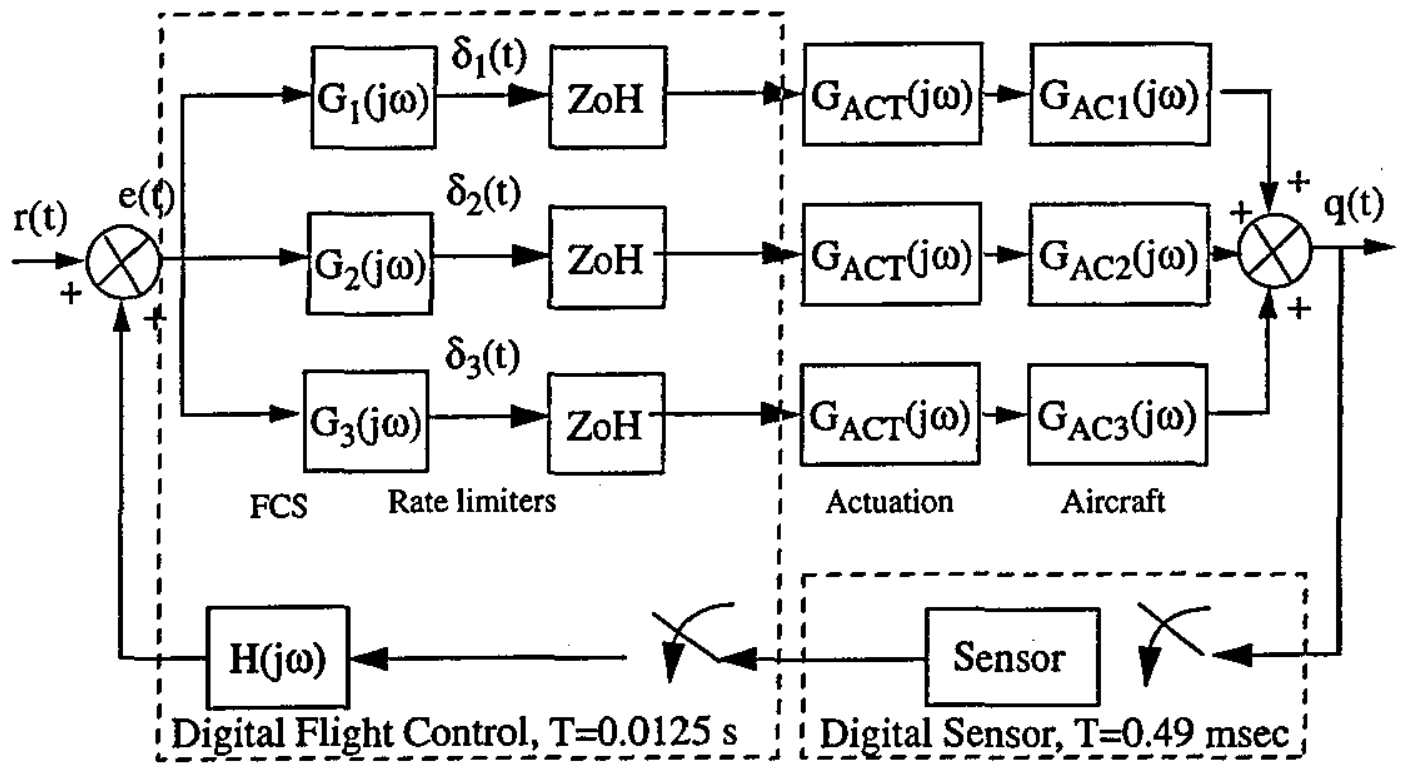

Figure 6.3 - Aircraft block diagram for digital system

Consider the response of the flexible aircraft and actuator model to inputs from the three control surface actuators. The maximum responses for each signal path for a frequency range of $0-200 \mathrm{~Hz}$ can be evaluated as shown in Figure 6.4, Figure 6.5 and Figure 6.6. The flight conditions examined are the zero altitude, zero speed case and Mach 0.836000 feet condition as before, with the maximum open-loop gain being obtained following the current design procedures. Including the zero-order-hold attenuation on these figures demonstrates the large amount of attenuation provided at high-frequencies. In particular, components close to integer multiples of the sampling frequency are greatly attenuated. This will have a significant impact on the aliasing of such response on to rigid-body frequencies as will be demonstrated later. 


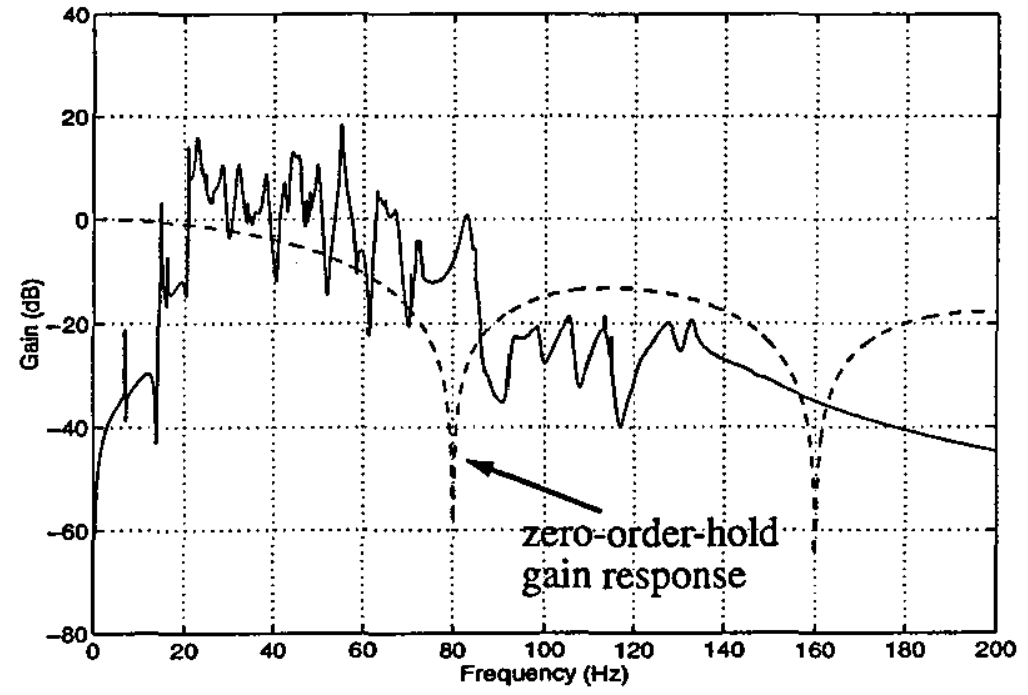

Figure 6.4 - Maximum envelope gain response, inboard flap actuator input and aircraft pitch rate (all flight conditions)

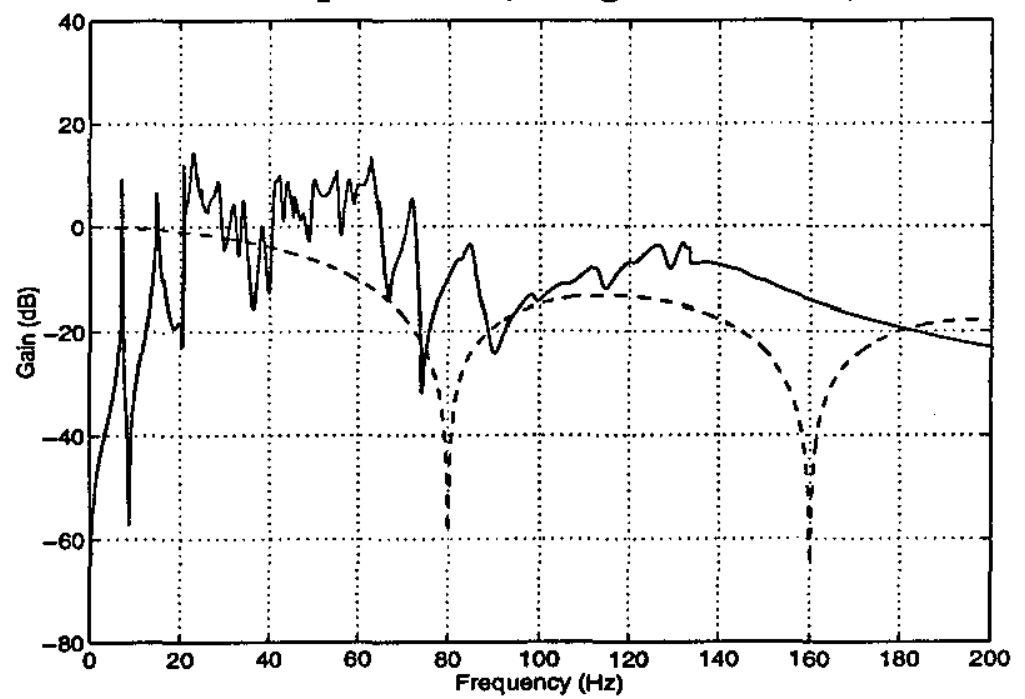

Figure 6.5 - Maximum envelope gain response, outboard flap actuator input and aircraft pitch rate (all flight conditions)

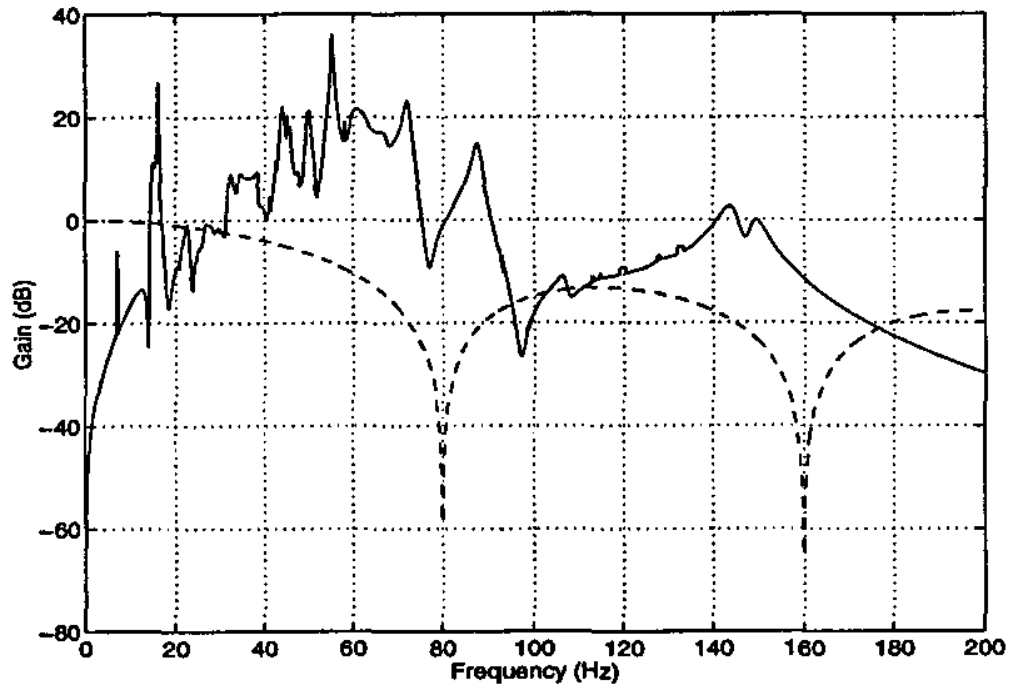

Figure 6.6 - Maximum envelope gain response, foreplane actuator input and aircraft pitch rate (all flight conditions) 


\subsubsection{Effect of aliasing}

Neglecting the sensor dynamics initially, the effect of the sampling of the aircraft response by the flight control computer can be deduced following the derivation of Chapter 5. For the frequency responses as shown in Figure 6.4,Figure 6.5 and Figure 6.6 , this effect will be to aliase the high-frequency response, resulting in the frequency responses of Figure 6.7, Figure 6.8 and Figure 6.9. The attenuation of the zero-orderhold on the input signal has been taken into account before the foldback of the highfrequency aliases. These results represent the maximum open-loop gain of the system between the actuator input and the sampled aircraft response for each control path. These results assume that the actuator input signal is in the form of a sampled and held sine wave signal. One point to note is that it has been assumed that the high-frequency signals are aliased to coincide exactly with the low-frequency signals. This produces the worst case response, which is required by current design procedures. For comparison, the maximum envelope neglecting digital effects has been included on these figures.

Comparing the aliased and unaliased responses of Figure 6.4, Figure 6.5 and Figure 6.6, the effect of the digital processing is evident. In the case of the inboard flap input, the high-gain mode at around $63 \mathrm{~Hz}$ in Figure 6.4 folds down onto the $17 \mathrm{~Hz}$ region in Figure 6.7. This could make the design of the structural-mode filters more difficult. The same effect is clear in the foreplane response, where a high-gain mode present in the input response at approximately $55 \mathrm{~Hz}$ can be seen to be aliased to a frequency of approximately $25 \mathrm{~Hz}$. This could again cause problems in the design of the structural-mode filters, particularly in the design of the low-pass filter.

Another point to note from the aliased responses is that a certain amount of the structural response has been aliased down on to the rigid aircraft frequency region ( 0 $5 \mathrm{~Hz}$ ) which could cause problems with obtaining the desired performance and stability-margins for the rigid aircraft. Fortunately this effect has been reduced by the high attenuation of the zero-order-hold function at frequencies close to integer multiples of the sampling frequency. 


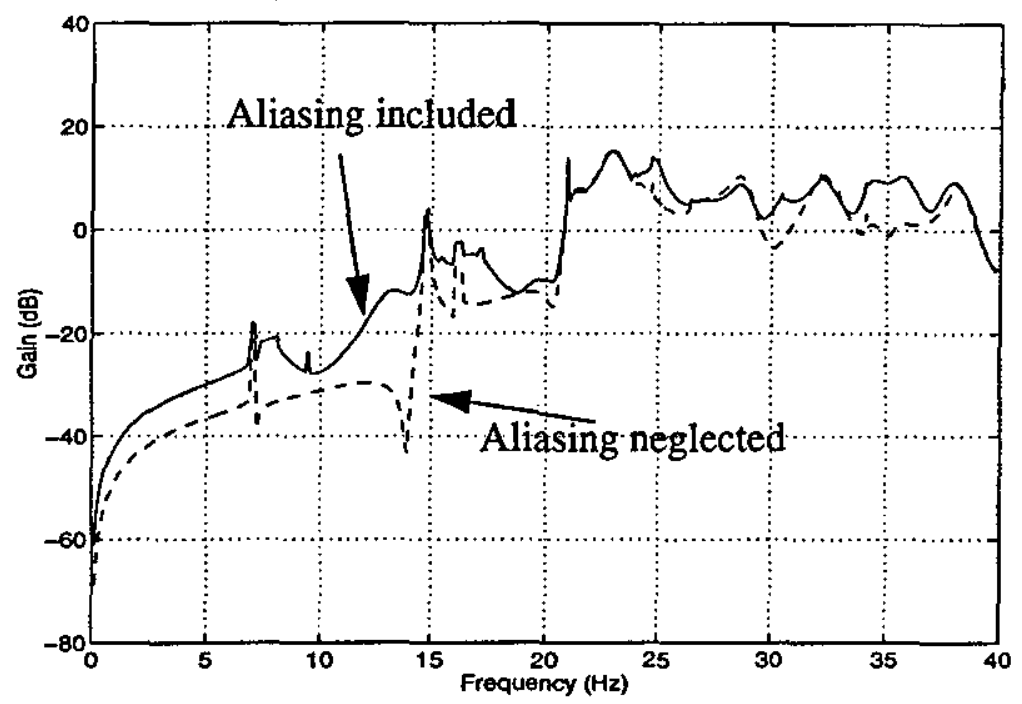

Figure 6.7 - Maximum envelope gain response, inboard actuator input and sampled aircraft pitch rate (all flight conditions)

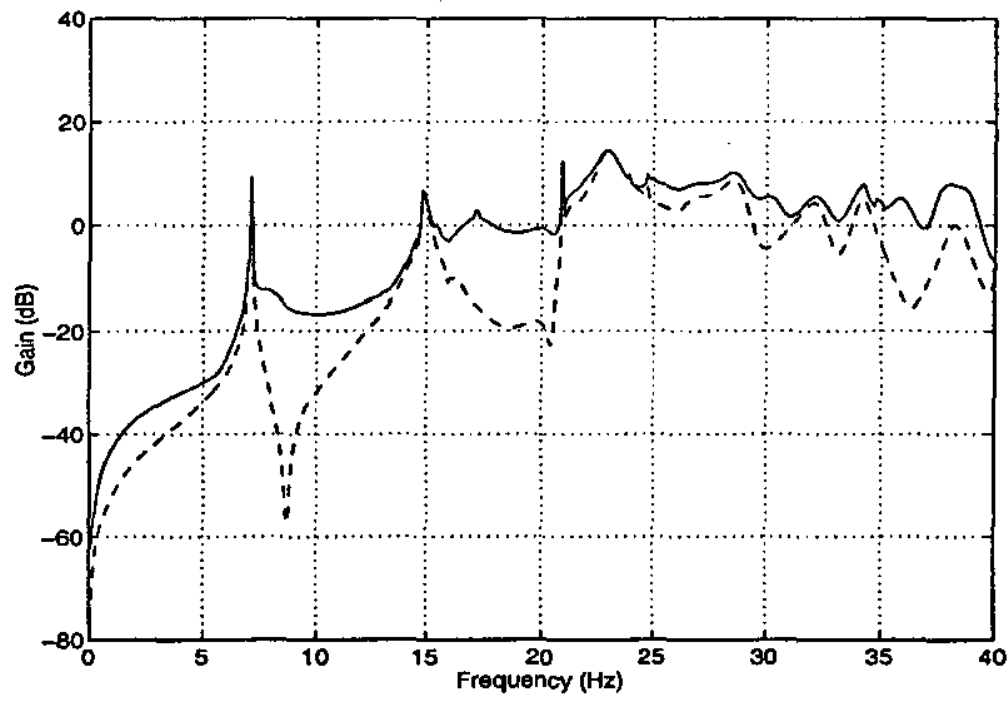

Figure 6.8 - Maximum envelope gain response, outboard actuator input and sampled aircraft pitch rate (all flight conditions)

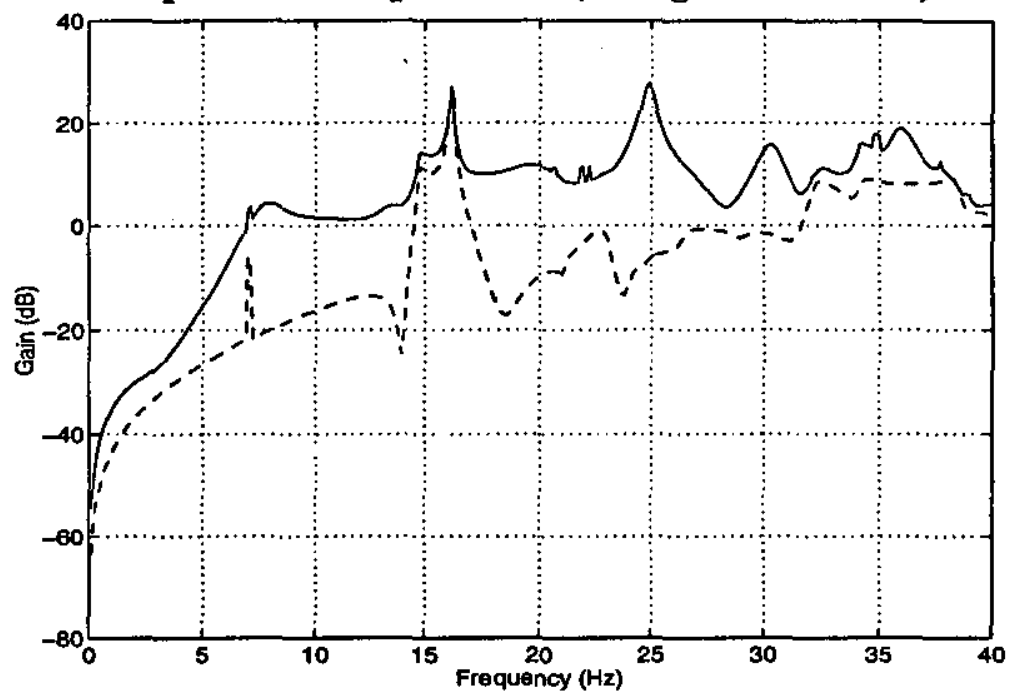

Figure 6.9 - Maximum envelope gain response, foreplane actuator input and sampled aircraft pitch rate (all flight conditions) 


\subsubsection{Production of structural-mode filter attenuation requirements}

The above results represent the maximum open-loop response between actuator input and sampled aircraft pitch rate response for the three control paths. In order to produce the attenuation requirements for the structural-mode filters, it is necessary to consider the effect of the flight control system itself on these responses. Considering the frequency response of the flight control computer in terms of the response to digital aircraft pitch rate signals, it is possible to produce frequency responses for the system for each signal path as shown in Figure 6.10 and Figure 6.11. These figures represent the gain response of the flight control system in terms of the primary components of the input and output sampled signals.

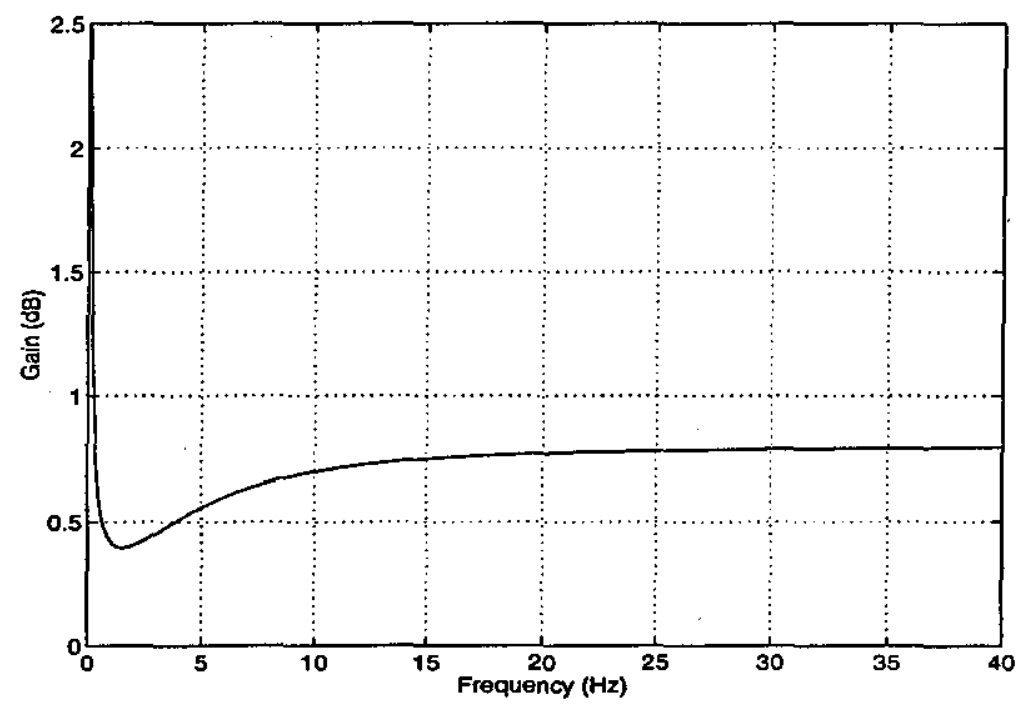

Figure 6.10 - Magnitude frequency response of flight control system for inboard/ outboard flap signal path

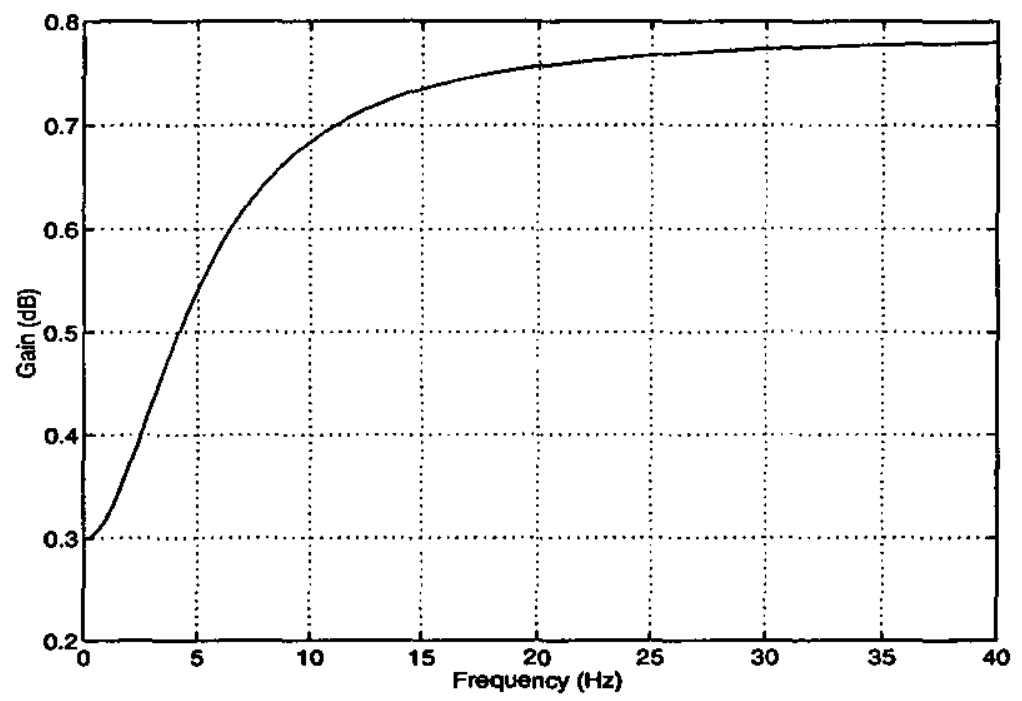

Figure 6.11 - Magnitude frequency response of flight control system for foreplane signal path 


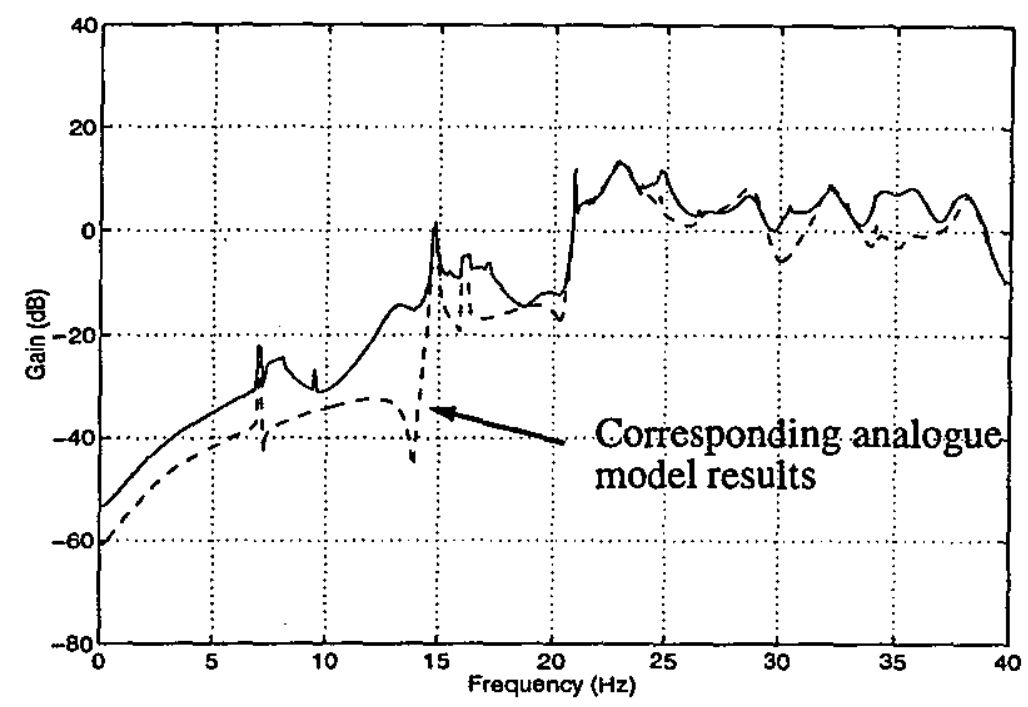

Figure 6.12 - Maximum envelope gain response between inboard actuator input and flight control computer output

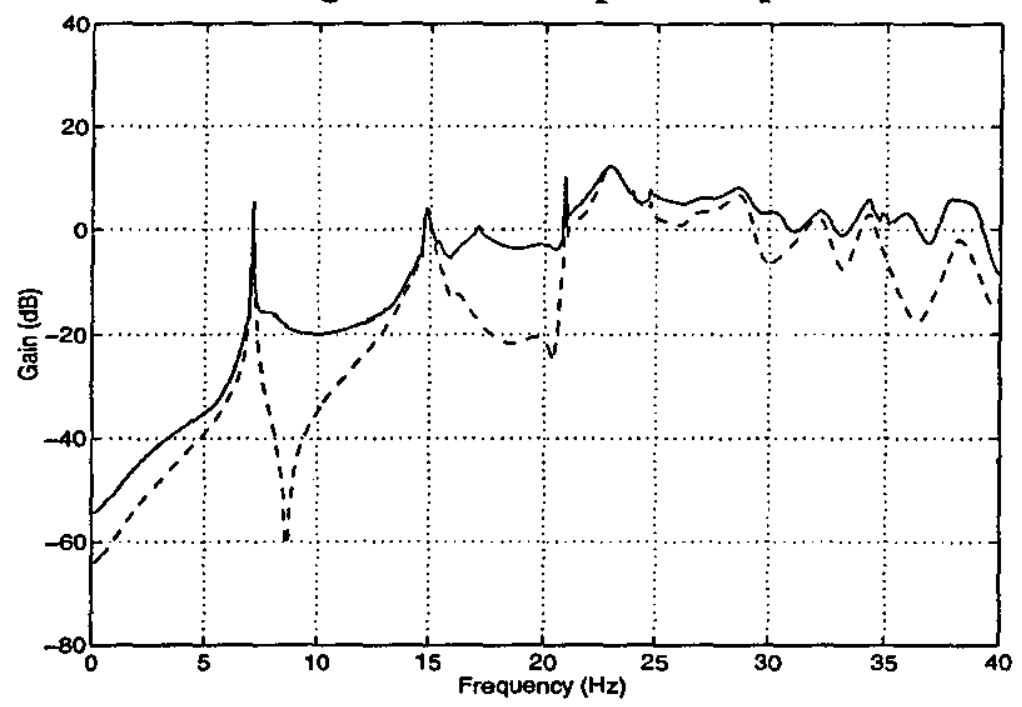

Figure 6.13 - Maximum envelope gain response between outboard actuator input and flight control computer output.

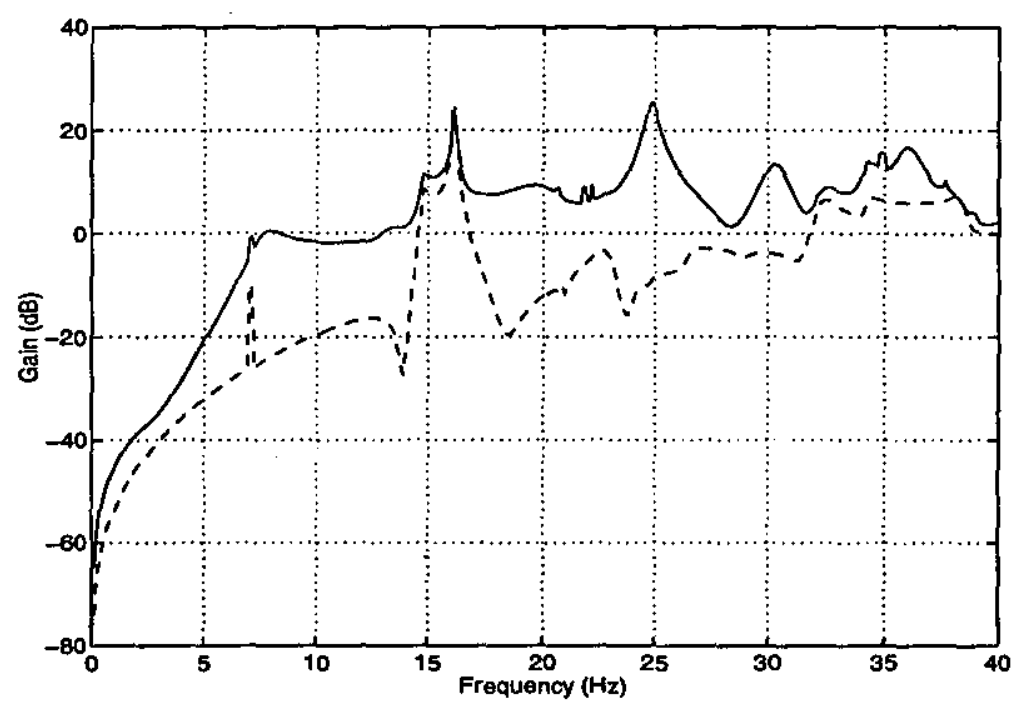

Figure 6.14 - Maximum envelope gain response between foreplane actuator input and flight control computer output 
Combining the above responses for the digital flight control system with the gain envelopes for the signal paths from actuator inputs to flight control computer inputs results in the maximum response envelopes for the system as a whole. These represent the gain of the system between the primary component of the input signal to the actuator and the primary component of the flight control system output signal. These responses are shown in Figure 6.12, Figure 6.13 and Figure 6.14. It is possible to combine these three signal path responses to obtain the maximum envelope of response of the digital system assuming that all signal paths act in-phase as for the analogue model. This maximum envelope of response is shown in Figure 6.15, which defines the attenuation requirements for the structural-mode filters for the digital system neglecting the effect of the sensor dynamics.

Comparing the attenuation requirements for the two systems, some of the affects due to the digital nature of the system are evident in the overall attenuation requirements. In particular the aliased modes at approximately $25,30 \mathrm{~Hz}$ and $35 \mathrm{~Hz}$ can be identified.

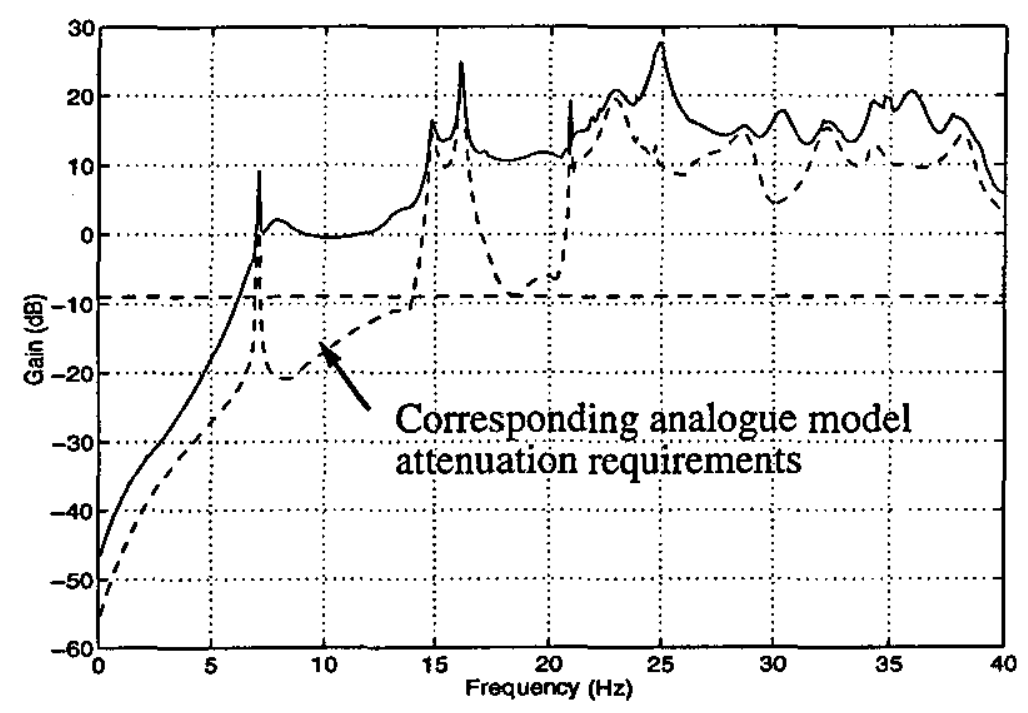

\section{Figure 6.15 - Structural-mode filter attenuation requirements for the a digital system neglecting sensor dynamics}

Although the effect of the digital nature of the control system are evident, the overall peak values of the attenuation requirements are only slightly different from the equivalent results for the analogue system. The only significant difference in the responses can be seen at a frequency of $25 \mathrm{~Hz}$. As mentioned earlier, this is due to the aliasing of the $55 \mathrm{~Hz}$ structural-mode excited by the foreplane input. The fact that the responses are similar at most of the other modes relies on the fact that none of the aliased modes fall on one of the lower frequency modes. If this were the case then the system response at the low-frequency mode could be greatly amplified as a result of the digital system aliasing.

Alternatively, if one of the high-frequency aliased modes had a particularly high-gain 
in comparison with the other modes then the attenuation requirements for the digital system might be significantly greater than for the analogue system. However, in this case the attenuation requirements are not significantly different.

\subsubsection{Inclusion of sensor dynamics}

The above attenuation requirements were obtained assuming that the sensor dynamics could be neglected. It has been shown in Chapter 5 however that the averaging process of the sensor has a significant effect in attenuating the high-frequency modes. As a result, the sensor dynamics form the third of the required elements as shown in Figure 6.1 .

Assuming that the aliasing as a result of the sensor sampling frequency $(2048 \mathrm{~Hz})$ can be neglected, then the sensor dynamics can be represented by the digital transfer function of equation (5.34). It has been shown in Chapter 5, that in magnitude at least, this digital filter behaves as a zero-order-hold for a sampling frequency of $85.3 \mathrm{~Hz}$. It is possible to include the attenuation of the sensor averaging into the earlier analysis to obtain the attenuation requirements for the structural-mode filters including the sensor dynamics, with the results for the analysis being as shown in Figure 6.16 below.

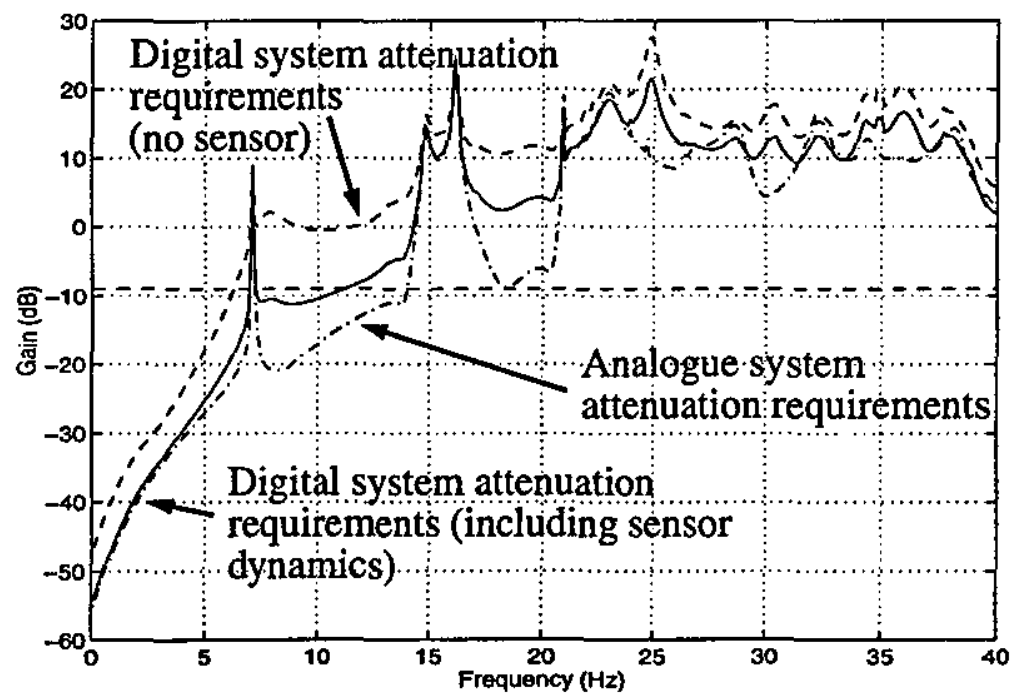

\section{Figure 6.16 - Structural-mode filter attenuation requirements for a digital system including sensor dynamics}

Comparing the attenuation requirements for the three cases included in Figure 6.16, the addition of the sensor averaging has reduced the gain of the system. The response for the digital system, including sensor averaging, approaches the attenuation requirements for the analogue system. This effect is particularly evident at the rigid aircraft frequency range $(0-5 \mathrm{~Hz})$, where the maximum response envelope is almost the same as for the analogue system. This is a result of the large amount of attenuation provided by the sensor averaging at the frequencies approaching the sampling frequency. This is in addition to the attenuation of the zero-order-hold at these frequencies. 


\subsubsection{Application of structural-mode filters}

Assuming that the structural filters will be implemented within the sensor, the filters designed for the analogue system may be converted to their digital equivalents. Application of such filters meets the attenuation requirements of the digital system as shown in Figure 6.17. This is not surprising, as the differences in the attenuation requirements between analogue and digital system are small. In addition, implementing the structural-mode filters at the sensor sampling rate results in the frequency response of the analogue and digital filters being almost identical up to the frequencies under consideration here. The phase lag introduced by the digital filters at this sampling frequency is also almost identical to that introduced by the analogue equivalents.

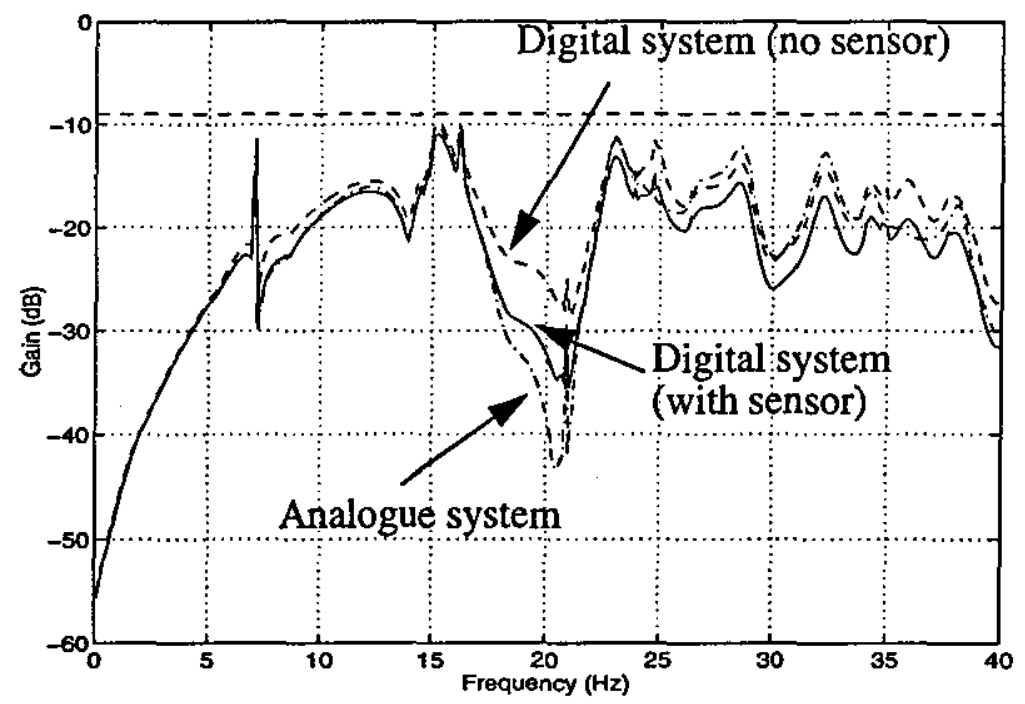

\section{Figure 6.17 - Open-loop frequency response for digital system - filtering in AMSU prior to $80 \mathrm{~Hz}$ foldback}

To demonstrate the importance of the location of the filters within the feedback path, consider the following example. If the filters are converted for implementation within the flight control system, at the $80 \mathrm{~Hz}$ sampling frequency, it can be seen from Figure 6.18 that the clearance requirements are no longer met. As a result, the placing of the structural-mode filters within the feedback path, and the correct interpretation of their positioning, is crucial to the design process. This can be seen to good effect by considering the effect of the low-pass filter on the overall response envelope.

It can be seen from Figure 4.19 that the low-pass filter provides significant attenuation to the high-frequency modes, with particular attenuation at a frequency of around $50 \mathrm{~Hz}$. If the attenuation of the filter was only added after the foldback of the response, then any structural-modes originally at a frequency of $50 \mathrm{~Hz}$ for example would only be subjected to the attenuation of the low-pass filter at the modes alias of $30 \mathrm{~Hz}$. This would result in a much higher gain for that particular mode as is evident from Figure 6.17 and Figure 6.18. If the attenuation of the filter was added before 
foldback, as is correct for an implementation within the sensor, then the mode at $50 \mathrm{~Hz}$ would be subjected to the attenuation of the filter at this frequency. As a result, the final maximum gain response envelope of the system is much lower.

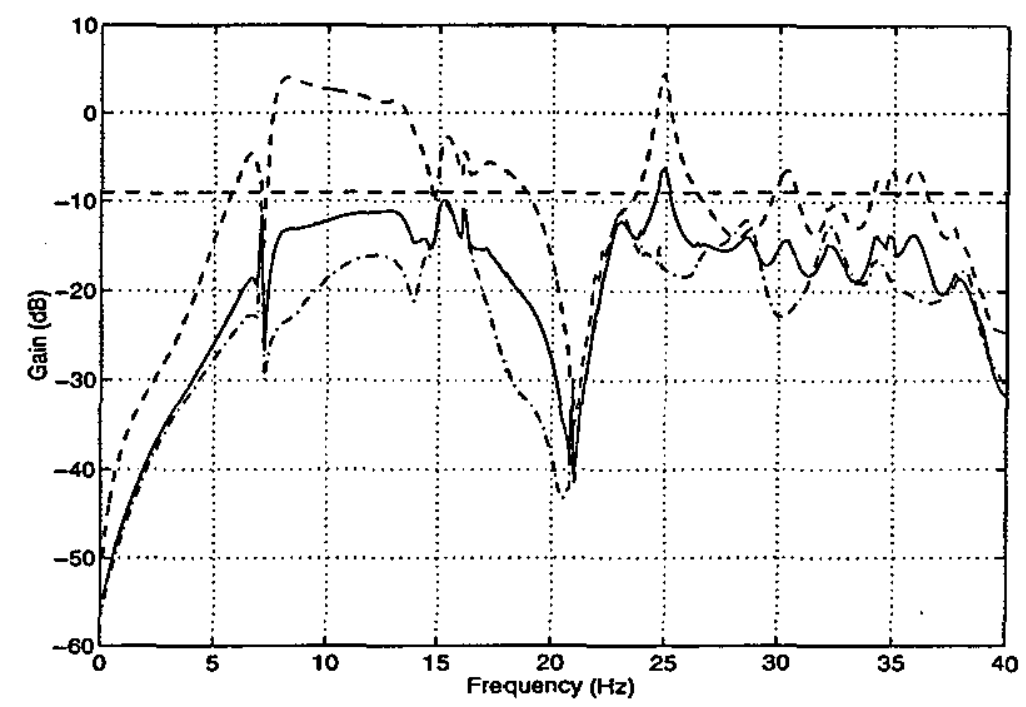

Figure 6.18 - Open-loop frequency response for digital system - filtering in FCS after $80 \mathrm{~Hz}$ foldback

\subsubsection{Effect of zero-order-hold and sensor phase lag}

Taking all of these factors into consideration, it would be reasonable to assume initially that the digital filters would thus meet all of the requirements for the digital system. In particular the requirement that the clearance boundary for the response of the rigid-body aircraft could be met. Since the phase lags introduced by the digital structural-mode filters match those introduced by their analogue equivalents, then it could be expected that this would be the case.

Unfortunately, the zero-order-hold and sensor averaging functions introduce phase lags and computation delays themselves, as can be seen from the relevant sections of Chapter 5. Taking these phase lags into consideration, and reproducing the open-loop Nichols plot for the digital system results in the response as shown in Figure 6.19.

It is clear from the above analysis that in the case of the digital system, the additional phase lags introduced by the zero-order-hold and sensor signal processing introduce an additional parameter into the aeroservoelastic problem. In this case, the extra phase lag introduced is not sufficient to cause violation of the clearance boundary. In the presence of significant computational delays however, this may not be the case. In reality, the digital effects would be taken into consideration in the initial design of the flight control system. This would result in a different flight control system than the one described in Chapter 4, such that the phase lags introduced by the digital nature of the control system were compensated for by suitable phase advance filtering. In doing so however, the gain of the system would be increased, resulting in a higher gain to the structural-modes. Clearly, their is a case for the direct digital design of the controller 
from the outset, enabling correct specification of the attenuation requirements for the structural-mode filters.

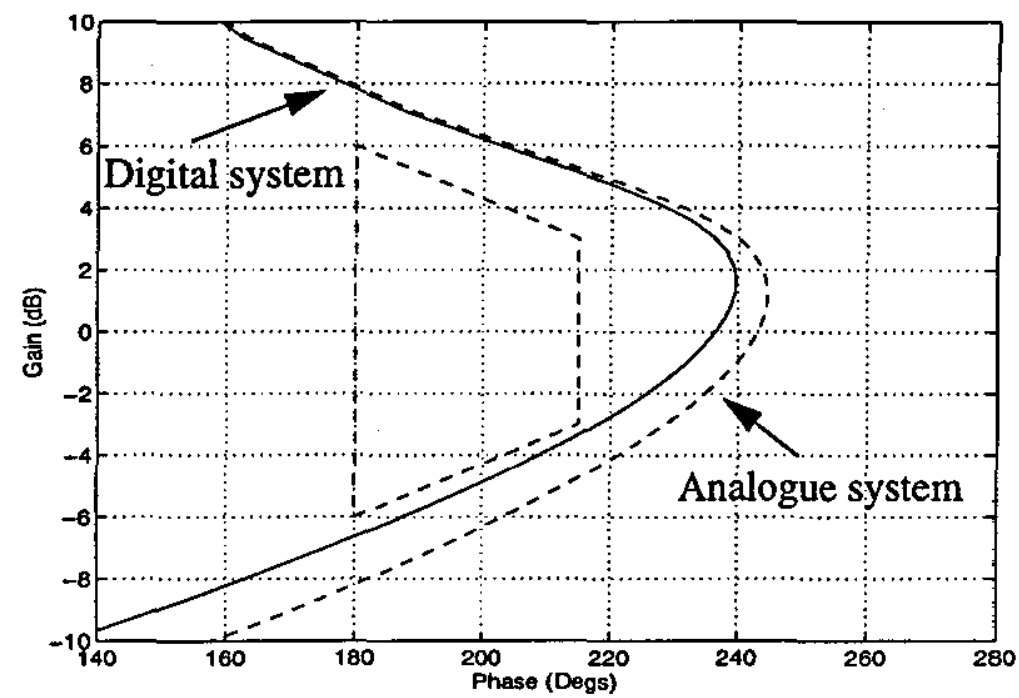

Figure 6.19 - Open-loop Nichols plot showing error signal stability-margins for digital system

\subsubsection{Conclusions}

To conclude, the application of digital effects to a typical aeroservoelastic system has been shown to be an important design consideration. Although in this case the aliasing of a high-frequency mode does not result in a potential instability, the need to consider such an occurrence is clear. In addition, the example has demonstrated that the inclusion of sensor dynamics within the analysis plays an equally important role. Finally, the position of the structural filters within the feedback path has been shown to have a crucial effect on their design and function.

\subsection{Evaluation of alternative design assumptions}

\subsubsection{Introduction}

In Chapter 4 and section 6.2, the problem of aeroservoelastic interactions occurring within the aircraft system was addressed under the current design assumptions employed by British Aerospace. The reasoning behind these assumptions has been discussed in Chapter 2. The main assumptions that have been employed in the earlier analysis concern the effect of aliasing of the high-frequency modes on to the lowfrequency response, and the effect of the three separate signal paths on the overall aeroservoelastic problem. In the following section it is intended to evaluate the effect of these assumptions on the structural-mode attenuation requirements.

\subsubsection{Aliasing effects on structural-mode attenuation requirements}

In the design of the structural-mode filters for the full-order digital system, the effect 
of aliasing on the structural-mode attenuation requirements was taken into account assuming that the signal aliases were exactly in-phase with their low-frequency counterparts. Clearly, this is a very conservative approach.

Theoretically, this foldback of the response should be completed over an infinite frequency range, low-frequency signals (that is below the Nyquist frequency) having an infinite number of aliases.

In practice, this is impossible, and it is necessary to decide upon a suitable frequency point above which the aliasing of higher frequency signals can be neglected. In the earlier case, this frequency point was chosen to be at $200 \mathrm{~Hz}$, it being considered that the attenuation of the remaining signals was sufficient to make the effect of higher frequencies negligible.

The high-frequency components of any signal within the digital system are mainly attenuated by the actuation system, the sample-and-hold effect and the sensor dynamics. In addition, if the actuation system becomes non-linear in nature, possibly due to saturation for example, then further high-frequency signal components could be produced as a result of the harmonic/subharmonic generation characteristics of such non-linearities ${ }^{28}$. This effect will be discussed in Chapter 7.

Consider the model of the system, with the full-order flexible aircraft model and digital effects included. The attenuation requirements for the system assuming inphase addition of the aliases up to a frequency of $200 \mathrm{~Hz}$, is shown in Figure 6.20 . In addition, the attenuation requirements for the analogue system are included for comparison.

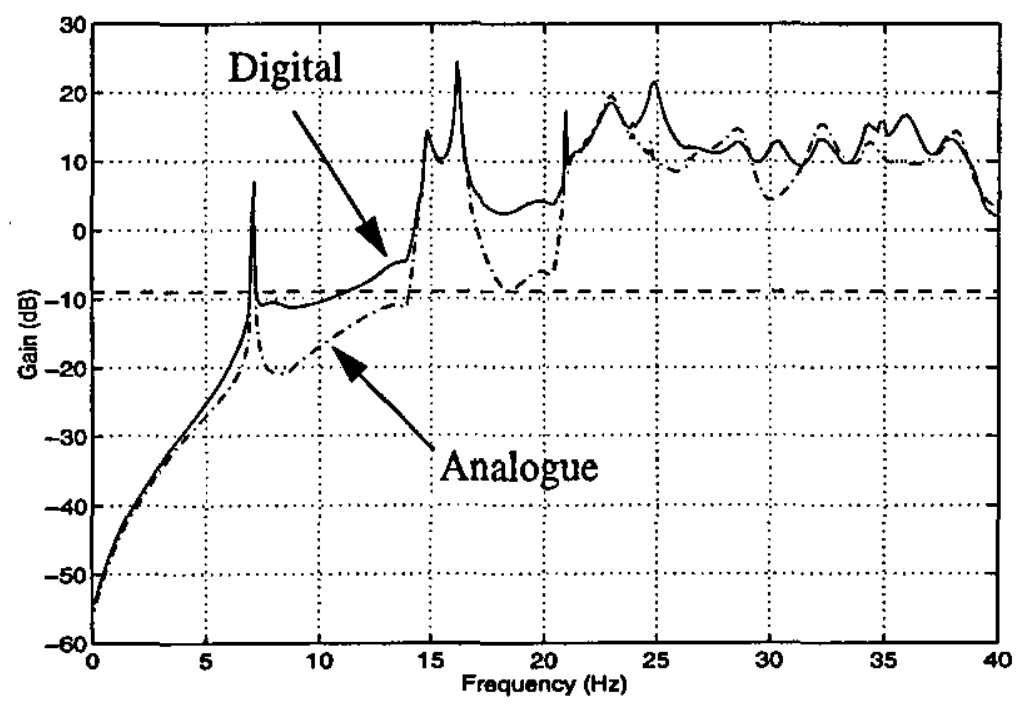

Figure 6.20 - Structural-mode attenuation requirements for digital system assuming in-phase addition of high-frequency aliases

As discussed earlier, the aliasing has little effect on the overall attenuation requirements of the system since the attenuation requirements rely on only the maximum values of the response which occurs at the modal frequencies. Since none 
of the high-frequency structural-modes aliases on to a low-frequency mode, the effect of the aliasing is limited.

Considering the aliasing of structural-modes of frequency greater than the sampling frequency, the frequency response of the zero-order-hold function alone dictates that at best a high-frequency mode suffers three times the attenuation of a low-frequency counterpart. This can be seen from the magnitude response of the zero-order-hold function corresponding to a sampling frequency of $80 \mathrm{~Hz}$ as shown in Figure 6.21 . For example, the gain of the zero-order-hold function at a frequency of $120 \mathrm{~Hz}$ is 0.21 which corresponds to a gain of 0.64 at a frequency of $40 \mathrm{~Hz}$. For other frequency values the difference in gain is more pronounced.

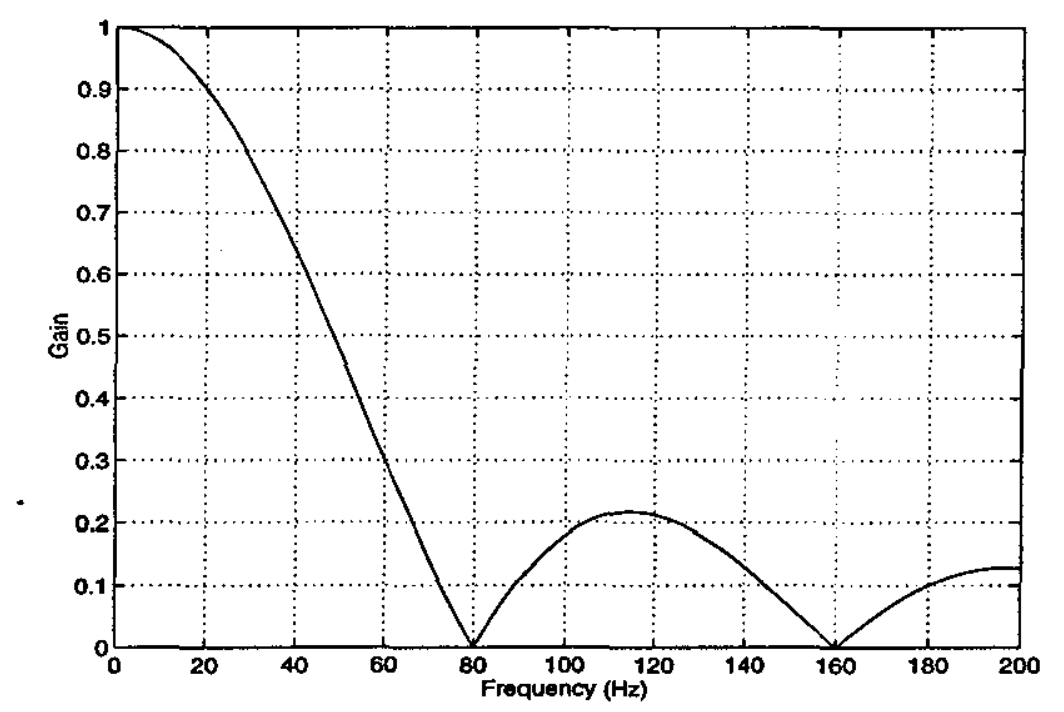

\section{Figure 6.21 - Gain response of Zero-order-hold function for sampling frequency of $80 \mathrm{~Hz}$}

This attenuation of the Zero-order-hold function for frequencies greater than the sampling frequency thus amounts to at least an extra $9.5 \mathrm{~dB}$ of attenuation on these high-frequency modes. Considering that the attenuation of the sensor averaging process represents a similar amount of attenuation, and that the actuation system will also attenuate such modes, their relevance within the aeroservoelastic problem is virtually zero.

As an example, consider the worst case, where there exists a low-frequency mode at the Nyquist frequency of $40 \mathrm{~Hz}$, and a high-frequency mode at a frequency of $120 \mathrm{~Hz}$, which aliases with identical phase on to the $40 \mathrm{~Hz}$ mode. Supposing that even after attenuation by the actuation system the high-frequency mode still has an equal gain to that of the low-frequency mode. From the above analysis, this high-frequency mode will be subjected to an additional $9.5 \mathrm{db}$ of attenuation to that of the low-frequency mode. Assuming that the attenuation of the sensor signal averaging process will have a similar difference in attenuation between the two frequencies as is the case, the highfrequency mode will be subjected to approximately $19 \mathrm{db}$ of extra attenuation 
compared with the low-frequency alias. As the phases are assumed to be identical, the addition of the low-frequency signal and the alias of the high-frequency component will result in the total response being only $0.92 \mathrm{~dB}$ higher than the low-frequency response alone. Considering that for the actuator model used here, the difference in gain between a frequency of $40 \mathrm{~Hz}$ and a frequency of $120 \mathrm{~Hz}$ is approximately $30 \mathrm{~dB}$, the effect of the mode at $120 \mathrm{~Hz}$ on the response at the $40 \mathrm{~Hz}$ mode will be negligible.

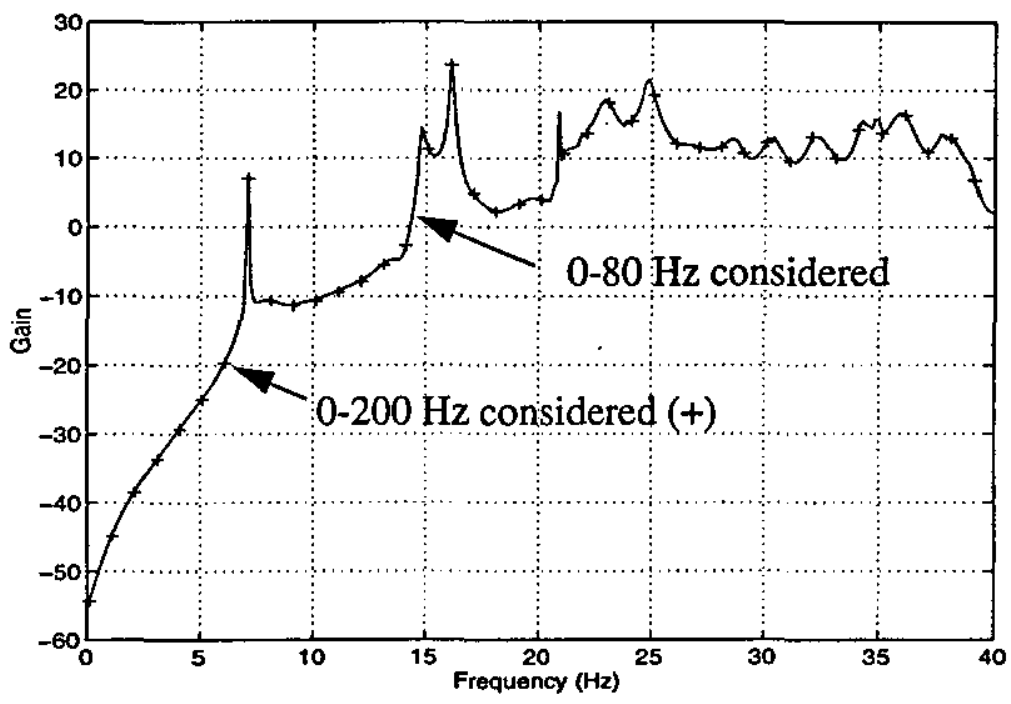

Figure 6.22 - Structural-mode attenuation requirements for digital system considering the structural-modes of frequency less then the sampling frequency

A comparison of the structural-mode attenuation requirements for the digital system for ranges up to $80 \mathrm{~Hz}$ and up to $200 \mathrm{~Hz}$ is shown in Figure 6.22. From the figure, it can be seen that the structural-modes of frequency greater than the sampling frequency have no effect on the attenuation requirements of the system.

Consider the effect of the aliasing of the signal components of frequencies between the Nyquist frequency and the sampling frequency. It can be seen from Figure 6.20, that the aliasing of these frequencies does have an impact on the structural-mode attenuation requirements. From the frequency response of the zero-order-hold function as shown in Figure 6.21, for frequencies between the Nyquist frequency and the sampling frequency, the difference in zero-order-hold attenuation can be small.

Considering a frequency of $41 \mathrm{~Hz}$ for example, which has an alias at $39 \mathrm{~Hz}$. The difference due to the zero-order-hold attenuation between these two frequencies is negligible, whereas for frequencies further away from the Nyquist frequency the difference becomes larger.

As discussed earlier, the actual effect of the aliasing on the overall attenuation requirements of the system is negligible in the case of this aircraft model. This is due to the fact that the structural-mode filters are designed to attenuate the peak values of the response to the desired clearance boundary. In this case these peaks are not changed by the aliasing effect, instead only additional peaks corresponding to the 
high-frequency aliases are generated. These new peaks fail in this case to make a significant impact on the attenuation requirements. It is because of this that the original analogue structural-mode filters are still adequate in their digital forms.

The attenuation requirements for the digital system are therefore not increased to such an extent by the aliasing of the high-frequency modes to warrant a redesign of the structural-mode filters from the earlier analogue versions. This is only the case for this system model however. Clearly, the aliasing effect of the system response between the Nyquist frequency and the sampling frequency on to the low-frequency range should be considered, the existence of aliased modes falling on to existing low-frequency modes being of most concern.

\subsubsection{Consideration of Phase effects}

In the analysis completed so far, and in the calculation of the structural-mode attenuation requirements earlier, it was assumed that the aliased components acted inphase with the low-frequency components. Obviously, this might not be the case, with there being a possibility that the aliased component will be exactly out of phase with the low-frequency component causing cancellation to take place. It has been discussed in Chapter 2 however that due to uncertainty in the modelling of the flexible aircraft particularly in terms of phase response, it is not possible to take the phase into account. As a result, the most pessimistic assumption must be made. For purposes of analysis however, it is possible to take the phase response of the flexible aircraft model into account, enabling a comparison to be made between the pessimistic viewpoint of assuming all aliases act in-phase, and the actual model results using the phase to calculate the true response.

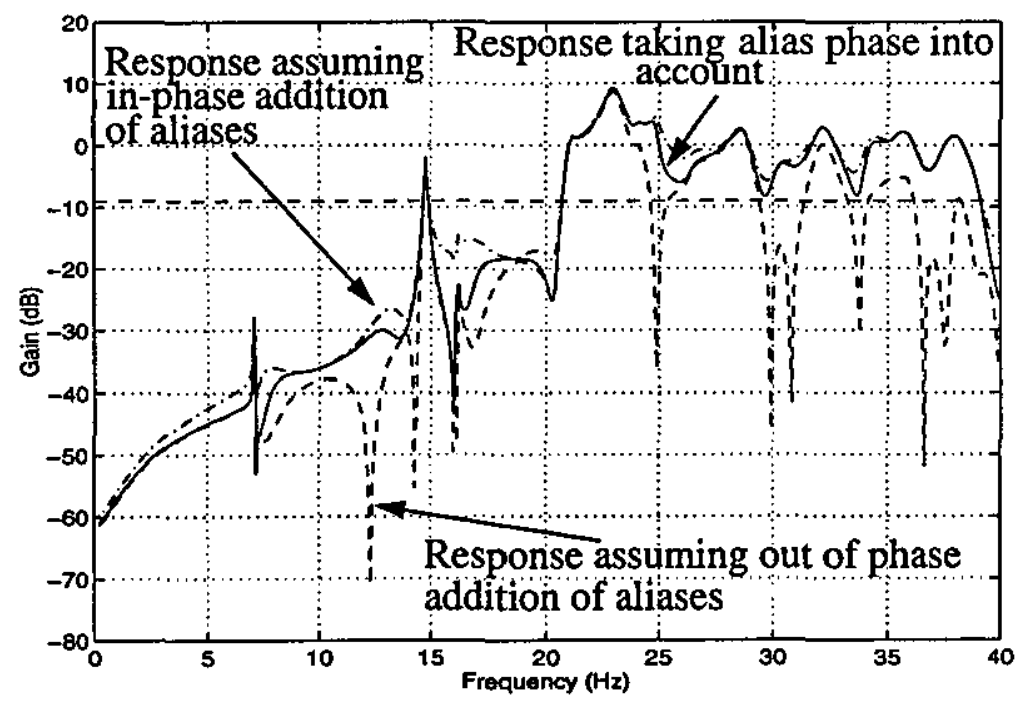

Figure 6.23 - Open-loop response of digital system to an input on to the Inboard flap actuator for differing alias phase assumptions for flight condition of Zero speed Zero altitude

Producing the open-loop responses for the digital system in response to an input on 
the inboard flap actuator gives the results as shown in Figure 6.23 for a flight condition of zero altitude, zero speed. The effect of the differing assumptions regarding the phase of the signal aliases can clearly be seen from the above figure. The response taking into account the phase of the aliased signals passes between the boundaries of the responses assuming in and out of phase addition of the aliased signals.

As with the effect of the aliasing, it can be seen that in this case, there is little advantage to be gained in taking the phase into consideration when folding back the higher frequency signals. Provided that no modes are aliased on to a low-frequency mode, then the phase of the aliases is of little importance. The gain associated with a modal response is far in excess of any inter-modal response that may be aliased on to it. It will only be when the aliasing of a high-frequency mode results in two modal responses being superimposed that the phases will become significant. This effect can be seen for the inter-modal response in Figure 6.23, where for frequencies away from the modal frequencies, the difference between the phase strategies is more significant. This is as a result of the two superimposed components being of a more comparable amplitude.

Producing the overall attenuation requirements for the system, and assuming in-phase addition of the signal paths, results in the attenuation requirements as shown in Figure 6.24 .

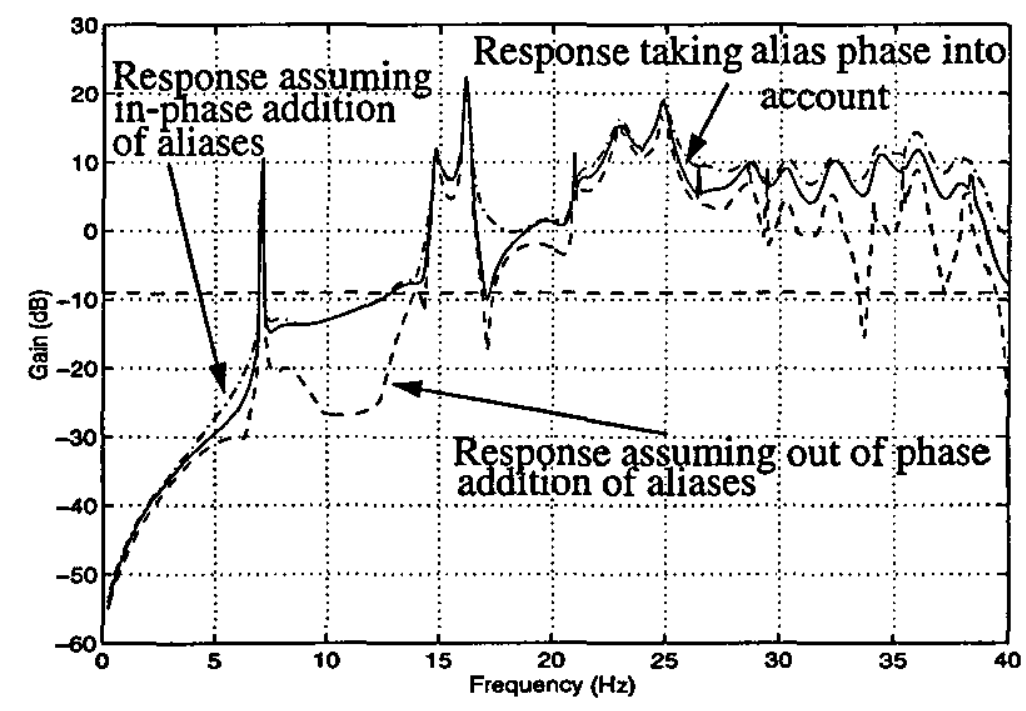

\section{Figure 6.24 - Structural-mode filter attenuation requirements for digital system using different alias phase assumptions}

It is clear from the above figure, that in terms of the overall structural-mode attenuation requirements, there is little advantage in taking the phase of the aliased component into consideration. The major areas where there is a significant difference between the requirements corresponding to the various assumptions concerning phase, are the frequency regions corresponding to inter-modal response of the system. As a result, the main peaks of response which define the attenuation requirements of the 
structural-mode filters are only changed slightly by considering the phase of the aliased components.

The biggest differences in the requirements which are of interest are at frequencies of around 33 and $37 \mathrm{~Hz}$. In the case of the response at around $33 \mathrm{~Hz}$, there is a lowfrequency mode and an aliased high-frequency mode very close together. As discussed, this could result in the two components being of similar magnitude and consequently, the phase of the aliased component will be significant. In this case, the aliased component cancels out the low-frequency mode to some extent, reducing the response from the maximum at this frequency.

Considering the differences at around $37 \mathrm{~Hz}$, once again, there are low-frequency and high-frequency aliased modes close together. Another factor that may increase the chances of the two components being of similar magnitude is the fact that the difference in attenuation between a low-frequency component and its high-frequency alias is reduced as the Nyquist frequency is approached. This could explain why there is a greater difference between the attenuation requirements as derived using the various phase assumptions in the higher frequency region.

It seems from the results obtained using this model at least, that the effect of the alias phase on the attenuation requirements of the problem is small, provided that there are no superposition of modal responses in the frequency ranges approaching the Nyquist frequency.

\subsubsection{Effect of consideration of signal path phase on structural-mode attenuation requirements}

In the designs so far, it has been assumed that the system response to each of the signal paths acts exactly in-phase for the purposes of the calculation of the structural-mode filter attenuation requirements. As discussed in Chapter 2, this assumption is made due to the uncertainty in the modelling of the flexible aircraft structure.

Now, it is intended to investigate the effect of this assumption on the attenuation requirements for the structural-mode filters, using the system model developed earlier. From the current system model, it is possible to produce the system response to each of the inputs at each of the flight conditions as before. Assuming now that the phase response of the system can be used in order to generate the response of the system as a whole, it is possible to calculate the system response for each flight condition. The phase relationships between each signal path will then be taken into account when producing the overall response. For the case where the in-phase aliasing is assumed, this results in the response of the aircraft system at the two flight conditions being as shown in Figure 6.25. 


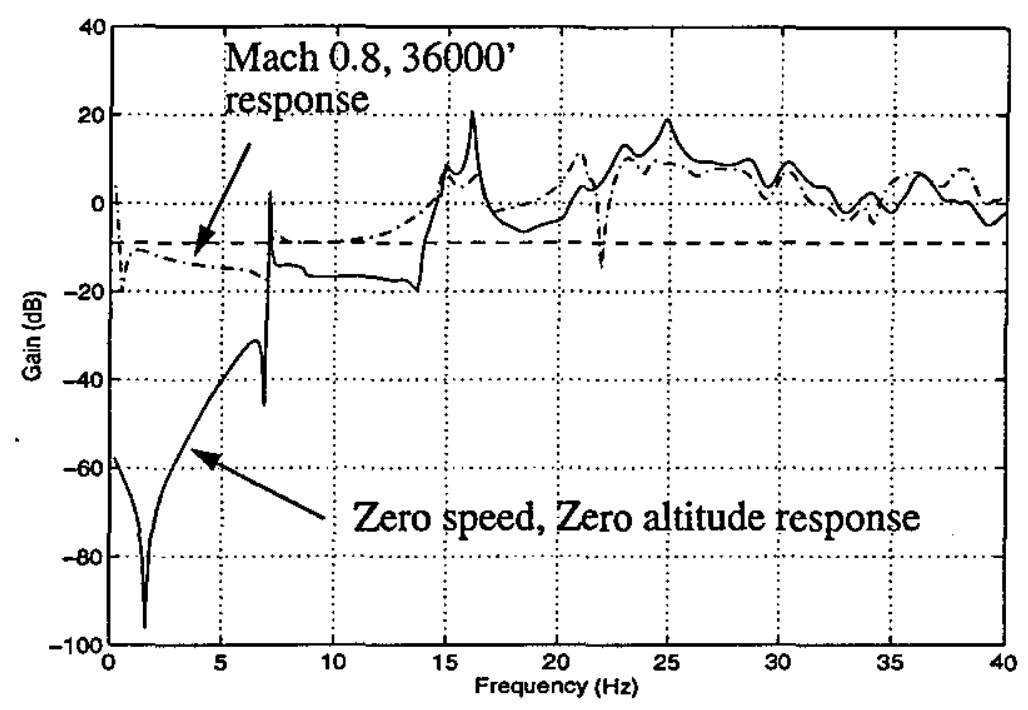

Figure 6.25 - Total open-loop system response taking signal phase paths into consideration, but assuming in-phase aliasing

Using these results to produce the maximum response envelope for the system, the attenuation requirements under these assumptions can be produced as shown in Figure 6.26 .

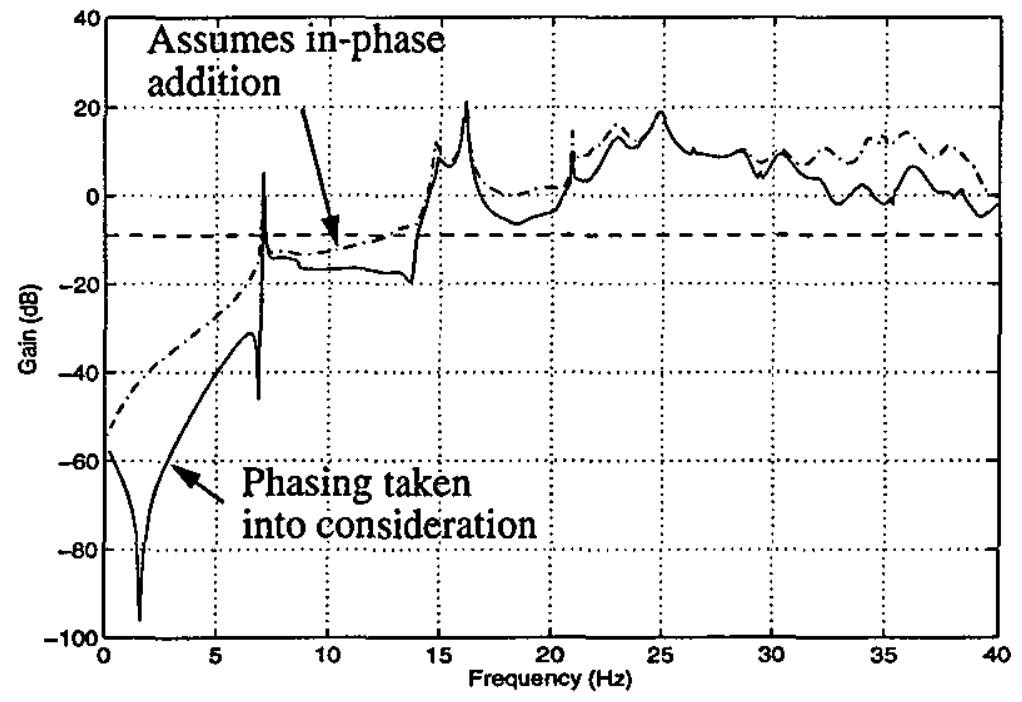

\section{Figure 6.26 - Attenuation requirements for digital system taking signal path phases into consideration}

Figure 6.26 shows that there is only a small advantage to be gained from taking the phases of the separate signal paths into consideration. The maximum modal levels, which dictate the structural-mode attenuation requirements are changed only slightly when the signal path phases are included.

Once again, for there to be a significant difference in modal gain values after the inclusion of the signal path phase, the contribution of each signal path to the overall modal response must be comparable. As an example, consider the first wing bending mode at a frequency of around $7.1 \mathrm{~Hz}$. It has already been discussed that the outboard 
flap control surface excites this mode the greatest. The aircraft response to the three inputs shows this well as can be seen in Figure 4.10, Figure 4.11 and Figure 4.12. The inclusion of the signal path phase into the response has almost no effect on the overall modal gain of this mode, the response due to the inboard flap and foreplane being swamped by the response due to the outboard flap.

Although the difference in modal gain values is small in general, there are certain advantages to be gained from taking the phase response of the system to the three inputs into consideration. The mode at approximately $37 \mathrm{~Hz}$ for example, which is one of the two "critical" modes effecting attenuation requirements of the structural filters, shows a reduction in modal gain of approximately $7 \mathrm{~dB}$. This reduction in gain could be critical for the optimum design of structural-mode filters.

If the phase response of the system was used as a whole, such that both the correct phase response during aliasing and signal path phases were taken into consideration, the overall attenuation requirements for the system would be as shown in Figure 6.27.

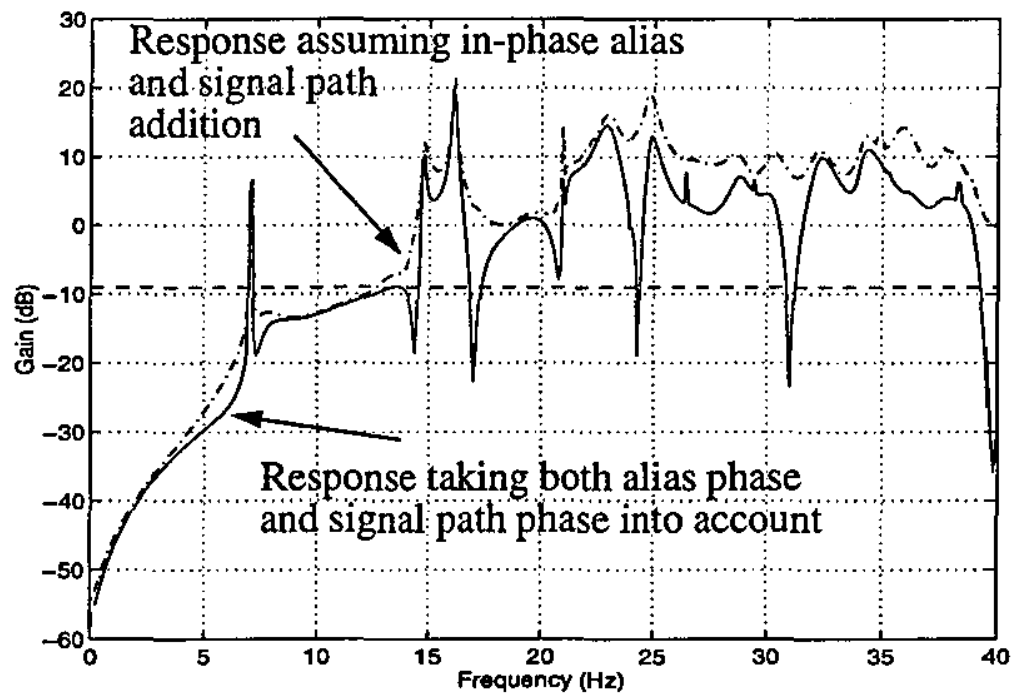

Figure 6.27 - Attenuation requirements for digital system taking both alias phase and signal path phase into account

By taking account of the phase response of the system in terms of both aliasing and signal path addition, it can be seen that there is a small advantage to be gained in terms of the attenuation required at the higher frequencies, where there seems to be a decrease in requirements of approximately $4 \mathrm{~dB}$.

Considering the attenuation requirements for the low-frequency structural-modes, there seems to be little advantage to be gained by taking the phases into account except at the mode at approximately $15 \mathrm{~Hz}$, which shows a decrease in gain of approximately $2 \mathrm{db}$. It can be seen from Figure 4.10, Figure 4.11 and Figure 4.12 that there exists two modes at approximately this frequency, the first fuselage bending mode and the first wing torsion mode. It seems that when the signal path phases have been taken into account, there has been some cancellation of response between these 
two modes. The effect of the aliasing results in additional cancellation of the response.

These results demonstrate that there is a certain advantage to be gained from taking the system phase response into account when calculating the structural-mode attenuation requirements. In terms of the notch filter that was designed earlier to attenuate the modes at a frequency just below $15 \mathrm{~Hz}$, it is clear that in the case of this model, the inclusion of the phase response would result in reduced requirements for this particular notch filter. The requirements for the other two notch filters are changed little by the consideration of system phases.

Considering the attenuation requirements for the low-pass filter for this model, the small change in requirements for the higher frequency modes would be particularly advantageous. This is because the low-pass filter introduces the greatest amount of phase lag into the system and any small reduction in attenuation requirements could reduce the phase lag of the filter significantly.

\subsection{Conclusions}

Consideration of the digital nature of the control system has demonstrated its effect on the aeroservoelastic problem. Application of the theoretical results of Chapter 5 to the aircraft model has demonstrated the potential for structural instability as a result of aliasing.Although in the case of this model, such aliasing did not result in the need to redesign the structural-mode filters, the need to consider the digital nature of the system from the outset is evident. It has also been demonstrated that any consideration of the effects of the digital nature of the control system should take account of the important role of the sensor dynamics in the attenuation of the structural-modes. A full consideration of the location of the structural-mode filters within the system has also been shown to be of importance

It has been demonstrated that there generally is no need to consider the aliasing of response from frequencies greater than the sampling frequency. This is due to the combination of attenuation introduced by the actuators, sample-and-hold and sensor dynamics.

Results have demonstrated a small reduction in the structural filter attenuation requirements as a result of the consideration of both alias and signal path phase. Although such a reduction in attenuation would be advantageous, it might not be worth the large amount of additional design work and testing required to verify such phase responses. In the case of a high-frequency mode being aliased on to a lowfrequency mode however, such consideration of phase response might significantly improve the structural filter attenuation requirements. Similarly, for structural-modes which are excited equally by two or more control surfaces, consideration of signal 
path phase could result in a large reduction in the filter attenuation requirements

Finally, these results were obtained for a flight control system identical to that designed for the analogue system case. In reality, this would not be the case, extra phase advance filtering being required in the digital case to account for the phase lags introduced by the sample-and-hold, sensor signal processing and computational delays. Such an increase in the phase advance filtering would undoubtedly result in an increase in the gain of the system. As a consequence, the attenuation requirements of the structural-mode filters would be increased. Clearly, the need for direct digital design of the control system, and subsequent allowances for the effects of the digital nature of the control system on the aeroservoelastic problem is important. 

Chapter 7

\section{Effect of Structural}

Feedback Signals on

Actuator Performance 


\subsection{Introduction}

The important role of the actuation system within the aeroservoelastic interaction cannot be overestimated. Firstly, the actuator provides the link between the flight control system and the structure of the aircraft itself. Inertial excitation of the aircraft structure has been seen to be the major contribution to the overall aeroservoelastic effect. This can be seen from Chapter 4, where the zero speed case resulted in the highest open-loop gain for the majority of the structural-modes. Secondly, the performance of the actuator in response to signals from the FCS forms an integral part of the rigid-body stabilisation. Any decrease in actuator performance from that assumed in the FCS design process could lead to the stability of the rigid-body aircraft being compromised. For example, an increase in the phase-lag of the actuator could result in unsatisfactory rigid-body stability-margins. Finally, the attenuation of the high-frequency structural-modes by the actuation system can be of great beneficial effect in preventing unwanted aeroservoelastic interactions.

As mentioned earlier, the highly non-linear nature of a servo-hydraulic actuation system can have serious consequences on aircraft control when the actuator is subjected to high-frequency input signals as well as the lower frequency flight control system demand signals. In order to achieve a greater understanding of the aeroservoelastic interaction, the performance of the actuator in the presence of structural feedback signals will be assessed. Such a situation could arise if a structuralmode resulted in a high-frequency noise signal being fed back to the FCS.

In the following chapter a suitable model of the actuation system will be developed for combination with the model of the flexible aircraft developed in Chapter 3.

\subsection{Actuation system modelling}

\subsubsection{Introduction}

As with most dynamic systems, the level of detail that can be used in developing a mathematical model depends on the requirements of the problem. In this case, the nonlinear nature of the actuation system is of utmost importance as its ability to respond to signals of more than one frequency is of great interest. As a result, a comprehensive model is required that represents the main nonlinear elements within the actuation system. Naturally, some assumptions still have to be made in order for the model to run efficiently in the SIMULINK environment.

The basic aim of the model is to represent the dynamics of the actuation system such that the relationship between the FCS demand signal for control surface angle 
and the actuator control surface angle demand is represented. This relationship can be seen from Figure 7.1, where the input signal to the actuation system is the output from the flight control system. This input signal is in the form of the control surface angle demands, which can be interpreted as ram displacement demands. The output from the actuation system is in the form of hydraulic ram displacements. This translates to control surface angle demands, through the application of the particular lever arm between the hydraulic ram and the control surface itself.

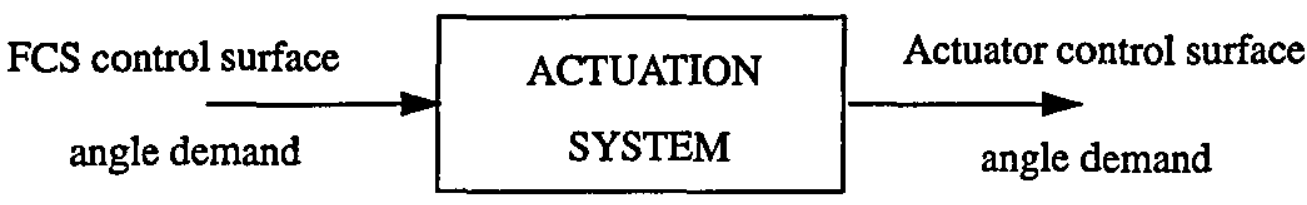

\section{Figure 7.1 - Actuation system simplified block diagram}

It has already been shown in Chapter 3 that the control surfaces themselves will suffer from vibrations as a result of the dynamic relationship between actuator output and the actual control surface angle achieved. These control surface dynamics were included in the flexible aircraft model developed earlier, the inputs to the model being the demanded control surface angles from the actuation system.

In the following section, to develop a model is developed to represent the input/ output relationship for the actuator as shown in Figure 7.1.

\subsubsection{Basic actuation system components}

The principles of servo-hydraulic actuation is covered in many suitable texts ${ }^{64-65}$, which will not be repeated here. Instead it is sufficient to break the system down into its components and consider each component separately.

The basic components of a typical aircraft servo-hydraulic actuation system are shown in Figure 7.2. In this case, the system comprises of four main blocks, namely the actuator control system, main-valve actuation, the main-valve block itself and the main-ram. The input to the system is the main-ram demand signals from the FCS and output is the main-ram position. It is possible to obtain equations to describe the dynamics of each of these blocks in turn. The following sections describe the deduction of suitable equations for each of these system blocks, leading to the full non-linear actuation system model to be used in later work.

As mentioned earlier, the level of detail that is required in a mathematical model depends on the problem to be solved and the environment in which it is to be used. In this case, certain assumptions will be made in order to obtain a non-linear actuation system model that operates efficiently within the SIMULINK environment whilst 
providing the required validity.

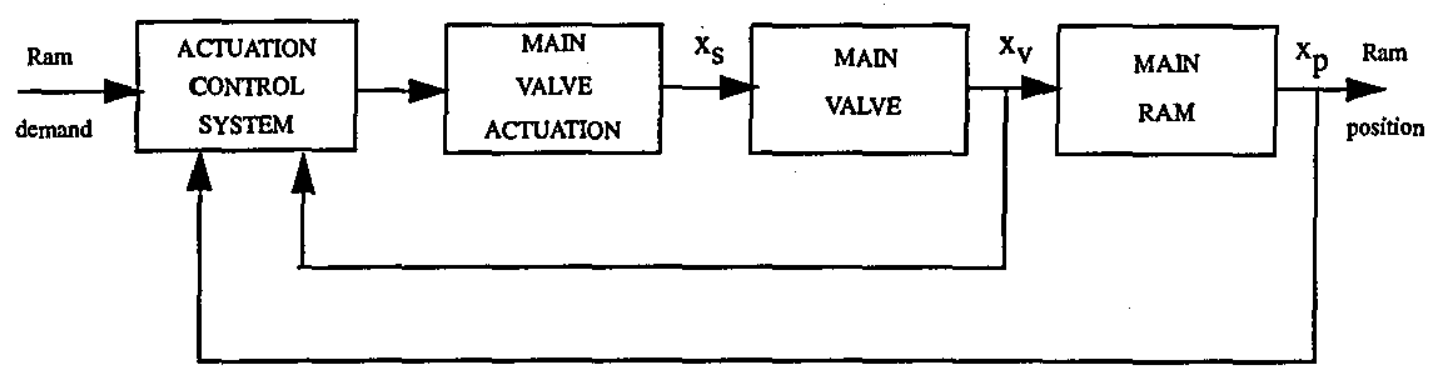

Figure 7.2 - Actuation system block diagram

\subsubsection{Main-valve actuation}

The control of the motion of the main-valve, which in turn controls the flow of hydraulic fluid to and from the main-ram chambers, can be achieved in many ways ${ }^{66}$. In this case, the model will be that for an electro-hydraulic servo-valve (EHSV), the main-valve being controlled by hydraulic fluid flow from a servo-valve, whose spool is moved by an electrical torque motor. This is representative of the main-valve actuation present on the Jaguar FBW taileron actuator.

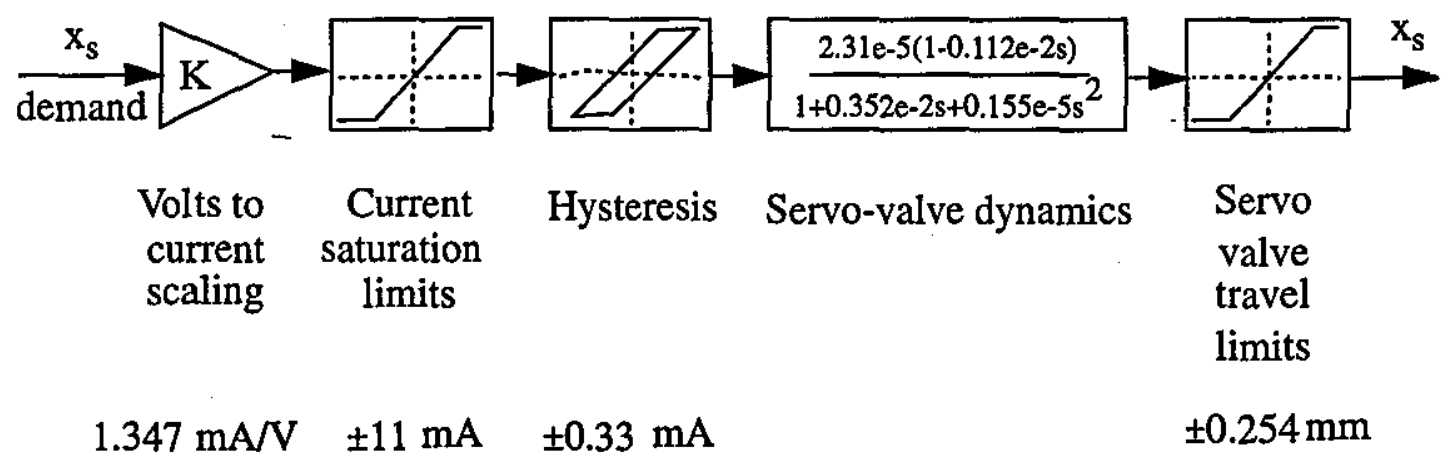

\section{Figure 7.3- Block diagram representation of an electro-hydraulic servo-valve}

A block diagram representation of a typical EHSV is included as Figure 7.3, which has been adapted from reference 66. This diagram shows the main nonlinearities in the system, namely the current saturation and hysteresis in the electrical actuation, and the servo-valve spool travel limits. The servo-valve dynamics themselves are generally modelled by matching a linear transfer function of suitable order to actual test results. The servo-valve dynamics for the Jaguar taileron actuator are given as in Figure $7.3^{67}$, which also includes the gains and limits for the taileron actuator ${ }^{68}$. The values of these gains and limits are given in the component manufacturers specification. This figure thus represents the non-linear dynamics of the main-valve actuation. 


\subsubsection{Main-valve dynamics}

The control of the main-ram of a typical hydraulic actuation system is generally accomplished using one or more main-valves, which govern the flow of the hydraulic fluid to and from the main-ram chambers. The motion of the main-valve itself is also effected by the motion of hydraulic fluid to and from its own chambers, and it is the modelling of this flow and the resultant motion of the main-valve itself that is of interest in this section. An in depth discussion of the characteristics of valve flow can be found in reference 66 . From reference 66, for a servo-valve with four ports as shown in Figure 7.4 below, the valve flow equations can be written as

$$
\begin{gathered}
q_{a}=C_{f} A_{a} \sqrt{\left|P_{1}-P_{R}\right|} \operatorname{Sign}\left(P_{1}-P_{R}\right) \\
q_{b}=C_{f} A_{b} \sqrt{\left|P_{S}-P_{1}\right|} \operatorname{Sign}\left(P_{S}-P_{1}\right) \\
q_{c}=C_{f} A_{c} \sqrt{\left|P_{s}-P_{2}\right|} \operatorname{Sign}\left(P_{S}-P_{2}\right) \\
q_{d}=C_{f} A_{d} \sqrt{\left|P_{2}-P_{R}\right|} \operatorname{Sign}\left(P_{2}-P_{R}\right) \\
q_{1}=q_{b}-q_{a} \\
q_{2}=q_{d}-q_{c}
\end{gathered}
$$

where,

$\mathrm{C}_{\mathrm{f}}$ is the general flow coefficient

$A_{x}$ is the effective area of port $x$ open to flow

$P_{S}, P_{R}$ are the supply and return pressures respectively

$\mathrm{P}_{1}, \mathrm{P}_{2}$ are the main-valve chamber pressures

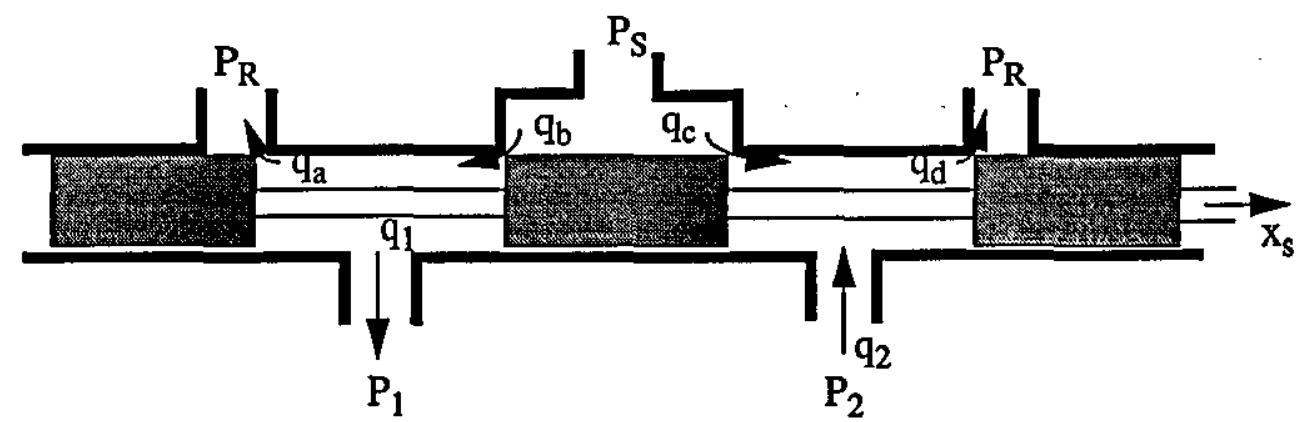

\section{Figure 7.4 - Typical servo-valve configuration}

The general flow coefficient will be assumed to be constant for now, although it is known to vary slightly with port opening. This variation will be taken into 
consideration later in the modelling process. The effective area of each port open to the flow can be calculated from relationships developed in reference 66 which depend on the presence of valve overlap, shaped valve ports and null position leakage flow. Suffice to say, that the values of $A_{x}$ can be calculated for any given spool position, $x_{s}$ from the valve manufacturers data.

Using the above equations enables the fluid flow to and from the main-valve chambers to be calculated. From Figure 7.5, this flow to/from the main-valve chambers will result in a pressure variation over the main-valve spool, resulting in movement of the spool. From reference 66 , the pressure changes occurring within the chambers of the main-valve spool can be calculated from

$$
\begin{gathered}
\dot{P}_{1}=\frac{N}{V_{1}}\left(q_{1 R}-A_{p 1} x_{v}-q_{x}\right) \\
\dot{P}_{2}=-\frac{N}{V_{2}}\left(q_{2 R}-A_{p 2} \dot{x}_{v}-q_{x}\right)
\end{gathered}
$$

where,

$\dot{\mathrm{P}}_{1}, \dot{\mathrm{P}}_{2}$ are the time rates of change of $\mathrm{P}_{1}$ and $\mathrm{P}_{2}$

$\mathrm{N}$ is the bulk modulus of the hydraulic fluid (assumed constant)

$q_{1 R}, q_{2 R}$ are the resultant flow in/out of the main-valve spool chambers

$\dot{x}_{v}$ is the velocity of the main-valve spool

$A_{p 1}, A_{p 2}$ are the main-valve piston areas

$\mathrm{V}_{1}, \mathrm{~V}_{2}$ are the chambers volumes either side of the main-valve spool piston

$\mathrm{q}_{\mathrm{x}}$ is the leakage flow across the piston

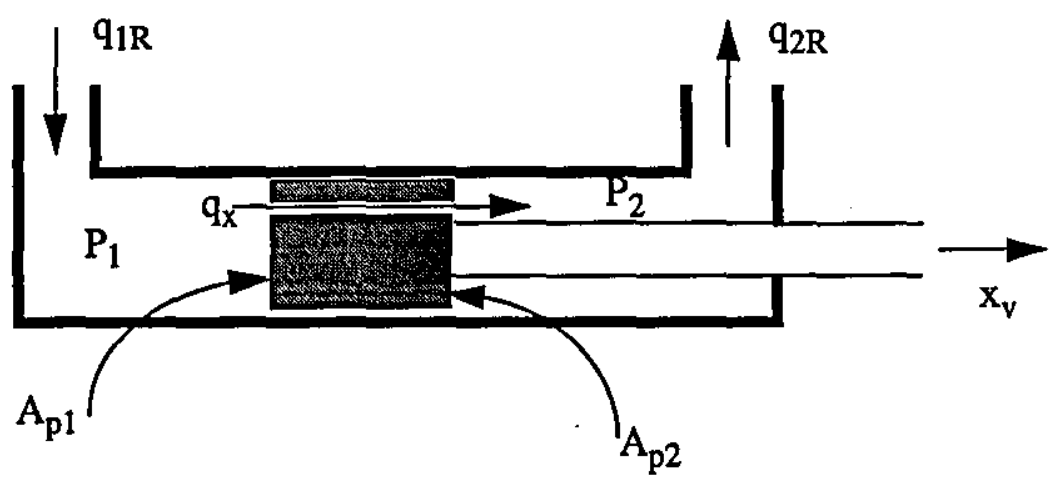

Figure 7.5 - Main-valve spool actuation

It is therefore possible to calculate the rate of change of the pressures acting on the main-valve spool piston as a result of the displacement of the servo-valve spool. These rates can be integrated over time to obtain the resultant pressures acting on the mainvalve spool. The displacement of the main-valve can be obtained with due regard to the dynamics of the main-valve spool. Modelling of the dynamics of the main-valve 
spool itself can vary in complexity between a simple second-order undamped linear system to a full non-linear consideration of system friction, backlash, damping, rate and position limits.

\subsubsection{Main-Ram Dynamics}

The above analysis for calculating the main-valve spool displacements as a result of a servo-valve spool displacement can be essentially repeated in order to calculate the motion of the main-ram as a result of the displacement of the main-valve spool. The only significant difference between the two systems is that the main-ram may be subjected to significant loading as a result of the attached control surface inertias and resultant aerodynamic loading. In the case of a comprehensive ram loading model however, where the effect of the control surface dynamics and aerodynamic loading on the actuator ram are taken into consideration, it would be necessary to feedback the states corresponding to the control surface positions from the flexible aircraft model as developed in Chapter 3.

\subsubsection{Actuator Control System}

From Figure 7.2, the remaining system component to be described is the actuator control system. In most cases, this system consists of two main elements, namely position control loops for the main-valve and main-ram. The main-valve position control loop is generally an integral part of the actuator system itself, whereas the main-ram position control loop generally forms part of the aircraft flight control system, and may be subject to the digital nature of the aircraft flight control system if applicable.

In this form the actuator control system can be considered to consist of an outer and inner loop as shown in Figure 7.2. The feedback sensors for these control loops are generally LVDT position sensors, the transfer functions of which are usually accepted to be simple gains. Further gains are incorporated into the feedback path along with first or second-order demodulation filters, and where required, anti-aliasing filters. Finally, input scaling is frequently required in order to convert from control surface positions demand by the flight control system to ram positions and EHSV drive voltages.

\subsubsection{Model Simplification}

The above sections give an insight into the complexities involved in modelling the actuation system modelling. The following model was produced using data for the Jaguar FBW aircraft taileron actuation system, which represents the actual actuator hardware available for testing throughout this work.

Initially, it is assumed that the actuator ram is unloaded, and free from friction and 
backlash. The main-valve dynamics are assumed to be representable by a simple flow gain which simplifies the transfer function between main-valve position, $x_{v}$, and servo-valve position, $\mathbf{x}_{s}$, to that of an integrator and a gain only. The calculation of this flow gain, covered in detail in reference 66 , is generally calculated using linearised versions of the valve flow and hydraulic equations given earlier under certain assumptions regarding spool dynamics and port geometries. This enables the mainvalve spool velocity, $x_{v}$ for example, to be written as a function of the servo-valve displacement, such that

$$
\dot{x}_{\mathrm{v}}=\mathrm{G}_{\mathrm{MV}} \mathrm{x}_{\mathrm{s}}
$$

Combining the model of the EHSV given in Figure 7.3 with the above relationship to describe the main-valve dynamics and a model of the main-ram dynamics and actuator control system results in a nonlinear model of the actuator. Such a model is suitable for incorporation into the SIMULINK model of the aircraft system developed in Chapters 4 and 6. The block diagram of the model is shown in Figures 7.6 and 7.7.

Some of the additional elements included in the model include the main-valve velocity and travel limits and the main-ram travel limits. In addition, scheduling of the flow coefficient of equations (7.1) to (7.4) with port opening is included according to reference 68 . Finally, the servo-valve displacement, $x_{s}$, is augmented at small values of drive current in accordance with reference 68 .

An additional element that has been included in the model as shown in Figure 7.6 is the software rate limiting of the input demand signal. It is common practice to include a rate limiter on the demand signal within the flight control system in order to protect the actuation system from potentially damaging inputs. This has been included within the actuation system model in order to retain all nonlinear elements of the system within a single system element. 


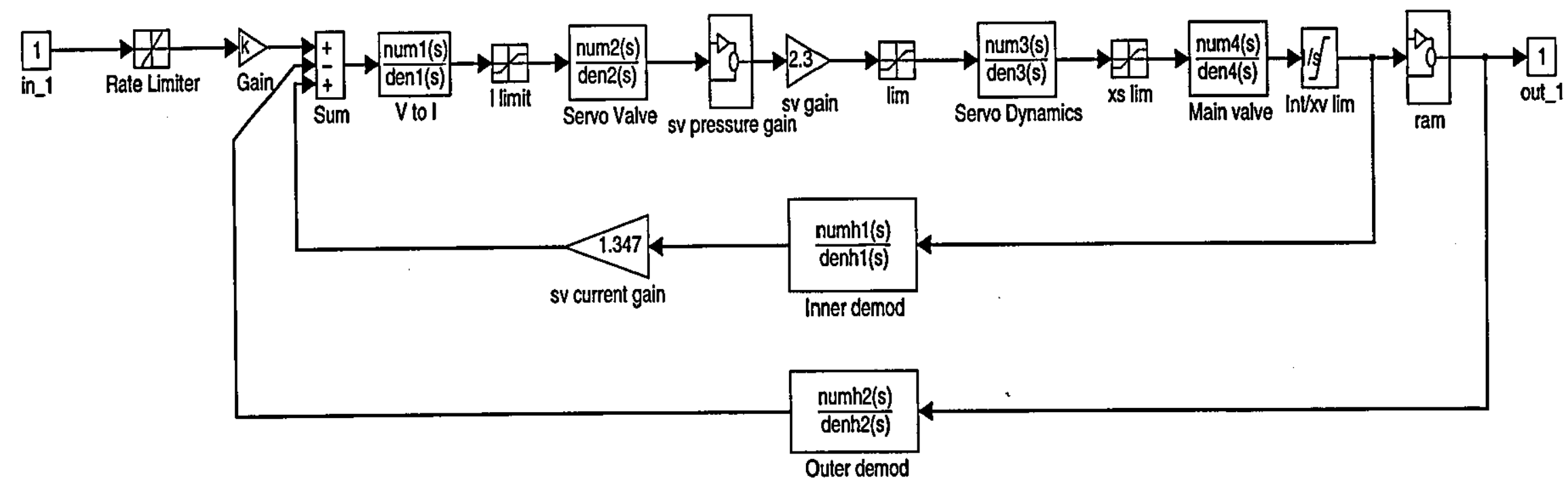

Figure 7.6 - Jaguar FBW taileron actuator system model - Overview 


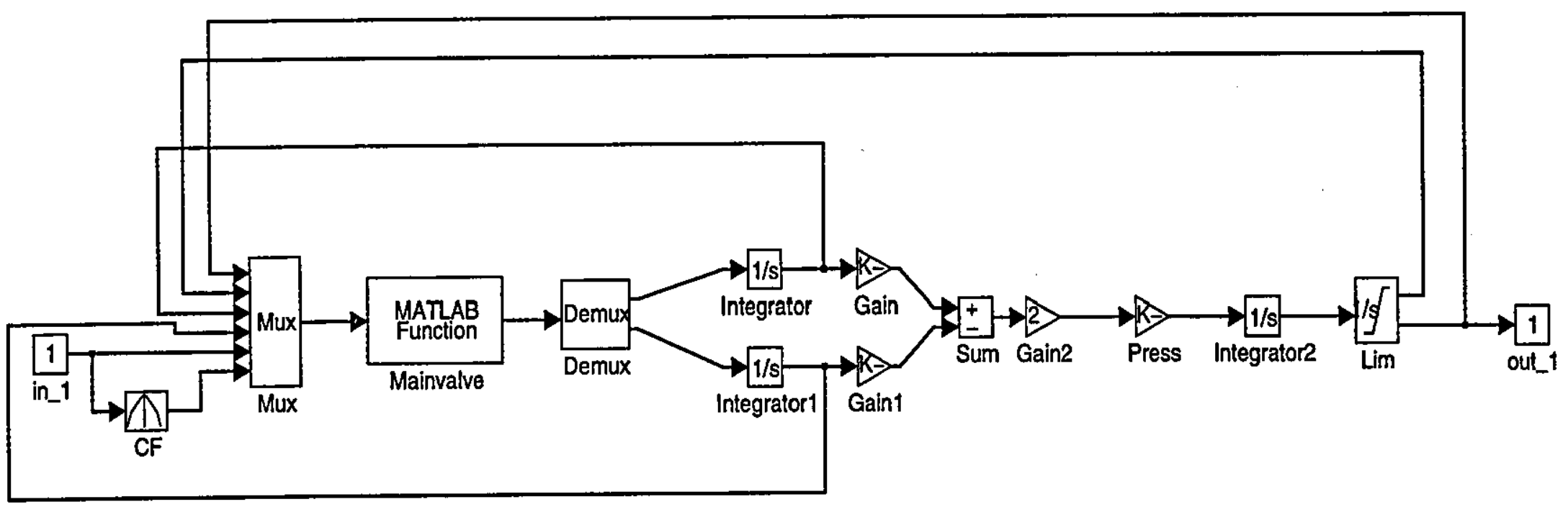

Figure 7.7 - Jaguar FBW Taileron actuator system model - Main-ram dynamics 


\subsection{Comparison of actuation system model with experimental results}

\subsubsection{Introduction}

In order to assess the validity of the actuation system model, comparison was made with results obtained from testing a Jaguar FBW taileron actuator. The following section describes the test rig in some detail and presents results of model validation tests carried out on the rig.

\subsubsection{Description of experimental rig}

The test rig consisted of the Jaguar FBW Taileron actuator itself, with the necessary electrical drive amplifiers and feedback controller that would be present within the aircraft. The electrical test set ${ }^{69}$ provides all of the necessary electrical requirements of the actuator such as servo-valve drive current and the main-valve and main-ram control loops as shown in Figure 7.2. In addition to this equipment, the test rig also consisted of a transfer function analyser and personal computer, which performed frequency response analysis as well as logging time response data. The personal computer also introduced a rate limiting function between the transfer function analyser and actuator itself by means of a suitable A/D-D/A card and software. A schematic diagram of the test rig is shown in Figure 7.8.

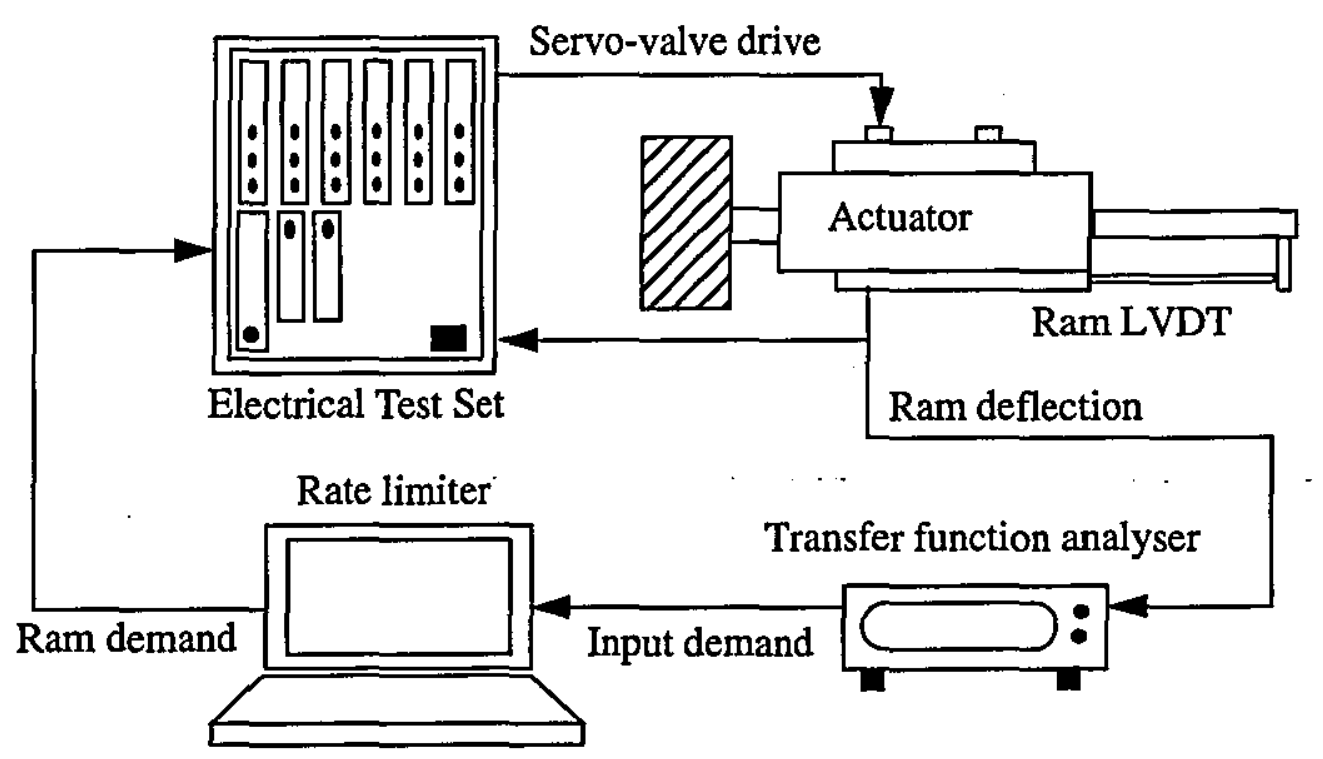

Figure 7.8 - Schematic diagram of actuator test rig

From the figure, the transfer function analyser provides the input demand signal which is sampled by the personal computer. This signal is then rate limited by the computer before being output to the test set. This signal thus represents the ratelimited ram demand signal. The electrical test set derives the required servo-valve 
drive signal to the actuator itself according to the main-valve and main-ram feedback signals and the demanded ram displacement. The output signal from the actuator representing ram displacement is then passed back to the transfer function analyser for calculation of the frequency response.

As mentioned earlier, the personal computer is also responsible for data logging of both input and output signals, enabling time response comparisons to be made with the simulated results. It should be noted that initially at least, the sample time of the personal computer was small enough to neglect digital effects. It will be demonstrated later however that the sample time can be set so as to represent the case of a digital implementation of the flight control laws. For initial model validation however, such a digital input case will not be examined.

\subsubsection{Comparison of test results with predicted frequency response}

In order to validate the analytical model of the actuator, frequency response testing was completed over a wide range of input amplitudes. The need to consider such a range of amplitudes results from the highly nonlinear nature of the actuator. It should be noted that in performing these frequency response tests, the actuator was under no static load, and variations in hydraulic supply pressure and fluid temperature were assumed to be negligible.

Frequency response results for both analytical model and test rig are included as Figure 7.9 to Figure 7.12 .
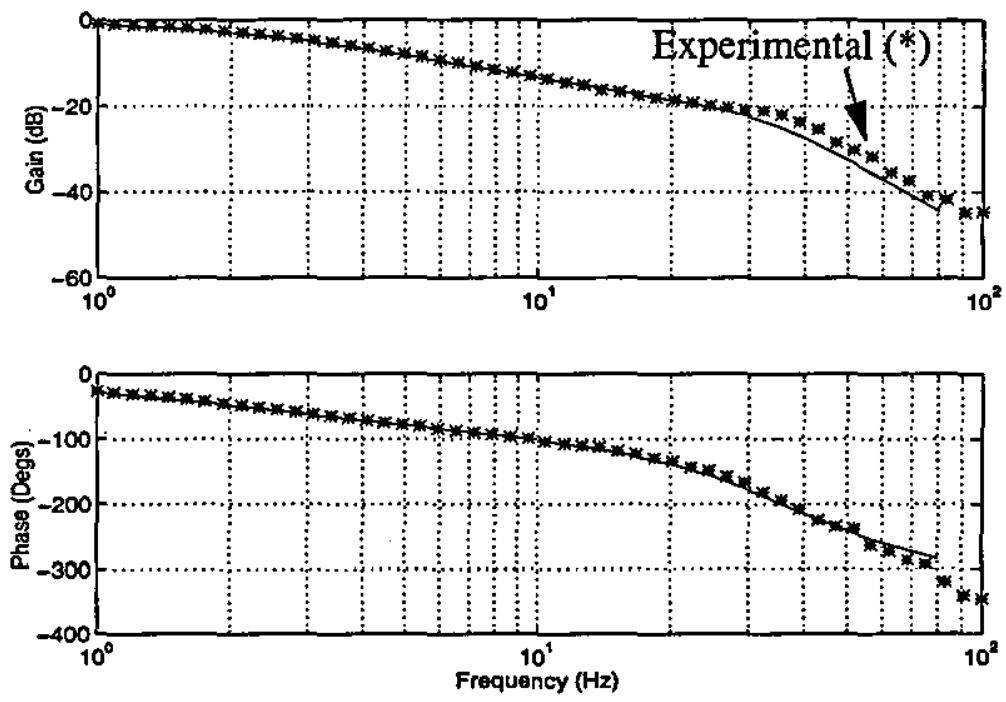

Figure 7.9 - Frequency response comparison, input amplitude $=0.13 \mathrm{~mm}$ 

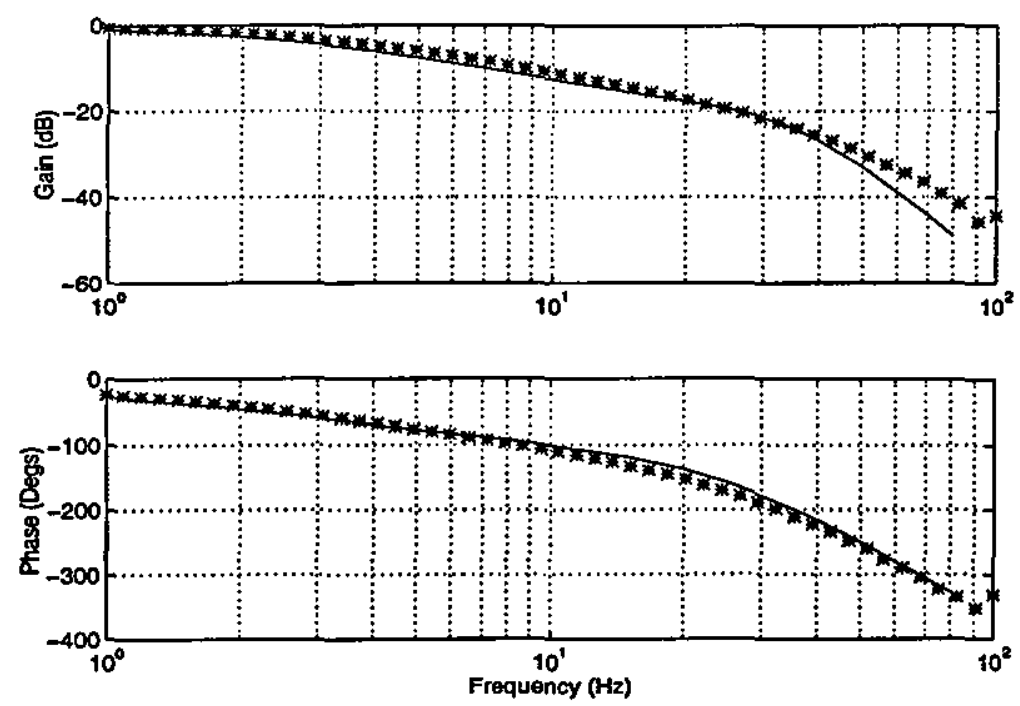

Figure 7.10 - Frequency response comparison, input amplitude $=1.0 \mathrm{~mm}$
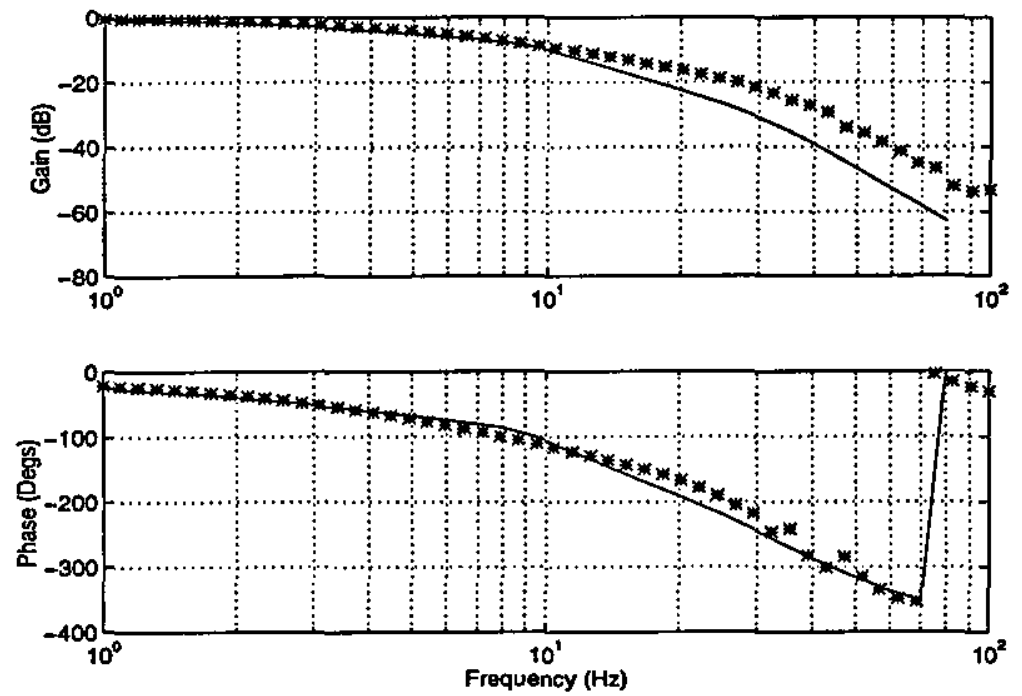

Figure 7.11 - Frequency response comparison, input amplitude $=5.0 \mathrm{~mm}$
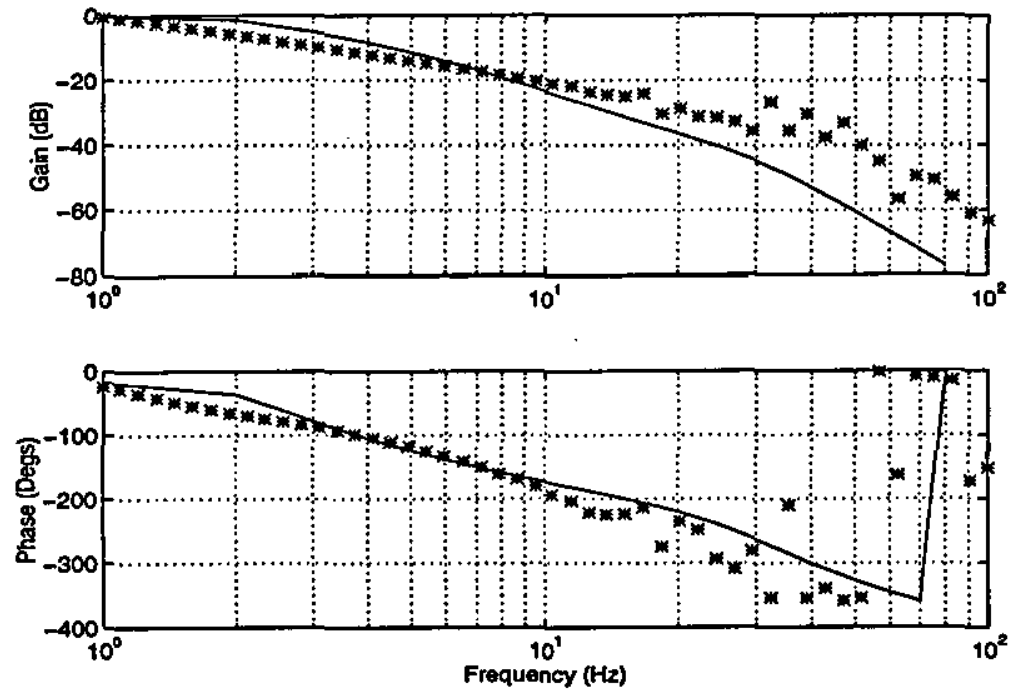

Figure 7.12 - Frequency response comparison, input amplitude $=50.0 \mathrm{~mm}$ 
From the results, it can be seen that the analytical model is a good representation of the actual actuator available for test. As a result, the nonlinear actuator model can be applied in the later sections with sufficient confidence in the models fidelity.

\subsection{Effect of structural-mode feedback signals on actuator performance}

\subsubsection{Introduction}

The role of the actuation system in the attenuation of structural-mode signals can be easily appreciated from the magnitude of the frequency response of a linearised actuator model as shown in Figure 7.13. The attenuation produced at high frequencies by the actuation system will have a crucial effect on the propagation of these highfrequency structural-mode signals around the closed loop. Obviously, if the actuator provides a high degree of attenuation, then the aeroservoelastic problem will be reduced. This however implies a lower bandwidth resulting in reduced performance. Relying on the attenuation of the actuators in solving the aeroservoelastic problem can also introduce serious effects on the stability of the rigid-body aircraft as a result of structural-modes falling within the bandwidth of the system.

In the following sections, it is intended to investigate the effect of high-frequency signals on this low-frequency performance, and also on the attenuation effect of the actuator at structural frequencies.

\subsubsection{Linearity boundary for non-linear actuation system model}

The effect of the system's non-linearities on actuator performance is important in the analysis of the total aircraft system's stability. If the non-linearities were not present, or the system was operating at such a point as to make their effect negligible, then the system stability can be readily assessed using classical linear theory.The importance of being able to predict the region of linear behaviour for an actuation system is therefore apparent, and this can be accomplished using simple linear theory ${ }^{29}$. Consider the block diagram of the actuation system for the actuator shown in Figure 7.14, where the main-valve flow equations have been linearised resulting in a linear transfer function between main-valve position and main-ram position. In addition, the software rate limiter at the input is neglected initially. This process results in a model consisting entirely of linear elements and saturations. 


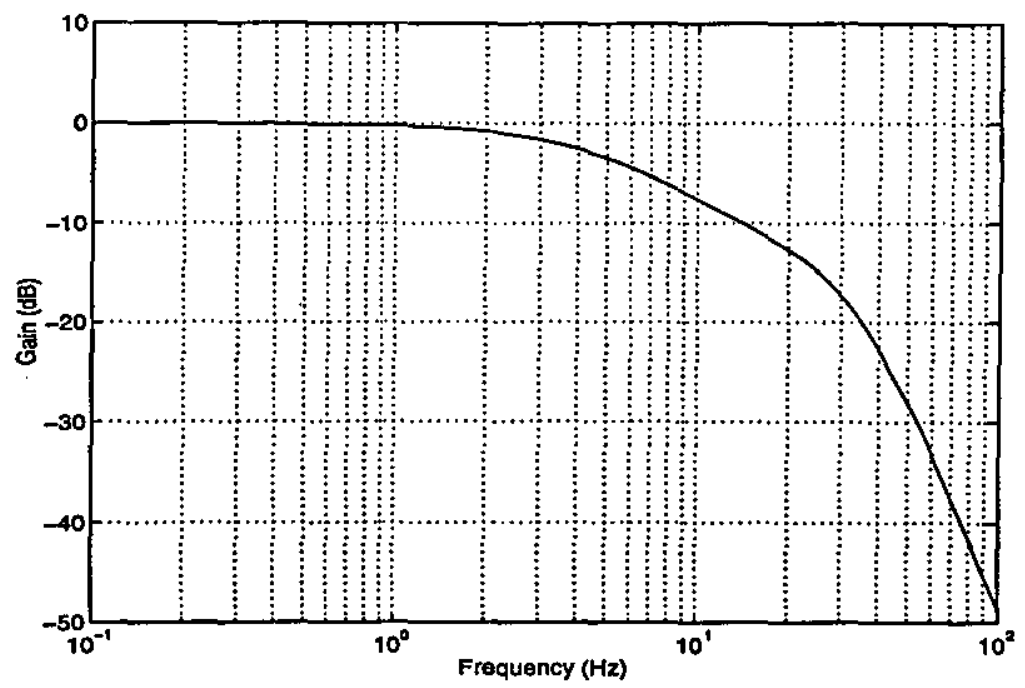

Figure 7.13 - Linearised actuator magnitude frequency response

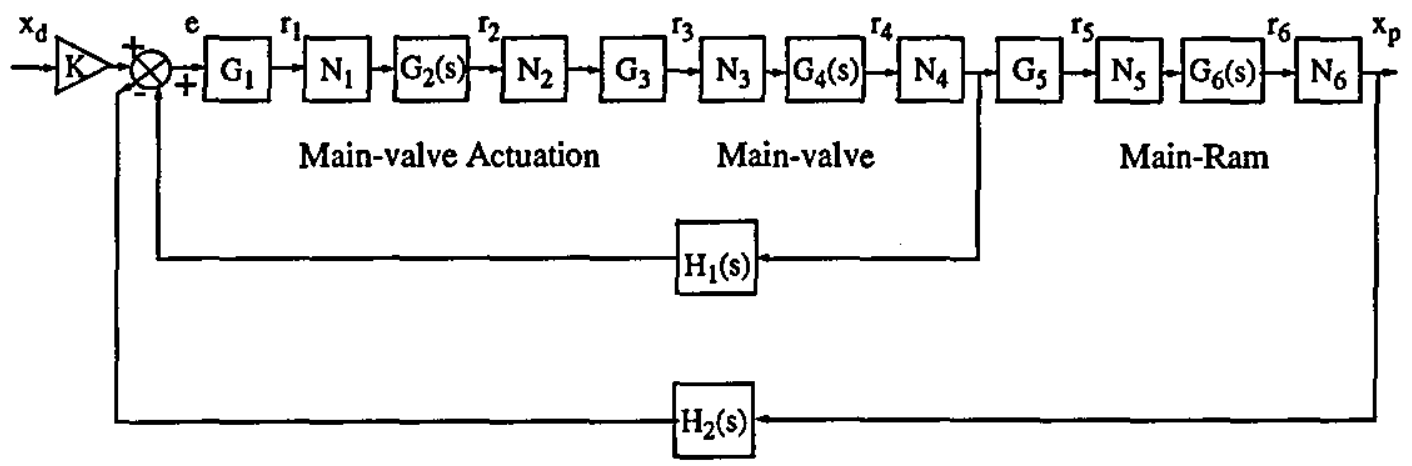

Figure 7.14 - Simplified actuator block diagram

For the output of ram position, $x_{p}(t)$, as a function of demanded ram position, the closed-loop error transfer function can be produced for an operating point within the linear region, such that the saturation non-linearities $\left(\mathrm{N}_{1}, \mathrm{~N}_{2}, \ldots \mathrm{N}_{6}\right)$ can be assumed to be simply equivalent to unity gain. Assuming this to be the case, the closed-loop error transfer function can be seen to be

$$
\frac{E(s)}{X_{d}(s)}=\frac{K}{1+\left(H_{2}(s)-\frac{H_{1}(s)}{G_{5} G_{6}(s)}\right) \prod_{n=1}^{6} G_{n}(s)}=F(s)
$$

Therefore, for example, the transfer function of servo-valve current, $r_{1}(t)$, to demanded main-ram displacement, $x_{d(t)}$

$$
\frac{R_{1}(s)}{X_{d}(s)}=G_{1}(s) F(s)
$$

can be obtained. The above transfer function only holds provided that none of the saturation limits are exceeded. Considering the servo-valve current, $r_{1}(t)$ for example, this signal must not exceed the level set by the non-linearity $\mathrm{N}_{1}$ in Figure 7.14. The 
magnitude of the input signal at which this level will be exceeded can be obtained from equation (7.11). For example, in the frequency domain

$$
\left|X_{d}(j \omega)\right|_{\text {MAX }}=\frac{\left|R_{1}(j \omega)\right|_{\text {MAX }}}{G_{1}(j \omega) F(j \omega)}
$$

which represents a frequency dependent linearity boundary for the system assuming saturation will occur at $\mathrm{N}_{1}$ first, and that all other saturation levels have not been reached. Repeating the above steps for each non-linearity in turn, will build up a series of linearity boundaries for the system.

This procedure has been completed for the Jaguar taileron actuator model as shown in Figure 7.14, with the resultant linearity boundary being as shown in Figure 7.15 .

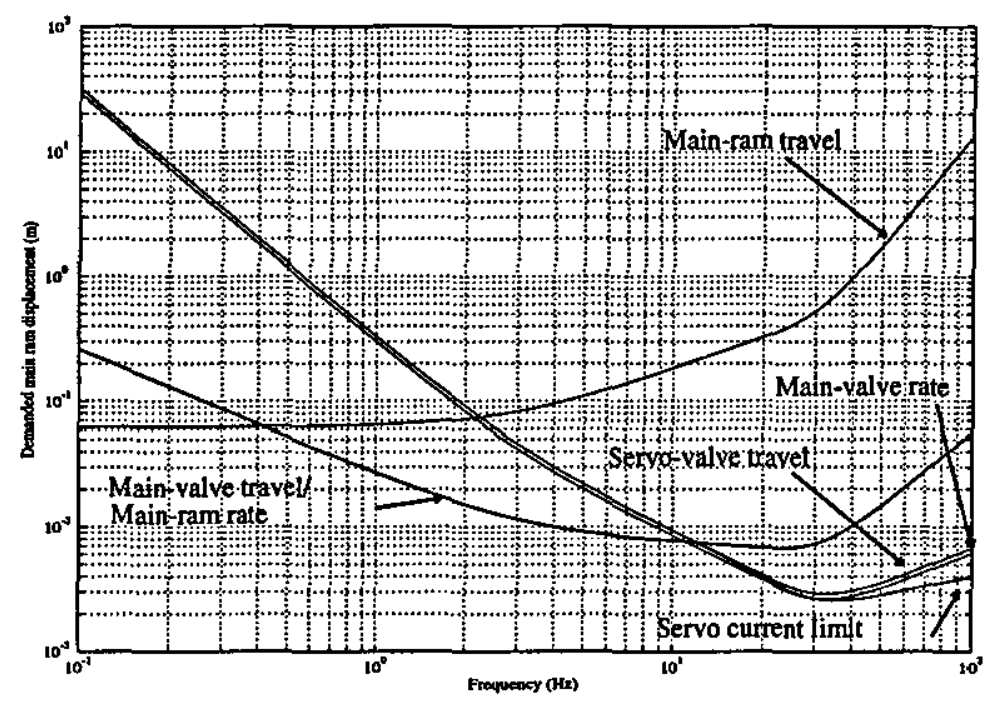

Figure 7.15 - Single input linearity boundaries for simplified actuator model

The figure demonstrates that there exists a region in which the actuator will behave in a linear manner, the region being bounded by several curves depending on which of the component saturations of the actuation system is critical at the particular input frequency. For example, from Figure 7.15, at low frequencies the critical limit is given by main-ram travel, as would be expected. As the input frequency reaches approximately $0.4 \mathrm{~Hz}$ however, the dominant limit becomes that of main-ram rate/ main-valve travel up to a frequency of approximately $10 \mathrm{~Hz}$ where the limits of the servo-valve become dominant.

When the actuator is subjected to a high-frequency input signal superimposed over a low-frequency signal, the performance of the actuator in terms of linear behaviour would be reduced, since the presence of the high-frequency signal causes the margins from the linearity boundaries to be reduced. For example, suppose that the actuator was subjected to a high-frequency sinusoidal signal of a certain amplitude. In responding to this signal, the actuator servo current for example might reach $50 \%$ of 
its limiting value. This would leave only the remaining $50 \%$ available for response to a lower frequency FCS signal before signal clipping would take place as a result of the saturation non-linearity. The system would operate in a nonlinear manner from this point on. The same reasoning applies to all of the saturations within the system model.

As a numerical example, for a high-frequency excitation signal of typical structural-mode frequency $50 \mathrm{~Hz}$ and amplitude at actuator input of $1.5 \mathrm{~mm}$, the linearity boundary for a second superimposed signal is as shown in Figure 7.16, along with the original single-input linearity boundary. The linearity boundary for the case with the high-frequency excitation signal was produced by considering the reduction in the saturation limits available in response to the primary signal as a result of the presence of the high-frequency signal. As expected, the linearity boundary is lowered by the presence of the high-frequency signal, but only by very small amounts in the low-frequency ranges, where the main-ram and main-valve travel limits dominate. This effect is a result of the low-pass characteristics of the main-valve and main-ram. The problem then lies in the high-frequency ranges, where the high-frequency excitation signal reduced the linearity boundary significantly, and where a further high-frequency input signal could easily cause the servo-valve limits to be reached and the region of non-linear actuator behaviour to be entered.

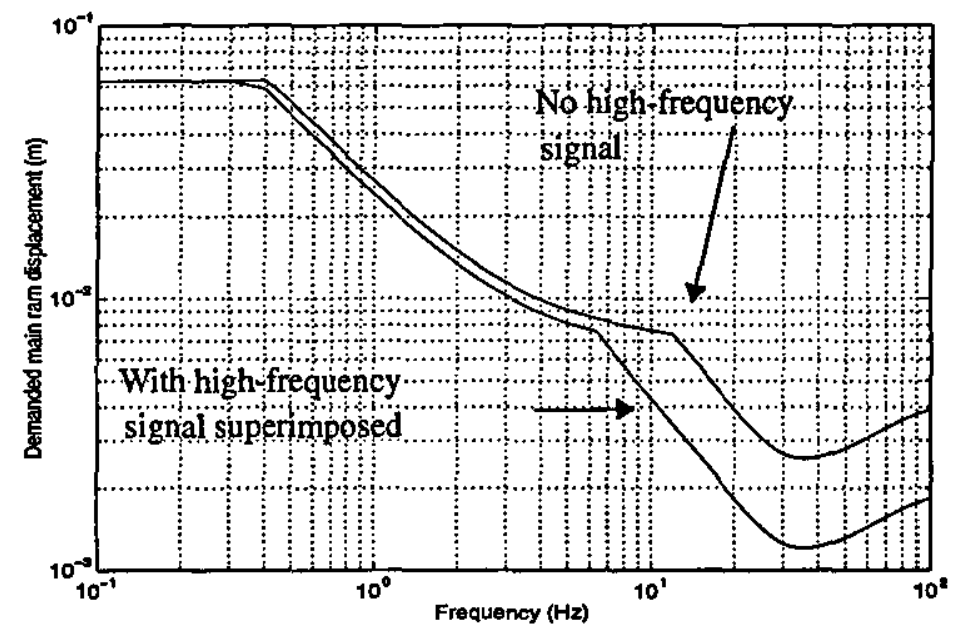

Figure 7.16 - Linearity boundaries for Jaguar FBW taileron actuator model with and without $50 \mathrm{~Hz}, 1.5 \mathrm{~mm}$ excitation signal

\subsubsection{Effect of structural-mode signals on low-frequency actuator per- formance}

The above analysis seems to indicate that the effect of the structural-mode signals on the low-frequency performance of the actuator will be small, since the linearity boundary at low frequencies is changed little by the addition of the structural-mode feedback signal. The effect of structural-mode signals on the performance of the actuator at low frequencies can be demonstrated by calculating the frequency response 
of the full nonlinear actuator model to low-frequency demand signals, when subjected to an additional high-frequency structural-mode signal ${ }^{29}$.
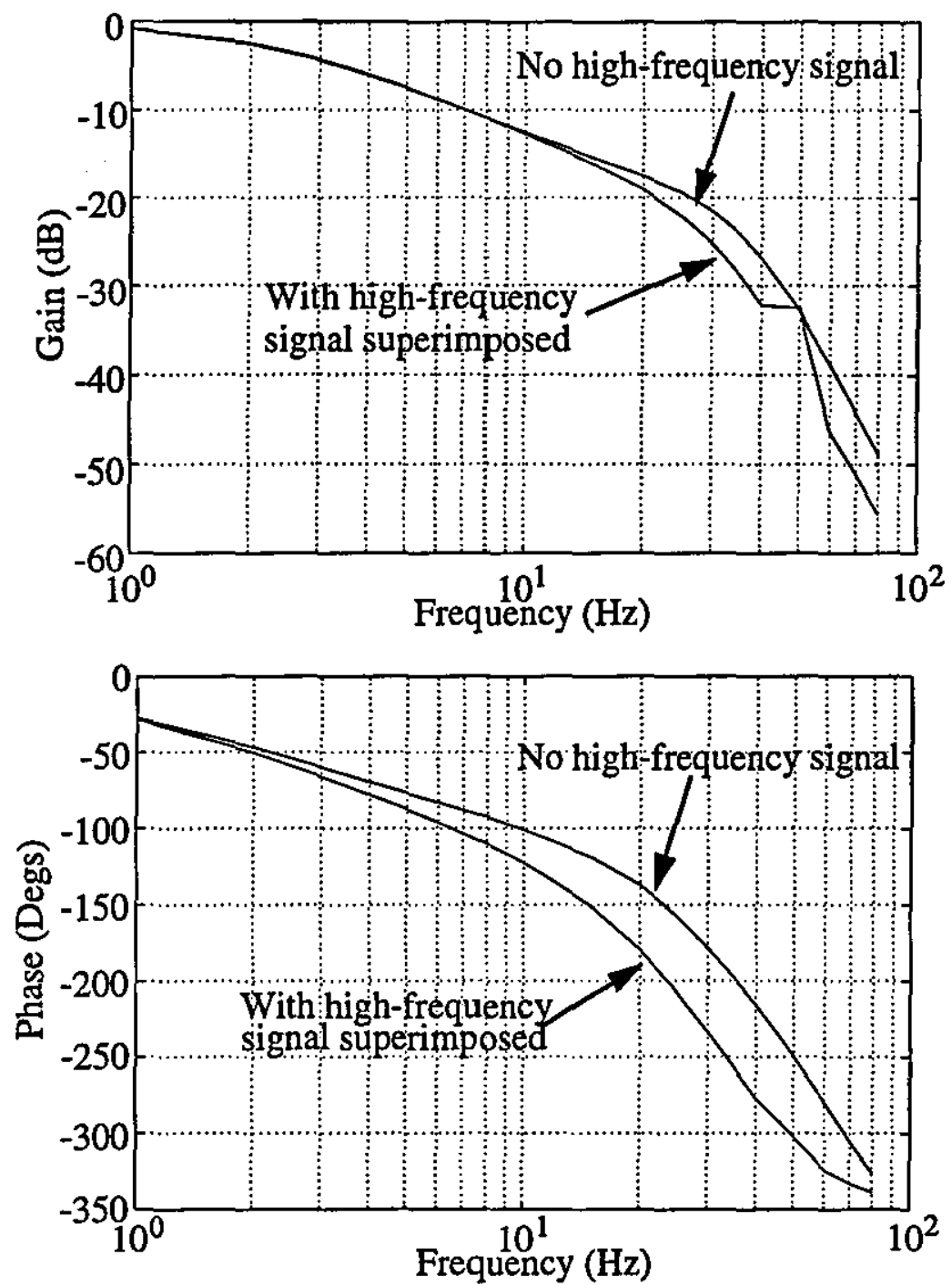

Figure 7.17 - Frequency response for nonlinear model with and without a $50 \mathrm{~Hz}$, $1.5 \mathrm{~mm}$ excitation signal

As a specific example, the frequency response of the nonlinear actuator model subjected to the earlier structural signal of frequency $50 \mathrm{~Hz}$ and amplitude $1.5 \mathrm{~mm}$ is shown as Figure 7.17. In this case, the frequency response corresponds to a demanded actuator displacement of $1.0 \mathrm{~mm}$ which is a typical demand amplitude for aircraft stabilisation by the FCS. The frequency response was calculated from the time response of the nonlinear actuator model to the combined input signals. The two input signals were generated with zero phase angle such that reinforcement of the demand signal by the structural signal would occur for a demand signal of $50 \mathrm{~Hz}$ frequency.

It is clear from Figure 7.17, that the structural signal did not greatly affect the gain of the actuator at low frequencies verifying the expectation from earlier calculations. It is also clear, however, that the phase response at low frequencies has been affected. The presence of the structural signal induces extra phase lag into the system even 
though Figure 7.16 predicts that none of the valve saturations will be reached under these operating conditions. This extra phase lag would be detrimental to flight control system performance as a whole, although the magnitude of the effect will obviously be dependent on the amplitude of the structural signal and its frequency.
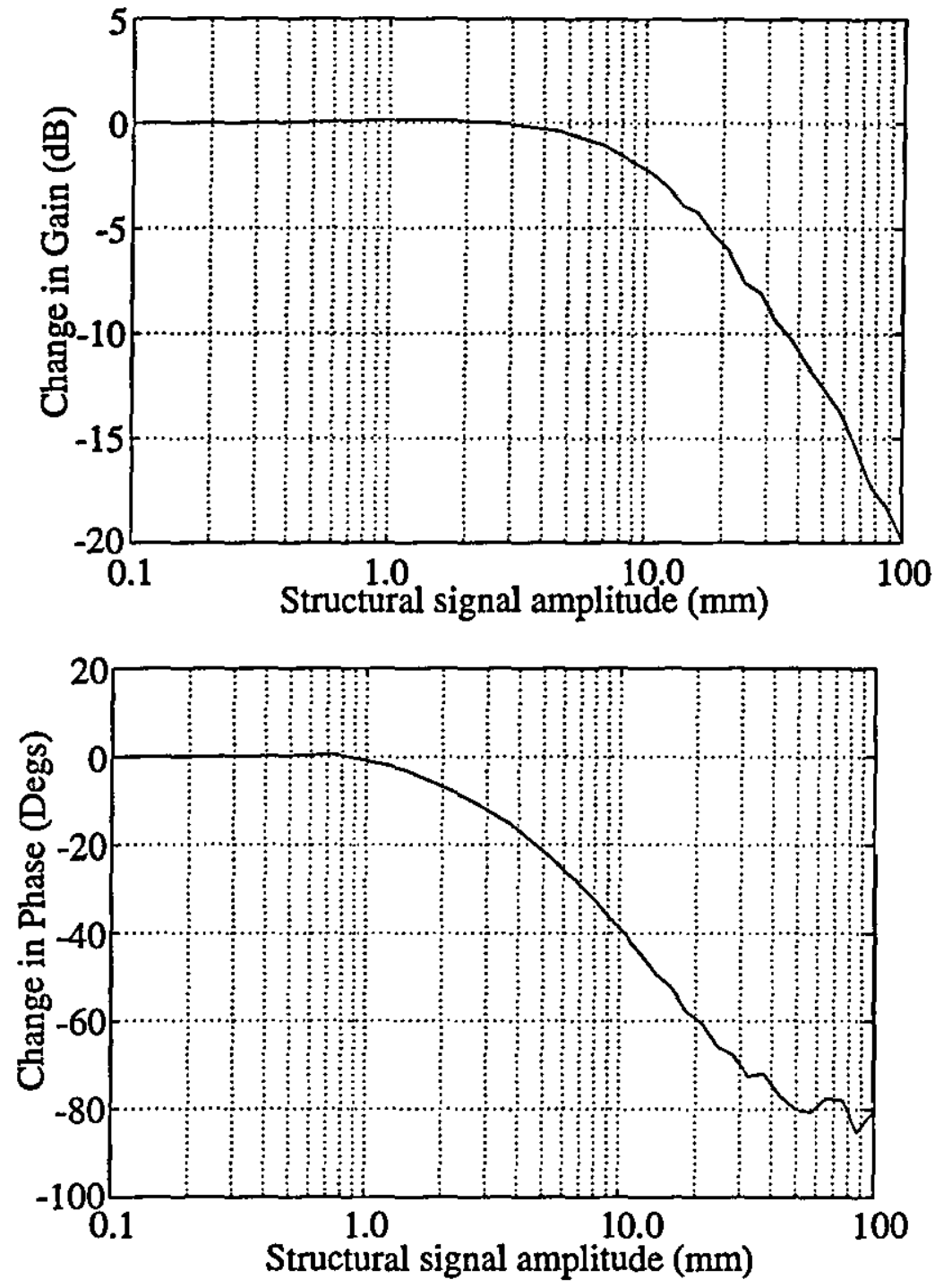

Figure 7.18 - Actuator gain and phase response changes for a $2 \mathrm{~Hz}$ demand signal and $50 \mathrm{~Hz}$ structural signal of varying amplitude

In order to demonstrate how the amplitude of the structural signal affects the above results, the gain and phase response of the actuator for a demand signal of amplitude $1.0 \mathrm{~mm}$ and frequency $2 \mathrm{~Hz}$ was calculated for the nonlinear model in the presence of a $50 \mathrm{~Hz}$ structural noise signal of varying amplitude. From Figure 7.18, as the amplitude of the structural signal is increased, the effect on the performance of the actuator becomes more pronounced as would be expected. It is worthwhile noting however, that it may be possible to specify a maximum level for the structural signal amplitude that could be tolerated for satisfactory aircraft control. This observation could in turn lead to a reduction in the attenuation requirements for the structuralmode filters. A possible application of this result is discussed in Chapter 8. 

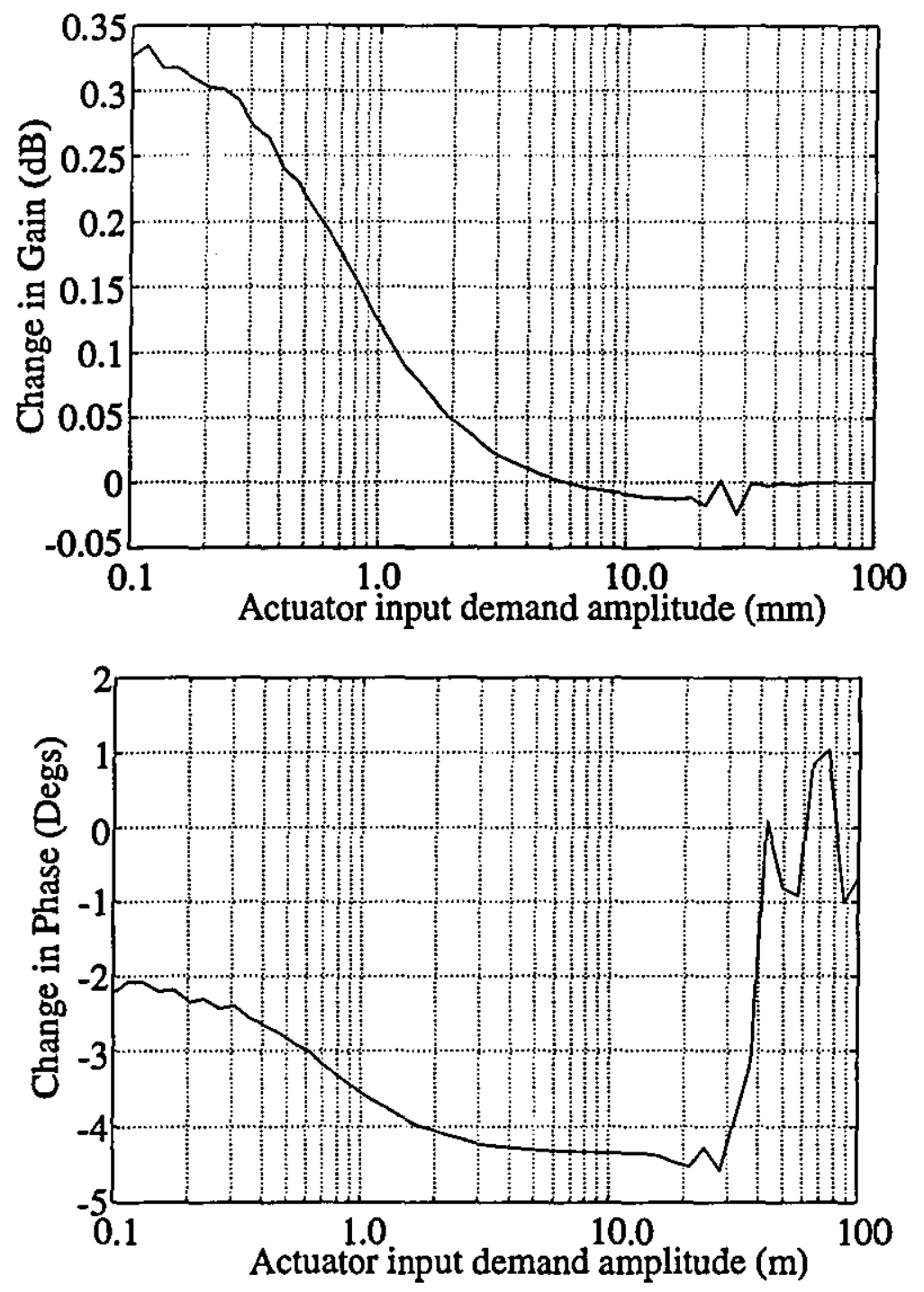

Figure 7.19 - Actuator gain and phase response changes for a $2 \mathrm{~Hz}$ demand signal of varying amplitude and $50 \mathrm{~Hz}$ structural signal

The previous analysis has been for a particular amplitude of actuator demand signal, $1.0 \mathrm{~mm}$, and no account had been taken of how the amplitude of this signal affects the results. This effect can be demonstrated by examining the actuator frequency response when subjected to a $50 \mathrm{~Hz}$ structural signal of amplitude $1.5 \mathrm{~mm}$ for varying amplitudes of actuator demand signal. This amplitude and frequency is representative of a filtered high-frequency structural feedback signal at actuator input. The results included in Figure 7.22 demonstrate the changes in gain and phase for an actuator demand signal of frequency $2 \mathrm{~Hz}$ of varying amplitude as a result of the presence of a high-frequency structural-mode signal of constant amplitude.

The results shown in Figure 7.22 indicate that the affect of the structural signal on the gain is more pronounced for input demands of small amplitude. For large magnitude demand signals leading to saturation of the valve in their own right, the effect of the structural signal is minimal in terms of gain change as would be expected. In terms of the effect of the structural signal on the phase response of the actuator, the 
results demonstrate that the change in phase is more pronounced for larger input demand signals. Once saturation of the valve travel limits is reached due to the combined input signal however, the change in the phase response of the actuator as a result of the presence of the structural signal is minimal.

The overall effect of the input signal amplitude on the results shown in Figure 7.18 is small however, as the changes in gain and phase are only of the order of $0.4 \mathrm{~dB}$ and 5 degrees respectively. This, however, will be dependent on the structural signal amplitude and frequency.

Although the results presented are limited in that they have only considered a structural noise signal at a single frequency and a single phase relationship between the two input signal components, they still demonstrate some interesting points. Firstly, the addition of a significant phase lag to the actuator response as a result of the structural-mode signal demonstrates how the problem of aeroservoelasticity can result in a reduction in the ability to control the rigid-body aircraft itself. The effect of the amplitude of the structural signal on the results demonstrates however that a certain level of structural signal may be acceptable for satisfactory control of the aircraft. However, further investigation is required into the effect of multiple high-frequency signals on the performance of the actuator.

\subsubsection{Effect of structural feedback signals on software rate limiting}

In order to explain the reason behind the unexpected increase in the phase lag of the actuation system, consider the effect of a software rate limiter at actuator input ${ }^{30}$. Such a rate limiter is typically present within the digital flight control computer, its purpose being to protect the actuator itself from excessive demand signals. It is possible to include such a rate limiter in the production of the linearity boundary for the actuation system, the input amplitude at which the rate limit is exceeded being simply a function of the input frequency and the rate limit value. For example, for an input signal of the form

$$
x=A \sin \omega t
$$

The rate demand can be expressed as

$$
\dot{x}=A \omega \cos \omega t
$$

Therefore, during any one period, the maximum rate demand is $A \omega$. For a given rate limit value, $\left.\dot{x}\right|_{\max }$, the input amplitude at which rate limiting will first occur is given by

$$
\hat{A}=\frac{\left.x\right|_{\max }}{\omega}
$$

The resulting linearity boundary for a rate limit of $60 \% / \mathrm{sec}(0.234 \mathrm{~m} / \mathrm{sec}$ ram 
demand) is as shown in Figure 7.20, which includes the linearity boundary for the actuation system without rate limiting for comparison. This value of the rate limit corresponds with that used on the Jaguar FBW aircraft.

From the figure, it is clear that the linearity boundary has reduced significantly at the higher frequencies. In fact, considering the $50 \mathrm{~Hz}$ structural noise signal, it is clear that the actuator will be rate limited for this signal alone. It can be deduced that the effect of the structural feedback signal is to cause premature rate limiting of the actuator input signal.

Another point to note from the figure is that the rate limiter becomes the dominant limit for input frequencies greater than $3.5 \mathrm{~Hz}$. Since structural-modes are generally of higher frequency than this, it can be deduced that the rate limiting function has an important role in the effect of structural feedback signals on actuator performance.

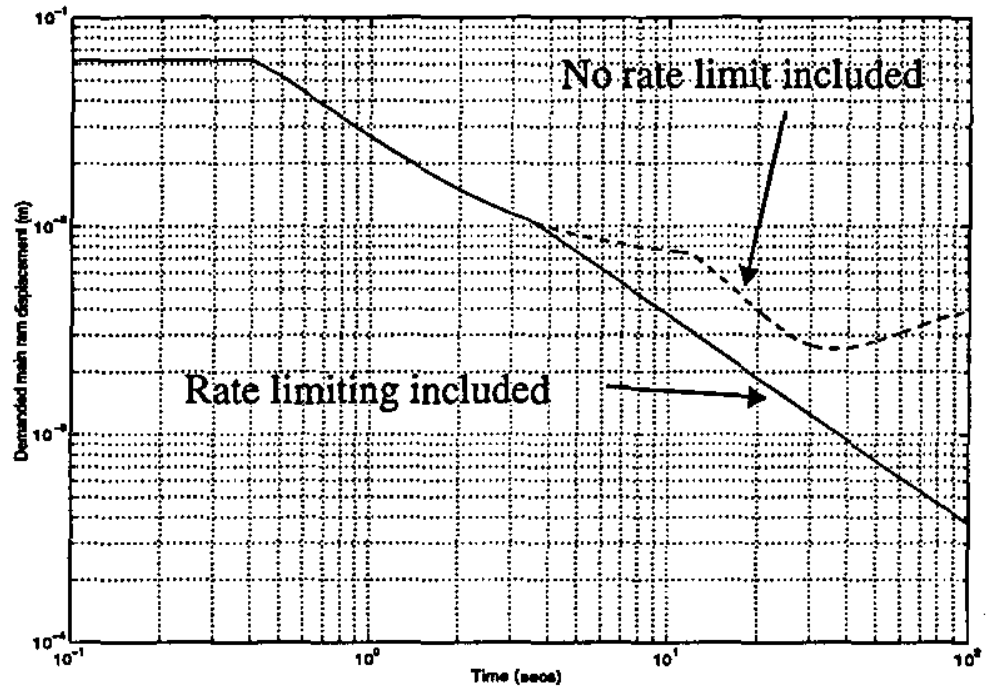

\section{Figure 7.20 - Linearity boundary for Jaguar FBW Taileron actuator model including $60 \%$ sec rate limiter}

The effect of rate limiting on actuator performance can be demonstrated by examining the input and output time responses of such a rate limiter in isolation. For example, consider a single input signal of frequency $50 \mathrm{~Hz}$ and amplitude $1.5 \mathrm{~mm}$. The results of Figure 7.20 predict that such an input signal will result in the rate limiting of the input signal to the actuator by the flight control computer software. This will result in the actual input signal to the actuator being as shown in Figure 7.21.

These results demonstrate how the presence of a structural signal of sufficient amplitude and frequency to cause rate limiting can result in a decrease in gain and increase in phase lag of the actuation system as a whole.

Earlier results demonstrated that the effect of the structural feedback signal on actuator performance was dependent on the amplitude of the structural signal as would be expected. It is now clear that once the amplitude of the structural signal is sufficient to cause the onset of rate limiting, then the performance of the actuator will decrease 
rapidly in response to a low-frequency pilot demand signal.

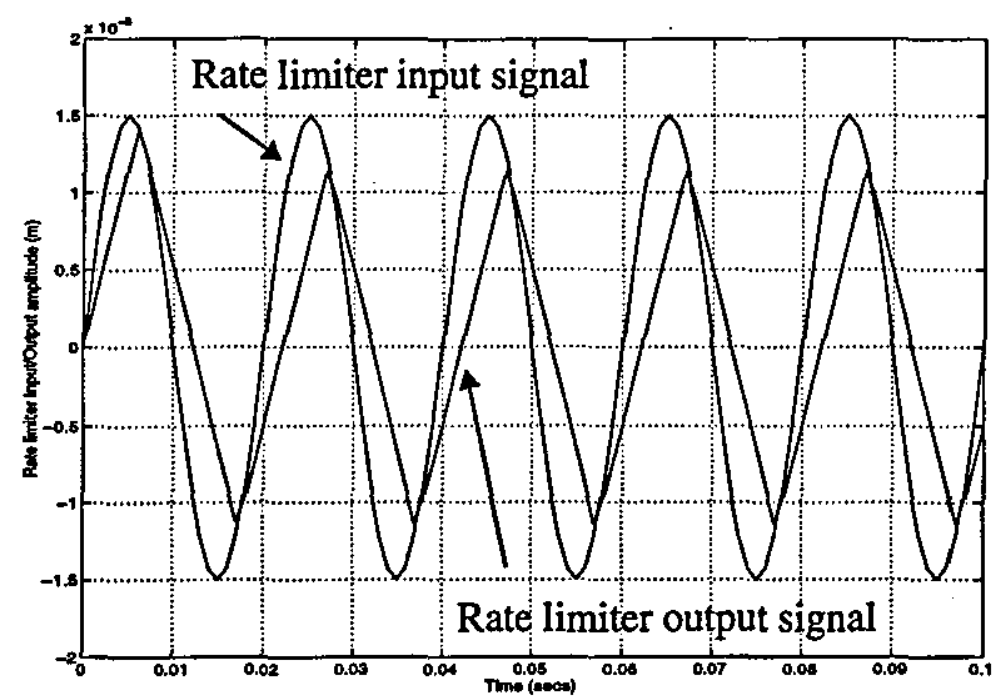

Figure 7.21 - Rate limiter input/output time response characteristics for $50 \mathrm{~Hz}$, $1.5 \mathrm{~mm}$ input signal

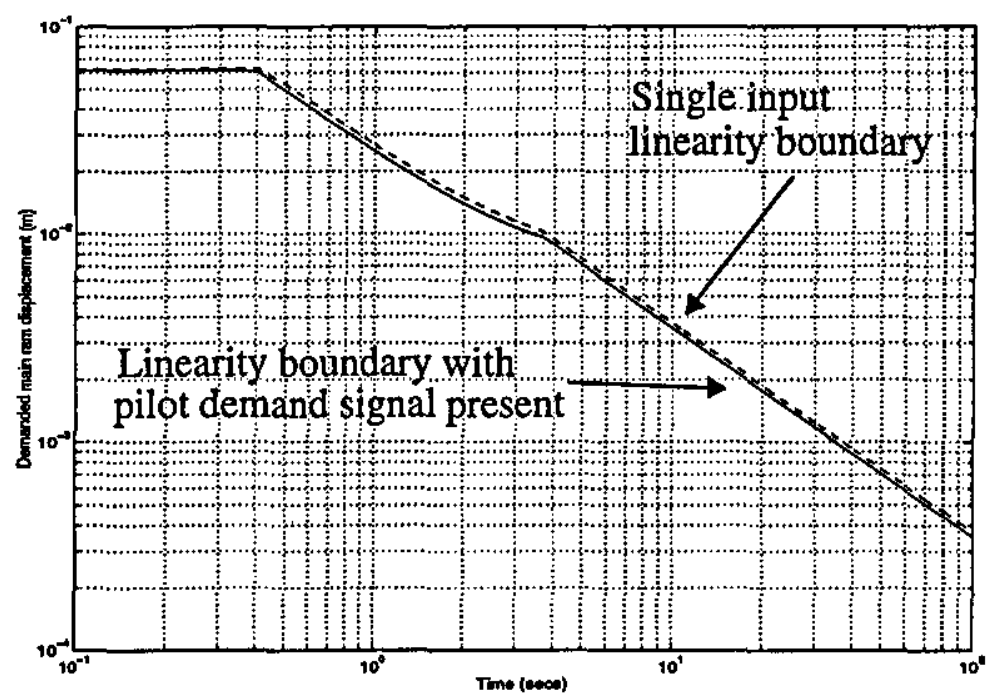

Figure 7.22 - Actuator linearity boundary in the presence of a $1.0 \mathrm{~mm} 2 \mathrm{~Hz}$ pilot demand signal.

In fact, the amplitude of structural signal at which rate limiting will occur in the presence of a low-frequency pilot demand signal can be easily predicted. Assuming the presence of a $2 \mathrm{~Hz}$ pilot demand signal resulting in a actuator ram demand of amplitude $1.0 \mathrm{~mm}$, the linearity limit for the actuator can be produced once again as shown in Figure 7.22.

The first thing to note from the above results is that the linearity boundary is changed little by the presence of such a pilot demand signal. Such a demand signal would not be expected to result in a large change in the boundary however, since it would not require a large amount of valve displacement or a large demanded ram rate to follow the input signal.

From the figure, it is possible to deduce the amplitude of the structural signal at 
which the actuator performance would begin to deteriorate as a result of the onset of input signal rate limiting. For example, for a structural signal of frequency $50 \mathrm{~Hz}$, the above results predict that the combined input signal would be rate limited once the amplitude of the structural feedback signal exceeded a ram demand of $0.7 \mathrm{~mm}$. This amplitude matches well with the results of Figure 7.18 which show the onset of the increase in the phase lag of the system at this amplitude.

To conclude, it can be seen that for a structural-mode of a particular frequency, there will exist an amplitude above which the input signal to the actuator is being rate limited by the flight control system. This has been shown to result in a decrease in the gain of the actuation system at high input frequencies. More importantly however, the presence of the structural signal can result in an increase in the phase lag of the actuation system at low frequencies which would have a detrimental effect on FCS performance.

\subsubsection{Dual input response of software rate limiter in isolation}

In order to examine the effect of structural feedback signals on the software rate limiter in more detail, consider the rate limiter in isolation once again. It is possible to produce frequency responses for the rate limiter alone in the presence of two sinusoidal input signals. As an example, consider the rate limiter subjected to a lowfrequency demand signal of frequency $3 \mathrm{~Hz}$ and amplitude $1 \mathrm{~mm}$ superimposed with a range of high-frequency signals. The resulting response of the rate limiter is as shown in Figure 7.23.

The results demonstrate the effect of a particular frequency and amplitude of structural signal on the performance of the rate limiter. Naturally, these results only apply to this particular low-frequency demand amplitude and frequency. Although these results are limited in this way, they still demonstrate a very useful point. From the figure, it can be seen that it may be possible to specify a maximum allowable level of structural noise at a particular frequency. For example, suppose that it was decided that a phase lag of up to 10 degrees at $3 \mathrm{~Hz}$ could be tolerated in terms of satisfactory rigid-body stability-margins. From the figure, it would be possible to specify a maximum boundary for the structural noise amplitude at actuator input. 

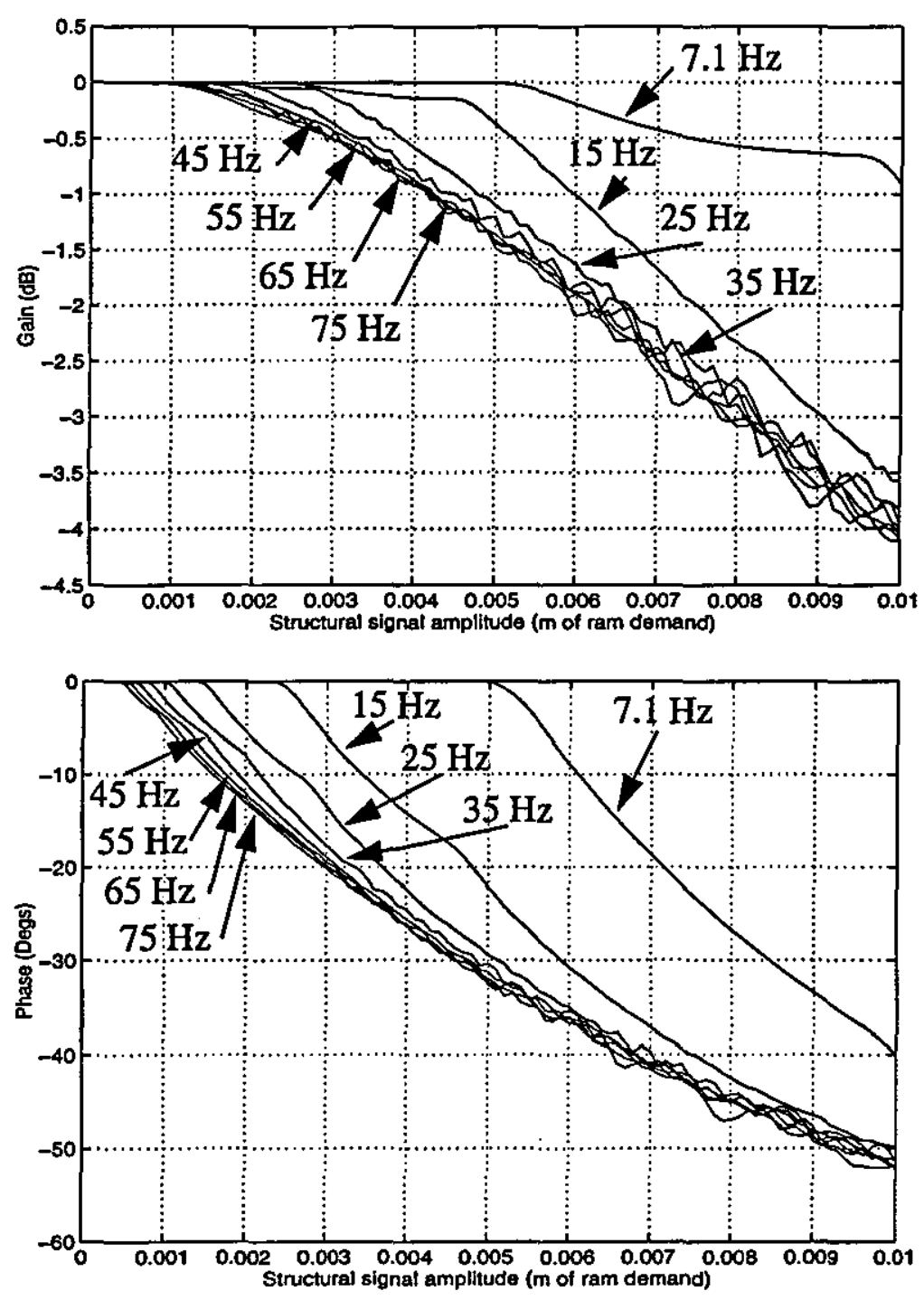

Figure 7.23 - Response of rate limiter in the presence of a $1.0 \mathrm{~mm} 3 \mathrm{~Hz}$ demand signal and structural signals of varying amplitude and frequency

\subsubsection{Effect of main-valve port profile on actuator performance in the} presence of structural feedback signals

The above discussion has centred on the effects of structural signals on actuator performance once the rate limiting function of the flight control system has been exceeded. If the structural feedback signal is not of sufficient frequency or amplitude to cause this however, the use of the linearity boundary of Figure 7.20 would seem to indicate that the actuator performance would not be effected. This is not necessarily the case however as will be shown in the following section.

Consider the case of a structural signal of frequency $7 \mathrm{~Hz}$ and amplitude $3 \mathrm{~mm}$ superimposed on a pilot demand signal of amplitude $1 \mathrm{~mm}$. Although in this case the structural signal is of larger amplitude than in the earlier case, the combined signal will not violate the linearity boundary at typical pilot demand frequencies. As a result, 
it would be expected from the earlier analysis that the performance of the actuator in response to the pilot demand signal would be unchanged at these frequencies. Figure 7.24 shows the frequency response of the actuation system in response to the pilot demand signal under such input conditions.
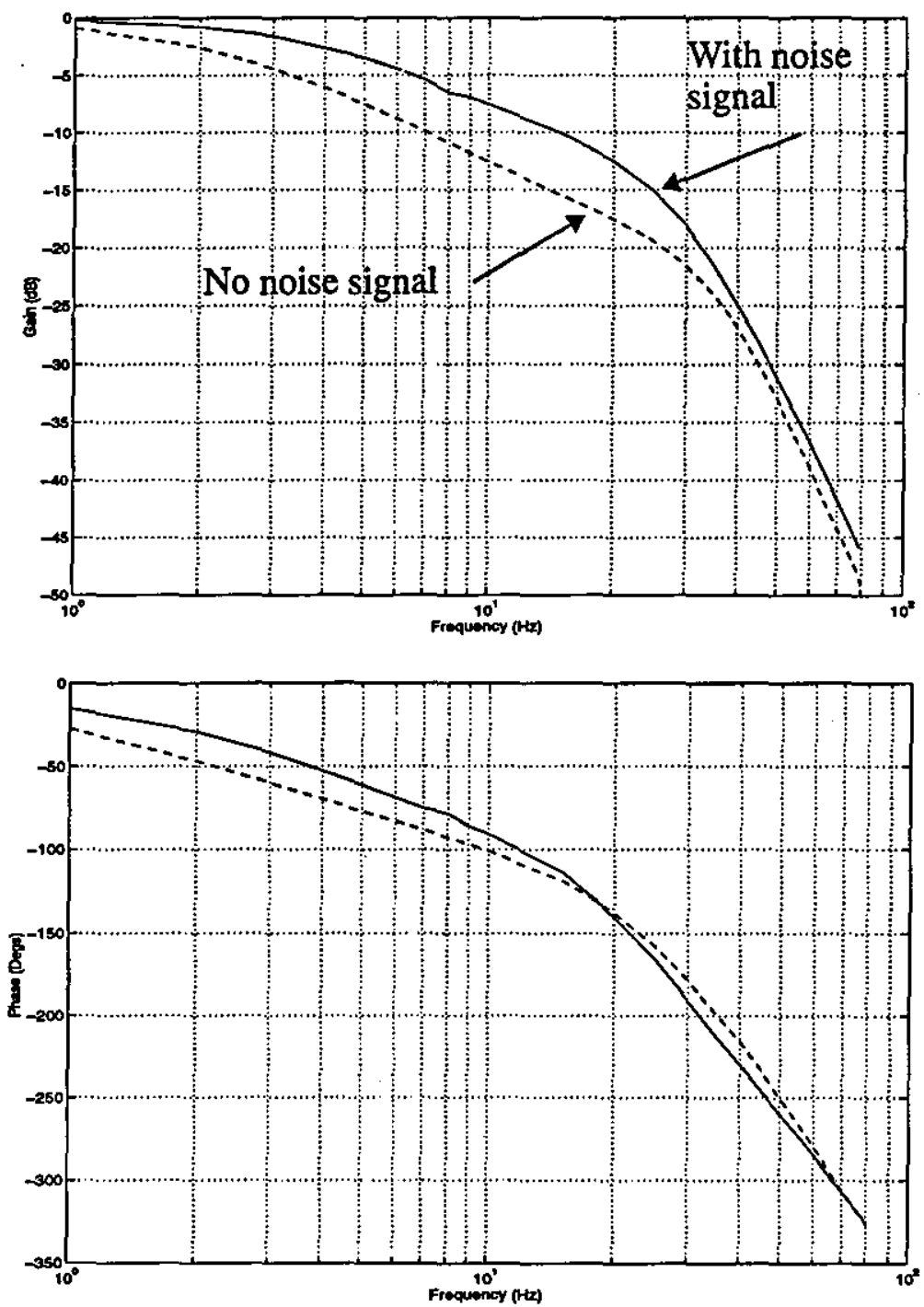

Figure 7.24 - Actuator frequency response with $7 \mathrm{~Hz}, 3 \mathrm{~mm}$ structural noise signal

From the figure, it is clear that the addition of the structural noise in this case has actually improved the performance of the actuator in response to the pilot demand signal. The effect of the structural noise signal has been to give an small increase in gain and significant decrease in phase lag of the system up to an input frequency of approximately $20 \mathrm{~Hz}$. This frequency corresponds with the onset of rate limiting of the combined input signal, resulting in the increase in phase lag and decrease in gain associated with this occurrence.

These results demonstrate that under certain input conditions, the presence of a structural-mode feedback signal could actually be beneficial to the performance of the actuator. It should be noted however that such a decrease in phase lag and increase in 
gain could also result in an unsatisfactory rigid-body response. In addition, the presence of the structural signal will result in a reduction in the effective rate limit boundary in response to the pilot demand signal.

In order to explain the cause of such a performance improvement in the presence of a structural noise signal, consider the effect of the main-valve port profile on actuator response $\mathrm{s}^{30}$. In most case, the main-valve ports are trapezoidal in shape as shown in Figure 7.25. The effect of this shaping of the main-valve ports is that the main-ram rate as a function of main-valve displacement is of the form shown in Figure 7.26. If the main-valve ports were rectangular in profile, then the relationship between main-valve displacement and main-ram rate would be as shown in Figure 7.26 .

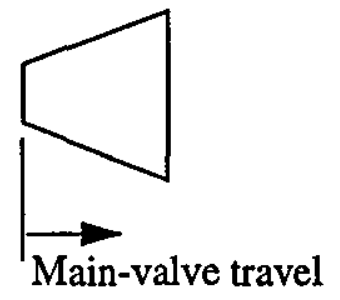

Figure 7.25 - Main-valve port profile for FBW Taileron actuator

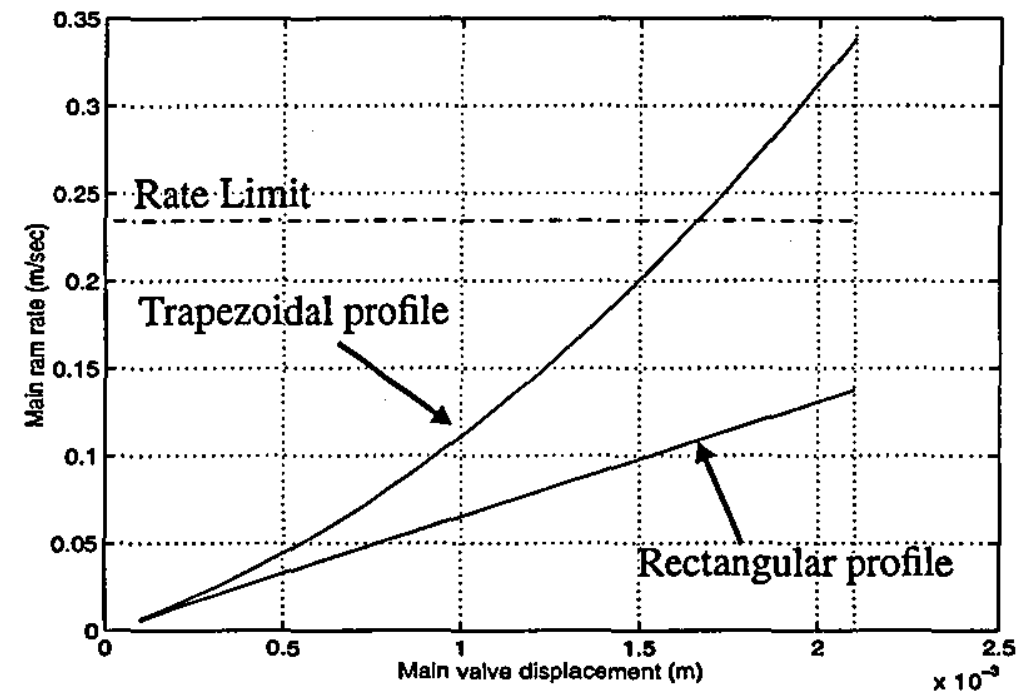

Figure 7.26 - Main-ram rate as a function of main-valve displacement

The importance of this shaping of the main-valve port is that the rate of change of main-ram rate with respect to main-valve displacement increases with main-valve displacement. This results in the increase in actuator performance in the presence of suitable structural-mode feedback signals. Consider the case of the pilot demand signal alone, which for a low-frequency input would result in only a small displacement of the main-valve, where the gradient of the function in Figure 7.26 is small. In the presence of a suitable structural-mode signal however, the main-valve displacement may be high as the actuator attempts to follow the structural feedback signal. As a result, any motion of the main-valve in response to the pilot demand 
signal can be reasonably assumed to result in a higher ram rate than for the case of the pilot demand signal alone. Such a higher ram rate would result in a decrease in the phase lag of the system in response to the pilot demand signal as has been demonstrated.
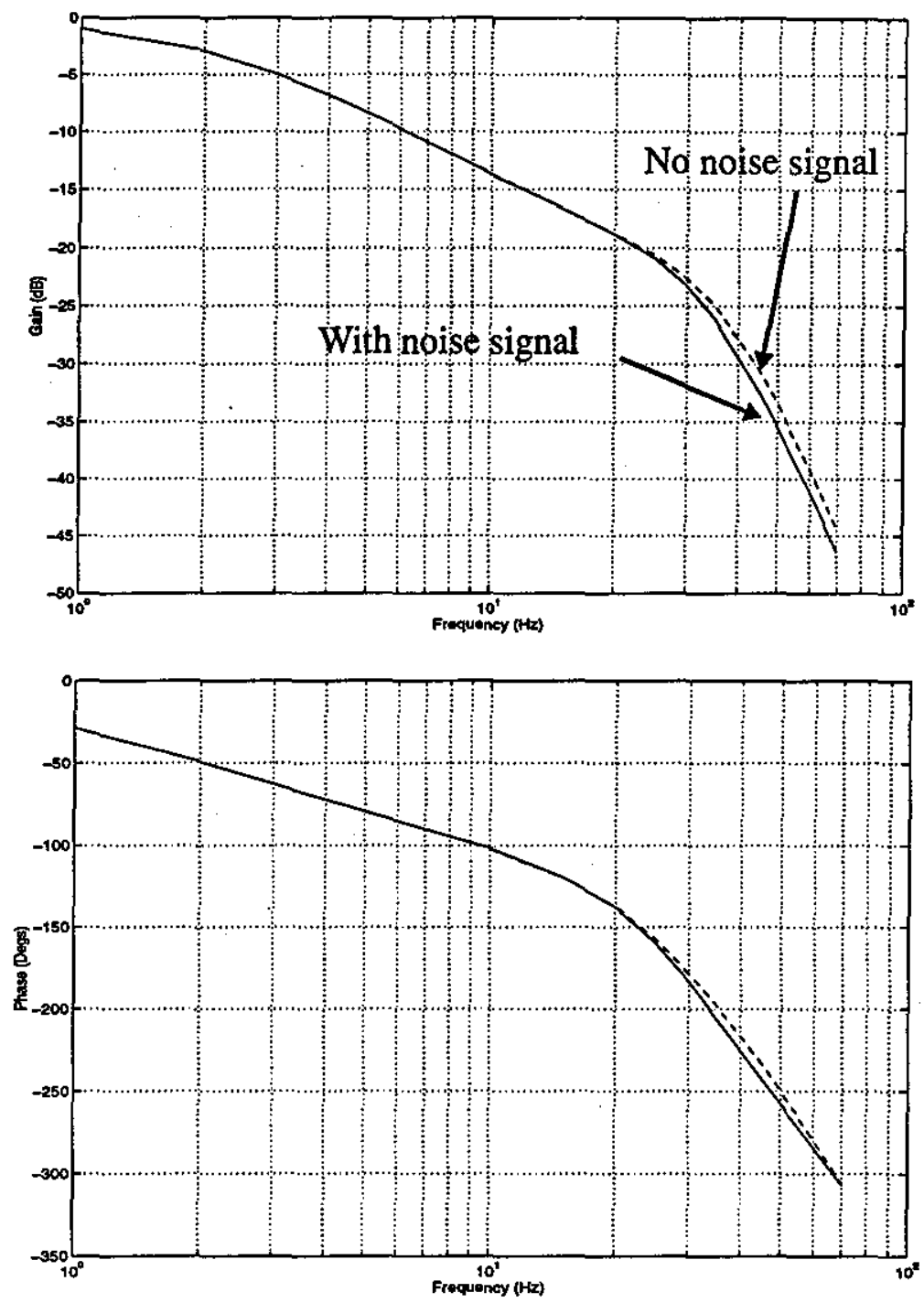

Figure 7.27 - Actuator frequency response with $7 \mathrm{~Hz}, 3 \mathrm{~mm}$ structural noise signal when main-valve ports are rectangular in profile

In order to demonstrate that it is indeed the main-valve profile that is responsible for the results of Figure 7.24, the results were repeated for an actuator whose mainvalve port was of rectangular profile. If the above reasoning were correct, then the frequency response with and without the $7 \mathrm{~Hz}$ structural signal should be identical, as is the case as can be seen in Figure 7.27. The slight differences in the two sets of the results at high frequencies is simply due to the earlier onset of rate limiting when the structural signal is present. It is clear therefore that it is indeed the shaping of the main-valve ports that is responsible for the actuator performance improvement in the presence of certain structural feedback signals.

In order for the actuator performance to be improved by the presence of the 
structural-mode signal, the above reasoning demonstrates that the structural signal must result in a significant displacement of the main-valve in comparison with the effect of the pilot demand signal alone. As a result, it would be expected that the effect of the shaping of the main-valve ports would be more prominent for small pilot demand amplitudes. In addition, the combined signal must not exceed the rate limit, as once this occurs, the actuator's ability to follow the pilots demand input has been shown to reduce rapidly.

\subsubsection{Conclusion}

Two mechanisms which result in actuator performance changes in the presence of structural feedback signals have been identified and demonstrated. Firstly, the software rate limiter which is generally adopted within the flight control system can result in a reduction in the gain and increase in the phase lag of the actuation system as a whole. This is as a result of the combined input signal exceeding the rate limit. Examination of a rate limiter in isolation has demonstrated however that a certain level of high-frequency rate limiting may be acceptable before the performance of the actuator at low-frequencies is seriously affected. This indicates that a certain level of structural feedback may be acceptable in terms of rigid-body stability-margins.

The second mechanism through which the feedback of high-frequency structural signals can affect actuator performance has been identified as the shaping of the mainvalve ports. It has been shown that this subtle effect can actually result in an increase in the gain and a decrease in the phase lag of the actuation system. This mechanism therefore opposes the effect of the rate limiter. It should be noted however that the beneficial effect of the port shaping in very reliant on the ratio of the demanded rates by both low-frequency demand and high-frequency signal. In addition, the effect of the rate limiting is more pronounced, resulting in rapid cancellation of the beneficial effect once rate limiting has occurred.

\subsection{Prediction of actuator performance changes in the presence of structural feedback signals}

\subsubsection{Introduction}

The above section has demonstrated that the presence of a structural-mode feedback signal at the input to the actuator can effect its performance in two main ways. Firstly, the combination of the structural signal with a low-frequency pilot demand signal may exceed the software rate limit. This results in a decrease in gain and an increase in the phase lag of the actuator. Opposing this increase in phase lag however is the secondary effect of the structural signal brought about by the shaping 
of the main-valve ports. In some circumstances, this shaping of the main-valve port can lead to an actual improvement in actuator performance in the presence of structural feedback signals.

Applying this knowledge of the effect of structural signals on actuator performance may now allow the prediction of actuator performance under such circumstances.

\subsubsection{Prediction of actuator performance changes in the presence of struc- tural feedback signals}

To predict the performance changes of the actuator in the presence of structural feedback signals, consider the linearity boundary of Figure 7.20, and the component boundaries from which it is generated as shown in Figure 7.28 below.

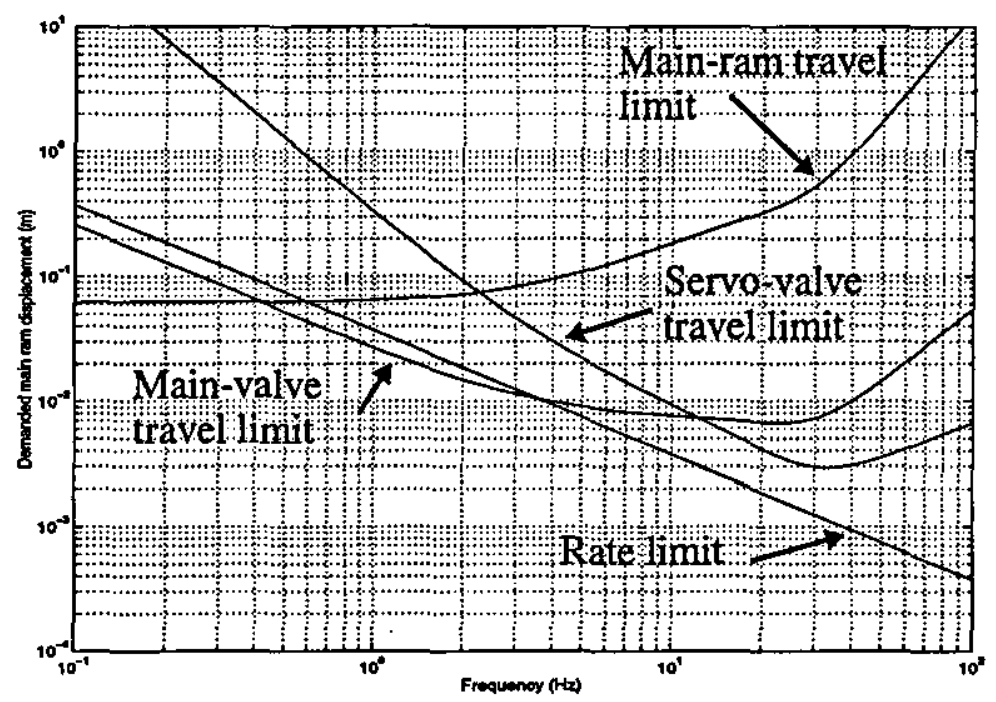

Figure 7.28 - Component linearity boundaries for FBW taileron actuator

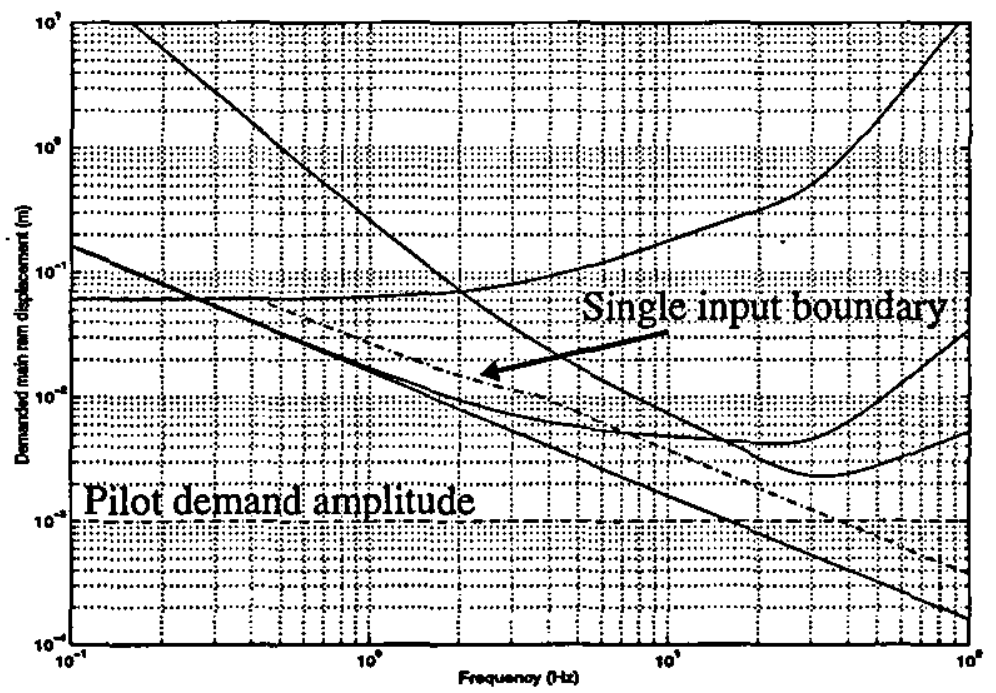

Figure 7.29 - Actuator linearity boundary in the presence of a $7 \mathrm{~Hz}, 3 \mathrm{~mm}$ structural feedback signal

Of particular interest are the two boundaries representing the main-valve travel limit and the rate limit. The rate limit is the lower boundary in this case for input 
signals of frequency greater than $3.5 \mathrm{~Hz}$. Consider the previous example of a structural signal of frequency $7 \mathrm{~Hz}$ and amplitude $3 \mathrm{~mm}$. From Figure 7.28, such a signal will result in approximately $40 \%$ of the maximum main-valve displacement, and $60 \%$ of the rate limit. As a comparison the effect of a $1 \mathrm{~Hz}$ pilot demand signal of amplitude 1 $\mathrm{mm}$ is approximately $4 \%$ of maximum main-valve displacement and $3 \%$ of the rate limit.

As a result, it would be expected from the earlier discussion that the actuator performance would be improved under these input conditions, since the rate limit is not being exceeded, and the structural signal would result in significant main-valve displacement in comparison with the pilot demand signal. Production of the linearity boundary for the actuator in the presence of the structural signal results in the boundary as shown in Figure 7.29. From the figure, it is possible to predict that the actuator performance will be improved up to an input frequency of approximately 17 $\mathrm{Hz}$, from where the combined input signal will be rate limited. This compares well with the frequency response results as shown in Figure 7.24.
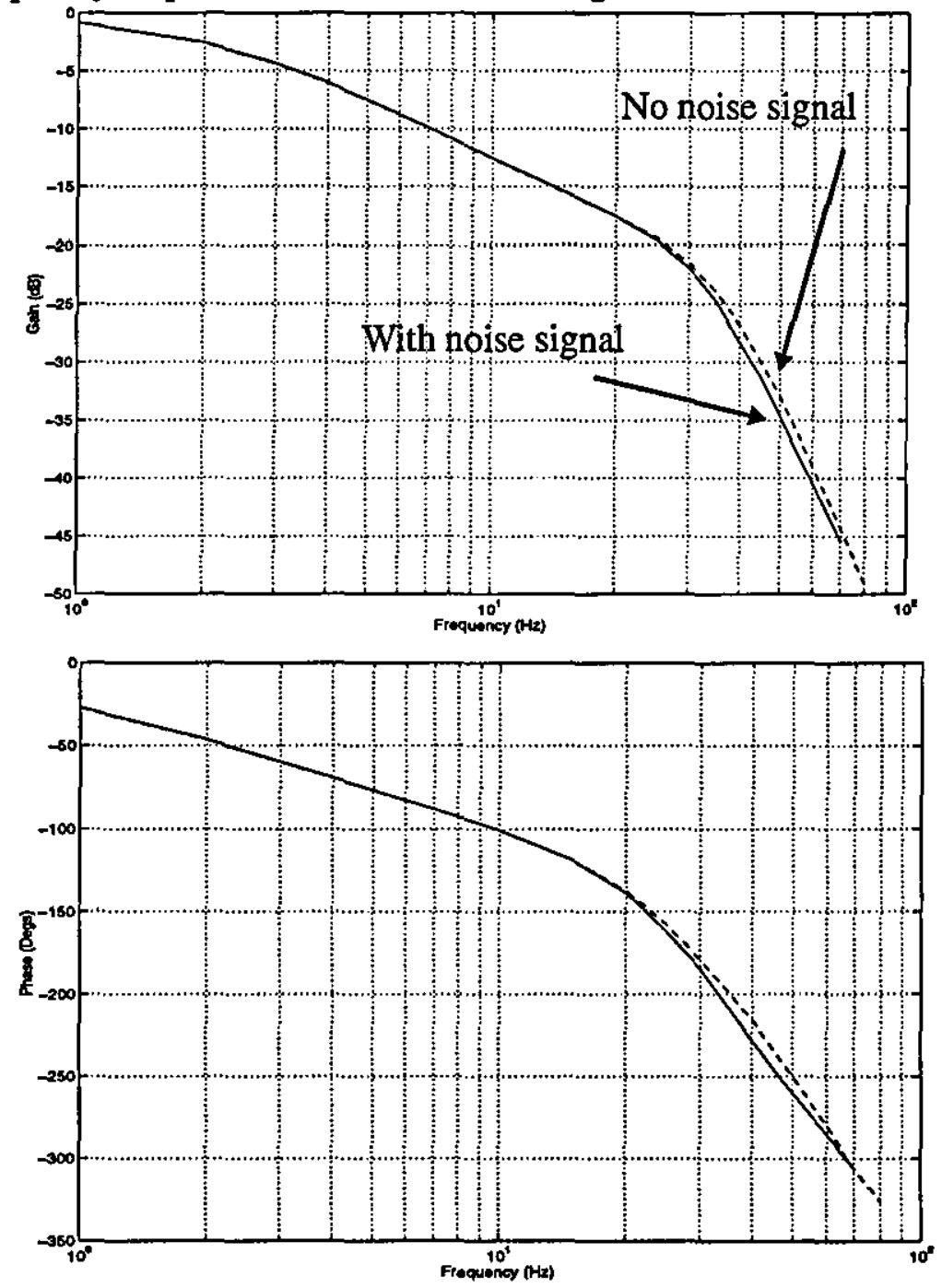

Figure 7.30 - Actuator frequency response with $50 \mathrm{~Hz}, 0.5 \mathrm{~mm}$ structural noise signal 
For other combinations of structural signal and pilot demand, it is possible to judge what effect the structural signal will have on actuator performance based on the production of linearity boundaries such as those shown in Figure 7.29. One point to note is that in order for the rate limit not to be violated, the amplitude of the structural signal must decrease with increasing frequency. However, consideration of the boundary for main-valve travel limit in Figure 7.28 shows that the amplitude of the motion of the main-valve in response to the structural signal will decrease rapidly as a result. For example a structural signal of frequency $50 \mathrm{~Hz}$ and amplitude $0.5 \mathrm{~mm}$ will only result in a main-valve motion of amplitude $3 \%$ of the maximum value. As a result, the effect of such a signal on the performance of the actuator to the $1 \mathrm{~mm}$ pilot demand signal will be negligible in terms of low-frequency response. One effect that such a structural signal will have however, is that it will encourage the onset of rate limiting; the effective rate limit being reduced to $33 \%$ of its original value. The frequency response of the actuator in such conditions is shown in Figure 7.30.

The results of Figure 7.30 confirm the predictions, with the two responses being identical up to approximately $20 \mathrm{~Hz}$, where the presence of the structural signal results in the input signal being rate limited for a lower pilot demand frequency than for the single input case.

It has been shown that the change in actuator performance is related to the amplitude of main-valve displacement in response to the structural signal. To demonstrate this further, reconsider the linearity boundaries of Figure 7.28. For any given structural frequency, it is possible from Figure 7.28, to specify a structural signal amplitude that results in a particular percentage of the maximum main-valve displacement. For example, for a structural frequency of $20 \mathrm{~Hz}$, a signal of amplitude $1 \mathrm{~mm}$ would result in a main-valve deflection of $25 \%$ the maximum value. Using this approach, it is possible to select a range of structural signal frequencies and amplitudes that all result in the same main-valve displacement, and hence will result in the same change in actuator performance up to the onset of rate limiting. This process has been completed for a range of structural signals resulting in a 20\% mainvalve displacement, with the resulting frequency response for the actuator shown in Figure 7.31.

Figure 7.31 demonstrates that it is indeed the amplitude of displacement of the main-valve in response to the structural signal that dictates the changes in actuator performance up to the onset of rate limiting. In the above cases, the rate limiting of the input signal was disabled, resulting in the identical responses for high input frequencies. If the rate limiting was included in the analysis, the frequency responses would alter depending on the input frequency at which the combined signal would exceed the rate limit. In the case of the $50 \mathrm{~Hz}$ structural signal for example, the rate 
limit would be exceeded for this signal alone.
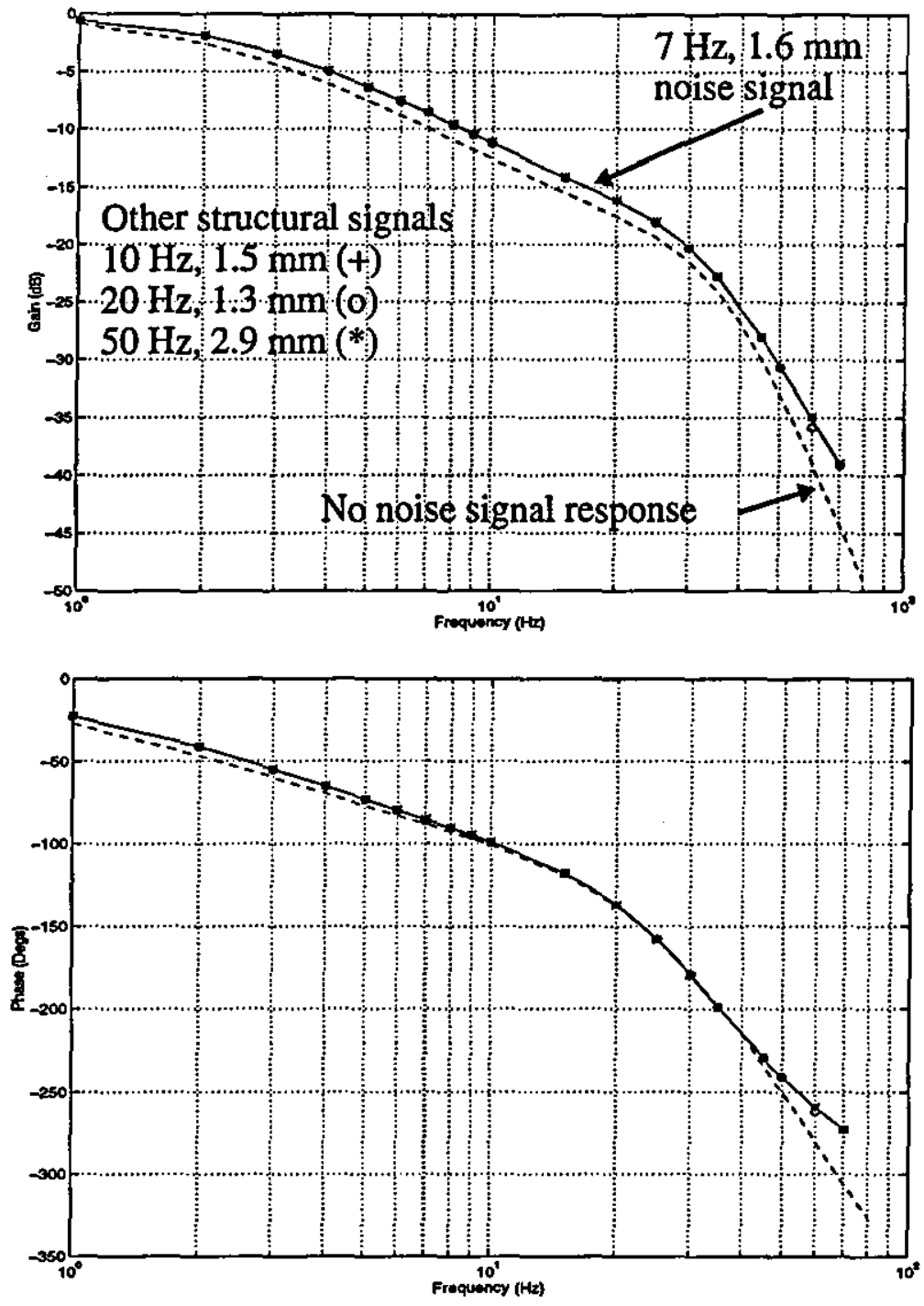

Figure 7.31 - Actuator frequency response in the presence of structural signals that result in a $20 \%$ main-valve displacement

The advantage of this approach is that it allows the performance of the actuator in the presence of a structural signal to be predicted from the frequency response of the actuator in the presence of a comparable (i.e. giving the same main-valve displacement amplitude) structural signal, and the frequency response of the rate limiter to the combined input signal. As an example, consider the case of a structural signal of frequency $7 \mathrm{~Hz}$ and amplitude $3 \mathrm{~mm}$ superimposed on a pilot demand signal of amplitude $1 \mathrm{~mm}$ as in Figure 7.24. Using frequency response results for a structural signal giving comparable main-valve deflection and the frequency response of the rate limiter in isolation, the results are as shown in Figure 7.32.

The results demonstrate that the performance of the actuator in response to a pilot demand signal in the presence of an additional structural feedback signal can be predicted $^{30}$. This prediction requires response data for the actuator subjected to the same pilot demand signal and a comparable structural feedback signal, but such 
results could be simply produced for a set of percentage main-valve displacements. In addition, the performance of the rate limiting function in response to the actual combined input signal is required. It should be noted however, that additional limits exist within the actuator that have not been taken into consideration here. The effect of the travel limit for the servo-valve should be considered in particular, although the linearity boundaries of Figure 7.28 indicate that this limit should not be encountered when the rate-limiting function is operational.
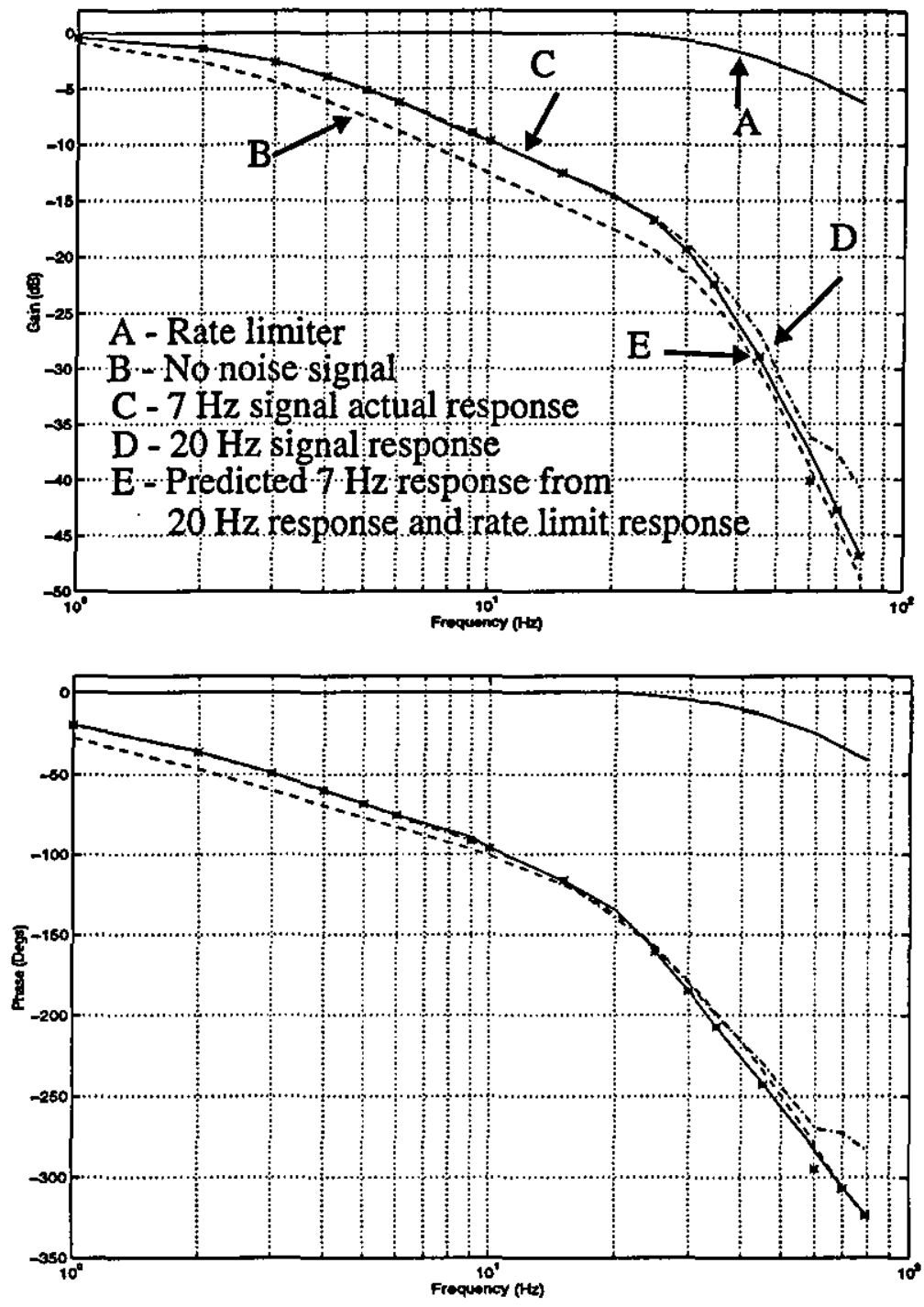

Figure 7.32 - Predicted and actual actuator frequency response in the presence of a $7 \mathrm{~Hz}, 3 \mathrm{~mm}$ structural feedback signal

\subsubsection{Conclusion}

The highly nonlinear nature of the actuation system has been shown to result in performance changes in the presence of structural feedback signals. Such performance changes have been shown to be predictable from a consideration of the two mechanisms involved. 


\subsection{Comparison of actuator model with experimental results in the presence of structural feedback signals}

\subsubsection{Introduction}

In order to increase confidence in the modelling of the actuator, experimental verification of some of the previous results was carried out. The test rig as shown in Figure 7.8 was modified to include a signal generator as a source of the required structural-mode feedback signal. A schematic diagram of the resulting test rig is included as Figure 7.33.

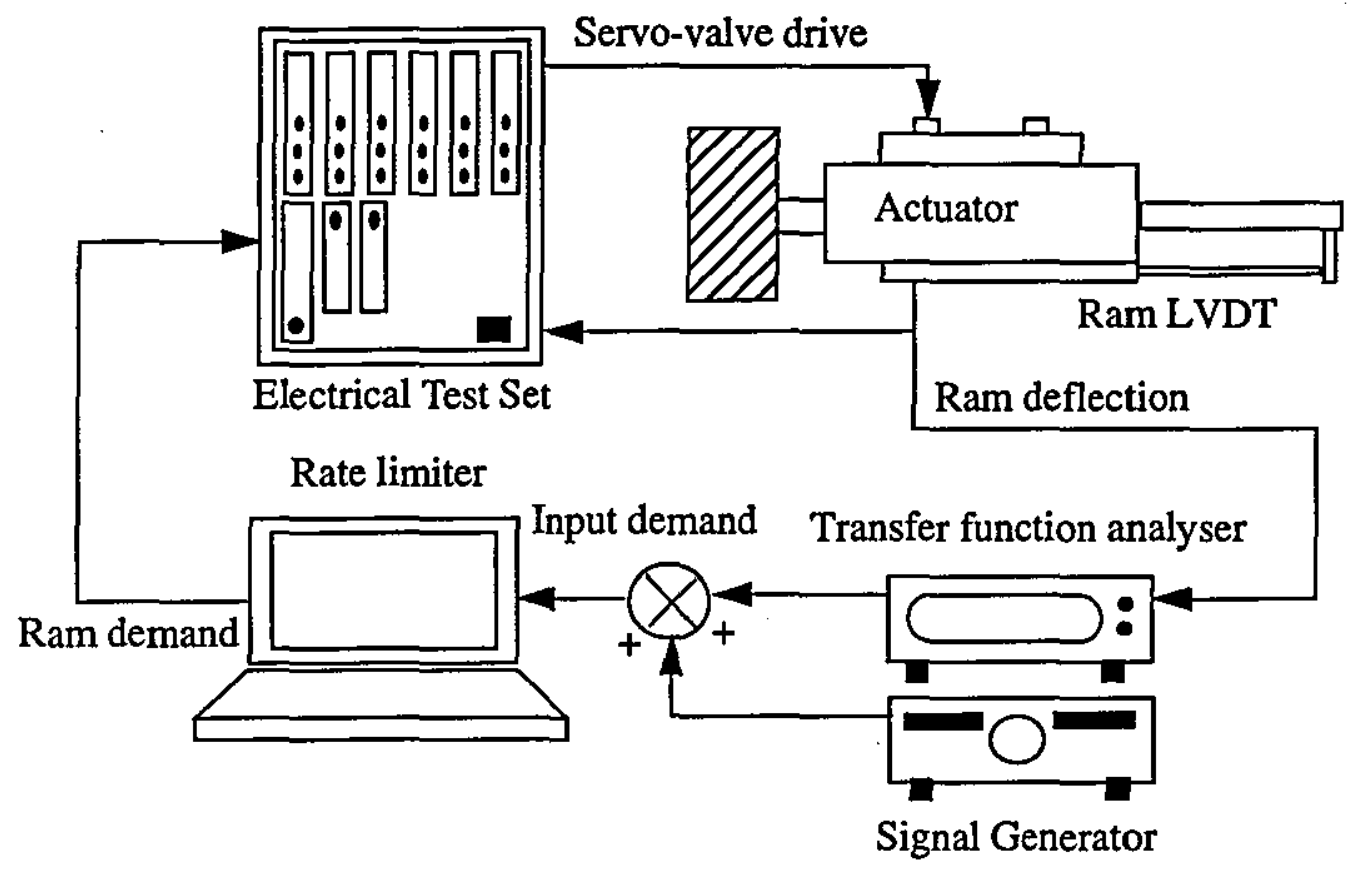

Figure 7.33 - Schematic diagram of test rig for dual input response tests

The testing procedure was identical to that described in section 7.3, except that the required structural feedback signal was generated by means of a signal generator. This signal was then summed with the demand signal from the transfer function analyser to form the total actuator demand signal. This summation of the two signals was completed within the computer, which also performed the rate limiting and data logging as before.

\subsubsection{Actuator performance changes in the presence of a $50 \mathrm{~Hz}$ structural feedback signal}

To demonstrate the effects of a high-frequency structural feedback signal on actuator performance, the frequency response of the actuator was obtained in the presence of a $50 \mathrm{~Hz}$ noise signal. As a comparison with the modelled results of Figure 7.17, the pilot demand amplitude was $1.0 \mathrm{~mm}$, and the amplitude of the $50 \mathrm{~Hz}$ noise signal was $1.5 \mathrm{~mm}$. The resulting frequency response is shown in Figure 7.34. 

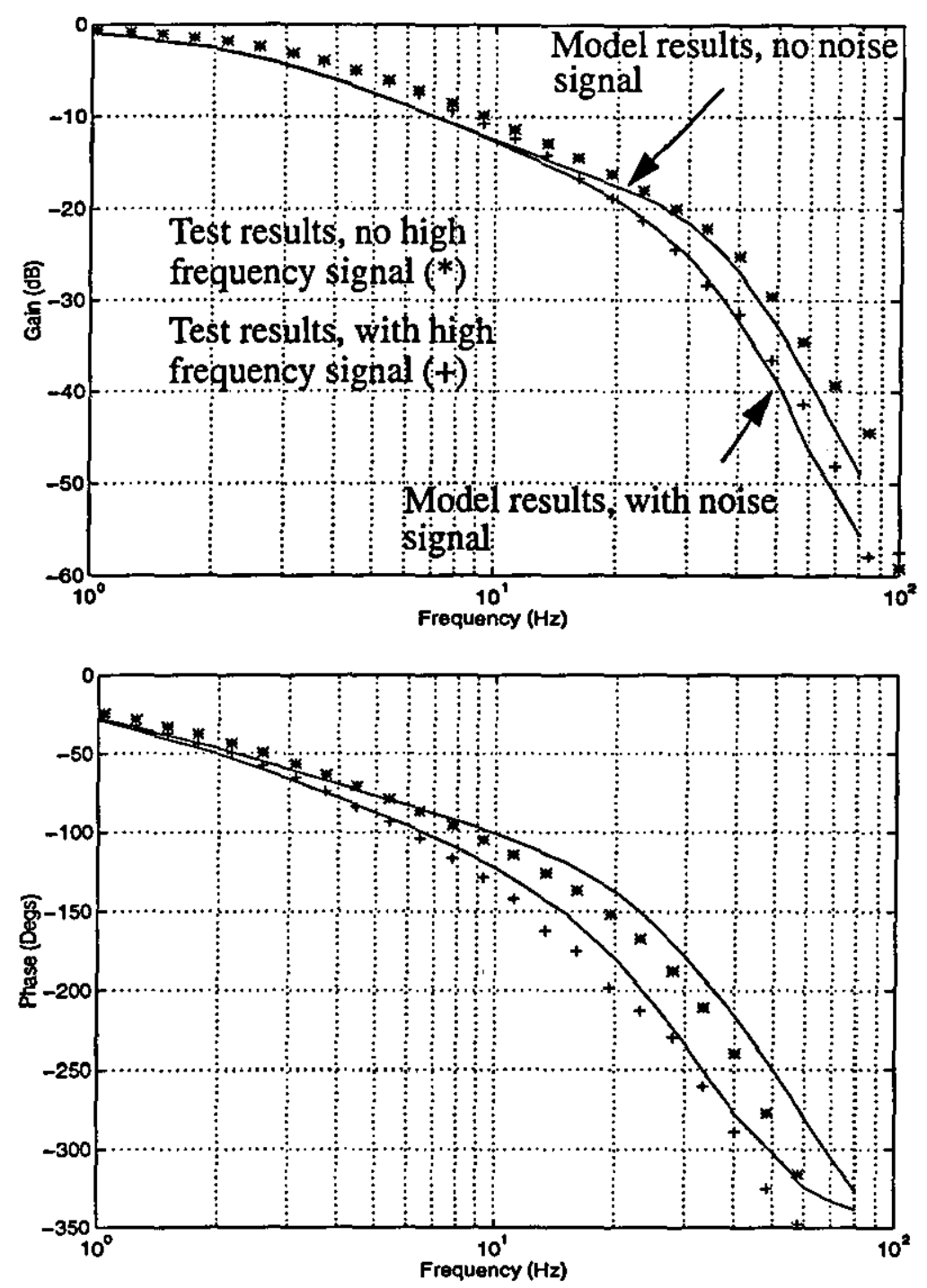

Figure 7.34 - Frequency response for Jaguar FBW taileron actuator with and without a $50 \mathrm{~Hz}, 1.5 \mathrm{~mm}$ noise signal

The above results confirm that for such a noise signal, the phase lag of the actuator is increased due to the rate limiting of the combined input signal. A good match between the experimental and simulation results is also demonstrated, leading to further confidence in the modelling of the actuation system.

These results are limited in that they consider only one noise signal amplitude. To demonstrate how the amplitude of the $50 \mathrm{~Hz}$ signal effects the response of the actuator, frequency response tests were carried out for a range of structural feedback amplitudes for comparison with the results from simulation as shown in Figure 7.18. The corresponding results are included as Figure 7.35. 

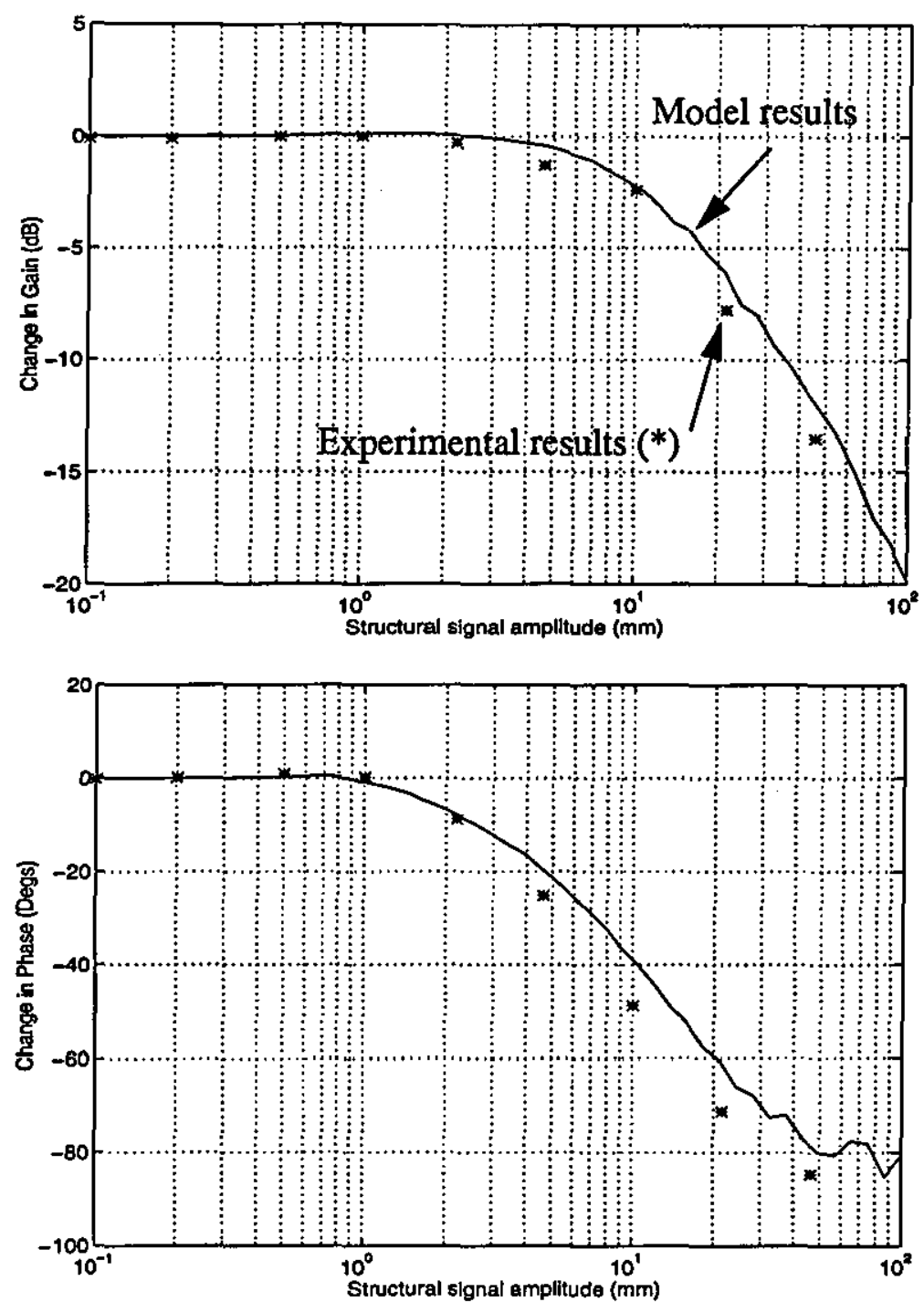

Figure 7.35 - Actuator gain and phase response changes for a $2 \mathrm{~Hz}$ demand signal and $50 \mathrm{~Hz}$ structural signal of varying amplitude

The modelled frequency response changes can be seen to represent well those changes experienced on the actual actuator. As expected from the modelled results, the phase lag and reduction in gain of the system increases rapidly once the rate limit has been exceeded. In this case, such a violation of the rate limit occurs for a structural amplitude of $0.7 \mathrm{~mm}$ which corresponds well with the actual results.

To demonstrate how the amplitude of the pilot demand signal influences the results, the frequency response of the actuator was produced with varying input demand amplitudes in the presence of a structural signal. The results are included as Figure 7.36. 

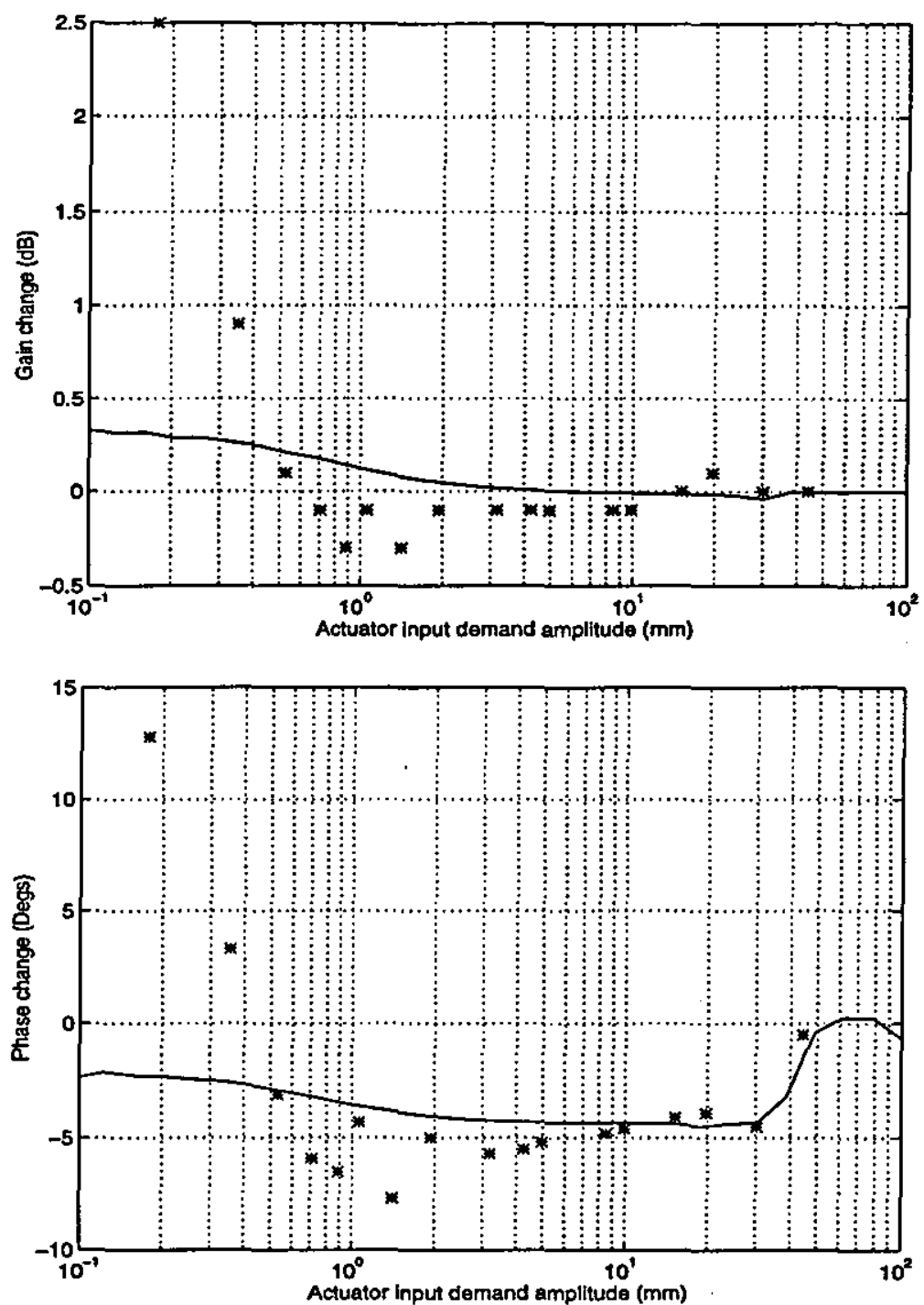

Figure 7.36 - Actuator gain and phase response changes for $\mathrm{a} 2 \mathrm{~Hz}$ demand signal of varying amplitude and $50 \mathrm{~Hz}$ structural signal

Although the previous comparisons between experimental and simulation results have shown a good match, the above results demonstrate a significant difference for very small amplitudes. The most likely cause of this difference in the results is due to un-modelled nonlinearities within the actuator itself. The presence of friction and deadzones in particular would result in such changes in performance in the presence of a high-frequency signal. In such a case, the high-frequency signal results in the actuator breaking-free of these effects. In fact, it is common practice to incorporate a high-frequency dither signal at the input to the actuator to achieve exactly this effect. In the case of the simulated results, these nonlinearities were not represented which resulted in the small change in actuator performance in the presence of the structural feedback signal. 


\subsubsection{Actuator performance changes in the presence of a $7 \mathrm{~Hz}$ structural feedback signal}

In order to demonstrate the effect of the shaping of the main-valve ports, experimental frequency response results were obtained for a pilot demand signal of amplitude $1.0 \mathrm{~mm}$ in the presence of a structural signal of frequency $7 \mathrm{~Hz}$ and amplitude $3.0 \mathrm{~mm}$. For these input conditions, the software rate limiter will not be exceeded for up to an input frequency of $16 \mathrm{~Hz}$. These results are compared with the simulation results in Figure 7.37.
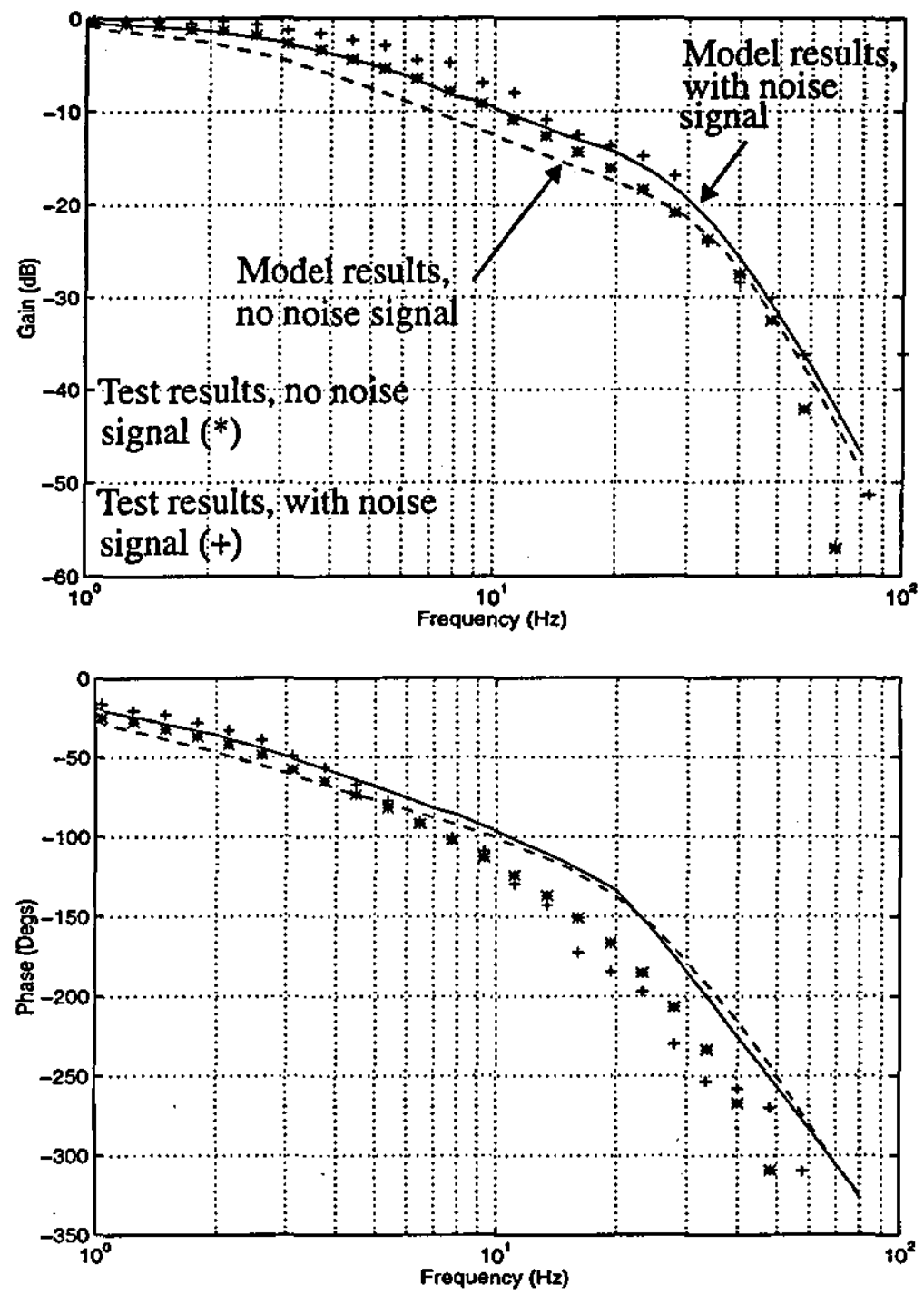

Figure 7.37 - Actuator frequency response with $7 \mathrm{~Hz}, 3 \mathrm{~mm}$ structural noise signal

The results confirm an increase in the performance of the actuator due to the presence of the structural signal. These effects, in terms of an increase in gain and decrease in phase lag, are particularly evident for demand frequencies of up to $5 \mathrm{~Hz}$. 


\subsubsection{Conclusions}

Although limited in scope to the effects of only particular structural feedback signals, the experimental results confirm the findings of the model analysis. In particular, the experimental results demonstrate that a certain level of structural noise may be acceptable for ensuring satisfactory rigid-body control.

\subsection{Effect Of subharmonic generation on aircraft response}

\subsubsection{Introduction}

One aspect of the nonlinear nature of the actuation system which has not yet been addressed is that of subharmonic generation on the aircraft response ${ }^{29}$. It is well documented that certain nonlinear elements when subjected to two input signals will result in an output signal with component frequencies below either of the two input signal frequencies ${ }^{27,70}$. If this is the case with aircraft actuation systems, then it is clear that problems involving the control of the aircraft could result. If for example, a structural-mode signal interacted with a flight control system demand signal to produce a control surface motion at a low-frequency, then undesirable aircraft response could be induced.

\subsubsection{Use of the dual input describing function to predict subharmonic generation}

The use of the single input describing function (SIDF) and dual input describing function (DIDF) is well documented in the analysis of nonlinear systems. In particular, the SIDF and DIDF methods have been successful in the analysis of the stability of nonlinear systems ${ }^{27}$ and in the prediction of limit cycling ${ }^{28}$. Methods also exist for the calculation of the closed-loop frequency response of nonlinear systems ${ }^{71}$. The use of these techniques is limited however to simple systems of a low-pass nature, where the component signals generated by the nonlinearity can be neglected. In the case of the model of this actuation system however, there are multiple nonlinearities as can be seen from the block diagram of Figure 7.14. As a result, the input signal to a particular nonlinearity may well have many component sinusoids. It is still possible however to use the DIDF to predict the generation of component frequencies for the output signal for the nonlinear actuation system model.

According to the theory relating to the dual-input describing function ${ }^{27}$, the autocorrelation function for the output for a general nonlinearity $n(x, y)$ subjected to two sinusoidal inputs can be expressed as 


$$
R_{0}(\tau)=\sum_{s=0 k}^{\infty} \sum_{k=0}^{\infty} \alpha_{s k}^{2} \varepsilon_{s} \varepsilon_{k} \cos s \omega_{a} \tau \cos k \omega_{b} \tau
$$

where $\varepsilon_{n}$ is the Neumann factor; $\varepsilon_{n}=1$ for $n=0$, and $\varepsilon_{n}=2$ for $n=1,2, \ldots$

For an input of the form $\mathrm{x}+\mathrm{y}$ where,

$$
\begin{gathered}
x(t)=a \operatorname{acos} \omega_{a} t \\
y(t)=b \cos \left(\omega_{b} t+\phi\right)
\end{gathered}
$$

the coefficient $\alpha_{\mathrm{sk}}$ can be expressed as

$$
\alpha_{s k}=\frac{1}{2 \pi} \int_{-\infty}^{\infty} N(j \omega) j^{s+k} J_{s}(a \omega) J_{k}(b \omega) d \omega
$$

where $J_{n}$ is the Bessel function of order $n$, and $N(j \omega)$ is bilateral Laplace transform of the nonlinear characteristics ${ }^{27}$.

The importance of the derivation in this case is that it allows prediction of the component frequencies of the output. In the case of the actuator model, the multitude of nonlinearities make it difficult to predict the amplitude of the output signal components, but it can be seen from equation (7.16) that the output signal will contain frequencies dictated by the expression $\cos s \omega_{\mathrm{a}} \cos \mathrm{k} \omega_{\mathrm{b}}$ for all integer combinations of $\mathrm{s}$ and $\mathrm{k}$ between zero and infinity.

Simple trigonometrical identities thus reveal that the output signal will contain components of frequency $\left(s \omega_{a} \pm k \omega_{b}\right)$.

It is possible therefore to predict the component frequencies in the output from a nonlinear system such as the actuation system model used here. One point to note however, is that the coefficient $\alpha_{\text {sk }}$, which governs the amplitude of the component signals reduces to zero for saturation type nonlinearities when $s+k$ is even ${ }^{27}$. Thus in this case the component frequencies can be predicted as being $\left(s \omega_{a} \pm k \omega_{b}\right)$ for all integer $s$ and $k$ between zero and infinity provided that $s+k$ is odd.

As an example, consider the nonlinear actuation model subjected to two sinusoidal inputs of frequency $3.05 \mathrm{~Hz}$ and $7.1 \mathrm{~Hz}$, the lower frequency being representative of a flight control system demand signal, and the upper frequency being representative of a low-frequency structural-mode such as the first wing bending mode.

From the above analysis, some of the lower frequency output signal components can be predicted as shown in Table 7.1. 


\begin{tabular}{c|c|c|c}
\hline $\mathrm{s}$ & $\mathrm{k}$ & $\begin{array}{c}\text { Frequency }(\mathrm{Hz}) \\
\mathrm{s+k}\end{array}$ & $\begin{array}{c}\text { Frequency }(\mathrm{Hz}) \\
\mathrm{s}-\mathrm{k}\end{array}$ \\
\hline 1 & 0 & 3.05 & 3.05 \\
\hline 0 & 1 & 7.1 & 7.1 \\
\hline 2 & 1 & 13.2 & 1.0 \\
\hline 1 & 2 & 17.25 & 11.15 \\
\hline 4 & 1 & 19.3 & 5.1 \\
\hline 1 & 4 & 31.45 & 25.35 \\
\hline
\end{tabular}

Table 7.1 - Example actuator output component frequencies

From the table it is clear that the nonlinear operation of the actuator will result in the production of an infinite number of output component signals at frequencies both above and below the input frequencies.

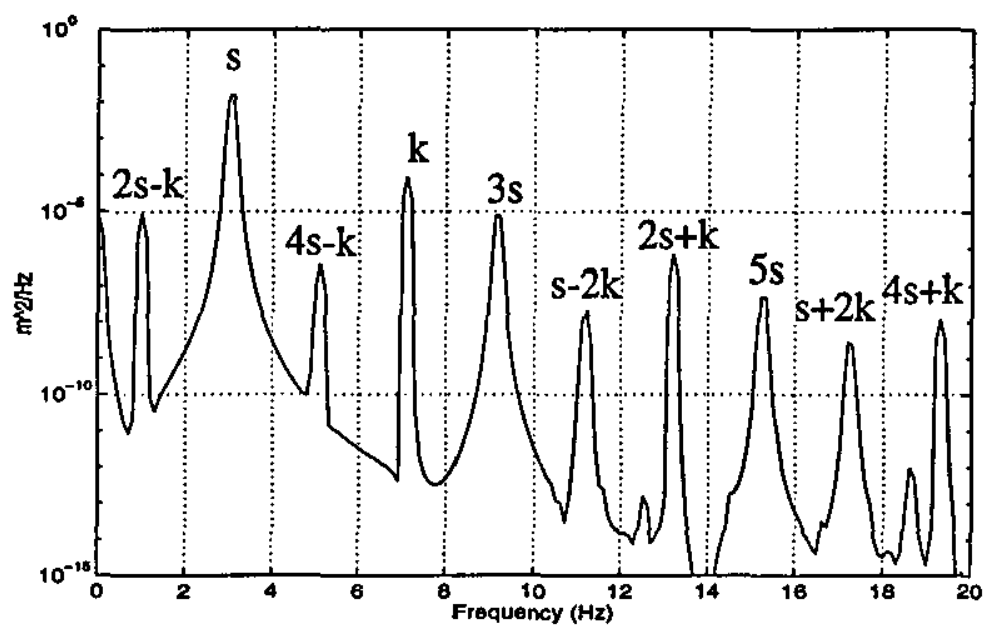

Figure 7.38 - Nonlinear model example output power spectra

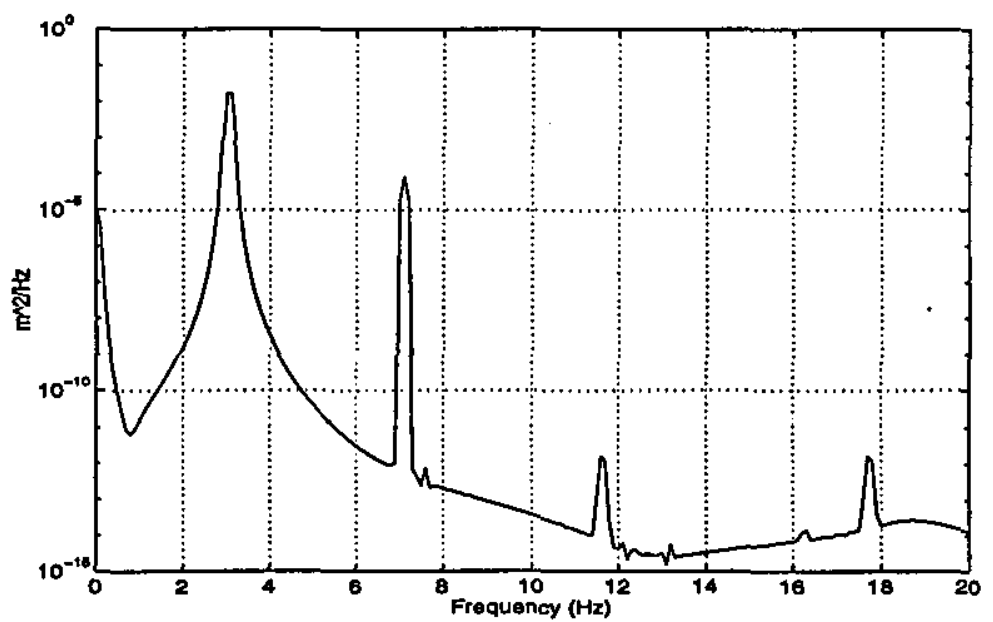

Figure 7.39 - Linearised model example output power spectra 
Applying such input signals to the actuator model, with demand amplitudes of 5.0 $\mathrm{mm}$ at $3.05 \mathrm{~Hz}$ and $0.5 \mathrm{~mm}$ at $7.1 \mathrm{~Hz}$, results in the power spectra of the output signal as shown in Figure 7.38, with some of the intermodulation components marked. As a comparison, the spectra of the output for a linearised model is given in Figure 7.39. Considering those component signals of high frequency the potential for excitation of the aircraft structural-modes is obvious.

It is clear from this example that the highly nonlinear nature of the actuation system can result in subharmonic frequencies being generated as a result of the interaction between the flight control system demand signal and any structural-mode signal that is present at actuator input.

\subsubsection{Effect of subharmonic components on aircraft response}

As an example of how this might effect the aircraft system as a whole, consider the case where the model of the nonlinear actuator is incorporated into a full system model of the aircraft. In this case, the aircraft model is a rigid-body representation with three control surface modes. The structural-mode feedback is represented by a suitable signal injected as sensor noise. This approach to examining the effect of a structural-mode feedback signal will be examined in Chapter 8 .

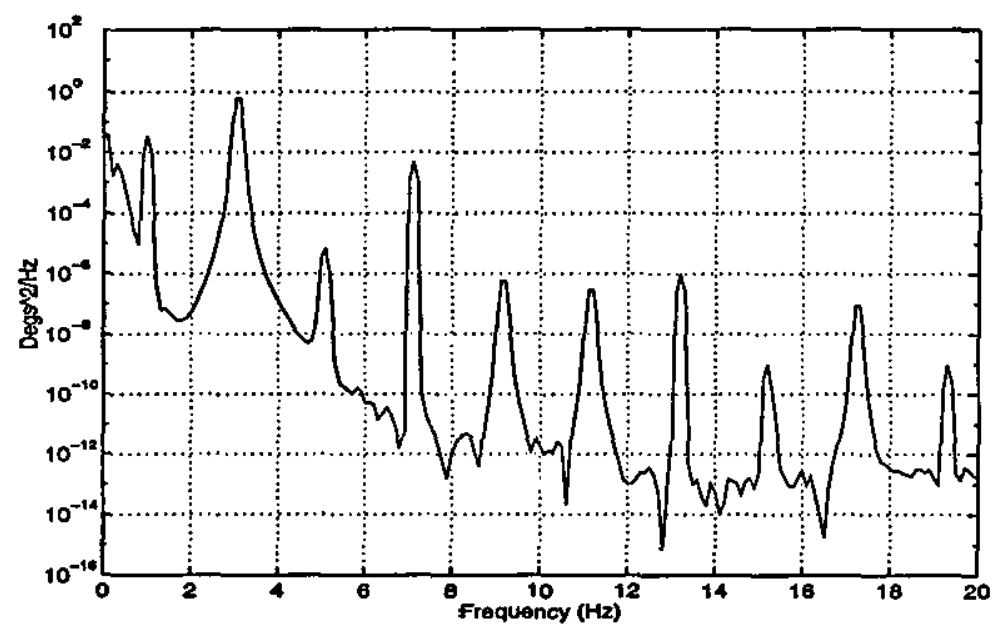

Figure 7.40 - Incidence angle power spectra

Applying equivalent inputs to the full system model results in a power spectra for the aircraft incidence as shown in Figure 7.40, where it is clear that the creation of a signal component in the actuator output at $1 \mathrm{~Hz}$ has resulted in aircraft response at this frequency. This can be seen in the incidence time response plot of Figure 7.41.

Comparing the power spectra plots of Figures 7.38 and 7.40, demonstrates that the low-pass nature of the rigid-body aircraft attenuates significantly the higher-frequency components, whilst the $1 \mathrm{~Hz}$ intermodulation component is still clearly visible in the response. 


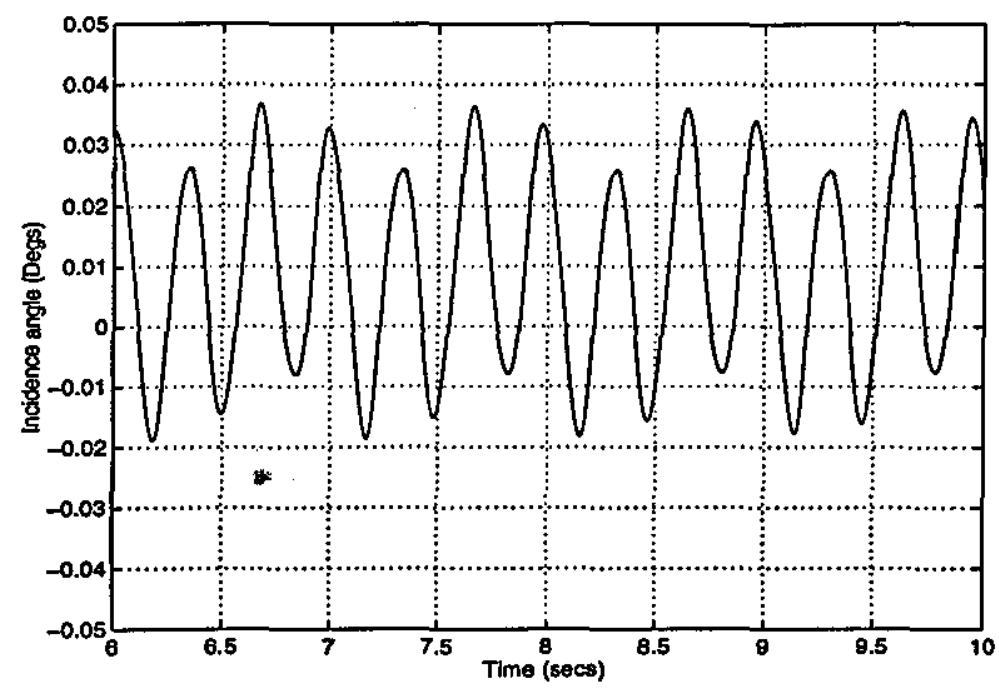

Figure 7.41 - Incidence angle time response

\subsubsection{Effect of higher frequency structural-modes on subharmonic genera- tion}

The above example concentrated on the effect of a low-frequency structural-mode signal on the aircraft response. Supposing now that the structural-mode frequency was at a frequency of $31.5 \mathrm{~Hz}$, whilst the flight control system demand signal remained at $3.05 \mathrm{~Hz}$. From the above analysis, it would be expected that a component would again exist at $1 \mathrm{~Hz}$ as a result of $\mathrm{s}=10$ and $\mathrm{k}=1$ in the term $\left(\mathrm{s} \omega_{\mathrm{a}} \pm \mathrm{k} \omega_{\mathrm{b}}\right)$. If the power spectra for the output of the actuator is produced for these input conditions, the component that exists at $1 \mathrm{~Hz}$ is negligible, and as a result, the effect on the aircraft's response is also negligible.

The reason for this effect lies in the amplitude coefficient $\alpha_{\text {sk }}$ of equation (7.19). From equation (7.19), it can be seen that the component amplitude is a function of the values of $s$ and $k$ through the order of the Bessel functions. Evaluating integrals of the form of equation (7.19) for increasing values of $s$ and $k$ for a saturation nonlinearity, for example, results in Figure 7.42. The figure demonstrates that as the order of the Bessel functions is increased, the magnitude of the component will decrease significantly. For the numerical example, it can be seen from Figure 7.42 that the $1 \mathrm{~Hz}$ component generated for input signal frequencies of $3.05 \mathrm{~Hz}$ and $7.1 \mathrm{~Hz}(\mathrm{~s}=2, \mathrm{k}=1)$ would have an amplitude approximately 40 times greater than the same component generated by input signals of frequency $3.05 \mathrm{~Hz}$ and $31.5 \mathrm{~Hz}(\mathrm{~s}=10, \mathrm{k}=1)$.

Hence it is clear that significant subharmonic components will only be generated when the structural-mode frequency is relatively low. This obviously depends however on the amplitude of the two input signals. In addition, input signals that are commensurate (i.e. $\omega_{\mathrm{a}} / \omega_{\mathrm{b}}$ is a ratio of integers) cause further problems as different 
combinations of $\mathrm{s}$ and $\mathrm{k}$ result in the same frequency component at output.

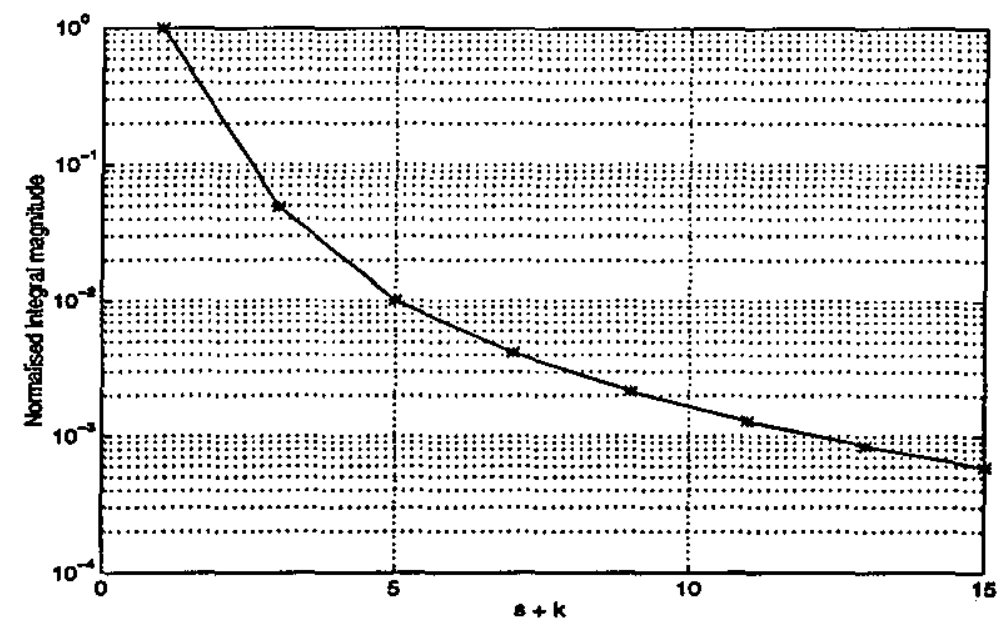

Figure 7.42 - Relative component amplitudes with increasing Bessel function order

\subsubsection{Subharmonic generation in a digital system}

The presence of two or more input signals to the actuator is not restricted to the existence of an unstable structural-mode and pilot demand signal. If the flight control system were digital as is usually the case, then aliases of the fundamental frequency will exist at the actuator input.

In the case of a typical frequency for a pilot demand signal, such an alias will be of a value close to the sampling frequency. As a result, subharmonic generation within the rate limiter and actuation system is unlikely. Suppose however that a structural oscillation with a frequency of $25 \mathrm{~Hz}$ existed within the system. The digital nature of the control system would result in an alias with a frequency of $55 \mathrm{~Hz}$ also existing at output from the flight control system. The combination of these two signals within the rate limiter and actuator could then lead to an output component with a frequency of 5 $\mathrm{Hz}$. Such a situation will be discussed in section 7.8.

\subsubsection{Conclusion}

The theoretical background to the generation of harmonics and sub-harmonics by a nonlinear system has been outlined. It has been demonstrated that low-frequency structural-modes may result in significant sub-harmonic aircraft response as a result of interactions between the structural feedback and pilot demand signals. In addition, the possible generation of subharmonic response in the presence of structural feedback as a result of the digital nature of the control system has been highlighted. 


\subsection{Performance boundary for nonlinear actuation system}

\subsubsection{Introduction}

As has been discussed, until recently any analysis of an aeroservoelastic system involved only linear actuation system models. One consequence of this would be that the amplitude of any unstable structural-mode would be unbounded, with obvious catastrophic results. In reality, servo-hydraulic actuation systems are nonlinear in nature, there being limits to servo-valve displacement, main-valve displacement and main-ram travel in particular. These limits can be interpreted as constraints on the main-ram acceleration, velocity and displacement respectively. Naturally, for any given actuator demand frequency, there will exist a demand amplitude at which the actuator performance is being limited in terms of these constraints ${ }^{72,73}$. The following section investigates the existence of such a performance boundary and its possible effects on the nature of the aeroservoelastic interaction.

\subsubsection{Actuator performance boundary for analogue system}

Consider the case of a very low-frequency demand signal. In such a situation, the actuator will be limited only by the limit on ram travel. Once this limit has been exceeded any increase in demand amplitude will not be met. For a higher frequency demand signal, a point exists where the main-valve becomes fully open, equating to the main-ram travelling at its maximum rate. Thus ram displacement would be limited by this maximum rate and the time period of the demand signal.

In addition to these performance limits which are determined by the hardware, it is common practice to incorporate rate limits in the software within the FCS so as to protect the actuator from potentially damaging demands. The effect of such rate limiting on actuator performance can be demonstrated by examining the input and output time responses of such a rate limiter in isolation as shown in Figure 7.21. From the figure, any subsequent increase in the amplitude of the input signal to the rate limiter will not result in an increase in the amplitude of the output waveform which of course forms the demand signal for the actuator. Consequently, the rate-limiting in the software is responsible in part for limiting the performance of the actuator.

The performance limit for a taileron actuator model is shown in Figure 7.43. This figure represents output amplitudes obtained from a nonlinear actuation system model for increasing demand amplitude. From the figure it is clear that the responses for increasing input amplitudes converge on a single boundary representing the performance limit for the actuator. In this case, the digital nature of the flight control system was neglected. This means that the input signal to the actuator itself will not be 
of the "stepped" form typical of the output signals from a zero-order-hold.

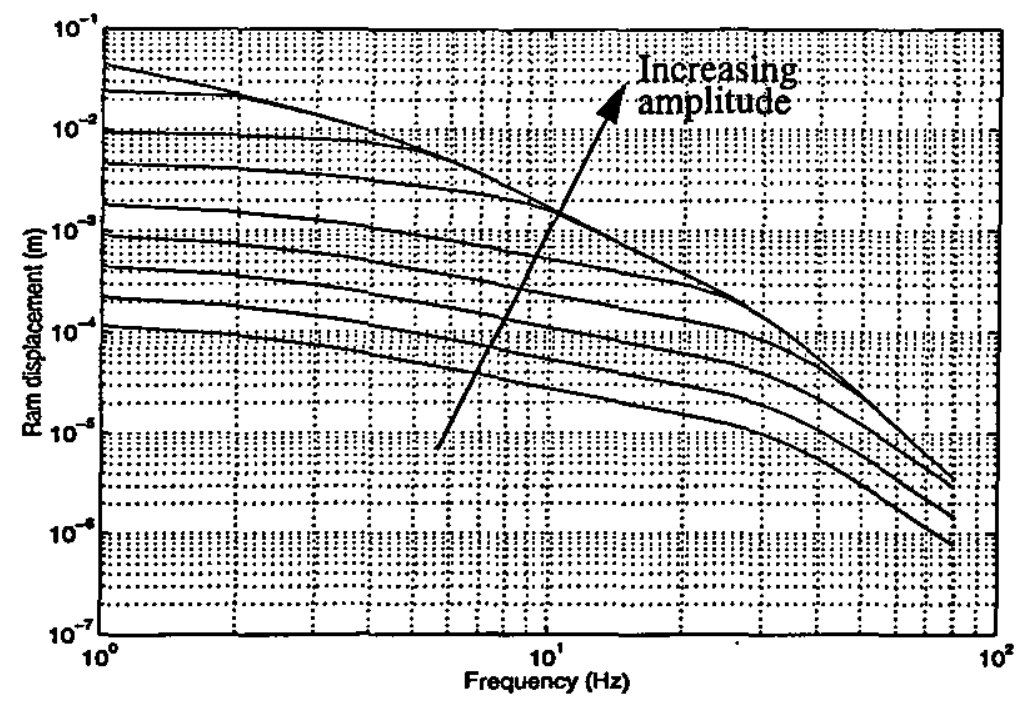

Figure 7.43 - Jaguar FBW taileron actuator performance limit for nonlinear model

The contribution of the differing hardware and software limits to this performance boundary can be demonstrated as shown in Figure 7.44. These results represent the performance boundary for the nonlinear actuation system model as each of the hardware and software limits is introduced into the model.

From the above results, the major cause of the performance limit of the actuation system is the software rate limiting of the input signal. This is to be expected as the purpose of the software rate limiting is to prevent main-valve saturation. Considering the boundary for travel limit of the main-ram alone, the results show that there is no change in the boundary with input frequency. As the servo-valve and main-valve limits are introduced, the results demonstrate that the performance limit is lowered from the simple travel limit case, as would be expected.

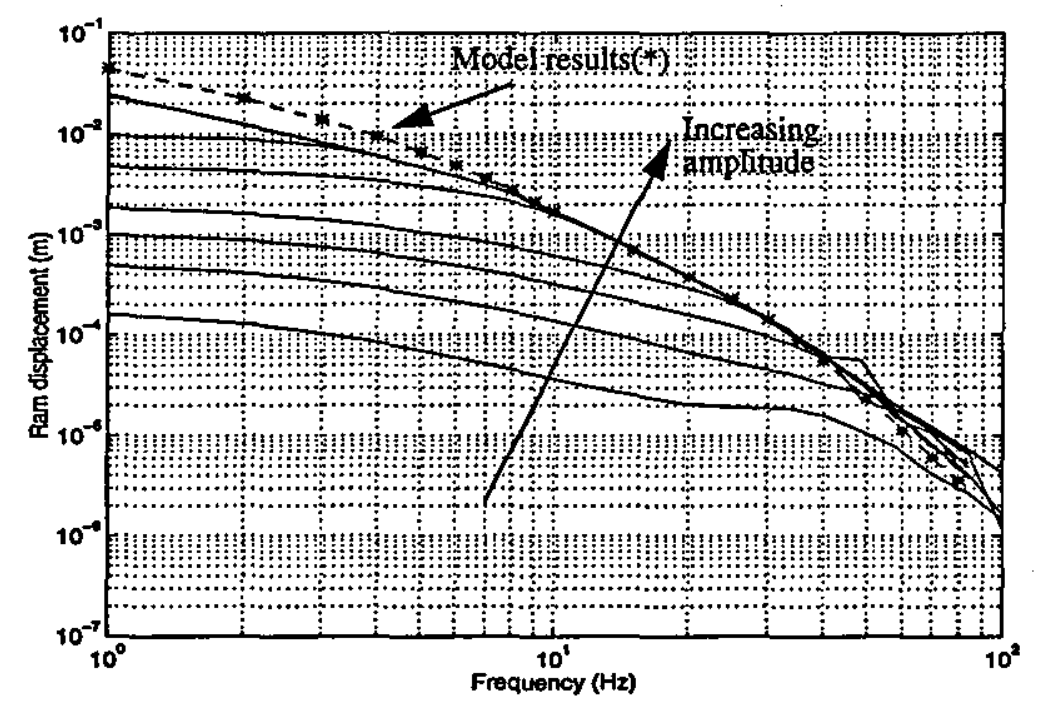

Figure 7.44 - Performance limit of actuation system model

Experimental single-input frequency response tests on the Jaguar FBW taileron 
actuator have confirmed the results obtained from simulation as shown in Figure 7.45, where the output amplitudes for the main-ram have been obtained for increasing demand frequency and amplitude. In addition, the performance boundary as predicted from simulation, is included in Figure 7.45. It can be seen that there is good correlation between the experimental and simulated results.

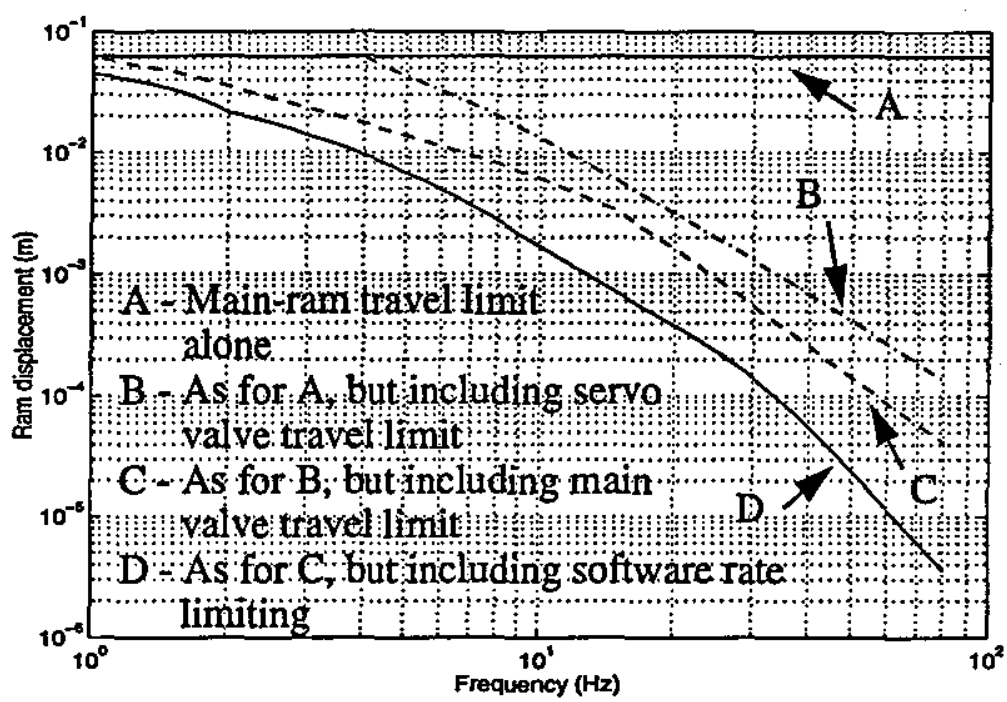

Figure 7.45 - Jaguar FBW taileron actuator performance limit.

\subsubsection{Actuator performance boundary for a digital system}

In the case of a fully digital system, the input signal to the actuator comes from a zero-order-hold. The consequence of this is that the fundamental frequency of such a signal cannot be higher than the Nyquist frequency of the digital system. In addition, the presence of high-frequency harmonics within the input signal will effect the actuator's performance in response to the fundamental frequency in the same way as the structural feedback signals can affect its performance ${ }^{14}$. As a result of these effects, the performance boundary for the actuator within a digital system may be different from that for the actuator within an analogue system.

To demonstrate this, the performance boundary for the actuator model subjected to an output signal from a zero-order-hold is included as Figure 7.46 . The production of this boundary involved the actuator model being subjected to a large amplitude demand signal. Prior to reaching the actuator however, the demand signal was passed through a zero-order-hold and digital rate limiter. From previous discussions of the digital nature of the control system, the sampling frequency for both zero-order -hold and rate limiter was $80 \mathrm{~Hz}$. 


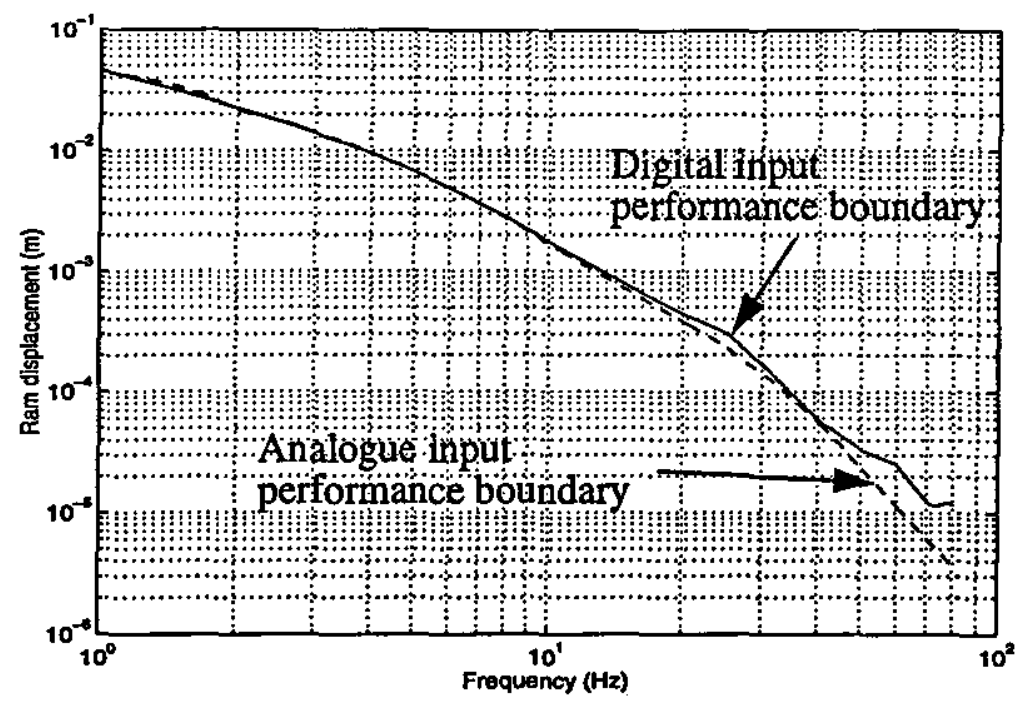

Figure 7.46 - Actuator model performance boundary for digital input signal

The results demonstrate that there are small differences in the performance boundary between the two cases. These differences exists at high input frequencies, where the performance boundary for the digital system is slightly higher than for the analogue input case. This difference in the two responses can be attributed to the generation of harmonics and subharmonics by the sample-and-hold and rate limiting processes. To demonstrate this, consider the case of an input signal of frequency 25 $\mathrm{Hz}$. From Figure 7.46 there is a distinct difference in the performance boundary at this frequency. The time responses for both the analogue and digital cases showing the output from the rate limiter are shown in 7.47.

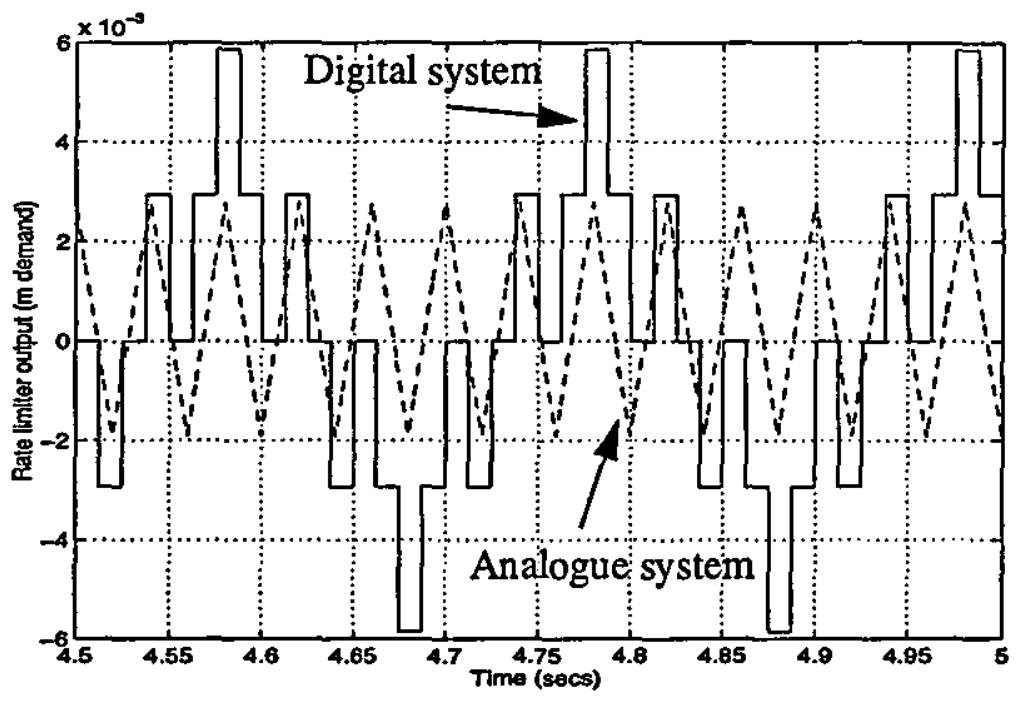

Figure 7.47 - Rate limiter output signal for digital system, $25 \mathrm{~Hz}$ input frequency

The time response demonstrates that the digital nature of the rate limiter can introduce harmonics and subharmonics of the input signal. In this case, for an input signal of frequency $25 \mathrm{~Hz}$, the sample-and-hold process will introduce a component of frequency $55 \mathrm{~Hz}$. After rate limiting has occurred, these two components will create a 
$5 \mathrm{~Hz}$ subharmonic component as can be seen from Figure 7.47. As a result, the actuator response to the original $25 \mathrm{~Hz}$ signal is increased due to the effect of the shaping of the main-valve ports as has been discussed earlier. This effect is more prominent for input frequencies of around $25 \mathrm{~Hz}$, because it is these frequencies which generate the lowest frequency subharmonic components after sampling and rate limiting has occurred.

For input frequencies greater than the Nyquist frequency, the initial sampling results in aliasing of the input signal. As a result, the rate limiter performs its operation on the low-frequency alias of the input signal. For example an input signal of frequency $60 \mathrm{~Hz}$ will result in an output signal from the rate limiter with a fundamental frequency of $20 \mathrm{~Hz}$. In addition to this fundamental frequency, this output signal will also contain a component at a frequency of $60 \mathrm{~Hz}$ due to the sampleand-hold process. The time response for the rate limiter is given in Figure 7.48.

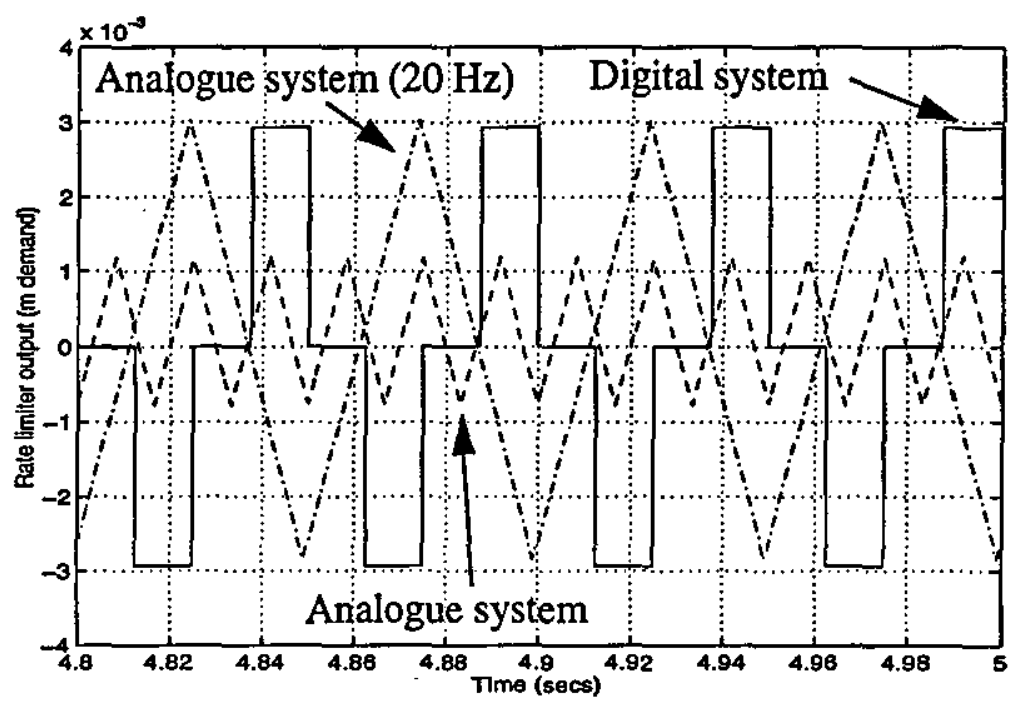

Figure 7.48 - Rate limiter output signal for digital system, $60 \mathrm{~Hz}$ input frequency

As a comparison to the digital response, the response for the analogue rate limiter to a $20 \mathrm{~Hz}$ input frequency is also included in Figure 7.48. The results demonstrate that the rate limiter in the digital case is responding to the $20 \mathrm{~Hz}$ fundamental frequency once sampling has occurred. The output signal may therefore contain a higher amplitude component at the original $60 \mathrm{~Hz}$ input frequency than would be the case for the analogue system. In addition to this effect, the shaping of the main-valve ports may result in a higher gain in response to the $60 \mathrm{~Hz}$ component signal.

Experimental results demonstrating the performance boundary for the Jaguar FBW taileron actuator for digital input signals are given in Figure 7.49. As for the analogue system, the match between experimental and simulation is good. 


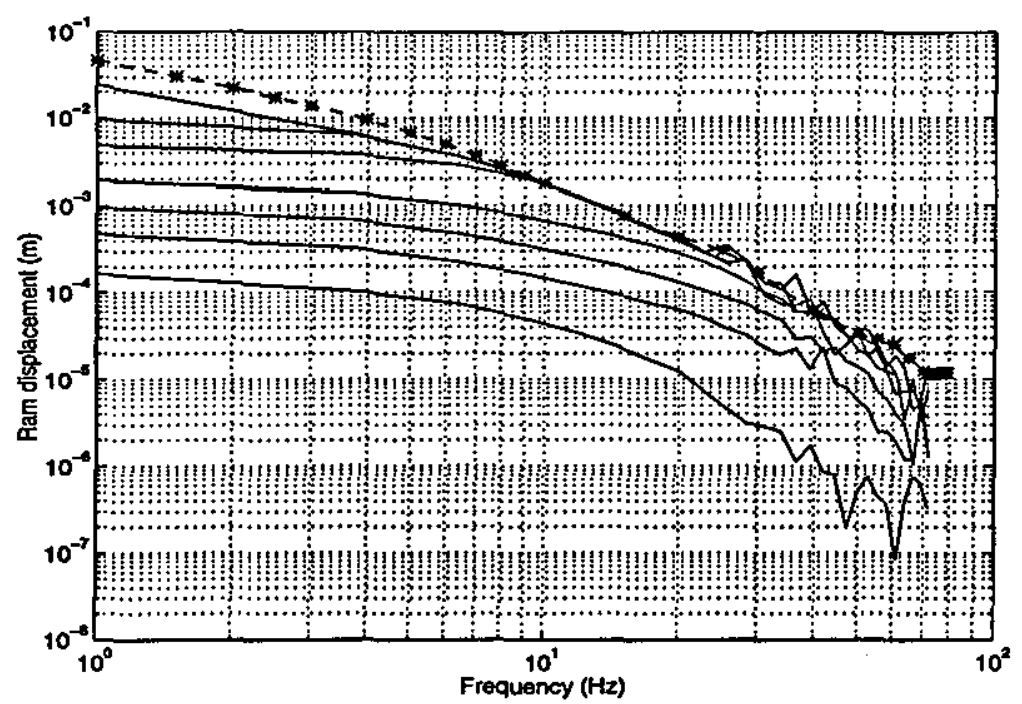

Figure 7.49 - Performance boundary for Jaguar FBW taileron actuator for digital input signals

\subsubsection{Conclusion}

The existence of a performance boundary for the actuation system has been demonstrated. The fact that the actuator ram has an upper limit on its displacement at any given input frequency indicates that an unstable structural-mode could not result in an unbounded response and catastrophic failure. Instead, it would seem that there would exists an upper limit to this structural excitation as a result of control surface motion.

\subsection{Conclusions}

The nonlinear nature of the actuation system has been shown to introduce several new elements to the aeroservoelastic problem. Firstly, the performance of the actuation system has been shown to change in the presence of structural feedback signals. The main cause for such performance changes being the software rate limiting and shaping of the main-valve ports. Although the profile of the main-valve ports can result in improved performance in the presence of structural feedback signals, it is the phase lag introduced by the software rate limiting that is of most concern.

A method of predicting response of the actuator in the presence of high-frequency noise has been demonstrated, although a pessimistic analysis can be obtained from a consideration of the software rate limiter in isolation. Such analysis has been completed for a rate limiter for a range of structural frequencies and amplitudes. These results indicate primarily that a certain level of structural noise may be acceptable at the input to the actuator before performance becomes unsatisfactory. 
A secondary effect of the nonlinear nature of the actuation system has also been demonstrated. This effect involves the possible generation of subharmonic response in the presence of structural feedback. It has been shown that such subharmonic generation is limited to low-frequency structural feedback signals. Alternatively, however, the digital nature of the control system can result in subharmonic generation for structural frequencies of approximately $25 \mathrm{~Hz}$ as a result of interactions between such frequencies and their aliases.

Finally, the nonlinearities inherent within the actuation system have been shown to result in a performance limit for the actuator. The importance of such a performance limit is that there is a physical constraint on the excitation of the aircraft structure as a result of the control surface motion. The consequence of this is that an unstable structural response cannot result in an unbounded oscillation. 
Chapter 8

Limit-cycle Prediction and Specification of Alternative Clearance Requirements 


\subsection{Introduction}

The previous chapter has discussed the effect of the nonlinear nature of the actuation system on the aeroservoelastic problem. It has been shown that the presence of structural-mode feedback signals can result in a reduction of actuator performance. Naturally, such a reduction would be detrimental to satisfactory rigid-body control of the aircraft. Importantly however, the results of Chapter 7 demonstrate that a certain level of structural noise may be tolerated before it creates an unacceptable decrease in the actuator's performance ${ }^{72,73}$.

In order for these results to be of use, a method of predicting the amplitude of structural signals within a system must be devised. For an unstable linear system, the amplitude of the resultant oscillation is unbounded. As a result, examination of the effects of a particular structural signal amplitude is meaningless in that there is no upper limit on this amplitude. In the real system however, where the actuation system is highly nonlinear, this chapter will show that such an unbounded oscillation will not occur. Instead, any resultant oscillation will have an upper limit to its amplitude.

Chapter 7 introduced the concept of the actuator performance boundary, which represents the maximum output of the actuator at any particular input frequency. This boundary is as a result of the nonlinearities inherent within the actuator itself, and the software rate limit within the FCS. As a result of this performance boundary, it would seem sensible to expect that the structural excitation as a result of actuator motion would also be limited in amplitude.

This chapter investigates the result of a structural-mode that is unstable in the closed-loop through an application of nonlinear system theory to both a simple example system, and the aircraft system model developed in earlier chapters. The possible advantage of such a consideration in terms of an alternative structural-mode clearance procedure will then be considered.

\subsection{Description of limit-cycle prediction technique}

\subsubsection{Introduction}

It has been shown that the nonlinear nature of the actuation system plays a vital role in the propagation of aeroservoelastic signals around the aircraft closed-loop. The existence of a performance boundary for the actuator, and the nonlinear nature of the actuation system in general, indicates that an unstable aeroservoelastic interaction would result in a limit cycling condition rather than a divergent instability. If such a limit cycling condition could be predicted then its effect on the aircraft system as a whole could be assessed. 


\subsubsection{Limit-cycle criteria and prediction}

The existence of limit-cycles in a nonlinear system can be predicted from a solution of its characteristic equation, with the nonlinear elements replaced by their describing functions ${ }^{71}$. In order to simplify the analysis, the only nonlinearity that will be considered is the software rate limit function. As a result, the actuator itself is assumed to behave linearly downstream of the rate limiter. As discussed in Chapter 7, the purpose of the rate limiter within the control software is to prevent saturation of the actuator main valve and as such, the rate limiter is the main nonlinearity which limits the performance of the actuator.

Consider the system as shown in Figure 8.1 in which an actuator is used in a position control system in which the load exhibits a structural-mode within the bandwidth of the closed-loop system.

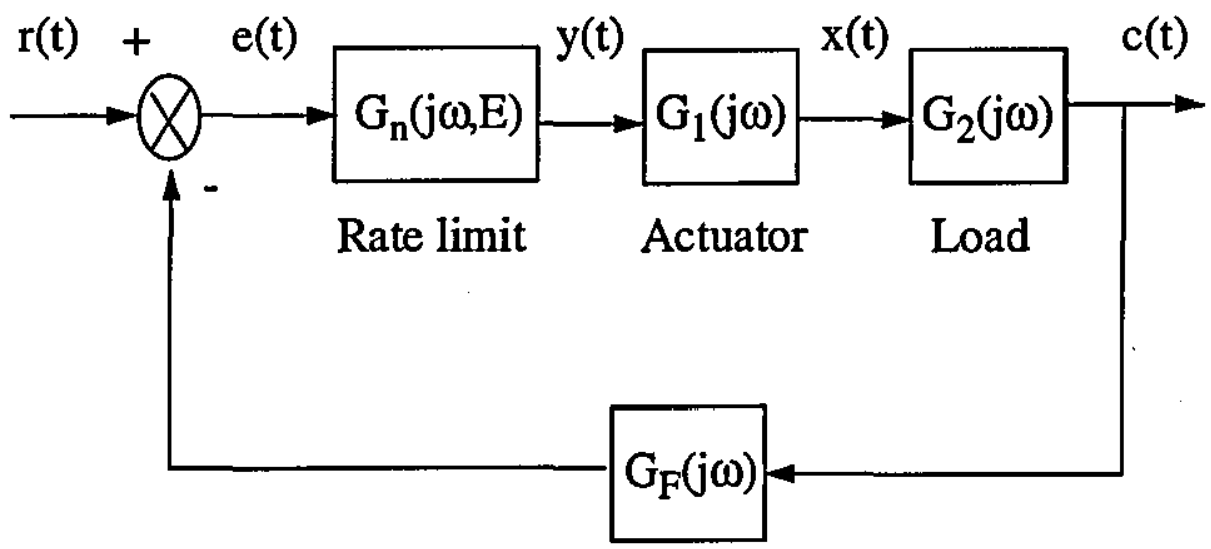

Structural Filters

Figure 8.1-System Block Diagram

In this case, the characteristic equation for the system can be written as

$$
1+G_{n}(j \omega, E) G_{1}(j \omega) G_{2}(j \omega) G_{F}(j \omega)=0
$$

The solution of the characteristic equation gives the limit-cycle condition, which can be predicted from a re-arrangement of Equation (8.1)

$$
G_{1}(j \omega) G_{2}(j \omega) G_{F}(j \omega)=\frac{-1}{G_{n}(j \omega, E)}
$$

Provided that the describing function for the rate limiter can be derived, and that the linear components within the system can be adequately modelled, it will be possible to predict the existence of limit cycling conditions within the system.

\subsubsection{Derivation of describing function for rate limit function}

In order to derive a describing function for the rate limiter, consider the input/ output characteristics of such a rate limiter as shown in Figure 8.2. In this case, the 
characteristics are shown after a length of time sufficient for a steady relationship to be achieved. In addition it is assumed that the input signal is a pure sinusoid which triggers the rate limiter to an output waveform which is triangular ${ }^{73}$.

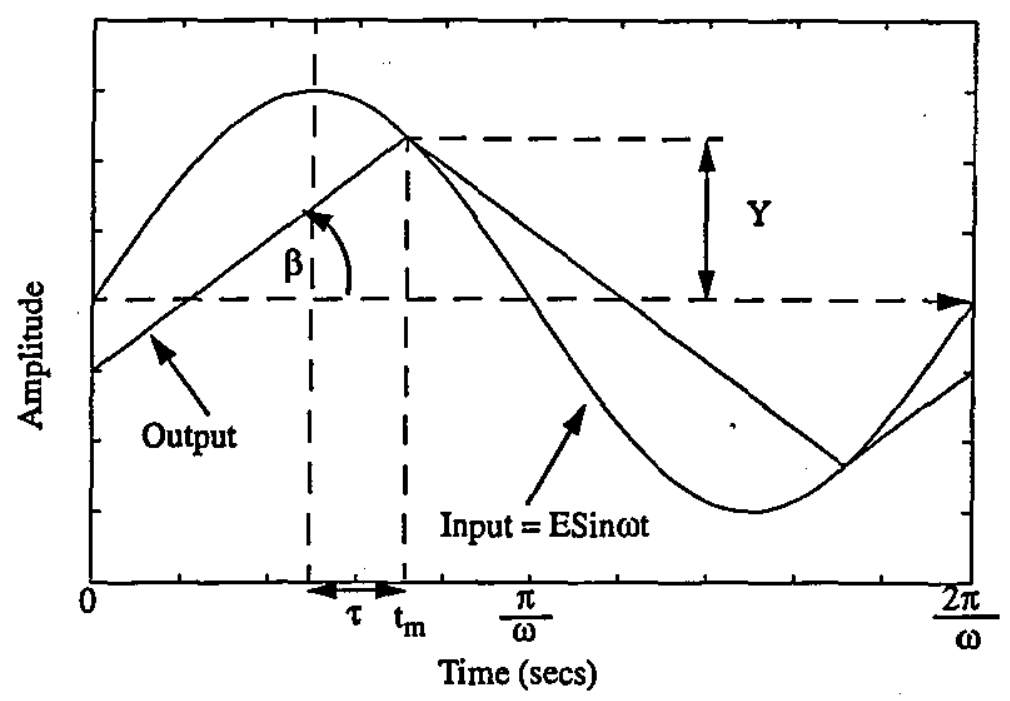

Figure 8.2- Input/Output characteristics of rate limit function

From Figure 8.2, the amplitude of the triangular output waveform can be derived as

$$
Y=\frac{\pi \beta}{2 \omega}
$$

where $\beta$ is the maximum rate as shown in Figure 8.2.

Fourier analysis of such a triangular waveform of amplitude $Y$, reveals that the amplitude of the fundamental is

$$
\left.\mathrm{Y}\right|_{\omega}=\frac{4 \beta}{\omega \pi}
$$

with an infinite number of harmonics. Neglecting these higher-order harmonics, the gain of the rate limiter can be expressed as

$$
\left|G_{n}(j \omega, E)\right|=\frac{4 \beta}{\omega E \pi}
$$

for an input sinusoid of the form as shown in Figure 8.2. (The generation of higher harmonic components in the actuator output signal, along with the possible generation of low-frequency sub-harmonic components has been discussed in detail in Chapter 7.)

In order to derive the phase response of the rate limiter, consider the input/output relationship of Figure 8.2 once again. From the figure, it can be seen that the phase lag between the two signals can be represented by the time delay, $\tau$. In order to obtain an expression for this time delay, it is necessary to locate the time at which the input 
signal is equal to the output signal, such that

$$
E \sin \omega t_{m}=\frac{\pi \beta}{2 \omega}
$$

Considering that $t_{m}$ occurs after $t=\frac{\pi}{2 \omega}$, the above equation can be solved for $t_{m}$ such that

$$
\mathrm{t}_{\mathrm{m}}=\frac{\pi}{\omega}-\frac{1}{\omega} \operatorname{asin} \frac{\pi \beta}{2 \mathrm{E} \omega}
$$

Therefore, the time delay, $\tau$, can be expressed as

$$
\tau=\frac{1}{\omega}\left(\frac{\pi}{2}-\operatorname{asin} \frac{\pi \beta}{2 \mathrm{E} \omega}\right)
$$

Finally, since the phase lag between the input and output signals can be expressed as

$$
\angle G_{n}(j \omega, E)=-\tau \omega
$$

the describing function of the rate limiter under the assumptions applied earlier is therefore

$$
\begin{gathered}
\left|G_{n}(j \omega, E)\right|=\frac{4 \beta}{\omega E \pi} \\
\angle G_{n}(j \omega, E)=-\left(\frac{\pi}{2}-\operatorname{asin} \frac{\pi \beta}{2 E \omega}\right)
\end{gathered}
$$

Since the gain of the rate limiter will never be greater than unity, and the phase of the rate limiter will never be greater than zero, limitations can be applied to the above expressions. This results in the requirement that

$$
E \omega \geq \frac{4 \beta}{\pi}
$$

for the gain expression to be valid, and

$$
E \omega \geq \frac{\pi \beta}{2}
$$

for the phase expression to be valid.

In order to verify the above derivation, the frequency response for such a rate limiter was obtained from time domain simulation. In this case, the rate limit was set such that $\beta=1.0$, and the input signal was of amplitude, $E=0.1$. The resulting frequency response compared with that predicted from the above describing function 
is as shown in Figure 8.3.
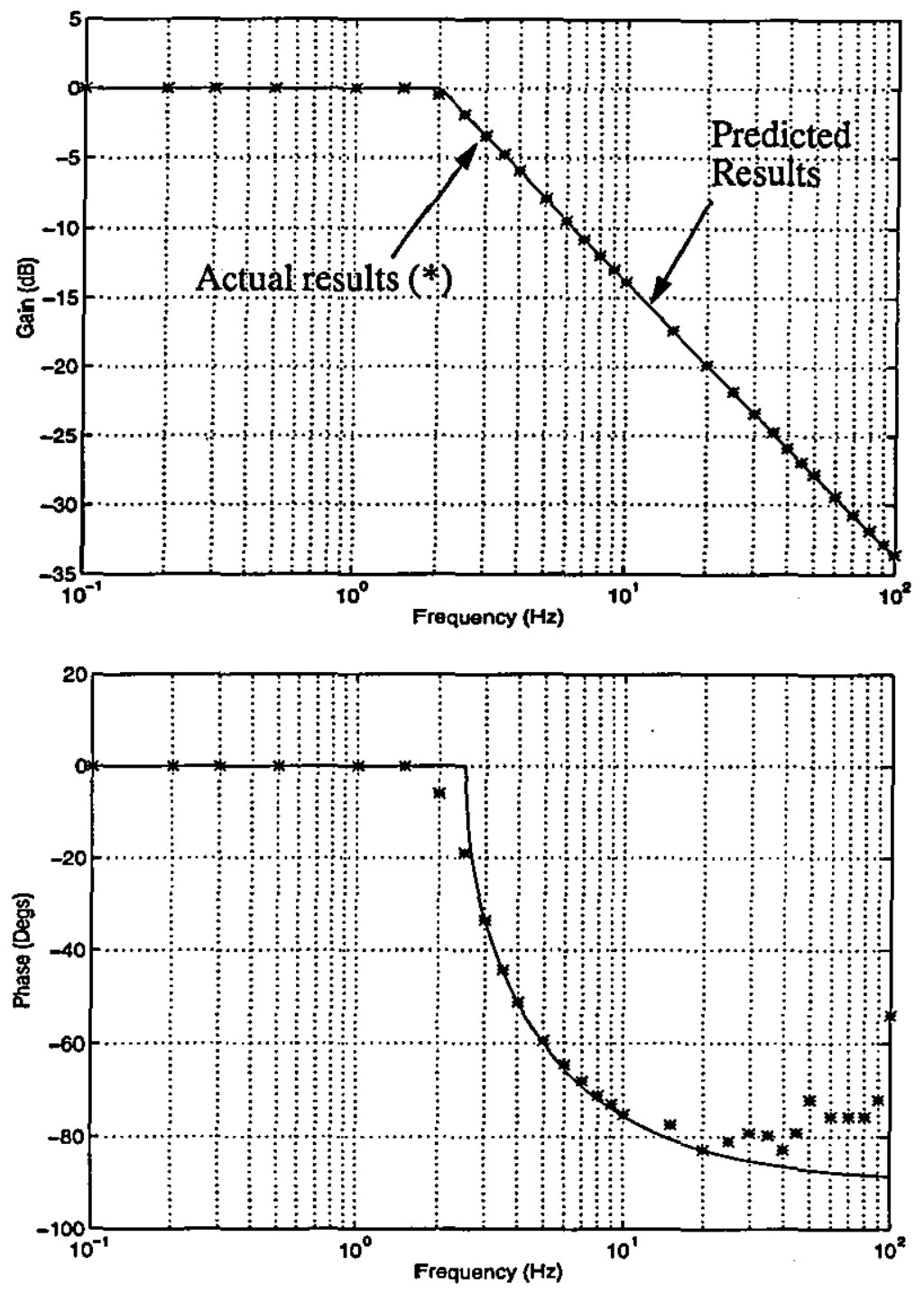

Figure 8.3 - Example rate limit frequency response

From the figure, the describing function for the rate limiter can be seen to give a good match to the results obtained from simulation. As expected, there are slight discrepancies between the two sets of results at approximately $2 \mathrm{~Hz}$. This corresponds with the region in which the value for the rate limit has been exceeded, but the condition corresponding to the triangular waveform of Figure 8.2 has not been reached. Since the describing function was derived assuming this output waveform, the results are slightly in error. It should be noted that the discrepancies between the phase responses at high frequencies is due to errors in the calculation of the phase response from the simulated results.

The above expressions therefore allow the prediction of the existence of the limit cycling condition from the solution of the characteristic equation for the system as given in Equation (8.2). This is provided that the linear elements of the system can be accurately modelled, or a frequency response obtained from suitable testing. 


\subsubsection{Prediction of limit-cycles in an example system}

In order to demonstrate the use of the describing function in the prediction of limit-cycles, consider the system as shown in Figure 8.1, whose characteristic equation is as given in equation (8.2). Suppose now that the linear elements of the system could be represented by the transfer functions

$$
\begin{gathered}
G_{1}(s)=\frac{1}{(0.026 s+1)\left(0.00005917 \mathrm{~s}^{2}+0.007693 \mathrm{~s}+1\right)} \\
G_{2}(\mathrm{~s})=\frac{4000}{\mathrm{~s}^{2}+\mathrm{s}+4000} \\
\mathrm{G}_{\mathrm{F}}(\mathrm{s})=1
\end{gathered}
$$

where the element $G_{1}(s)$ represents a typical servo-hydraulic actuator, and $G_{2}(s)$ represents a lightly damped modal system.

In the absence of the rate limiter, the system is unstable in the closed-loop resulting in an unbounded oscillatory response in the presence of an initial disturbance. For the system including the rate limiter however, the characteristic equation can be solved in order to predict any resulting limit-cycle. One method of solution of the resulting characteristic equation is to plot both sides of equation (8.2) on a Nyquist diagram and find the intersection of the two loci. Unfortunately, the describing function for the rate limiter is both frequency and input amplitude dependent, resulting in an infinite number of loci. However, solutions of the characteristic equation can still be located through suitable iterative techniques.

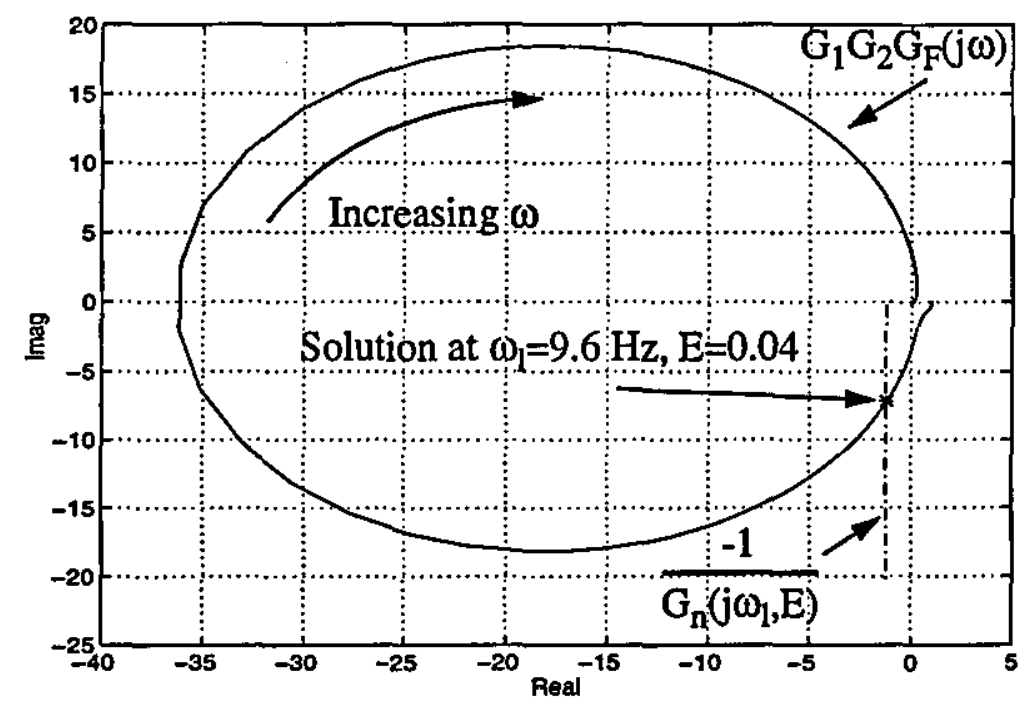

Figure 8.4 - Nyquist diagram showing limit-cycle solution

The corresponding Nyquist diagram for the example system is shown in Figure 8.4. In this case, the nonlinear characteristics have been plotted for a single frequency, $\omega_{1}$, which intersects the locus for $G_{1} G_{2} G_{F}(j \omega)$ at $G_{1} G_{2} G_{F}\left(j \omega_{1}\right)$. This point represents 
the solution of the characteristic equation, and therefore predicts the frequency and amplitude of the resulting limit-cycle. From Figure 8.4, the value of the frequency at which the two loci intersect is $9.6 \mathrm{~Hz}$, and the corresponding limit-cycle amplitude, $\mathrm{E}$, is 0.04 . Figure 8.5 shows the intersection of the two loci in more detail.

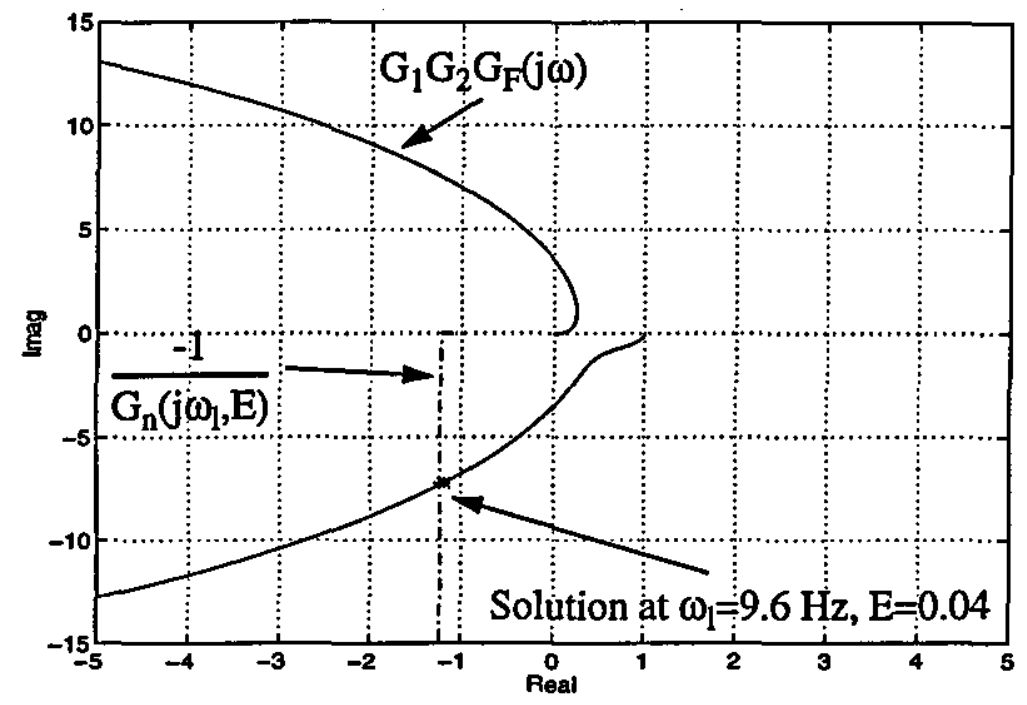

Figure 8.5 - Nyquist diagram showing limit-cycle solution

A simulation of the system results in the limit-cycle shown in Figure 8.6. From the figure, the limit-cycle frequency is $9.64 \mathrm{~Hz}$ with an amplitude of $E=0.038$. These results match well those predicted by the describing function technique.

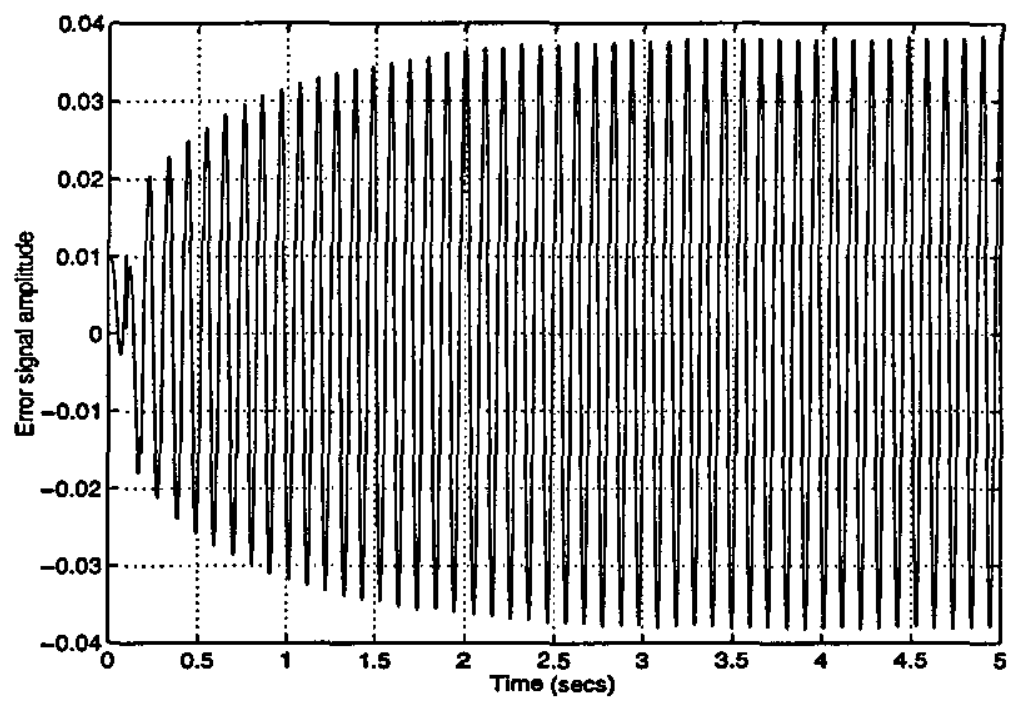

Figure 8.6 - Time domain simulation results of example system

From this simple example at least, the prediction of limit-cycles within a system involving a rate limiter seems feasible. The following section extends this analysis to consider a system based on the aircraft system model developed earlier.

\subsubsection{Limit-cycle prediction in an aircraft system}

The previous section demonstrated an application of describing function theory to the prediction of limit-cycles. However, the example system used was relatively 
simple compared with the aircraft system model developed earlier. Assuming the aircraft flight control system to be analogue, and that the sensor dynamics can be neglected at present, the block diagram for the aircraft system can be considered as shown in Figure 8.7. The three control-surface actuators are assumed to be identical as before, and have been linearised in order to make use of the earlier describing function analysis.

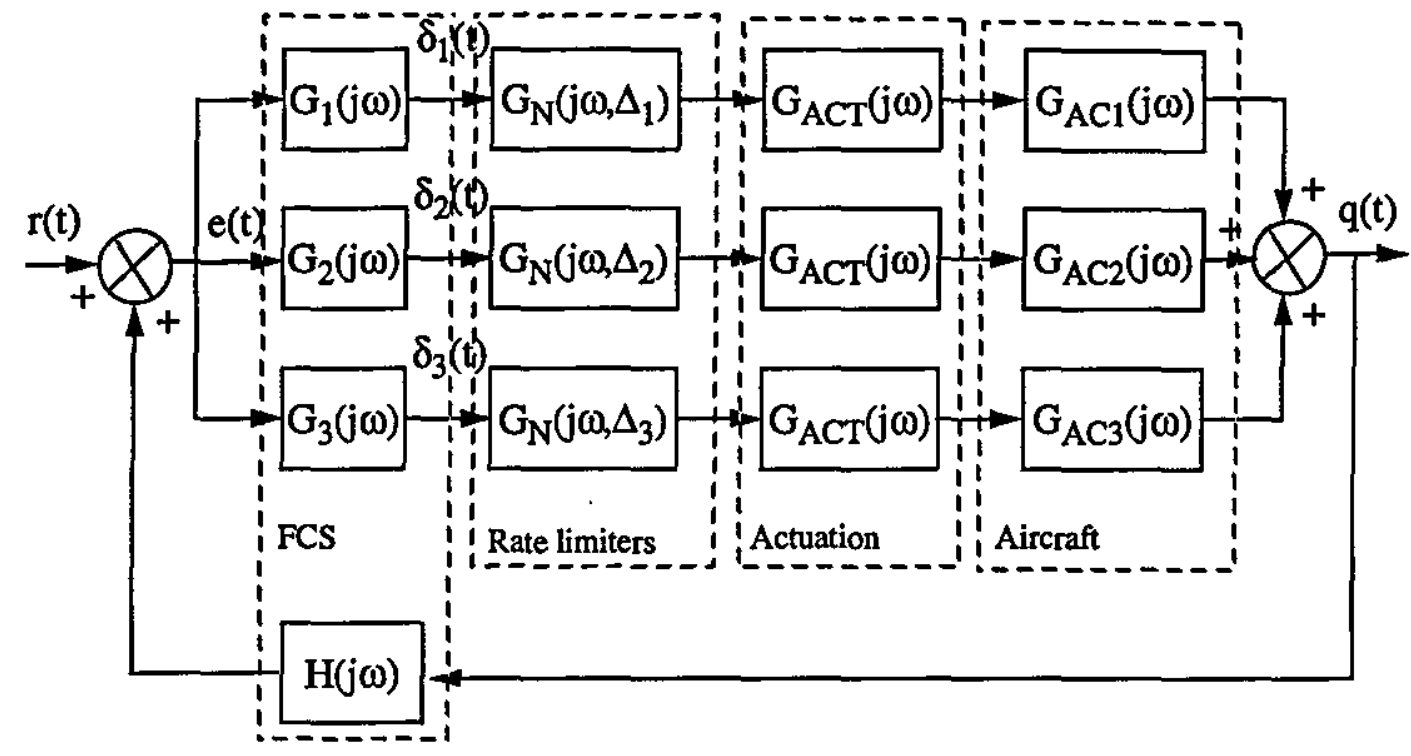

Figure 8.7 - Aircraft system block diagram

The derivation of the characteristic equation for the system is straightforward, and results in the equation

$$
1-H(j \omega) G_{A C T}(j \omega) G_{T}\left(j \omega, \Delta_{1}, \Delta_{2}, \Delta_{3}\right)=0
$$

where

$$
\begin{array}{r}
G_{T}\left(j \omega, \Delta_{1}, \Delta_{2}, \Delta_{3}\right)=G_{A C 1}(j \omega) G_{1}(j \omega) G_{N}\left(j \omega, \Delta_{1}\right)+ \\
G_{A C 2}(j \omega) G_{2}(j \omega) G_{N}\left(j \omega, \Delta_{2}\right)+G_{A C 3}(j \omega) G_{3}(j \omega) G_{N}\left(j \omega, \Delta_{3}\right)
\end{array}
$$

Now, the amplitude of the input signals to the rate limiters $\left(\Delta_{1}, \Delta_{2}, \Delta_{3}\right)$ can be derived from the error signal and the particular FCS path transfer function, such that for example

$$
\Delta_{1}=\left|G_{1}(j \omega)\right| E
$$

This enables the characteristic equation for the system to be expressed as a function of $\omega$ and $E$ only, as was the case for the earlier example. Although the resulting equation is more complex than for the earlier example, the principle is exactly the same in that a solution of the characteristic equation will predict the existence of limit-cycles within the system. The characteristic equation is therefore 


$$
H(j \omega) G_{A C T}(j \omega)=\frac{1}{G_{T}(j \omega, E)}
$$

where

$$
\begin{gathered}
G_{T}(j \omega, E)=G_{A C 1}(j \omega) G_{1}(j \omega) G_{N 1}(j \omega, E)+ \\
G_{A C 2}(j \omega) G_{2}(j \omega) G_{N 2}(j \omega, E)+G_{A C 3}(j \omega) G_{3}(j \omega) G_{N 3}(j \omega, E)
\end{gathered}
$$

and

$$
\begin{gathered}
\left|G_{N x}(j \omega, E)\right|=\frac{4 \beta}{\omega\left|G_{x}(j \omega)\right| E \pi} \\
\angle G_{N x}(j \omega, E)=-\left(\frac{\pi}{2}-\operatorname{asin} \frac{\pi \beta}{2\left|G_{x}(j \omega)\right| E \omega}\right)
\end{gathered}
$$

The solutions of this characteristic equation for a reduced-order model of the flexible aircraft with 10 modes are as shown in Table 8.1. These solutions were obtained using the same principal as shown in Figure 8.4. The results therefore predict that there exist five possible operating points for the system, each point representing a limit-cycle of differing amplitude and frequency.

\begin{tabular}{|c|c|}
\hline$\omega(\mathrm{Hz})$ & $\mathrm{E}$ \\
\hline \hline 16.0 & 0.0142 \\
\hline 16.4 & 0.0332 \\
\hline 24.0 & 0.0371 \\
\hline 66.0 & 0.0040 \\
\hline 73.6 & 0.0106 \\
\hline
\end{tabular}

\section{Table 8.1 - Predicted aircraft system limit-cycles}

A time domain simulation of the system for an arbitrary initial disturbance, results in a limit-cycle as shown in Figure 8.8. The amplitude and frequency of the actual limit-cycle is 0.035 and $16.4 \mathrm{~Hz}$ respectively. This compares well with the second of the predicted limit-cycles in Table 8.1 .

The above results demonstrate that it is possible to predict the existence of limitcycles even in a complex system such as the aircraft model shown in Figure 8.7. In this case, the theoretical analysis predicted the existence of five possible limit-cycle conditions. In reality, the system will operate at only a single limit-cycle condition. The actual limit-cycle that occurs in practice can vary between the five possible conditions of Table 8.1. 


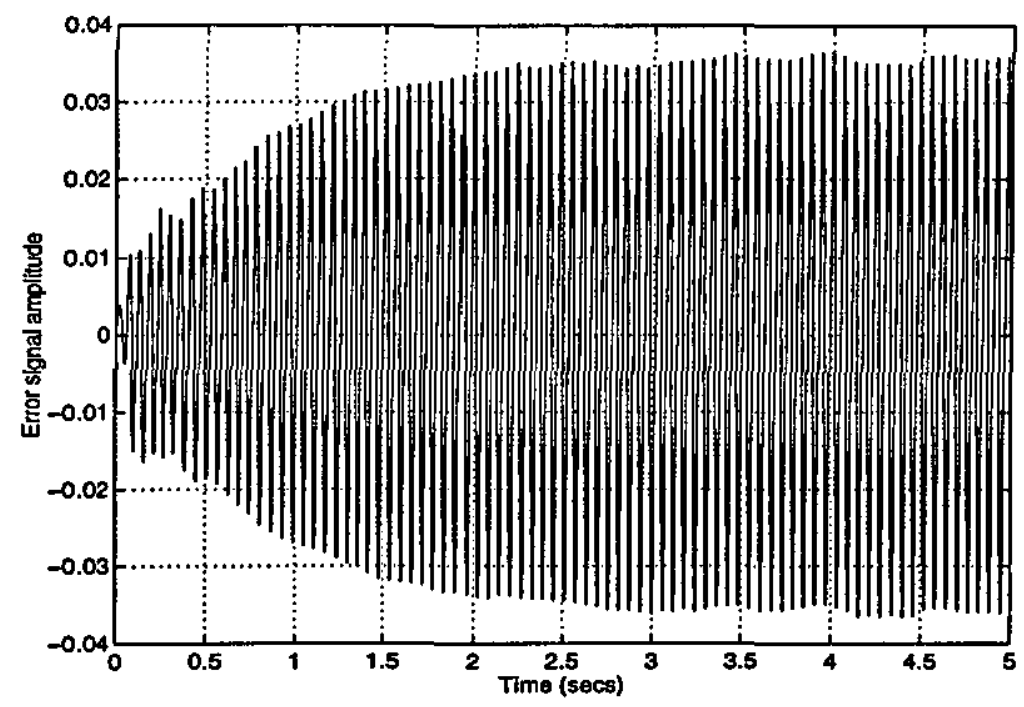

Figure 8.8 -Limit-cycle condition for reduced order aircraft model

\section{$\underline{8.2 .6 \text { Conclusions }}$}

In conclusion, it is possible to predict the existence of limit-cycles within a typical aeroservoelastic system from a consideration of the describing function of the software rate limiter. Such an application of the describing function allows the characteristic equation of the system to be solved to predict the system's limit-cycles. This prediction is reliant however on the accurate linearisation and modelling of the remaining elements of the system.

\subsection{Prediction of limit-cycles in the presence of phase uncertainty}

\subsubsection{Introduction}

The above section has described a method for predicting the existence of limitcycles in a nonlinear system. In this case the only nonlinear element that has been considered is the software rate limiter. Comparison of the predicted limit-cycles with those obtained from time domain simulation has shown that such a simplification of the nonlinearities still produces a good estimate of the limit-cycle frequency and amplitude.

Unfortunately, this procedure relies on the existence of reliable frequency response data for all the linear elements of the system. In the case of the real aircraft system, this is not the case as has been discussed in Chapter 2 . Although ground vibration tests provide reliable measurements of the open-loop gain of the aircraft system, there exists a large degree of uncertainty in the phase response of the system. This is due in part to uncertainties in the modelling of the unsteady aerodynamics and also in the 
phase relationships between the many possible signal paths that exist within a typical flight control system. At present, clearance procedures allow for this uncertainty in the phase by neglecting its influence on the stability of the system and by assuming inphase addition of all the signal paths.

If the phase response of the system cannot be relied upon, then the use of these limit-cycle prediction techniques is restricted. The following section discusses to what extent the limit-cycle condition can be analysed in the presence of such uncertainties.

\subsubsection{Limit-cycle prediction in the presence of phase uncertainty}

Consider the characteristic equation of the aircraft system as given in Equation (8.20). If no phase information is available, then the solution of the characteristic equation becomes one of gains only, such that

$$
|H(j \omega)|\left|G_{A C T}(j \omega)\right|=\frac{1}{\left|G_{T}(j \omega, E)\right|}
$$

where

$$
\begin{gathered}
\left|G_{T}(j \omega, E)\right|=\left|G_{A C 1}(j \omega)\right|\left|G_{1}(j \omega)\right|\left|G_{N 1}(j \omega, E)\right|+ \\
\left|G_{A C 2}(j \omega)\right|\left|G_{2}(j \omega)\right|\left|G_{N 2}(j \omega, E)\right|+\left|G_{A C 3}(j \omega)\right|\left|G_{3}(j \omega)\right|\left|G_{N 3}(j \omega, E)\right|
\end{gathered}
$$

and

$$
\left|G_{N x}(j \omega, E)\right|=\frac{4 \beta}{\omega\left|G_{x}(j \omega)\right| E \pi}
$$

Whereas in the earlier case there was a single solution, there now becomes an infinite number of possible solutions. In reality, the actual solution that exists is dependent on the phase response. Since this phase response is not reliably known, then it has to be assumed that a limit-cycle could occur at all frequencies

Substituting for equation (8.26) into equation (8.25) where appropriate, the characteristic equation can be expressed as

$$
|H(j \omega)|\left|G_{A C T}\right|(j \omega)=\frac{1}{\frac{4 \beta}{\omega E \pi}\left(\left|G_{A C 1}(j \omega)\right|+\left|G_{A C 2}(j \omega)\right|+\left|G_{A C 3}(j \omega)\right|\right)}
$$

which can be re-arranged to result in an equation for the amplitude of the limitcycle in terms of the error signal, such that

$$
E=\frac{4 \beta}{\omega \pi}\left|G_{A C T}(j \omega)\right|\left(\left|G_{A C 1}(j \omega)\right|+\left|G_{A C 2}(j \omega)\right|+\left|G_{A C 3}(j \omega)\right|\right)|H(j \omega)|
$$

Consideration of equation (8.28) reveals that the limit-cycle amplitude at any given frequency is simply the maximum output of the rate limiter multiplied by the 
loop gain between this point and the point at which the limit-cycle amplitude is desired. In this case, where the limit-cycle amplitude is given in terms of the error signal, the amplitude is as given by equation (8.28). In addition, the worst case is assumed where the three signal paths are assumed to act in accordance with current design methodology. It would also be possible to account for changes in flight condition within the form of equation (8.28) by augmenting the aircraft gain terms accordingly.

Now, consider that

$$
\frac{4 \beta}{\omega \pi}\left|G_{A C T}(j \omega)\right|=X(j \omega)
$$

where $X(j \omega)$ is the maximum output of the linear actuator and rate limiter combination at any given frequency. It is therefore possible to apply the actuator performance boundary as demonstrated in Chapter 7 to the prediction of the limitcycle amplitudes by substituting the envelope for $X(j \omega)$ in Equation (8.28). This enables the amplitudes of the limit-cycles to be predicted from the performance limit of the actuator and the gain response of the remaining linear elements of the system. In addition, the linearisation of the actuator is no longer necessary in order to predict such limit-cycle amplitudes. The presence of uncertainty in the phase response has therefore restricted the prediction of the limit-cycles within a system to the estimation of only the limit-cycle amplitudes.

\subsubsection{Application of the prediction of limit-cycle amplitude to an aircraft system}

In order to demonstrate the prediction of the maximum limit-cycle amplitudes in a typical system, consider the aircraft system model as shown in Figure 8.7. In this case however, the model will contain the nonlinear actuation system model developed in Chapter 7 as opposed to the linear model used earlier. In addition, all of the structuralmodes will be included in the analysis.

From equations (8.28) and (8.29), the elements required to calculate the limitcycle amplitudes at the error signal position are as shown in Figure 8.9 and 8.10. 


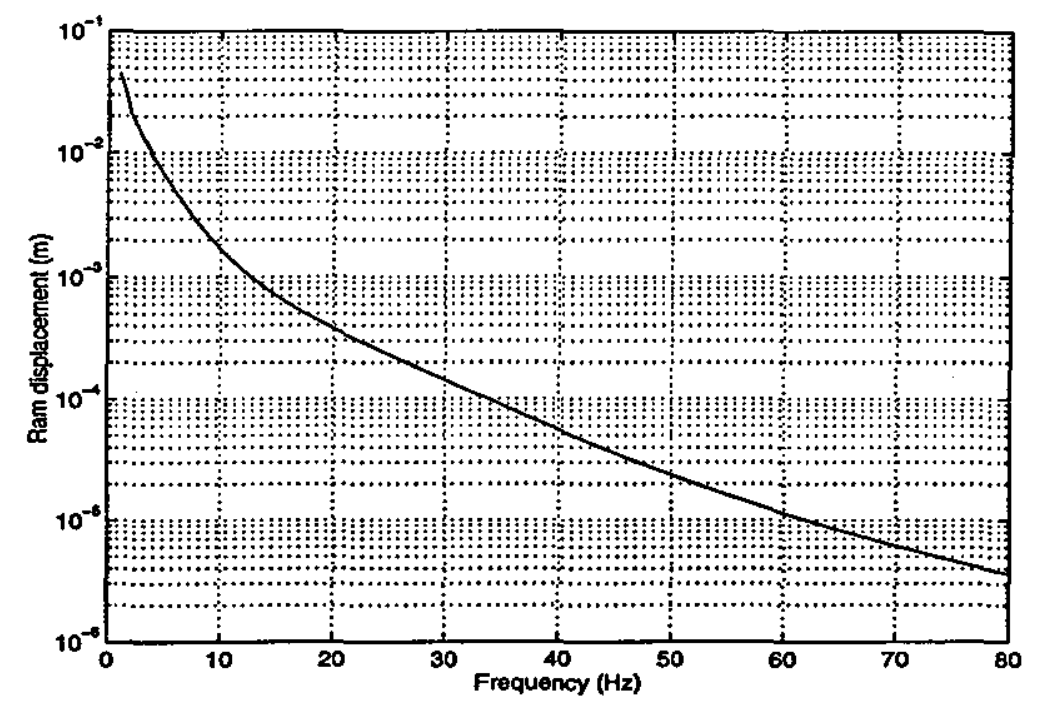

Figure 8.9 - Actuator performance limit, $X(j \omega)$

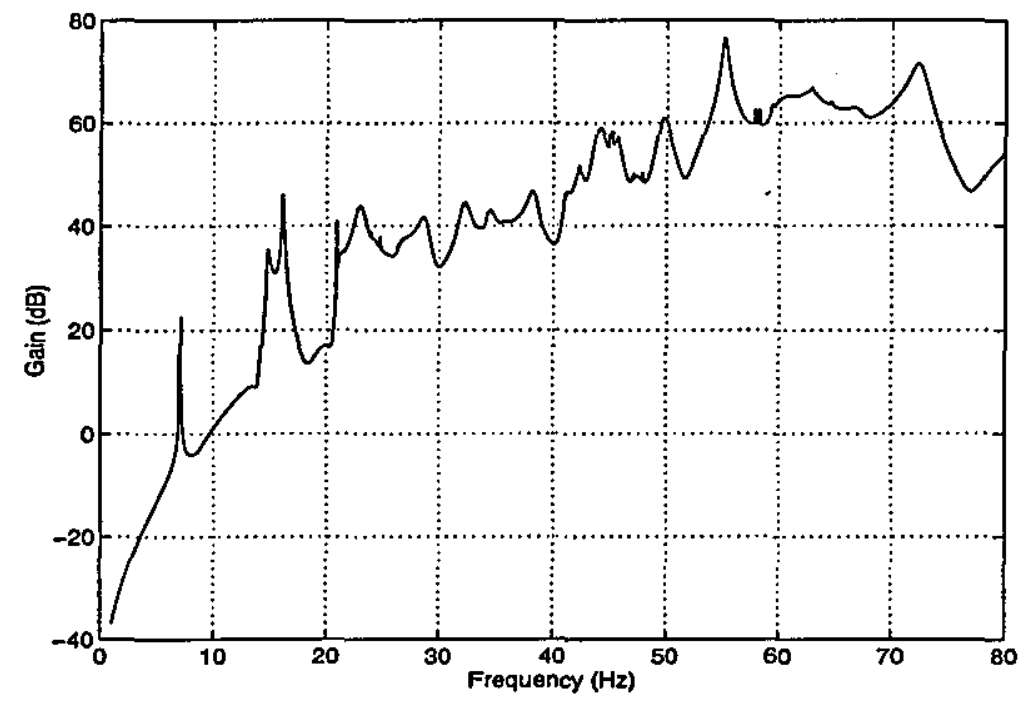

Figure 8.10 - Loop gain, $\left(\left|\mathrm{G}_{\mathrm{AC1}}(j \omega)\right|+\left|\mathrm{G}_{\mathrm{AC} 2}(j \omega)\right|+\left|\mathrm{G}_{\mathrm{AC}}(j \omega)\right|\right)|\mathrm{H}(j \omega)|$

Combining the above two figures according to equation (8.28) along with the necessary scaling between ram extension and control surface motion results in a maximum boundary for the limit-cycle amplitude at the error signal location ${ }^{72,73}$. This boundary is as shown in Figure 8.11. 


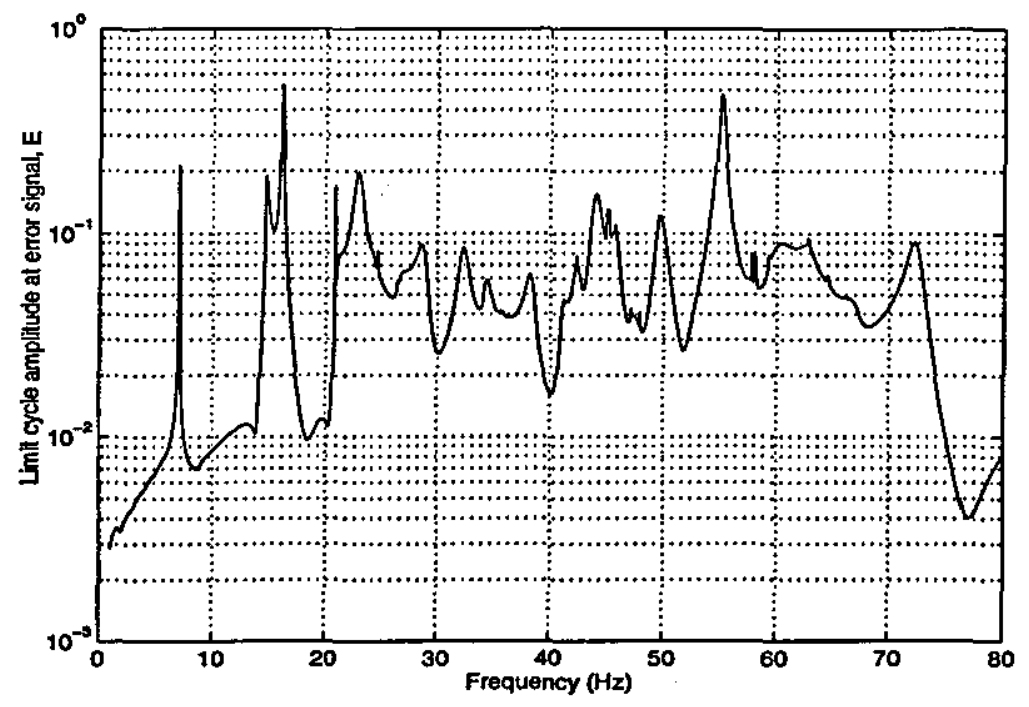

Figure 8.11 - Maximum limit-cycle amplitude at error signal, E

The above theory has demonstrated that the maximum amplitude of the limitcycles within the system can be quickly defined using the performance boundary of the actuation systems and the gain response of the aircraft structure and flight control system. The ability to predict such amplitudes enables their effect on the control of the aircraft's rigid-body motion to be assessed. In reality, the existence of limit-cycles within the system would not be tolerated for long periods of time. Such conditions would have serious consequences both in terms of the fatigue life of the aircraft structure and in terms of the wear of actuator components. In addition, were a limitcycling condition to arise, a large amount of power would be dissipated within the flight control system in responding to it. As a result, it is desirable to ensure that such limit cycling conditions do not arise under normal circumstances.

\subsubsection{Conclusions}

The existence of uncertainty in the phase in the modelling of the aircraft structure has been shown to result in a restriction in the solution of the characteristic equation. Fortunately, this uncertainty in phase does allow for the prediction of the maximum amplitude of the limit-cycles however. In addition, the solution of the characteristic equation in the presence of this uncertainty allows the application of the actuator performance boundary as obtained in Chapter 7. The consequence of this is that any errors introduced by the linearisation of the actuator can be avoided.

\subsection{Prevention of limit-cycles}

\subsubsection{Introduction}

Sections 8.2 and 8.3 have discussed in some detail the prediction of limit-cycles 
within nonlinear systems. In particular, systems where the phase response of the system is unreliable have been considered. In the case of the aircraft system however, limit-cycling conditions must be avoided. The effects of a limit-cycling condition existing within the aircraft system are many. Firstly, the results of Chapter 7 have demonstrated that actuator performance can be affected as a result of the existence of high-frequency signals at its input. Even if it were shown that the amplitude of the limit-cycle was not sufficient to cause unsatisfactory rigid-body response, highfrequency inputs to the actuator could result in significant wear. In addition, the power consumption of the actuator in terms of electrical power required to move the servo valves and in producing the required hydraulic flow would be significantly increased. Persistent limit-cycling would have the potential to create an oscillatory loading of the aircraft's structure with a consequent reduction in fatigue life.

The following section describes how limit-cycles can be prevented in the case of the aircraft system under consideration, where the phase response is not known.

\subsubsection{Criteria for the prevention of limit-cycles}

Returning to the system of section 8.2.4, the system as shown in Figure 8.1 has the characteristic equation

$$
G_{1}(j \omega) G_{2}(j \omega) G_{F}(j \omega)=\frac{-1}{G_{n}(j \omega, E)}
$$

Consider now that the gain of the rate limiter, $G_{n}(j \omega, E)$, which can never be greater than unity by definition. As a result, the magnitude of the right hand side of equation (8.30) can never be less than unity. The result of this in terms of the Nyquist diagram is that the locus of the right hand side of equation $(8.30)$ originates from the $(-1,0)$ point and never enters the unit circle. In order to prevent a possible limit-cycle condition, it is therefore adequate to ensure that the locus of the left hand side of equation (8.30) remains within the unit circle. If this is achieved, then the two loci cannot intersect and no limit-cycle can occur. This can be demonstrated graphically as shown on the Nyquist diagram of Figure 8.12. 


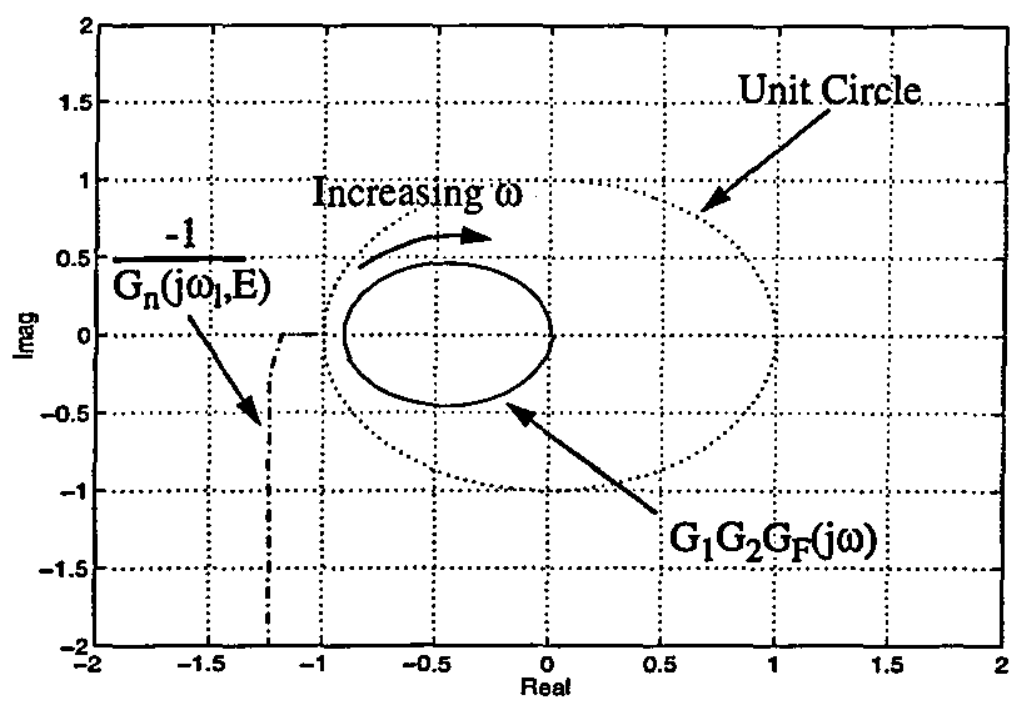

Figure 8.12 - Nyquist diagram for arbitrary system

In this contrived example, the linear elements of the example system considered in section 8.2.4 have been attenuated by a gain sufficient to bring the response within the unit circle. Although the inclusion of such a gain in the system may not result in the required closed-loop response, it will ensure that a limit-cycling condition may not occur.

Such a criteria may be satisfied in the case of the aircraft system even in the presence of phase uncertainty. Since the criteria only depend on the open-loop gain of the system, phase effects are unimportant. If phase information were available at certain structural frequencies, it may be possible to relax this requirement. For example, if reliable phase information was available for the $7 \mathrm{~Hz}$ structural-mode, and it was found that it did not cross the locus of the rate limiter describing function, then a limit-cycle could not result.

In summary, provided that the open-loop gain of the system is less than unity, the Nyquist plot for the linear system cannot intersect the rate limit describing function at any point. As a result, the existence of a limit-cycle is not possible given the nature of this particular nonlinearity.

As with the current clearance procedure, suitable filters introduced into the feedback path may be used to reduce the open-loop gain to the required level. It is important to note that, should the model of the aircraft be in error, then the nature of the any potential limit-cycle is predictable. In addition, the results of Chapter 7 have demonstrated that if a limit-cycle does occur, its effect in terms of rigid aircraft response can be quantified. 


\subsection{Specification of an alternative clearance procedure}

\subsubsection{Introduction}

The previous sections have introduced the concept of limit-cycle prediction and prevention within an aeroservoelastic system. The ability to prevent the occurrence of limit-cycles under nominal conditions, and predict their effect in the case of their existence, allows an alternative clearance procedure to be suggested. The following section discusses such an alternative procedure

\subsubsection{Specification of an alternative clearance procedure}

The current clearance procedure as discussed and demonstrated in Chapters 2 and 4 respectively, assumes that the aircraft system can be considered to be linear. The result of this is that feedback filters are designed so as to ensure closed-loop stability by ensuring a maximum open-loop gain of $-9 \mathrm{~dB}$. This safety margin of $9 \mathrm{~dB}$ ensures that even in the presence of significant modelling errors, closed-loop stability will be assured. This large safety margin was applied due to uncertainty in the effect of an unstable structural-mode on the aircraft as a whole.

The previous sections have discussed in some detail the effect of the nonlinear nature of the actuation system on the aeroservoelastic problem. In particular, it has been demonstrated that due to the existence of the rate limiting function within the FCS, an unstable structural response may only result in a limit cycling condition. The criteria for the existence of such a limit-cycle have been introduced earlier. In the presence of uncertainty of the phase response however, it has been shown that limit cycling conditions may arise wherever the open-loop gain of the system exceeds $0 \mathrm{~dB}$.

The affect of allowing limit-cycles to exist in the nominal case has been discussed earlier, and as a result suitable filters should be incorporated into the system to give a maximum open-loop gain of less than $0 \mathrm{~dB}$. The question that remains however is to what extent should the open-loop gain be attenuated below this level in order take account of possible modelling errors.

Fortunately, in the event of modelling errors causing the open-loop gain to become greater than unity, any resulting limit-cycle can be predicted. For example, for the case of the system model being in error, any frequency at which the open-loop gain exceeds $0 \mathrm{~dB}$ may result in a limit-cycle. Importantly however, the amplitude of such a limitcycle can be predicted and its effect on the satisfactory control of the rigid-body aircraft assessed.

Suppose that the structural filters were designed so as to give a maximum open- 
loop gain of $-1 \mathrm{~dB}$. As a result, the phase lag introduced by these filters will be significantly less than that introduced by the - $9 \mathrm{~dB}$ filters developed in Chapter 4 . In the mominal case, these filters will ensure that a limit cycling condition could not arise. One consequence of this action however, would be that any error in the modelling of the system could result in a limit cycling condition occurring.

Consider a situation where an error in the modelling has indeed resulted in an inflight limit-cycle condition. Such a condition would be more likely at high aircraft incidence where the FCS gains are highest. Provided that such a possibility has been imvestigated in terms of the limit-cycle amplitude and its effect on the actuator performance, rigid-body stability will be maintained. The aircraft incidence could then be safely reduced, whereupon the limit-cycle would dissipate as a result of the reduction in $\mathrm{FCS}$ gain $^{73}$. If such an in-flight interaction were encountered, then it would be possible to correct the flexible aircraft model accordingly, redesigning the structural-mode filters so as to maintain the $-1 \mathrm{~dB}$ maximum open-loop gain for the nominal case. Provided that a suitable safety margin has been explored in terms of limit-cycle amplitude and its effects, the implementation of structural filters giving a $1 \mathrm{~dB}$ open-loop gain should be free of risk to the aircraft.

The proposed alternative design procedure can be represented by the flow diagram given in Figure 8.13. The initial stages of the design process are identical to that currently employed. Firstly, a flexible aircraft model is developed, which when combined with a model of the flight control system allows the production of an envelope showing maximum open-loop gain versus frequency. These results can be modified by actual ground test data when available. As for the current design method, it is assumed that all the signal paths act in-phase. The next stage in the design process is to design suitable structural-mode filters to meet the $-1 \mathrm{~dB}$ maximum open-loop gain requirement.

In parallel with this work, the performance limit of the actuation systems is derived both from modelling and bench tests of the actual hardware. Once this has been obtained, it can be combined with the model of the remaining elements within the system. This results in a specification of the maximum filtered system response, assuming the system model to be correct. As a check that all is well at this stage, if the amplitude of the structural feedback signals at the rate limiter are calculated under these conditions, the rate limit should not be exceeded. 


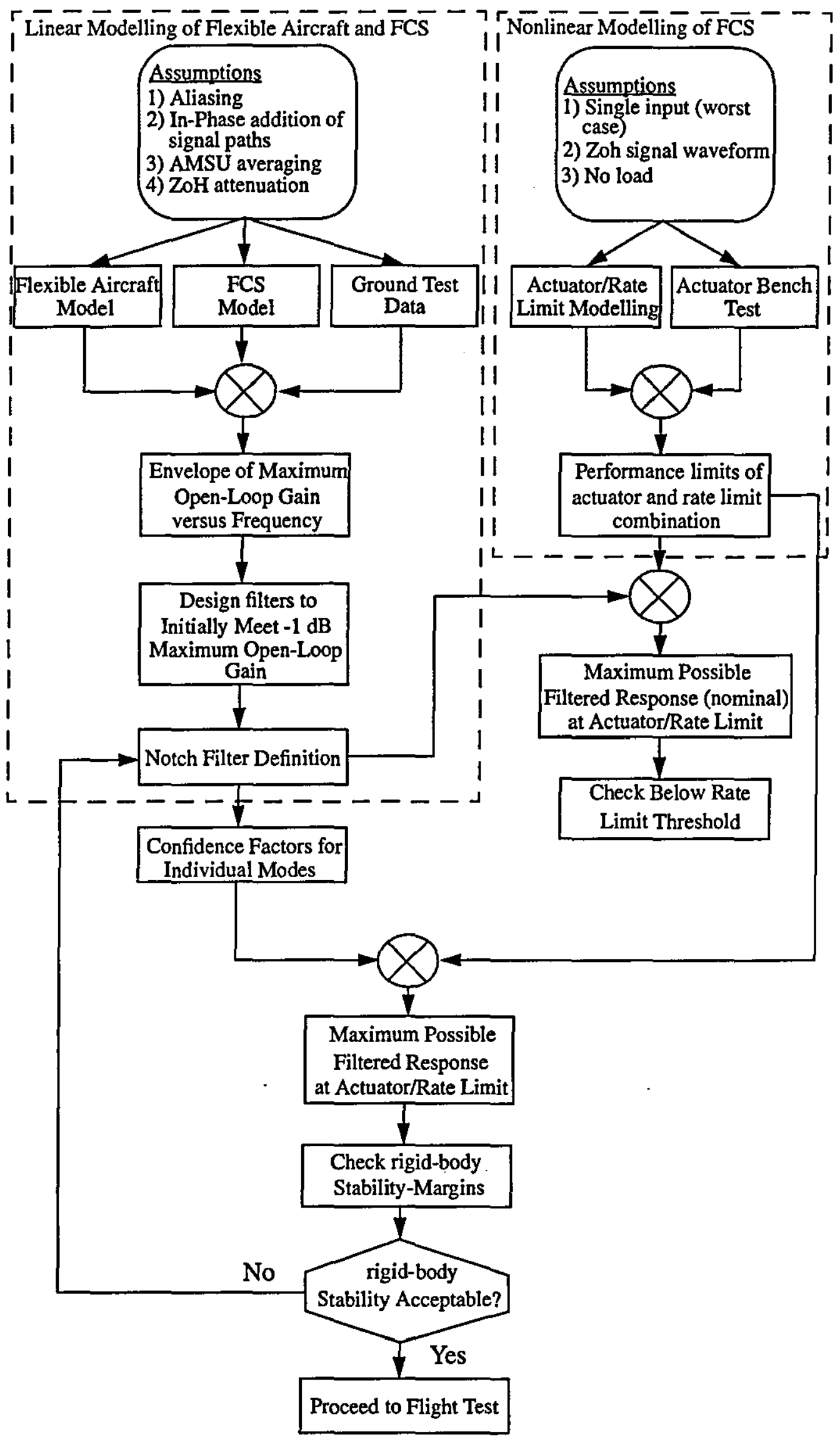

Figure 8.13 - Proposed aeroservoelastic design and clearance process 
The next stage in the design process is to consider the effect of any errors within the modelling of the system. This could be expressed in terms of an overall increase in gain, or a more specific gain increase for each structural-mode. For example, it may be felt that the system model might be in error by a certain factor. Alternatively, results from ground test and where possible in-flight tests, might lead to a greater confidence in the gain of particular structural-modes. Once obtained, such an "error" model may be used to predict the maximum possible filtered system response. This envelope will therefore permit the prediction of the amplitude of any limit-cycle that may exist within the system. Assuming such a situation to be the case, the effect of these limitcycles on rigid-body performance can be assessed from a consideration of their effects on actuator performance.

If it were found that none of the predicted limit-cycles caused unsatisfactory rigidbody response, then it would be safe to proceed to flight testing. Alternatively, if it were found that a particular limit-cycle had the potential for causing unsatisfactory rigid-body response, then the structural-mode filters should be compensated accordingly.

Although in the presence of modelling errors, the potential for limit-cycles may exist, it is still not certain that they will occur. The discussion of the criteria for limitcycles made earlier in the chapter has highlighted the need for the correct phase response before a limit-cycle occurs. Combining this requirement with the fact that the separate control paths will almost certainly not act in-phase as is assumed, makes the existence of an in-flight limit-cycle a remote possibility. Such conditions for the actual occurrence of a limit-cycle are highlighted within Figure 8.14.

In the following section, the alternative design procedure is demonstrated using the aircraft model developed in Chapter 4.

\subsection{Demonstration of alternative clearance procedure on analogue aircraft system}

\subsubsection{Introduction}

The above section has described an alternative procedure for aeroservoelastic design and clearance. This alternative procedure is based on the prevention of limitcycles within the nominal aircraft system, and the prediction of limit-cycle amplitudes should the system model be in error. The following section describes such a design procedure for the aircraft system model developed in Chapter 4. Initially, the digital nature of the control system will be neglected. 


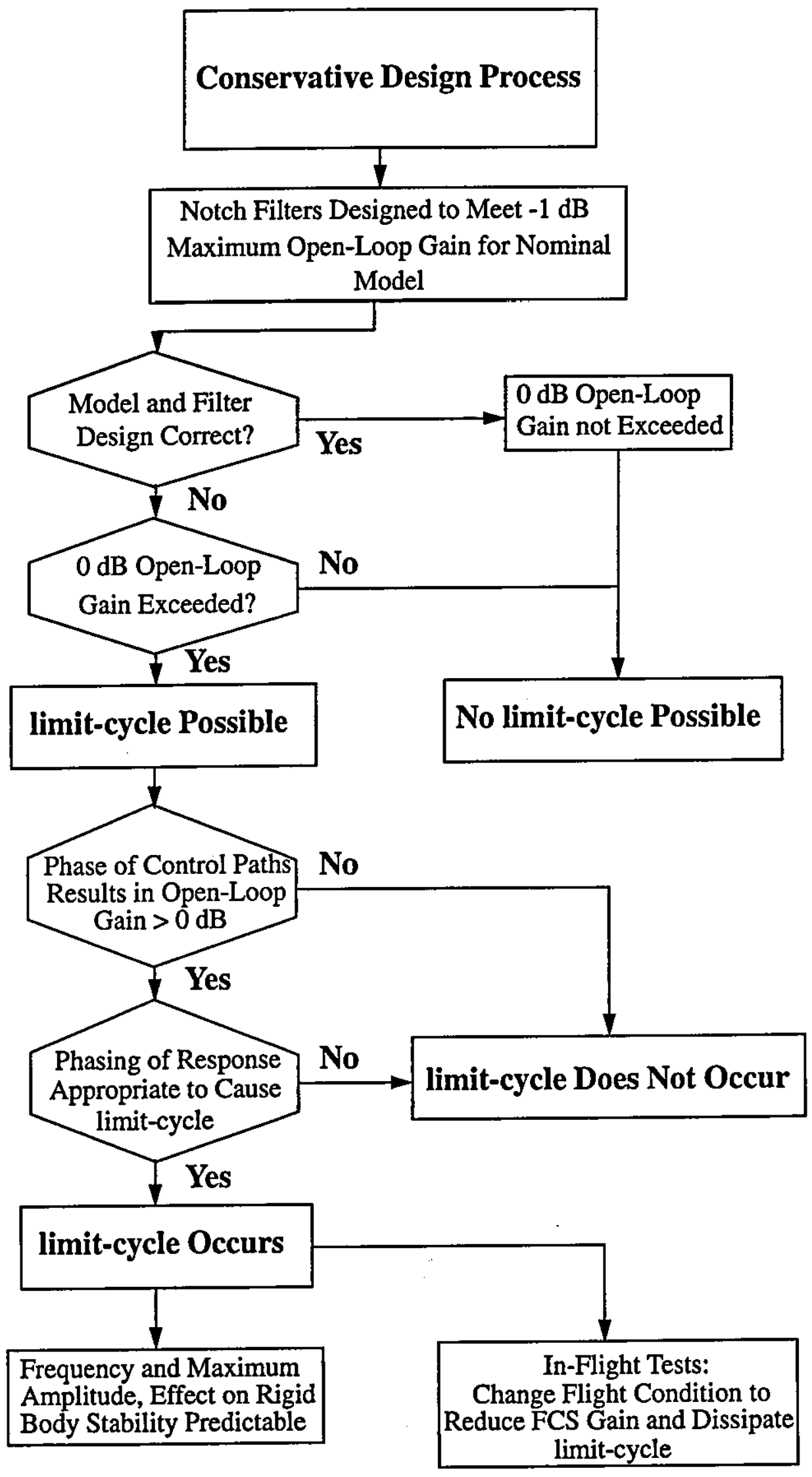

Figure 8.14 - Conditions for limit-cycle oscillation and implications 


\subsubsection{Design of structural-mode filters}

In order to design suitable structural-mode filters under the alternative design procedure, it is necessary to produce a model of the aircraft system as for the current design procedure. Such a model has been developed in Chapters 3 and 4 . In order to prevent a limit-cycle condition arising, it has been discussed that it is sufficient to ensure that the open-loop gain of the system is less than unity. In order to achieve this, filters can be designed for implementation within the feedback path of the aircraft. Although this is identical to the current design procedure, it is important to note that in this case, filters are designed to give a maximum open-loop gain of $-1 \mathrm{~dB}$. This is in contrast to the current design procedure which results in a maximum open-loop gain of $-9 \mathrm{~dB}$.

Producing the maximum open-loop gain for the earlier aircraft model for all flight conditions results in a specification of the structural-mode filter attenuation as shown in Figure 8.15.

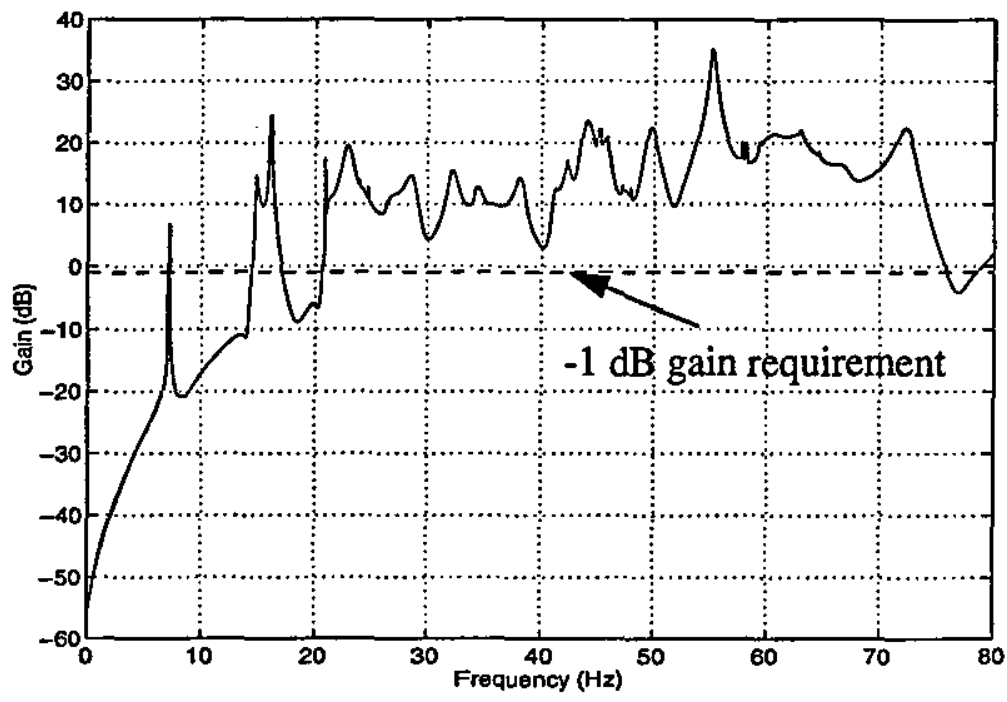

Figure 8.15 - Maximum open-loop modal response envelope for full flexible aircraft system model.

If suitable structural-mode filters are designed so as to meet the attenuation requirements defined in Figure 8.15, the resultant filters are

$$
\begin{gathered}
G_{s f 1}(s)=\frac{s^{2}+0.90 s+2018}{s^{2}+2.7 s+1968} \\
G_{s f 2}(s)=\frac{s^{2}+1.62 s+10250}{s^{2}+2 s+9990} \\
G_{s f 3}(s)=\frac{s^{2}+1.49 s+8636}{s^{2}+7 s+8420} \\
G_{s f 4}(s)=\frac{1.648 e-1 \times\left(s^{2}+5.3854 s+113290\right)\left(s^{2}+2.2307 s+19437\right)}{s^{2}+274 s+29821\left(s^{2}+50 s+13131 e 4\right)}
\end{gathered}
$$


where,

$\mathrm{G}_{\text {sf } 1}(\mathrm{~s})$ is a notch filter centred on $7.15 \mathrm{~Hz}$

$\mathrm{G}_{\mathrm{sf} 2}(\mathrm{~s})$ is a notch filter centred on $16.1 \mathrm{~Hz}$

$\mathrm{G}_{\mathrm{sf} 3}(\mathrm{~s})$ is a notch filter centred on $14.8 \mathrm{~Hz}$

$\mathrm{G}_{\mathrm{sf} 4}(\mathrm{~s})$ is a low-pass filter designed to attenuate the high-frequency modes

The procedure used in the calculation of the above filters is described in Chapter 4.

Applying these filters to the maximum open-loop gain as shown in Figure 8.15 results in the maximum open-loop gain for the filtered system as shown in Figure 8.16.

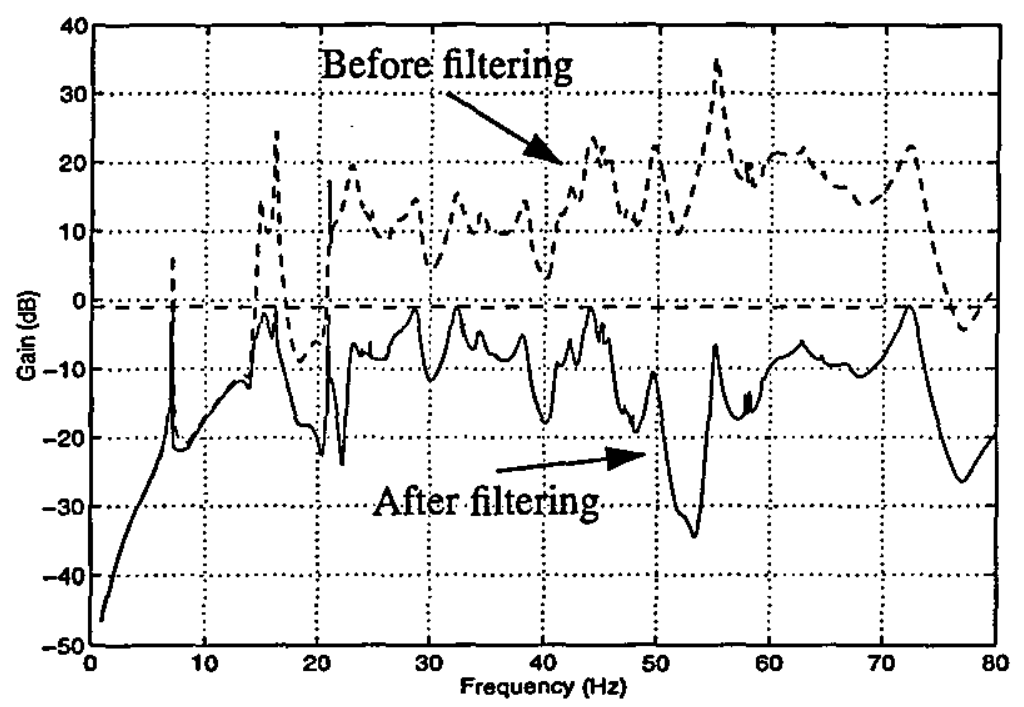

Figure 8.16 - Maximum open-loop gain for aircraft system after filtering

The above figure demonstrates that the required level of attenuation has been achieved resulting in the maximum open-loop gain of the system being less than -1 dB. As a result, inclusion of such filters into the aircraft system will ensure that a limit cycling condition cannot arise.

Examining the differences in frequency response between these filters and those designed to achieve a maximum open-loop gain of $-9 \mathrm{~dB}$ reveals the advantage of adopting such an alternative clearance procedure. The frequency responses as shown in Figure 8.17 demonstrate that there is a significant reduction in the phase lag of the filters at rigid-body frequencies. This fact can be clearly seen from the phase response of the filters at rigid-body frequencies as shown in Figure 8.18. 

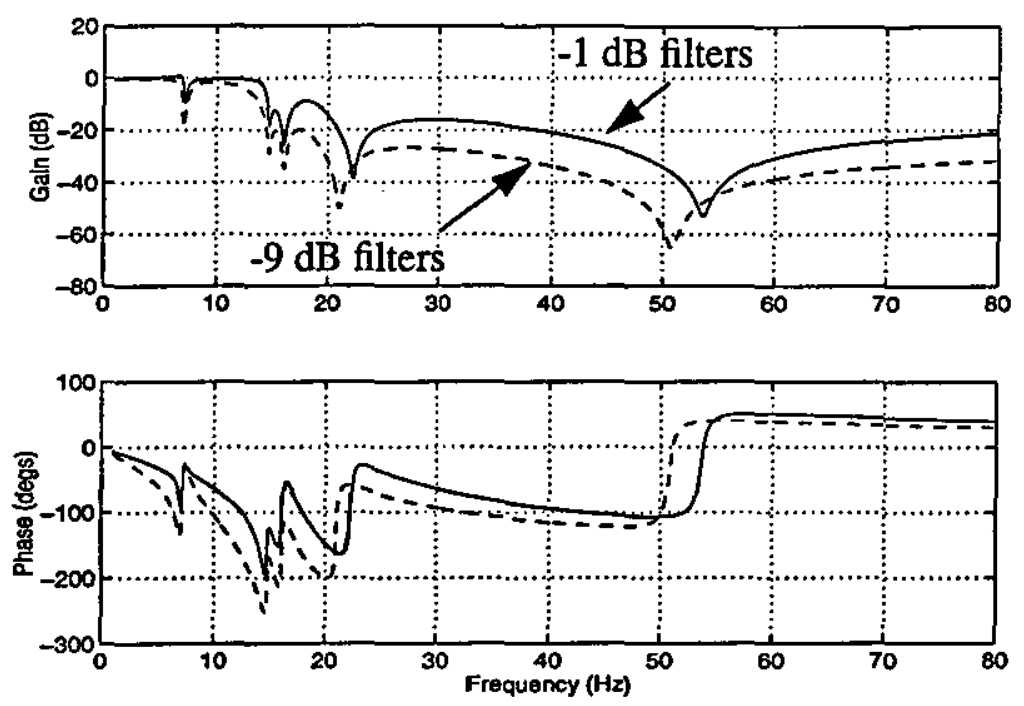

Figure 8.17 - Structural-mode filter frequency response for both $-1 \mathrm{~dB}$ and $-9 \mathrm{~dB}$ clearance requirements

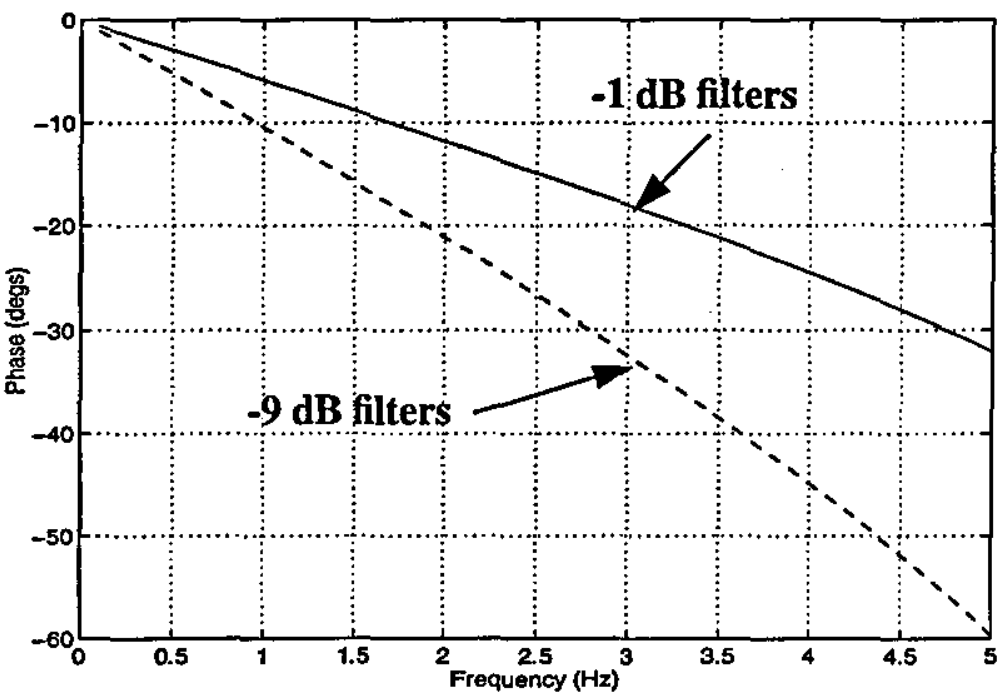

Figure 8.18 - Structural-mode filter phase response for both $-1 \mathrm{~dB}$ and $-9 \mathrm{~dB}$ clearance requirements

As a numerical comparison, the phase lag of the $-1 \mathrm{~dB}$ filters at a frequency of $3 \mathrm{~Hz}$ is -18.0 degrees. This compares very favourably to a phase lag of -32.4 degrees for the current $-9 \mathrm{~dB}$ filters at the same frequency. It can be seen that there is a significant advantage to be gained in applying a clearance requirement of $-1 \mathrm{~dB}$ for the structuralmodes. Such an advantage has been achieved at the expense of the system robustness to modelling errors however.

The ability of the $-1 \mathrm{~dB}$ filters to prevent a limit-cycle condition relies on the actual aircraft response being accurately modelled. Any increase in the system gain above that represented in Figure 8.15 may result in the open-loop gain of the filtered system exceeding $0 \mathrm{~dB}$. This could in turn result in a limit cycling condition. It is important therefore to assess what impact such a situation would have on rigid-body control. 
The ability to predict the possible outcome of an error in the modelling of the system is crucial to this alternative clearance procedure. For the linear system, it must be assumed that a structural-mode whose open-loop gain is greater than $0 \mathrm{~dB}$ would result in an unbounded structural oscillation in the closed-loop. The nonlinear nature of the system allows the prediction of any resulting limit-cycle and its effects, allowing a confident reduction in the structural-mode clearance requirements.

It should be noted that even if the gain of the open-loop system were to exceed 0 $\mathrm{dB}$, the existence of a limit-cycle is by no means certain. As has been discussed earlier, the existence of a limit-cycle is governed by consideration of both gain and phase. The consequence of this is, if the gain is greater than $0 \mathrm{~dB}$, a limit-cycle will only occur if the phase response of the system is appropriate. In terms of a Nyquist diagram, even though the response of the linear elements may exceed the unit circle, it may still not cross the locus of the rate limiter describing function at a compatible frequency.

\subsubsection{Limit-cycle prediction in the presence of system modelling errors}

The results of earlier sections have shown how it is possible to predict the maximum amplitude of any possible limit-cycles within a system. In this case, provided that the system gain is as modelled, then no limit-cycles will occur due to the presence of correctly designed structural-mode filters. In the presence of modelling errors however, the amplitude of any limit-cycle can be obtained and its effect on the rigid-body control assessed.

Consider the nominal system model as developed in Chapter 4, with no structuralmode filters in the feedback path. The resulting maximum amplitude of any limitcycle oscillation can be predicted as in section 8.3. This results in the maximum amplitude envelope as shown in Figure 8.11. If the $-1 \mathrm{~dB}$ structural filters were now incorporated into the system, then the maximum amplitude of any resultant limit-cycle can be predicted as shown in Figure 8.19. 


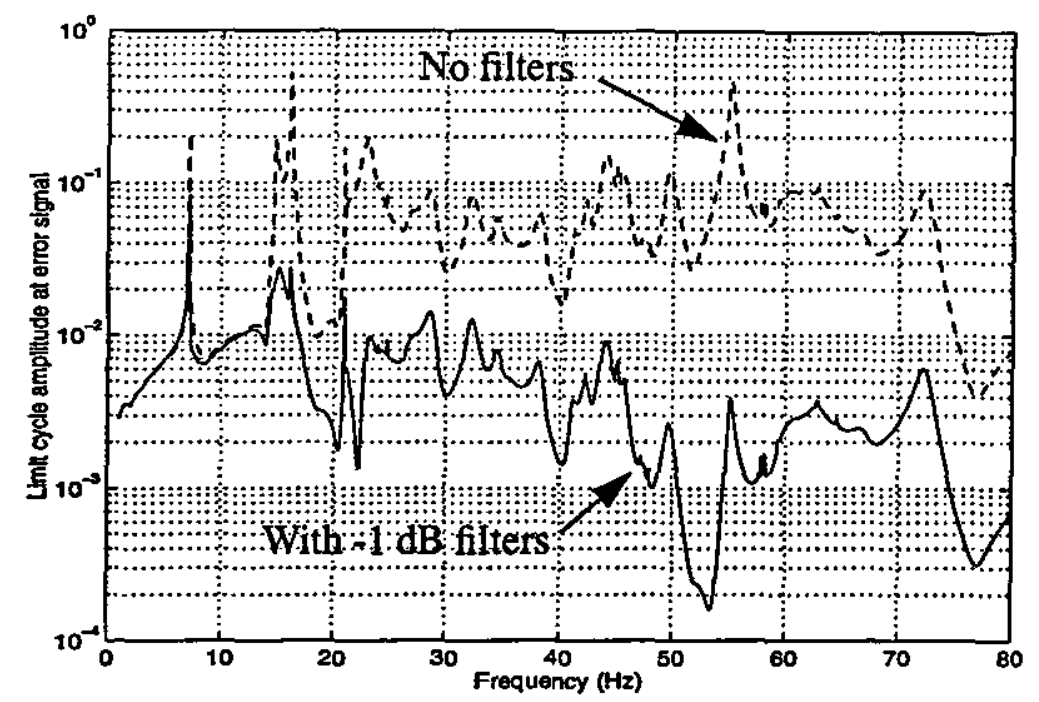

Figure 8.19 - Maximum limit-cycle amplitude at error signal - nominal model, -1 dB filter

Production of such an envelope in the case of the nominal model is purely an academic exercise. In reality, provided that the system gain is as modelled, then the -1 $\mathrm{dB}$ filters will prevent limit cycling. Production of the maximum amplitude envelope for the nominal model does allow the effect of modelling errors to be quickly assessed however.

Suppose for example that the open-loop gain of the system was in error by a factor of 2. From Figure 8.19, the amplitude of any possible limit-cycle can be easily obtained. The resulting amplitude envelope is as shown in Figure 8.20.

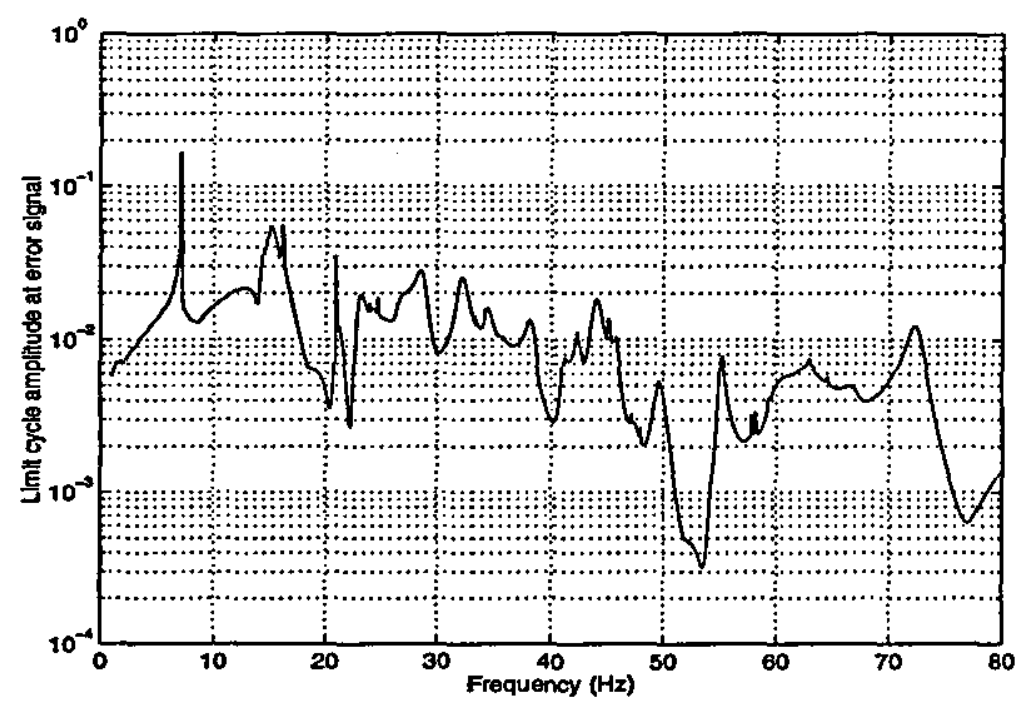

Figure 8.20 - Maximum limit-cycle amplitude at error signal - 2* nominal model, $-1 \mathrm{~dB}$ filters

Even though the system model is in error to such a degree however, it is still only possible for limit-cycles to occur where the open-loop gain of the system exceeds 0 dB. 


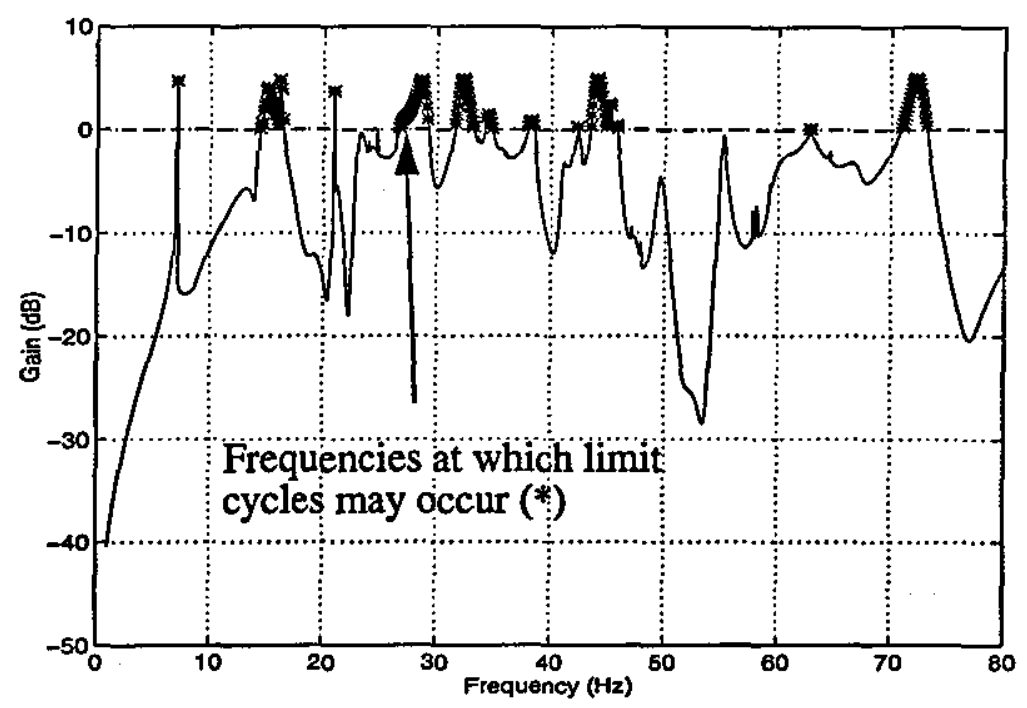

Figure 8.21 - Maximum open-loop gain for system with $2 *$ nominal gain and -1 dB filters in place

The open-loop gain response of such a system is as shown in Figure 8.21 where the it is possible to identify those frequencies at which a limit-cycle may occur as those at which the open-loop gain is greater than $0 \mathrm{~dB}$. Incorporating these results on to the specification of the maximum limit-cycle amplitude results in a prediction of the possible limit-cycle frequencies and amplitudes when the system gain is twice that of the nominal model. Such a prediction is shown in Figure 8.22.

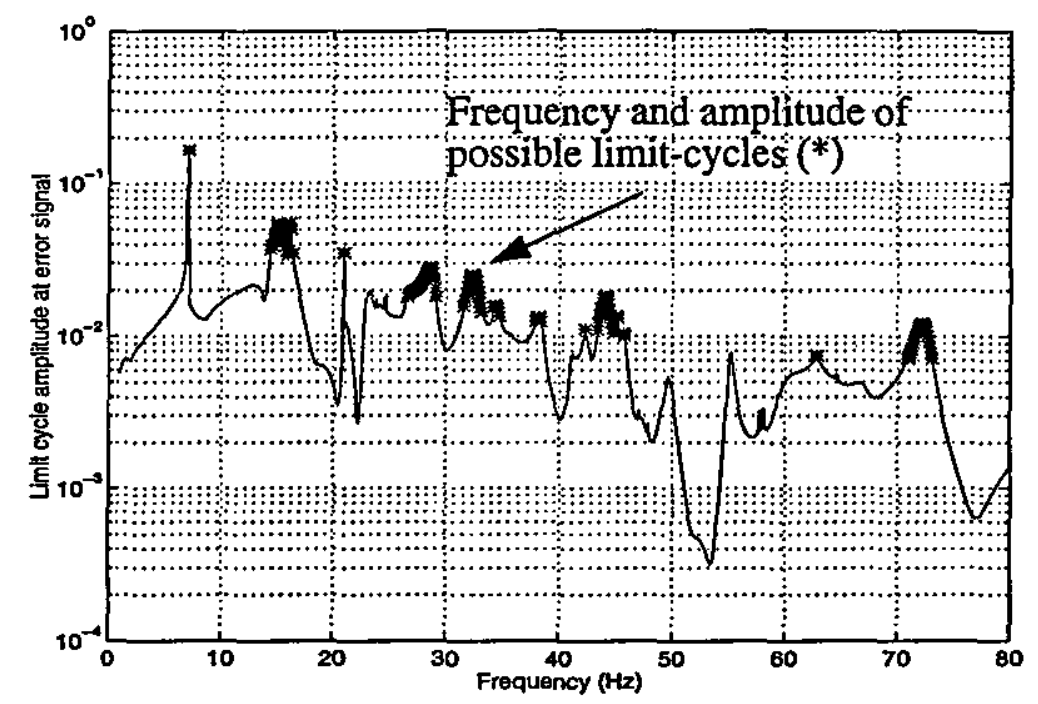

Figure 8.22 - Predicted limit-cycle frequencies and amplitudes for system with $2 *$ nominal gain and $-1 \mathrm{~dB}$ filters

It can be seen therefore that it is possible to predict both the frequency and amplitude of limit-cycles that may exist within the system given a particular level of error in the modelling of the system. In this case, this error was chosen as being a twofold increase in the open-loop gain of the structural-modes.

It is important to be able to assess the effect, if any, of such limit cycling 
conditions on the satisfactory rigid-body control of the aircraft. If it can be shown that satisfactory rigid-body control is maintained then the $-1 \mathrm{~dB}$ filters can be applied as designed. In this way, the condition where the system model is significantly in error can be explored and the safety of the system ensured.

\subsubsection{Estimation of the effect of a limit cycling condition on rigid aircraft stability}

The previous section has demonstrated how the frequency and amplitude of any possible limit-cycle within the system may be calculated for the case where the system model is in error. From Figure 8.22, the potential limit-cycles for the situation where the system gain is twice that modelled are given in Table 8.2.

The impact of such limit-cycles on the rigid-body control of the aircraft can be assessed using several methods. The effect of limit-cycles on the performance of the actuators alone can be assessed from a consideration of the results of Chapter 7, or from rig testing. These results can then be applied to the whole aircraft system in order to determine the effect of the limit-cycle on rigid-body stability-margins. A second method of assessing the effect of such limit-cycles on aircraft stability is to inject a signal of suitable amplitude and frequency into a simulation of the whole aircraft system. This method will be discussed in more detail later.

\begin{tabular}{|c|c|}
\hline $\begin{array}{c}\text { Frequency } \\
(\mathrm{Hz})\end{array}$ & $\begin{array}{c}\text { Amplitude } \\
\text { at error } \\
\text { signal }\end{array}$ \\
\hline \hline 7.1 & 0.16 \\
\hline 15.1 & 0.055 \\
\hline 20.9 & 0.035 \\
\hline 28.6 & 0.028 \\
\hline 44.0 & 0.019 \\
\hline 72.2 & 0.013 \\
\hline
\end{tabular}

Table 8.2 - Limit-cycle amplitude and frequencies

Considering the first method, the analysis of Chapter 7 has shown how the effect of structural feedback signals on actuator performance is due to two main mechanisms. These mechanisms are as a result of the software rate limiter and mainvalve port profile. It has been shown that the presence of the software rate limiter can result in a decrease in gain and increase in the phase lag of the system in the presence of structural noise. Opposing this effect to a certain extent, the effect of the mainvalve port profile is to cause a decrease in the phase lag of the system and a slight increase in gain in the presence of structural noise. If the effect of the mainvalve port 
shaping is neglected initially, then the effect of the limit-cycle condition can be approximated from a consideration of the software rate limiter alone.

Consider the system model as developed in Chapters 3 and 4, in particular the baseline system model developed in section 4.2.2. This consists of a linearised actuator and an aircraft model containing no structural-modes. If the open-loop frequency response of this system is produced, and the results plotted on a Nichols diagram, the rigid-body aircraft stability-margins can be assessed. Such a Nichols diagram is as shown in Figure 4.5. In this case however, the structural-mode filters need to be included in the analysis. The presence of the filters results in an introduction of a phase lag into the system as can be seen from Figure 8.23.

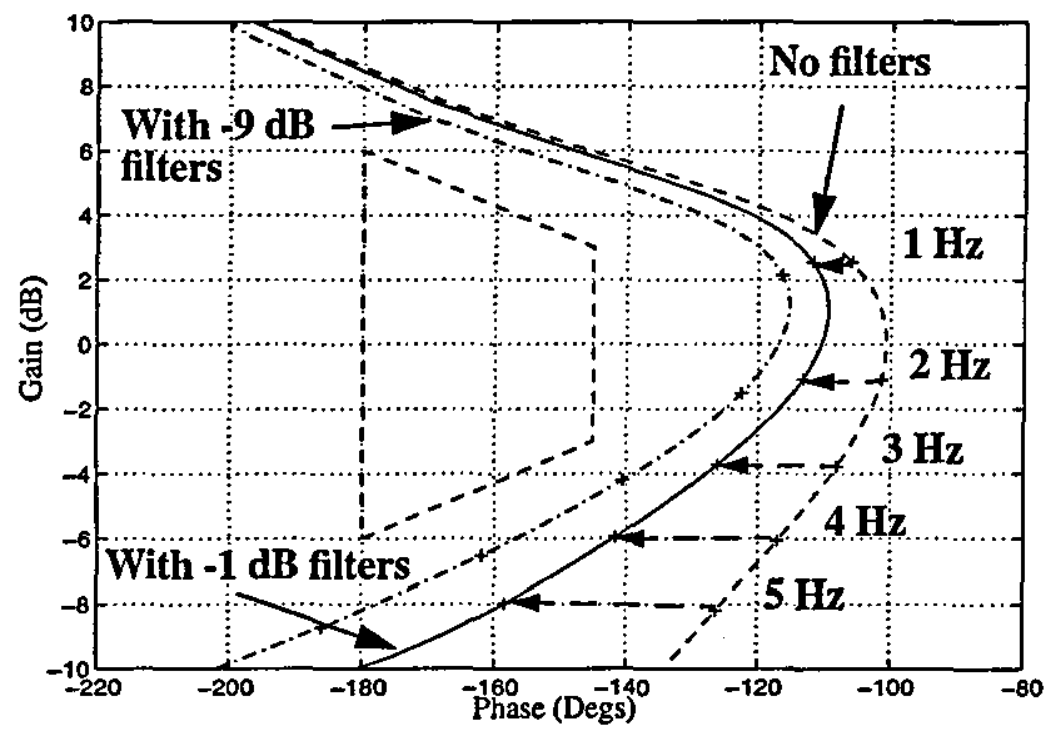

Figure 8.23 - Open-loop frequency response for baseline system model including -1 dB structural filters

The advantage to be gained in applying the $-1 \mathrm{~dB}$ filters can also be seen from the figure. The phase lag introduced by these filters is significantly less than that introduced by the $-9 \mathrm{~dB}$ filters.

In the presence of a limit cycling condition, it has been shown that there may be changes in the actuator response to low-frequency demand signals. Considering only the effect of the software rate limiting initially, it is possible to derive the gain and phase response of such a rate limiter in isolation. This response can then be incorporated in the above Nichols diagram to give a pessimistic assessment of the effect of a limit cycling condition on the control of the rigid-body aircraft.

For example, consider the limit-cycle condition (Table 8.2) for a frequency of 7.1 $\mathrm{Hz}$. Such a limit-cycle would result in an actuator demand signal of frequency $7.1 \mathrm{~Hz}$ and amplitude $10.9 \mathrm{~mm}$. This change in amplitude is as a result of the gain of the FCS between error signal and actuator demand signal (upstream of the software rate limiter). In addition, the lever arm scaling between actuator demand in terms of control surface angle and actual ram demand has been taken into consideration. Such a 
procedure can be completed for all of the limit-cycles specified in Table 8.2 resulting in the limit-cycle amplitude and frequencies as specified in Table 8.2.

\begin{tabular}{|c|c|c|}
\hline $\begin{array}{c}\text { Frequency } \\
\text { (Hz) }\end{array}$ & $\begin{array}{c}\text { Amplitude } \\
\text { of inboard/ } \\
\text { outboard } \\
\text { ram } \\
\text { demand } \\
\text { (mm) }\end{array}$ & $\begin{array}{c}\text { Amplitude } \\
\text { of } \\
\text { foreplane } \\
\text { ram } \\
\text { demand } \\
\text { (mm) }\end{array}$ \\
\hline \hline 7.1 & 10.9 & 10.7 \\
\hline 15.1 & 3.73 & 3.68 \\
\hline 20.9 & 2.38 & 2.34 \\
\hline 28.6 & 1.90 & 1.87 \\
\hline 44.0 & 1.29 & 1.27 \\
\hline 72.2 & 0.88 & 0.87 \\
\hline
\end{tabular}

Table 8.3 - Limit-cycle amplitude and frequencies

The difference between the ram demand amplitudes at the flaperon and foreplane inputs are due to the slight difference between the two control paths. Neglecting this difference, it is possible to approximate the impact of the limit-cycle on rigid-body control. Producing the frequency response of the rate limiter in isolation for an input consisting of a $1 \mathrm{~mm}$ low-frequency demand signal and the limit-cycles as defined in Table 8.2 gives the results as shown in Figure 8.24.
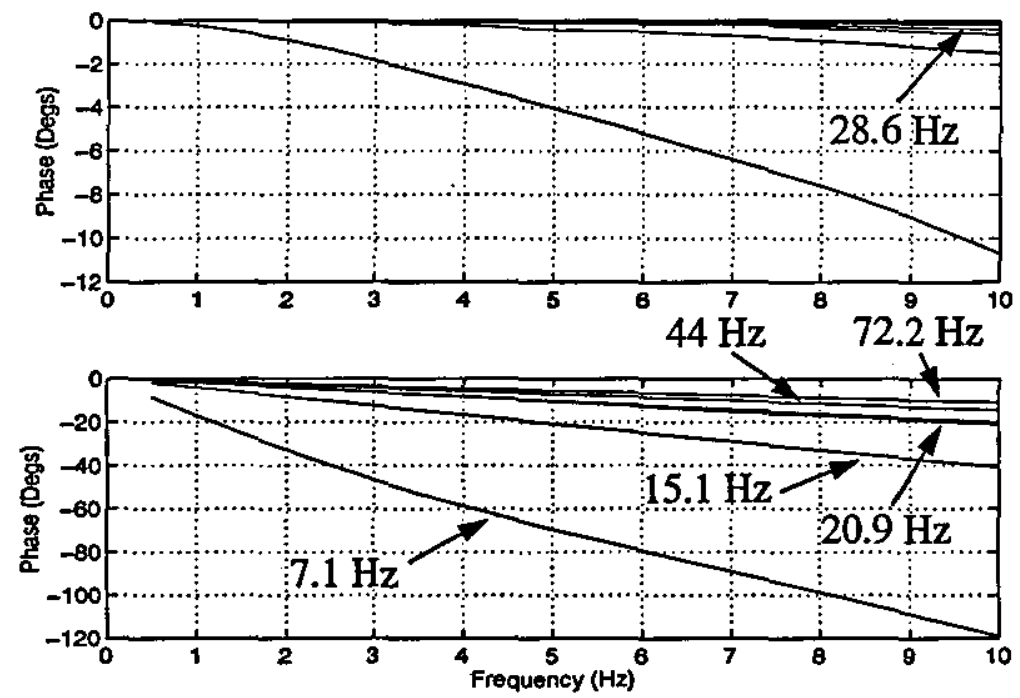

Figure 8.24 - Rate limiter frequency response in the presence of various limitcycle oscillations - 1mm low-frequency demand amplitude

From the figure, it is clear that it is the limit-cycle at a frequency of $7.1 \mathrm{~Hz}$ that would have the greatest effect on the rigid-body stability-margins. Combining these results with the open-loop frequency response of the remainder of the system results 
in the Nichols plot as shown in Figure 8.25. In this case, it is assumed that the effect of the rate limiter acts on all three signal paths equally. This enables its response to be simply added to that of the remainder of the system as shown in Figure 8.23.

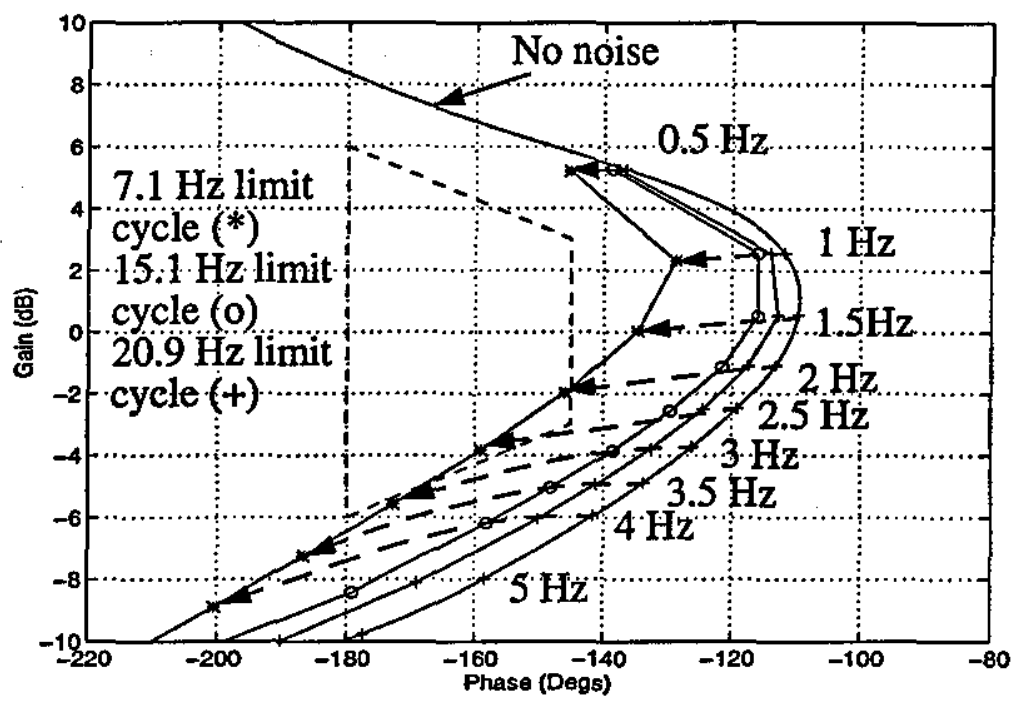
Figure 8.25 - Approximate open-loop frequency response in the presence of
limit-cycle oscillations

The reduction in gain and increase in phase lag of the system at rigid-body frequencies due to the response of the rate limiter can clearly be seen from Figure 8.25. Since the open-loop frequency response violates the rigid-body clearance boundary for the $7.1 \mathrm{~Hz}$ limit-cycle, it would be sensible to increase the attenuation of this structural-mode by the structural-mode filters.

It is important to realise however that the results of Figure 8.25 are pessimistic in that they neglect the effect of the mainvalve port shaping. In addition, the results have been produced assuming an error in the system modelling resulting in an open-loop gain of two times the nominal value. Under nominal circumstances such limit-cycles would not occur as has been discussed earlier. Considering that increasing the attenuation of the $7.1 \mathrm{~Hz}$ structural-mode would not significantly alter the phase lag of the structural filters as a whole, such an increase in attenuation would seem to be prudent. The fact that it is the low-pass filter that contributes the majority of the phase lag inherent within the structural-mode filters has been discussed in Chapter 4.

\subsubsection{Use of time domain system simulations in the assessment of the effect of limit cycling oscillations}

The method used in the production of Figure 8.25 makes several assumptions regarding the effect of the limit-cycle oscillation on the rigid-body control of the aircraft. In order to assess the effect more accurately, consider a time domain simulation of the system. From such a simulation, it is possible to calculate the openloop frequency response in the presence of a limit-cycle. Unfortunately, simulation of the full flexible aircraft model may not demonstrate the required limit-cycle due to the 
models particular phase response at that frequency. To evaluate the effect of a particular limit-cycle thus requires some means of artificially stimulating the required limit-cycle oscillation. Due to the low-pass nature of the rigid-body system, it is possible to represent the effect of a limit-cycle by injecting a signal of the required amplitude and frequency at a suitable point within the system ${ }^{11,12,72}$.

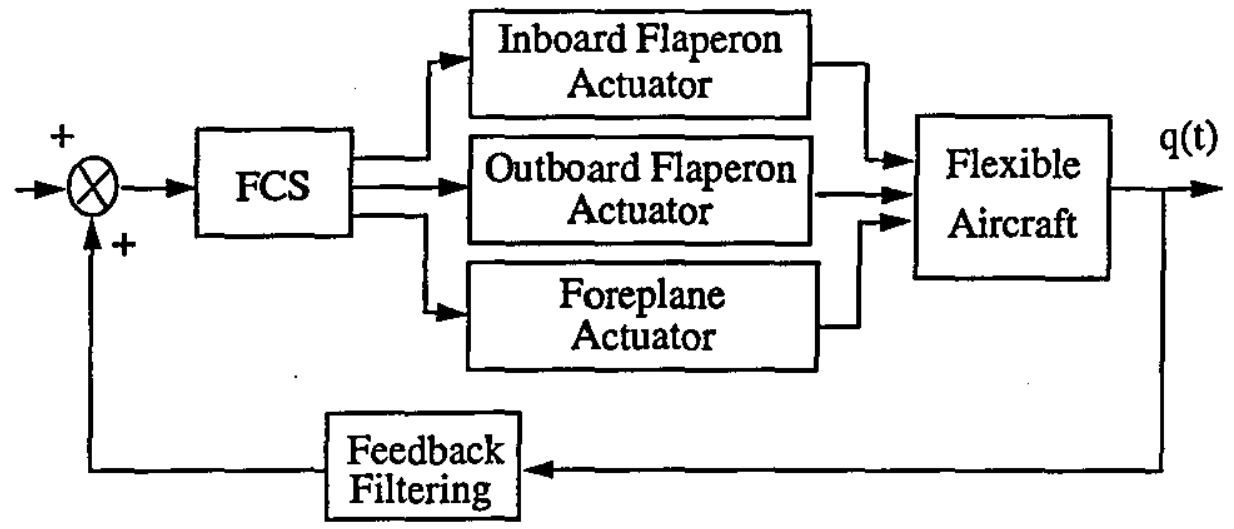

Figure 8.26 - Aircraft block diagram

Consider the case shown in Figure 8.26, where, the aircraft's pitch rate, as sensed by the aircraft motion sensor unit (AMSU), can be expressed as

$$
q(t)=q_{r p}(t)+q_{r s}(t)+q_{s p}(t)+q_{s s}(t)
$$

where -

$q(t)$ is the pitch rate as sensed by the AMSU

$\mathrm{q}_{\mathrm{rp}}(\mathrm{t})$ is the rigid-body response due to the pilot demand signal

$\mathrm{q}_{\mathrm{rs}}(\mathrm{t})$ is the rigid-body response due to the structural feedback signal

$\mathrm{q}_{\mathrm{sp}}(\mathrm{t})$ is the structural response due to the pilot demand signal

$\mathrm{q}_{\mathrm{ss}}(\mathrm{t})$ is the structural response due to the structural feedback signal

In a limit cycling condition, the maximum amplitude of the limit-cycle is governed by the actuator performance limits such that $\mathrm{q}_{s s}(\mathrm{t})$ can be defined. If in addition, the structural response due to the pilot demand signal, $\mathrm{q}_{\mathrm{sp}}(\mathrm{t})$, can be assumed negligible when compared with that due to the structural feedback signal, the system block diagram can be redrawn as in Figure 8.27. 


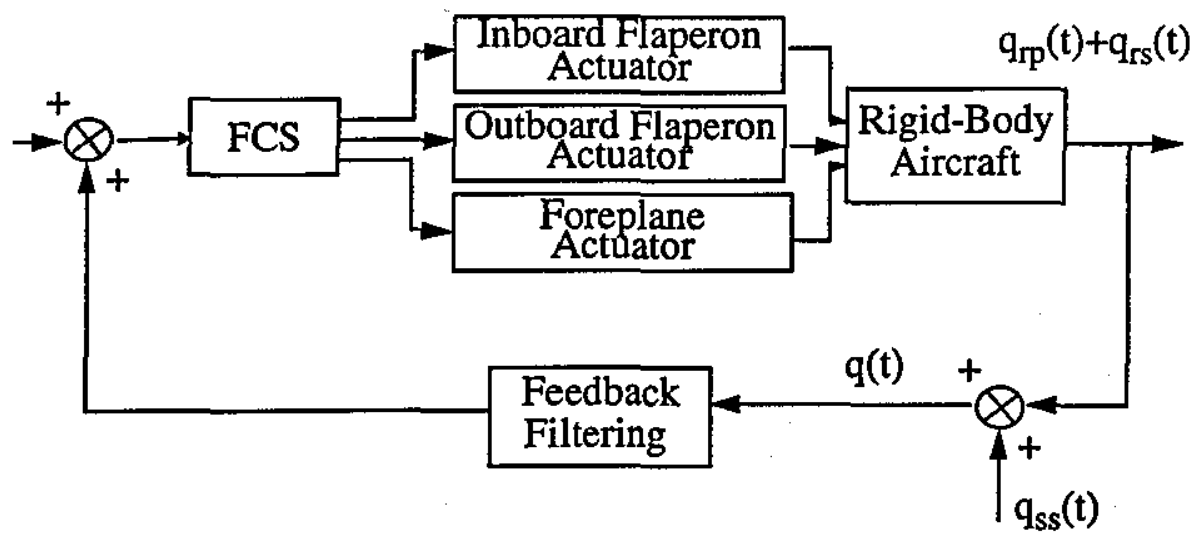

\section{Figure 8.27 - Aircraft block diagram showing limit-cycle signal represented by measurement noise}

As a result, the structural-mode can be represented by a signal of the equivalent amplitude and frequency injected into the system as measurement noise. The flexible aircraft is then replaced by a model of only its rigid-body dynamics as shown in Figure 8.27.

This approach allows the frequency, amplitude and phase of the limit-cycle to be easily varied, whilst allowing the response of the rigid-body aircraft system to be quantified as the performance of the actuation system changes.

In this case, the limit-cycle has been represented by a signal injected as measurement noise. There is no reason to prevent a suitable signal being injected at any point within the system however. In the case of the system under consideration, the possible limit-cycle amplitudes have been defined at the error signal. Their effect can therefore be assessed by injecting signals of the desired frequency and amplitude into a suitable time domain simulation.

Consider initially the case where there is no limit-cycle oscillation present. Calculating the frequency response of the system for a pilot demand signal equivalent to approximately $1.0 \mathrm{~mm}$ of actuator ram demand produces the results as shown in Figure 8.28. 


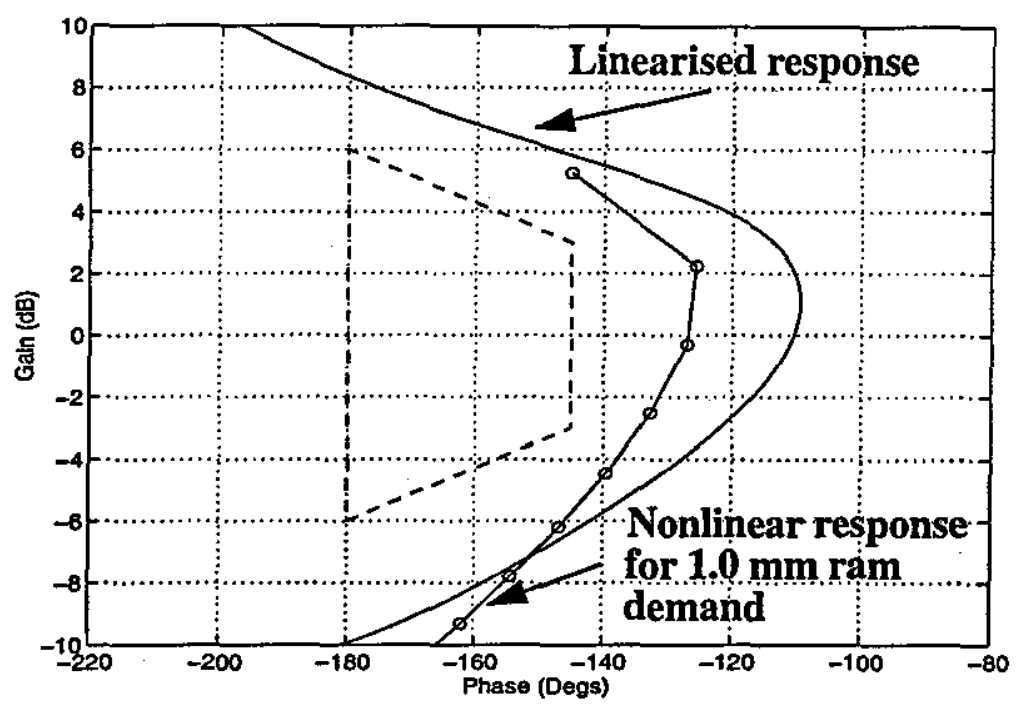

\section{Figure 8.28 - Open-loop frequency response as obtained from time domain simulation of nonlinear system}

The frequency response obtained from an actual time domain simulation of the system demonstrates the errors introduced by considering only a linearisation of the actuator dynamics. Since the performance of the actuator varies considerably as a function of demand amplitude, such a linearisation about a particular operating point will be prone to errors. In fact, the actuator performance can vary significantly for demand inputs of differing amplitude. This can be seen from the actuator frequency responses for the actuator included in Chapter 7.

In order to assess fully the impact of a limit cycling condition, it is necessary to consider the effect of such a limit-cycle over a range of low-frequency demand amplitudes. It has been demonstrated in Chapter 7 that the effect of a high-frequency signal on actuator performance will be dictated in part by the amplitude of the lowfrequency signal.

Consider once again the aircraft system as shown in Figure 8.27 with a pilot demand signal equivalent to an actuator ram displacement of $1.0 \mathrm{~mm}$. If the frequency response of the system is produced in the presence of the limit-cycles specified in Table 8.2, the results are as shown in Figure 8.29. 

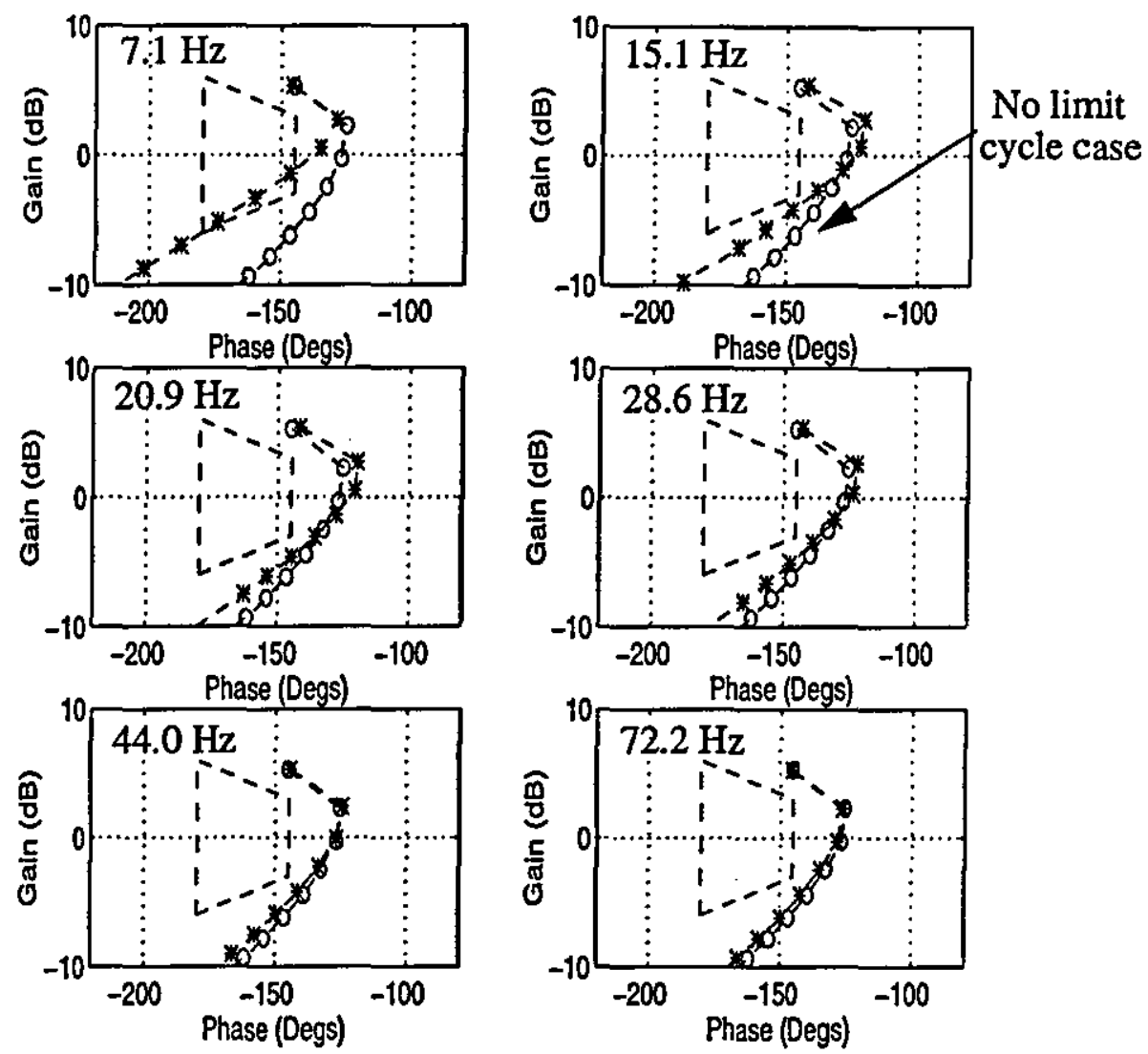

Figure 8.29 - Open-loop frequency response in presence of limit-cycles, pilot demand equivalent to $1.0 \mathrm{~mm}$ ram demand
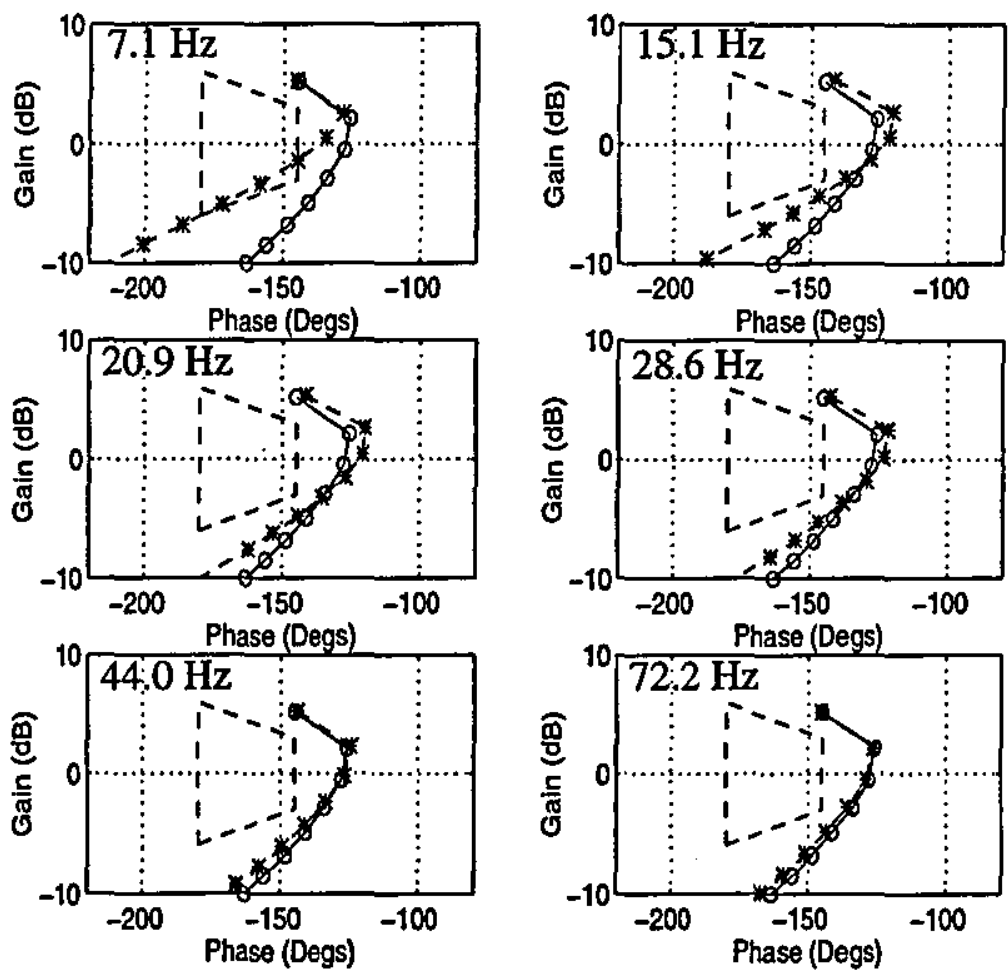

Figure 8.30 - Open-loop frequency response in presence of limit-cycles, pilot demand equivalent to $0.1 \mathrm{~mm}$ ram demand 

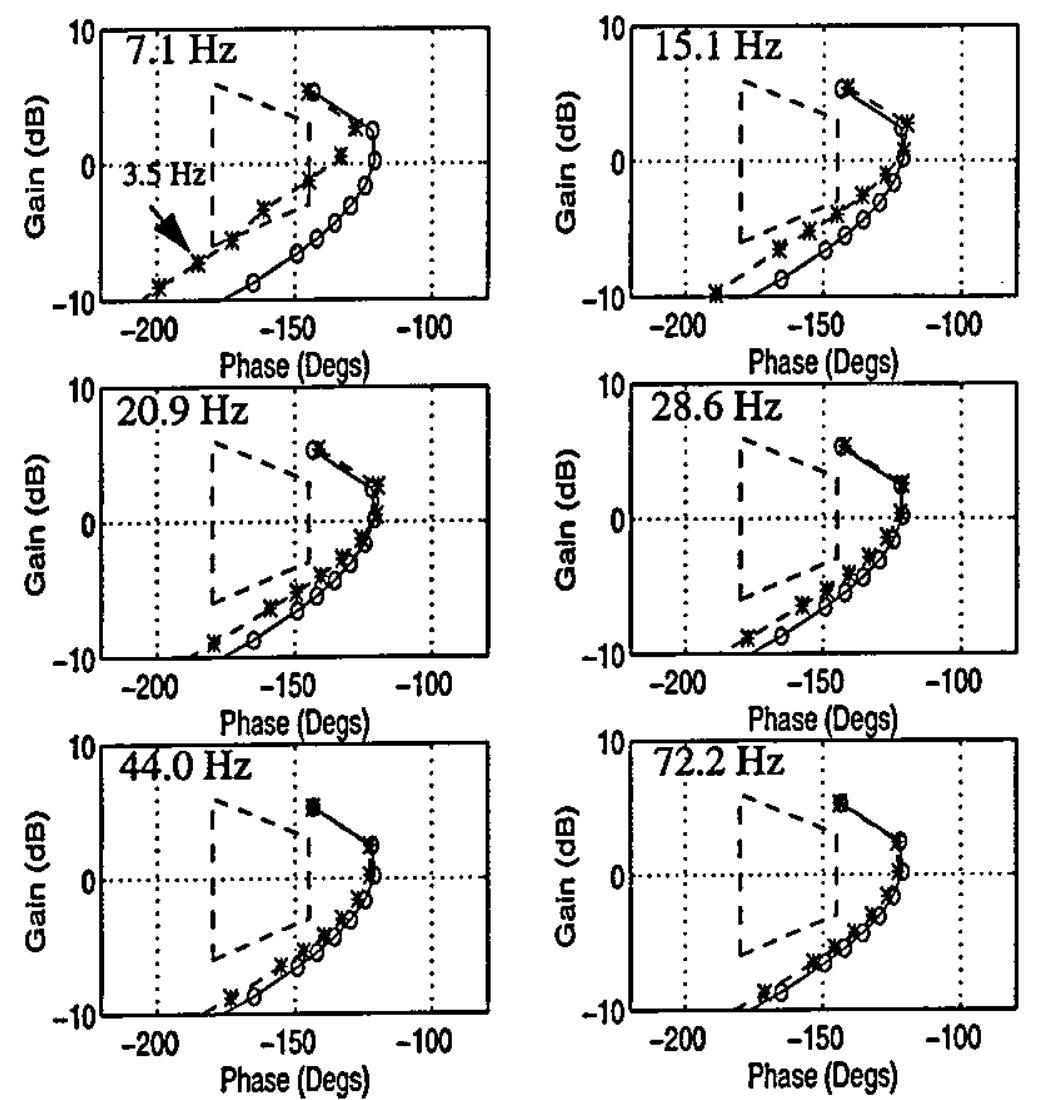

Figure 8.31 - Open-loop frequency response in presence of limit-cycles, pilot demand equivalent to $5 \mathrm{~mm}$ ram demand
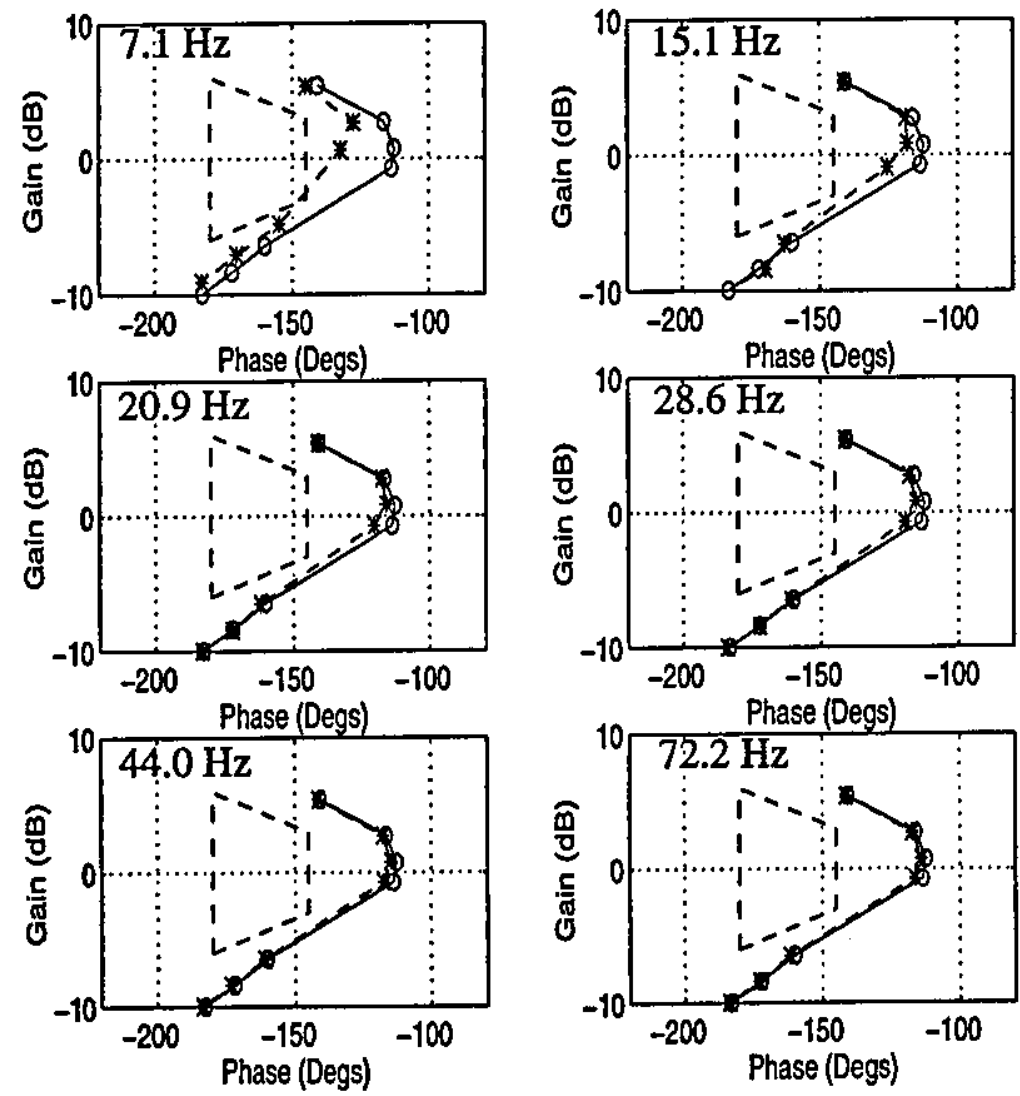

Figure 8.32 - Open-loop frequency response in presence of limit-cycles, pilot demand equivalent to $20.0 \mathrm{~mm}$ ram demand 
The results demonstrate that the effect of the limit-cycles on system performance is similar to the approximate results of Figure 8.25. Once again, it is the possible limitcycle at a frequency of $7.1 \mathrm{~Hz}$ that has the potential for causing unsatisfactory system response. It should be stressed however that this is still assuming the gain of the structural-modes to be twice that originally designed for. Even if this were the case, it is by no means certain that such a limit-cycle would occur. Neglecting such considerations however, it can be seen that it would be wise to increase the attenuation of this mode.

In the case of the $7.1 \mathrm{~Hz}$ limit-cycle, its effect in terms of additional phase lag is clear. As expected from the results of Chapter 7, the presence of such a limit-cycle has undoubtedly resulted in the demand signal to the actuator being rate limited. This in turn has led to the increase in the phase lag of the system. It is interesting to note that in the case of the $15.1 \mathrm{~Hz}, 20.9 \mathrm{~Hz}$ and $28.6 \mathrm{~Hz}$ limit-cycles, at low input demand frequencies there is actually a small decrease in the phase lag of the system. This can be attributed to the shaping of the main valve ports as has been discussed previously. As the input demand frequency increases, the combined signal begins to exceed the rate limit. Once this occurs, the increase in phase lag, characteristic of the onset of rate limiting, is evident. The fact that the higher frequency limit-cycles do not result in a violation of the clearance boundary is due to the fact that their maximum amplitudes are less than a third of the maximum amplitude of the $7.1 \mathrm{~Hz}$ limit-cycle. This effect is even clearer in the case of the $44.0 \mathrm{~Hz}$ and $72.2 \mathrm{~Hz}$ limit-cycles which have very little effect on the rigid-body clearance requirements. In this case, their amplitudes are less than one eighth of the maximum amplitude of the $7.1 \mathrm{~Hz}$ limit-cycle.

As mentioned previously, in order to fully assess the impact of such limit-cycles on rigid-body stability, it is necessary to consider a range of pilot demand amplitudes. The results of Figure 8.29 were produced for a pilot demand amplitude equivalent to approximately $1 \mathrm{~mm}$ of actuator ram demand. The results of Chapter 7 have demonstrated however that the effect of a high-frequency signal superimposed upon a low-frequency demand signal is dependent on the amplitude and frequency of both components. To demonstrate this, the frequency responses of the system were obtained for other pilot demand amplitudes. These results are included as Figure 8.30 to 8.32 and demonstrate the effect of the limit-cycles on the control of the rigid-body aircraft for a range of pilot demand inputs. As for the $1.0 \mathrm{~mm}$ pilot demand, it is the potential limit-cycle of frequency $7.1 \mathrm{~Hz}$ that has the ability to cause violation of the rigid-body clearance requirements.

Consider the results of Figure 8.30 which show the effect of the rate limits on the rigid-body performance for a pilot demand equivalent to $0.1 \mathrm{~mm}$ of ram demand. Once again, it is the $7.1 \mathrm{~Hz}$ limit-cycle that has the potential for causing unsatisfactory 
rigid-body response. In the case of this pilot demand amplitude, the effect of the shaping of the main valve port is more in evidence. This is particularly clear for the limit-cycles of frequency $15.1 \mathrm{~Hz}, 20.9 \mathrm{~Hz}$ and $28.6 \mathrm{~Hz}$. In the case of such a small pilot demand amplitude, the shaping of the main valve ports has a greater effect since there is a larger difference in demanded rate between the low-frequency signal and limit-cycle. This effect has been discussed in detail in Chapter 7. For larger demand amplitudes, the effect of the shaping of the main valve port is not noticeable as can be seen from Figure 8.32.

From Figure 8.32, the effect of the limit-cycles on rigid-body performance in the presence of large pilot demand amplitudes is not as pronounced as for the smaller amplitudes. This is particularly true for pilot demand frequencies greater than $2 \mathrm{~Hz}$. The cause of this is that for a pilot demand amplitude of $20.0 \mathrm{~mm}$, the rate limit is exceed by the pilot demand signal alone at a frequency of $1.9 \mathrm{~Hz}$. Because of this, any further addition of a high-frequency limit-cycle signal will have little effect on the actuator performance.

Another important factor that must be considered when assessing the impact of limit-cycles on rigid-body stability, is the tendency to generate subharmonic response. As has been discussed in Chapter 7, the existence of two input signals of different frequencies to the nonlinear actuator can result in the generation of subharmonic response. The production of the above results examines the response at the frequency of the primary input signal. As a result, no information is gained as to the effect of the limit-cycle in terms of subharmonic generation.

The analysis of Chapter 7 reveals that it is the structural-modes of low-frequency which can create significant subharmonic generation. As an example, consider the time response for the system used in the production of Figure 8.31. In this case, the pilot demand signal is equivalent to a ram demand of $5.0 \mathrm{~mm}$. Consider the case of the $7.1 \mathrm{~Hz}$ limit-cycle and a pilot demand signal of frequency $3.5 \mathrm{~Hz}$. From Figure 8.31, such conditions result in satisfactory rigid-body performance in terms of the stabilitymargins. If however the pitch rate time response of the system is plotted for these conditions, the time response is clearly unsatisfactory as can be seen from Figure 8.33. 


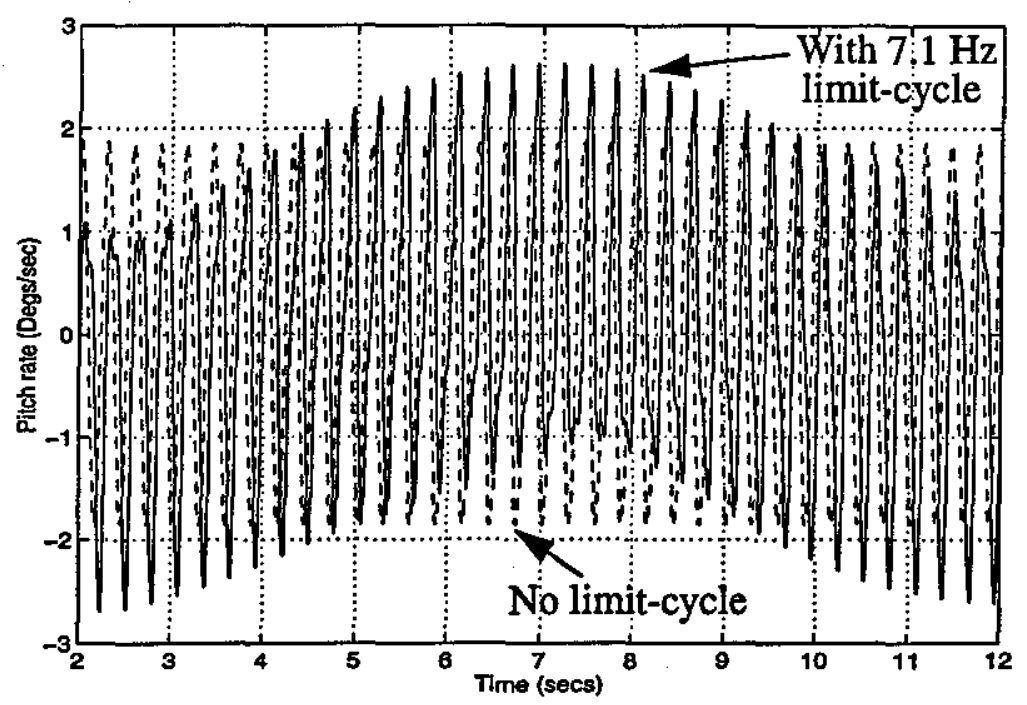

Figure 8.33 - Aircraft time response in presence of $7.1 \mathrm{~Hz}$ limit-cycle

The existence of the limit-cycle in this case has therefore resulted in the production of subharmonic aircraft motion. From the theory of Chapter 7, such a combination of pilot demand and limit-cycle frequencies could be expected to give a subharmonic response of frequency $0.1 \mathrm{~Hz}$. This is the case as can be seen from Figure 8.33. In this example, even though the rigid-body clearance requirements were met in the presence of the limit-cycle, an unsatisfactory aircraft time response resulted. It is important therefore to assess the impact of the limit-cycle in both the frequency and time domains.

8.6.6 Experimental verification of the effects of the limit-cycles on actuator performance

In order to verify these simulated results, it is possible to examine the actuator performance changes in the presence of the limit-cycles as defined in Table 8.2. As for the simulated results, it is necessary to consider a range of pilot demand amplitudes in order to assess the true effect of the limit cycling condition.

Using the actuator test rig as described in section 7.6, it is possible to assess the performance of the actuator in the presence of the structural noise signals. Results comparing the performance changes as measured on the real actuator with those predicted from the system simulations are included as Figure 8.34 to Figure 8.41. 

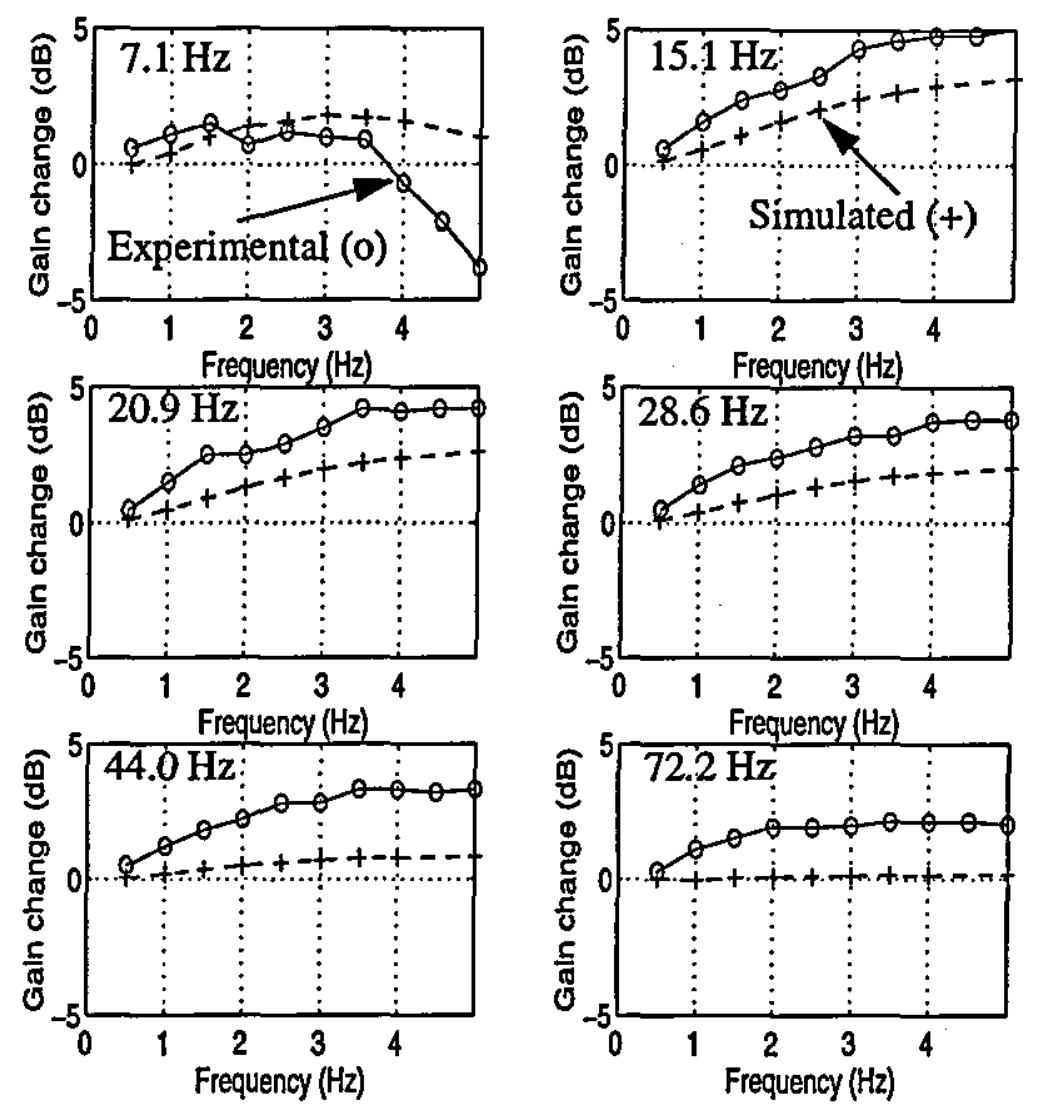

Figure 8.34 - Actuator gain response changes in the presence of limit-cycles, 0.1 mm ram demand
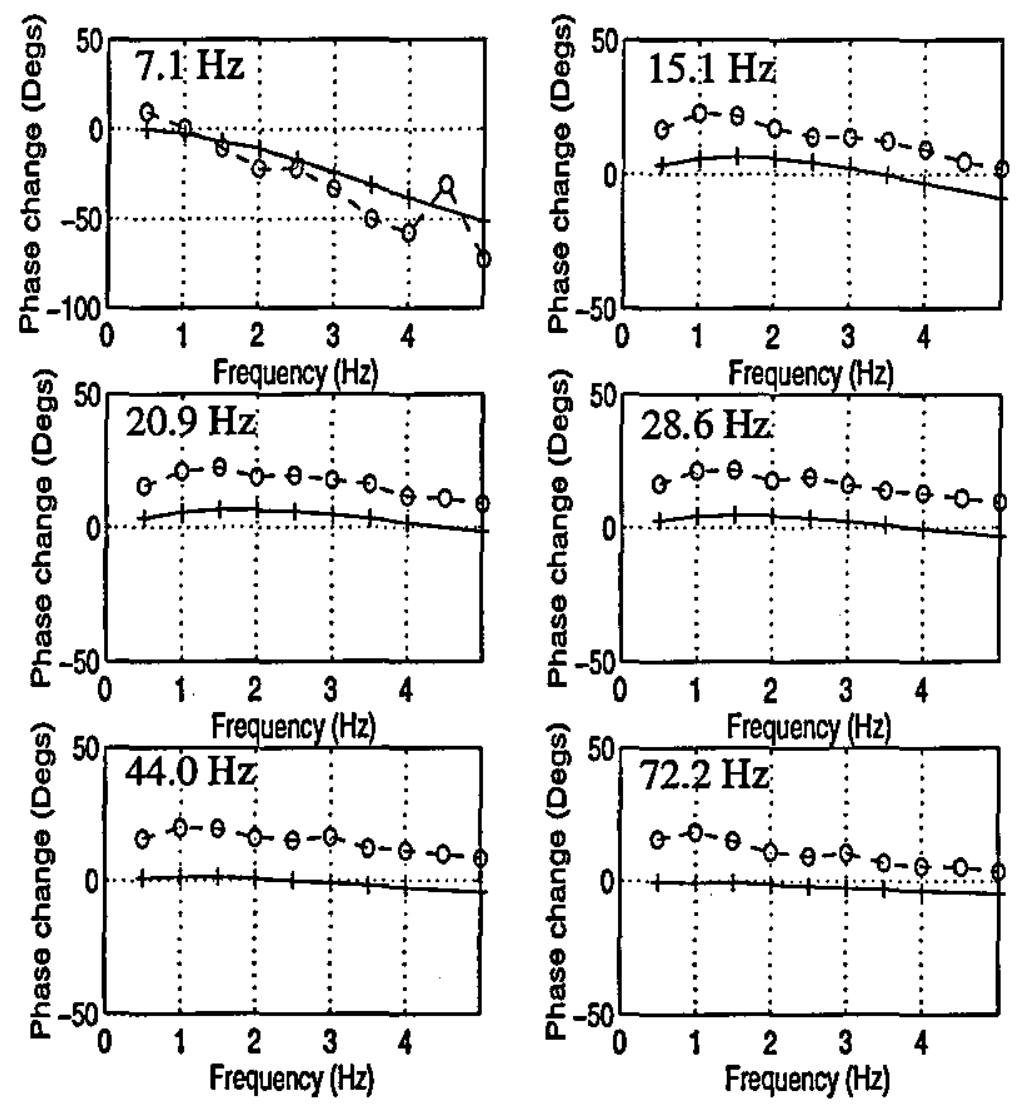

Figure 8.35 - Actuator phase response changes in the presence of limit-cycles, 0.1 mm ram demand 

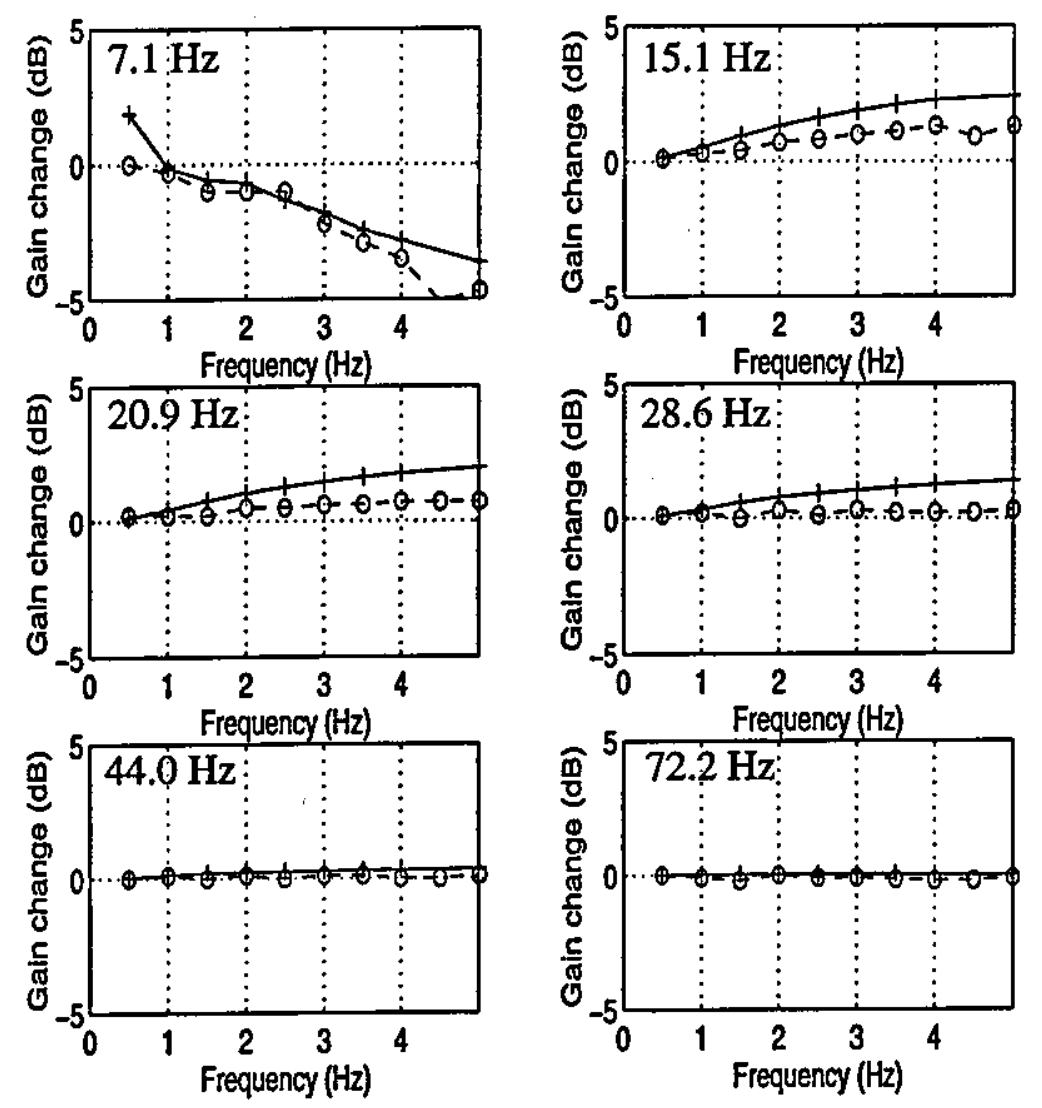

Figure 8.36 - Actuator gain response changes in the presence of limit-cycles, 1.0 mm ram demand
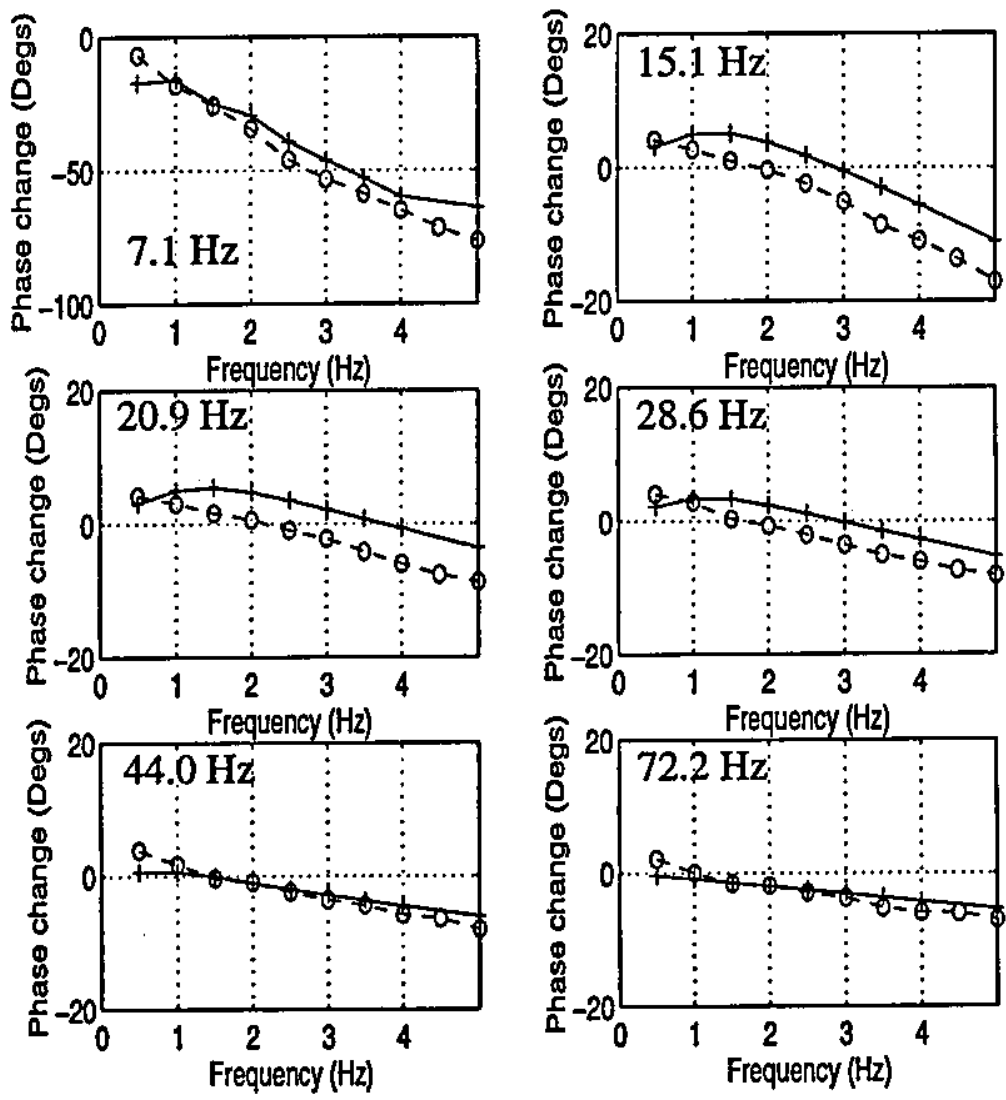

Figure 8.37 - Actuator phase response changes in the presence of limit-cycles, 1.0 mm ram demand 

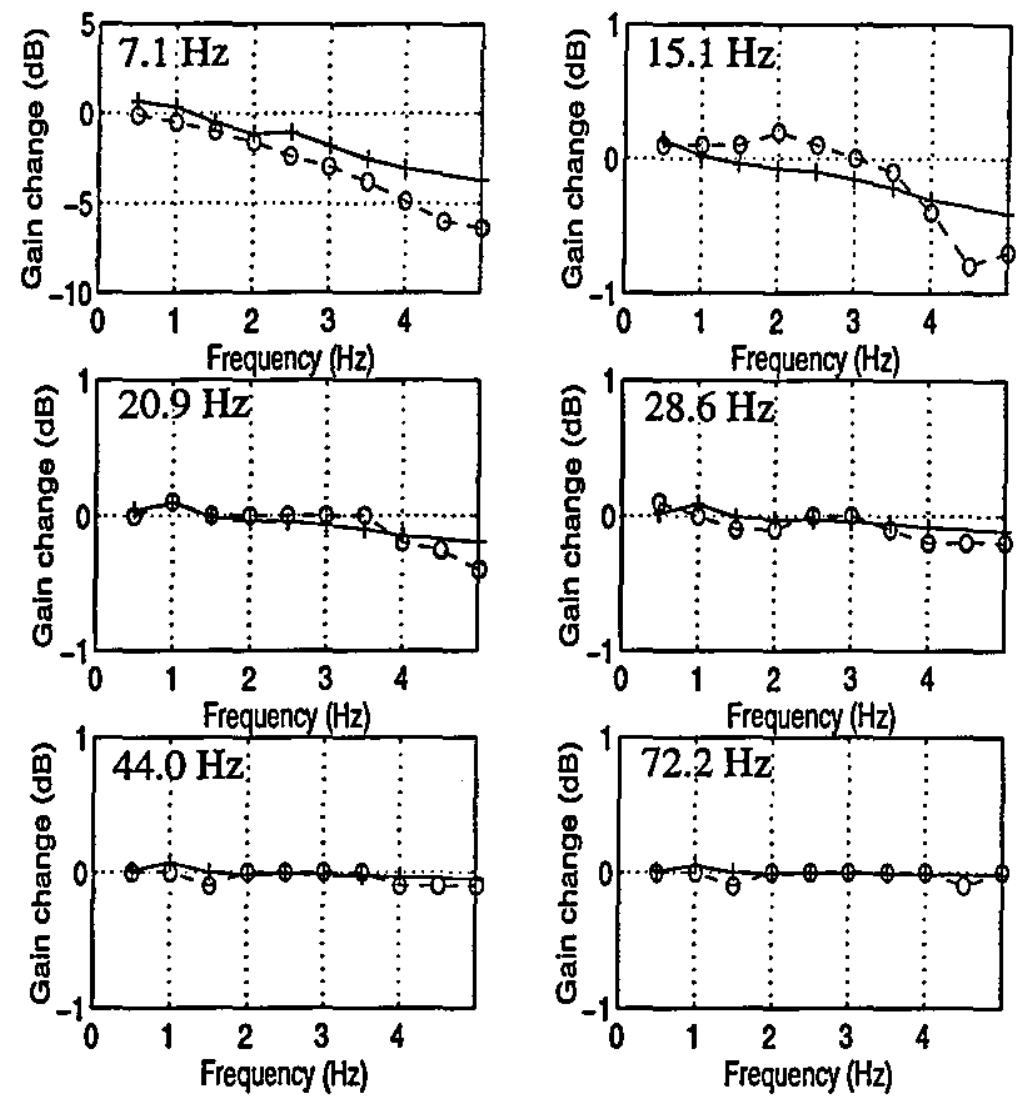

Figure 8.38 - Actuator gain response changes in presence of limit-cycles, $5.0 \mathrm{~mm}$ ram demand
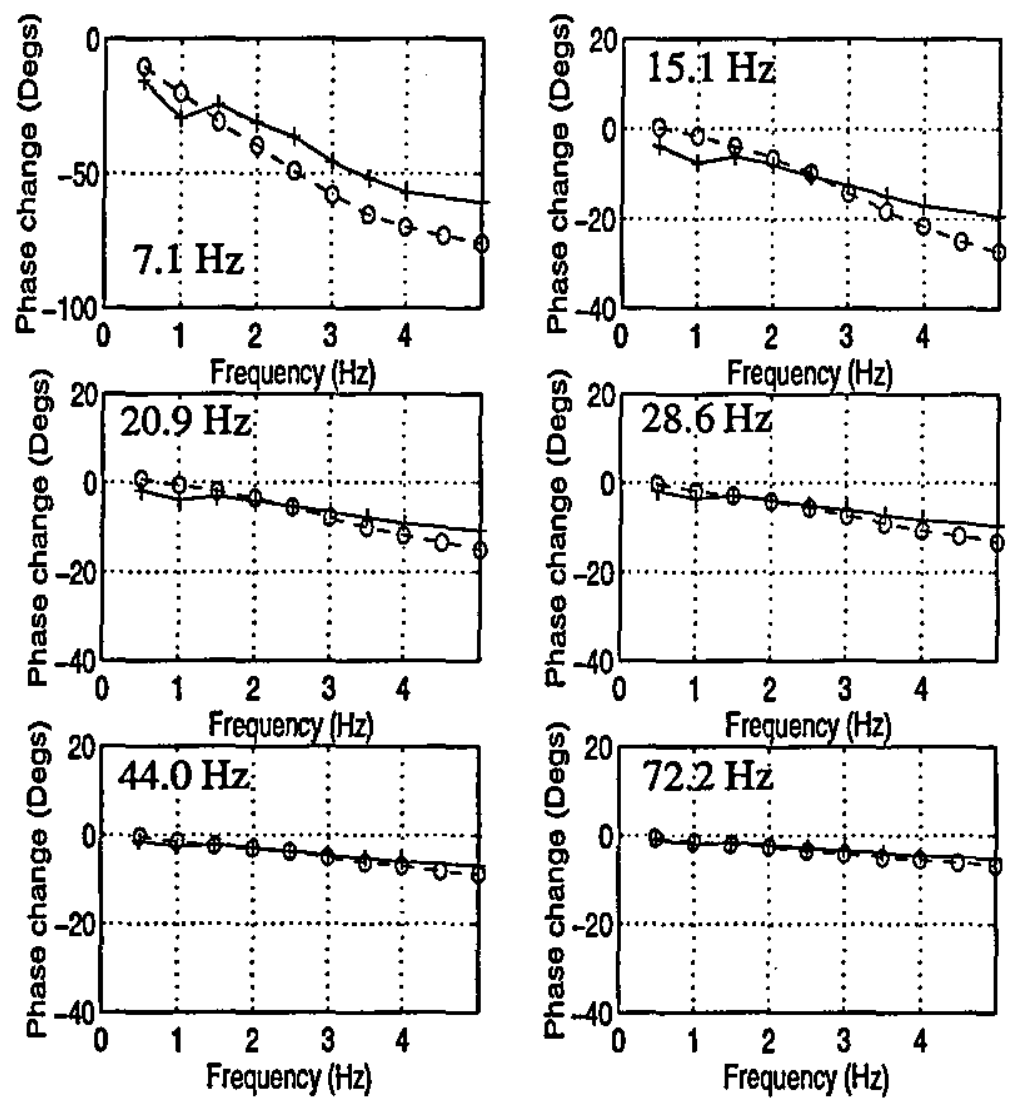

Figure 8.39 - Actuator phase response changes in the presence of limit-cycles, 5.0 mm ram demand 

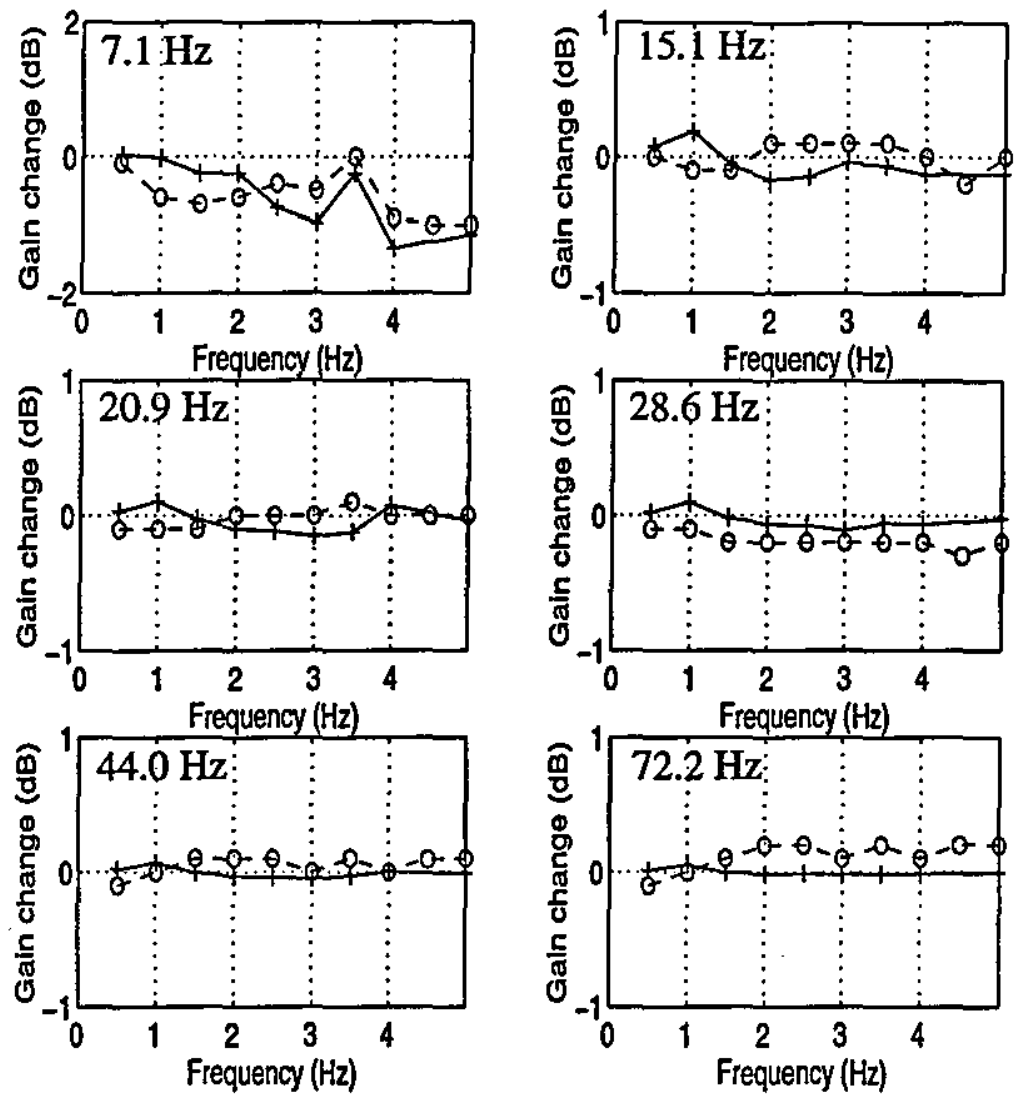

Figure 8.40 - Actuator gain response changes in the presence of limit-cycles, 20.0 $\mathrm{mm}$ ram demand
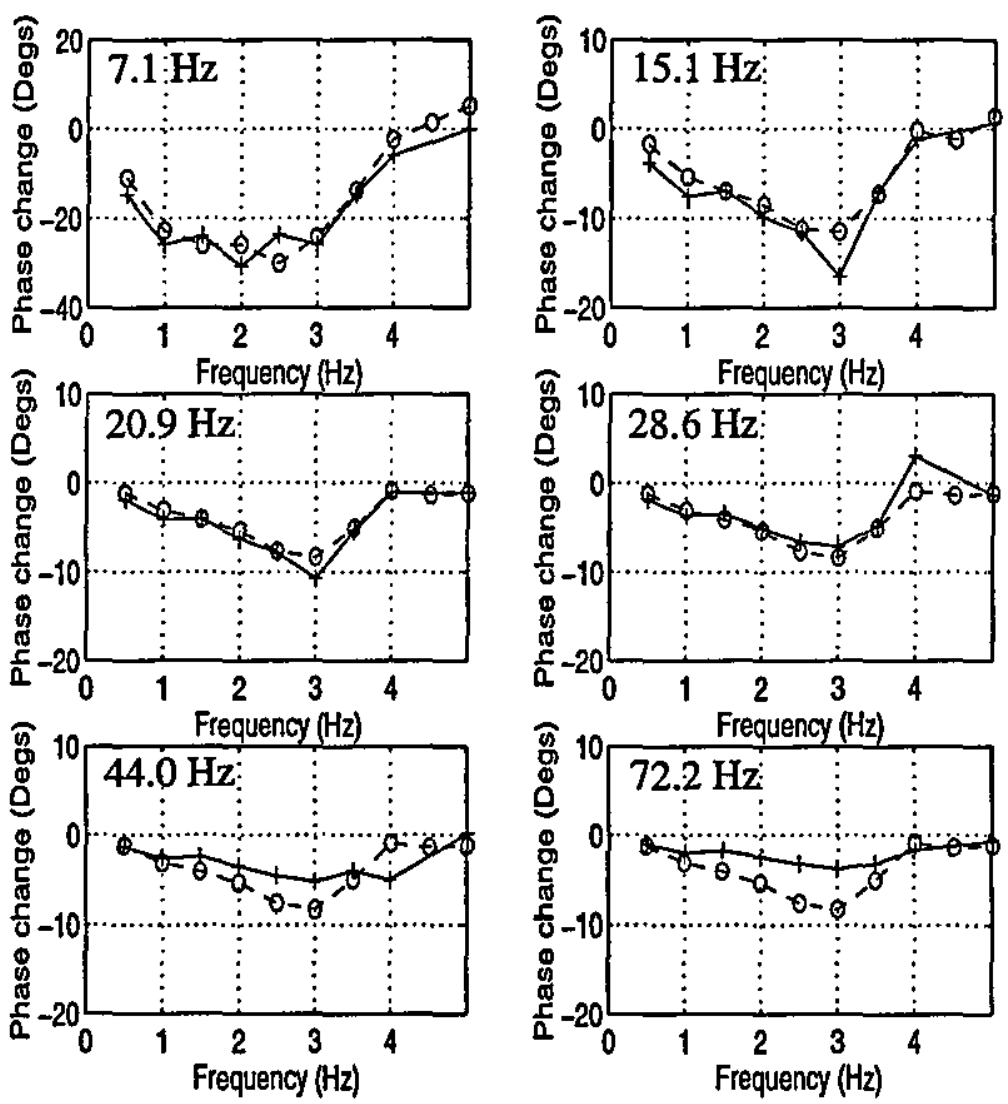

Figure 8.41 - Actuator phase response changes in the presence of limit-cycles, $20.0 \mathrm{~mm}$ ram demand 
The results of Figure 8.34 to Figure 8.41 confirm the effects of the specified limitcycles on actuator performance. As can be seen from the response of the whole system as shown in Figure 8.29 to Figure 8.32, the changes in actuator performance as a result of the presence of a limit-cycle are predictable. One point to note however, is that the comparison for the smallest ram demand amplitude of $0.1 \mathrm{~mm}$ is not as good as for larger ram demand amplitudes. It is believed that this is due to the presence of unmodelled nonlinearities within the actuator. For example, factors such as dead zone and frictional effects will be more relevant for small ram demand amplitudes. In the case of the simulated results, such affects are not modelled, and as a result, the change in actuator performance is not as large as for the experimental results. Considering the experimental results, the presence of the limit-cycle causes the actuator to break-free of these un-modelled nonlinear regions. Importantly however, the presence of the limit-cycle in this case has caused an improvement in actuator performance which is underestimated by the simulated results.

Overall therefore, it can be seen that the modelling of the actuator has predicted the effects of the limit-cycles on actuator performance. An alternative method of assessing the effects of such limit-cycles on the performance of the overall system is to apply results from actuator testing to the full system. For example, if the frequency response of the actuator can be obtained in the presence of the limit-cycle, then it can be applied to a model of the remaining elements within the system. Such a method would then eliminate to some extent the need for time domain simulations of the full aircraft system. This method will be applied in the later consideration of the digital nature of the control system.

\subsubsection{Conclusions}

To conclude, the ability to predict the frequency and amplitude of a potential limitcycle enables its affect on the rigid-body control of the aircraft to be assessed. This can be done in several ways, from an approximate analysis considering just the response of the rate limiter, to a time domain simulation of the whole system with the limit-cycle injected as measurement noise. An alternative approach has also been proposed whereby simulation or testing of the actuators in isolation could be applied to a linear model of the remaining elements of the system. The aim however is the same in all cases in that the effect of the predicted limit-cycles on the rigid-body stability of the system needs to be assessed.

In the examples shown here, a twofold error in the gain of the system was assumed at all frequencies. The results demonstrate that in this case, such an error could lead to poor stability if the $7.1 \mathrm{~Hz}$ structural-mode resulted in a limit-cycle. If it could be shown however that such an error was unlikely in the gain of this mode, then there would be no need to increase the attenuation of the structural filters at this frequency. 
Instead of assuming a particular level of error for all frequencies when investigating the effect of the limit-cycles, the likely error at each structural-mode could be assessed. For example, if it were felt that the gain of the $7.1 \mathrm{~Hz}$ structuralmode was reliably modelled, then the $-1 \mathrm{~dB}$ filters would ensure that a limit-cycle could never occur at this frequency. If however, it was felt that the modelling of the high-frequency modes was unreliable and could be subject to an error of up to (say) $300 \%$, then such a situation could be investigated using the earlier analysis. If it were found that a particular limit-cycle has the potential for causing violation of the clearance boundary or unsatisfactory time response, then the attenuation of this frequency by the structural-mode filters should be increased. For example, the above results demonstrated that the $7.1 \mathrm{~Hz}$ limit-cycle has the potential for causing unsatisfactory rigid-body response under the assumed conditions. If such an error in the gain of this mode was possible, then the attenuation of the structural filter at this frequency should be increased.

Once a set of structural filters are devised in such a manner, they can be implemented on the aircraft. In doing so, satisfactory rigid-body control will be maintained even in the presence of the assumed modelling errors. As has been discussed earlier, if an in-flight limit-cycle condition was then encountered, rigid-body control would be maintained. Such an in-flight limit-cycle would pin-point an error in the modelling of the system. Such an error could then be corrected, and the structural filters modified to maintain the $-1 \mathrm{~dB}$ maximum open-loop gain.

\subsection{Effect of digital representation}

\subsubsection{Introduction}

In Chapter 5, the digital nature of the typical modern control system was investigated. As a result, its effect on the current aeroservoelastic design procedure was assessed and discussed in Chapter 6. The above sections have introduced an alternative design procedure based on a knowledge of the nonlinear nature of the true aeroservoelastic system. In order to fully assess the alternative design procedure, it is necessary to consider the effect of the digital nature of the control system.

\subsubsection{Limit-cycle prediction in digital system}

Consider the aircraft system block diagram shown in Figure 8.7. In the presence of phase uncertainty, it has been shown that the occurrence of limit-cycles can be predicted from a solution of equation (8.27). This equation therefore predicts the amplitude of any limit-cycle that may exist within the aircraft system. Consider now 
the aircraft block diagram for the digital system in Figure 8.42 .

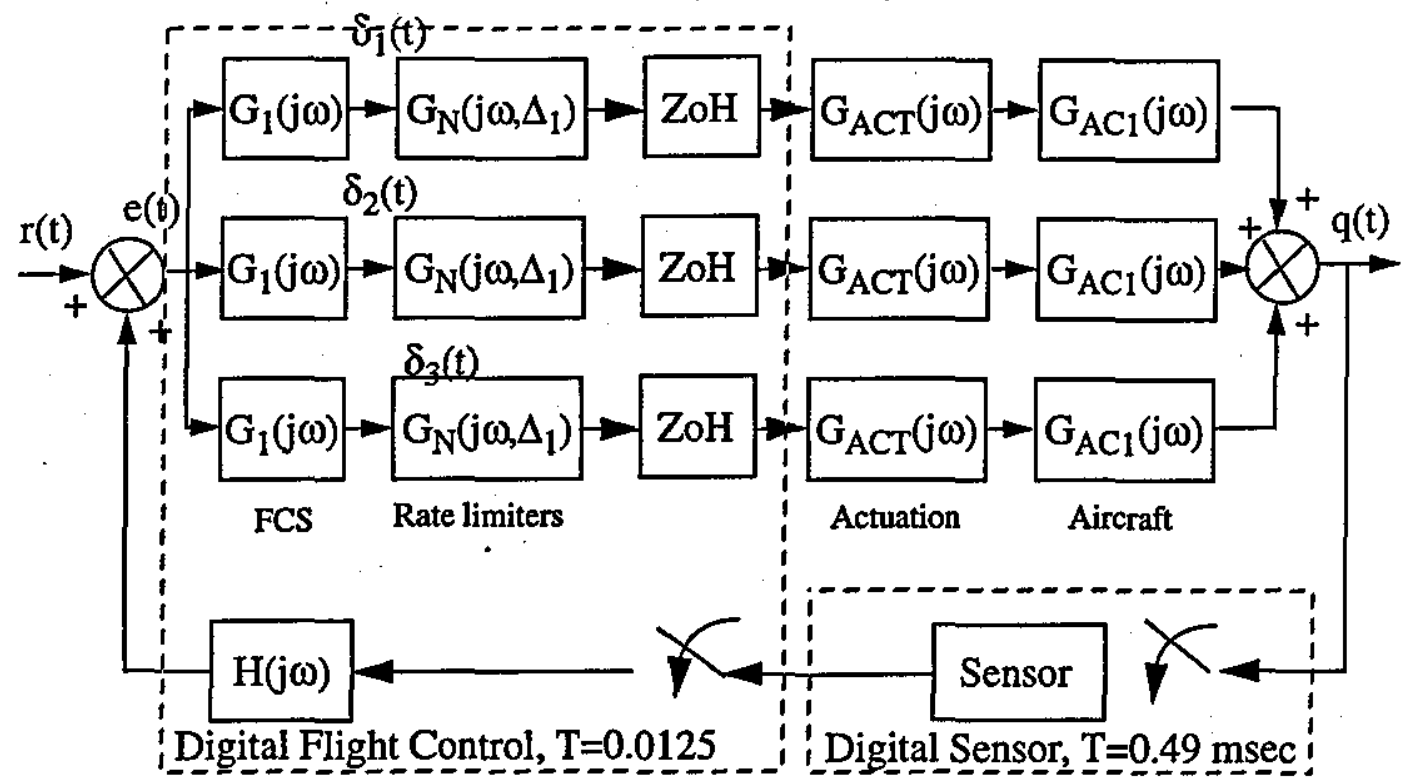

Figure 8.42 - Aircraft block diagram for digital system

Since the solution of equation (8.27) for the analogue system relies purely on the gain response of the various elements, the same applies to the digital system case. Naturally, the aliasing effect of the flight control system sampler must be taken into account as has been discussed in Chapter 6. In addition, in the digital system, the input waveform to the actuators are generated by zero-order-holds as shown. This results in the input signals to the actuators containing high-frequency aliases of the primary signal. The maximum performance boundary for the actuator is therefore slightly different from that for the analogue system. Such a performance boundary for the actuator within a digital system has been discussed in Chapter 7 .

\subsection{Demonstration of alternative clearance procedure on digital aircraft system}

\subsubsection{Introduction}

Consideration of the digital nature of the control system has been shown in Chapter 6 to be a vital element within the current aeroservoelastic design procedure. The aliasing of high-frequency modes, and the possibility of generating subharmonics as a result of the digital nature of the control system are of most concern ${ }^{14}$. The following section will examine the application of the proposed design procedure to a digital aircraft system.

\subsubsection{Design of structural-mode filters}

The design of structural-mode filters under the alternative design procedure requires the production of the filter attenuation requirements. In the case of the digital 
system, the results presented in Chapter 6 have demonstrated the need to consider the aliasing of the high-frequency response in the production of these attenuation requirements.

As for the analogue system described earlier, in order to ensure that limit-cycles would not arise in the nominal situation, filters will be designed to ensure a maximum open-loop gain of $-1 \mathrm{~dB}$.

Producing the maximum open-loop gain for the earlier aircraft model for all flight conditions results in the specification of the structural filter attenuation as shown in Figure 8.43. In this case, the aliasing of the response has been taken into consideration, as have the attenuation of the zero-order-hold and sensor dynamics. In line with the current design procedure and the results of Chapter 6 , it has been assumed that all signal paths act in-phase, and that the foldback of the high-frequency response is in-phase with the low-frequency response.

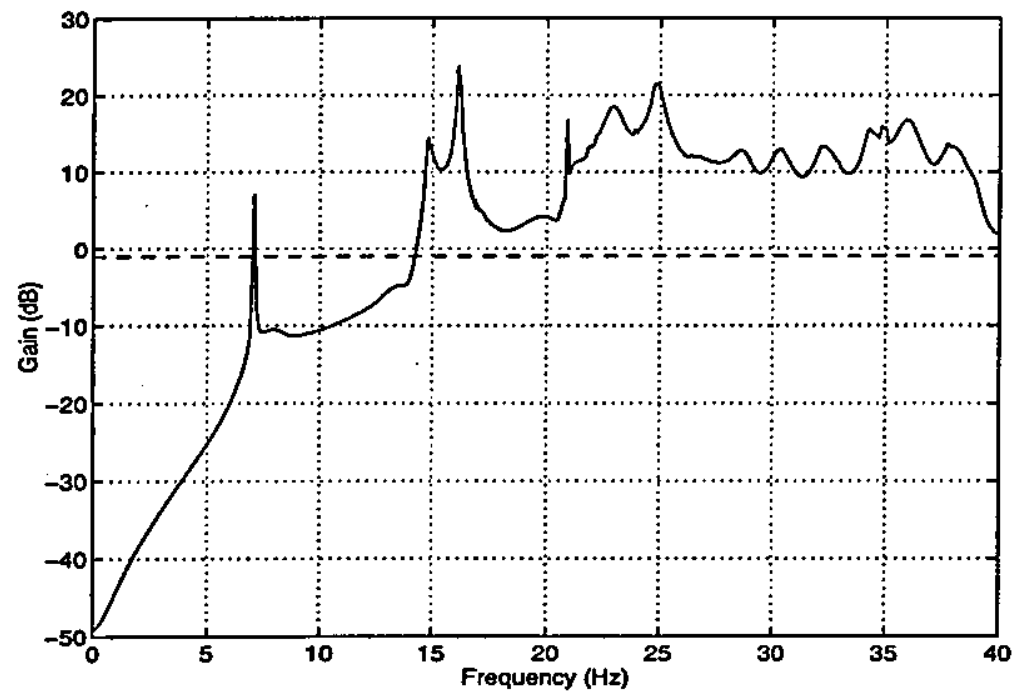

Figure 8.43 - Maximum open-loop gain envelope for digital aircraft system model

Applying the analogue $-1 \mathrm{~dB}$ filters, as defined in equations (8.31) to (8.34), within the sensor, results in the maximum open-loop gain response as shown in Figure 8.44. Clearly these filters, which were designed for application within the analogue system, still meet the $-1 \mathrm{~dB}$ clearance requirement when the control system is digital. In this case, the effect of the aliasing of the high-frequency modes is not sufficient to require a re-design of the structural-mode filters. In fact, the attenuation of the zero-order-hold and sensor dynamics has resulted in the maximum gain response for the digital system after filtering being lower than that for the analogue system. 


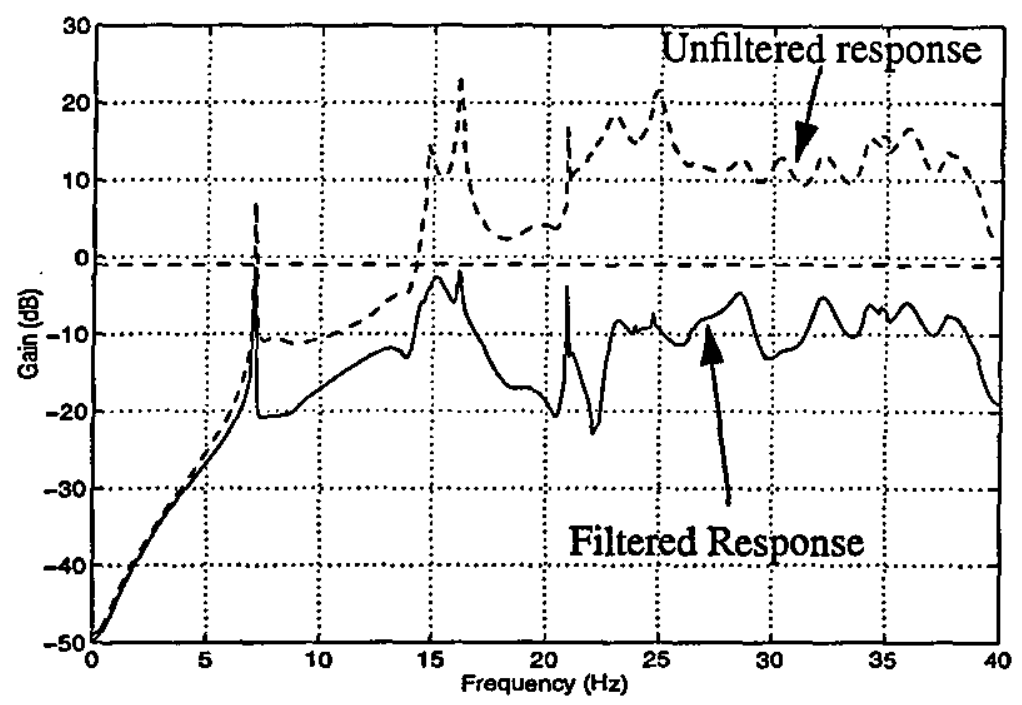

Figure 8.44 - Maximum open-loop gain envelope for digital aircraft system after filtering

\subsubsection{Limit-cycle prediction for digital system in the presence of model- ling errors}

As for the analogue case, provided the system has been accurately modelled, then no limit-cycles will occur within the digital system in the presence of the defined structural-mode filters. If the system model is in error however, the potential for limitcycles should be addressed.

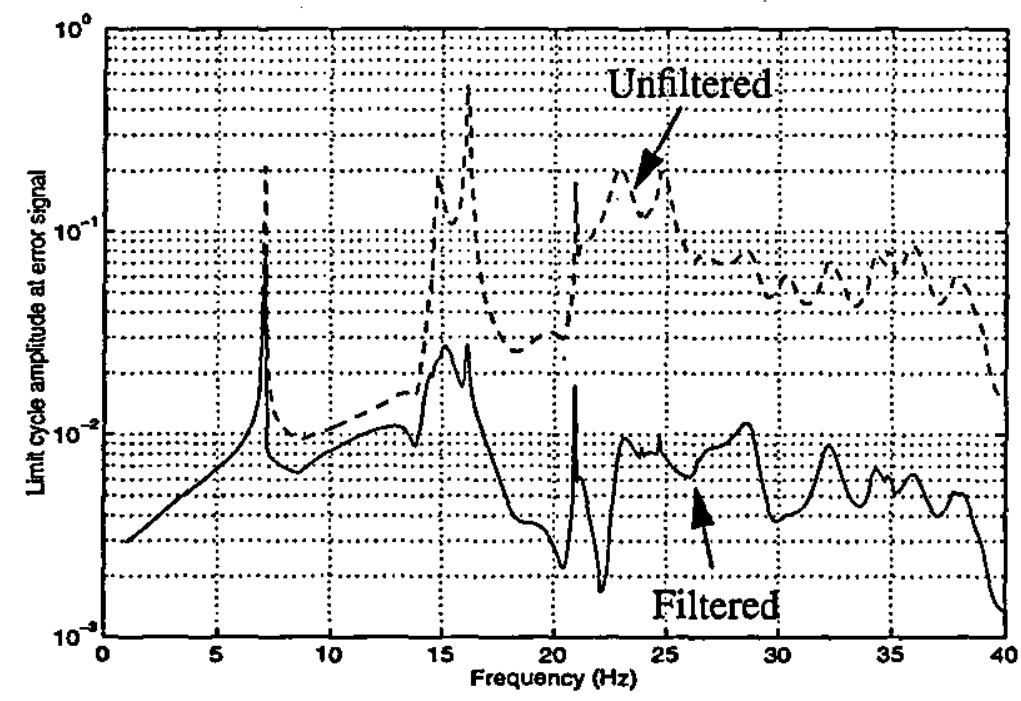

Figure 8.45 - Maximum limit-cycle amplitude at error signal for digital system nominal model, $-\mathbf{1} \mathrm{dB}$ filters

Consider the nominal model of the system. Disregarding the fact that limit-cycles cannot actually occur, the maximum amplitude of limit-cycle oscillation can be defined from a consideration of the actuator performance boundary and open-loop system gain. For the digital system, this results in the maximum amplitude of limitcycle being defined by Figure 8.45 . 
Suppose now that it was considered that the system model might be in error by a factor of two. To ensure that such a situation could not result in unsatisfactory rigidbody response, the effect of any resulting limit-cycles must be considered. In such a situation, the maximum amplitude of the potential limit-cycles is as shown in Figure 8.46

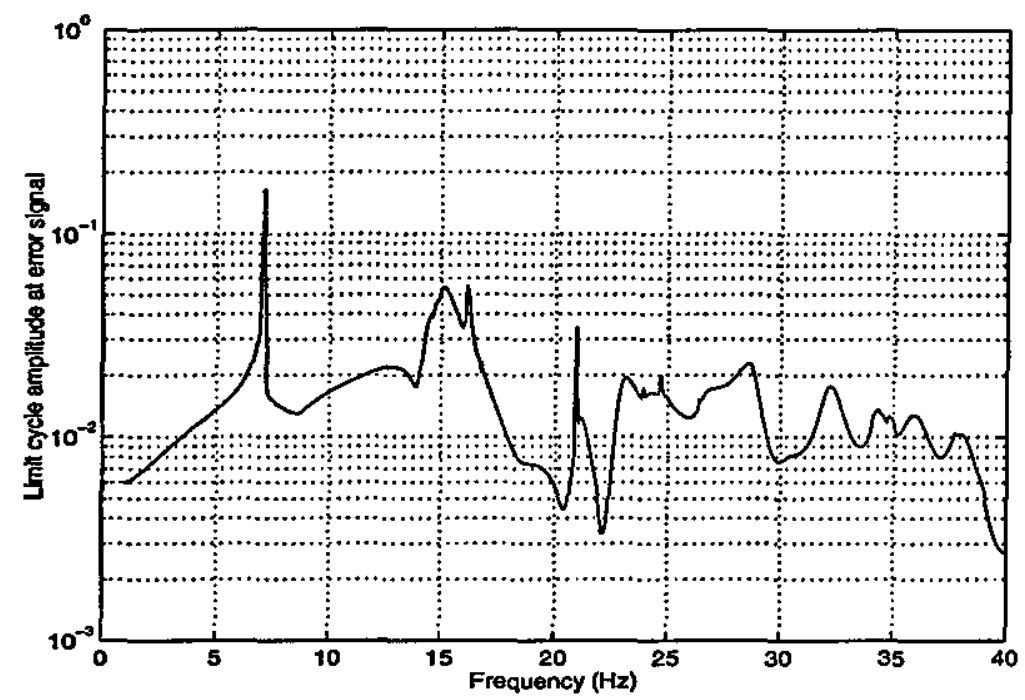

Figure 8.46 - Maximum limit-cycle amplitude at error signal for digital system $2 *$ nominal model gain, $-1 \mathrm{~dB}$ filters

It has been discussed previously that limit-cycles may only occur when the openloop gain of the system is greater than $0 \mathrm{~dB}$ due to the nature of the rate limiting nonlinearity. Production of the open-loop frequency response for the digital system assuming a twofold error in the gain results in the specification of potential limitcycles. Such an open-loop gain response is shown in Figure 8.47.

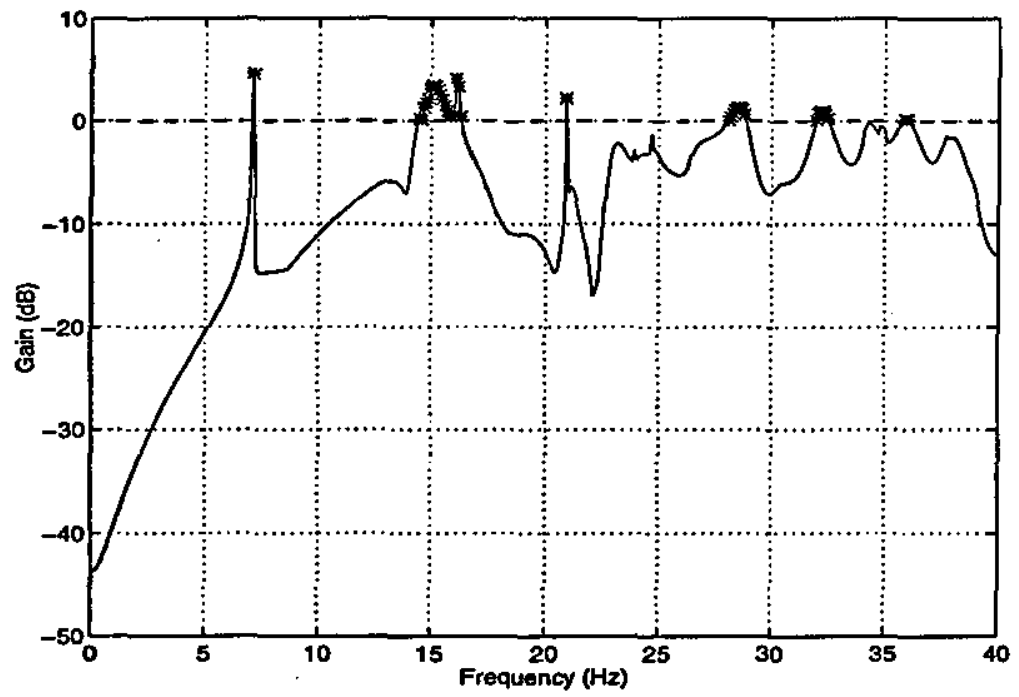

Figure 8.47 - Maximum open-loop gain for digital system with $2 *^{*}$ nominal gain and $-1 \mathrm{~dB}$ filters in place

Combining the results of Figure 8.46 and Figure 8.47 produces a prediction of both the frequency and amplitude of the limit-cycles that may occur within the digital 
system. (This assumes that there is a twofold error in the gain of the model.) For the model of the digital system, the potential limit-cycles are as shown in Figure 8.48

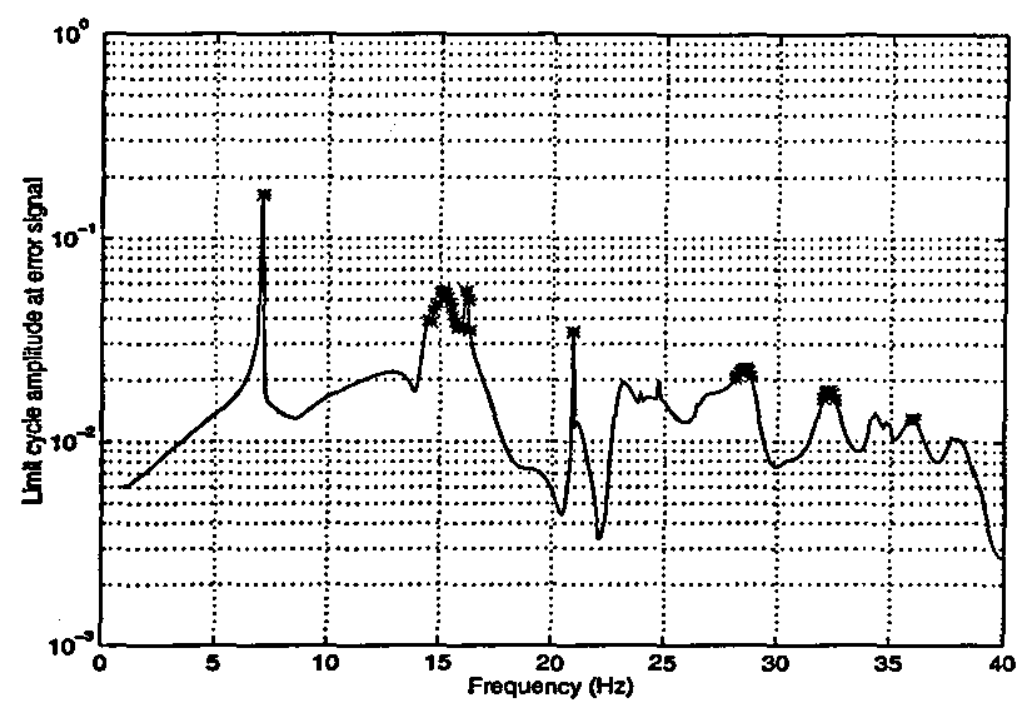

Figure 8.48 - Predicted limit-cycle frequencies and amplitudes for digital system with $2 *$ nominal gain and $-1 \mathrm{~dB}$ filters

\subsubsection{Assessment of the effect of limit-cycle oscillations within the digital system}

Once the frequencies of any potential limit-cycles have been identified and their maximum amplitudes calculated, their effect on the satisfactory control of the rigidbody aircraft can be assessed. Three possible methods of accomplishing this have already been introduced. In this case, a method will be adopted whereby simulation or experimental results examining the response of the actuators in isolation will be applied to a model of the remainder of the system. In this way, the effect of the limitcycles on rigid-body performance can be deduced without the need to resort to time domain simulations of the full system. From Figure 8.48, suitable potential limitcycles for testing can be selected. In this case, those limit-cycles are as defined in Table 8.2.

Testing of the Jaguar FBW actuator for a range of low-frequency ram demands in addition to the above limit-cycles was completed. In addition, the same tests were also completed for the actuator model. Both the actuator itself and model were subjected to the combined input signal after processing by a sample-and-hold and a rate limiter. In order to be representative of the digital system, the sampling frequency was set to 80 $\mathrm{Hz}$. A comparison of the experimental and simulated results are included as Figure 8.49 to Figure 8.56 . 


\begin{tabular}{|c|c|c|c|}
\hline $\begin{array}{c}\text { Frequency } \\
\text { (Hz) }\end{array}$ & $\begin{array}{c}\text { Amplitude } \\
\text { at error } \\
\text { signal }\end{array}$ & $\begin{array}{c}\text { Amplitude } \\
\text { of inboard/ } \\
\text { outboard } \\
\text { ram } \\
\text { demand } \\
\text { (mm) }\end{array}$ & $\begin{array}{c}\text { Amplitude } \\
\text { of } \\
\text { foreplane } \\
\text { ram } \\
\text { demand } \\
\text { (mm) }\end{array}$ \\
\hline \hline 7.1 & 0.16 & 10.9 & 10.7 \\
\hline 15.1 & 0.055 & 3.73 & 3.68 \\
\hline 20.9 & 0.034 & 2.31 & 2.27 \\
\hline 28.6 & 0.023 & 1.56 & 1.54 \\
\hline 32.2 & 0.018 & 1.22 & 1.20 \\
\hline 35.9 & 0.013 & 0.88 & 0.87 \\
\hline
\end{tabular}

Table 8.4 - Limit-cycle amplitude and frequencies

As for the analogue system, it can be seen that the simulated responses match well with the experimental results. Once again, the responses demonstrate the changes in gain and phase expected as a result of the presence of the limit-cycles. It is the potential limit-cycle at a frequency of $7.1 \mathrm{~Hz}$ that causes the greatest change in actuator performance as was the case for the analogue system. The relatively large difference between the experimental and simulated responses for the smallest pilot demand amplitude mirrors the results found for the analogue system. As discussed previously, it is believed that these differences originate from un-modelled nonlinearities within the actuator.

To examine how such changes in actuator performance will effect the rigid-body stability-margins, it is possible to apply the results of Figure 8.49 through Figure 8.56 to an aircraft system model. Since actual actuator test data is available, it will be this that will be utilized. Producing the open-loop frequency response for the digital system by applying the actuator frequency responses which were obtained experimentally to a model of the remaining linear elements results in Figure 8.57 to Figure 8.60. 

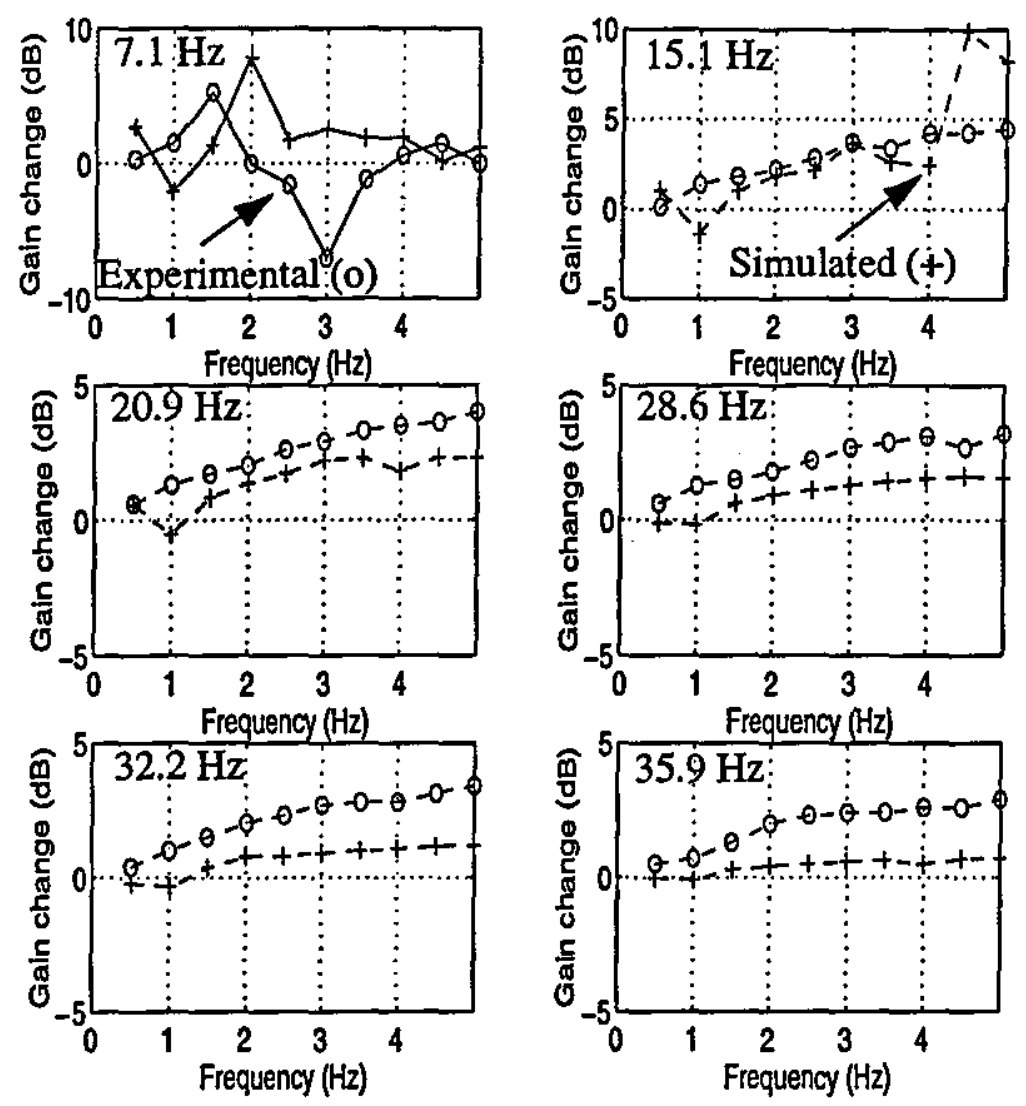

Figure 8.49 - Actuator gain response changes in the presence of limit-cycles, 0.1 mm ram demand (digital system)
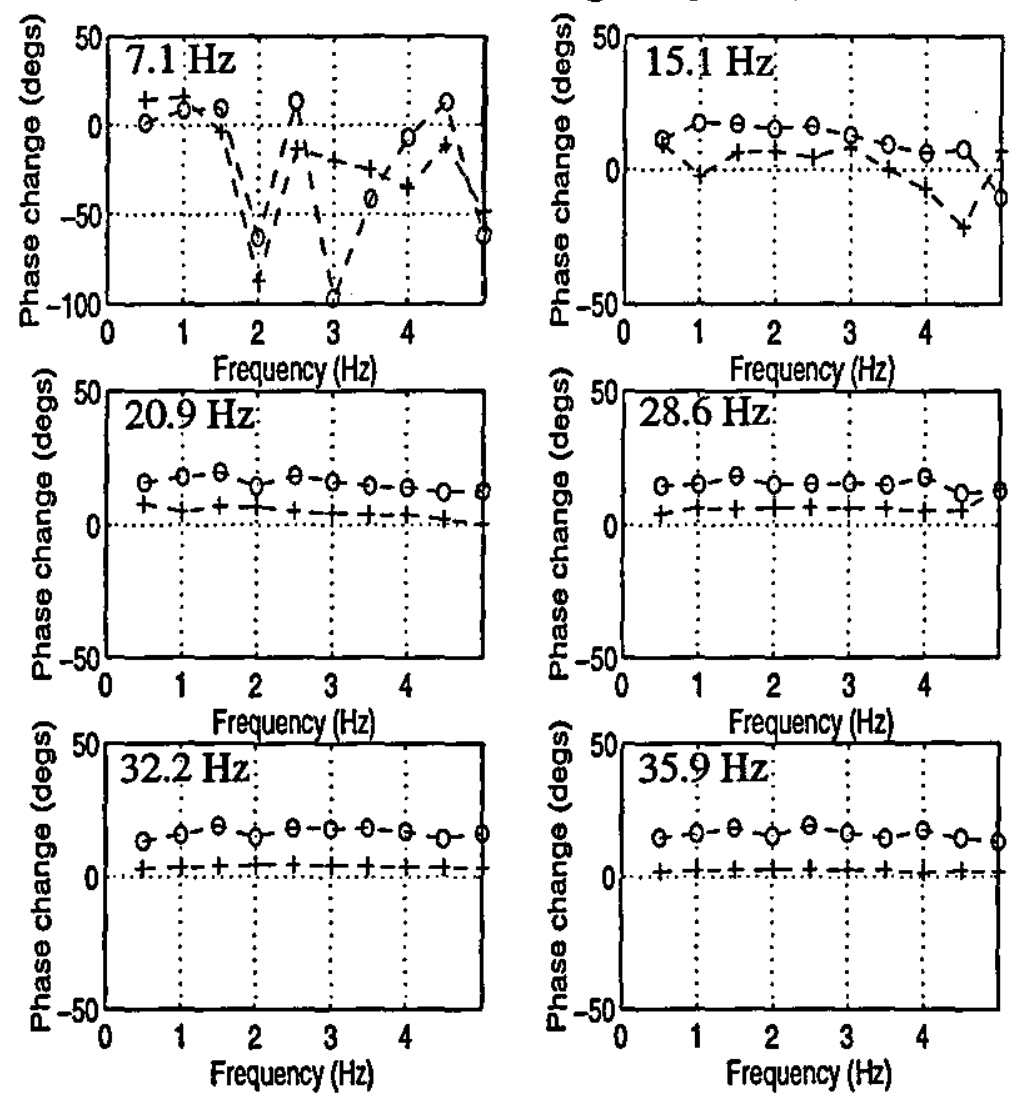

Figure 8.50 - Actuator phase response changes in the presence of limit-cycles, 0.1 mm ram demand (digital system) 

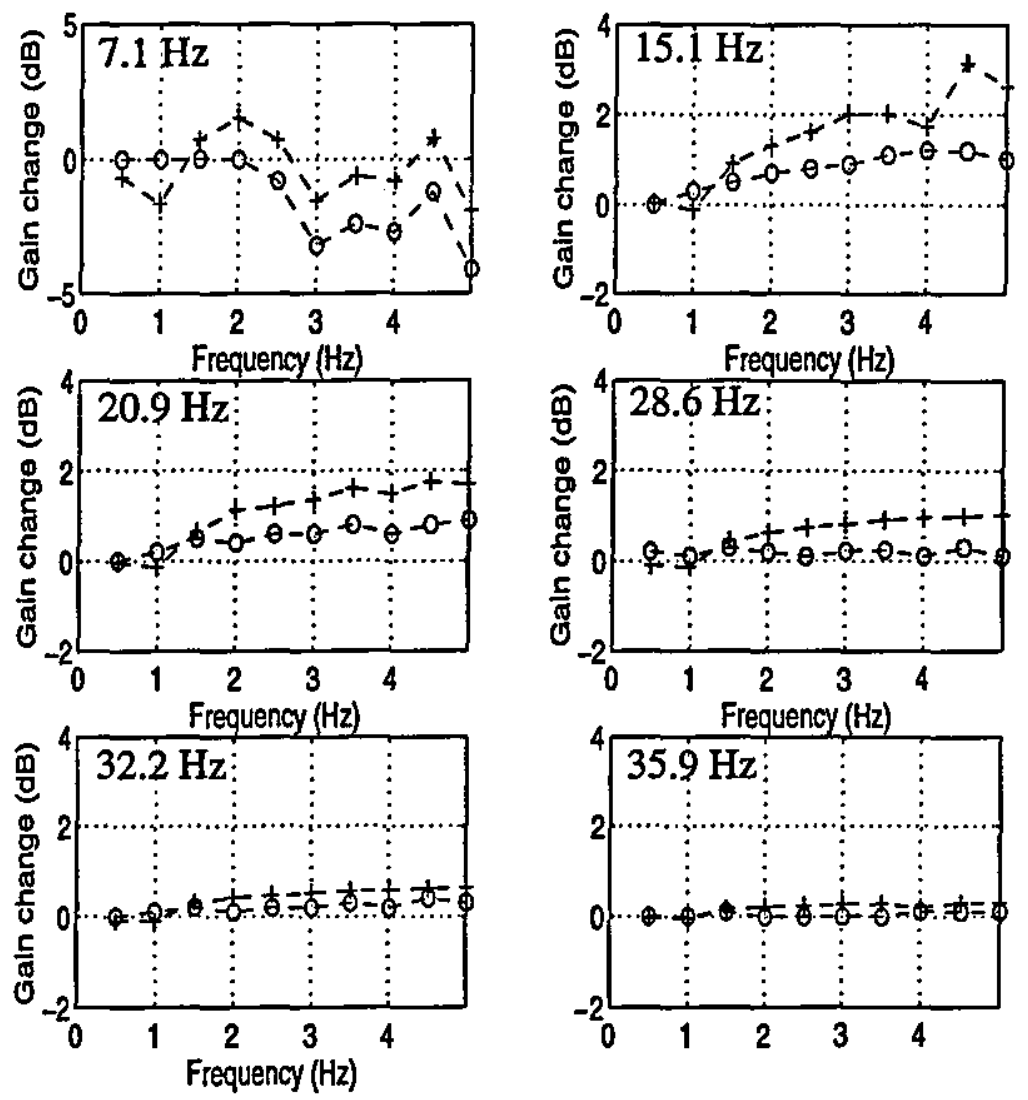

Figure 8.51 - Actuator gain response changes in the presence of limit-cycles, 1.0 mm ram demand (digital system)
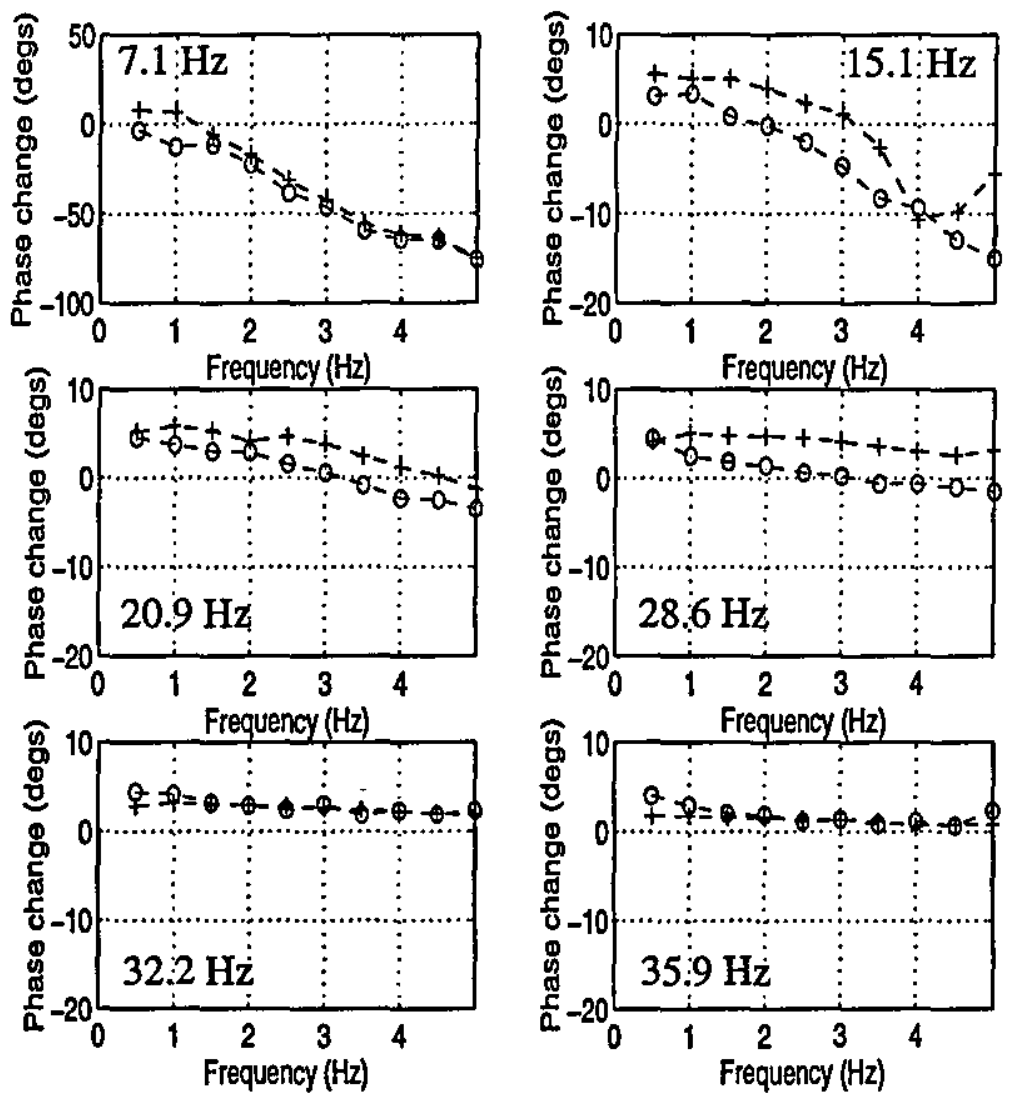

Figure 8.52 - Actuator phase response changes in the presence of limit-cycles, 1.0 $\mathrm{mm}$ ram demand (digital system) 

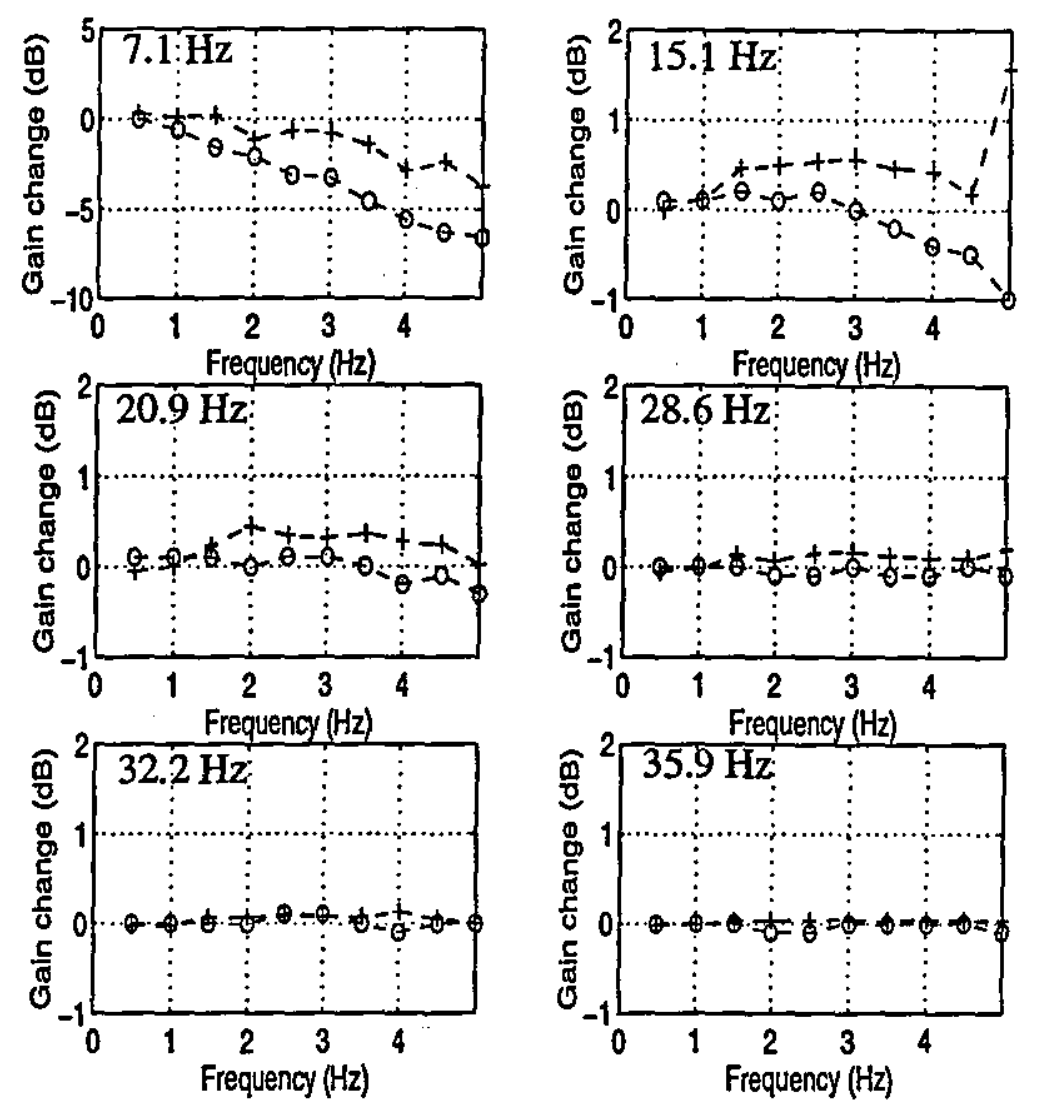

Figure 8.53 - Actuator gain response changes in the presence of limit-cycles, 5.0 mm ram demand (digital system)
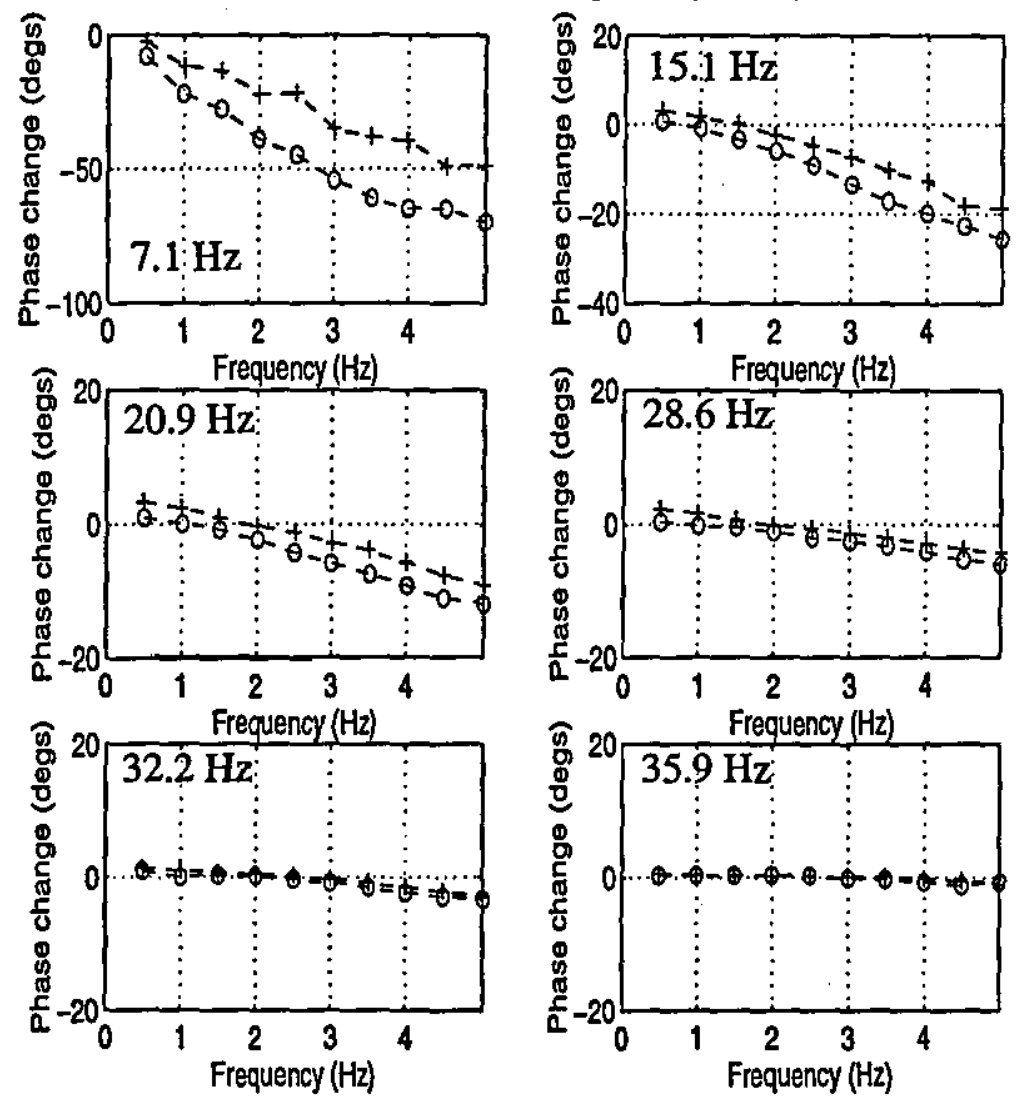

Figure 8.54 - Actuator phase response changes in the presence of limit-cycles, 5.0 mm ram demand (digital system) 

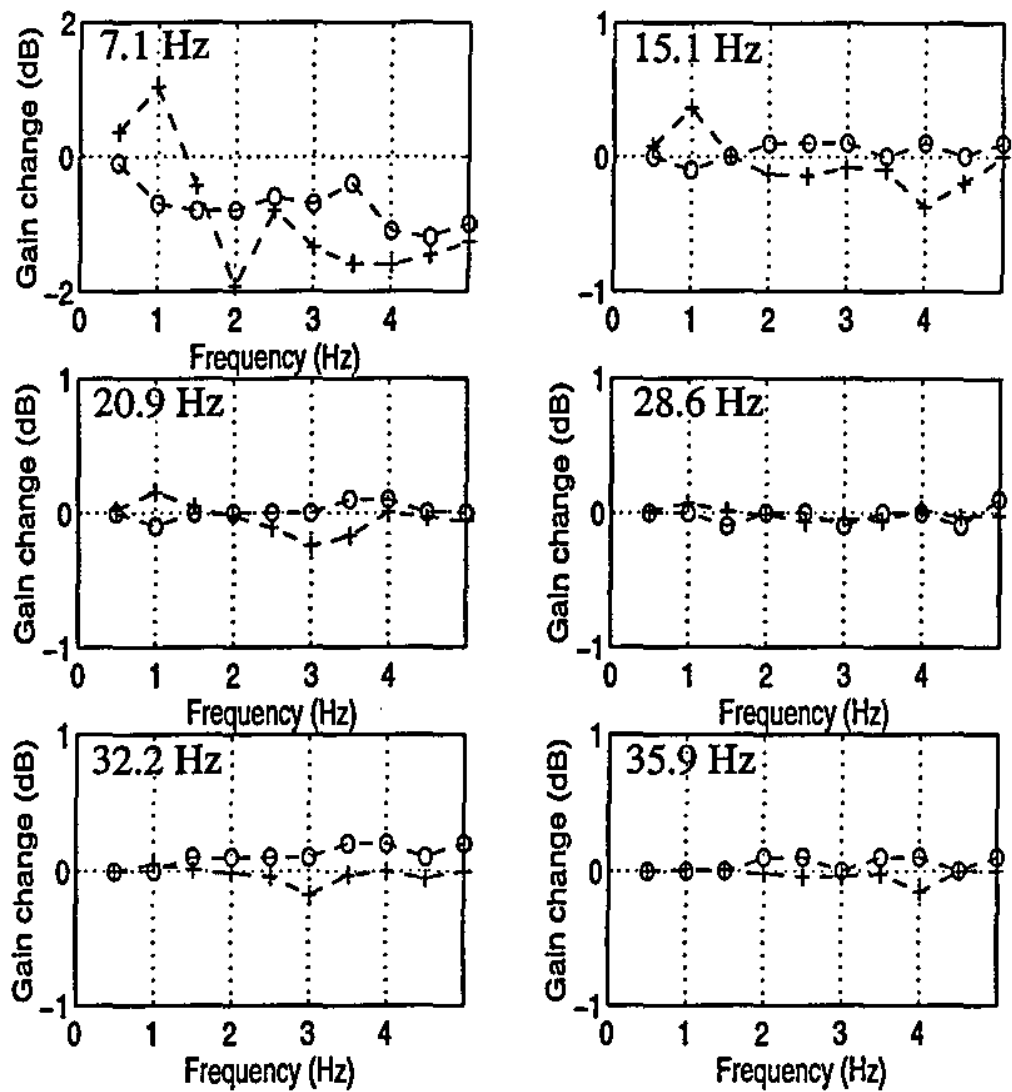

Figure 8.55 - Actuator gain response changes in the presence of limit-cycles, 20.0 mm ram demand (digital system)
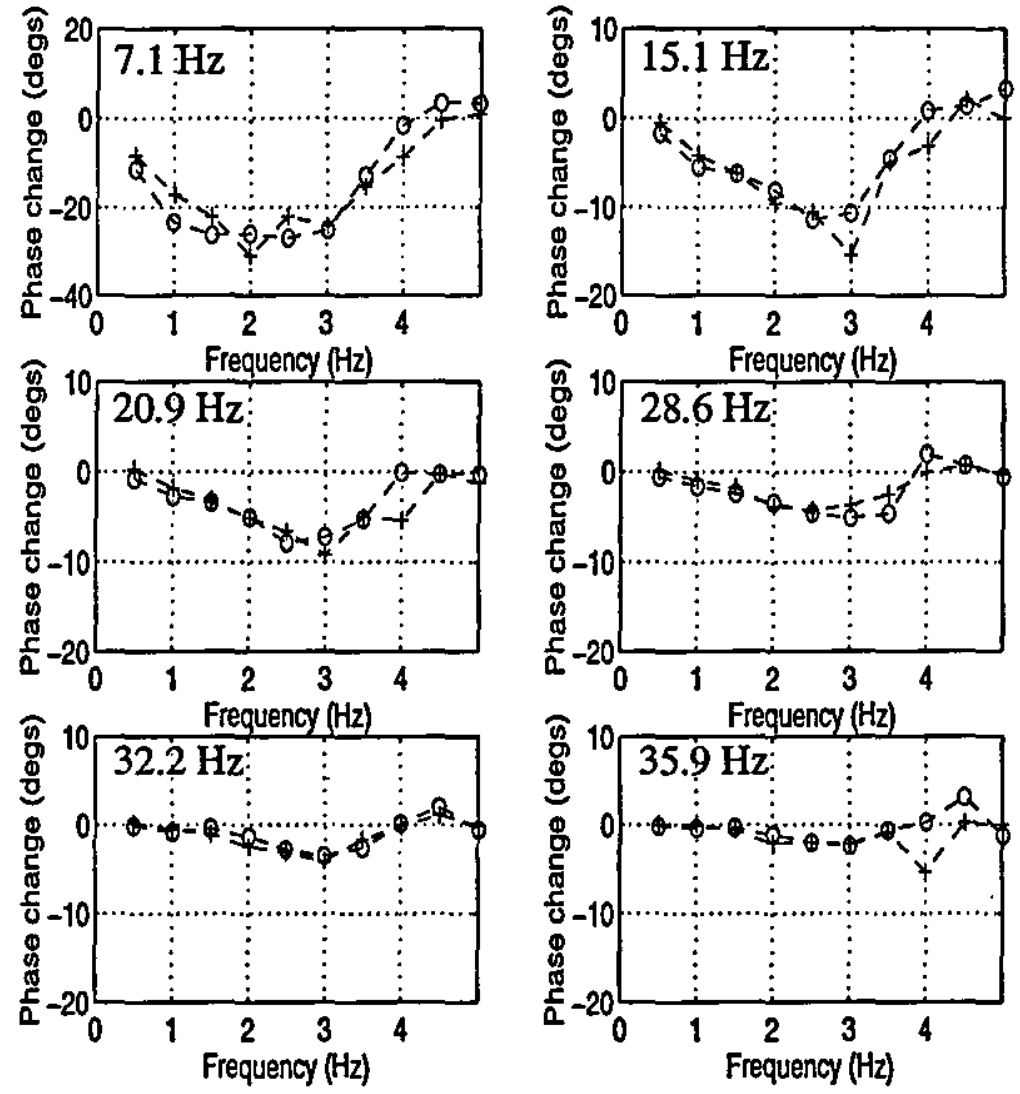

Figure 8.56 - Actuator phase response changes in the presence of limit-cycles, $20.0 \mathrm{~mm}$ ram demand (digital system) 

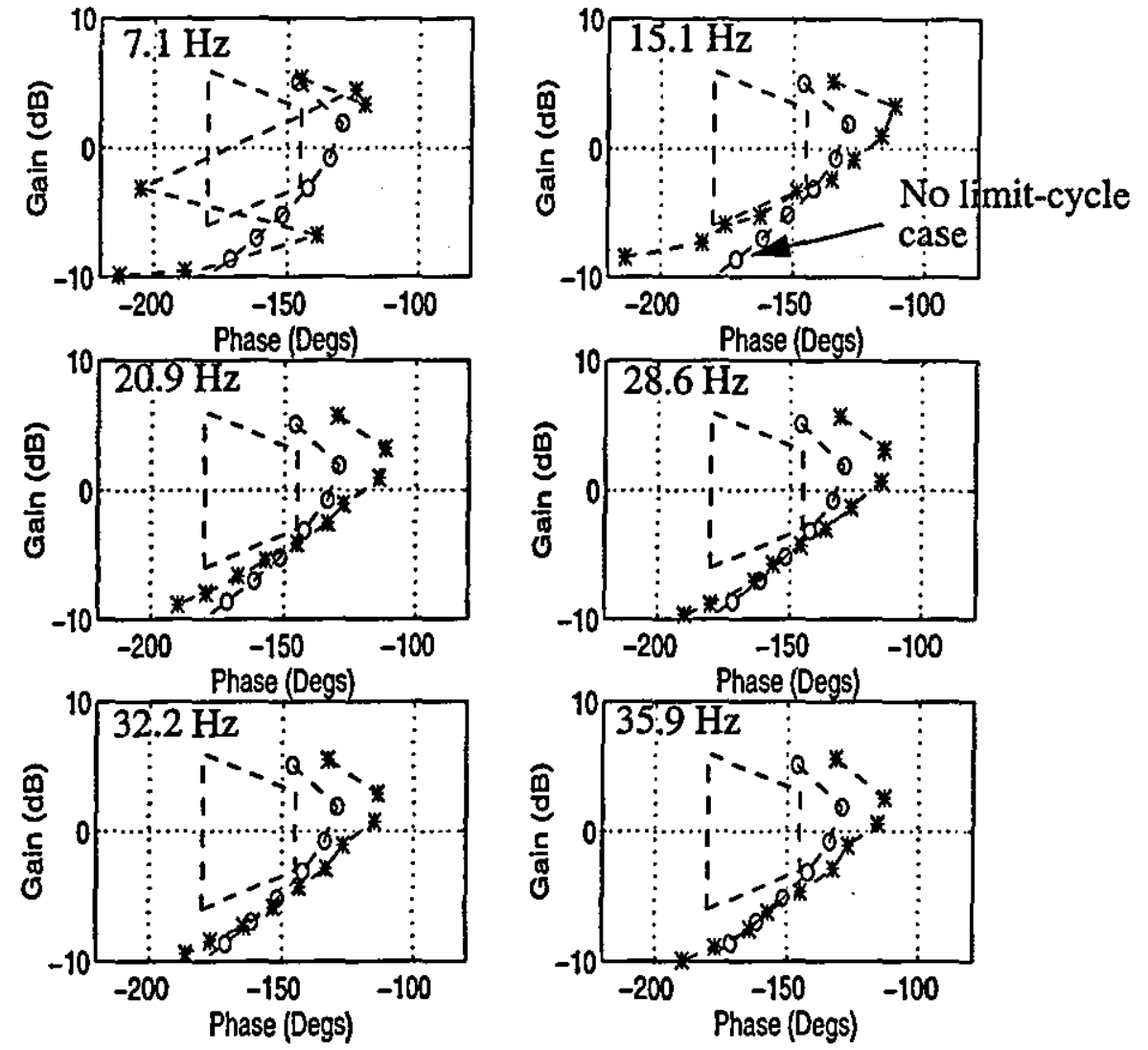

Figure 8.57 - Open-loop frequency response in presence of limit-cycles, pilot demand equivalent to $0.1 \mathrm{~mm}$ ram demand (digital system)
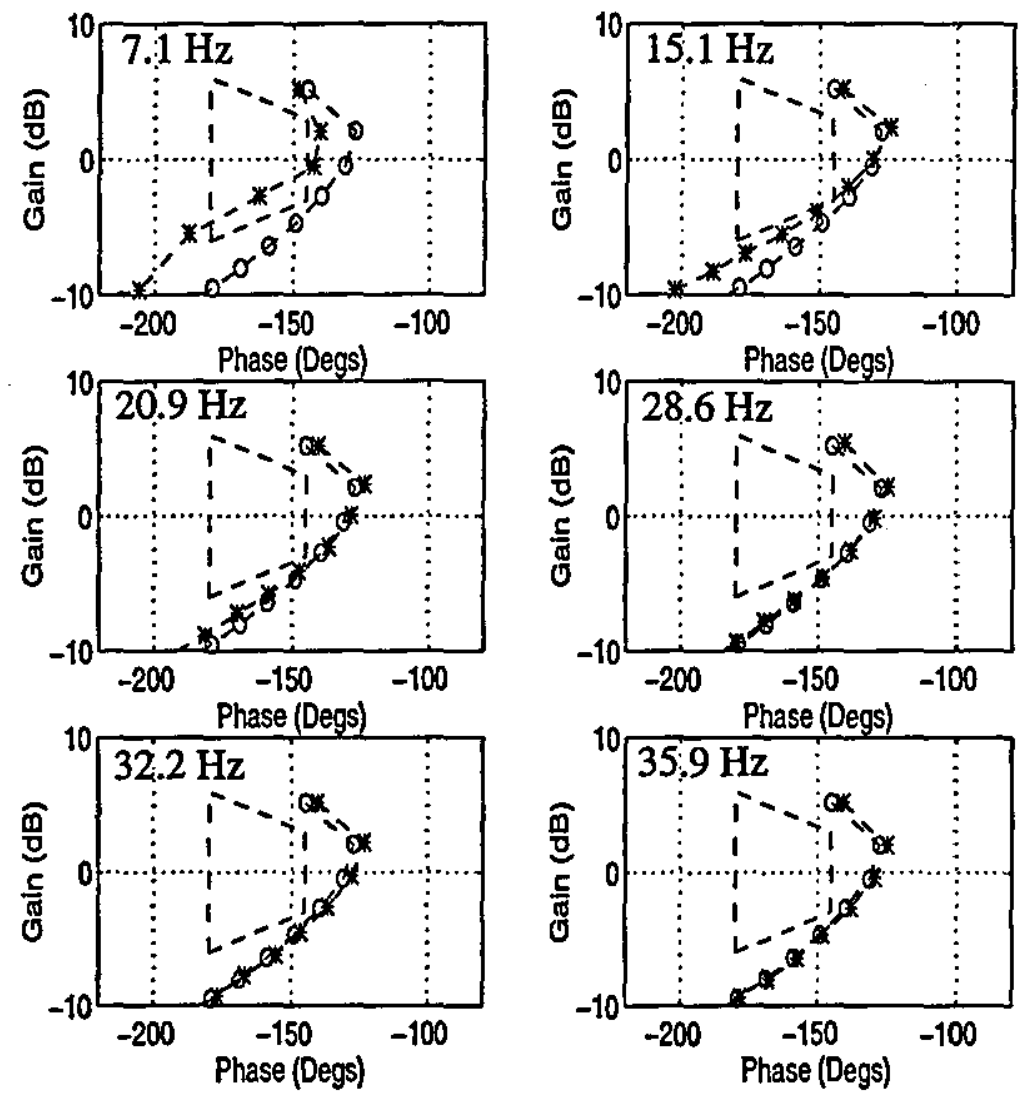

Figure 8.58 - Open-loop frequency response in presence of limit-cycles, pilot demand equivalent to $1.0 \mathrm{~mm}$ ram demand (digital system) 

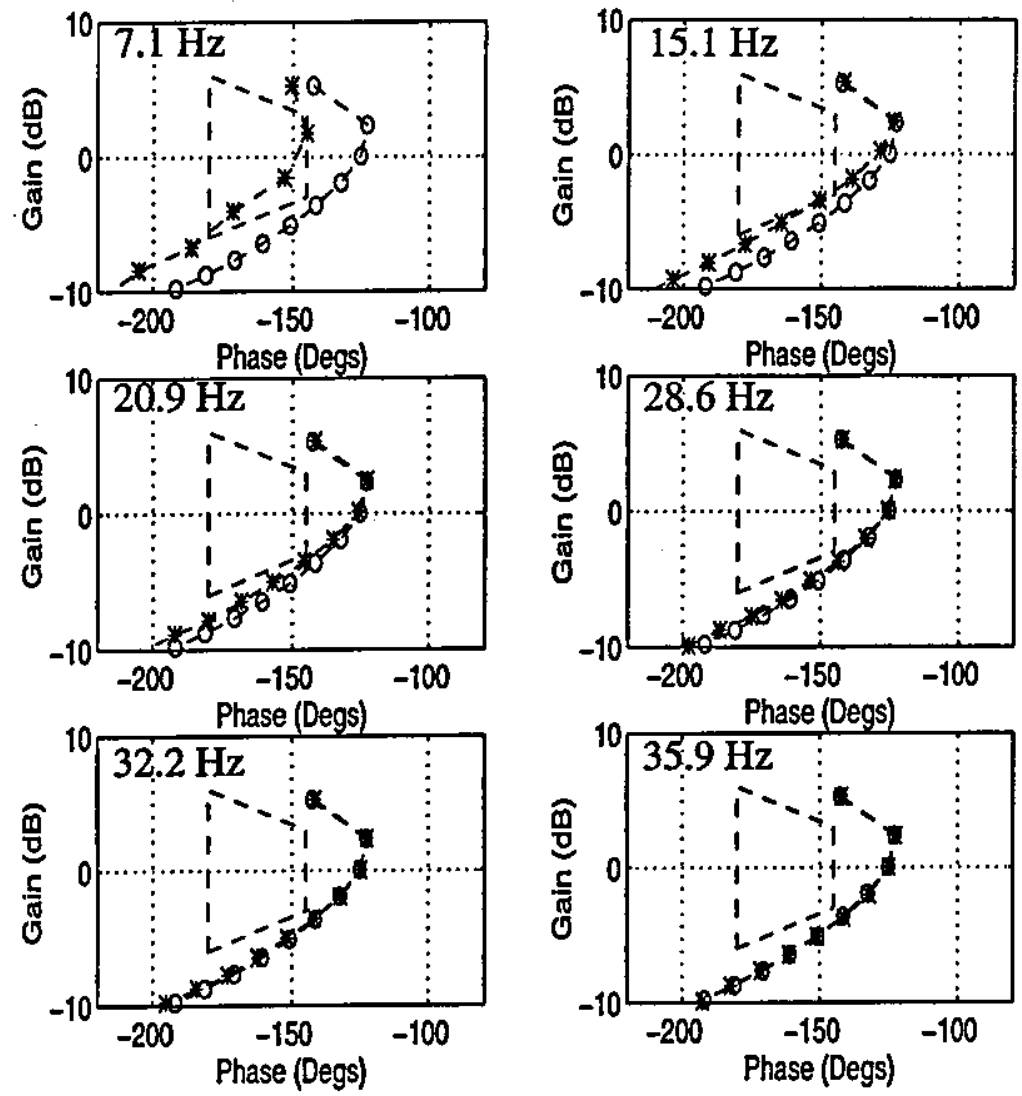

Figure 8.59 - Open-loop frequency response in presence of limit-cycles, pilot demand equivalent to $5.0 \mathrm{~mm}$ ram demand (digital system)
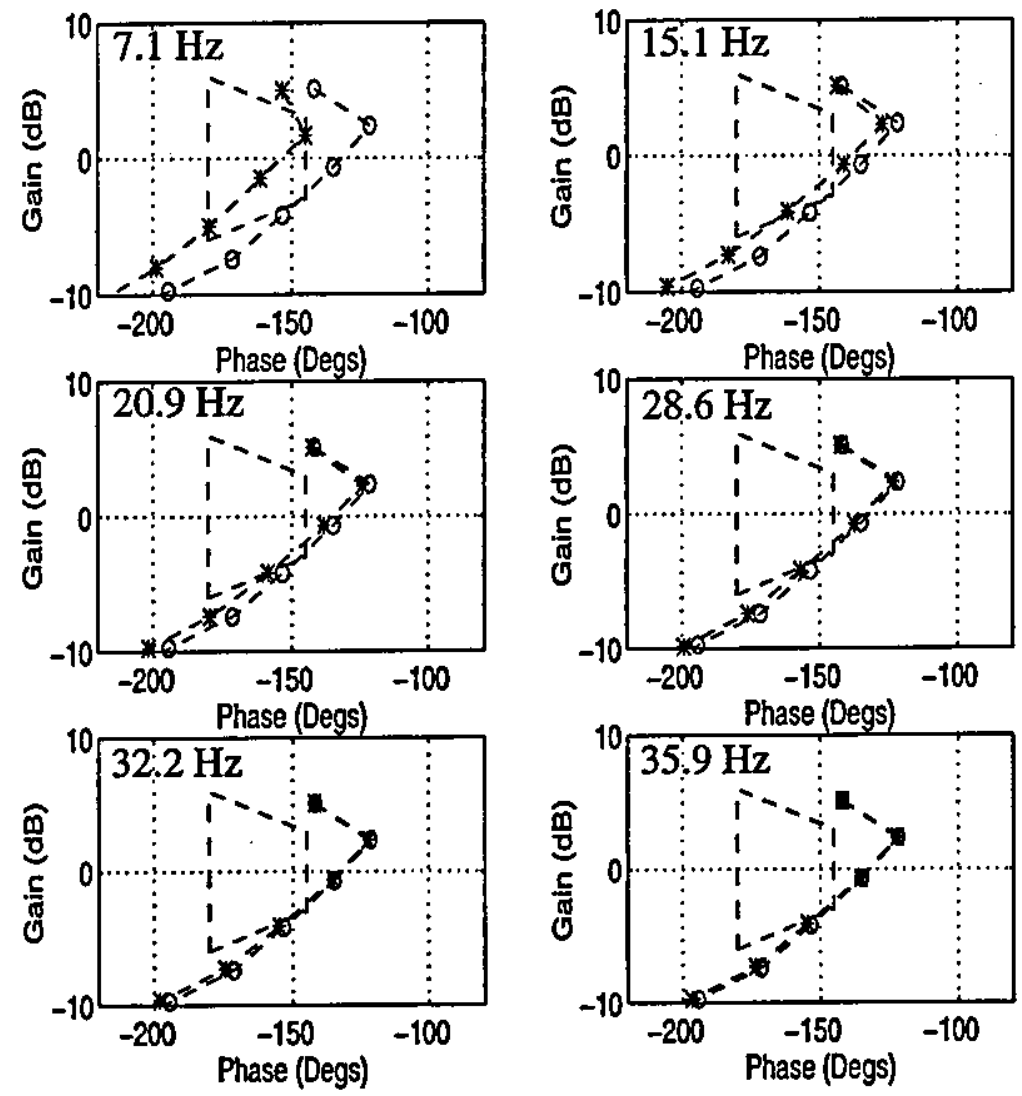

Figure 8.60 - Open-loop frequency response in presence of limit-cycles, pilot demand equivalent to $20.0 \mathrm{~mm}$ ram demand (digital system) 
The above Nichols plots showing the open-loop frequency response for the digital system, demonstrate several interesting points. Firstly, as for the analogue system, it is the potential limit-cycle of frequency $7.1 \mathrm{~Hz}$ that causes the most serious change in stability-margins for the rigid-body aircraft. This is to be expected, since this limitcycle has the largest potential amplitude due to the actuator performance limit. In this case however, it can be seen that the all of the potential limit-cycles cause violation of the clearance boundary at large pilot-demand amplitudes, as can be seen from Figure 8.60. This is due in the main, however, to the effect of the dynamics of the sampleand-hold and sensor. The phase lags introduced by these two elements has resulted in only a very small margin between the clearance boundary and the frequency response in the absence of any limit-cycles. In fact, it can be seen from Figure 8.60 that this response just crosses the lower corner of the clearance boundary.

In reality, the phase lags introduced by the sample-and-hold and sensor dynamics will be compensated for within the flight control system. Throughout this research, the flight control system has remained unchanged from that developed in Chapter 4 to allow comparisons to be made between the levels of structural excitation. In this case, this has resulted in the violation of the clearance boundary for the digital system for large pilot demand amplitudes. This would not be the case if the digital nature of the control system were taken into account initially. It is important to note however, that the inclusion of extra phase advance filtering within the flight control system would increase the gain of the structural-modes. As a result, the potential limit-cycle amplitudes will be higher than those examined here.

However, this example demonstrates that it is possible to assess the effects of the predicted limit-cycles on the stability-margins of the rigid-body under digital control.

\subsubsection{Conclusions}

The inclusion of the digital nature of the control system within the alternative design procedure has been demonstrated. In this case, as for the analysis of Chapter 6 , the aliasing of the high-frequency response did not result in the need to re-design the structural-mode filters. This does not indicate however that the digital nature of the control system can be neglected within the aeroservoelastic design procedure. As for the analysis of the current design procedure, the digital nature of the control system must be taken into account from the outset.

\subsection{Conclusions}

The nonlinear nature of the typical aeroservoelastic system has been shown to result in the potential for limit-cycle oscillations as opposed to an unbounded structural response as is currently assumed. From a consideration of the describing 
function technique for nonlinear system, it has been shown that the nature of such limit-cycles can be predicted. In the presence of uncertain phase information however, this prediction is restricted to the amplitude of any potential limit-cycle and the frequency range over which they may occur.

Consideration of the nature of the software rate limiting function has shown that in order to prevent unwanted limit-cycle oscillation, it is sufficient to ensure that the open-loop gain of the remaining linear elements of the system is less than $0 \mathrm{~dB}$. This ability to predict and prevent limit-cycles in the nominal situation has led to the proposal of a reduction in the current structural-mode clearance requirement. Instead of the current $-9 \mathrm{~dB}$ maximum open-loop gain at structural frequencies, it is proposed that a maximum open-loop gain of $-1 \mathrm{~dB}$ be allowed. Such an approach will result in a significant reduction in the phase lag introduced by the required structural-mode filtering.

Adopting this approach to aeroservoelastic clearance will result in poorer robustness to modelling errors than for the current design procedure. In this case however, the effect of modelling errors can be predicted. This will lead to a specification of the maximum limit-cycle amplitude in the presence of quantified modelling errors. In turn, the effect of such limit-cycles on rigid-body stabilitymargins can be assessed. Provided that satisfactory rigid-body stability is maintained, the aircraft may be flown without risk.

The alternative design procedure has been demonstrated for the aircraft system under consideration. The results of this analysis have demonstrated how it is possible to predict the potential for limit-cycles and to ensure that rigid-body stability is maintained even in the presence of modelling errors. 


\section{Chapter 9}

Experimental Evaluation of Alternative Clearance Requirements 


\subsection{Introduction}

The previous chapter has introduced an alternative aeroservoelastic design procedure based on a knowledge of any limit cycling condition that may arise within a system. The ability to predict such limit-cycles, assess their effect on rigid-body stability and define the attenuation necessary to prevent them provides a useful tool for the flight control system designer.

Although the design procedure has been demonstrated as applied to an aircraft model, it is necessary to demonstrate its use on an actual test system ${ }^{73}$. Naturally, the availability of a complete aircraft system for test is limited. It is still possible however to demonstrate both current and alternative design procedures on a suitable test rig.

The following chapter describes the test rig in detail, and presents the results of tests examining both the current and proposed design procedures.

\subsection{Description of the test rig}

Chapter 7 discussed in detail the form of the test rig used for the testing of the actuation system in isolation. In order to demonstrate a typical aeroservoelastic system however, the rig had to be modified so that the actuator was exciting a suitable structure. In addition, the motion of this structure then had to influence the demand signal to the actuator in order to promote a suitable interaction. A diagrammatic representation of the test rig is shown in Figure 9.1.

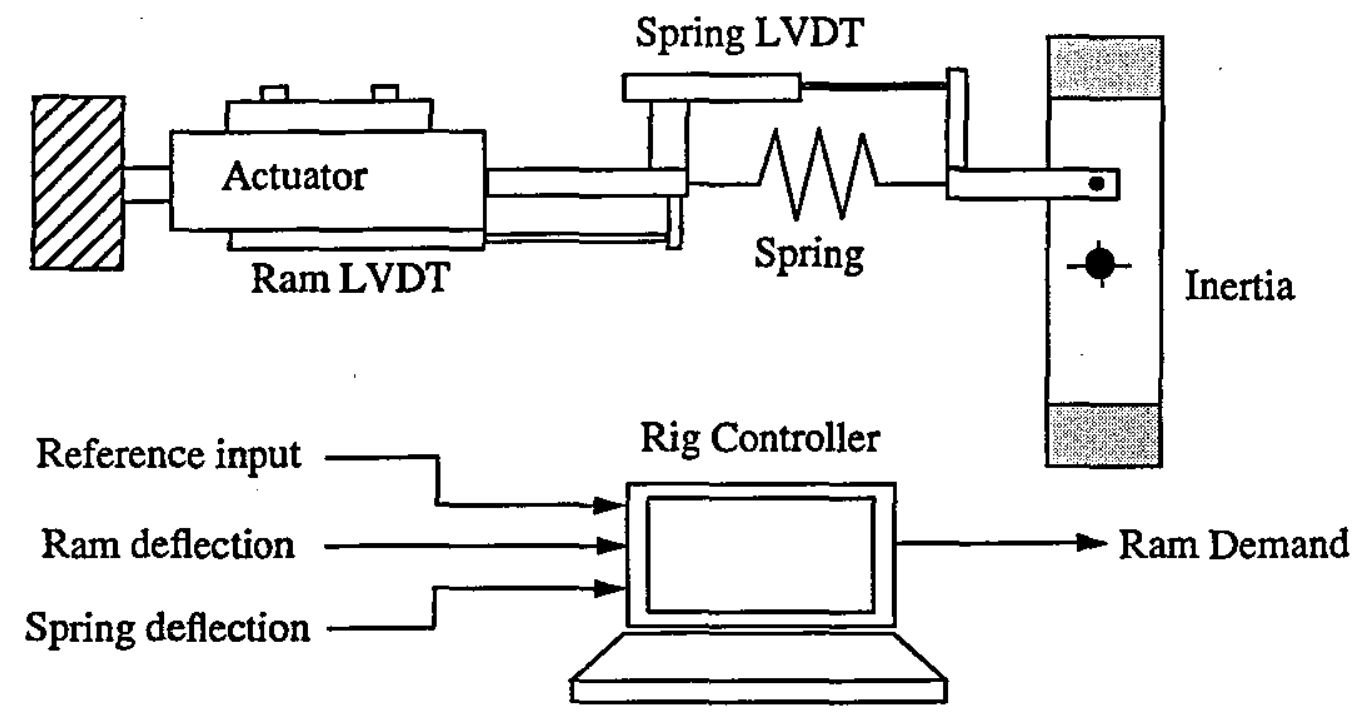

Figure 9.1 - Diagrammatic representation of test rig

In this case, the transfer function analyser and electronic test set shown in Figure 7.8 are omitted for simplicity. In the case of the test arrangement, the personal computer has a much larger role to play than in the testing of the actuator in isolation. 
In this case, the computer acts as a control system, feeding back both ram position and spring deflection to the actuator input in addition to executing the rate limiting algorithm. A block diagram of the system is shown in Figure 9.2.

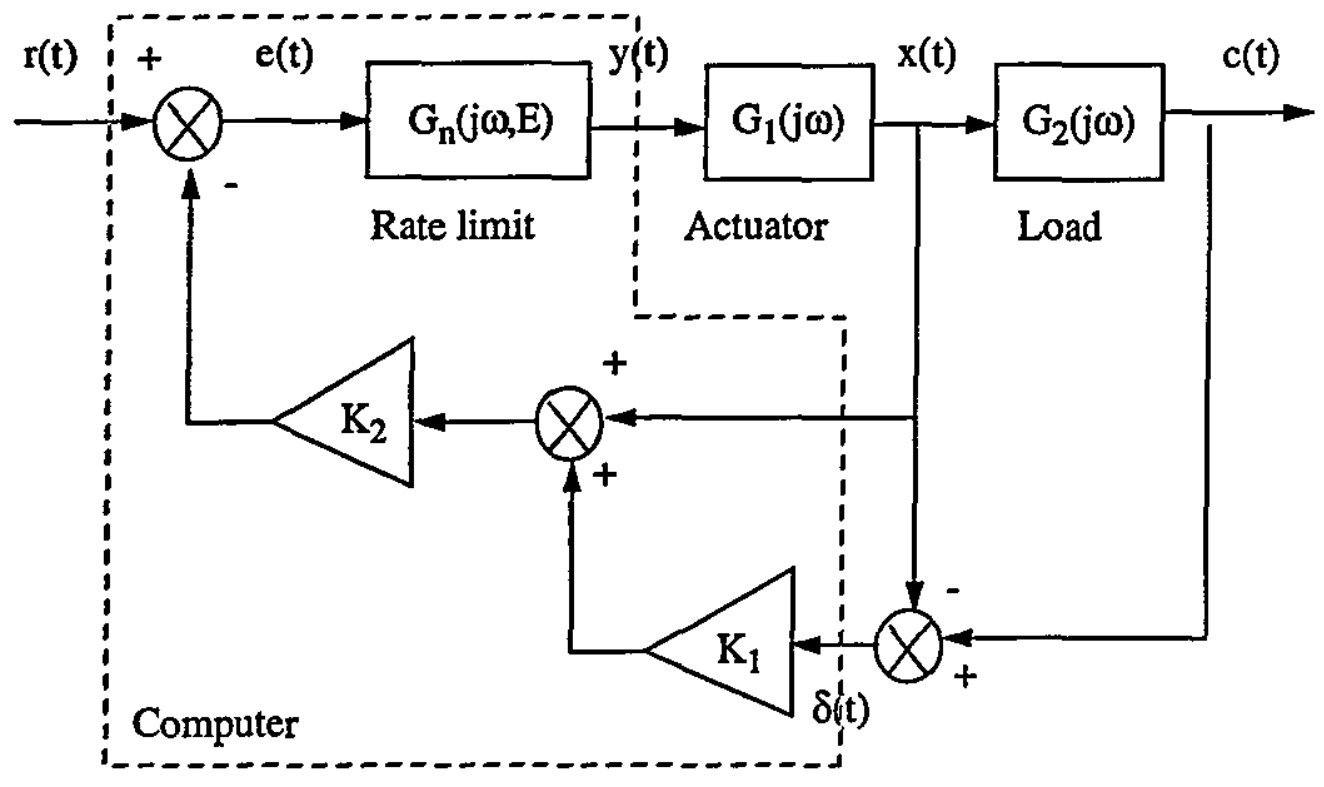

Figure 9.2 - Block diagram of test rig

The test rig consists of a Jaguar FBW taileron actuator driving a load made up of a pivoted mass-spring system as shown in Figure 9.1. The LVDT shown in Figure 9.1 was arranged so as to measure the deflection of the spring, as signified by the signal $\delta(t)$. This signal was then combined with the actuator ram deflection, $x(t)$ within the computer before feedback to the actuator input. The two gains in the feedback path, $K_{1}$ and $K_{2}$, were included so as to vary the characteristics of the system in order to generate a suitable structural interaction.

Although the rig itself is simple in comparison with an entire aircraft system, it exhibits many characteristics of the full system. The nonlinear actuator is driving a load which exhibits a structural-mode. This structural-mode is sensed by the control system and fed back to the actuator input as in the aircraft. The purpose of the feedback loop for ram position was to represent the rigid-body dynamics of the system, such that if $\mathrm{K}_{1}=0$ in Figure 9.2, then the controller would only be influenced by the rigid-body dynamics.

\subsection{Derivation of a system model}

\subsubsection{Introduction}

As with the aircraft system under consideration in the earlier chapters, one of the first steps in the design of suitable structural-mode filters, is the development of a representative system model. This model may be developed analytically at first, and 
then updated when suitable testing of the system can be completed. Once such a system model is obtained, it is possible to design any necessary structural-mode filters in accordance with either the current or proposed alternative design procedure.

\subsubsection{Analytical derivation of a system model}

In order to develop a suitable system model, consider the block diagram as shown in Figure 9.2. Assuming that the rate limiter can be neglected for now, and that the actuator can be represented by the linear model given in equation (4.1), the only system element that requires modelling is the load, $G_{2}(j \omega)$.

Consider the load as shown in Figure 9.3.

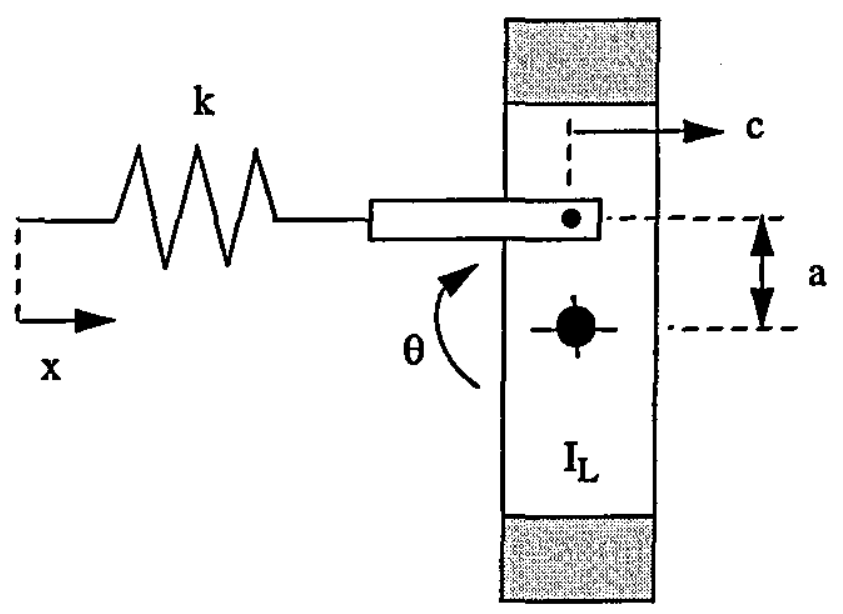

Figure 9.3 - Test rig loading system

Neglecting any damping inherent within the system, the equation of motion of the load can be derived as

$$
\frac{\mathrm{I}_{\mathrm{L}}}{\mathrm{a}} \ddot{\mathrm{c}}=k(x-c) \mathrm{a}
$$

for small $\theta$. This leads to the transfer function of the load, $G_{2}(j \omega)$, becoming

$$
G_{2}(j \omega)=\frac{C(s)}{X(s)}=\frac{\frac{k a^{2}}{I_{L}}}{s^{2}+\frac{k a^{2}}{I_{L}}}
$$

In this case, the spring stiffness, $\mathrm{k}$, can be calculated to be $640 \mathrm{kNm}$, and the inertia of the load, $\mathrm{I}_{\mathrm{L}}$, can be calculated to be $2.01 \mathrm{Kgm}^{2}$. Assuming an inherent damping of $\zeta=0.01$ as for the aircraft structure, the transfer function of the load can be derived as

$$
G_{2}(j \omega)=\frac{7161}{s^{2}+1.6925 s+7161}
$$


Combining this model of the load with the remaining system elements results in a linear model of the test rig. In order to validate the model, and update the load transfer function if required, it is necessary to perform open-loop frequency response tests for comparison with the simulated results.

\subsubsection{Comparison of simulated open-loop frequency response with test results}

Frequency response results for both the rig model and the rig itself are included as Figure 9.4. In this case, the two feedback gains, $\mathrm{K}_{1}$ and $\mathrm{K}_{2}$, are set at 5.0 and 0.1 respectively. In addition, in order to represent the situation that would be found in a typical aircraft ground test, the direct feedback of $x(t)$ to the gain $\mathrm{K}_{2}$ is disabled. This effectively switches the rigid-body dynamics out of the open-loop frequency response test, mimicking what occurs in the aircraft ground test situation.
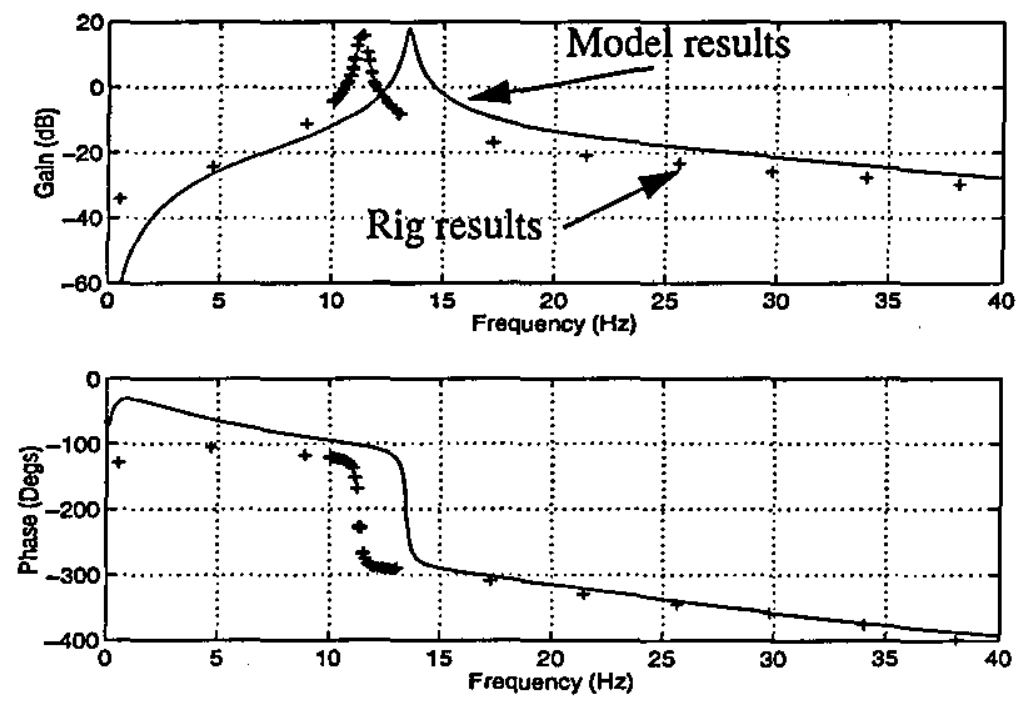

\section{Figure 9.4 - Test rig open-loop frequency response comparison}

The results demonstrate that there is an error between the simulated results and the actual test rig results. There are many possible explanations for this, the most likely of them being that the assumptions made in calculating the transfer function of the load are not valid. These assumptions include the assumed value of structural damping, and assumptions in the calculation of the spring stiffness and load inertia. Naturally, changes in these three parameters will change the frequency and gain of the resonant peak of the system. The results demonstrate however that an approximation to the structural-mode can be obtained from simple theory.

Compensating the dynamics of the load in terms of its inertia, stiffness and damping allows the simulated results to match more closely the results from the rig tests. The resulting load transfer function, $G_{2}(j \omega)$, is

$$
G_{2}(j \omega)=\frac{5050}{s^{2}+2 s+5050}
$$


Recalculating the model frequency response for the updated load model produces the results as shown in Figure 9.5.
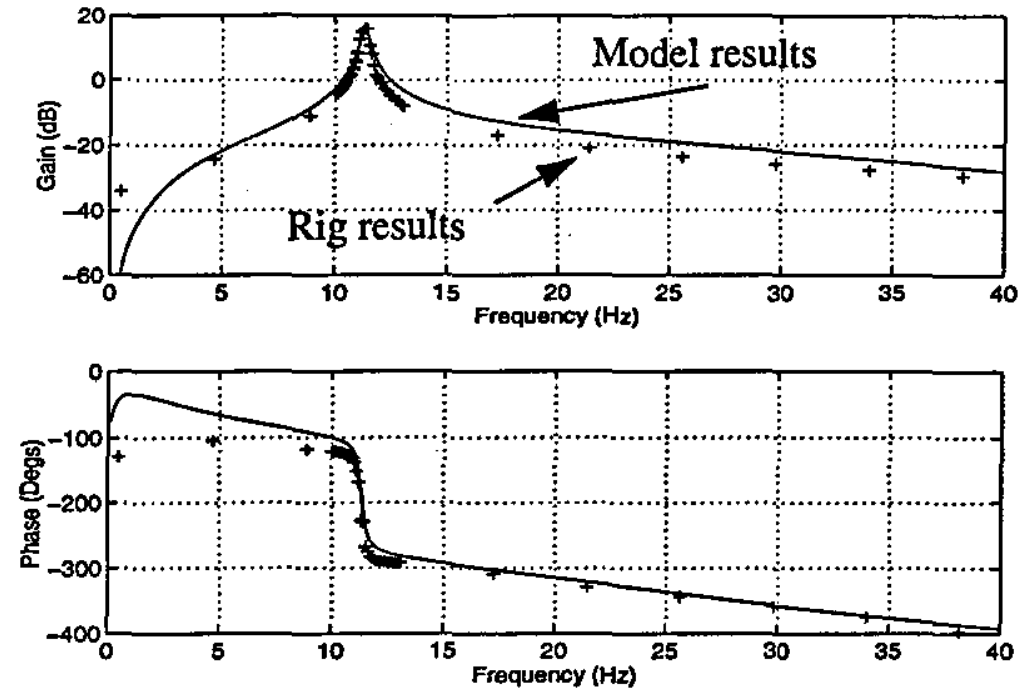

Figure 9.5 - Test rig open-loop frequency response comparison

The results show that the revised load model produces a good representation of the actual rig results.

Although the development of the system model to match the test results is not strictly necessary for the design of the structural-mode filters to take place, in this case, such a model will be used to demonstrate some of the results of Chapter 8 . In the case of the aircraft system, once ground test results are available, the structural-model of the aircraft is not required. Instead, only the model of the unsteady aerodynamic effects is used to augment the results from the ground tests for the structural dynamics.

Before the design of any structural-mode filters takes place, it is instructive to examine the unfiltered closed-loop system response.

\subsection{Limit-cycle prediction for the test system}

\subsubsection{Introduction}

The possible existence of limit-cycles within nonlinear aeroservoelastic systems has been discussed in some detail in Chapter 8. The application of nonlinear theory has allowed the successful prediction of limit-cycles in both the simple system used in the example, and in the full aircraft system model. The existence of such limit-cycles has only been demonstrated within simulated systems however. In order to demonstrate that such a limit cycling condition does arise, it is useful to examine the closed-loop behaviour of the test rig before the application of any structural-mode filters. 


\subsubsection{Limit-cycle prediction for test system}

The prediction of limit-cycles within a nonlinear system has been discussed in Chapter 8. This procedure requires the derivation of the system's characteristic equation. Considering the block diagram for the test rig as shown in Figure 9.2, it is possible to derive the characteristic equation of the system to give

$$
1+G_{n}(j \omega, E)\left\{G_{1}(j \omega) K_{2}-G_{1}(j \omega) K_{1} K_{2}+G_{1}(j \omega) G_{2}(j \omega) K_{1} K_{2}\right\}=0
$$

The solution of the characteristic equation identifies a limit-cycle condition, which can be predicted from a re-arrangement of equation (9.5) giving

$$
G_{1}(j \omega) K_{2}\left\{\dot{l}-K_{1}+G_{2}(j \omega) K_{1}\right\}=\frac{-1}{G_{n}(j \omega, E)}
$$

The describing function of the rate limiter, $G_{n}(j \omega, E)$, has been derived in Chapter 8. Since the left hand side of the above equation is simply a function of the linear elements of the test rig, the solution of the characteristic equation is relatively straightforward to obtain. However, since the describing function of the rate limiter is dependent on both input amplitude and frequency, there exists an infinite number of loci. However, only one loci for the nonlinearity intersects the frequency response at a compatible frequency, as in the Nyquist diagram of Figure 9.6.

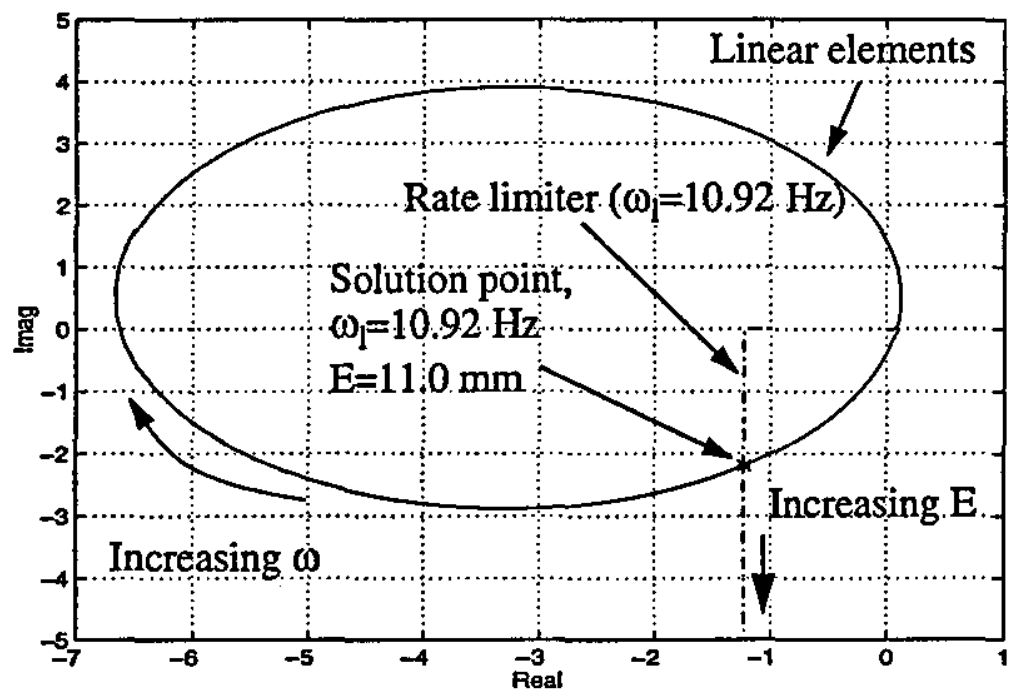

Figure 9.6 - Characteristic equation solution

In this case, the nonlinear characteristics have been plotted for a single frequency, $\omega_{1}$, which intersects the response of the linear elements at $\omega_{1}$. This point represents the solution of the characteristic equation, and therefore predicts the frequency and amplitude of the resulting limit-cycle. From Figure 9.6, nonlinear theory predicts that a limit-cycle will occur on the test rig at a frequency of $10.9 \mathrm{~Hz}$ and of amplitude equivalent to $11.0 \mathrm{~mm}$ of demand at the error signal, $e(t)$. Simulation of the test rig with the model of the linear actuator results in a limit-cycle of exactly the same 
frequency and amplitude.

If the linear actuator model is replaced by the nonlinear model developed in Chapter 7, the resulting limit-cycle is as shown in Figure 9.7.

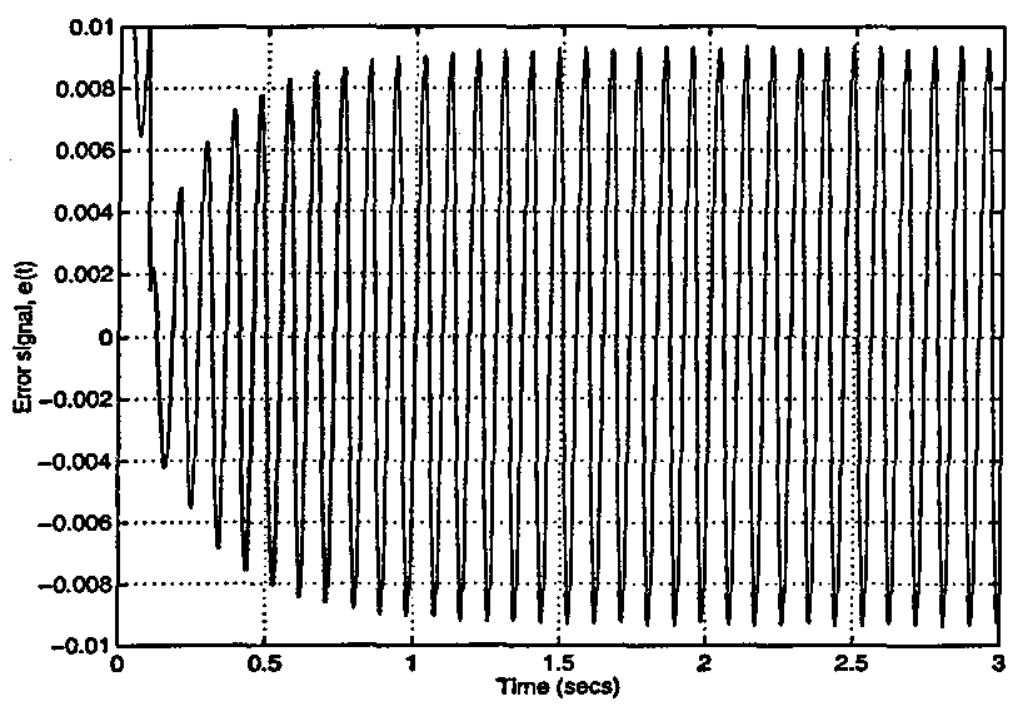

Figure 9.7 - Simulated test rig limit-cycle

From the figure, the frequency of the limit-cycle is $10.9 \mathrm{~Hz}$ with an amplitude of $9.5 \mathrm{~mm}$ at the error signal, $e(t)$. The error between the predicted amplitude and the amplitude seen in the simulation is due to the differences between the linear actuator model used in the prediction and the true nonlinear actuator model used in the time domain simulation. The ability to predict the exact characteristics of the limit-cycle by solving the system characteristic equation is therefore limited by the accuracy of the linearisation of the actuator model.

To demonstrate that a limit-cycle does occur on the test rig in practice, the results from a closed-loop test are included as Figure 9.8.

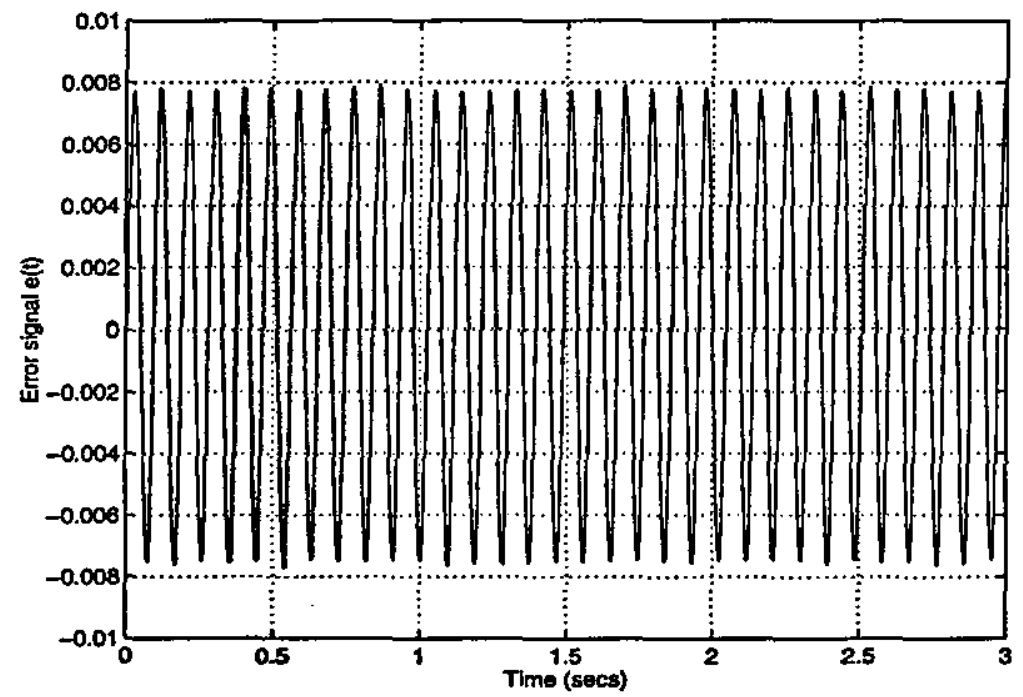

Figure 9.8 - Test rig limit-cycle

The experimental results show a limit-cycle of frequency $10.8 \mathrm{~Hz}$ and amplitude 
of $7.6 \mathrm{~mm}$ at the error signal, $\mathrm{e}(\mathrm{t})$. Clearly, their are slight differences between the simulated and experimental limit-cycles. In this case, the error is due to the inaccuracies in the modelling of both nonlinear actuator and load system. Although the load transfer function as given in equation (9.6) was matched to the open-loop test data, it is still only a second-order approximation to the actual load itself.

It has already been noted that the accuracy of the limit-cycle prediction using the characteristic equation is limited by the accuracy of the actuator linearisation. In the case of the aircraft system however, the uncertainty in the phase response of the system leads to a restriction in the use of the characteristic equation. Chapter 8 has discussed this problem in some detail, leading to the fact that in the presence of phase uncertainty it is only possible to predict the amplitude of any potential limit-cycle. Instead of refining the model of the test system, it will be assumed that the phase response of the system is unreliable. As a result, only the amplitude of any possible limit-cycles can be predicted.

Consider the solution of the characteristic equation for the test rig, (9.6), in the absence of phase information. The resulting equation is

$$
\left|G_{1}(j \omega)\right| K_{2}\left\{1-K_{1}+\left|G_{2}(j \omega)\right| K_{1}\right\}=\frac{1}{\left|G_{n}(j \omega, E)\right|}
$$

Substituting for the magnitude of the rate limit function, as given in equation (8.5), and solving for the amplitude of the error signal, E, gives

$$
\frac{4 \beta}{\omega \pi}\left|G_{1}(j \omega)\right| K_{2}\left\{1-K_{1}+\left|G_{2}(j \omega)\right| K_{1}\right\}=E
$$

Consider now that

$$
\frac{4 \beta}{\omega \pi}\left|G_{1}(j \omega)\right|=X(j \omega)
$$

where, $X(j \omega)$ is the performance boundary of the actuator as shown in Figure 7.45. The gain response of the remaining elements, $K_{2}\left\{1-K_{1}+\left|G_{2}(j \omega)\right| K_{1}\right\}$, can be obtained from experimental results. If such a procedure is completed, the predicted maximum amplitude of the limit-cycles that may exist within the system are as shown in Figure 9.9. 


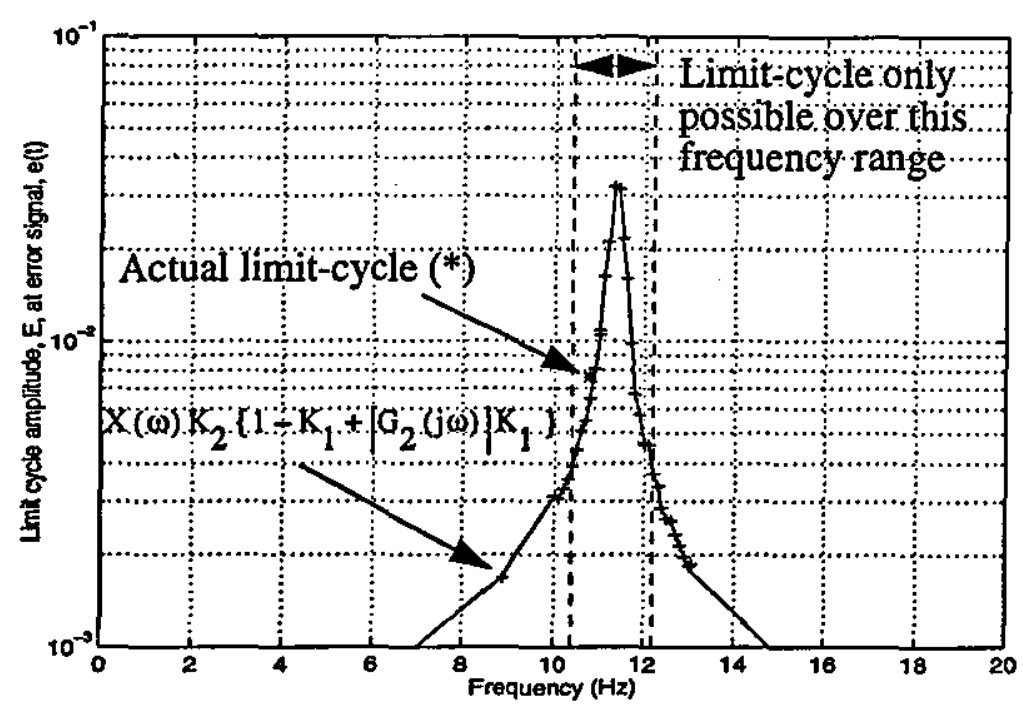

Figure 9.9 - Predicted limit-cycle amplitude for test rig

The results demonstrate that it is possible to predict the amplitude of the limitcycle that occurs on the test rig from a knowledge of the actuator performance boundary and the open-loop gain of the remaining linear elements. It is important to realise that since limit-cycles can only occur at frequencies where the open-loop gain is greater than $0 \mathrm{~dB}$, it is possible to predict a frequency range over which a limit-cycle may arise. This frequency range has been obtained from Figure 9.5, and is included on Figure 9.9. In addition, the prediction of the potential limit-cycle amplitudes and frequency range over which they may occur can be accomplished without the use of any analytical models. Instead, test results for both actuator performance boundary and open-loop system gain provide a simpler, and more accurate alternative.

Careful examination of the results of Figure 9.9 reveals that the amplitude of the actual limit-cycle that occurred on the test rig is slightly higher than that predicted. Since the actuator performance boundary has been derived experimentally, this suggests that the load system itself is slightly nonlinear. The open-loop gain of the system was obtained for a ram deflection of lower amplitude than was achieved in the limit-cycle condition. If the load exhibits a higher gain under such circumstances than for a smaller ram displacement, the difference in predicted and actual limit-cycle amplitudes would be the results. Clearly, such a consideration would need to be taken into account on an aircraft, but variations such as this could be addressed within the predicted error margins. 


\subsection{Design of structural-mode filters}

\subsubsection{Introduction}

The above section has demonstrated that it is possible to predict the amplitude of any potential limit-cycle within the system. Although this has proved useful in confirming the theory contained in Chapter 8 , such a situation should be avoided on an aircraft. To prevent such an interaction, structural-mode filters need to be applied within the feedback path. These filters will be designed in the following section to meet both the current and proposed alternative clearance requirements.

\subsubsection{Design of structural-mode filters}

Since actual test data for the open-loop frequency response of the rig is available, the design of the filters will be based on this data as opposed to any analytical modelling of the system. This is representative of the real aircraft situation, where the structural-modelling is superseded by ground tests once a prototype aircraft is available. In this case, the response of the system that will be used in the design of the structural-mode filters is as shown in Figure 9.5.

Since the system is relatively simple as there is only a single structural-mode, only one bandstop filter is required. Using the design procedure that was discussed in Chapter 4, it is possible to design a filter to meet both current and alternative clearance requirements.

The resulting filters, which meet the implementation limitations described in Chapter 4, are

$$
\begin{aligned}
& G_{S F 1}(s)=\frac{s^{2}+0.9929 s+5030.0}{s^{2}+12.8 s+4929.4} \\
& G_{S F 9}(s)=\frac{s^{2}+0.9929 s+5030.0}{s^{2}+32.0 s+4929.4}
\end{aligned}
$$

where, $\mathrm{G}_{\mathrm{SF} 1}$ is the notch filter designed to meet the proposed clearance requirement of a maximum open-loop gain of $-1 \mathrm{~dB}$, and $\mathrm{G}_{\mathrm{SF} 9}$ is the notch filter designed to meet the current clearance requirement which results in a maximum open-loop gain of $-9 \mathrm{~dB}$.

To demonstrate that the above filters meet the respective clearance requirement, the open-loop gain response for system including the notch filters are included as Figure 9.10 and Figure 9.11. 


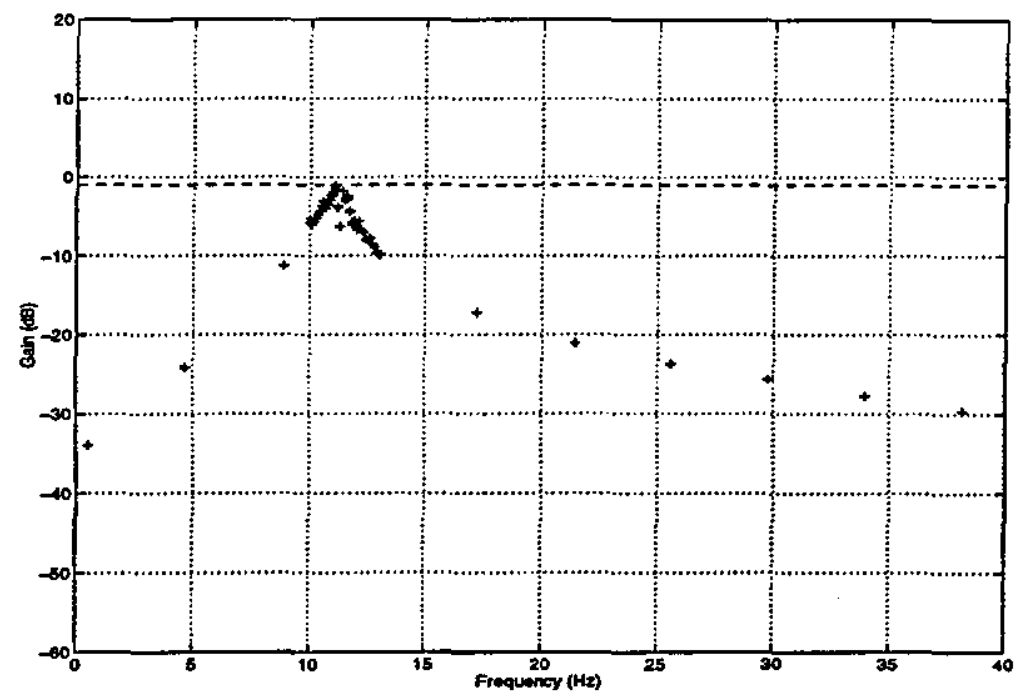

Figure 9.10 - Test rig open-loop frequency response in the presence of $-1 \mathrm{~dB}$ filtering

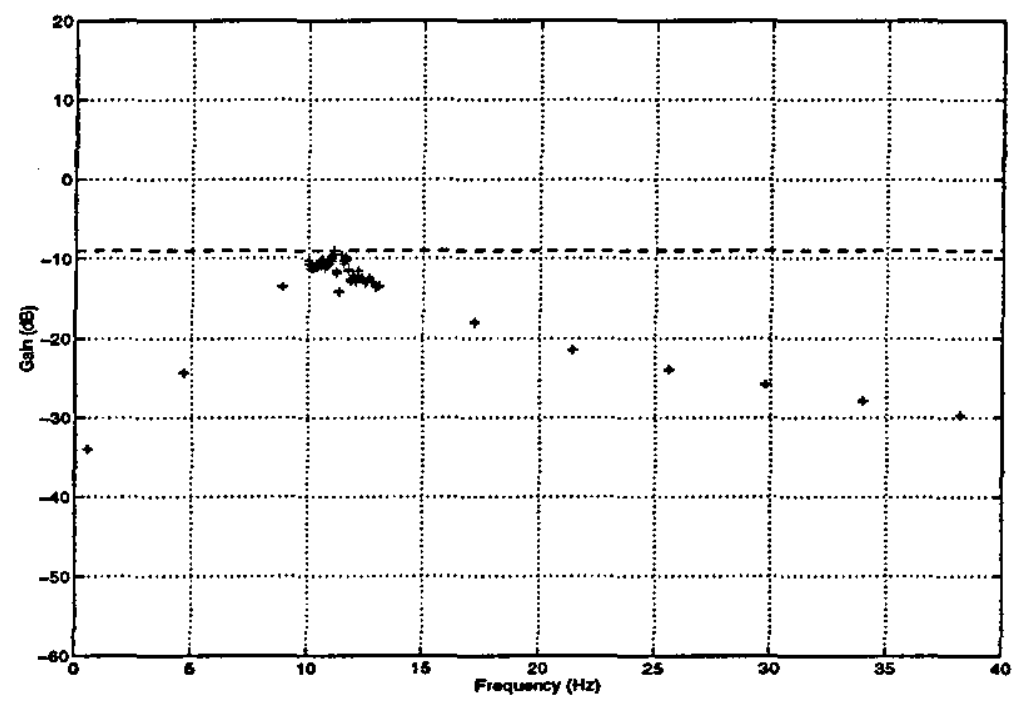

Figure 9.11 - Test rig open-loop frequency response in the presence of $-9 \mathrm{~dB}$ filtering

\subsection{Implementation of structural-mode filtering}

It was shown in Chapter 8 that provided that the open-loop gain of the system is not greater than $0 \mathrm{~dB}$ then no limit-cycle can occur. The results of Figure 9.10 and Figure 9.11 would predict therefore that the closed-loop system would show no limitcycling behaviour in the presence of either of the two structural-mode filters. When the filters were implemented within the digital computer as shown in Figure 9.12 this was found to be the case on the test rig itself for both filtering specifications. 


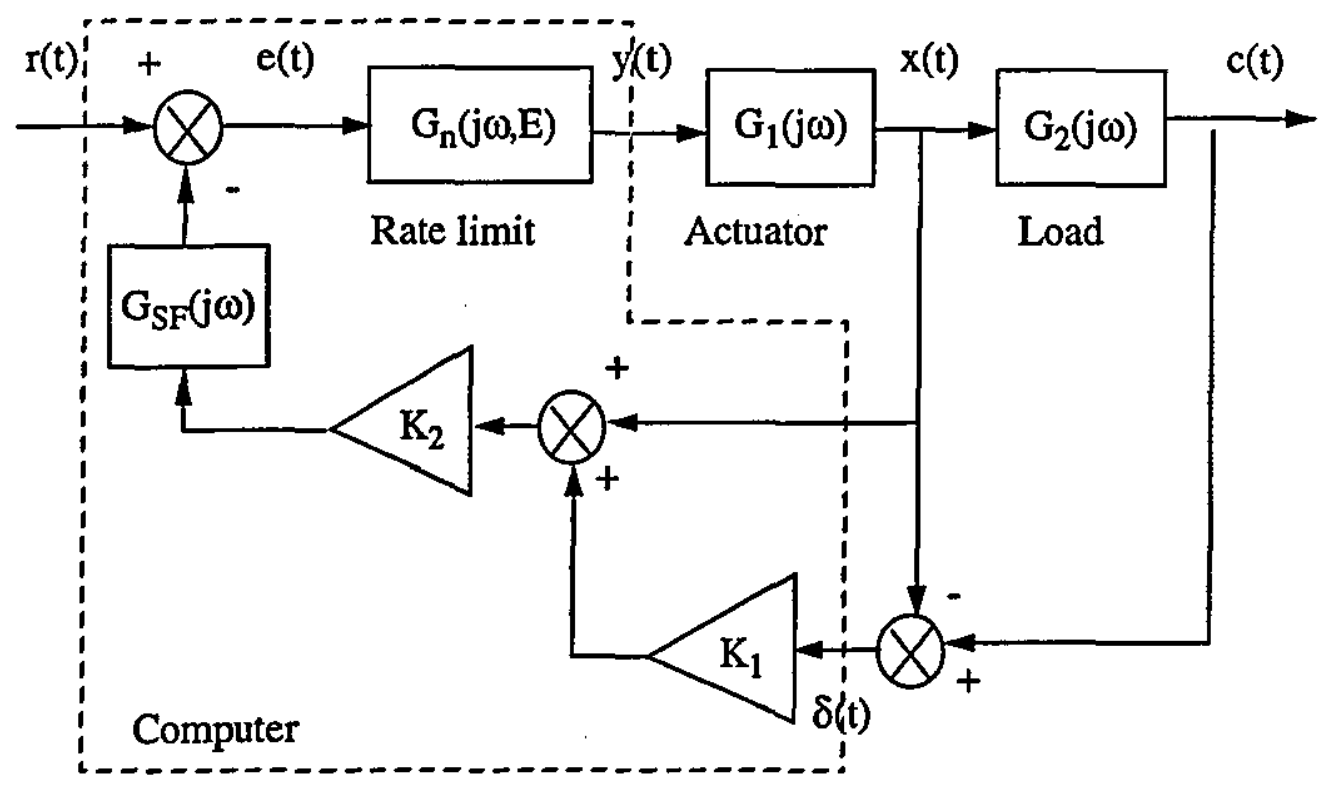

Figure 9.12 - Block diagram of test rig

To demonstrate this, the time response of the closed-loop system to a pilot demand signal of frequency $0.5 \mathrm{~Hz}$ and amplitude equivalent to $1.0 \mathrm{~mm}$ of ram deflection is as shown in Figure 9.13. In this case, the system contains the $-1 \mathrm{~dB}$ structural-mode filter in the feedback path. These results can be compared with the response of the system for the case where there is no filtering in the feedback path leading to a limit-cycle oscillation (Figure 9.14).
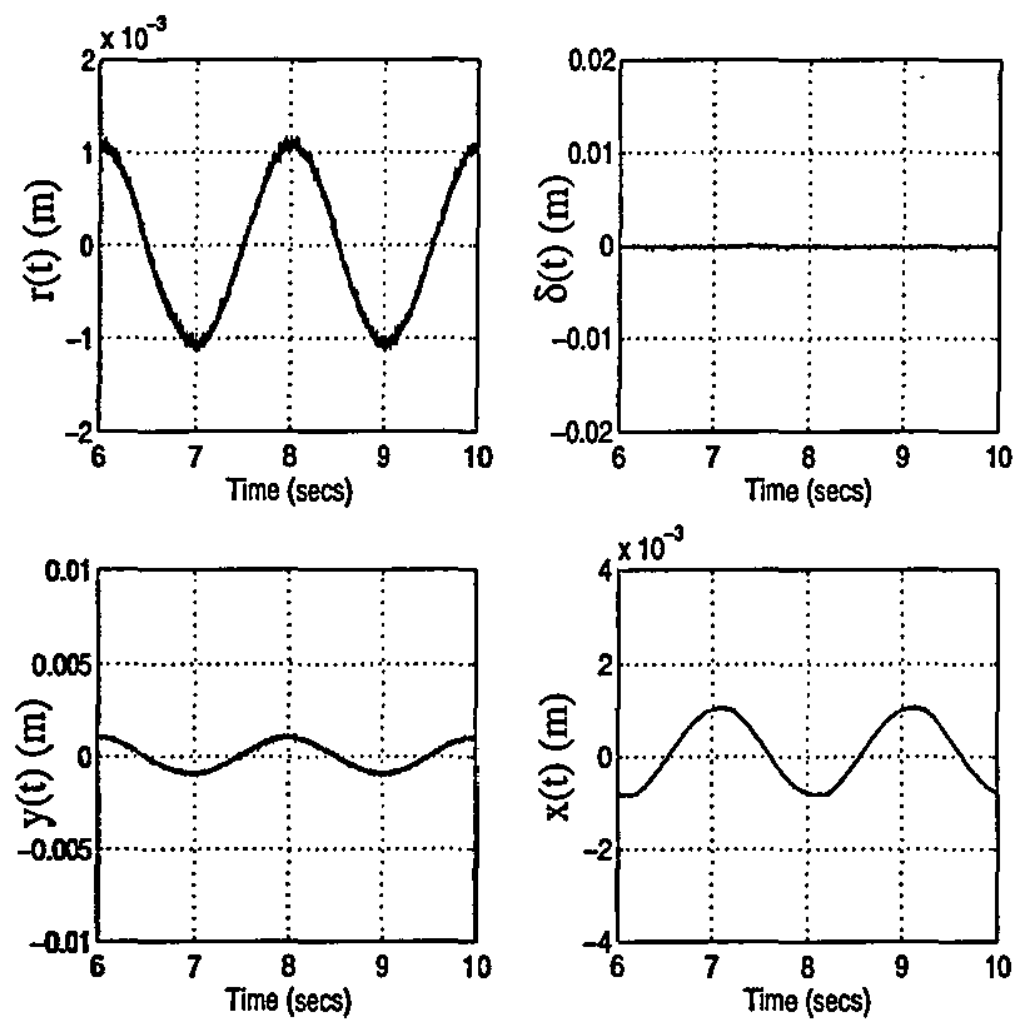

Figure 9.13 - Test rig time response for $0.5 \mathrm{~Hz}, 1.0 \mathrm{~mm}$ pilot demand signal with $-1 \mathrm{~dB}$ filtering 
Comparison of the results demonstrates that the filters designed to achieve a maximum open-loop gain of $-1 \mathrm{~dB}$ prevent the occurrence of the predicted limit-cycle. Importantly, even in the presence of the limit-cycling condition, the actuator ram follows the desired demand input as can be seen from Figure 9.14, demonstrating that a certain level of structural noise may be acceptable for satisfactory actuator performance.
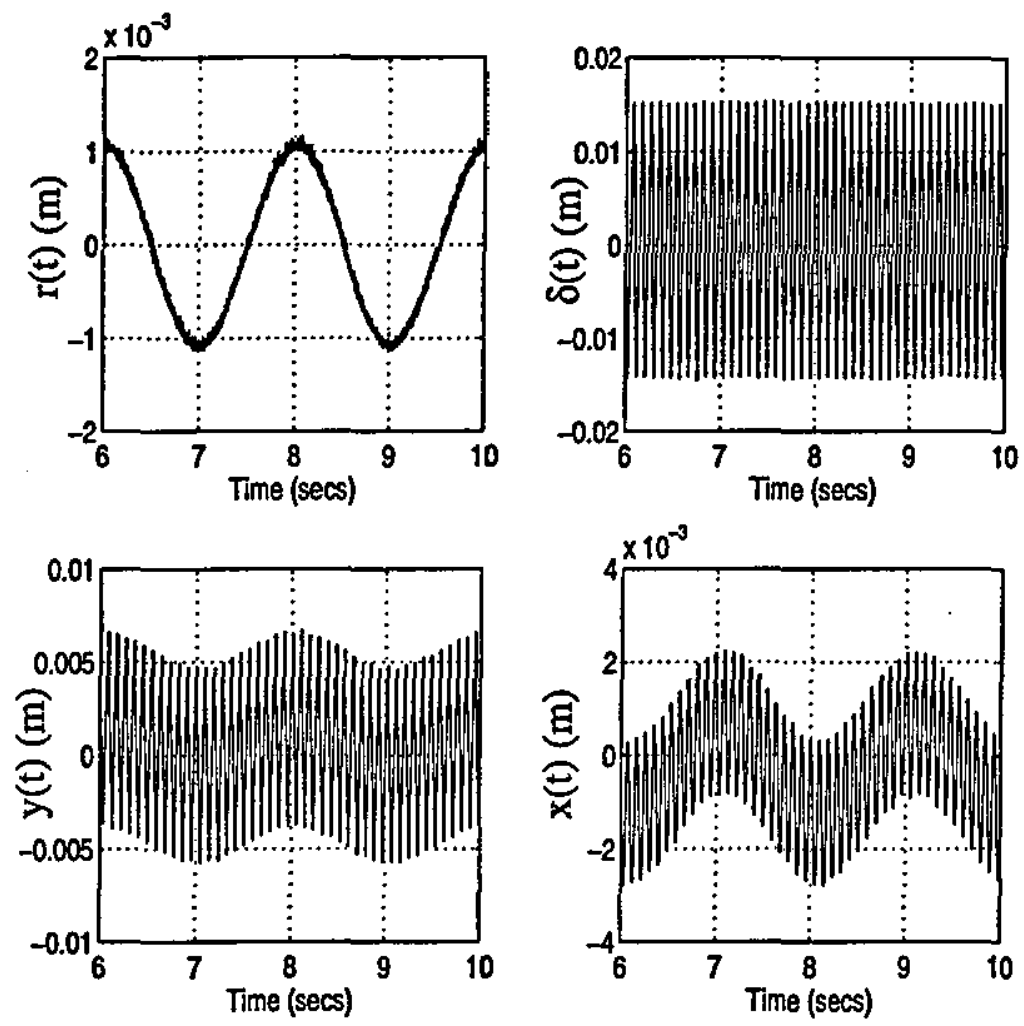

Figure 9.14 - Test rig time response for $0.5 \mathrm{~Hz}, 1.0 \mathrm{~mm}$ pilot demand signal with no feedback filtering

\subsection{Effect of structural-mode filters and limit-cycles on rigid-body performance}

Although the inclusion of the $-1 \mathrm{~dB}$ structural-mode filter within the feedback path has eliminated the occurrence of the limit-cycle, it has been achieved with some reduction in the performance of the rigid-body system. For the aircraft, the inclusion of the structural-mode filters resulted in significant phase lag being introduced within the system.

Consider the "rigid-body" for the test rig as shown in Figure 9.15. 


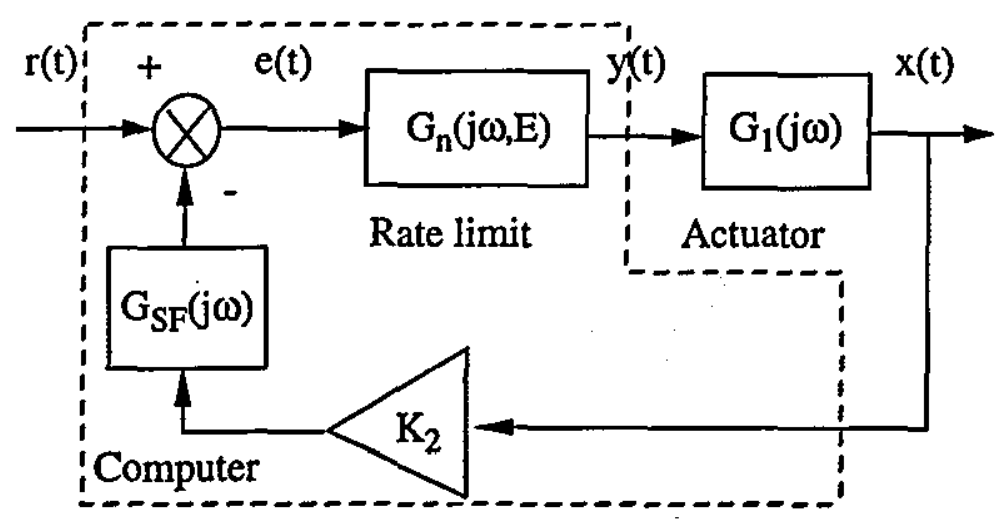

\section{Figure 9.15 - Block diagram of "rigid-body" test rig}

If the open-loop frequency response of the system is obtained, the results are as shown in Figure 9.16. This response represents the nominal open-loop response of the system. Inclusion of a structural-mode filter within the feedback path effects this response as shown in Figure 9.16. As expected, the filter designed to meet the $-1 \mathrm{~dB}$ clearance requirement introduces less phase lag to the system than the filter design to meet the current clearance requirement. Provided that the system remains as tested, and no errors are introduced, it can be seen that the filter designed to meet the proposed alternative clearance requirements prevents the occurrence of limit-cycles at a reduced cost when compared to the current design procedure.

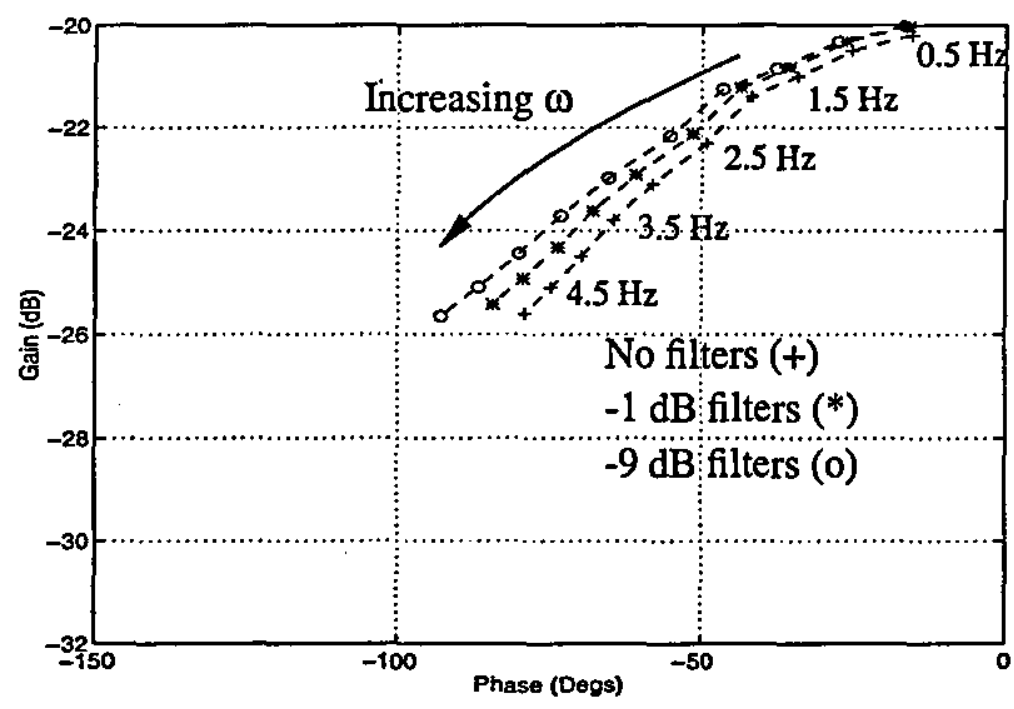

Figure 9.16 - Test rig open-loop frequency response including structural-mode filters

Suppose that it were felt that the limit-cycle predicted for the case of the closedloop system with no structural-mode filters could be tolerated in terms of system fatigue, wear, power consumption etc. It is instructive to examine how such a limitcycle effects the open-loop frequency response of the system. Initially, this can be estimated from a consideration of the rate limit function in isolation. If this analysis is completed for a limit-cycle of frequency $10.8 \mathrm{~Hz}$ and amplitude $7.6 \mathrm{~mm}$, the predicted 
open-loop frequency response is as shown in Figure 9.17. From the analysis of Chapter 7, it would be expected that the actual experimental results would match the predicted response reasonably well. This is the case as can be seen from Figure 9.17, where the actual open-loop frequency response of the test system in the presence of the limit-cycle is included for comparison.

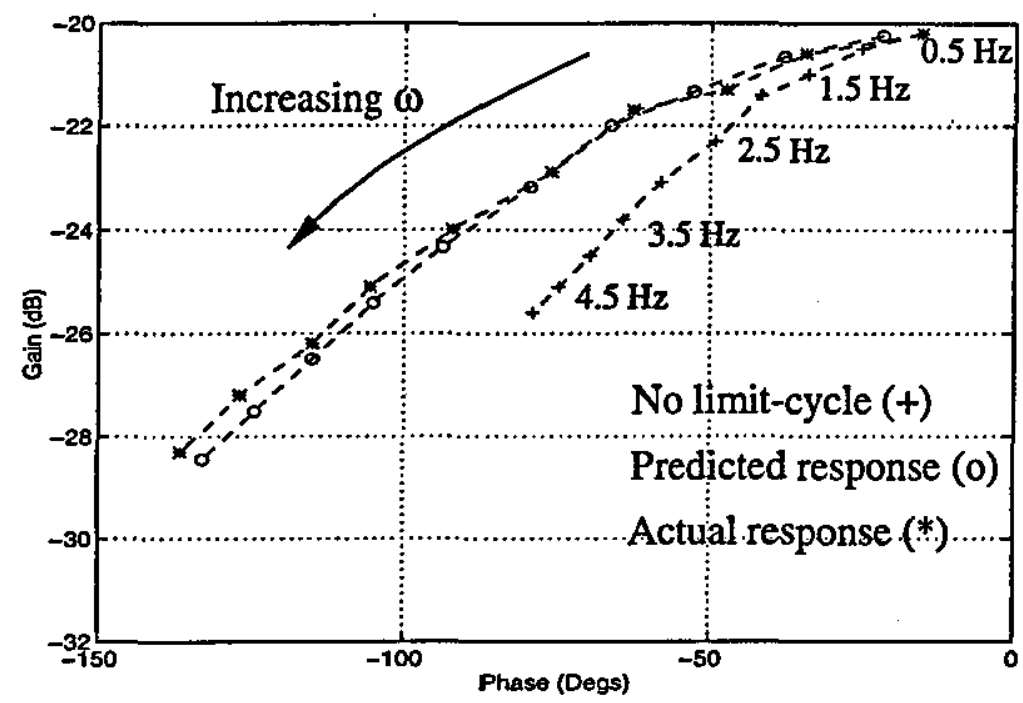

\section{Figure 9.17 - Test rig open-loop frequency response in presence of unfiltered limit-cycle}

The results demonstrate the large effect of the limit-cycle on the performance of the test rig as a whole. In particular, the significant phase lag introduced due to the limit-cycle can be seen from the results of Figure 9.17. Comparing these results with those of Figure 9.16 demonstrate that the cost of introducing structural-mode filters in terms of the induced phase lag is small when compared with the phase lag introduced as a result of the potential limit-cycle.

\subsubsection{Conclusions}

The above sections have demonstrated the prediction and prevention of limitcycles within a system typical of an aeroservoelastic system. In this case, reliable open-loop testing of the system has enabled the resulting filters to be designed with some confidence. Comparison of the phase lags introduced to the system by the different filtering strategies has demonstrated that the reduction in attenuation requirements of the structural-mode filters has resulted in an improvement in system performance when compared to the current clearance procedure. In addition, the effect of the predicted limit-cycle on system performance has been demonstrated.

As has been discussed in previous chapters, in the case of a real aircraft, the modelling and testing of the system cannot be relied upon entirely. As a result, it is necessary to consider what effect an error in the modelling/testing of the system would have on the potential for limit-cycles. 


\subsection{Prediction of limit-cycles in the presence of modelling error}

\subsubsection{Introduction}

The previous sections have applied the analysis of Chapter 8 to a test rig which shows an interaction between control system and structure typical of an aeroservoelastic interaction. In this case, the simplicity of the system and reliability of the open-loop frequency response testing results in a prevention of the potential limitcycle. Unfortunately, the safety critical nature of an aircraft's flight control system combined with the uncertainty in the reliability of the system modelling results in the need to consider the situation where the system model is in error.

\subsubsection{Prediction of limit-cycles in the presence of modelling error}

Consider for example, that the test rig is representative of a real aircraft, and that the open-loop frequency response given in Figure 9.5 was obtained from ground testing of the aircraft itself. Assuming that there is only a single control path under consideration, then implementation of the $-1 \mathrm{~dB}$ filter specified in (9.10) would prevent a limit-cycle oscillation occurring.

Consider the situation where, in-flight, the aerodynamic effects result in the gain of the structural-mode exceeding that assumed in the design of the structural-mode filter. In the case of the $-1 \mathrm{~dB}$ filtering, such an increase could well result in the potential for limit-cycling. Naturally, in the real aircraft case, the aerodynamic effects are represented within the initial design of the structural-mode filters. There exists however, the potential for error in this aerodynamic modelling and it is this that must be taken into consideration along with any potential errors in the testing of the aircraft structure and variations between different aircraft.

Returning to the test rig, consider a situation where it was felt that a twofold increase in the open-loop gain of the system at the structural frequencies could occur whether it be due to a particular operating condition, or an error in the open-loop testing of the system. Such a situation could be represented by an increase in the value of $K_{1}$ to 10.0 .

Assuming that the system can be adequately modelled using the transfer function of equation (9.4), it is possible to solve the characteristic equation for the case where the $-1 \mathrm{~dB}$ filter is in the feedback path, and the value of $K_{1}$ is set to 10.0 . The characteristic equation is therefore given by

$$
G_{1}(j \omega) G_{S F 1}(j \omega) K_{2}\left\{1-K_{1}+G_{2}(j \omega) K_{1}\right\}=\frac{-1}{G_{n}(j \omega, E)}
$$


whose solution can be represented on the Nyquist diagram of Figure 9.18.

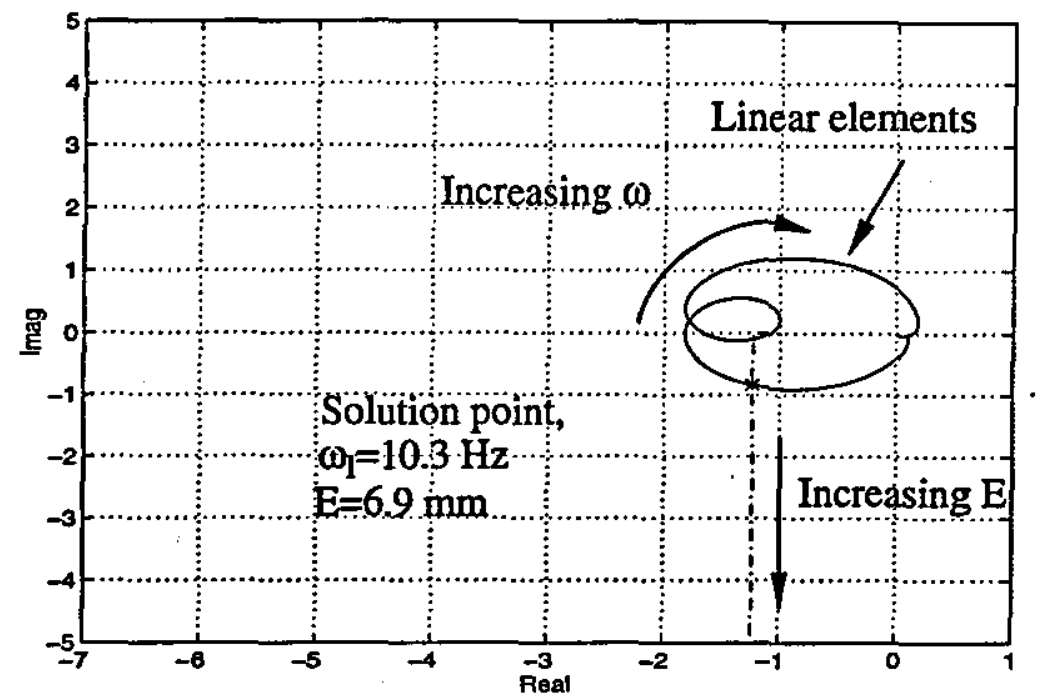

\section{Figure 9.18 - Characteristic equation solution, $K_{1}=10,-1 \mathrm{~dB}$ structural filters}

In reality, due to the assumed uncertainty in the phase response of the system, it is only possible to specify the amplitude and frequency range over which a limit-cycle may occur. Applying the performance limit of the actuator to the gain response of the remaining elements of the system results in the limit-cycle prediction as shown in Figure 9.19. As for the nominal situation represented by Figure 9.9, these results are based on the actual experimental open-loop frequency response of the system.

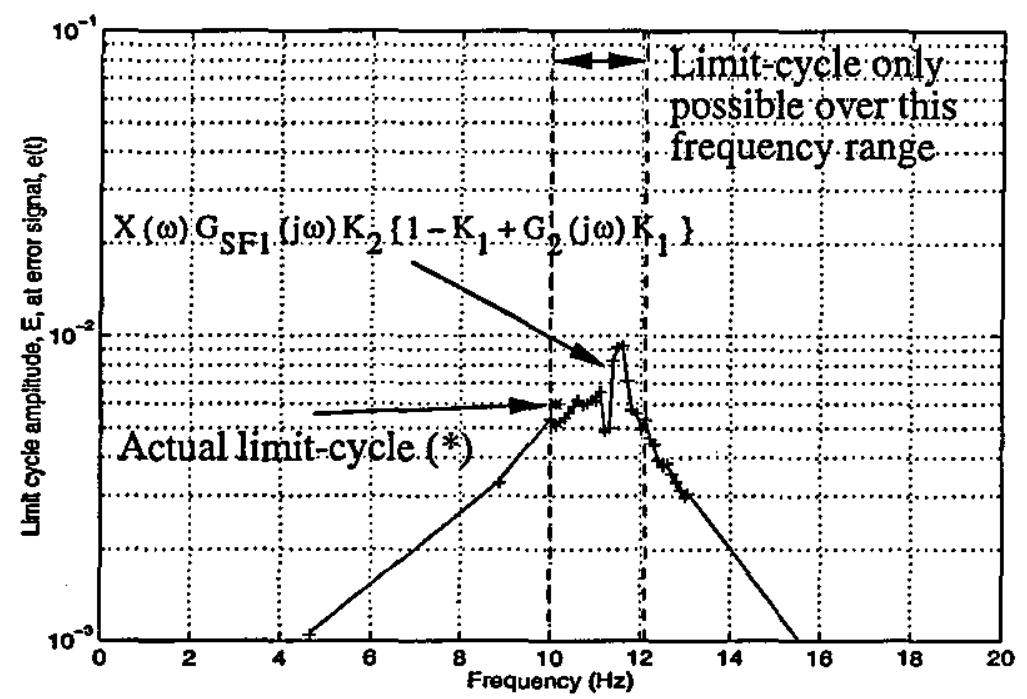

Figure 9.19 - Predicted limit-cycle amplitude for test rig with $K_{1}=10.0,-1 \mathrm{~dB}$ filters

Comparison of the results of Figure 9.19 with those for the nominal unfiltered system shown in Figure 9.9 reveals the effect of both the increase in system gain and the structural filter on the maximum amplitude of the predicted limit-cycles. As expected, the maximum amplitude of the potential limit-cycles is significantly reduced by the presence of the filters. One interesting point to note is the relatively high potential amplitude for a limit-cycle of frequency $11.5 \mathrm{~Hz}$. If such a situation were 
considered a possibility, then it might be considered prudent to increase the width of the structural notch filter. This would reduce the potential amplitude at this frequency.

It is possible therefore to predict the amplitude of limit-cycles that may occur within a typical system in the presence of an assumed modelling error. In this case, with the nominal $-1 \mathrm{~dB}$ filters in the feedback path, a twofold error in the open-loop gain of the system has the potential for causing the limit-cycles as specified in Figure 9.19. This prediction will, in turn, allow the effect of such a situation to be analysed in terms of satisfactory actuator performance.

\subsection{Effect of limit-cycle on system performance in the presence of modelling errors}

The ability to predict the amplitude and frequency range of any potential limitcycle within the test system even in the presence of modelling errors enables the safe application of the structural-mode filters to be assessed. Although the open-loop response of the test rig is reliably known, the previous section has demonstrated the prediction of the potential limit-cycle amplitude in the case of a twofold error in the gain of the structural-mode.

In order to assess whether these limit-cycles would have a significant impact on the satisfactory control of the "rigid-body", it is possible to apply one of the methods described in Chapter 8. Initially, an estimation of the effect of the potential limitcycles on the performance of the system can be gained from an analysis of the rate limiter in isolation. Taking the worst-case scenario, where the limit-cycle that actually occurs is assumed to be the one at $11.5 \mathrm{~Hz}$ in Figure 9.19, it is possible to predict the open-loop response as shown in Figure 9.20.

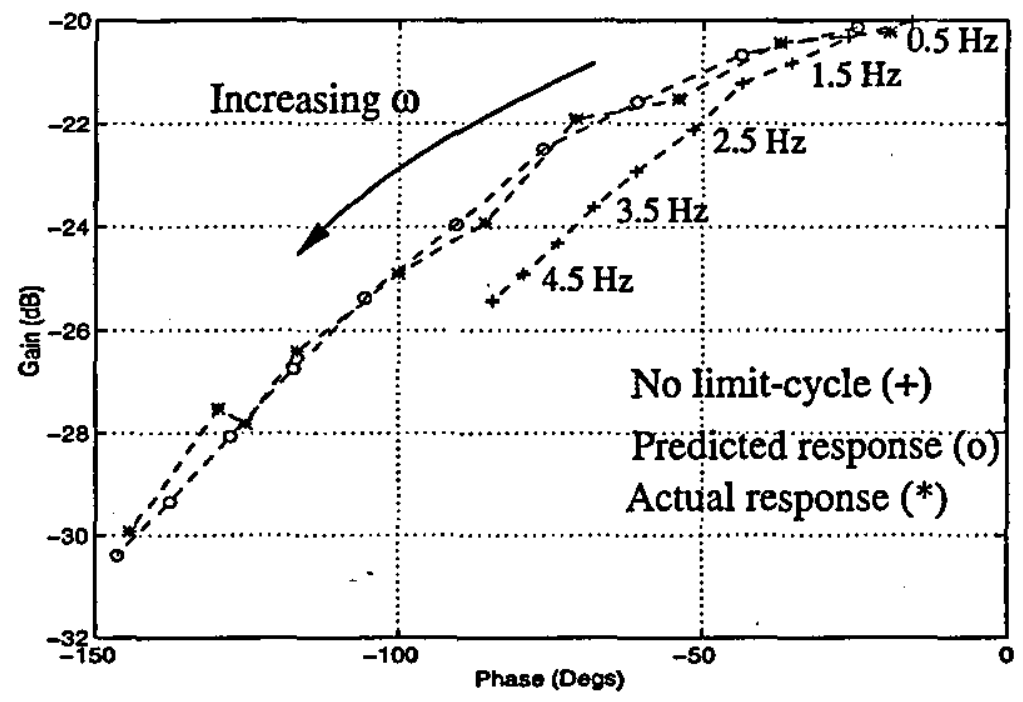

Figure 9.20 - Test rig open-loop frequency response including -1 $\mathrm{dB}$ filters and 11.5 Hz limit-cycle 
The results demonstrate the significant effect of such a limit-cycle on the performance of the system. It should be noted however that this disregards any alteration of the notch filter following the discussion of the previous section.

In reality, the actual limit-cycle that occurred on the test rig for a value of gain, $\mathrm{K} 2=10.0$, and in the presence of the $-1 \mathrm{~dB}$ structural filter, is as shown in Figure 9.19. Clearly, the limit-cycle that actually occurred is of significantly lower amplitude than the worst-case examined in Figure 9.20. Comparing the open-loop frequency response of the system in the presence of this $10.1 \mathrm{~Hz}$ limit-cycle (Figure 9.21) with that for the $11.5 \mathrm{~Hz}$ limit-cycle demonstrates that the actual change in system performance is significantly less than that predicted for the worst-case analysis of Figure 9.20.

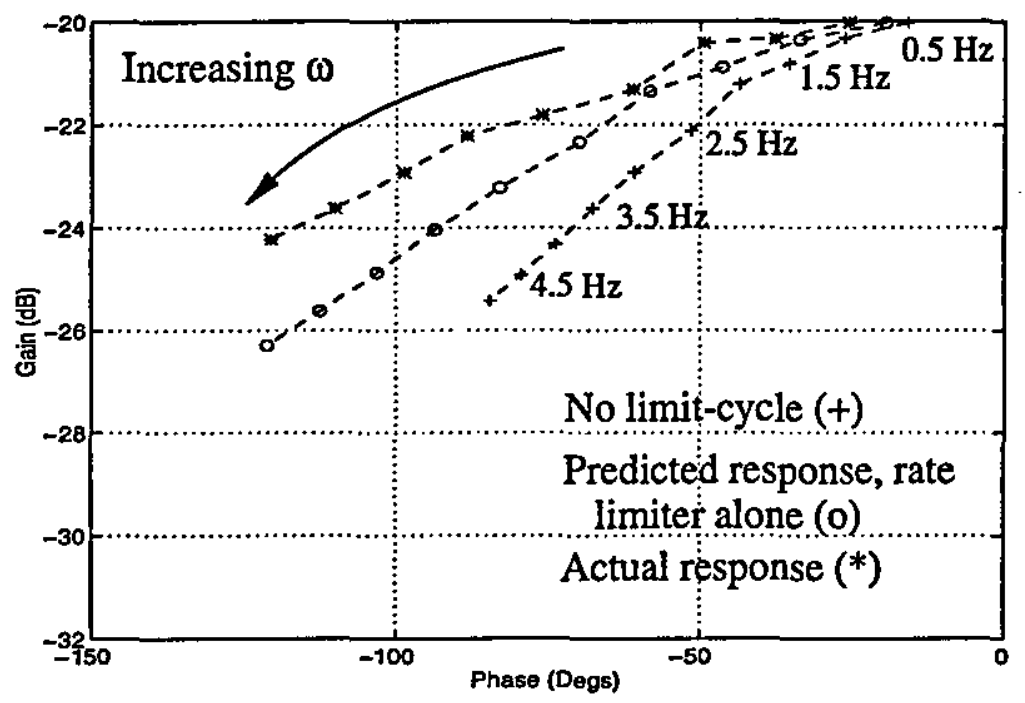

Figure 9.21 - Test rig open-loop frequency response including $-1 \mathrm{~dB}$ filters and $10.1 \mathrm{~Hz}$ limit-cycle

Estimating the change in the system gain and phase response from a consideration of the rate limiter alone is pessimistic as can be seen from Figure 9.21. In this case, the actual response seen on the test rig exhibits an increase in gain when compared with the nominal open-loop results. In addition, the extra phase lag predicted from the analysis of the rate limiter in isolation is not as evident in the actual response. These differences are almost certainly due to the shaping of the mainvalve ports and other un-modelled nonlinear effects.

Instead of estimating the effect of the $10.1 \mathrm{~Hz}$ limit-cycle from a consideration of the software rate limiter alone, results from an analysis of the actuator model developed in Chapter 7 provide a better approximation to the test results in this case. Such a comparison is included as Figure 9.22.

Clearly, in the case of an aircraft system, the best method for assessing the impact of a limit-cycle on the performance of the actuator and hence on the performance of the system, would be to test the actuator itself (as in Chapter 8). These results could 
then be incorporated into an analysis of the aircraft as a whole.

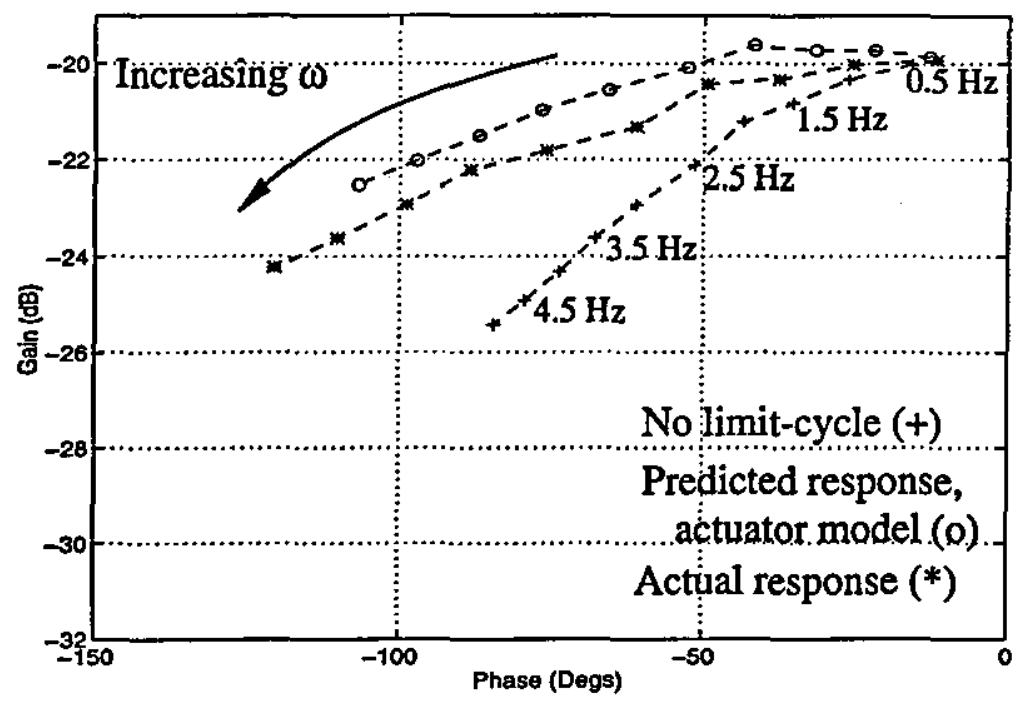

Figure 9.22 - Test rig open-loop frequency response including $-1 \mathrm{~dB}$ filters and 10.1 Hz limit-cycle

\subsubsection{Conclusions}

These results have demonstrated several important points. Firstly, as for the nominal system, it is possible to predict the amplitude and frequency range for the potential limit-cycles in the presence of modelling errors. Unfortunately in this case, a twofold increase in the open-loop gain results in the potential for a limit-cycle that causes a serious change in the system performance. The fact that this particular potential limit-cycle does not actually occur in practice is as a result of the phase response of the system. Clearly, for the aircraft, all potential limit-cycles must be considered in the presence of uncertainty in this phase response. It would be advantageous to be able to narrow down the region of potential limit-cycles from that shown in Figure 9.19. This would only be possible however given some estimate of the phase response. For example, in terms of the Nyquist plot showing the solution of the characteristic equation (Figure 9.6), provided that the phase response at a particular frequency could be guaranteed as not being in the same quadrant as the describing function for the rate limiter, then no limit-cycle would result at this frequency.

In this case, where such an estimate of system phase response cannot be considered, an alternative approach was discussed earlier where a widening of the notch filter could be applied. This would reduce the amplitude of the potential limitcycle at a frequency of $11.5 \mathrm{~Hz}$ at the cost of an increase in the phase-lag of the filter itself. As a result, it might be considered that satisfactory rigid-body control of the rig could be maintained in the presence of the assumed level of modelling errors. This would lead to confidence in the use of the $-1 \mathrm{~dB}$ filters in the nominal situation where modelling errors were possible. 
Importantly, the results of the rig testing have demonstrated that the design and clearance procedure discussed in Chapter 8 can be applied to a real system.

\subsection{Inclusion of digital effects}

The importance of the inclusion of digital effects within aeroservoelastic analysis has been highlighted in Chapters 5,6 and 8. In particular, where structural-modes exist at frequencies above the Nyquist frequency, the possibility of the digital system aliasing the response to within the bandwidth of the flight control system needs to be investigated. In this case, the test rig contains only a single structural-mode below the Nyquist frequency when sampling at $80 \mathrm{~Hz}$. As a result, the effect of the digital representation of the control system on the attenuation requirements for the test rig is negligible.

In order to verify that this is the case, the structural filter designed to meet the -1 $\mathrm{dB}$ attenuation requirements was applied within the control system for a sampling frequency of $80 \mathrm{~Hz}$. As expected, the limit-cycle that had occurred for the system in the absence of any structural-mode filtering was prevented.

\subsection{Conclusions}

This chapter has demonstrated the proposed clearance procedure as applied to a representative test rig. The procedure for predicting the amplitude and frequency range of any potential limit-cycle has been verified, with the experimentally observed limit-cycle results matching those of the predictions. In addition, the predicted effect of the limit-cycle on the performance of the actuation system was confirmed by the experimental results.

The implementation of suitable filtering to meet both the current and alternative clearance requirements resulted in the prevention of the potential limit-cycles. In the presence of significant modelling error however, which was artificially represented on the test rig, the presence of further potential limit-cycles was predicted and their effects assessed. These results were then confirmed from experimental results. Clearly, differences exist between the actuator performance changes predicted from the simulations, and those actually experienced from the rig testing. Naturally, this is due to inadequacies in the modelling of the actuator. These results demonstrate however that in the absence of a sufficiently accurate model, the actuator itself can be tested in the presence of any potential limit-cycle. These results can then be applied to modelling/simulation of the remainder of the system, enabling a prediction of the system performance changes in the presence of limit-cycles. In this way, the highly complex actuator is represented by actual test results, giving a form of hardware in- 
the-loop simulation of the whole system.

Although the test rig used in this analysis is far simpler than the real aircraft system, its use has enabled verification of the procedures outlined in Chapter 8 . In particular the prediction and prevention of limit-cycles, and the assessment of their effect on satisfactory actuation system performance has been confirmed. 
Chapter 10

Conclusions 


\subsection{Aeroservoelasticity}

The problem of an interaction between an aircraft's structural dynamics, flight control system and aerodynamics has been demonstrated. Although such interactions are rarely encountered in-flight, the fact that there is the potential for loss of aircraft control and/or structural failure results in the need for careful design of the flight control system. As the drive to produce lighter aircraft structures results in more structural flexibility, the potential for aeroservoelastic interactions will increase. This fact, coupled with the increasing use of high-authority digital flight control systems across a wider range of aircraft types means that the aeroservoelastic interaction may become as important as aeroelasticity in aircraft design.

Although this research has concentrated on the negative consequences of aeroservoelasticity, the potential for a positive contribution exists. For example, the close link between the aeroservoelastic and aeroelastic phenomena may mean that in future, the use of so-called smart structures will allow aircraft operation beyond the currently accepted flutter speeds.

One of the major problems that has been emphasised by this programme of research is the multi-disciplinary nature of the aeroservoelastic interaction. To the elements of structural dynamics and unsteady aerodynamics found in the study of aeroelasticity, is added the flight control system. This additional element introduces further complexity to the problem, involving consideration of nonlinear actuation and digital effects. Both of these additional elements have been of particular interest throughout this research.

\subsection{Discussion of the current design process}

Although both the current and proposed alternative design methods adopt a similar method of solution of the aeroservoelastic problem, their approach is considerably different. The current design procedure is based on a limited understanding of the interactions between the system elements. In particular, the uncertainty over the effects of an unstable structural-mode on the system dictates the need for conservative clearance requirements. This cautious approach is represented by the $-9 \mathrm{~dB}$ maximum open-loop gain at structural frequencies. One of the major reasons for such conservatism is the fact that in most modern combat aircraft, the flight control system is safety critical. As a result, any factor which prejudices the safety of the aircraft must be carefully considered.

An additional factor which influences the conservatism in the current design procedure is the uncertainty in the modelling of the flexible aircraft. Although ground 
testing of the prototype aircraft allows verification of the structural model, the determination of the correct in-flight aerodynamic forces is more complex. In fact, the availability of ground structural tests eliminates to some extent the need for the structural model at all, but in-flight aeroservoelastic testing is still very limited.

Uncertainty in the modelling of the aircraft introduces an additional element of conservatism to the current design process by assuming that the phase response of the system cannot be relied upon. This effects the analysis in several ways, the most important being the assumed in-phase addition of the signal paths within the flight control system. In the case of the aircraft system considered throughout this research, there were three signal paths involved in the longitudinal control of the aircraft. Although the actual phasing of the response might result in cancellation between the individual signal paths, uncertainty forces the worst-case scenario to be examined. Clearly, the current design process is severely restricted by both uncertainty in the effects of an unstable structural response and in the reliability of the aircraft modelling.

In the case of the current design procedure, the aircraft system, particularly the actuator, is assumed to be linear. Although this greatly simplifies both the system modelling and analysis, such an assumption can lead to a limited understanding of the true nature of the problem. As was shown in the example of Chapter 4, if the linear nature of the system is assumed, then an "unstable" structural-mode must result in an unbounded oscillation. Clearly, if this were the case, then catastrophic structural failure would occur. The need in this case to ensure structural-mode stability in all circumstances is clear. This results in the large margin of safety employed within the current clearance requirements. The price that must be paid for this large safety margin is in the phase lag introduced by the structural-mode filtering.

Although the filter design methods employed in Chapter 4 were simple, they demonstrated several important points. Firstly, iterations required in the design of these simple filters points to the need for an optimization of the filter design process. Such an optimization is generally adopted, the phase lag of the filters being minimised whilst ensuring that the attenuation requirements are met. This minimization of the phase lag introduced by the structural-mode filters is the driving force behind a relaxation of the current clearance requirements. In particular, since it has been demonstrated that the majority of the phase lag is introduced by the low-pass filter, it is desirable to reduce the attenuation requirements of this filter as much as possible. 


\subsection{Effect of digital nature of control system on the current design procedure}

In most modern flight control systems, the control algorithms are processed within a digital computer. In addition, the aircraft's motion sensor unit usually employs digital processing of the measured data. As a result, the need for a careful assessment of the sampled nature of the control system is evident. This was clear for the in-flight aeroservoelastic interaction on the X-29 aircraft ${ }^{15}$. The analysis of the digital nature of the typical aeroservoelastic system has demonstrated how the aliasing of highfrequency structural response by the flight control computer can result in an unstable interaction. This is in contrast to the analogue situation, where no such unstable interaction would result. The current design method takes such aliasing into account, folding back the high-frequency response to below the Nyquist frequency.

It is important that the attenuation of the sample-and-hold and sensor dynamics are taken into consideration during the design process. These two elements provide a substantial attenuation of the high-frequency modes and therefore reduce the problem of aliasing significantly. In addition, the high attenuation of the sample-and-hold and sensor dynamics for frequencies above the sampling frequency would mean that in general, consideration need only be given to the aliasing of structural-modes below this frequency.

The results of Chapter 6 demonstrate that in terms of the digital nature of the control system, there is little advantage to be gained in refining the current design procedure. In particular, the assumed in-phase addition of both the signal paths and high-frequency aliases results in attenuation requirements that are only slightly different from those for the system whose phase response is taken into account. Such a result is dependent however on several factors. Firstly, for this aircraft model, none of the high-frequency modes aliased directly onto a low-frequency mode. As a result, the response at the high-frequency mode tended to swamp that at its low-frequency alias, making the phasing between the two components irrelevant. A similar effect was seen when taking the phasing for the signal paths into account. For most of the "critical" structural- modes such as the wing bending mode, excitation in the main by a single control surface makes the phasing of the response from the other control surfaces redundant. Such factors however may not be applicable for a different aircraft structure, where consideration of the phase response of the system as a whole may be beneficial, and worth the significant increase in the required analysis. Alternatively, phase information available on particular structural-modes may be applied in order to specify particular filter attenuation requirements at that frequency.

An additional effect of the digital nature of the control system is the significant 
phase lags and computational delays that can be incurred. In the examples given throughout this research, the original controller designed for the analogue system was not modified in order to allow comparison between the attenuation requirements for analogue and digital systems. In reality, provided that the digital nature of the controller is taken into account from the initial design stages, the flight control system would compensate for these lags by virtue of a phase advance filter. The addition of such a filter would increase the gain of the structural-modes however, resulting in a higher attenuation requirement for the structural-mode filters.

Although for this example aircraft, the digital nature of the control system did not result in an unstable interaction, the possibility of one occurring is clear. As a result, the digital nature of the control system must be considered from the initial stages of any design process.

\subsection{Actuator nonlinearities}

One of the major assumptions made in the current design process is the linear nature of the system as a whole. In reality, the aircraft system is highly nonlinear, and one of the main sources of these nonlinearities are the servo-hydraulic actuators. One consequence of the nonlinear nature of the actuation systems is that an unbounded structural oscillation cannot occur. This is as a result of the performance limitations inherent within the actuators themselves and within the flight control system by virtue of the software rate limiting This limit to the performance of the actuation system manifests as an upper limit on ram displacement for any particular input frequency. Since the aeroservoelastic interaction is driven by the flight control system through control surface motion, it would seem logical to assume that the resulting structural oscillation will also be bounded.

A second effect of the nonlinear nature of the actuation systems is that the performance of the actuator in response to pilot demand or stabilisation signals is affected by the presence of structural feedback signals. The importance of this is that during the design of the flight control system, a specific actuator performance is assumed. If this performance is not achieved due to the presence of structural feedback signals, then the satisfactory control of the rigid-body aircraft may not be maintained. Although the current design procedure ensures that the amplitude of such structural feedback signals will be negligible, it is important that such changes in performance be considered. This is particularly true for alternative design procedures which may allow a higher level of structural feedback under certain circumstances. The main mechanisms for these performance changes have been identified from both simulation and testing as being the software rate limiting and the profile of the mainvalve ports. 
The effect of the software rate limiting is to introduce a possible reduction in gain and increase in phase lag at the frequency of the demand signal in the presence of high-frequency structural feedback. If the combination of the two input components results in a violation of the rate limit, then such an effect occurs. Importantly however, this increase in the phase lag of the actuation system at low-frequencies is dependent on the amplitude and frequency of both input components. The result of this is that even though the rate limiter may be exceeded, the phase lag introduced at low frequencies may be small. Clearly, a certain level of structural feedback may be acceptable before satisfactory performance of the actuation system is lost.

The second mechanism through which the presence of structural feedback may effect actuator performance is as a result of the shaping of the main-valve ports. In the case of the actuator modelled and tested, these ports were trapezoidal. The result of this is that under certain circumstances, the performance of the actuator to lowfrequency demand signals may actually be improved in the presence of a highfrequency structural feedback signal. The effect of the main-valve port shaping is therefore in opposition to that of the software rate limiting, although the latter is by far the most dominant. Simulation and test results have shown that the improvement in actuator performance as a result of the shaping of the main-valve port only occurs when there is a large difference between the demanded rate of the low-frequency and high-frequency signal. Such a situation may occur for a small amplitude pilot demand signal. Once the combined input exceeds the rate limit however, actuator performance quickly reduces to below that for the no-noise case. A pessimistic assessment of the effect of a structural signal on actuator performance may therefore be obtained from a consideration of the software rate limiting alone. Alternatively, a method whereby the performance of the actuator may be predicted from a consideration of these two mechanisms has been demonstrated.

In addition to the effect of the structural-mode feedback on actuator performance in the frequency domain, it has been shown that undesired actuator response may result in the time domain. The generation of subharmonic actuator response as a result of structural feedback has been demonstrated. In such a case, there may exist an output signal component of frequency below that of either of the two input components. In the case of an aircraft in-flight, this may at least lead to undesirable rigid-body motion, and at worse, excite one of the rigid-body modes of the aircraft. Clearly, in order to fully assess the impact of a particular structural feedback signal on rigid-body performance, it is necessary to consider both the time and frequency domain. Analysis of the generation of such a subharmonic response has demonstrated that it is the low-frequency structural-modes that have the highest potential for causing such an effect. In the case of a digital system however, low-frequency subharmonics may be generated by quite high structural-mode frequencies due to 
interactions with their aliases.

\subsection{Discussion of the alternative design procedure}

The development of an alternative design and clearance procedure has been based on the fact that the true aeroservoelastic interaction is not a linear phenomena as is currently assumed, but is highly nonlinear due in the main to the servo-hydraulic actuation systems and software rate limiting. The result of this is that aeroservoelasticity is not a problem involving potential divergent instability, but rather one involving the possible occurrence of limit-cycle oscillations at frequencies above typical rigid body frequencies. Consideration of the nonlinear nature of the system has demonstrated that the potential for limit-cycles can be recognized, the frequency range over which they may occur identified, and their amplitudes predicted even in the presence of phase uncertainty. The proposed alternative clearance procedure relies on these facts along with the assertion that the effect of such limit cycling conditions on rigid-body stability can be assessed from a consideration of changes in actuator performance in the presence of high-frequency feedback signals. The key elements of the aeroservoelastic problem are defined in Figure 10.1.

Since it is desirable to avoid limit-cycle oscillations under normal circumstances, the proposed clearance requirements suggests that filters are introduced within the feedback path to attenuate the open-loop gain of the system to below $-1 \mathrm{~dB}$ at structural frequencies. The actual design of these filters will be based on the current design procedure for the derivation of the flexible aircraft model. In this way, the current assumptions regarding signal path phase, digital effects and adoption of the worst-case flight condition will be maintained, preserving the conservatism inherent in such an approach. Under normal circumstances, limit-cycle oscillations will therefore not occur. In the presence of flexible aircraft modelling errors however, the potential for limit-cycles exists.

It is possible to assess such a situation beforehand from a consideration of the confidence in the fidelity of the modelling process. Such a consideration could be used to denote confidence levels in terms of an incremental gain on a mode-by-mode basis, or, as was adopted in Chapters 8 and 9, in terms of an overall gain error margin. Such an assessment can be based on whether a particular mode is represented by modelled or test results, whether the gain associated with it is highest in-flight or on the ground and whether test results for the same mode for differing aircraft are well matched. In addition, it will be possible to predict the flight conditions at which the limit-cycle oscillations will occur from a consideration of the scheduling of the controller gains with Mach number, altitude and incidence. 


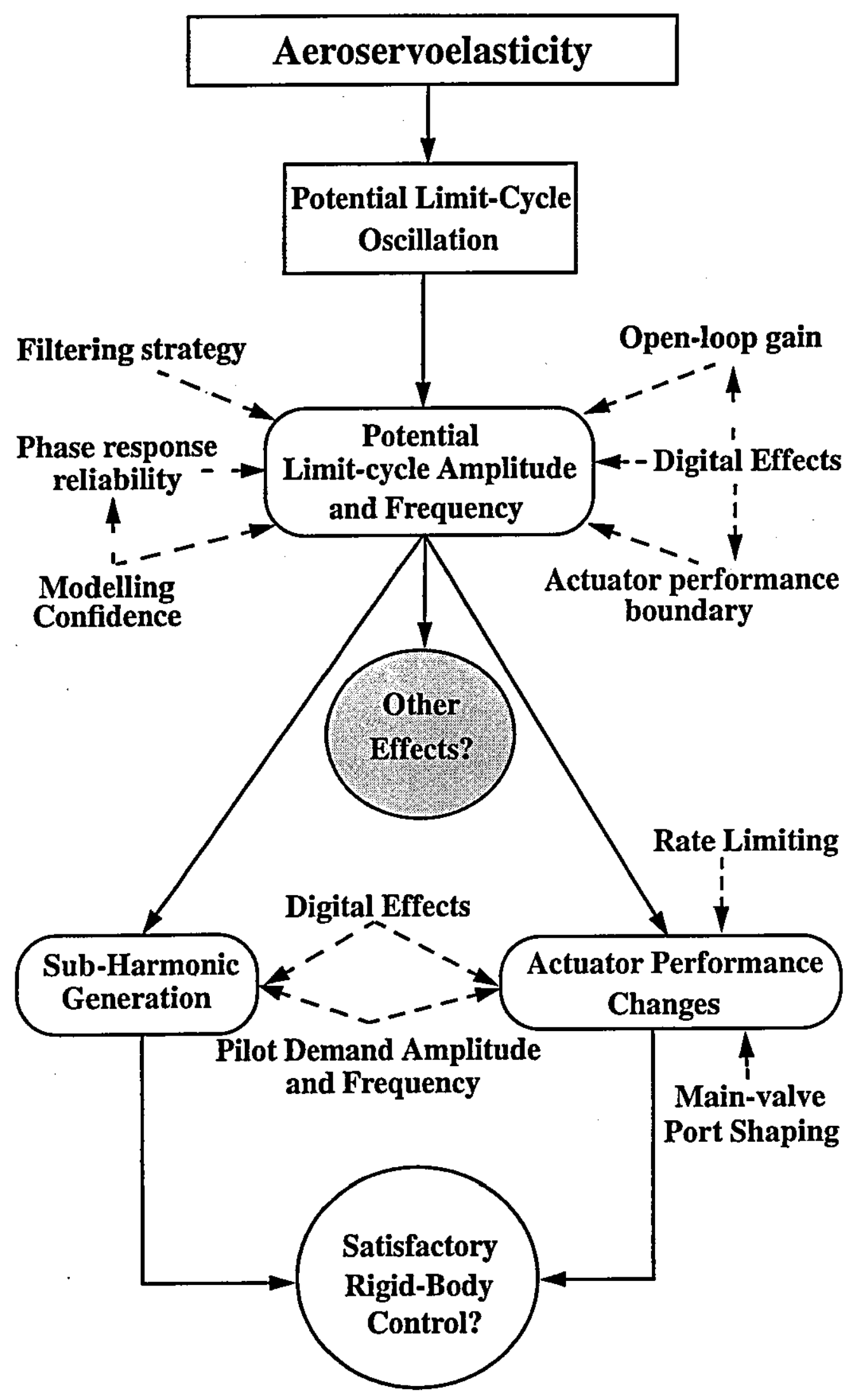

Figure 10.1 - Key elements of aeroservoelasticity 
Once this has been completed, the amplitude of any potential limit-cycle can be deduced and its effect on the rigid-body stability margins assessed. Provided satisfactory rigid-body control is maintained in the presence of such limit-cycles, the aircraft could be considered safe to fly for development work.

The proposed alternative clearance procedure therefore utilises the nonlinear nature of the control system in reducing the attenuation requirements of the structuralmode filters whilst ensuring satisfactory rigid-body control. The demonstration of the alternative clearance procedure on the test rig has validated its use, and shown considerable advantages over the current clearance requirement.

\subsection{Recommendations}

The main recommendation that has arisen from this programme of research is that a nonlinear assessment of the aeroservoelastic system will allow a reduction in the clearance requirements. Such a reduction will result in a significantly lower phase-lag being introduced by the structural-mode filters. As a consequence of this, the performance of the flight control system may be improved. Overall, the recommendations for an alternative clearance procedure can be summarized as follows

- Maintain the current flexible aircraft modelling process, in particular, the assumptions regarding in-phase signal path addition, and the adoption of the worst-case flight condition/stores configuration.

- Take account of the digital nature of the control system from the initial design stage. Preserve the current assumptions regarding the inclusion of sample-and-hold and sensor attenuation within the derivation of the maximum open-loop gain.

- Include any aliasing of the response in the analysis, along with the correct interpretation of the positioning of any structural-mode filters within the flight control system. Neglect any structural-modes whose frequency is higher than the sampling frequency.

- Design structural-mode filters so as to ensure a maximum open-loop gain of

$-1 \mathrm{~dB}$, minimizing the induced phase lag by the use of suitable optimization.

- Deduce confidence levels to the individual modes of the flexible aircraft in terms of an incremental gain.

- Obtain actuator performance boundaries for the individual actuation system from simulation and bench tests.

- Assuming the flexible aircraft model to be in error by the derived confidence 
levels, predict the potential for limit-cycle oscillations in terms of frequency range and amplitude. In addition, predict the range of flight conditions over which the limit-cycle oscillations will occur.

- Assess the impact of such limit-cycles on rigid-body stability margins through a combination of simulation, bench tests of the actuator and aircraft ground tests.

- Consider the impact of the potential limit-cycles on the generation of subharmonic aircraft response. In particular, careful consideration should be given to the effect of low-frequency structural-modes, which have been shown to have the greatest potential for sub-harmonic generation. In the case of a digital flight control system, structural-modes which combine with their highfrequency aliases to produce low-frequency subharmonic response should also be considered.

- Augment the attenuation of the structural-mode filters if necessary so as to ensure satisfactory rigid-body control even in the presence of the derived modelling errors.

- Proceed to ground test of the aircraft, initially ensuring that the open-loop gain of the system with the filters in place is below the $-1 \mathrm{~B}$ level. Check that the closure of the flight controller loops does not result in a limit-cycle oscillation even during taxiing tests of the aircraft.

- Proceed to flight-test, examining the areas of the flight envelope felt to be critical in terms of the potential for limit-cycle oscillations.

\subsection{Future Work}

The above recommendations are based on the results of this programme of research and as such are restricted by the scope of these results. In order to confirm these findings, and lend further support to the adoption of the proposed alternative clearance procedure, it is suggested that further work be completed. In particular, the progression of the results of Chapter 9 on to a more representative test rig would enable evaluation of the procedure on a more realistic structure. Ideally, such a test bed would make use an aircraft that was no longer cleared for flight, enabling testing of the structure without the fear of exceeding the fatigue loads. Such a test facility would enable confirmation of the prediction of the limit-cycle amplitudes, and the subsequent effect on actuator performance.

In addition to such a test facility, other areas of future work exist. These areas concentrate in particular on areas of this research programme where it is felt that a 
more thorough analysis would be beneficial. These areas are as follows:

- Dual and multiple input testing of the rate limiter. Since it is apparent that it is this function that dominates both the actuator performance boundary and the effect of limit-cycles an actuator performance, a more thorough analysis of its function seems sensible. This could examine both experimentally and analytically, the effect of differing amplitude and frequency of both pilot demand and limit-cycle signals. In addition, the effect of multiple input signals on the rate limiter could be addressed.

- A more complete analysis of the generation of subharmonic response as a result of the presence of two or more input signals to the actuator. This would enable better prediction of limit-cycles frequencies and amplitudes likely to cause such a response. In addition, subharmonic generation within a digital system should be examined in more detail.

- Consideration of other system nonlinearities. The analysis in Chapter 8 was based on the assumption that the dominant nonlinearity of the system was the software rate limiter. Although this has been shown to be the case from the analysis of Chapter 7, it would be of use to investigate the effect of further system nonlinearities on the prediction of limit-cycles.

- The effect of limit-cycles on other elements of the aircraft system should be considered as represented in Figure 10.1. In particular, the effect of a limitcycle on the fatigue life of the structure, the allowable life of the actuator and the hydraulic supply to the actuator itself. In addition, the effect of a limitcycle on the handling qualities of the aircraft should be considered.

- Assessment of phase uncertainty. It has been discussed that the potential for limit-cycle may be reduced if a certain level of confidence in the phase modelling can be gained. For example, if the phase response of the system can be assured as being within a particular quadrant of the Nyquist diagram for a certain structural-mode, then it may be shown that a limit-cycle is not possible even if the open-loop gain is greater than $0 \mathrm{~dB}$ at that particular frequency. Such an assessment may form part of a wider consideration of the assignment of confidence factors to a particular structural-mode.

- The implications of an increased open-loop gain at structural frequencies in terms of the closed-loop damping of the structure should be addressed. In particular, the likelihood of coupling between the flight control system and the flutter response of the aircraft, and the response of the structure in the presence of turbulence should be considered.

- The effect of aerodynamic loading of the actuator on the system response 
should be analysed. This research has assumed that the actuator is unloaded, but in the real case, it will be subjected to significant loading due to the aerodynamic forces on, and the inertia of, the control surfaces themselves. Consideration must be given as to how this would effect the performance boundary of the actuator, and the performance changes in the presence of a limit-cycle condition.

- Finally, this research has demonstrated the role of the software rate limiter in the prediction and prevention of limit-cycle oscillations. Alternative forms of software limiting such as acceleration limits and amplitude dependent rate limits could prove useful in the prevention of unwanted aeroservoelastic interactions.

Clearly, there exist several areas in which further investigation is required in order for the alternative design and clearance procedure to be adopted. Many of these areas would be examined in a specific aircraft design case however. Importantly, this research has examined the true nature of the problem of aeroservoelasticity. Through better understanding of the interaction, it may be possible to improve the performance . of the flight control system whilst ensuring aircraft safety. 


\section{References}

1 - Bisplinghoff, R. L., Ashley, H., "Principles of Aeroelasticity", John Wiley and Sons, 1962

2 - Scott, R. C., Weisshaar, T. A., "Controlling Panel Flutter Using Adaptive Materials", AIAA-91-1067, AIAA/ASME/ASCE/AHS/ASC 32nd Structures, Structural Dynamics and Materials Conference, Baltimore, April 8-10, 1991.

3 - Freymann, R., "Dynamic Interactions between active control systems and a flexible aircraft", AIAA-86-0960, AIAA/ASME/ASCE/AHS/ASC 27th Structures, Structural Dynamics and Materials Conference, San Antonio, May 1986.

4 - Zimmermann, H. "Aeroservoelasticity", $2^{\text {nd }}$ World Congress on Computational Mechanics, Stuttgart, August 27-31 1990, pp 719-735.

5 - Felt, L. R., Huttsell, L. J., Noll, T. E. and Cooley, D. E., “Aeroservoelastic Encounters", Journal of Aircraft, Volume 16, No. 7, July 1979. pp 477-483.

6 - Noll, T. E., "Aeroservoelasticity", AIAA-90-1073, AIAA/ASME/ASCE/AHS/ASC 31st Structures, Structural Dynamics and Materials Conference, Long Beach, April 241990.

7 - Arthurs, T. D., Gallagher, J. T., "Interaction between Control Augmentation System and Airframe Dynamics on the YF-17", AIAA-75-824, AIAA/ASME/ASCE/AHS/ ASC 16th Structures, Structural Dynamics and Materials Conference, Denver, May 27291975.

8 - Peloubet, R. P., Jr. "YF-16 Active-Control-System/Structural Dynamics Interaction Instability", AIAA-75-823, AIAA/ASME/SAE 16th Structures, Structural Dynamics, and Materials Conference, Denver, May 1975.

9 - Anonymous, "Fifth B-2 Joins USAF Test Fleet", Article, Aviation week \& Space Technology, 12 October 1992.

10 - Norris, G., "AMRAAM block placed on Lockheed F-16s", Article, Flight International, 20-26 October 1993.

11 - Pratt, R. W., Taylor, R., Caldwell, B. D., “Aeroservoelasticity: Key issues affecting the design of flight control systems", IEE Control'94, Warwick, March 1994.

12 - Taylor, R., Pratt, R. W., Caldwell, B. D., "An overview of the effects of aeroservoelasticity on the design of flight control systems", IEE Colloquium on multivariable methods for flight control applications, Digest No. 1994/132, London, May 1994. 
13 - McLean, D., "Gust load alleviation control systems: A feasibility study", Loughborough University of Technology, Department of Transport Téchnology Report, TT7076, 1976.

14 - Taylor, R., Pratt, R. W., Caldwell, B. D., "The effect of sampled signals on the flight control system of an agile combat aircraft with a flexible structure", IEEE/AIAA/ ASME/AIChE/ISA/SCS American Control Conference, Proc. Volume 1, pp505-509, Seattle, June 1995.

15 - Kehoe, M. W., Laurie, E. J. and Bjarke, L. J., "An In-Flight Interaction of the X29A Canard and Flight Control System", AIAA-90-1240, AIAA Dynamics Specialists Conference, Long Beach, April 5-6 1990.

16 - Caldwell, B. D., "Flight Control/ Structural Coupling - BAE Warton Experience in Aeroservoelasticity", L07 C428-059, IMechE Aerotech'92, Birmingham, January 14-17 1992.

17- Irving, J., "Description of the Notch Filter Design and Clearance Procedure Within the FCS Design Group", British Aerospace p.l.c. Company Report, BAE-WAE-RPGEN-FCS-000784, May 1992. [Unclassified]

18 - Rosenberg, K., "The Implementation of Structural Functions in Digital Flight Control Systems", IMechE Interactions between Aircraft Structural Dynamics \& Control Systems, March 1995

19 - Sallee, V. J., "ADAM 2.0 - An ASE analysis code for aircraft with digital flight control systems", AIAA-90-1077, AIAA Dynamics and Control of Large Structures Conference, May 8-10 1989.

20 - Cheng, P. Y., Hirner, T. J., "Automated procedures for aircraft aeroservoelastic compensation", AIAA-92-4606, AIAA Guidance, Navigation and Control Conference, August 10-12 1992.

21 - Cheng, P. Y., Hirner, T. J., "Aircraft Aeroservoelastic Compensation using Constrained Optimization", AIAA-92-2399, AIAA/ASME/ASCE/AHS/ASC 33rd Structures, Structural Dynamics and Materials Conference, Dallas, April 13-15 1992.

22- Schmidt, D. K., Newmann, B., "Multivariable flight control synthesis and literal robustness analysis for an aeroelastic vehicle", AIAA-90-3446, AIAA Guidance, Navigation and Control Conference, Portland, 1990.

23 - Colgren, R., " $\mathrm{H}_{\infty}$ design of future flight control systems", Proceedings of the 5th IEEE International Symposium on Intelligent Control, pp805-810, Philadelphia, 1990.

24 - Adams, W. M., Tiffany, S. H., "Development of a flutter suppression control law 
by use of linear quadratic gaussian and constrained optimization design techniques", 2nd International Symposium on Aeroelasticity and Structural Dynamics, Collected Papers, pp297-308, Aachen, April 1985.

25 - Breslin, S. G., Grimble, M. J., "The control of an ASTOVL (advance short takeoff and vertical landing) aircraft using $\mathrm{H}$ methods", IEE Colloquium on Multivariable methods for flight control applications, Digest No. 1994/132, London, 25 May 1994.

26 - Watts, A., "EAP Primary Actuator Performance Tests for In-Flight Flutter/Structural Coupling Mode Excitation System Feasibility Study", British Aerospace p.l.c. Company Report, BAE-WFC̣-RP-EAP-002074. [Unclassified]

27 - Atherton, D. P., "Nonlinear Control Engineering”, Van Nostrand Reinhold, 1975.

28 - Gibson, J. E., “Nonlinear Automatic Control”, McGraw-Hill, 1963.

29 - Taylor, R., Pratt, R.W., Caldwell, B. D., "The effect of actuator nonlinearities on aeroservoelasticity", AIAA-94-3628, AIAA Guidance Navigation and Control Conference, Scottsdale, August 1-3, 1994. Also to be published in AIAA Journal of Guidance, Control and Dynamics, January-February 1996.

30 - Taylor, R., Pratt, R. W., Caldwell, B. D., "Actuator performance changes in the presence of aeroservoelastic feedback signals", AIAA-95-3180, AIAA Guidance, Navigation and Control Conference, Baltimore, August 1995.

31 - Stirling, R, Cowling, D. A., "Implementation of Comprehensive actuation system models in aeroservoelastic analysis", Proc. of European Forum on Aeroelasticity and Structural Dynamics,1989, p567-576.

32 - Maharaj, D. Y., Rosenberg, G., Cowling, D. A., "The modelling of actuator bearing backlash in aeroservoelastic analysis and its effect on FCS/structural mode coupling", Proc. of European Forum on Aeroelasticity and Structural Dynamics, 1991.

33 - Brenner, M. J., "Actuator and aerodynamic modelling for high-angle-of-attack aeroservoelasticity", AIAA-93-1419, AIAA/ASME Structures, Structural Dynamics and Materials Conference, April 19-22 1993.

34 - Becker, J., "Aeroservoelastic Stability of Aircraft at High Incidence", AGARD Manoeuvring Aerodynamics Forum, November 1991.

35 - Voracek, D. F., Clarke, R., "Buffet induced structural/flight-control system interaction of the X-29A aircraft", AIAA-91-1053, AIAA/ASME/ASCE/AHS/ASC 32nd Structures, Structural Dynamics and Materials Conference, Baltimore, April 1991.

36 - Inman, D. J., "Control/structure interaction: effects of actuator dynamics”, AIAA- 
90-1223, AIAA Dynamics Specialists Conference, Long Beach, April 1990

37 - Youssef, H. M., Radovcich, N. A., "Aeroservoelastic modelling methodology for active control simulation", National Aerospace and Electronics Conference, Proceedings, Volume 1, pp487-494, Dayton, May 1984.

38 - Tiffany, S. H., Karpel, M., "Aeroservoelastic modelling and applications using minimum-state approximations of unsteady aerodynamics", AIAA-89-1188, AIAA/ ASME Structures, Structural Dynamics and Materials Conference, April 3-5 1989

39 - Felt, L. R., Kehoe, M. W., "Flight flutter testing at Ames-Dyden", 16th Society of Flight Test Engineers Annual Symposium, Proceedings, pp6.6-1 - 6.6-36, Seattle, July 1985.

40 - Patton, R. J., Taylor, P., Young, P., "Frequency domain techniques applied to the identification of helicopter dynamics", IMechE Control'88, Oxford, April 1988.

41 - Karpel, M., "Multi-disciplinary Optimization of Aeroservoelastic Systems Using Reduced-Size Models", AIAA Journal of Aircraft, Volume 29, No. 5, Sept.-Oct. 1992, pp939-946.

42- Karpel, M., "Reduced-order aeroelastic modelling via dynamic residualization", AIAA Journal of Aircraft, Volume 27, No. 5, May 1990, pp449-455.

43 - Silva, W. A., "A Methodology for using Nonlinear Aerodynamics in Aeroservoelastic Analysis and Design", AIAA-91-1110, AIAA/ASME/ASCE/AHS/ASC 32nd Structures, Structural Dynamics and Materials Conference, Baltimore, April 8-10, 1991.

44 - Nesline, F. W., Nesline, M. L., "Phase vs. gain stabilisation of structural feedback oscillations in homing missile autopilots",1985 American Control Conference, Proceedings Vol. 1, p 323-329.

45 - Cheng, P. Y., Chan, S. Y., Myers, T. T., Klyde, D. H., McRuer, D. T., "Aeroservoelastic stabilisation techniques for hypersonic flight vehicles", AIAA-91-5056, AIAA 3rd International Aerospace Planes Conference, Orlando, December 1991.

46 - Cheng, P. Y., Chan, S. Y., Myers, T. T., Klyde, D. H., McRuer, D. T., "Aeroservoelastic stabilisation technique refinement for hypersonic flight vehicles", AIAA-925014, AIAA4th International Aerospace Planes Conference, Orlando, December 1992.

47 - "Flight Control Systems - Design, Installation and Test of Piloted Aircraft, General Specification for" - MIL-F-9490D.

48 - Noll, T. E., Blair, M., Cerra, J., “ADAM, An aeroservoelastic analysis method for 
analog or digital systems", AIAA Journal of Aircraft, Volume 23, No. 11, November 1986.

49 - Pitt, D. M., Goodman, C. E., "FAMUSS: A new aeroservoelastic modelling tool", AIAA-92-2395, AIAA 33rd Structures, Structural Dynamics and Materials Conference, Dallas, April 1992.

50 - Adams, W. M., Tiffany, S. H., "ISAC: A tool for aeroservoelastic modelling and analysis", AIAA-93-1421, 34th Structures, Structural Dynamics and Materials Conference, La Jolla, April 1993

51 - Meirovitch, L., "Dynamics and control of structures", John Wiley and Sons, Inc., 1990

52 - Katz, J., Plotkin, A., "Low-speed Aerodynamics - From Wing Theory to Panel Methods", McGraw-Hill, Inc., 1991

53 - Dowell, E. H., Curtiss, H. C., Scanlan, R. H., Sisto, F., "A Modern Course in Aeroelasticity", Sijthoff \& Noordhoff, 1978.

54 - Gupta, K. K., Brenner, M. J., Voelker, L. S., "Integrated Aeroservoelastic Analysis Capability with X-29A Comparisons", AIAA Journal of Aircraft, Volume 26, No. 1, January 1989, pp84-90.

55 - Broadbent, E. G., "The Elementary Theory of Aeroelasticity", Bunhill Publications Ltd., 1954

56 - Blakelock, J. H., “Automatic Control of Aircraft and Missiles”, John Wiley and Sons, Inc., 1991

57 - McRuer, D., Ashkenas, I., Graham, D., “Aircraft Dynamics and Automatic Control", Princeton University Press, 1973.

58 - Beck, J., "Jaguar Fly By Wire Linear Taileron Actuation System Model for Frequency Response and Impedance", British Aerospace p.l.c. Company Report, FCSDGR 245, November 1985. [Unclassified]

59 - Little, J. N., Shure, L., "Signal Processing Toolbox for use with MATLAB - User's Guide", The Mathworks, Inc., August 1988.

60 - Leigh, J. R., “Applied Digital Control”, Second Edition, Prentice Hall, 1992.

61 - Lynn, P. A., "An Introduction to the Analysis and Processing of Signals", Third Edition, Macmillan Education, 1989.

62 - Franklin, G. F., Powell, J. D. and Workman, M. L., "Digital Control of Dynamic 
Systems", Second Edition, Addison-Wesley 1990

63 - Widger, H., "Transfer Function Representation of the Interface Between Rate Gyros and Flight Control Computer", British Aerospace p.l.c. Company Report, BAEWAE-RP-GEN-FCS-640, 1989. [Unclassified]

64 - Merrit, H. E., "Hydraulic Control Systems", John Wiley and Sons, 1967.

65 - Guillon, M., “Hydraulic Servo Systems”, Butterworths, 1969.

66 - Stirling Dynamics Ltd., “Actuator Modelling Standard Reference Document”, British Aerospace p.l.c. Company Document, SDL-103-TR-1, January 1992. [Unclassified]

67 - Beck, J., "Jaguar Fly By Wire Taileron Actuation System Model Matched to Frequency Response and Impedance", British Aerospace p.l.c. Company Report, AE/485 FCSDGR 210. [Unclassified]

68 - Beck, J., "Jaguar FBW Taileron Actuation System Tests at Dowty Boulton Paul", British Aerospace p.l.c. Company Report, AE/486 FCSDGR 211. [Unclassified]

69 - Dowty Boulton Paul, "Jaguar (FBW) Actuators, Electronic Test Equipment (P.343-07-1031), Functions and Operation", Dowty Boulton Paul Company Report, T.S.D./1027/A, October 1978.

70 - Gelb, A., Vander Velde, W. E., "Multiple Input Describing Functions and NonLinear System Design", McGraw-Hill, 1968.

71 - Nagrath, I. J., Gopal, M., “Control Systems Engineering”, John Wiley and Sons, 1982.

72 - Taylor, R., Pratt, R. W., Caldwell, B. D., "The application of actuator performance limits to aeroservoelastic compensation", AIAA-95-1195, AIAA/ASME/ASCE/AHS/ ASC Structures, Structural Dynamics and Materials Conference, New Orleans, April 1995.

73 - Taylor, R., Pratt, R. W., Caldwell, B. D., “An alternative approach to aeroservoelastic design and clearance", CEAS Aeroelasticity and Structural Dynamics Forum, Manchester, June 1995. Also to be published in IEE Proceedings, Control Theory and Applications. 
Appendix A

Structure of the Flexible

Aircraft model 


\section{A.1 Introduction}

The following appendix summarises the structure of the flexible aircraft model and actuator used throughout this research. Access to the actual numerical data for the models is restricted by British Aerospace Defence Ltd., and so cannot be included here. Any organization wishing to obtain the model data should contact the Flight Control System Design Group, Aerodynamics Department (W310P), British Aerospace Defence Ltd., Warton, Preston PR4 1AX.

\section{A.2 Structure of the Flexible Aircraft Model}

The state-space representation of the aircraft model is given by

$$
\begin{aligned}
& \dot{x}=\left[\begin{array}{cc}
-A^{-1}\left(D+\sigma V_{T} B\right) & -A^{-1}\left(E+\sigma V_{T}^{2} C\right) \\
I & 0
\end{array}\right] x+\left[\begin{array}{c}
A^{-1} F \\
0
\end{array}\right] u
\end{aligned}
$$

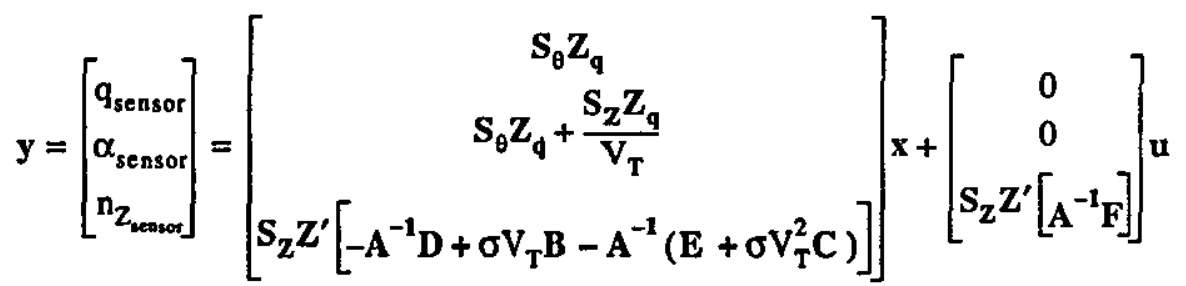

where,

$$
\begin{gathered}
\mathbf{x}=\left[\begin{array}{l}
\dot{q} \\
\mathbf{q}
\end{array}\right] \\
\mathbf{u}=\left[\begin{array}{l}
\delta_{i_{\text {dem }}} \\
\delta_{\text {ob }_{\text {dem }}} \\
\eta_{\text {dem }}
\end{array}\right] \\
\mathbf{z}_{\mathbf{q}}=\left[\begin{array}{ll}
\mathbf{Z}^{\prime} & 0
\end{array}\right] \\
\mathbf{z}_{\mathbf{q}}=\left[\begin{array}{ll}
\mathbf{0} & \mathbf{Z}^{\prime}
\end{array}\right]
\end{gathered}
$$

$S_{\theta}$ is a 40 by 1 vector such that $S_{\theta}(2 p)=1$ and all other elements are zero.

$S_{z}$ is a 40 by 1 vector such that $S_{z}(2 p-1)=1$ and all other elements are zero.

$\mathrm{p}$ is the sensor location on the aircraft centre-line according to Figure 3.10.

$\mathbf{Z}^{\prime}$ is the reduced transformation matrix as supplied by British Aerospace.

This reduced transformation matrix enables the displacements at twenty points along the aircraft centre-line, $d_{f}$, to be deduced from the generalised coordinate vector, 
q, so that

$$
\mathbf{d}_{F}=\left[\begin{array}{c}
z_{1} \\
\theta_{1} \\
z_{1} \\
\theta_{2} \\
\cdots \\
\cdots \\
z_{20} \\
\theta_{20}
\end{array}\right]=Z^{\prime} \mathbf{q}
$$

The remaining matrices of the model can be defined as

$$
\begin{aligned}
\mathbf{A} & =\mathbf{Z}^{\mathbf{T}} \mathbf{M Z} \\
\mathbf{E} & =\mathbf{Z}^{\mathbf{T}} \mathbf{K Z} \\
\mathbf{C} & =\mathbf{Z}^{\mathbf{T}} \mathbf{K}_{\mathbf{A}} \mathbf{Z} \\
\mathbf{B} & =\mathbf{Z}^{\mathbf{T}} \mathbf{D}_{\mathbf{A}} \mathbf{Z} \\
\mathbf{F} & =\left[\begin{array}{ccc}
0 & 0 & 0 \\
0 & 0 & 0 \\
\cdots & \cdots & \cdots \\
\cdots & \cdots & \cdots \\
0 & 0 & 0 \\
\mathrm{k}_{\delta_{\mathrm{bb}}} & 0 & 0 \\
0 & \mathrm{k}_{\delta_{\mathrm{ob}}} & 0 \\
0 & 0 & \mathbf{k}_{\eta} \\
0 & 0 & 0 \\
0 & 0 & 0
\end{array}\right]
\end{aligned}
$$

and the damping matrix, $\mathbf{D}$, is a diagonal matrix where,

$$
d_{i j}=2 \zeta \omega_{i}
$$

and

$$
\begin{aligned}
& \omega_{i}=\sqrt{\frac{e_{11}}{a_{11}}} \\
& \zeta=0.01
\end{aligned}
$$

The $\mathbf{A}, \mathbf{B}, \mathbf{C}, \mathbf{D}, \mathbf{E}$ and $\mathbf{Z}$ matrices were supplied by British Aerospace and corrected as part of this research as discussed in Chapter 3.

The structural inertia matrix, $\mathbf{A}$, is generalised according to equation (A.8), where the form of the real inertia matrix is given by 


$$
M=\left[\begin{array}{ccc}
M_{S} & 0 & 0 \\
0 & M_{C} & 0 \\
0 & 0 & M_{R}
\end{array}\right]
$$

where,

$\mathbf{M}_{\mathbf{S}}$ is the inertia matrix for the structure

$\mathbf{M}_{\mathbf{C}}$ is the inertia matrix for the control surfaces

$\mathbf{M}_{\mathbf{R}}$ is the inertia matrix for the rigid body dynamics

Similarly, the matrices $\mathbf{B}, \mathbf{C}$, and $\mathbf{E}$ are generalized versions of the actual aerodynamic damping, aerodynamic stiffness and structural stiffness matrices respectively.

Further details of the derivation of the flexible aircraft model are contained in Chapter 3.

\section{A.3 Structure of the actuation system model}

The structure of the actuation system model used throughout this research is shown in Figure A.1. and A.2. The calculation of hydraulic fluid flow through the main-valve is completed in the block titled "Mainvalve" in Figure A.2 in accordance with equations (7.1) to (7.8). In addition, the scheduling of the flow coefficient, $\mathrm{C}_{\mathrm{F}}$, is made with port opening as can be seen in Figure A.2. Finally, the servo-valve displacement is augmented at small values of servo-valve drive current in the block titled "sv pressure gain" in Figure A.1.

Further details of the derivation of the actuation system model are contained in Chapter 7. 


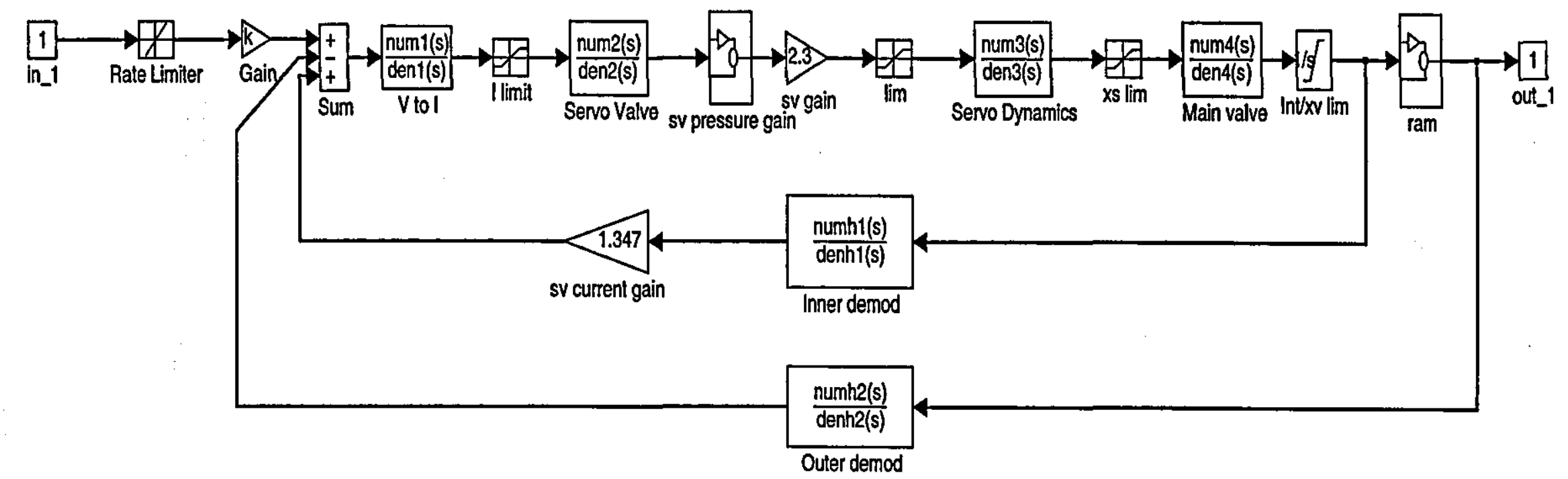

Figure A.1 - Jaguar FBW taileron actuator system model - Overview 


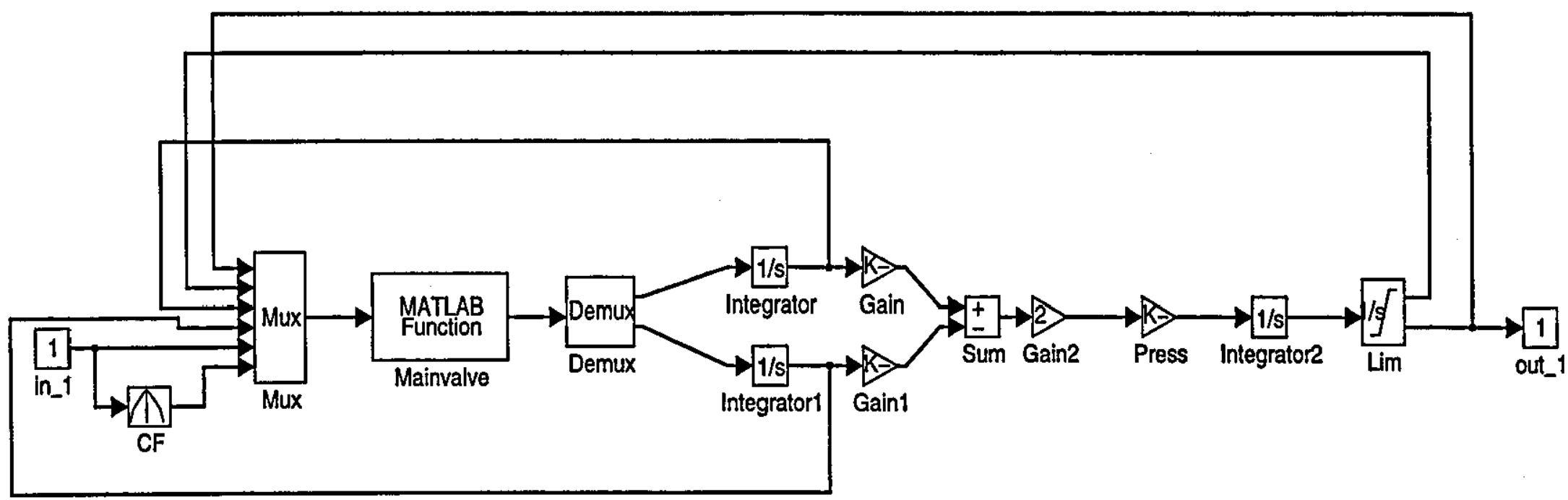

Figure A.2 - Jaguar FBW Taileron actuator system model - Main-ram dynamics 
